\title{
A NOVEL ROBOTIC FRAMEWORK FOR SAFE INSPECTION AND TELEMANIPULATION IN HAZARDOUS AND UNSTRUCTURED ENVIRONMENTS
}

DOCTOR OF PHILOSOPHY

AUTOMATION AND ROBOTICS

MARIO DI CASTRO

September 27, 2019 

CERN - EUROPEAN ORGANIZATION

FOR NUCLEAR RESEARCH

\title{
A NOVEL ROBOTIC FRAMEWORK FOR SAFE INSPECTION AND TELEMANIPULATION IN HAZARDOUS AND UNSTRUCTURED ENVIRONMENTS
}

\author{
DOCTOR OF PHILOSOPHY \\ AUTOMATION AND ROBOTICS
}

\begin{abstract}
Author: $\quad$ Mario Di Castro
Electronic Engineer

Advisors: Dr. Manuel Ferre Pérez

Universidad Politécnica de Madrid

Dr. Alessandro Masi

CERN - European Organization for

Nuclear Research
\end{abstract}

September 27, 2019 



\title{
A NOVEL ROBOTIC FRAMEWORK FOR SAFE INSPECTION AND TELEMANIPULATION IN HAZARDOUS AND UNSTRUCTURED ENVIRONMENTS
}

\author{
Autor: Mario Di Castro
}

\section{Tribunal}

Presidente:

Dr. Claudio Rossi

Secretario:

Dr. Paloma de la Puente

Vocal A:

Dr. Cristina Adorisio

Vocal B:

Dr. Antonio Giménez Fernández

Vocal C:

Dr. Raúl Marín Prades

Suplente A:

Dr. Marco Pezzetti

Suplente B:

Dr. Concepción Alicia Monje Micharet

Acuerdan otorgar la calificatión de:

Madrid,

de, 2019 



\section{Acknowledgments}

First of all, I would like to express my deep gratitude to my research supervisor, Professor Manuel Ferre Pérez, for his patient guidance, enthusiastic encouragement and useful critiques of this thesis. An immense thankfulness goes to my CERN supervisor, Doctor Alessandro Masi, who has believed in my potential and who has always supported me during the last years, being my mentor and my inspiration. Thanks to the unconditional support of Roberto Losito for the direction of the Engineering Department at CERN, that provides extraordinary opportunities and individual development to its scientists. I would like to express my great appreciation and deep respect to Professor Raul Marin for the support for writing of the thesis, help and profound discussions on teleoperation and robotic controls.

Also, I would like to offer my special thanks to my colleagues of the Mechatronics, Robotics and Operation Section within the Survey, Mechatronics and Measurement Group at CERN for their inestimable help in the building-up of this work. In the specific, Giacomo Lunghi, Luca Buonocore, Carlos Veiga Almagro, Leanne Attard, Jorge Camarero Vera, Zsolt Pasztori, Santiago Solis Paiva, Arturs Ivanovs and Maria Laura Baiguera Tambutti. My grateful thanks are directed to all collegues at CERN, and in the specific the Radioprotection group, that inspired me with problems and discussions.

My warmest thanks go to my children Giada, Lorenzo and Federico, for bringing joy and source of inspiration to reach what looks impossible. The deepest thanks goes to my wife Valeria, that has supported me during these years with trust and love, to her I dedicate this thesis. 


\section{Abstract}

Intelligent robotic systems are becoming essential for space applications, industries, $\mathrm{nu}-$ clear plants and for harsh environments in general, such as the European Organization for Nuclear Research (CERN) particles accelerator complex and experiments. Robotics technology has huge potential benefits for people and its ultimate scope depends on the way this technology is used. In order to increase safety and machine availability, robots can perform repetitive, unplanned and dangerous tasks, which humans either prefer to avoid or are unable to carry out due to hazards, size constraints, or the extreme environments in which they take place. Nowadays, mechatronic systems use mature technologies that allow their robust and safe use, even in collaboration with human workers. Over the past years, the progress of robots has been based on the development of smart sensors, artificial intelligence and modular mechanical systems. Due to the multiple challenges that hazardous and unstructured environments have for the application of autonomous industrial systems, there is still a high demand for intelligent and teleoperation systems that give the control of a robot (slave) to a human operator via haptic input devices (master), as well as using human-supervised telerobotic control techniques. Modern techniques like simulation and virtual reality systems can facilitate the preparation of ad-hoc mechatronic tools and robotic intervention including recovery scenarios and failure mode analysis.

The basic contribution of this thesis is the development of a novel robotic framework for autonomous inspections and supervised teleoperations in harsh environments. The proposed framework covers all aspects of a robotic intervention, from the specification and operator training, the choice of the robot and its material in accordance with possible radiological contamination risks, to the realization of the intervention, including procedures and recovery scenarios. In a second set of contributions, new methods for mutirobots maintenance operations are developed, including intervention preparation and best practices for remote handling and advanced tools. The third set of contributions is built on a novel multimodal user-friendly human-robot interface that allows operator training using virtual reality systems and technicians not expert in robot operation to perform inspection/maintenance tasks. In this thesis, we exploit a robotic system able to navigate autonomously and to inspect unknown environments in a safe way. A new real-time control system has been implemented in order to guarantee a fast response to environmental changes and adaptation to different type of scenarios the robot may find in a semi-structured and hazardous environment. The proposed new robotic control system has been integrated on different robots, tested and validated with several robotic interventions in the CERN hazardous particle accelerator complex. 


\section{Resumen}

Los sistemas robóticos inteligentes se están convirtiendo en algo esencial para las aplicaciones espaciales, industriales, las centrales nucleares y los entornos hostiles en general, tales como las complejas instalaciones del acelerador de partículas de la Organización Europea para la Investigación Nuclear (CERN) y sus experimentos. La tecnología robótica tiene un enorme potencial para el beneficio de las personas y su ámbito de aplicación final depende de la forma en que esta es utilizada. Con el fin de incrementar la seguridad y disponibilidad de las maquinas, los robots pueden llevar a cabo tareas repetitivas, inesperadas y peligrosas, las cuales prefieren ser evitadas por los humanos o bien, son incapaces de realizarlas debido a tales peligros, limitaciones de espacio, o los entornos extremos donde tienen lugar. En la actualidad, los sistemas de mecatrónica utilizan tecnologías consolidadas que les permite un uso robusto y seguro, incluso en colaboración con trabajadores humanos. En los últimos años, el avance de los robots se ha basado en el desarrollo de sensores inteligentes, inteligencia artificial y sistemas mecánicos modulares.

Debido a la multitud de desafíos que los entornos peligrosos y desestructurados generan a la hora de aplicar sistemas industriales autónomos, todavía existe una amplia demanda de sistemas inteligentes y tele-operados que entregan el control de un robot (esclavo) a un operador humano a través de dispositivos de entrada hápticos (maestro). Técnicas modernas como los sistemas de simulación y la realidad virtual pueden facilitar la preparación de herramientas mecatrónicas puntuales e intervenciones robóticas, incluyendo escenarios de recuperación y modo de análisis de fallos.

La contribución base de esta tesis es el desarrollo de un novedoso framework robótico para inspecciones autónomas y teleoperaciones supervisadas en entornos hostiles. El framework propuesto cubre los aspectos de una intervención robótica al completo, desde la especificación y adiestramiento del operador, la elección del robot y sus materiales acorde con los posibles riesgos de contaminación radiológica, hasta la realización de la intervención, incluyendo procedimientos y planes de recuperación. En un segundo conjunto de contribuciones, se desarrolla un método para las operaciones de mantenimiento mediante multirobots, incluyendo la preparación de las intervenciones y las mejores prácticas para la manipulación remota y herramientas avanzadas. El tercer grupo de contribuciones se basa en una nueva interfaz multimodal humano-robot fácil de usar, la cual permite entrenar operadores inexpertos en operaciones robóticas, haciendo uso de sistemas y técnicas de realidad virtual, para tareas de inspección y/o mantenimiento. En esta tesis, explotamos un sistema robótico capaz de navegar de forma autónoma, inspeccionando entornos desconocidos de una forma segura.

Un nuevo sistema de control en tiempo real ha sido implementado con el fin de garantizar 
una respuesta rápida a los cambios del entorno y la adaptación a los diferentes tipos de escenarios que el robot podría encontrar en un entorno hostil y semiestructurado. El nuevo sistema para el control de robots propuesto, ha sido integrado en diferentes robots, además de testeado y validado en un gran número de intervenciones robóticas dentro de las instalaciones del complejo acelerador de partículas del CERN. 


\section{Contents}

Acknowledgment

Abstract $\quad$ V

Resumen $\quad$ vi

1 Introduction $\quad 1$

1.1 Remote Interventions . . . . . . . . . . . . . . . . . . . . . . 3

1.2 Motivation . . . . . . . . . . . . . . . . . 5

1.3 Thesis Hypothesis and Contributions . . . . . . . . . . . . . . . 7

1.4 List of Publications . . . . . . . . . . . . . . . . . . . . . . 8

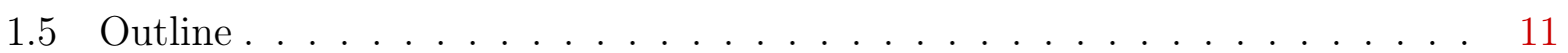

2 State of the Art on Robotic Systems 12

2.1 Artificial Intelligence . . . . . . . . . . . . . . . . . . . . . 17

2.2 Telemanipulation . . . . . . . . . . . . . . . . . . . . 19

2.3 Haptic Telepresence . . . . . . . . . . . . . . . . . . . . . . 19

2.4 Hands on Preparation for Robotic Intervention . . . . . . . . . . . . . . . . 20

2.5 Training . . . . . . . . . . . . . . . . . . 25

2.6 Ethical Aspects in Robotics . . . . . . . . . . . . . 26

3 The CERNTAURO Framework for Remote Handling 29

3.1 Control System Architecture . . . . . . . . . . . . . . . . . . . 29

3.2 Methods for Multirobots Maintenance Operations . . . . . . . . . . . 36

3.3 Communication . . . . . . . . . . . . . . . . . . 50

4 Key Functionalities for Advanced Remote Handling Performances 55

4.1 Environmenthal Perception and Measurements . . . . . . . . . . . . . 55

4.2 Autonomous Navigation for Workplace Approach . . . . . . . . . . . . . . . 76

4.3 Cooperative Multi-Agents Scenarios . . . . . . . . . . . . . . . . . . 89

5 Operators Behaviour and Performance Metrics 94

5.1 Human-Robot Interface . . . . . . . . . . . . . . . . . . . . . . . . 94

5.2 Teleoperation Factors . . . . . . . . . . . . . . . . . . . . . . . . 99

5.3 VERO: Virtual Simulation Environments for Operator Training . . . . . . 116

5.4 Comparative Analysis and System Usability Tests . . . . . . . . . . . . 131 
6 Results and System validation 141

6.1 Autonomous Navigation and Inspections in CERN Accelerators Area . . . . 141

6.2 Tracking-based Depth Estimation System . . . . . . . . . . . . . . . . . . . 141

6.3 Structural Monitoring of Tunnel Areas . . . . . . . . . . . . . . . . . . . 145

6.4 Human Detection and Remote Respiration Monitoring . . . . . . . . . . . . 149

6.5 Learning by Demonstration Results . . . . . . . . . . . . . . . . . . . . 156

6.6 Metallic Objects Detection and 6D Pose Estimation . . . . . . . . . . . . . 159

6.7 Radiation Dose Measurements with i-TIM . . . . . . . . . . . . . . . 162

6.8 Challenging Teleoperated Tasks in Harsh Environments . . . . . . . . . . . 162

7 Conclusions and Future Lines of Research 168

$\begin{array}{ll}\text { A Novel Robots Developed } & 173\end{array}$

$\begin{array}{ll}\text { B Experimental Setups } & 190\end{array}$

$\begin{array}{lr}\text { C Master Handler } & 194\end{array}$

$\begin{array}{ll}\text { D Advanced Tools } & 201\end{array}$

E CERN Remote Handling Code of Practice 205

$\begin{array}{lll}\text { F } & \text { TInspect Modules Description } & 231\end{array}$

$\begin{array}{ll}\text { G Software Code } & 257\end{array}$

$\begin{array}{ll}\text { List of Figures } & 260\end{array}$

$\begin{array}{ll}\text { List of Tables } & 272\end{array}$

$\begin{array}{ll}\text { Bibliography } & 274\end{array}$ 


\section{Chapter 1}

\section{Introduction}

The revolution of Industry 4.0 involves a modern manufacturing style, known as Intelligent Manufacturing [1], which focuses on customized production and massive customization instead of the classic massive production. A key component of Industry 4.0 is the global industrial movement named Internet of Things (IoT) [2, 3, 4], which changed the way a wide range of industries approach the procurement, processing, and distribution of raw materials and finished products.

The production in capital-intensive industries has created needs for robustness, dexterity, and cost efficiency, especially in the field of automation and mechatronics. Robotics technology has huge potential benefits for people and its ultimate scope depends on the way this technology is used. The goal of the presented work is to apply robotics technologies to improve the quality and safety of human workers in dangerous environments improving the availability of the machines. Nuclear plants like Fukushima [5], power plants like ITER [6], as well as particle accelerator facilities [7], such as the European Organization for Nuclear Research (CERN) [8], The European X-ray free-electron laser (XFEL) [9], the Deutsches Elektronen-Synchrotron (DESY) [10], the Spallation Neutron Source (SNS) [11], or FERMILAB [12], present harsh environments, several kilometers of underground and semi-structured accelerator areas with thousands of different items of equipment which need to be inspected and maintained. Due to the presence of human hazards mainly produced by radiation and high magnetic fields, the plants and accelerators need to be inspected and maintained remotely, possibly using robots. Power plants and semi-structured particle accelerator environments present different constraints like accessibility, long distances, objects with various pose and occlusion in cluttered areas.

In addition, the machine's equipment are delicate and expensive and in most cases, the equipment owners and/or machine experts need to operate the robots for the remote maintenance. This aspect requires a robotic system with a user-friendly human-machine interface, possibly equipped with haptic devices that allow the operator long operation without too much stress.

Industrial robots today are mainly assigned to repetitive and grunt work without much flexibility or even intelligence and they are not adapted to harsh and semi-structured environments. The gap between requirement and reality introduces industrial-level mobile 
manipulation robotic technology featured with task flexibility, robotic mobility and learning ability [13]. However, these solutions are environment specific and cannot be seamlessly adapted to different kinds of environment without a non-negligible cost in terms of hardware and software alterations. Operating robots for maintenance in dangerous environments on costly machines requires skilled and well trained, dedicated shift operators [14]. This is costly, highly time-consuming and is mainly due to the non-intuitive human robot interfaces present on industrial robot mobile manipulators. In specific remote tasks such as brazing or milling, expert technicians are unable to operate robots due to lack of teleoperation experience. Thus, a user-friendly human robot interface is required that increases the process transparency [15], reduces the operator's fatigue and does not require a pool of well-trained robotic operators which, in some cases, are dedicated to a specific robotic task.

Another crucial aspect to take into account for environments where a quick robotic intervention is needed to increase the machine uptime and reduce the operational cost, is the ability to mitigate incidents during robotic operations. For example, the robot must be able to recover an object lying on the ground or a tool, which may accidentally fall, an aspect that is not present in many of the industrial solutions. It also has to be able to be recovered by another robot or machine in case of failure. Taking into account these aspects, the robot must be lightweight and recoverable by another robot or turned off remotely in a safe way.

Moreover, in environments where radiological contamination is a risk, the materials of which the robots are composed of are fundamental to avoid radiological contamination and unwanted radiological waste with the risk that an expensive robot might become impossible to be used again. Regarding the design of robots for hazardous environments, some specific requirements are needed, such as waterproof mechanical components, security skeleton, wheels adapted to the environment, and lifting system, in order to enhance the efficiency of the mobile manipulator. This allows further use of a robot after an intervention, enabling the robot to be easily cleaned [16]. Expensive machines in hazardous and semi-structured environments in general present maintenance and dismantling challenges, which require a mobile manipulator system with vision, intelligence, and automation, which is not currently available as an industrial solution.

This work presents a novel robotic framework for autonomous inspection and supervised teleoperations in semi-structured and harsh environments. The framework covers all the aspects of a robotic intervention, from the low-level control, the requirements analysis and the intervention simulation to the on-the-field operation including procedures, tools and recovery scenarios. The framework has been developed following the continuous integration model and it has been used in real intervention while research has been performed and new features were constantly integrated into the main system core. The unique infrastructure and environments present in semi-structured and harsh areas, as well as the unique requirements, need the unique, modular and reconfigurable CERNTAURO robotic system. The proposed system is adaptable and modular from mechanics, electronics and software architecture point of view presenting also a user-friendly and multimodal human-robot interface. 


\section{$1.1 \quad$ Remote Interventions}

Remote interventions are often needed to decrease human exposure to hazards and intervention time and most of the time they require remote handling tasks. Remote Handling is an action of using a mechatronic device to execute a remote controlled or human-supervised tasks. In the last decades, mechatronics and, in the specific, robot developments, have been through several steps of evolution (see Table 1.1) [17]. Mobile manipulators are robotic systems consisting of one or more robot arms mounted on a mobile platform, which allows the system to perform tasks with locomotion and manipulation abilities. Remotely operated systems allow human beings to work effectively from a safe place. Applications of such robotic systems in hazardous and semi-structured environments cover a wide range of scenarios such as underwater, where remotely operated vehicles (ROVs) [18] and autonomous vehicles (AUVs) [19, 20, 21, 22] are mostly used by oil and gas industries for inspection and installation tasks [23, 24, 25]. Various other applications can be found in aerial fields [26, 27]. These include robotic solutions for observation [28], military operations [29, 30, 31, 32], civilian and private applications [33] and payload delivery in the space field, for open space manipulation and robotic exploration vehicles [34, 35, 36, 37, 38], in mining fields [39, 40], military fields [41, 42], such as landmine eradication or bomb detonation $[43,44]$, as well as in nuclear and radioactive fields [45, 46].

Table 1.1: Robotic Revolution for Past and Future Years

\begin{tabular}{||c|c|c||}
\hline Robotic revolution & Year & Type \\
\hline \hline 1.0 & 1970 & Industrial robots \\
\hline 2.0 & 1980 & Service robots \\
\hline 3.0 & 2000 & Personal robots \\
\hline 4.0 & 2010 & $\begin{array}{c}\text { Connected robots (IoT)/ } \\
\text { Industry 4.0 }\end{array}$ \\
\hline 5.0 & 2020 & Intelligent robots \\
\hline 6.0 & 2030 & $\begin{array}{c}\text { Singularity robots } \\
\text { Self-sufficient robots }\end{array}$ \\
\hline
\end{tabular}

Particularly for the radioactive field, the first remote handling was developed in the 1940s when the discoveries in atomic physics led to the possibility of exploring the nature of the materials associated with ionizing radiation in more detail. The first system developed was a mechanical-master-slave manipulator performing kinaesthetic and tactile feedback [47]. The mechanical coupling between the master and the slave device constituted a non-negligible problem considering that the two devices were separated by a distance of about 10 meters. This problem was largely overtaken during the late 1960s and the early 1970s, when the advent of new technologies in electronics and computing led to the physical separation of the master and the slave, developing the first electrical master-slave manipulator [48]. During 
this period, research and innovation in the field of the telemanipulation defined the control system hierarchies and structures in telerobotics [49], producing several industrial products for the following years. From the 1980s onward, the development of mobile manipulators has gone through several stages, concentrating on different key components in both hardware and software. During that period, many mobile manipulators have been developed, of which MORO [50], Rob@Work [51], Little Helper [52], PR2 [53], TUM Rosie [54], KUKA OmniRob and KMR iiwa are the most representative mobile manipulators ones (see Figure 1.1).

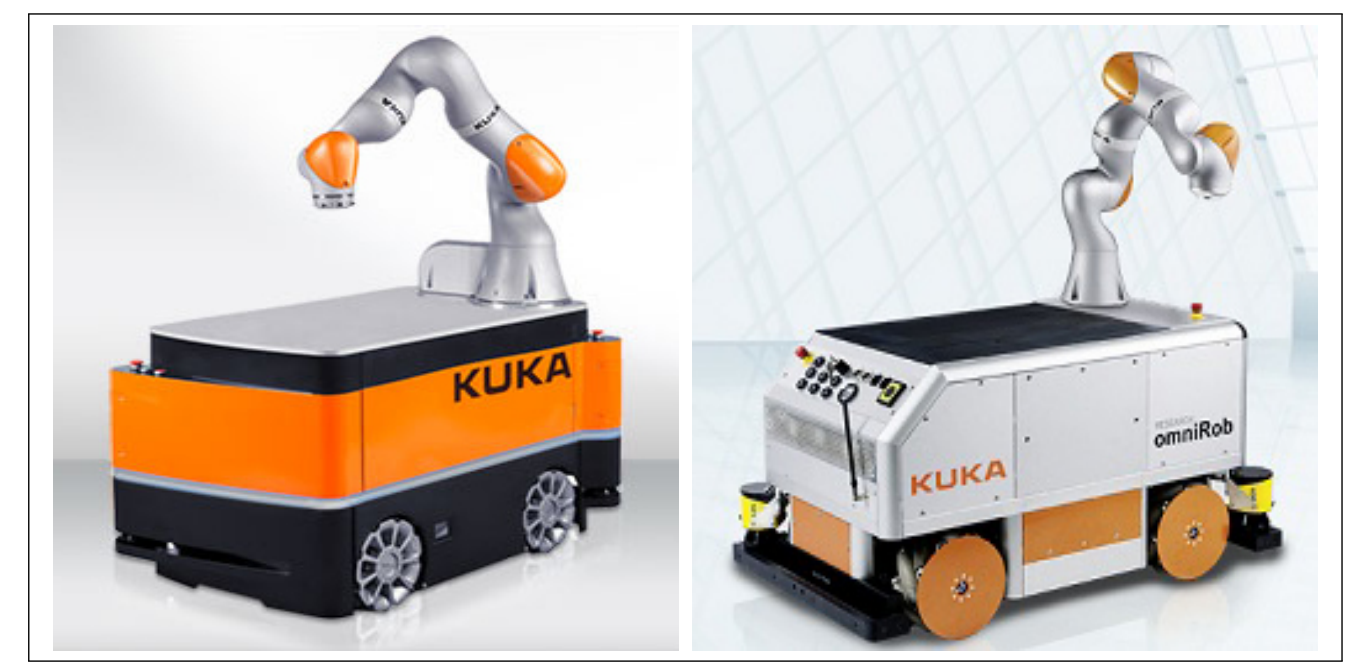

Figure 1.1: Kuka Omnirob mobile manipulator (adapted from [55])

Robust military vehicles were built in the last decades [56], satisfying the demands of demining and bomb disposal, but lacking the scalability and the possibility to integrate custom control and artificial intelligence which are both mandatory in intelligent robotic systems. Unlike the many types of industrial robots deployed in industrial environments [57], robots ready to be used in semi-structured and hazardous environments are not abundant and in general, they are built for specific needs. In recent years, significant progress has been made in advancing the state-of-the-art in the same way as mobile manipulators.

Technologies such as vision-based navigation [58], teleoperation [59, 60, 55] and collision free motion planning for telemanipulation are widely studied and applied on robotic platforms with satisfactory results. Several European projects include robust mobility and dexterous manipulation, such as FirstMM [61], TAPAS [62], and VALERI [63], for which the results reveal that research in mobile manipulation follows the logical integration of navigation, perception, teleoperation, manipulation and learning. 


\subsection{Motivation}

Although important progress was made in the field, there is still much work left in the application of mobile manipulation in harsh and semi-structured environments.

Industrial ready-to-use solutions do not exists and robots used and developed for research purposes are not enough safe and reliable to be applied in these environments or they are designed and built for specific tasks or places.

Particle accelerators and nuclear plants present many variable environments that would need many specific robots for each task or location demanding huge budget and manpower resources to adapt and maintain the robotic systems (see Figure 1.2).
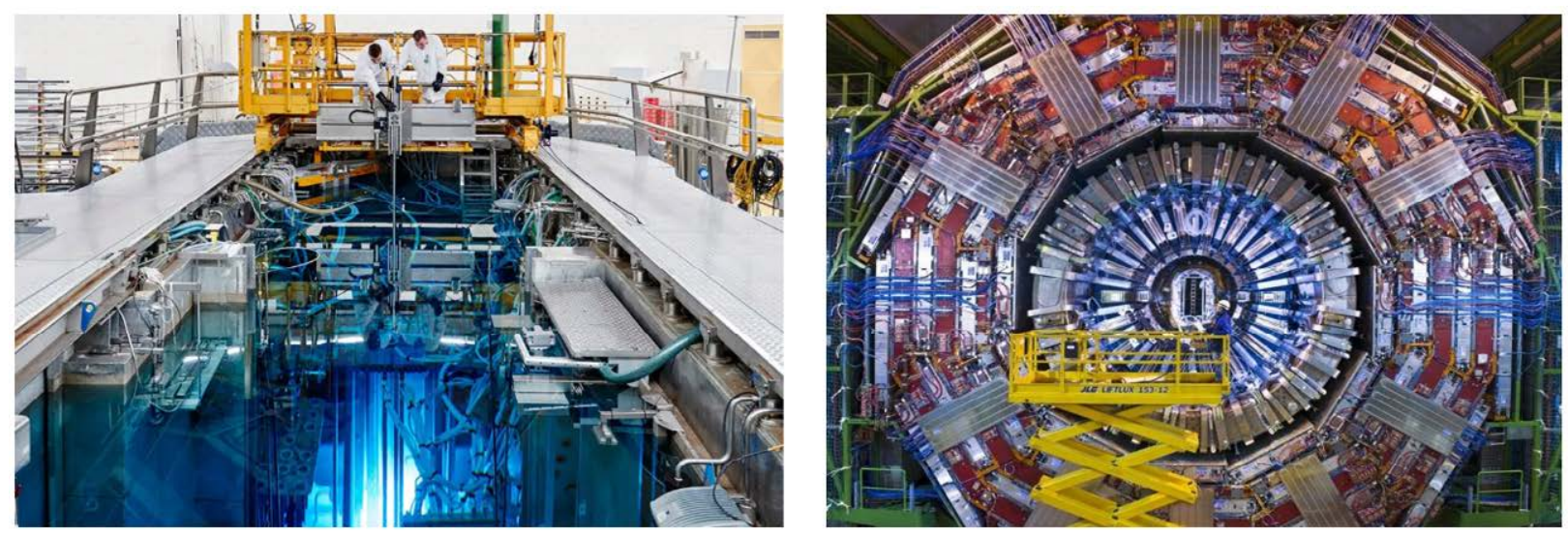

Figure 1.2: The Thorium nuclear reactor (left) [64] (adapted from www.newscientist.com) and the Compact Muon Solenoid experiment (right) [65]

The main idea behind this thesis work, on which the CERNTAURO framework is built, is to develop a modular and adaptable robotic systems from all the mechatronics aspects, having the possibility of making the human, the machine and the interface a unique system that works together. The system could be adaptable to different environments, as well as to different operator skills. In addition, extended control plants and experiment need structured documentation and code of practice to apply safe and reliable maintenance plants in hazardous places. The CERNATURO system integrates a code of practice for remote handling that has been designed and commissioned following the numerous real interventions performed at CERN, while the research part of this work was performed, profiting also of the continuous integration model adopted for this work. Robotic control systems that covers all the aspects of the robotic interventions, from its preparation until the realization and the robots operation including recovery scenarios, do not exist. Consequently, thanks to the various novelties and added values of the CERNTAURO system with respect to the state of the art, the presented framework in its integrity could be considered as benchmark for robotic control systems in harsh and semi-structured environments.

In the following, examples of robotic missions needed at CERN, and in general particle accelerators facilities as well as in nuclear and power plants, are listed: 
1. Autonomous inspection of semi-structured tunnels

2. Nondestructive testing inspections

3. Safe use of robots in confined spaces

4. In-situ maintenance and repair using teleoperation

5. Personnel escort and health monitoring

These five examples provide a reasonably diverse overview motivating the development of customized robotic controls and systems. Challenges include the need for software flexibility, modularity and safety. Furthermore, the complication of the process of teaching a robot and programming its features, demonstrate how much work is left as well as adapting the developed solution for different needs. TABLE 1.2 summarizes the most common harsh and semi-structured environment challenges and the main and unique CERNTAURO control features to tackle these challenges, including the possibility to control the robot via SMS using GSM-operated remote control systems.

Table 1.2: Semi-structured and Harsh Environments Challenges and the unique CERNTAURO control features to tackle them

\begin{tabular}{|c|c|}
\hline Challenge & Unique CERNTAURO control feature \\
\hline $\begin{array}{l}\text { Variable environment. } \\
\text { Unknown infrastructure }\end{array}$ & $\begin{array}{l}\text { Modular controls and robotic platforms. } \\
\text { System in use while research is performed }\end{array}$ \\
\hline $\begin{array}{l}\text { Maintenance needs to be performed } \\
\text { by equipment owners } \\
\text { (non expert robots operators) }\end{array}$ & $\begin{array}{c}\text { User friendly human-robot } \\
\text { interface and haptics feedback. } \\
\text { Virtual reality for intervention preparation }\end{array}$ \\
\hline $\begin{array}{l}\text { Limited intervention time } \\
\text { in very long distances }\end{array}$ & $\begin{array}{l}\text { Variable speeds according to the time needs. } \\
\text { Passivation of unused on-board sensors and devices }\end{array}$ \\
\hline Unexpected Obstacles & Autonomous navigation and obstacle avoidance \\
\hline $\begin{array}{l}\text { Environmental hazards } \\
\text { (e.g. radiation) }\end{array}$ & $\begin{array}{l}\text { Augmented reality navigation } \\
\text { using virtual obstacles }\end{array}$ \\
\hline $\begin{array}{l}\text { Precision localization during } \\
\text { environmental measurement }\end{array}$ & $\begin{array}{l}\text { Precise on board localization using modular sensor } \\
\text { fusion according to real time measurements } \\
\text { using radio maps and visual odometry }\end{array}$ \\
\hline Delicate equipment and safety & Anti-collision and fail-safe system, recovery scenarios \\
\hline $\begin{array}{l}\text { Poor communication signal, } \\
\text { time fluctuations due to } \\
\text { indoor and metallic environment }\end{array}$ & $\begin{array}{l}\text { Time delay passivation, control through SMS, } \\
\text { notifications/alarms via SMS. } \\
\text { Multimodal communication through } \\
\text { SMS, } 2 \mathrm{G}, 3 \mathrm{G}, 4 \mathrm{G} \text { and Wifi }\end{array}$ \\
\hline Supervision needed from several places & Web based multicast monitor module \\
\hline
\end{tabular}




\subsection{Thesis Hypothesis and Contributions}

Taking into account the motivation of the problem and the necessities of remote handling in hazardous facilities, the main hypothesis of the thesis can be stated as follows:

- Modularity: In order to have a reliable and safe remote handling capabilities in industrial plants, as well as in hazardous and unstructured environments, the framework that drives the remote handling devices must have a control system that is modular and adaptable to different hardware and environmental challenges, like space constraints and communication channels. The framework must be capable of integrating different functionalities, like for example vision techniques based on advanced cameras system in order to give a comfortable and efficient view of the workplace to the robot operators. In cases where metallic objects are predominant in a working place and/or there is the presence of smoke, thermal cameras or radar systems could be used to endorse the environmental perception helping the robot embedded control as well as the operator recognition of a workplace. Modularity could be obtained by adopting framework structures based on core-periphery models that guarantee flexibility and reusability of the different modules and building blocks.

- Autonomy: The robotic systems that should operate in large workspaces and/or for long periods of time, in order to increase the robustness and the safety of the robot navigation, must have an embedded artificial intelligence that can guarantee a satisfactory level of autonomy and environmental perception. Simultaneous navigation and mapping algorithms, endorsed with visual odometry techniques, could be a suitable solution to allow autonomous navigation of robotic systems in unstructured and space-confined areas.

- Human operator performance: Another important aspect to take into account for remote handling tasks, is the human operator performance metrix and the psychological stress for the preparation and the execution of the operation. To help the robotic operators to be more comfortable during the task executions, the human robot interface must be intuitive and user-friendly, trying to mitigate and balance the amount of useful information to pass to the operator without providing too much and useless data.

The main contributions of this thesis are related to the analysis, design and validation of framework for remote handling in irradiated facilities. This framework has been designed according to the current state of the art of technology and validated at CERN facilities. 


\subsection{List of Publications}

The contributions of the presented work have been shared with the research community by means of the following international journal papers and conferences.

- Di Castro, Mario, Manuel Ferre, and Alessandro Masi. "CERNTAURO: A Modular Architecture for Robotic Inspection and Telemanipulation in Harsh and SemiStructured Environments." IEEE Access 6 (2018): 37506-37522.

- Di Castro, Mario, Jorge Camarero Vera, Alessandro Masi, and Manuel Ferre. "Object Detection and 6D Pose Estimation for Precise Robotic Manipulation in Unstructured Environments." Volume 495 of the Lecture Notes in Electrical Engineering series, Springer, 2019, DOI 978-3-030-11292-9_20, (c) 2020.

- Di Castro, M., Almagro, C. V., Lunghi, G., Marin, R., Ferre, M., \& Masi, A. (2018, October). Tracking-Based Depth Estimation of Metallic Pieces for Robotic Guidance. In 2018 IEEE/RSJ International Conference on Intelligent Robots and Systems (IROS) (pp. 5503-5508). IEEE.

- Di Castro, Mario, Manuel Ferre, Raul Marin, Giacomo Lunghi, Alessandro Masi, "A Multidimensional RSSI Based Framework for Autonomous Relay Robots in Harsh Environments", presented at IEEE IRC19.

- Di Castro, M., ML Baiguera Tambutti, M. Ferre, R. Losito, G. Lunghi, and A. Masi. "i-TIM: A Robotic System for Safety, Measurements, Inspection and Maintenance in Harsh Environments." In 2018 IEEE International Symposium on Safety, Security, and Rescue Robotics (SSRR), pp. 1-6. IEEE, 2018.

- Di Castro, Mario, Alessandro Masi, Simone Gilardoni, Roberto Losito, Maria Laura Baiguera Tambutti, and Giacomo Lunghi. "LHC train control system for autonomous inspections and measurements." (2018): THPHA061.

- Di Castro, Mario, Alessandro Masi, Luca Rosario Buonocore, Manuel Ferre, Roberto Losito, Simone Gilardoni, and Giacomo Lunghi. "A dual arms robotic platform control for navigation, inspection and telemanipulation." (2018): TUPHA127.

- Di Castro, Mario, David Blanco Mulero, Manuel Ferre, and Alessandro Masi. "A Real-Time Reconfigurable Collision Avoidance System for Robot Manipulation." In Proceedings of the 3rd International Conference on Mechatronics and Robotics Engineering, pp. 6-10. ACM, 2017.

- Di Castro, Mario, Jorge Camarero Vera, Alessandro Masi, and Manuel Ferre. "Novel Pose Estimation System for Precise Robotic Manipulation in Unstructured Environment." In ICINCO (2), pp. 50-55. 2017. 
- Attard, Leanne, Carl James Debono, Gianluca Valentino, and Mario Di Castro. "Visionbased change detection for inspection of tunnel liners." Automation in Construction 91 (2018): 142-154.

- Attard, Leanne, Carl James Debono, Gianluca Valentino, and Mario Di Castro. "Tunnel inspection using photogrammetric techniques and image processing: A review." ISPRS journal of photogrammetry and remote sensing 144 (2018): 180-188.

- Angrisani, L., Arpaia, P., Gatti, D., Masi, A., \& Di Castro, M. (2018, August). Augmented Reality monitoring of robot-assisted intervention in harsh environments at CERN. In Journal of Physics: Conference Series (Vol. 1065, No. 17, p. 172008). IOP Publishing.

- Ivanovs, Arturs, Mario Di Castro, Agris Nikitenko, Manuel Ferre, Alessandro Masi, Toms Torims, "Multi-sensor Based Low-Cost System for Real-Time Human Detection and Remote Vital Parameter Acquisition", presented at IEEE IRC19.

- Saliba C., K. Bugeja M., G. Fabri S., Di Castro M., Mosca A. and Ferre M. (2018). A Training Simulator for Teleoperated Robots Deployed at CERN.In Proceedings of the 15th International Conference on Informatics in Control, Automation and Robotics Volume 2: ICINCO, ISBN 978-989-758-321-6, pages 283-290. DOI: 10.5220/0006849302930300

- Grech, L., Valentino, G., Di Castro, M., \& Almagro, C. V. (2018, August). Collision Avoidance System for the RP Survey and Visual Inspection Train in the CERN Large Hadron Collider. In 2018 IEEE 14th International Conference on Automation Science and Engineering (CASE) (pp. 817-822). IEEE.

- Attard, Leanne, Carl James Debono, Gianluca Valentino, and Mario Di Castro. "Image mosaicing of tunnel wall images using high level features." In Proceedings of the 10th International Symposium on Image and Signal Processing and Analysis, pp. 141146. IEEE, 2017.

- Attard, L., Debono, C. J., Valentino, G., di Castro, M., \& Tambutti, M. L. B. (2018, October). An RGB-D video-based wire detection tool to aid robotic arms during machine alignment measurement. In 2018 IEEE International Conference on Imaging Systems and Techniques (IST) (pp. 1-6). IEEE.

- Attard, L., Debono, C. J., Valentino, G., di Castro, M., Osborne, J. A., Scibile, L., \& Ferre, M. (2018, October). A comprehensive virtual reality system for tunnel surface documentation and structural health monitoring. In 2018 IEEE International Conference on Imaging Systems and Techniques (IST) (pp. 1-6). IEEE.

- Lunghi, Giacomo, Raul Marin Prades, Mario Di Castro, Manuel Ferre, and Alessandro Masi. "An RGB-D based Augmented Reality 3D Reconstruction System for Robotic Environmental Inspection of Radioactive Areas." In ICINCO (2), pp. 233-238. 2017. 
- Lunghi, Giacomo, Raul Marin Prades, and Mario Di Castro. "An Advanced, Adaptive and Multimodal Graphical User Interface for Human-robot Teleoperation in Radioactive Scenarios." In ICINCO (2), pp. 224-231. 2016.

- Chenf, Fei, Boyang Gao, Mario Selvaggio, Zhijun Li, Darwin Caldwell, Keith Kershaw, Alessandro Masi, Mario Di Castro, and Roberto Losito. "A framework of teleoperated and stereo vision guided mobile manipulation for industrial automation." In 2016 IEEE International Conference on Mechatronics and Automation, pp. 1641-1648. IEEE, 2016. 


\subsection{Outline}

Chapter 1 introduces the instruments used in this thesis. The context of the work is shown as well as the problematic that have raised the needs for this work. a state of the art for current deployed robotic system is done and the main contributions are shown as well as the outline of the thesis.

Chapter 2 presents the state of the art in robotic systems. Technologies like artificial intelligence and telemanipulation are introduced, including the importance of the haptic telepresence. in addition to operator training and ethical aspects in the use of robots.

Chapter 3 is the backbone of the work presenting the novel framework architecture. The proposed control system is introduced including new methods used in this thesis for multirobots maintenance operation. The intervention preparation methods and the code of practice for remote handling generated in this work are also presented. The code is based on the operational experience of more than 130 robotic intervention performed at CERN in the last 5 years. Part of the presented work is composed by algorithms for data communication and energy management.

Chapter 4 introduces the features of the proposed framework that is endorsed with an autonomous navigation system and a human recognition system in hazardous environment, as well with a relays robot architecture to extend the network coverage and to navigate in cluttered environments. Novel modules of the proposed framework like environmental reconstruction and object detection, as well as collision avoidance, are presented. Features of the proposed framework are also learning by demonstration techniques and vision based change detection systems for plants structural monitoring. In addition, the new architecture for cooperative multi-agents is presented, including a novel live scripting system in order to generate custom control strategies during the robotic operations.

Chapter 5 describes the operator performance metrix in using the proposed novel system. The novel human-roobt interface, used to operate all the robots integrated in the CERNTAURO framework and endorsed with image super-resolution techniques, is presented. Techniques to endorse the proprioception during teleoperation are shown, as well as a novel virtual-reality-based simulation system for safety assessments, robots integration and operator training. Finally the chapter shows a comparative analysis in accomplishing remote handling tasks by using industrial robotic systems and CERNTAURO based robotic solutions

Chapter 6 shows the results of the application of the proposed framework in real intervention done at CERN. Results like tunnel structural monitoring, as well as tracking-based depth estimation of metallic objects and application of learning by demonstration techniques are presented.

Chapter 7 concludes this thesis and the appendixes complete the presented work describing the experimental results, the novel robots and tools developed, as well as the details of the vision based structural monitoring system and the useful-to-share software codes developed. 


\section{Chapter 2}

\section{State of the Art on Robotic Systems}

Currently there are a great variety of robotics systems from robots for elder-caring [66] to space robotics [67]. In the following an introduction to robots applied in different domains is presented, underlying the state of the art of robots for radioactive environments.

\section{Robotics for Industrial applications}

Advanced robotics and automation are potential game-changing technologies for strengthening the manufacturing sector, particularly for small and medium-sized enterprises and automotive industry continues to be the largest user of industrial robots. Based on mechanical configuration, industrial robots can be classified into six major types namely: articulated robots, cartesian robots, SCARA robots, delta robots, polar robots and cylindrical robots. Apart from mechanical configuration, industrial robots can also be categorized based on motion control, power supply control and physical characteristics. The most commonly used robot configurations for industrial automation, include articulated robots, SCARA robots and gantry robots.

Typical applications of industrial robots include welding, painting, ironing, assembly, pick and place, palletizing, product inspection, and testing, all accomplished with high endurance, speed, and precision [68].

\section{Robotics for Infrastructures}

Infrastructure robots are becoming essential for inspection and maintenance of civil and general infrastructure. Inspections and repair of miles of critical infrastructure across urban, suburban, and rural areas fall well within the "dull, dirty, or dangerous" work ideal for automation. Robots can operate in dangerous and challenging locations, such as inside underground pipes, or at height underneath bridges, or on live roads to perform inspection, repair, maintenance, and removal tasks. Robotic systems help in improving speed, quality and timeliness of infrastructure engineering, while reducing direct costs, economic impacts, material waste, energy usage, environmental damage, and risk to human operators [69]. These type of robots are built specifically for dedicated places and they need trained operators to be effective. 


\section{Robotics at ITER}

ITER (International Thermonuclear Experimental Reactor) is an international nuclear fusion research and engineering megaproject, which will be the world's largest magnetic confinement plasma physics experiment [70]. ITER is being designed to have a good integration with different robotic systems to be able to work with the radiation of a fusion reactor. Between the robotic systems of ITER [71] can be found a blanket remote handling system [72], a divertor remote handling system [73] (see Figure 2.1), a cask and plug remote handling system [74], an in vessel viewing system [75], a neutral beam remote handling system [76] and a remote handling for hot cell [6]. Thes systems have to work in areas with high radioactivity, as the blanket remote handling system which will be operated in a high radiation environment (250 Gy/h max.) and must stably handle the blanket modules, which weigh $4.5 t$ and are more than $1.5 \mathrm{~m}$ in length, with a high degree of position and posture accuracy [77]. The facility is structured and adapts to the needs of the telemanipulator systems.
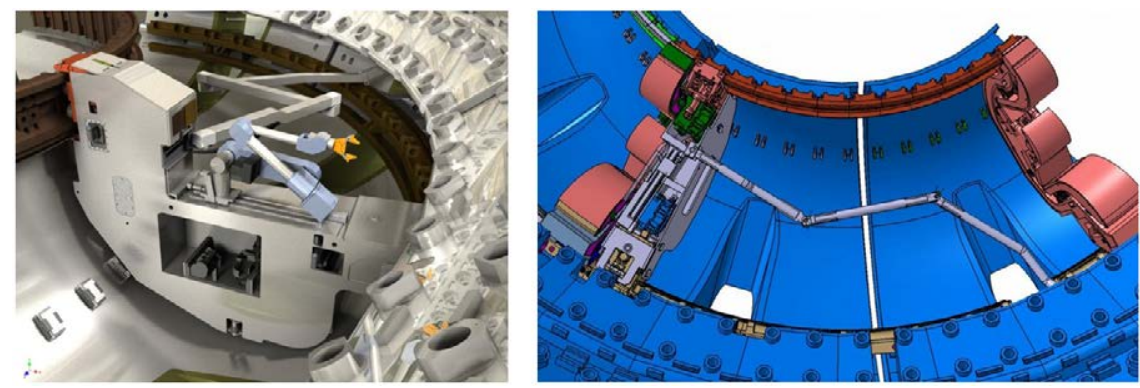

Figure 2.1: A 3D image of the remote handling system for the ITER divertor on the left and the Divertor cassette placement inside the vacuum vessel by means of the cassette toroidal mover (cable handling device deployed) on the right (Credit: Assystem)

\section{Robotics at JET}

The Joint European Torus (JET) is the flagship for European Fusion Research [78]. This study seeks to address the issue of the need for remote handling in the process of recreating nuclear fusion as a limitless source of clean energy. The JET remote handling system employs a "man in the loop" approach with the robotic Boom and Master Slave Servo-Manipulator (MSM) system providing the operator with a pair of "remote hands" inside the JET Torus. All remote handling tooling and components are designed to be handled by the "remote hands" in a manner similar to an operator actually working within the JET Torus (see Figure 2.2). Such an approach requires a high degree of operator training, together with systematic methodologies for remote operations task development, tooling and component validation [79]. The facility is structured and adapts to the needs of the telemanipulator systems. 

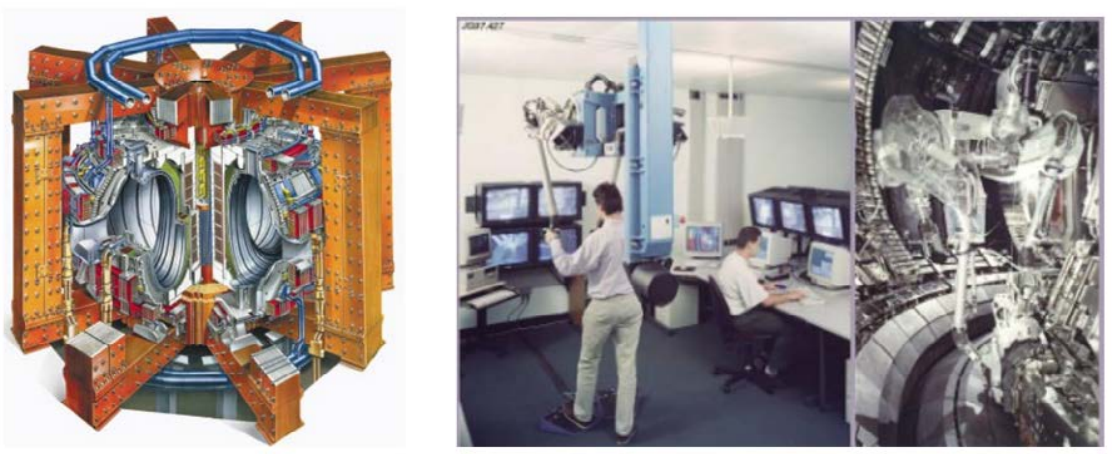

Figure 2.2: JET Torus (left) and remote handling approach using the MASCOT system [80] (Credit: www.euro-fusion.org)

\section{Robotics at SNS}

The Spallation Neutrino Source (SNS) facility is the latest step in the development of accelerator-based neutron research facilities [11]. One thousand beam pulses will be bunched in a ring and then directed to a flowing liquid mercury target that will convert the protons to a pulse of approximately 5 x 1015 neutrons. The neutrons will then be slowed to useful energies and guided to 24 instruments where scientists from around the world will have the opportunity to undertake basic research in materials science. For this facility has been developed a remote handling robotic system [81] based on Telerob EMSM-2B and a mobile vehicle equiped with a servomanipulator (see Figure 2.3). The facility is structured and adapts to the needs of the telemanipulator systems.
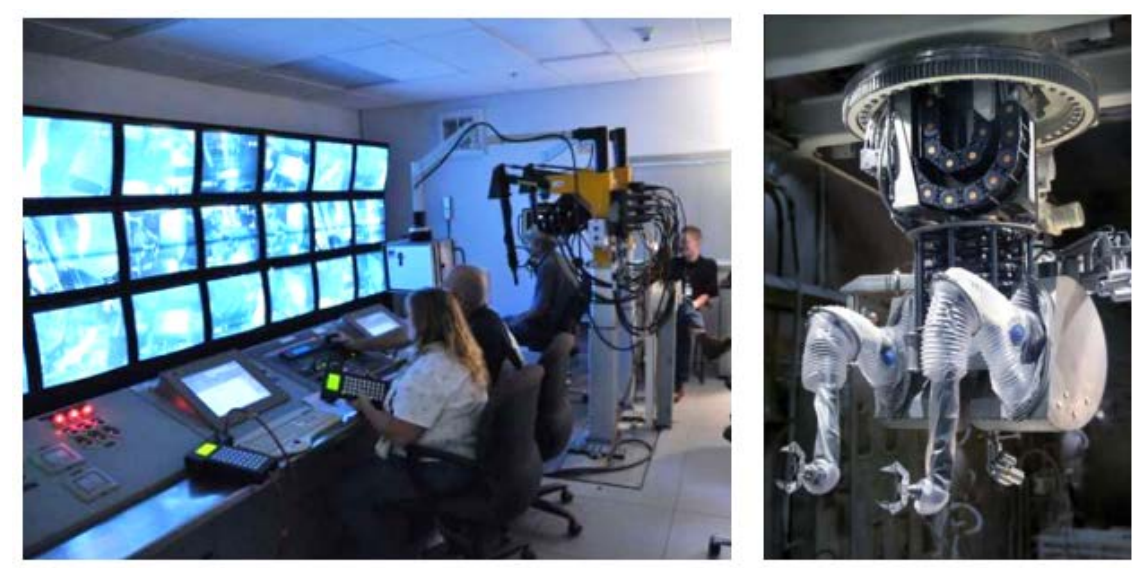

Figure 2.3: Remote handling control room (left) and the Telerob EMSM 2B telemanipulator system in use at SNS (Credit: www.ornl.gov) 


\section{Robotics for the Fukushima Daiichi Nuclear Reactor Disaster}

The Fukushima Daiichi nuclear reactor disaster triggered by the March 2011 tsunami that devastated parts of Japan serves as a reality check on our capacity to use robots effectively for hazardous tasks [82]. Beyond the carefully engineered environments that characterize the nuclear material handling industry, the disappointing performance of several robots [83] has demonstrated just how far we still have to go [57]. The disaster exposed the need for prior planning and continuous training, rehearsal and cooperation between research and development agencies, defence disaster relief agencies, robotic systems manufacturers and engineers at the hazardous facilities where the robots would be used. Continuous updating of robot systems is needed: some robots that could have been deployed relied on obsolete electronic and computer components that could not be replaced. Following this disaster, we can expect a significantly increased effort to deploy robots over the coming years to deal with the consequences of similar events (see Figure 2.4). The Fukushima Daiichi disaster and other operations following the March 2011 tsunami demonstrated that, as in the other applications discussed above, robots can still only be expected to perform a niche role. Robots have only performed consistently in such environments after decades of development, and investment in developing trained operators with appropriate maintenance and support facilities. Our ability to realistically predict the capabilities of robotic operations in unforeseen hazardous environments is still very limited.

While for industrial structured environments autonomous robots could do different tasks, for safety reasons in harsh unstructured and semi-structured environments, telerobotics is the preferred approach. These dangerous environments have the necessity to use the humanin-the-loop approach in a multimodal way.
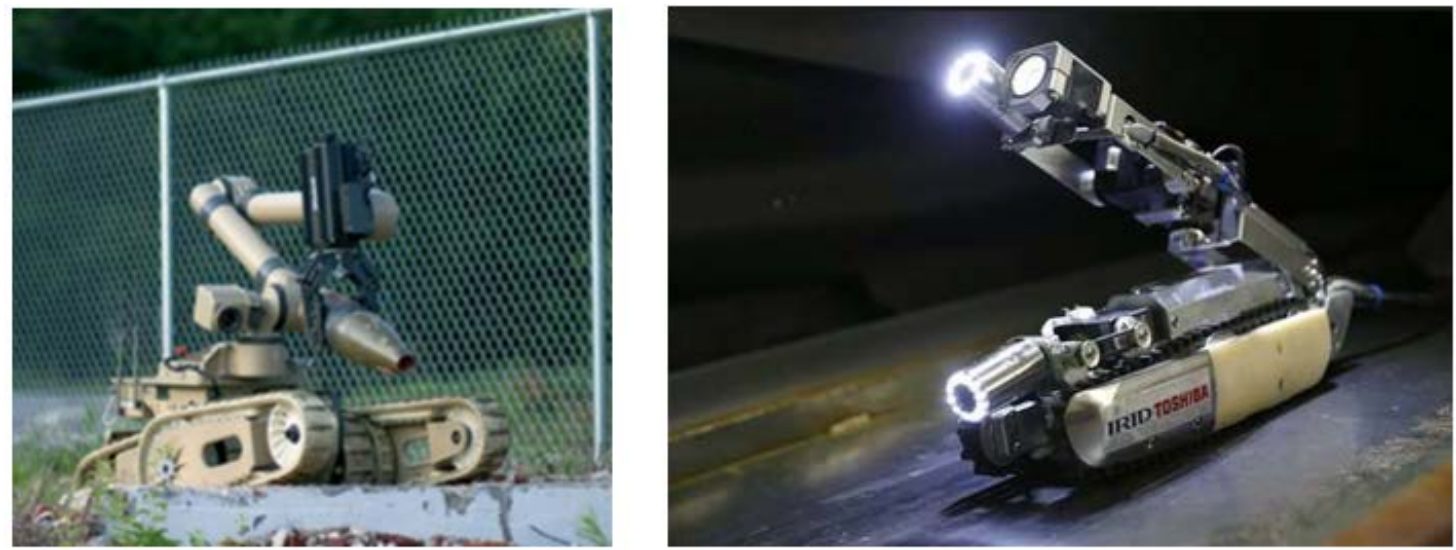

Figure 2.4: Examples of robotic systems entered into the Fukushima nuclear plant. The irobot (left, credit: www.popularmechanics.com) and the Toshiba Scorpion (right, credit: www.toshiba-energy.com) 


\section{Robotics for Deactivation and Decommissioning Systems}

One of the most complex forms of hazardous operations is the deactivation and decommissioning $(\mathrm{D} \& \mathrm{D})$ of defunct facilities where nuclear radiation or toxicity hazards preclude human presence. D\&D can be thought of as remote demolition for the most part. Some operations are crude such as knocking down building structures and debris removal. Other operations may involve careful disassembly of equipment and devices, size reduction and packaging of handling/storage. These operations are essentially the inverse of remote maintenance and require the dexterous use of tools and handling of objects [57]. Several robotic systems have been developed for this D\&D tasks in nuclear applications [84] and for inspection and maintenance [85].

\section{Nuclear Spent Fuel Reprocessing Plant}

In nuclear fuel reprocessing facilities, technology was developed to chemically separate and recover fissionable plutonium from spent nuclear fuel [86]. In these facilities, remote handling equipments have to deal with severe constraints:

- Maximum integrated dose for 1 year between 104 and 105 Gy.

- Decontamination capability using potentially aggressive products.

- Operation at high temperature $\left(508^{\circ} \mathrm{C}\right)$.

- Electromagnetic compatibility.

- Volume and weight compatible with remote operations.

- Compliance with safety and quality standards.

- Diagnosis and maintainability constraints.

- Compatibility with waste management.

For this task industrial robots can be adapted to the constraints listed here above. That includes the development of radiation tolerant electronic parts, force-feedback control software for master-slave teleoperation, and the integration of the overall technologies in order to build a remote handling system [87].

\section{Human Supervisory Control}

While for industrial structured environments autonomous robots could do different tasks, for safety reasons in medical applications, as well as in harsh unstructured and semi-structured environments, telerobotics is the preferred approach. Robotic surgery and dangerous environments have the necessity to use the human-in-the-loop approach in a multimodal way [88] [89]. 
Telerobotics includes master-slave interactions and also semi-autonomous operations that are composed by human supervised controls of autonomous behaviours. These applications are often endorsed during the last years by artificial intelligence technologies and learning by demonstration techniques [90] [91].

\subsection{Artificial Intelligence}

AI (artificial intelligence) is the simulation of human intelligence processes by machines, especially computer systems. These processes include learning (the acquisition of information and rules for using the information), reasoning (using rules to reach approximate or definite conclusions) and self-correction. Particular applications of AI include expert systems, speech recognition and machine vision [92].

In this section it will focus in deep learning and all its techniques. Deep learning allows computational models that are composed of multiple processing layers to learn representations of data with multiple levels of abstraction. Deep learning discovers intricate structure in large data sets by using the backpropagation algorithm to indicate how a machine should change its internal parameters that are used to compute the representation in each layer from the representation in the previous layer. Deep convolutional nets have brought about breakthroughs in processing images, video, speech and audio, whereas recurrent nets have shone light on sequential data such as text and speech[93].

Here we will focus in the deep learning techniques for computer vision and reinforcement learning. Both of them currently are being applied a lot in the field of robotics and autonomous systems.

\section{Computer Vision using Deep Learning}

One of the main applications is image classification [94, 95, 96, 97, 98, 99], where objects are identified in a picture. In the field of robotics is also important to know where is the object in the picture. For this demands, several algorithms of object detection have been developed $[100,101,102,103,104,105,106,107,108,109,110,111]$, an example is shown in Figure 2.5. Techniques for video classification have been developed[112, 113] during the last years, as well as tracking detection objects, very useful for assisted tele-operations [114, 115, 116].

In the tunnel areas communications could be very limited and a live visual feedback can be compromised. Useful techniques to overcome this problems are efficient compression algorithms [118] and super-resolution ones in which a low resolution set of images are converted into higher resolution ones (see Section 5.2) [119, 120, 121, 122, 123, 124, 125]. The image objects detection using bounding boxes sometimes could very inaccurate needing image segmentation techniques to increase the accuracy, then algorithms of semantic segmentation can be used [126, 127, 128, 129, 130, 131, 132, 133]. 


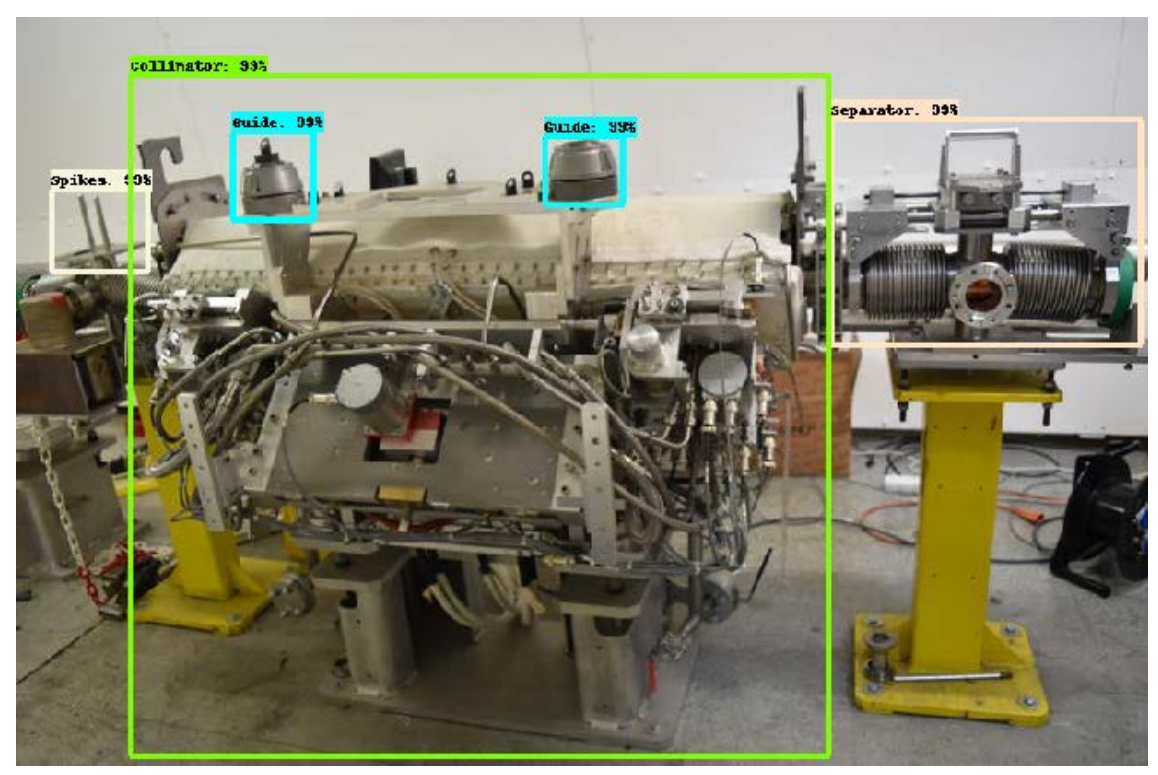

Figure 2.5: Detection of different objects in a LHC collimator[117] using Faster R-CNN[110]. The bounding boxes in the picture highlight the detected specific components on a LHC collimator.

\section{Deep Reinforcement Learning}

Reinforcement learning is learning what to do-how to map situations to actions - so as to maximize a numerical reward signal. The learner is not told which actions to take, but instead must discover which actions yield the most reward by trying them. In the most interesting and challenging cases, actions may affect not only the immediate reward but also the next situation and, through that, all subsequent rewards. These two characteristics - trialand-error search and delayed reward - are the two most important distinguishing features of reinforcement learning [134]. The combination of reinforcement learning with deep learning techniques is called Deep Reinforment Learning, it started in 2013 with the publication of a paper in which a computer reaches human-level playing Atari games [135, 136].

After Atari games more harder games were tried to reach human level and further, as Go [137], chess, shogi [138], StarCraft [139] and Dota2 [140]. Currently Deep Reinforcement Learning is a research field with a huge number of researchers participating in it and it's been used to teach robots how to move and execute actions [141, 142, 143, 144, 145, 146]. One of the techniques that use Deep Reinforment Learning is Deep Imitation Learning, this allows to teach robot how to move using demonstrations [147, 148]. Apart of robotics and games is being use in control of different systems [149].

\section{Others Deep Learning Techniques}

Deep learning has been also applied to other fields as, for example, Natural Language Processing (NLP). Natural language processing [150] (NLP) is a theory-motivated range 
of computational techniques for the automatic analysis and representation of human language. NLP enables computers to perform a wide range of natural language related tasks at all levels, ranging from parsing [151, 152, 153, 154, 155] and part-of-speech tagging [156, 157, 158, 159], to machine translation [160, 161, 162, 163] and dialogue systems [164, 165, 166, 167, 167]. These techniques are very useful to increase the multimodality of Telerobotic human-robot interfaces.

\subsection{Telemanipulation}

Teleoperation, telemanipulation and telerobotics in general have the capabilities of carrying out tasks in a remote environment using proper robotic systems.

Telemanipulation systems have been the first applications of the robotic technology, mainly because of the interest for the nuclear research back in the 50's. Since then, huge developments have been achieved, both from the technological point of view (materials, processing power etc.) and from the theoretical one [168].

Recently more research has been focusing on experimenting on different venues of telemanipulation. The design of low weight, high torque BLDC motors has resulted in a breaktrough in aerial manipulation. Several systems were designed, which consist of a quadcopter, which is capable of moving in euclidian space and a low weight robotic arm with less then 6 degrees of freedom [169, 170, 171]. These robots allow the user to perform interventions in hard to reach, disaster areas. The use of quadcopters is still limited by their small effective range.

The Fukushima disaster also showed the need for robotic systems which can operate underwater. Many nuclear powerplants are located close vincinity of sea water in order to provide them with sufficient cooling water. In case of emergencies and repears the intervention might need to take place underwater. There are only a few systems capable of telemanipulation underwater. Most of them are based on a submarine, equipped with a manipulator [172, 173, 174, 175]. A special bimanual humanoid robot was also designed for more complicated interaction underwater [176]. The underwater teleoperation is very challenging, because of the low bandwith and high distortion of the signal. Normally sonar is used, which provide around 10kbps bandwidth and high delays (order of seconds depending on the distance), which are also very fluctuating. Recently radiofrecuency techniques are starting to be used, providing some kbps and low and constant delays, with short distances. Another technique, that could also be used in the LHC is VLC (Visual Light Communication), which provides high bandwidth and low delay, needing direct view. Compression techniques are very important in these scenarios [177].

\section{$2.3 \quad$ Haptic Telepresence}

Haptic telepresence can be considered as a subtopic of telemanipulation. In general telemanipulation provides visual feedback to the operator. Haptic telepresence additionally gives a 
haptic feedback to the user. Haptic feedback can be useful to perform complicated motions in an unknown environment.

The most widespread haptic feedback devices use vibrations. They usually use eccentric rotating mass actuators (ERM), which consist of a motor and an unbalanced weight. Another family of actuators is called Linear Resonant Actuators (LMA). LMA actuators are based on the interaction of AC current magnetic field and a permanent magnet. These motors have small size and can be fit into wrist watches and mobile phones. Their main drawback is that their haptic resolution is minimal. They can only signal a few different states, such as vibrating less or more. On the robot side the signal source for these actuators come from high precision microscopes and accelerometers. This method has been used in telesurgery [178].

The most articulated way to create haptic feedback is by the usage of force-feedback. During force-feedback, through a mechanism forces are exerted on the user. Unlike the technology based on vibrations, force-feedback can create a much detailed, lifelike sensation for the user. They have been in use in flight and car simulators for over decades. These simulators are usually big and expensive. Newer devices, such as the Phantom Omni [179] can be used for CAD modeling and graphic design. Their disadvantage is that they need sophisticated mechanisms with several degrees of freedom. Each degree of freedom needs a separate actuator.

During teleoperation with force-feedback the delay in the control loop can introduce unstable behaviour. There has been much work done on the stability analysis of control loops with delays $[180,181,182]$.

Recently several efforts have been carried out to create non-contact haptic devices. Disney Research has created AIREAL [183], a device based on air vortex rings. A recent start-up company Ultrahaptics [184] have created a device based on the use of ultrasound vibrations. These devices are unfortunately still at an early stage of development.

\subsection{Hands on Preparation for Robotic Intervention}

A part of the basic utilities concerning the robot setup which are necessary and specific for each intervention (see Appendix D) such as: single or double robotic arm, lifting stage, tools (cutter, pumps, etc), devices accelerometers, radioactivity sensor, thermal camera, etc), kind of gripping solution, wheels, communication protocol (WI-FI, 4G, Bluetooth, etc); which is a common part for any task, it is necessary to pay especial attention to the environment where intervention shall be carried out.

Thus, due to the fact interventions at CERN involved all possibles scenarios, the procedure has to focus on the adaptability and maintenance of the equipment as far as practicable, with the aim to reduce the waste and cost, not either for the robotic platform but also for the facilities. Moreover, radioactive environments brings the necessity to pay attention in specific topics beyond the normal situations, hence contingency plans become essential, and the analyse of possible accident scenarios is a fundamental aspect to be taken into consideration (see Section 3.2). All of these matters to consider are explained below: 
- Space: it must be taken into account for accessibility of the equipment necessary to perform the task. This includes cranes, manipulator, robots, robotic arms, cameras etc. Both the RH equipment as the human operator should have enough space to long the path to the work space.

- Protective covering: Joints and mechanisms should be covered in harsh environments to avoid humidity, dust, radiation, etc; especially if these will require hands-on maintenance or are difficult to decontaminate. The protective coatings must be removable and replaceable. However, this protective coating do not have to impair the operation.

- Tooling design: the requirements depend importantly on the component itself and the type of facility where the task is going to be performed. For those environments that are radioactive, it is priority to try to reduce the degree of contamination that the tool could receive as far as practicable. Hence the following design considerations are important for easy decontamination, waste reduction, and possible reuse of the equipment used in a radioactive/activated environment:

- Material equipment: The equipment, not to be reused, may be constructed of lower grade materials not necessarily resistant to the decontamination reagents. However, if require a re-use continues the material, this should be radiation resistant (if is possible). In addition, the materials for the equipment that have to be decontaminated, should be resist degradation by the decontamination fluids and procedures.

- Decontamination equipment: The material part not covered and non-easy replaceable should be decontaminated until acceptable levels. Another part such as bearings and connectors are susceptible to damage by decontamination solutions, in this case could be removed and replaced.

- Components: the number of components and complexity of the systems should be reduced as much as possible. The use of few, simple and well-verified components are preferable. Moreover, simple and easy handled mechanism are preferable that complex elements.

An adequate visualization and lighting system is crucial in the work-space, where remote operations are required. If not, the remote work could not be done. Likewise, real time is very important, since most remote operations rely on real time visual feedback to an operator. This information is the main link between the operator and the remote operation being performed. For no real time task, it is possible to gather visual data for postmortem visualization.

This system includes the incorporation of lights, cameras and visualization systems such as TV or virtual reality glasses. Where lights and cameras are inside of the work area and the operator in the safety control area uses the TV, displays or VR glasses (see Section 5.2). Sufficient lights and cameras should be placed from several different angles so to adequately lighting and visualized the task components and features such as pan, tilt or zoom on some 
of cameras and lights are often necessary to provide views of all or most areas and to aid in the interventions (see Figure 2.6). This visualization system should allow a view of the entire area and components, creating greater immersion in the environment. If not, the operator could not perform remote tasks or control the environment or, classical visual helper-assistance systems could get difficulties in time to provide the proper visual feedback. CERNTAURO considers this constraint and a tracking system capable to estimate the target distance in harsh conditions was developed [185] overcoming these kind of situations, which is explained in Section 4.1.

In other cases, when remote maintenance is not necessary, the cameras are used as visual support and help to obtain more information about the environment, due to this, and the high important of the visual feedback (mentioned above) in the task performance, it deserves a special mention within the preparation.
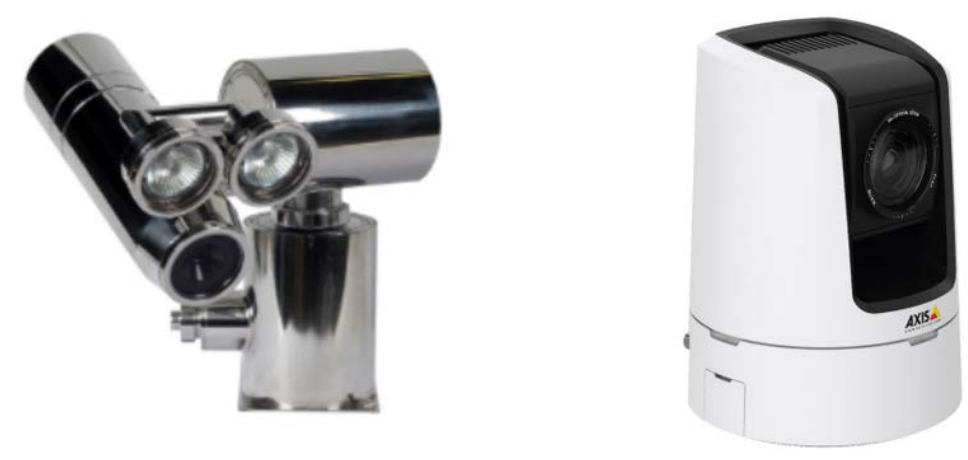

Figure 2.6: Example of camera and light for an extreme environments from Diakont (left) and a pan, tilt and zoom camera axis-V5915 (right). Both commercial camera systems are integrated into the CERNTAURO framework and are the most used for robotic operations at CERN.

There are three types of cameras to obtain as much information as possible from the environment during maintenance operations. These cameras are: RGB to visualize the environment in 2D, RGBD to visualize the environment with depth (3D) and thermal camera to visualize the temperature.

In the same manner, we can find two different types of visual elements positioning:

- Fix elements: Displays and TV are fixed in the control room or control area. In the work area, visualization and illumination system could be fixed in the walls and elements. It allows a whole overview of the work zone. This cameras and light should be a pan and tilt movement.

- Portable elements: cameras and lights are placed in mobile elements. For example, they could be integrated in trolley, servo-manipulators, crane or robot vehicle. These should be able to provide images or video in different points of the work area. Viewing and illumination has to be totally integrated with the requirements of the remote handling equipment and tools, taking care of the shadows and vision barriers from the 
components, accessibility, (dis)assembling procedures, and the degree of visual detail required for the particular procedure.

Is it important the quality and quantity of the cameras This questions are replied by the specifications of each task. Although multiple camera views in different point are required to adequately complete most remote maintenance tasks and obtain successes results, it is important to take into account the effects of radiation on this equipment, and how this can affect it and costs. The higher the quality of the camera, the better the results will be, but a balance must be found with the factors below:

- Radiation affect to the quality of the image.

- Avoid highly reflective surfaces where possible or put anti-reflection painting, if not, the problem can be dealt with the tracking-based depth estimation deployed in Section 4.1 .

- Provide colour differences where possible.

- Regulate the light.

- The bandwidth, which is usually the biggest constraint of the system.

It is recommended to use commercial cameras and lights, due to its high availability in case of failure. In addition, the selection of the camera depends on the environment and other requirements, being possible to choose commercial cameras resistant to radiation. However, sometimes it is preferable to select a normal camera that is not resistant to radiation, and replace it when necessary. This is due to the high price of special cameras for that purpose.

When dealing with interventions in harsh environments, being these human or robotic interventions, it is fundamental to maintain and to guarantee the safety of the humans, of the equipment, of the robots, and so on providing robust failure mode analysis and ecovery scenarios.

The analysis of requirements carried out previously to the intervention, which consider either the facilities as the environment, shows the intervention features and the robot specification that will be necessary. All of this allow to generate the proper risk analysis where, critical aspects like the environments where radiological contamination is a risk, or the materials of which the robots are composed of are fundamental to avoid radiological contamination and unwanted radiological waste with the risk that an expensive robot might become impossible to be used again.

Regarding the design of robots for hazardous environments, some specific requirements must be undertaken, such as waterproof mechanical components, security skeleton, wheels adapted to the environment, and lifting system, in order to enhance the efficiency of the mobile manipulator. This allows further use of a robot after an intervention, enabling the robot to be easily cleaned [16]. Moreover, once the framework suggests the appropriate robot to use for a requested intervention, the functional requirements' designs are conducted through 
a safety aspect implementation, including failure mode identifications using clustering analysis and recovery scenario procedures. Then, this design is taken into account to perform the interventions, which will be carried out on unstructured areas with high importance and cost surroundings which must be cared for.

These two fundamental steps are strictly iterative during the first phase of an intervention preparation. Safety or recovery scenario procedures can influence, and in some cases, adjust the requirements because of technological limitations. Machine and human safety aspects need to be taken into consideration, for example the risk of radioactive environments or places with a high magnetic field presence that can negatively influence the normal operational behaviour of mechatronic systems.

In addition to a robust robotic design for an efficient behaviour, it is mandatory to design the robots to make them easily cleanable and decontaminated, when operating in radioactive environment. Nevertheless, the requirements depend importantly on the component itself and the type of facility. Reducing the degree of contamination implies the improvement of processes, systems and several components. So the following design considerations are important for easy decontamination in a radioactive/activated environment:

- Decontaminable surfaces:

- Smooth or polished surfaces are favourable. For example, electro-polishing is fast, inexpensive and effective for complex structures.

- Try to avoid holes, deep grooves, discontinuous welds, cracks, crevices, porous materials and joints that trap dust.

- Apply coatings or liners on porous materials, for example concrete.

- The cleaning of the component should be greater when the probability and degree of contamination is higher.

- Protective covering: Joints and mechanisms should be covered in contaminated environments, especially if these will require hands-on maintenance or are difficult to decontaminate. The protective coatings must be removable and replaceable. However, this protective coating do not have to impair the operation.

- Decontamination equipment: The material part not covered and non-easy replaceable should be decontaminated until acceptable levels. Another part such as bearings and connectors are susceptible to damage by decontamination solutions, in this case could be removed and replaced.

- Materials: The equipment, not to be reused, may be constructed of lower grade materials not necessarily resistant to the decontamination reagents. However, if require a re-use, this should be radiation resistant. In addition, the materials for the equipment that have to be decontaminated, these should be resist degradation by the decontamination fluids and procedures. 


\subsection{Training}

The complexity of the private company robots tele-manipulation is regarding to the complexity of the platform. This becomes in two parallel challenges listed below:

- Control training, industrial robots use a complex control panel, which requires high exhaustive operator training in order to learn how the robot works and how to use the whole set of tools carried by it. This complexity changes regarding to the robot setup, since some parameters like the number of degrees of freedom leads the sophistication of the control panel, which might give the chance to work on world coordinates, increasing so the learning time exponentially.

- Performance experience, the relationship of the hardship is inversely proportional to the complexity of the control panel of the robot. That means that it is going to be more difficult to reach the goal tasks as long as the controller being easier to use and vice versa. Unfortunately, the only way to overcome this handicap is spending a high number of hours of field training.

Therefore, the CERNTAURO's controller reproduces the real robot performance on the operator's side, providing a perfect feedback with whole set of sensors (torque, cameras, etc.) for either rookie or expertise operator, knowing in real-time the state of the robot and what to do as next step in intuitive way, without the necessity to get training before. Thus, the differences between the un-trained and expertise operators shall be (unavoidably the experience) the innate psychic skills such as: confidence, analysis capacity, level of stress, etc.

During the design and development of a robotic system it is important to take into account some aspects that are related to the operator and the type of interaction that he or she has with the robot. Such aspects give additional guidelines for the design, both in terms of interactional and behavioral choices and in terms of functionalities. For example, in the matter of this work, all the definitions and concepts defined in this section are taken into account afterwards for the implementation of the Human-Robot Interface.

One of the first steps in the development of a unified interface is the definition of the users and the use context. As previously stated, the need of enlarging the operator's pool is of primary interest in CERN Robotics Operations strategy, in order to take profit from expertise of skilled technicians that know in detail the scientific and engineering environment.

Normally, three main categories of operators can be defined [186]:

- Expert operators, who have a long experience in telerobotics, followed training courses and already performed several interventions.

- Project involved operators, who did not acquire experience in the teleoperation field, but they were involved in the projects development, acquiring, therefore, a detailed representation of the robotic system. 
- Entry-level operators, who are experts on the equipment that requires the intervention, and never had the possibility to use the telerobotic system before. This category is the largest and most interesting one, since it contains the experts of the equipment that requires the intervention.

The robotic system described in this work takes into account these three categories of operators. Furthermore, due to the criticality of the operation performed, the system should take into account the human error as much as possible. Historically, there has been a lack of scientific research on human error, which focused instead on the correct behavior of a human. Research on this topic started only in the early 80s, for example with studies on human behavior in nuclear power industry [187] and in air traffic control [188]. Another aspect to take into account is the mental workload and stress caused to the operator during an intervention. In particular, mental workload and stress affect the operator's working memory during the operation. According to [189], an actor uses two types of memory, the short-term memory, faster and more resource demanding, and the long-term memory, slower but of higher capacity. While interacting with an interface, an operator makes extensive use of the short-term memory which, therefore, must not be overloaded by the interface: in fact the short-term memory can store up to 7-9 conceptual elements. In addition, this number drastically reduces in presence of stress. In the short-term memory, the operator must store the status of the environment in which the operation is performed and the status of the robot: since the complexity of the status of the environment is not controllable, the interface should optimize the representation of the status of the robot. For example, there is no need to constantly display the battery level of the robot, which can be shown through a message (or additionally a sound in case of critical levels) only when passing certain thresholds. Another possibility is to limit the number of simultaneous visible cameras, in order to drive the operator to select only cameras that provide an effective feedback.

\subsection{Ethical Aspects in Robotics}

The increasing use of robotics promises a host of benefits that are compelling and imaginative, but as with other emerging technologies, they also come with risks and new questions that society must confront. Therefore, Robot ethics, often referred to by "roboethics", should be given utmost importance. This concerns ethical problems that occur with the use of robots. Questions, such as whether robots pose a threat to persons, whether some uses of robots are problematic and how robots should be designed such as they act 'ethically' should be addressed in "roboethics". In [190], the flourishing role of robots in society is described and a survey of the numerous related ethical and social issues is presented.

Establishing policies and procedures regarding robotics safety is of utmost importance. Rules should be strictly enforced and their violations should result in disciplinary action. Only trained employees should have access to a robot and its workstation, unauthorized personnel should never enter the robot's area. In addition, lockout procedures and control panel actions should be employed. 
Moreover, with robotics, the safety issue is with their design and software. Designing robotic workstations, adequate clearance distances should be established. Even though safety rails, chains, ropes and floor markings are useful as cautionary reminder, they do not provide absolute guarding. Considering this, presence sensing devices must be installed such that when humans are present within a predefined area, the robot is triggered to either operate at a reduced speed or halt motion entirely. Such devices may include pressure floor mats, ultrasonic sensors on the robot's arm and light curtain installations. Furthermore, limit switches and fixed stops near an axis of rotation and translation and emergency stops should be considered. In addition to this, safety analysis to assess existing hazards caused by robots should be conducted and periodic inspections, examining mechanical parts for wear and tear and electronic parts for authenticity should be carried out. While a software bug usually does not result in significant harm in office applications, even a minor software flaw in machinery, such as a car or a robot, could lead to fatal results. Taking this into consideration, thorough testing and simulations should be done before actual deployment. Hacking is also becoming an associated concern of robotics, thus software and network security should be carefully considered.

Recent developments in the robotics world has made robots more user friendly, intelligent, and cost-effective. Considering these benefits, it is intuitive that robots have found jobs in every field, from industrial manufacturing to the medical field. Today's workplace is rapidly changing and so is its workforce. An increasing number of jobs that were once performed by people are now conducted by robots.

In many cases, however, robots are simply taking over jobs that are repetitive, tedious, have a low margin for error or require heavy lifting. Furthermore, robots' use is increasing due to their ability to carry out tasks that would be extremely dangerous for humans. Not only can robots operate in environments where humans cannot, but they can also take on challenges that are hazardous. Robots can explore beneath the sea and in deep space. Robots have also been tested for a varying range of emergency response applications, dealing with floods, wildfires, and other situations. They can also be used in search operations after earthquakes or tsunamis. Robots can move around on a military field or in a contaminated environment involving dust, noise and solvents as well as handle hazardous materials, all of which would otherwise involve high risks to humans.

Robotics can play important roles in harsh and dangerous environments while assisting the human team to accomplish critical and complex tasks in hazardous situations such as during rescue and risky operations [191] and harsh environments [192]. Among hostile conditions where robots are a necessity, one finds high temperatures, freezing cold, humidity, high pressure level, low oxygen level, high radioactivity or reduced accessibility in confined spaces. For instance, in radioactively contaminated areas, a robot can be useful to achieve tasks which are impossible to complete by a human operator as the latter cannot access the site. Such interventions may include vision inspection for leakages, oil refilling, material loading, screwing, drilling and replacing components on machines.

Unlike, what the movies present us, robots are unable to think and make decisions; they are only tools to aid people in getting things done. Robots are machines with programmed movements that allow them to move in certain defined directions or sequences. Even though 
artificial intelligence has given robots more ability to process information and to "learn", they are still limited by the information and functions input by the humans themselves. Thus, it is very important to realise that robots are increasingly being used, not to replace humans on their jobs but to assist them avoid their exposure to hazardous environments and dangerous situations. 


\section{Chapter 3}

\section{The CERNTAURO Framework for Remote Handling}

The CERNTAURO framework is composed by several modules, described in this chapter, that allow its safe and robust deployment on several different robots developed in this thesis work (see Appendix A). The proposed system has been built following the continuous integration model. Research and developments, as well as operational feedbacks and new requirements, were constant inputs during the realization of this framework system infrastructure (see Figure 3.1). For the control system, software testing techniques can be applied to mechatronic systems in order to guarantee a correct functioning of the single modules of the framework through updates. In order to provide constant software reliability through time, the framework implements unit tests procedures. Furthermore, the structure of the test libraries developed in this work imposes a certain structure of the framework as well, guaranteeing its modularity.

\subsection{Control System Architecture}

The introduction, state of the art and problem formulation exposed in the previous chapters contributes to the necessity of developing a brand-new user-friendly robotic solution, modular and adaptable to the different robots and different scenarios presented in the harsh and semi-structured environments, like the CERN accelerator complexes or nuclear facilities.

Based on a core-periphery model [193], the Central Operating System (COS) proposed in the novel CERNTAURO framework was developed using $\mathrm{C}++$ and it is interfaced with the different mechatronic parts of a robotic system (see Figure 3.2). The framework is based on several modules with a deterministic loop time allowing their combination to be real time compatible.

In Figure 3.3, the overall architecture of the CERNTAURO framework is presented, while in Figure 3.4, the system stack diagram is presented. The two main agents in this architecture are the Human-Robot Interface, responsible of allowing a user-friendly operation to the operator through multimodality, and the robot in the field, which contains all 


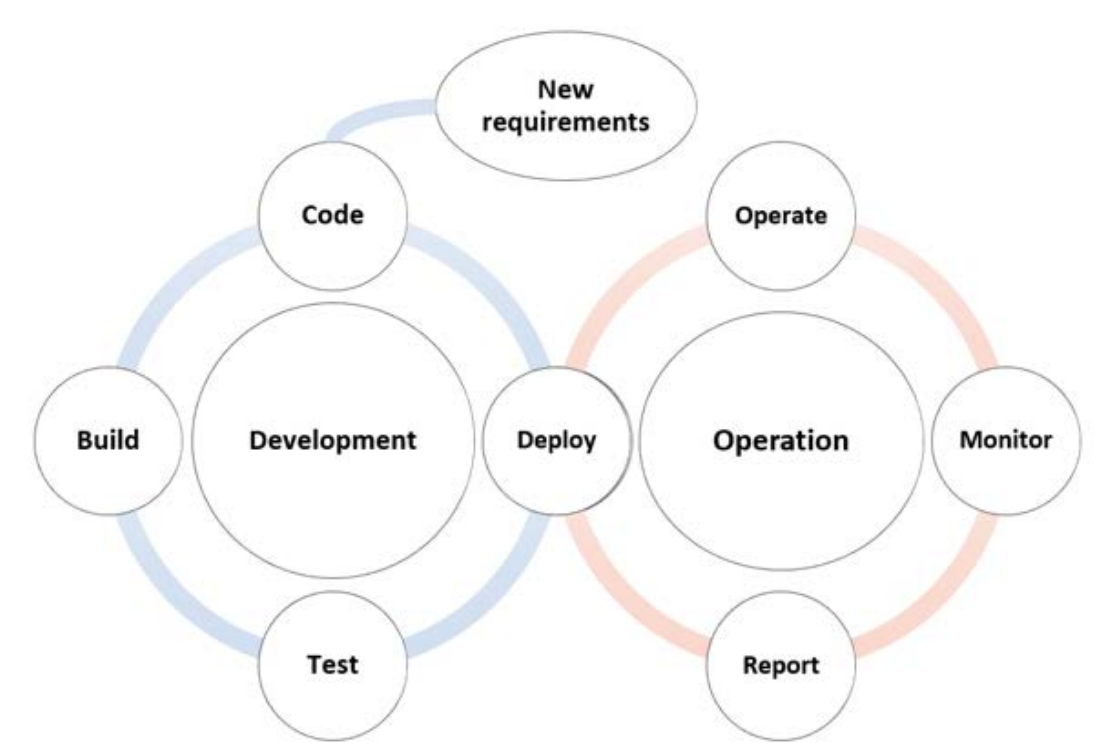

Figure 3.1: The continuous integration model used for the CERNTAURO framework implementation. The development phase is deployed in operation once it has been judged to be robust enough to allow a safe and reliable robotic application on the field. Operational feedbacks after each interventions drive future developments towards robustness and safety.

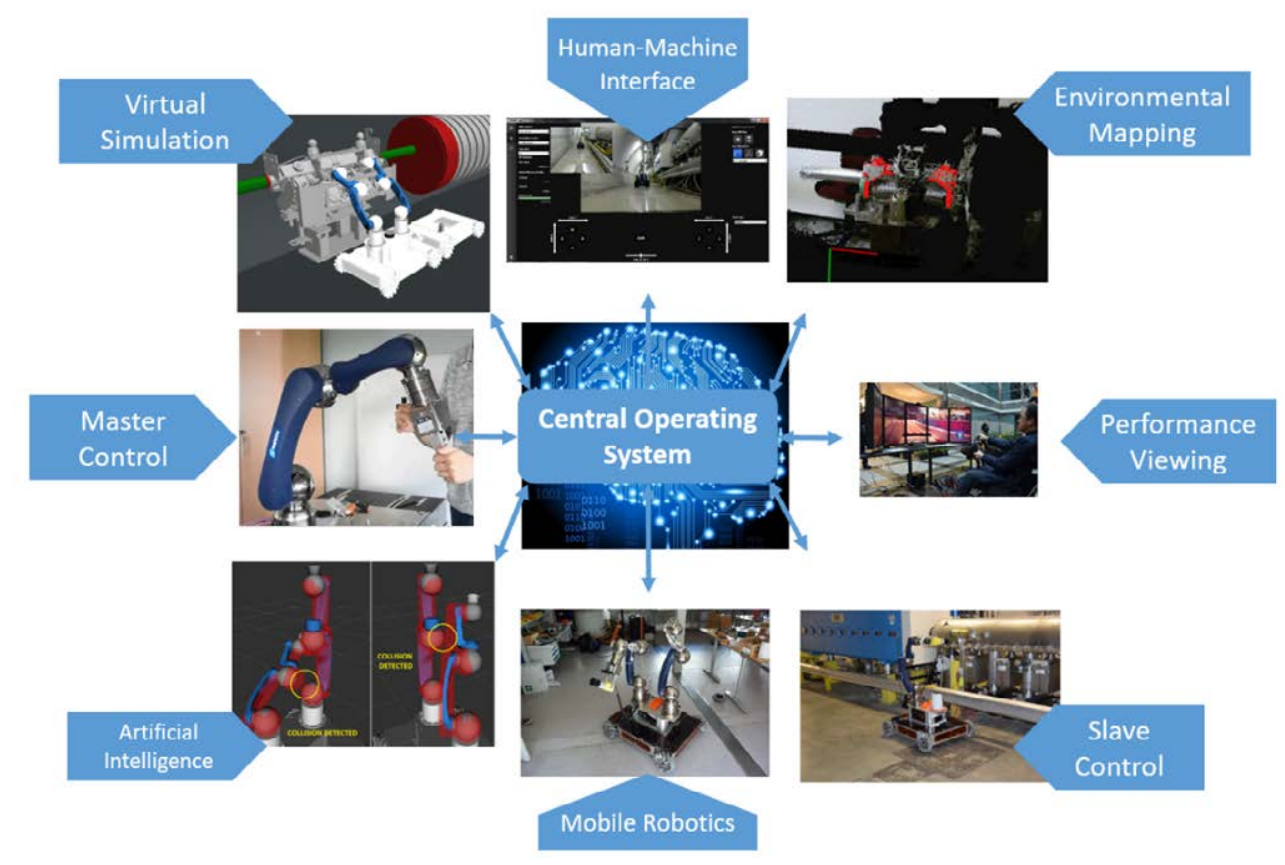

Figure 3.2: A high-level block diagram with pictures representation of the Central Operating System (COS) that has been developed following the core-periphery model. Each model interacts with the others always through the core part of the COS. 


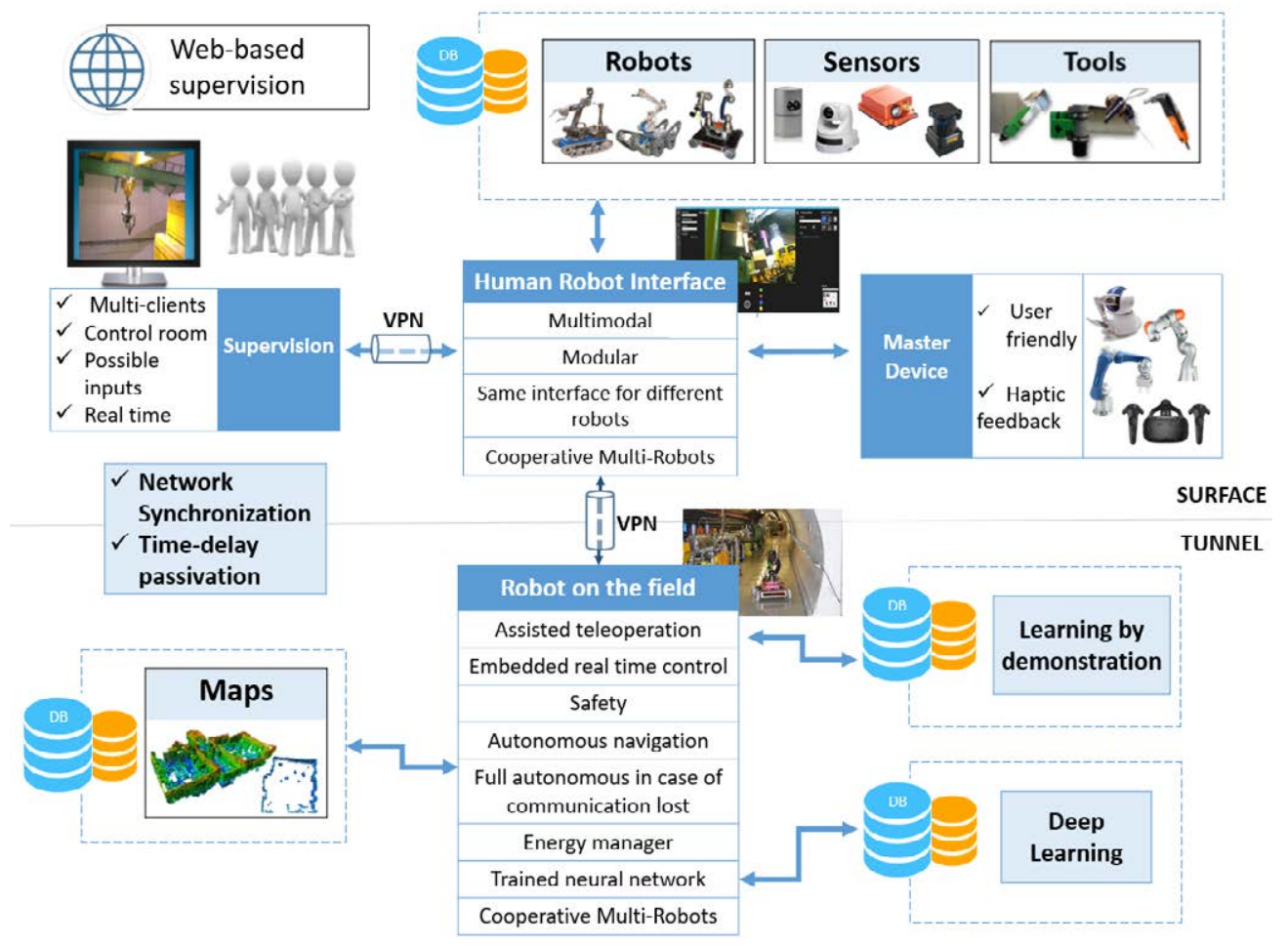

Figure 3.3: The overall architecture of the CERNTAURO framework. The robot on the field has an onboard intelligent control featuring capabilities like autonomous navigation and energy management. The comunication in between the field/tunnel and the surface is guaranteed through a virtual private network to allow data encryption and safety. The novel human robot interface developed integrates several features like multi-robots cooperation and connection with different supervision layers including multi-client and web applications.

the embedded intelligence of the robot, necessary for allowing safe operation in the remote environment. The Virtual Reality, the intervention preparation and all the other modules, integrate these two main agents in the complete intervention pipeline in order to guarantee the safest and quickest possible operation. A virtual private network (VPN) using PPTP connection with CHAP encryption has been implemented for communication safety. The proposed novel robotic framework allows single or cooperative operators to control different robots by using the same human-robot-interface and gives the possibility to perform tasks using multiple collaborative robots (see Figure 3.5).

The control architecture of the proposed framework is designed to be modular and failsafe. In a semi-structured and hazardous environment, normally the communication link between the operator and the robot is not reliable as it is based on $3 \mathrm{G} / 4 \mathrm{G} / \mathrm{WiFi}$ and on standard internet protocols (TCP, UDP). Therefore, to guarantee safety and autonomy, the entire control loop should be closed on the on-board robot control system.

The CERNTAURO systems implement two types of control: 


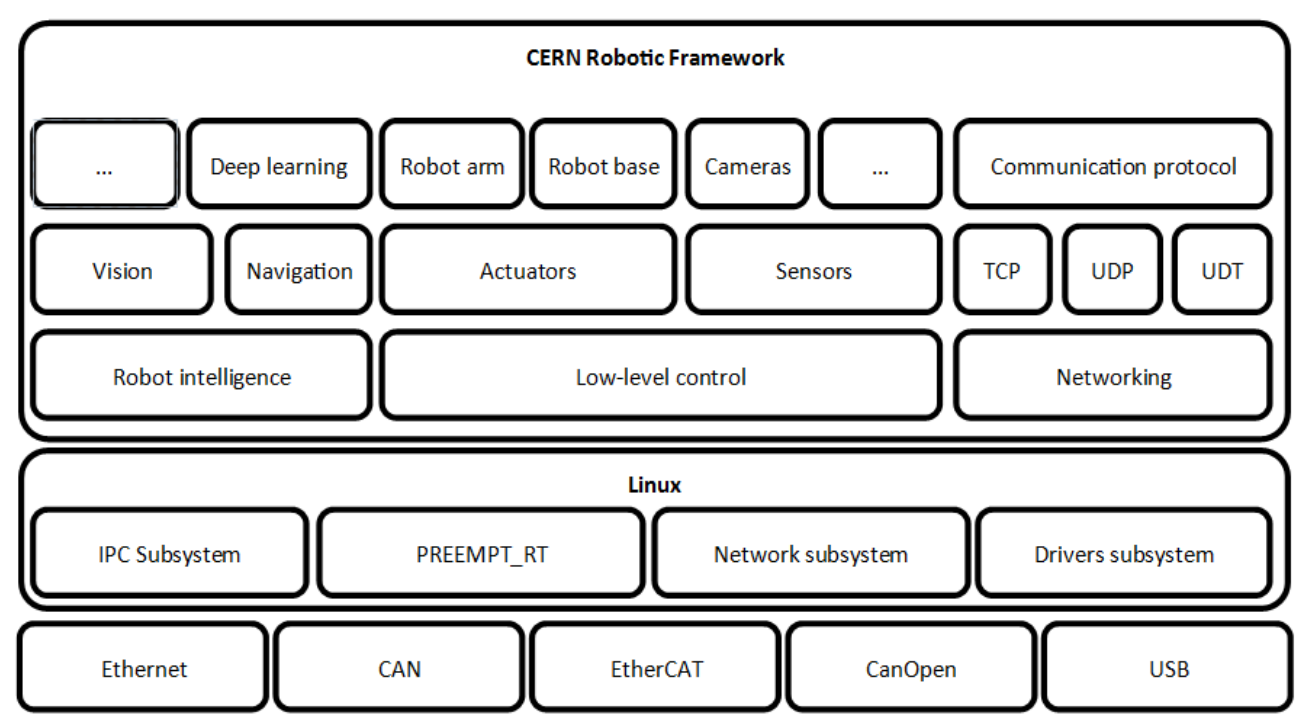

Figure 3.4: The CERNTATURO system stack diagram. The bottom layer is composed by fieldbus modules and drivers that comunicate with the hardware and the real-time linux based operating system. On top of this, the low-level control including the networking and the robot intelligence provide robot controls interfacing with the sensors and the actuators. The last layer is composed mainly by the artificial intelligence and comunication modules.

1. Supervisory control, in which the closed loop control is entirely on the robot side and the human operator receives feedback from the robot (visual, haptics etc.) and sends commands to it through a Human Robot Interface (HRI).

2. Fully automatic control, in which the closed loop control is entirely on the robot side and the human operator receives only feedback from the robot.

This structure provides a major functional specification for the design of the control system, in which all the modules must be interconnected. The control system must be always able to provide safe commands to the actuators, regardless of the state of the connection with the operator. The proposed control system is portable, modular and is divided in different layers (see Figure 3.6)

The Hardware Abstraction Layer (HAL) provides an interface between the system and the hardware. This layer is fundamental for the development of a modular and an adaptable system since it provides an abstraction interface between the hardware and the upper layers. Furthermore, the HAL also allows the use and test of modules belonging to upper layers by using simulated hardware. This is extremely important for the system validation, operational procedures, and recovery scenarios and for the offline operators' training, who can operate a simulated robot in advance as a preparation for the real intervention. The control layer provides all the control strategies for the actuators including the robotic arm controls (e.g. position control, trajectory control, kinematic control, torque control) and the robotic platform control (e.g. speed regulation, omnidirectional control). The supervision 


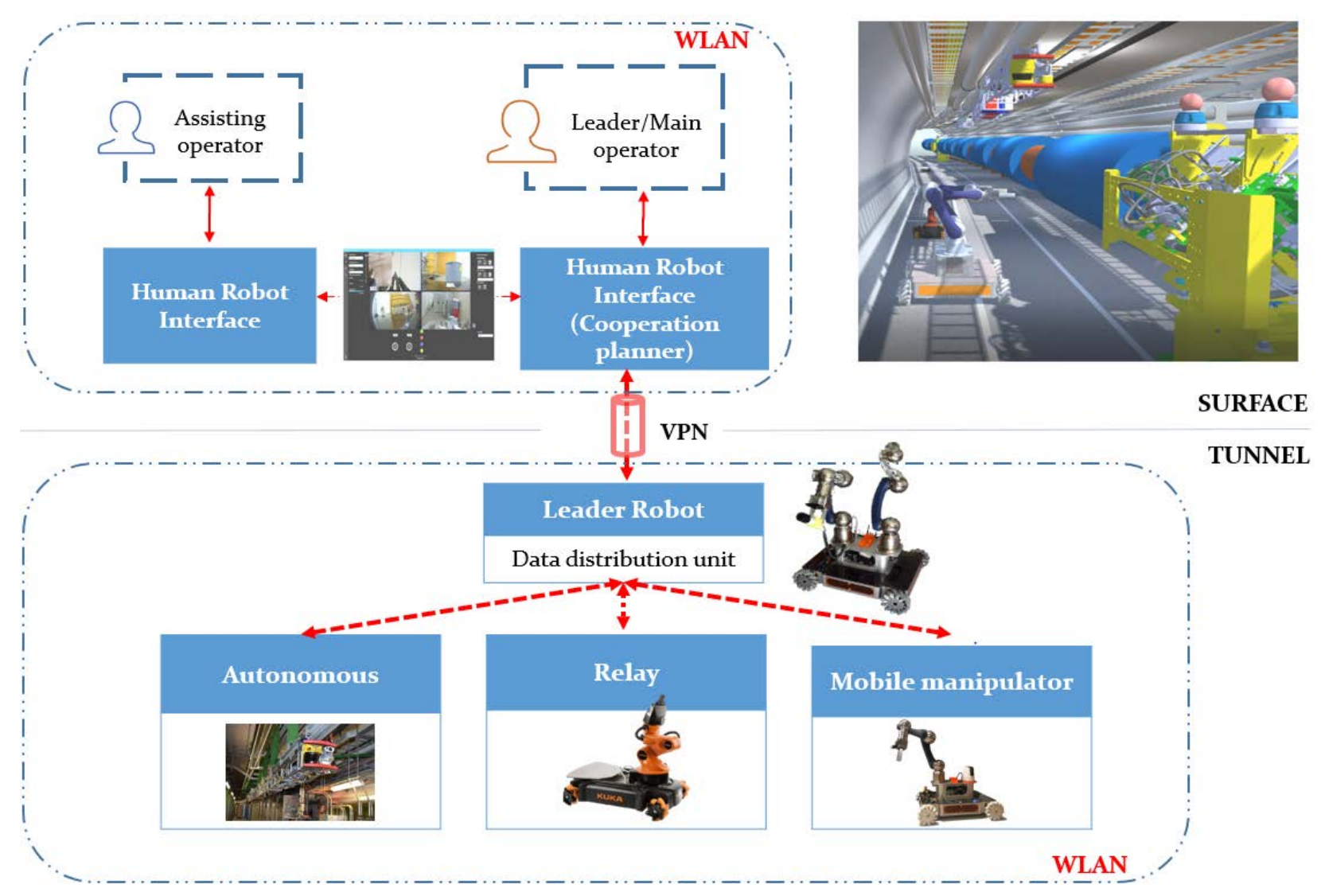

Figure 3.5: CERNTAURO Architecture for Cooperative Multirobot Interventions. Simulation in virtual reality of collaborative robots running CERNTAURO framework (top-right). The architecture for Cooperative multirobot is based on a Leader robot that on the field distributes and manage the different intervention tasks. The Human Robot Interface manages all the high level cooperation planning using also intervention scripting tools. The human control of the CERNTAURO framework for the robotic interventions consists in a leader/main operator and an assisting operator. 


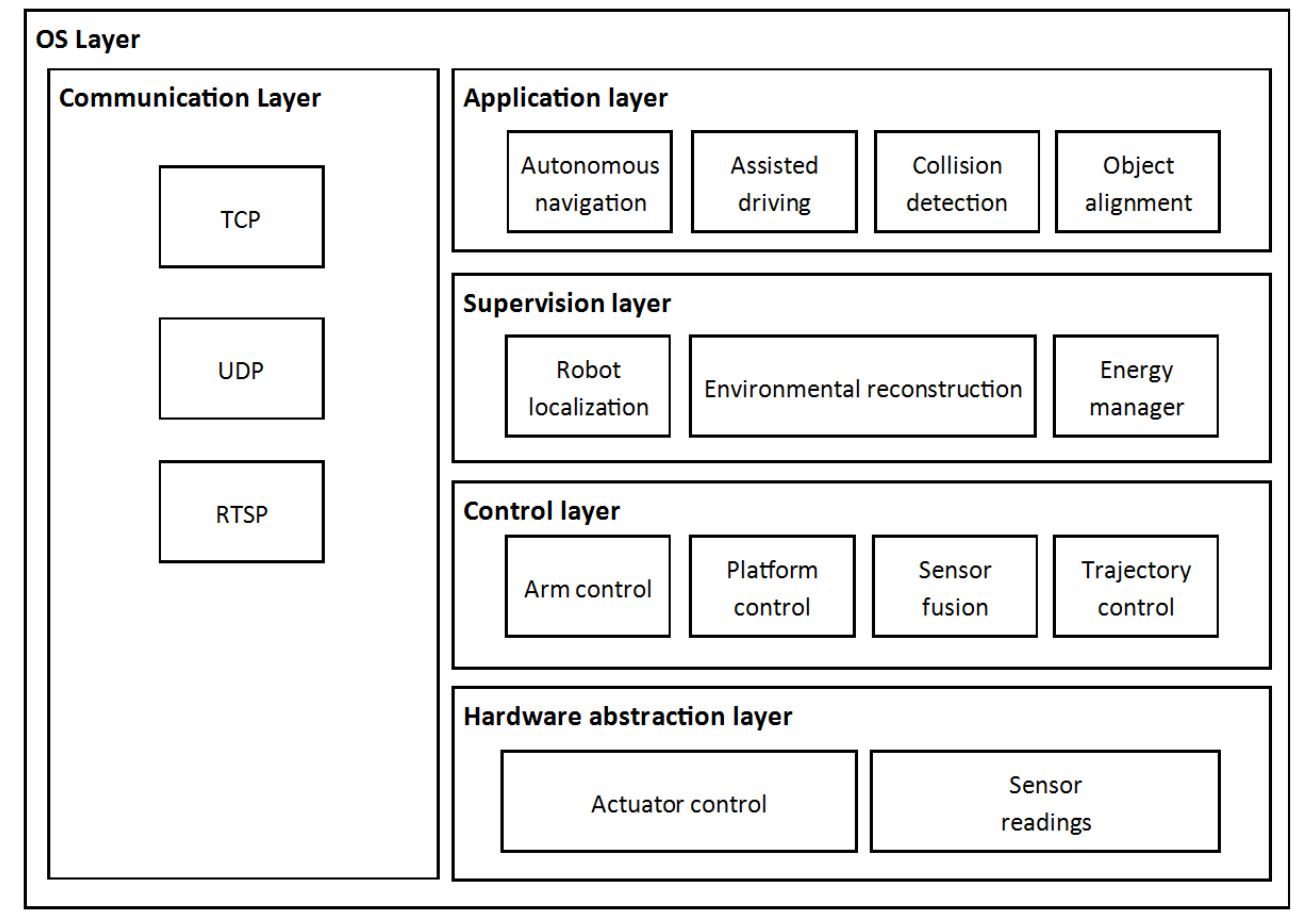

Figure 3.6: CERNTAURO control system architecture divided in layers allowing modularity and adaptability of the control system to different hardware. The Hardware Abstraction Layer provides an interface between the system and the hardware. The control layer provides all the control strategies for the actuators. The supervision layer contains all the modules which are responsible for determining the complete state of the system in each moment. The application layer contains all the features that the robot can provide such as autonomous navigation. The communication layer provides all the communication methods with the HRI while the OS layer provides all the functionalities in order to maintain all the modules properly active and interconnected. 


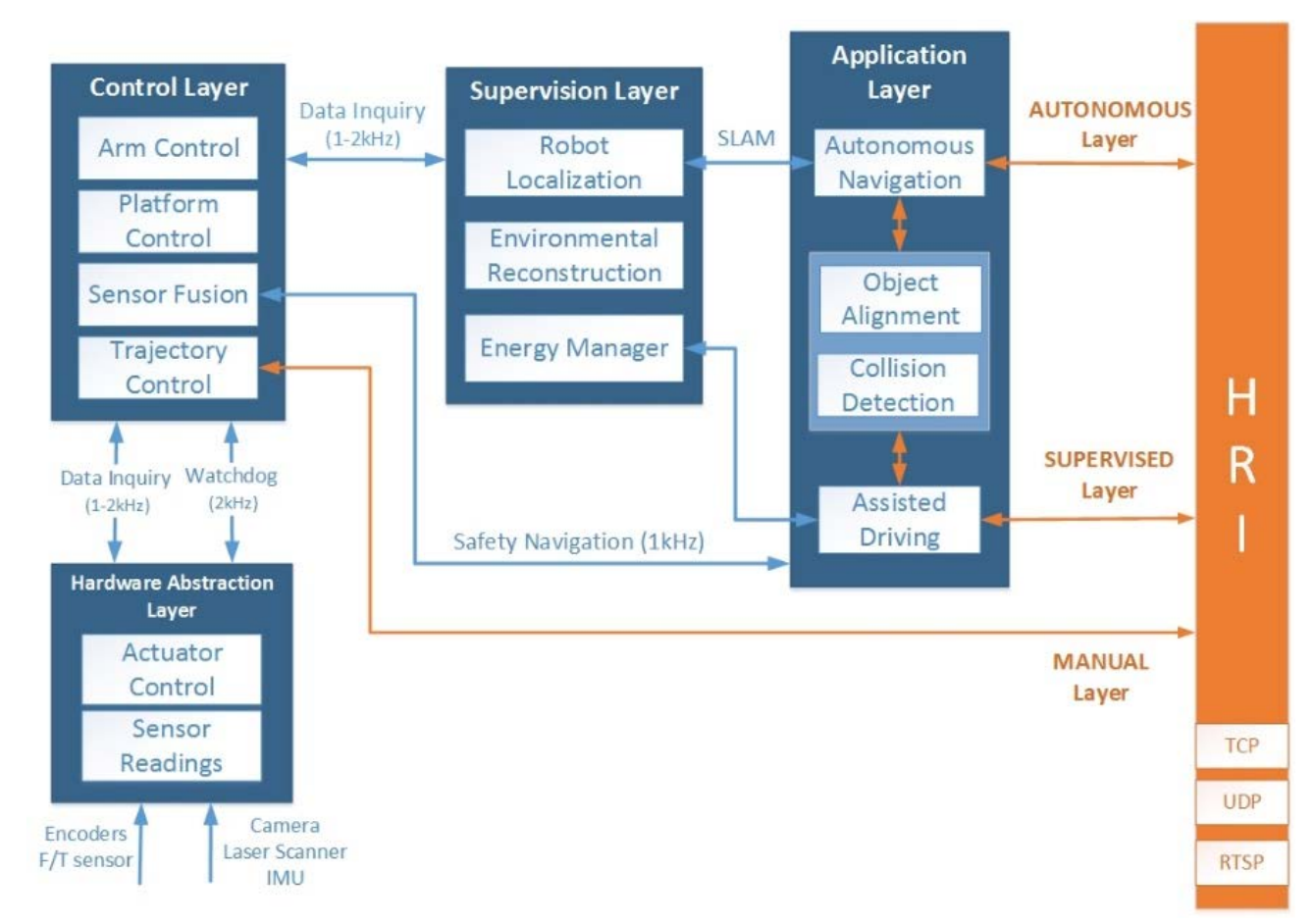

Figure 3.7: Software communication diagram that describes the control system communication in between the different layers described in Figure 3.6

layer contains all the modules which are responsible for determining the complete state of the system in each moment. It contains therefore the robot localization, environmental reconstruction, the battery management, the communication optimization, etc. Finally, the application layer contains all the features that the robot can provide such as assisted and autonomous navigation [194], collision avoidance [195], autonomous object recognition and alignment [196], as well as a sequencer of multiple operations. Each part is treated as a HAL with a standard interface for the COS. This allows flexibility and portability of the proposed software, which can be adapted to different robotic types and hardware. In Figure 3.7, the software communication diagram implemented in the proposed framework is presented.

In addition to the mentioned control layers, there are two layers which span the entire system: the communication layer and the OS layer. The communication layer provides all the communication methods with the HRI including methods for timestamp synchronization between the HRI and the robot, as well as communication compression and optimization methods. The communication layer is connected with all the modules, since each module can provide and receive, if requested, data to and from the HRI. The com-munications layer canalso act as a sensor,by using the RSSI, for localization (see Section 4.3) Above all, the OS layer provides all the functionalities in order to maintain all the modules properly active and interconnected. All the modules communicate together through Inter Process Communication (IPC) but not all the modules are active simultaneously and modules can be activated or deactivated automatically or by the operator according to the needs. When 
a new module is activated or deactivated, the OS layer manages the reconnection and the redirection of the IPC. For example, the operator can choose to drive the robotic platform by sending direct commands or by the use of the assisted driving module in the application layer. The driving commands also go through the energy management to optimize the power consumption of the system. Therefore, when the operator chooses to use the assisted driving, the OS layer automatically modifies all the affected IPC connections in order to adapt to the new control configuration.

The OS layer also provides watchdog functionalities; each module has its own priority and its own criticality. According to its criticality and priority, if the module suddenly stops, the watchdog detects this stop and manages it, by restarting it or by interrupting other modules in order to maintain the system in a safe state. In addition to energy management algorithm implemented in the CERNTAURO control architecture (see Section 3.2), a sleep mode possibility has been implemented. If the communication is lost, for example during a teleoperation task, and the battery are emptying, the control system puts the hardware in sleep mode avoiding the complete shut-down of the system and it will wait for the communication to be back available. As it has been conceived and realized, the COS is multi-platform and compatible with real-time controllers, features which are absent in the most common robotic control systems, such as in ROS [197].

Thanks to its implementation, the COS is light and it can manage several sub-tasks like SLAM [198] [199], autonomous navigation, data acquisition, robotic controls among others, in parallel and on-board. The proposed novel control system has been conceived also to be a complement of ROS (see Table 3.1).

\subsection{Methods for Multirobots Maintenance Operations}

When preparing for a robotic intervention, several methods and procedures should be applied to guarantee the safety and the efficiency of the tasks to be performed by the robots. For the presented novel robotic control framework, a new series of procedures, in the form of modules, have been designed and commissioned for the robotic operation in CERN accelerators facilities. These modules, in order, are the functional requirements, the failure modes and recovery scenarios, the best practice, the procedures preparation and the operator training. The modules and procedures are explained in the following subsections. In order to achieve a successful robot operation, the first vital step is the full acknowledgement of the functional intervention requirements. It is important to distinguish between a machine-related task and its possibilities in reality. The proposed CERNTAURO framework can be integrated into different robots, and this feature has driven the design of the Functional Requirements Module (FRM). The FRM is an automatic decision tree algorithm that helps the intervention preparation by suggesting the choice of the robot from functional requirements like type of requested task, payload, compliancy, dexterity, hazards and intervention time. The FRM takes information of the existing mechatronic configurations and tooling possibilities from the robots and tooling pools, where the most important features of each device and tool are listed and classified according to the priority of executing different 
Table 3.1: SOME CERNTAURO ADDED VALUES WITH RESPECT TO ROS

\begin{tabular}{||c|c|c||}
\hline Feature & ROS & CERNTAURO \\
\hline \hline Multiplatform & NO & YES \\
\hline Computational needs & Needs a middle level computer & $\begin{array}{c}\text { Runs on industrial NUCs } \\
\text { and embedded PCs } \\
\text { Control full real-time } \\
\text { system compatible }\end{array}$ \\
\hline $\begin{array}{c}\text { Safety: control tolerant to } \\
\text { environmental disturbance } \\
\text { (e.g. radiation) }\end{array}$ & NO & $\begin{array}{c}\text { YES. Triple modular } \\
\text { redundancy to avoid } \\
\text { single event effect due } \\
\text { to radiation }\end{array}$ \\
\hline Real-Time messaging & YES but needs some updating & YES \\
\hline $\begin{array}{c}\text { Reconfigurable GUI with } \\
\text { different robots }\end{array}$ & NO & YES \\
\hline 3D Setup tool & NO & YES, VERO module \\
\hline Data security & NO & YES \\
\hline $\begin{array}{c}\text { Energy Management } \\
\text { Software verification } \\
\text { and validation }\end{array}$ & NO & YES \\
\hline Final scope & $\begin{array}{c}\text { Intended for university } \\
\text { and R\&D applications }\end{array}$ & $\begin{array}{c}\text { Intervention in real } \\
\text { scenarios as well as R\&D }\end{array}$ \\
\hline
\end{tabular}

tasks (see Table 3.2 and Table 3.3). The module then automatically suggests the robots and the tools to use for the desired intervention (see Figure 3.8). The FRM also provides the possibility to combine different robotic components. For instance, integrating different robotic arms on different UGVs or in the case of relay robot needs, it can suggest the use of different robots as WiFi relays to extend the communication coverage [200]. On the database of the robotic pools, the entire features of each robot are listed, but the proposed automatic module can dynamically configure types of robots and tool suggestions mixing the basic ones with different properties.

Table 3.2: A reduced example of a database fixed table of the CERNTAURO Robotic Pool

\begin{tabular}{|c|c|c|c|c|}
\hline Robot name & $\begin{array}{c}\text { Payload } \\
{[\mathrm{kg}]}\end{array}$ & $\begin{array}{c}\text { Angular } \\
\text { max speed } \\
{[\mathrm{rad} / \mathrm{s}]}\end{array}$ & $\begin{array}{c}\text { Linear } \\
\text { velocity } \\
{[\mathrm{m} / \mathrm{s}]}\end{array}$ & $\begin{array}{c}\text { Dexterity } \\
{[\text { from A }} \\
\text { to F] }\end{array}$ \\
\hline $\mathrm{A}$ & 5 & 15 & 0 & $\mathrm{~B}$ \\
\hline $\mathrm{B}$ & 7 & 40 & 1.5 & $\mathrm{C}$ \\
\hline $\mathrm{C}$ & 50 & 5 & 2 & $\mathrm{E}$ \\
\hline
\end{tabular}


Table 3.3: A reduced example of a database table of the CERNTAURO Tooling Pool

\begin{tabular}{|c|c|c|c|c|}
\hline Tool name & $\begin{array}{c}\text { Torque } \\
{[\mathrm{Nm}]}\end{array}$ & $\begin{array}{c}\text { Angular } \\
\text { max speed } \\
{[\mathrm{rad} / \mathrm{s}]}\end{array}$ & $\begin{array}{c}\text { Linear } \\
\text { velocity } \\
{[\mathrm{m} / \mathrm{s}]}\end{array}$ & $\begin{array}{c}\text { Dexterity } \\
{[\text { from A }} \\
\text { to } \mathrm{F}]\end{array}$ \\
\hline $\mathrm{A}$ & 24 & 15 & 0 & $\mathrm{~B}$ \\
\hline $\mathrm{B}$ & 4 & 40 & 1.5 & $\mathrm{C}$ \\
\hline $\mathrm{C}$ & 5 & 5 & 2 & $\mathrm{E}$ \\
\hline
\end{tabular}

As an example, if a fine grasping and manipulation is needed, the FRM will most likely suggest using Robot B (high dexterity required) with Tool C, while it would choose Robot $\mathrm{C}$ with Tool A if a high payload was needed. The module can also indicate if a robot and/or a tool that can fulfil the demanded tasks are not present in the robotic pool, indicating then the need to design or modify new robots and tools.

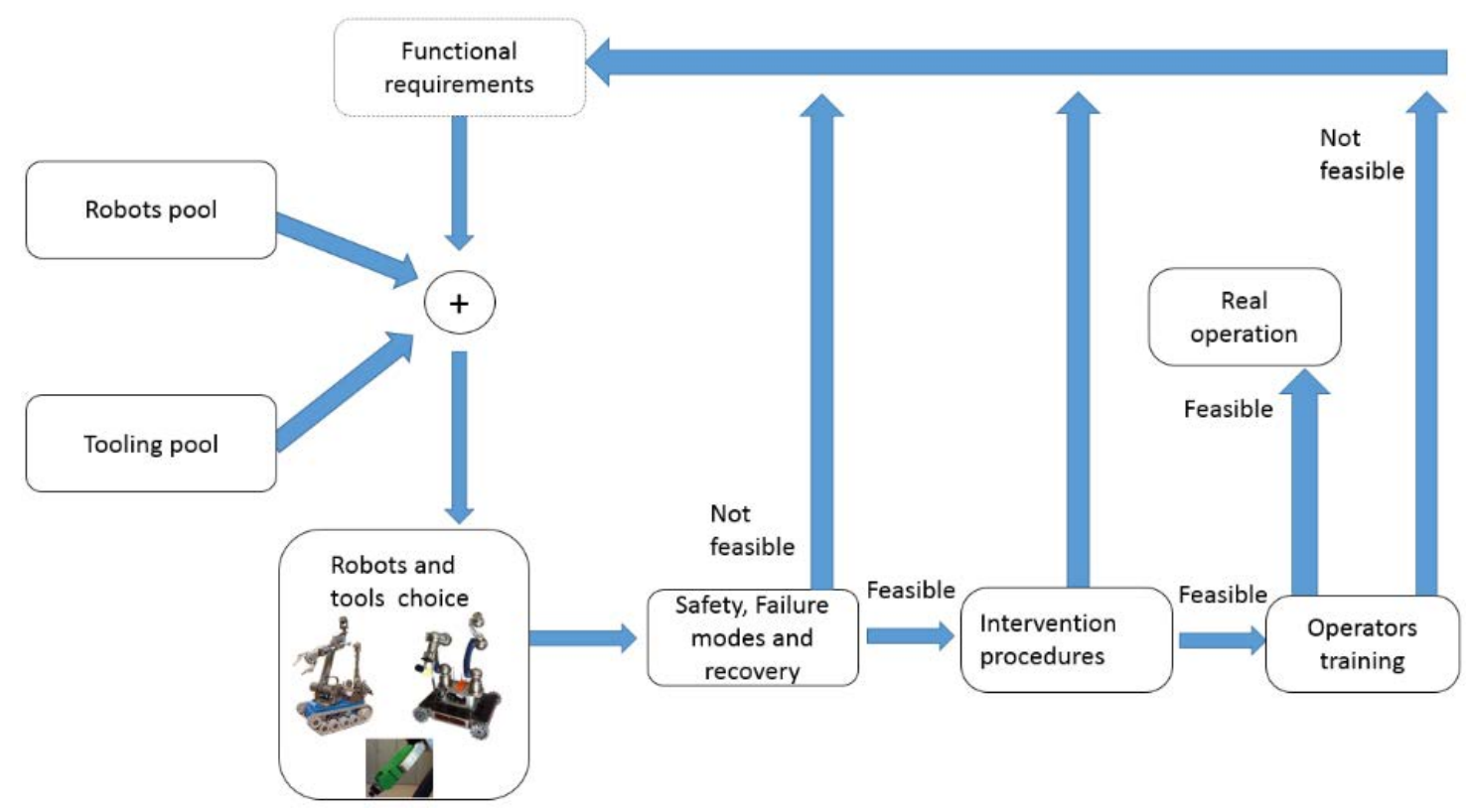

Figure 3.8: Pipeline scheme of a CERNTAURO robotic intervention preparation

Once the framework suggests the appropriate robot to use for a requested remote intervention, the functional requirements' designs are conducted through a safety aspect implementation, including failure mode identification using clustering analysis and recovery scenario procedures. These two fundamental steps are strictly iterative during the first phase of an intervention preparation: safety or recovery scenario procedures can influence, and in some cases, adjust the requirements because of technological limitations. Machine and human safety aspects need to be taken into consideration, for example the risk of radioactive environments or places with a high magnetic field presence that can negatively influence the normal operational behaviour of mechatronic systems. 
For the intervention preparation, the CERNTAURO framework has been equipped with the Virtual Environment for intelligent Robotic Operations (VERO) module, which takes in input of all relevant CAD models and drawings. From this data, a virtual environment for simulation is created using Unity $[201,202]$ as a framework for virtual environment simulation. As a framework for robotic mission planning and training, this module is fundamental for the design of the robotic procedures, tools and recovery scenarios that are vital in harsh and hazardous environments. According to the tasks and missions requested, a training program for the robot and the operators has to be set up. Part of the CERNTAURO framework is the Robot Operator Training (ROOT) module which, according to the difficulty of the mission and the dexterity demanded, suggests the type of training and learning that the operators and the robots must undertake. During the offline training, time and operator stress are monitored. One of the main requirements during the development of this system was to create an environment which is usable for different types of operators. Therefore a classification of the operators was done and different categories of operators were defined [186] according with their robotic operation skills and experience. The development of the system then took into account these categories, resulting in a system that is usable and learnable for entry-level operators but also provides all the advanced features required by an expert operator. From the low-level robot control to the Human-Robot Interface, the entire development was done in a way to provide a complete and involving experience to any type of operator. A robotic simulator framework using Unity and Gazebo [203] was created. In this simulator, the entire robot pool in all its configurations is integrated, not only in its mechanical aspects, but also in its control and sensor systems, including cameras. In this simulator, it is then possible to include the intervention area model, which is obtained by integrating $\mathrm{CAD}$ drawings or real 3D reconstructions obtained from previous inspections of the same area by the use of RGB-D cameras or pictures used in a Structure from Motion pipeline [204]. This model contains all the details of the real environment including cable positions, unexpected objects, etc.). The simulator provides an interface, which allows an operator to control any robot through the multimodal Human-Robot Interface. Consequently, the operator will train using exactly the same interface which will be used during the real intervention, providing a more accurate experience during the training phase. During the training period, intervention procedures are established by following best practices in remote handling, as well as the definition of the need in terms of tools and recovery scenarios in case of accidents. After each intervention, the robots are checked for possible radiological contamination following procedures included in the proposed framework [205]. The robots of the CERNTAURO pool are modified to be airtight avoiding contaminated dust to enter inside them minimizing contaminated waste [206]. New techniques for sealing are under study, having undewater techniques as references (e.g. bluerov cilinders for cameras [207]) .In addition, the materials of the robots are chosen to minimize the robots radiological activation $[208,209]$. The CERNTAURO framework includes a debriefing template document to be filled by the operators and the clients and its goal is to analyze eventual problems or matters which can be improved for future robotic tasks. This preventive work for future robotic tasks is fundamental in places where robotic services are needed.

During the preparation of the presented framework, a robotic team at CERN including 
students and technical staff has been built and lead by the author of this thesis.

Based on the experience at JET and SNS, to ensure the quality and the efficiency of the remote handling $(\mathrm{RH})$ and robotic support that the team has guaranteed for the whole CERN community during the last years, the robotic team formation has been created with the following stakeholders:

- The RH team leader that manages the project.

- The RH equipment design engineer.

- The RH system engineer.

- The RH simulations student.

- The RH operation engineers and students.

- The RH operation supervision engineer.

- The plant operator (if present).

Experiences in Remote Handling $(\mathrm{RH})$ for maintenance, for example at CERN, JET and SNS, have demonstrated that the remote manipulation tasks are made safer and faster if the plants handling maintenance needs are taken into consideration from the design phase of its development cycle.

The continuous development model of the presented work has been tasted and validated in several occasions in CERN accelerators facilities and it has demonstrated that the feasibility of handling tasks on devices not designed previously for RH compatibility ranges from being impossible to being possible but at significant cost in time and money. In the cases at CERN where remote handling was performed on non- $\mathrm{RH}$ compatible plant, the time needed to perform the tasks has been of the order of $2-5$ times longer than similar tasks involving plant which had been designed with $\mathrm{RH}$ compatibility. The amount of time required to intervene in zones not designed to be RH compatible, strongly depends on the level of knowledge of the environment and on the modularity of the remote system that could be adopted. In addition, the remote handling tasks, even on machine not fully compatible with remote handling devices, are made much easier if safe and robust procedures are in place, setting-up standards for RH in particular challenging tasks. These mentioned aspects have given the motivation to create the modular robots and the robust intervention procedures presented in this work.

Based on the several intervention done at CERN using the CERNTAURO framework, we can state that, at design stage, the main elements needed to ensure a RH compatibility of a plant are as follows:

- Use of only RH compatible mechanical interfaces, electrical connectors, fluid couplings, flanges, fixing, patch panels etc. 
- Use of only RH compatible size (this strongly depends on the type od RH devices/robots that need to be adopted.)

- Use of self-alignement pins, lifting attachments, gripping elements, visual cues etc.

- elimination of all non-captive parts, use of removable/replaceable threaded inserts, inclusion of mechanical compliance on pipework to facilitate alignment.

- Accessibility around the machine to be manipulated taking into account the dynamic of the remote manipulator, the end-effector, tools and general equipment.

- Clearance aroud the machine to guarantee viewing.

- avoid reflecting surfaces to avoid light reflections in the viewing sytems.

- Guarantee machine safety protecting delicate and sensitive equipment.

- Guarantee accessibility to eventual recovery machines.

- Design a realistic failure mode scenario to generate robust procedure and eventually adjust the $\mathrm{RH}$ requirements.

At the manufacturing and build stage, the main elements needed to ensure a RH compatibility of a plant are as follows:

- adoption of RH authorized quality requirements e.g dimensional tolerances on bolt threads, surface finish and dimensional tolerances of mating parts etc.

- Qualification tests of plant for RH authorization.

Remote handling requirements must take into account designs optimized to reduce human exposure to hazards during the lifetime of the equipments. Optimisation may be in terms of speeding up hands-on interventions or by ensuring design is compatible with remote handling techniques. To be noted that in many cases, remote handling equipment available is not always known at time of design. For RH tasks, good design practices for installation guidance, fasteners and connectors must be considered. Iterations and application of principles to real project cases including a quantification of cost and benefits must be taken into account.

Thanks to the operational experience and $\mathrm{R} \& \mathrm{D}$ provided by this thesis work, a code of practice document, described in this section and named CERNRHCOP (CERN remote handling code of practice), has been released. The code of practice is also the basic document for the ITHACA (Intervention in Highly Activated Area of Hi-Lumi LHC) working group created for the High Luminosity LHC project at CERN [210]. Originally this document has been based to the ITER remote handling code of practice and during the year has been adapted to new technologies and CERN needs. The CERNRHCOP contains information related to industry best practice in designing machine for remote handling compatibility 
based on CERNremote handling standards. This document is intended to be used as a reference guide by all personnel who have an interface and influence in the successful outcome of the CERN maintenance activities. These stakeholders include not only the remote handling responsible engineers but also the personnel responsible for the design of the machines. In general the efficiency and safety of remote handling operations is not only related to the design and operation of the remote handling $(\mathrm{RH})$ equipment itself, but also most significantly on the RH compatibility of the items being handled. The remote handling compatibility of the CERN plant can be assured by the application of the methodology specified and described in the CERNRHCOP, together with the application of approved standards. Important aspects like positioning of patch panels, accessibility and labeling must be considered. The proposed code of practice guidelines aims to guide the choice and the design of different interfaces and connectors that could be used for robust remote handling task execution. An example of a proposed electrical connector suitable for remote handling is shown in Figure 3.9. More info on the CERNRHCOP module are detailed in Appendix E.
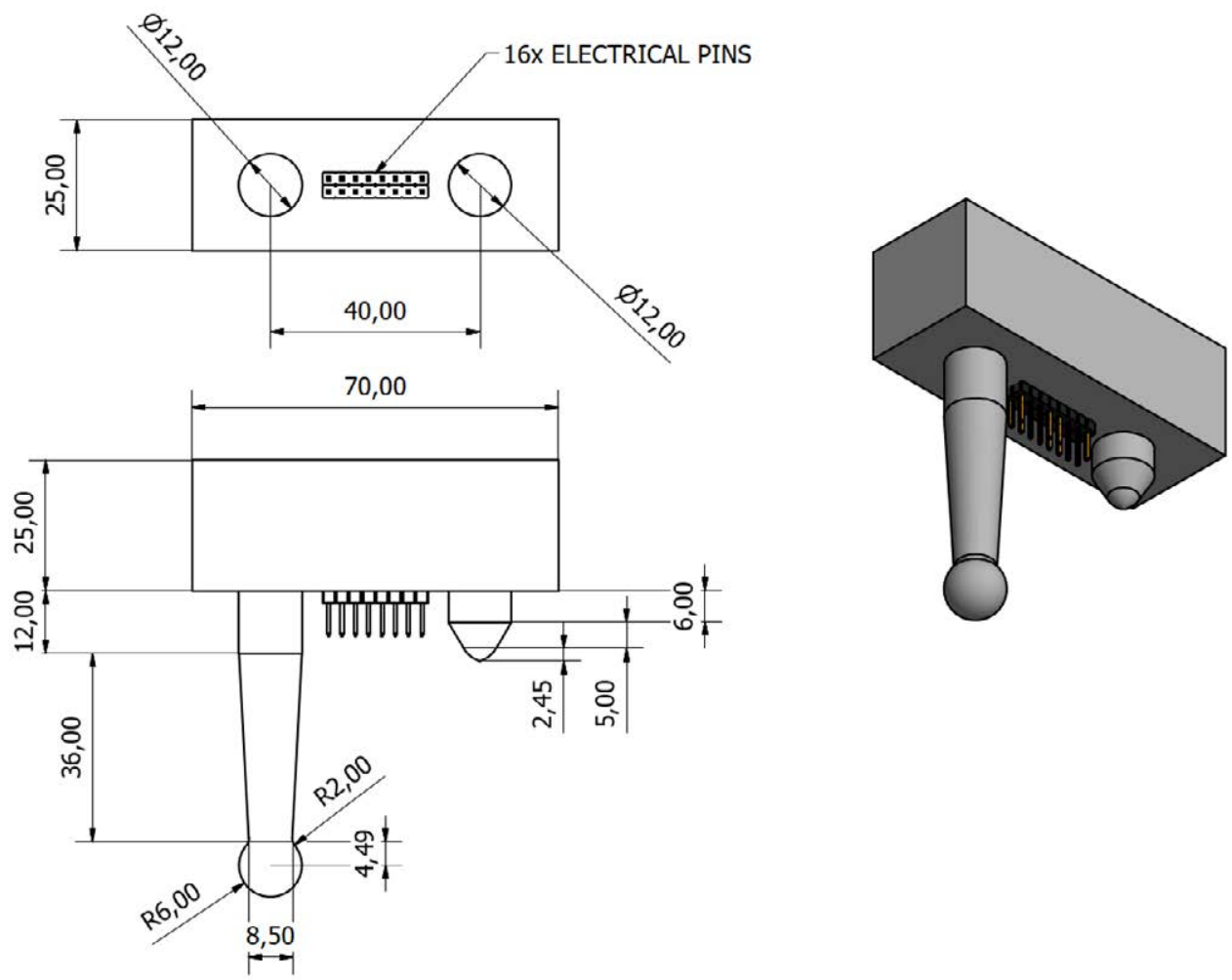

Figure 3.9: Dowel for electrical connectors example

\section{Energy management}

Energy management is fundamental when assessing teleoperated, semi-autonomous and autonomous tasks on battery powered robots. It is important to quantify the residual energy 
available in order to always allow a safe return of the robot to the base station. This is possible only by knowing the power consumption profile of each component of the robot and by knowing the discharge characteristic of the batteries.

The first step is to estimate the battery state of charge. The state of charge (SoC) estimation runs on the robot microcontroller, which is responsible of monitoring charging and discharging current of the batteries as well as their voltage. State of charge is one of the most important parameters, describing the battery system. Knowledge about its value and ability to make a correct estimation provide necessary basics for building an energy management system, capable to ensure significant energy savings. The field of state of charge computation represents a widely explored area with several stable methods developed for reliable state of charge estimation. However, due to the chemical nature of the battery, the challenge for precise calculations is still of current interest for research [211, 212].

An improved Coulomb counting method was chosen for state of charge estimation as it provides a satisfactory accuracy for the type of tasks performed by the robot. The procedure, based on integrating the current over the specific time period, includes an averaging method for instantaneous current measurements, suitable for non constant charge and discharge rates [213].

$$
S O C(t)=\frac{C\left(\widetilde{I}_{T, f_{s}}\right) S O C(t-\delta t)-\left(\widetilde{I}_{T, f_{s}}(t-\delta t)+\widetilde{I}_{T, f_{s}}(t)\right) \frac{\delta t}{2}}{C}
$$

The main advantage of the chosen method is that the only required input is the signal from the current sensor providing measurements of the out-flowing current with the fixed sampling rate. However, in order to understand when the battery is charging and when it is not, measurement of the in-flowing current is recommended to be obtained. This will give more uncertainty to the state of charge algorithm about the moment to reset the state of charge value to $100 \%$.

One of the most critical part of the previous equation is $C\left(\widetilde{I}_{T, f_{s}}\right)$, called rated capacity, which is usually available on the battery datasheet. For more accurate estimations, it is possible through a constant-current discharge process to estimate this function, which is dependent not only by the current discharge but as well by the environmental temperature and other factors. Furthermore, the system is sensitive to the current sensors accuracy. Once the state of charge is available, it is possible to estimate the power model of the robot. In the matter of this work, the power model of the robotic arm with payload and the power model of the motion of the CERNbot have been treated separately. The power model of the sensors, instead, it is considered as a constant value.

Knowing the power model of the robotic arm and the platform is necessary in order to predict the power consumption of the robot in future movements and optimise it when necessary.

\section{Robot arm power model}

For the purpose of creating the power consumption model of the arm, the experiments needed to be designed that ensure accurate and reliable data collection. According to the initial 


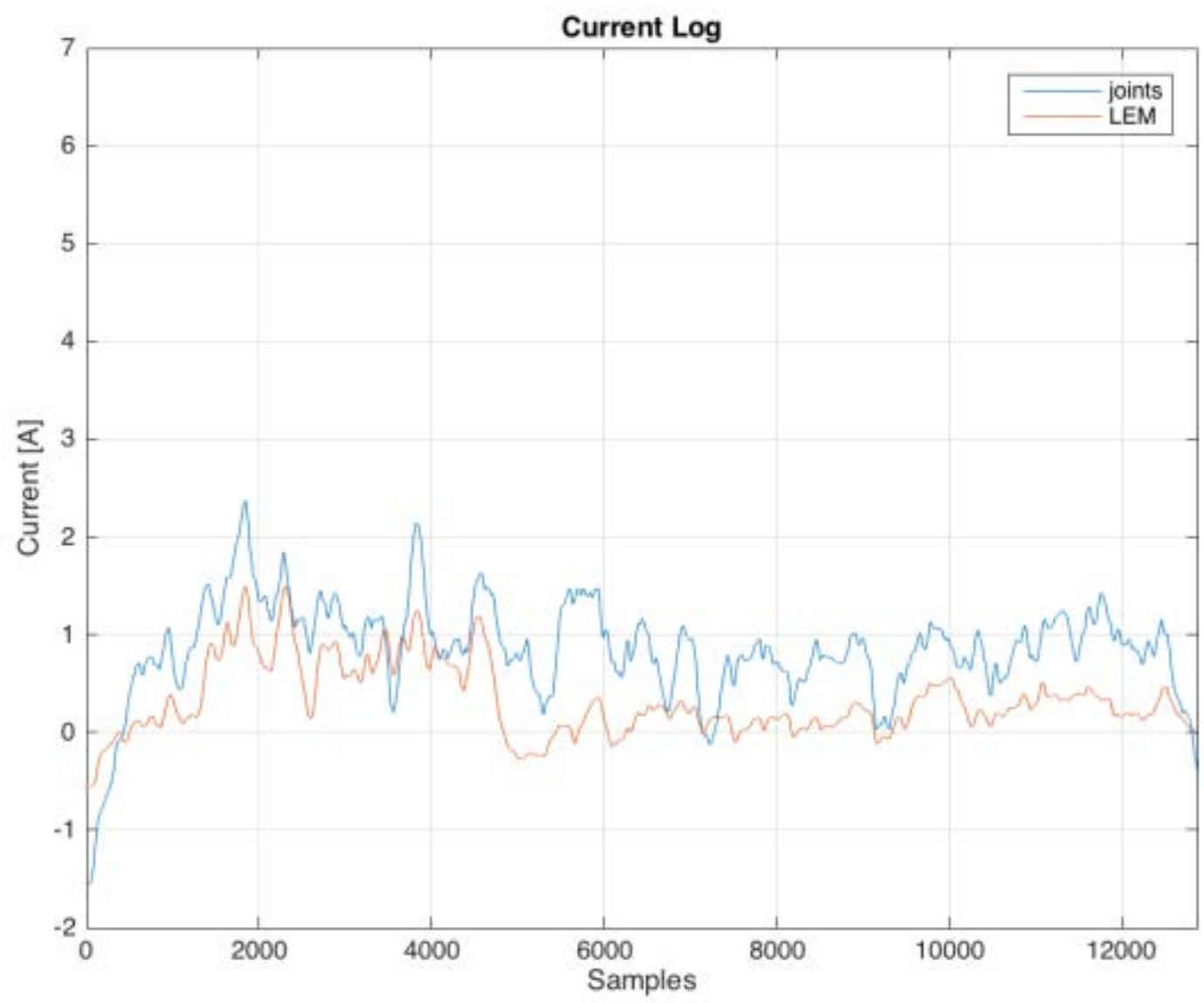

Figure 3.10: Results comparison of current measurements from LEM and arm sensors

hypothesis, robotic arm was considered being a highly non-linear system, which would make creation of a white box model very complicated. Furthermore, literature research showed that there are no established models that can reflect the power consumption by the arm joints, including possible influence of joints behaviour on each other. Therefore, the decision was taken to build a black box model based on experimental data. The estimation of the robotic arm power model has been done by performing several trajectories and logging position, velocity and current sensed by the internal robotic arm controller. Furthermore, in order to validate the accuracy of the internal current sensors, an external current sensor to measure the overall current consumption has been added. Figure 3.10 shows a comparison of the overall current measured by the internal sensors, compared with the current measured by the external sensor (LEM).

The logged data has been used afterwards to train a GPML algorithm for system identification. Two black-box model have been tested (see Figure 3.11). The first one returns in output the current of each joint, while the second one returns in output the overall current consumed by the robotic arm.

The training of the GPML model for the identification of these two black box system 


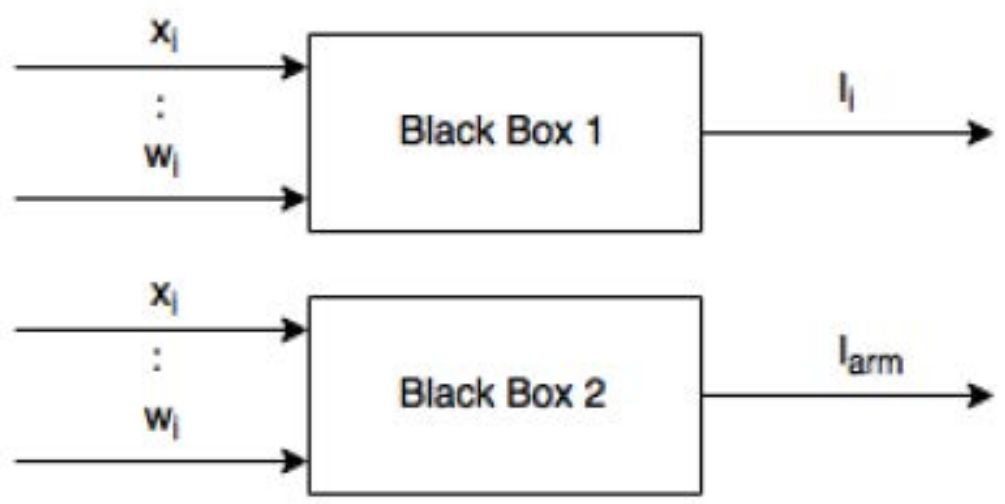

Figure 3.11: Robotic arm black box models

shows how the second system is better identified and it is able to predict better the current consumed by the robotic arm (see Figure 3.12).

\section{CERNbot power model}

Differently than the robotic arm, it is possible to use some already known models for the estimation of the CERNbot power model such as the mechanical model based on the Newton laws or a polynomial model for DC electric motor. Furthermore, various black box models have been tested in order to take in consideration as well all the non-linear effects of the motion.

Both the mechanical and the polynomial model, as well as various black-box models have been evaluated in terms of computational speed, accuracy, robustness and complexity.

According to the results shown in Figure 3.14, the fastest model is the mechanics one, while the slowest one is the GPML. Though it processes the same amount of input data, the latter one needs to pass through the learning stage of the algorithm which requires estimation of hyperparameters at every input. On contrary, mechanics model uses the friction's dependence on the velocity, which has a simple linear form and needs to be calibrated only once. Similarly, BJ SI model has a high run time as it goes through optimization procedure. Optimal set of model orders is determined by using the for loop, which compares achieved goodness of fit after every iteration. Complexity and robustness were defined as non-numerical criteria, which are judged based on the development of the algorithm. GPML model makes higher number of references to itself, while computing the means and kernels, in contrast, for example, to the polynomial model which uses only one calibration cycle. Finally, accuracy criteria demonstrates that SI models have the best precision out of all created models, as their goodness of fit has a significant value. However, one should keep in mind that SI models, as well as GPML models, are subjected to high dependency on the fed-in data. This means that achieved accuracy can be influenced by the particular range of dataset chosen, while mechanics and polynomial model are calibrated using the whole dataset. In order to choose the motion model all criteria should be considered simultane- 


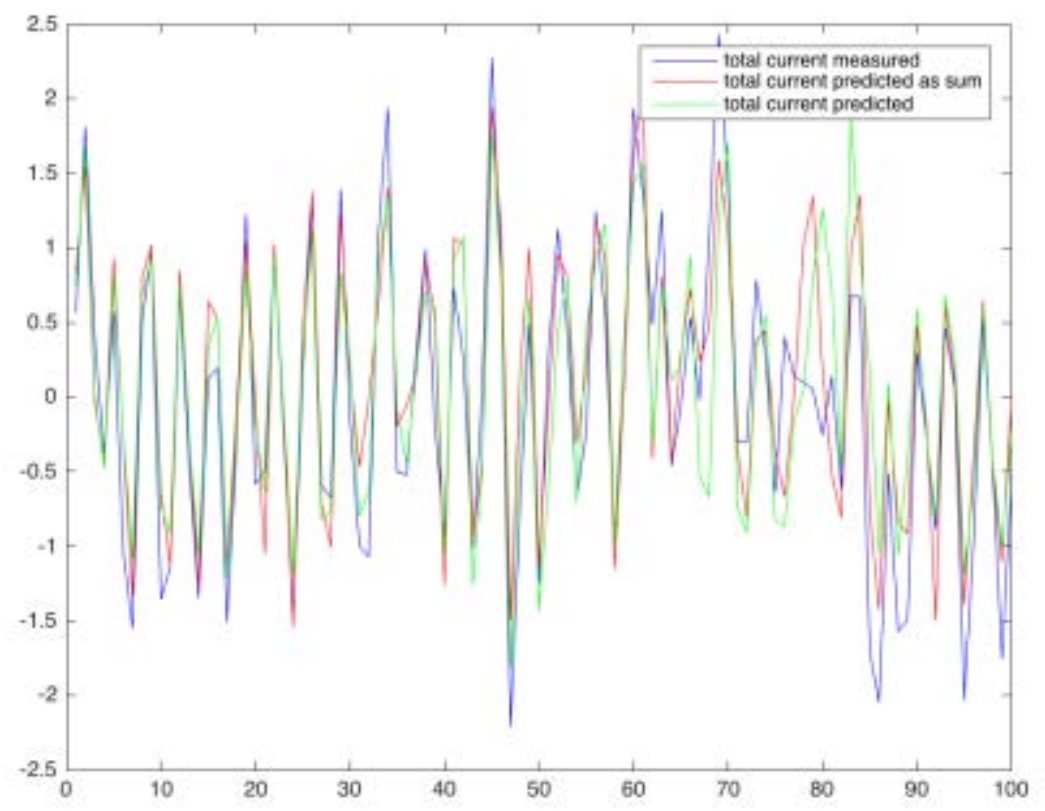

Figure 3.12: Comparison of two different black box models of the arm

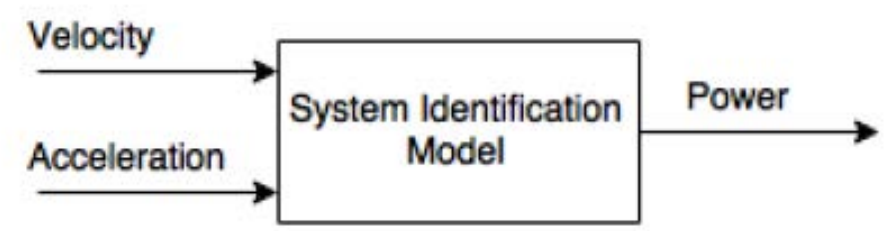

Figure 3.13: Black box motion model for System Identification 


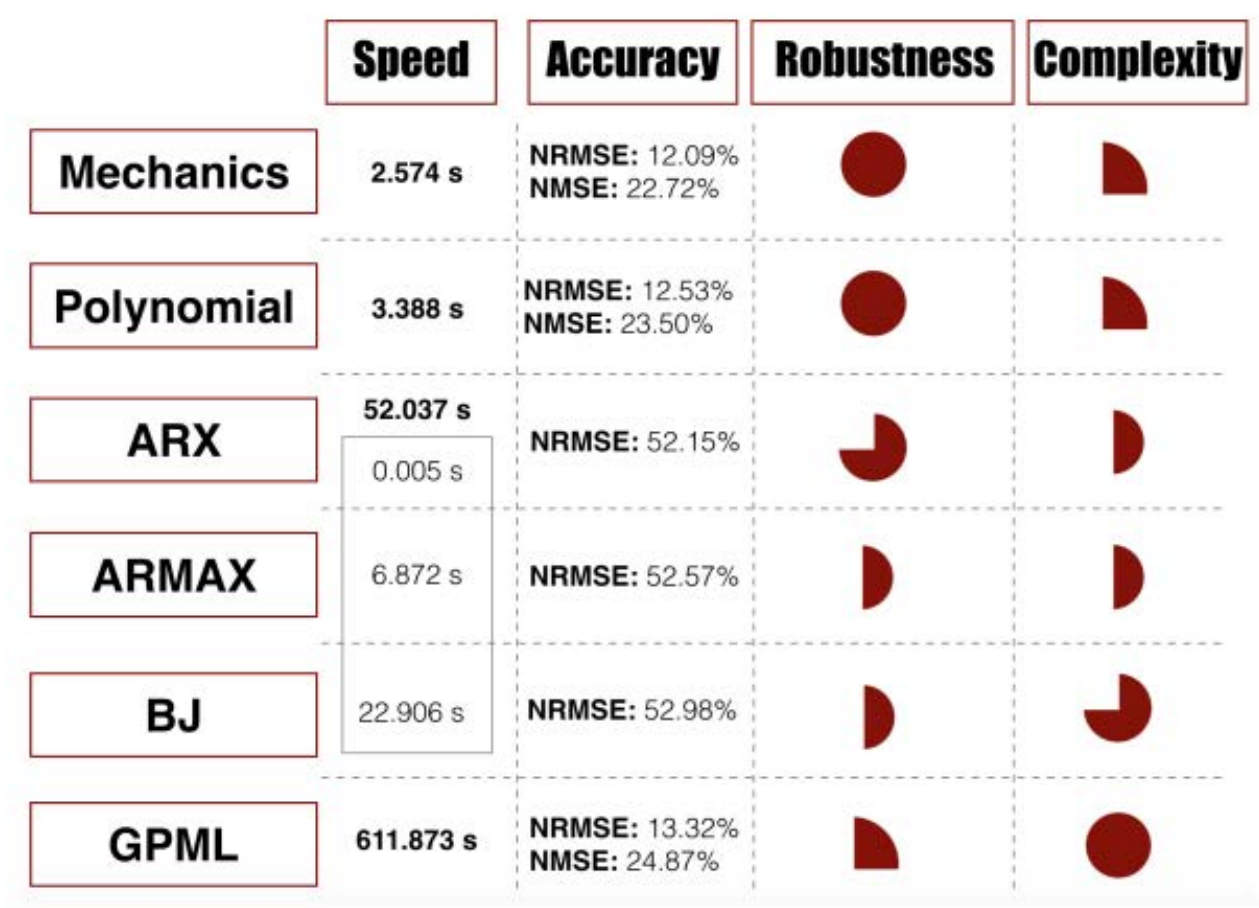

Figure 3.14: Comparison of motion models

ously and the trade-off between qualitative and operational criteria needs to be achieved. Thus, following the main goal that power consumption model serves as the platform for further optimization procedures, the polynomial model is chosen. It demonstrates satisfactory goodness of fit while having a high speed and simplicity of implementation. Moreover, it can be represented by a single equation, which makes it suitable for cost function development.

\section{Motion planning optimization}

Motion control for mobile robots represents a wide field of research with two primary objectives: trajectory planning, including obstacle avoidance [214, 215], and motion profile planning. The latter received a lot of attention from researchers in the recent years, as the problem of autonomous and semi-autonomous navigation is directly related to the fact that the amount energy, possible to be stored in the on-board batteries of the robot, is finite. Due to this, a rigorous motion planning should be performed in order to ensure efficient operation. Conditions of choosing the motion plan are subjected to the optimum dilemma: traversing the path with the prolonged mission duration for the sake of achieving considerable energy savings or neglecting energy usage in the interest of performing the actions as fast as possible. Both of these cases constitute extreme solutions of the problem, compromising the mission achievement. Therefore, the optimal motion plan can be determined as a trade-off between the time duration and power consumption, which leads to a multiple objective optimization problem. Satisfaction of both criteria can be achieved by finding an 
optimal velocity profile, which is unique for a given trajectory length and traversing time [216, 217]. Different approaches exist for its determination, mainly characterized as direct and indirect [218]. However, each of them has its own drawbacks such as low accuracy of numerical solution and probability of being blocked at the "pseudominima" for the direct methods and low convergence areas for indirect methods. Nevertheless, the algorithms developed resulted in definition of two main types of optimal velocity profiles [219, 220]. In the real applications trapezoidal velocity profile, comprised of acceleration, cruising and deceleration parts, is the most commonly used, as it is relatively easy to be constructed [221, 222, 223]. Despite of this, when considering energy savings, the minimum energy profile, representing a parabolic continuous function, is often compared with the trapezoidal profile [217]. Velocity profiles represented by sigmoid functions didn't receive yet a wide usage due to their computational complexity [221]. Overall, the choice of one velocity profile over the other is dictated by the pre-vailing objectives of the mission to be performed by mobile robot, which means the higher priority of either time or energy minimization. Additionally, it is worth mentioning that precisely defined power model of the robot and correct definition of the constraints of the physical system improve the accuracy of optimal velocity profiles found. Nonetheless, fluctuations of the energy consumed still can occur due to unaccounted losses in the system, deviations in following the predefined velocity profile or unexpected external influences coming from the operational environment.

Minimum energy profile Minimum energy profile appears several times in the literature, with the full mathematical explanation behind the model of the profile and proposed algorithms for its determination [224, 225]. However, all the papers concern only the case with zero initial and final velocities, therefore current thesis presents the definition of the minimum energy profile that takes into account nonzero values of these parameters. Let's assume no constraint on the maximum linear velocity of the base is introduced, though it is known that in reality maximum velocity bound exists because of mechanical limitations of the system or safety rules of working in the tunnel. If the solution for the unconstrained problem does not violate the real velocity maximum constraint, then it can be used to construct the optimal velocity profile. Otherwise, the constrained problem needs to be solved.

Trapezoidal velocity profile Being the simplest profile to construct, trapezoidal velocity profile is characterized by the high potential for energy savings [223, 217, 225]. However, the algorithms proposed in the literature, similarly to the minimum energy profile, are not generalized to nonzero initial and terminal velocities. Thus, current work provides a complete solution to determine position, velocity, acceleration and objective function in the formalism of trapezoidal velocity profile with user defined values for velocities at $t=0$ and $t=t_{f}$.

Figure 3.15 depicts typical trapezoidal velocity profile where main parameters and variables are demonstrated. Here $v_{0}$ and $v_{f}$ are the initial and the terminal velocities respectively, which can be both set by the user through the related input. Decision variables are present as $a_{\text {max }}$, which is the value of acceleration assumed being equal to the value of deceleration, 


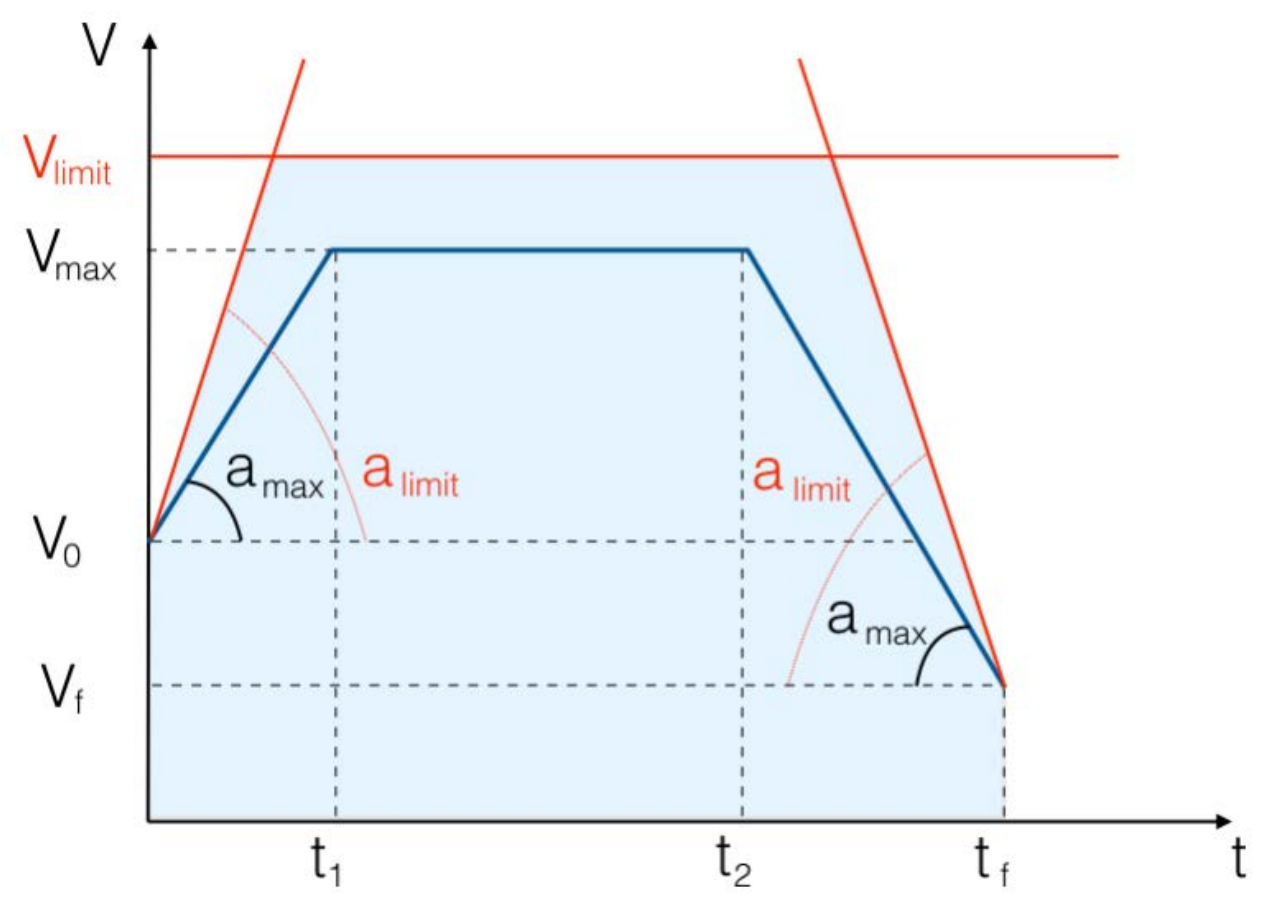

Figure 3.15: Trapezoidal velocity profile

and $v_{\max }$ which is a stabilised velocity at time $t_{1} t_{2}$. Terminal time $t_{f}$ can be either imposed by the user or calculated by the optimization procedure to ensure the fastest trajectory traverse. Values of limit velocity $v_{\text {limit }}$ and acceleration $a_{\text {limit }}$ constitute the constraints of the optimization problem. The area highlighted in blue is equal to the distance traversed during the mission and acts as one of the internal constraints to check the accuracy of the solution found. When the battery of the robot is fully charged, timing becomes the main issue of the movement security. As at the beginning of the mission available amount of energy stored is large, there is no need to economize the consumption, especially if it's known beforehand that the mission duration will not exceed the battery lifetime. Instead, shortening the operation time and finding how much time it actually takes to traverse the trajectory becomes interesting. Therefore, the separate case needs to be studied, where the robot uses maximum speed with the fastest acceleration profile.

Figure 3.16 demonstrates the geometrical approach of finding the final time to traverse the trajectory $t_{f}$. One can notice that due to actuator limitations, time t1 is fixed as it's unequivocally defined by the intersection of acceleration and velocity lines. Thus the distance left to travel after the acceleration phase $t_{1}$ can be found as following:

$$
\text { Distance }_{t_{1} t_{f}}=x_{f}-\frac{\left(v_{\max }-v_{0}\right) v_{0}}{a_{\max }}-\frac{1}{2} \frac{\left(v_{\max }-v_{0}\right)^{2}}{a_{\max }}
$$

Similarly due to the slope of $a_{\max }$, the area of the deceleration triangle is fixed, implying the equality of the areas $B t_{f} t_{2}$ and $C t_{f}^{\prime} t_{2}^{\prime}$. Following that, time period $t_{2}$ to $t_{f}$ is fixed as 


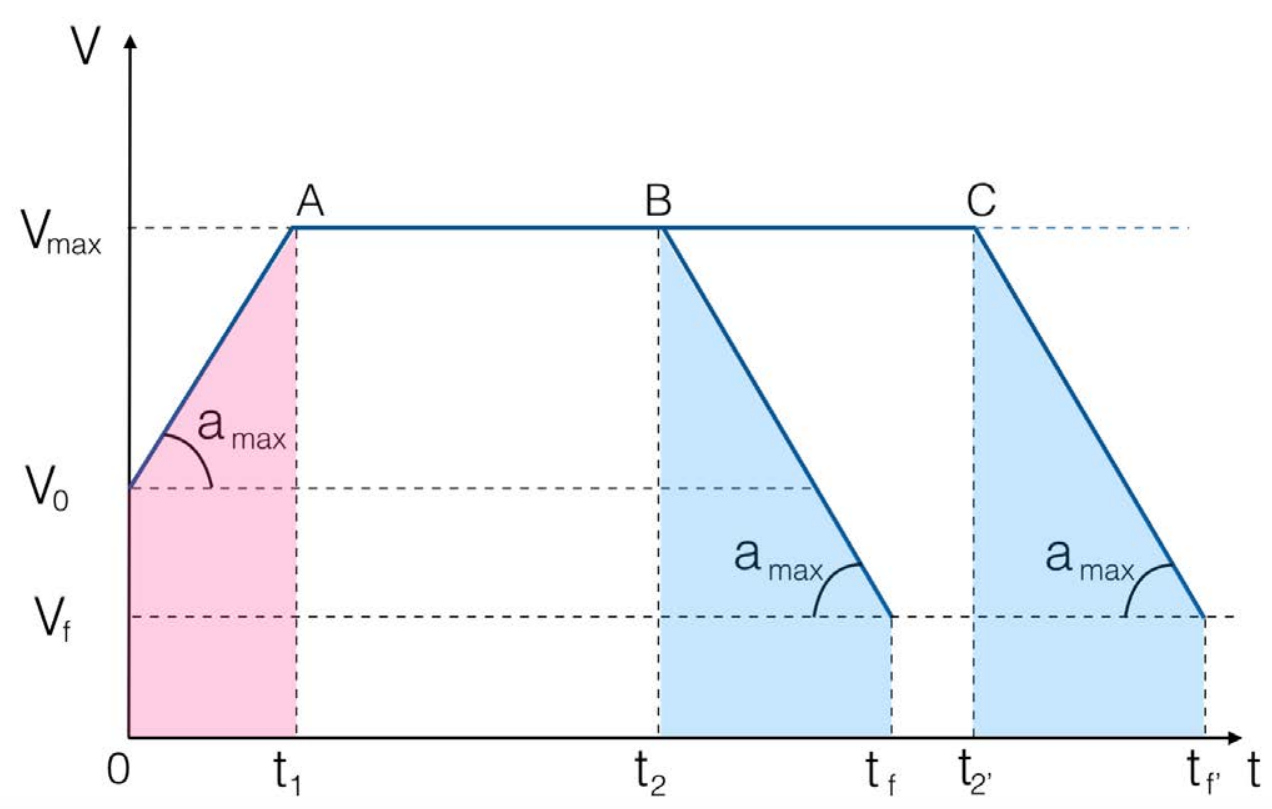

Figure 3.16: Geometrical approach to search for the $t_{f}$

well. Indeed, if the phase is shifted further in time, then the trajectory length is defined only by the area of the rectangle $A B t_{2} t_{1}$ :

$$
\text { Distance }_{t_{1} t_{f}}=\text { Distance }_{t_{1} t_{f}}-\frac{1}{2} \frac{\left(v_{\max }+v_{f}\right)\left(v_{\max }-v_{f}\right.}{a_{\max }}
$$

Taking the above mentioned into account, the final traverse time can be found as following:

$$
\left\{\begin{array}{l}
t_{1}=\frac{v_{\max }-v_{0}}{a_{m} a x} \\
t_{2}=t_{1}+\frac{\text { Distance }_{1} t_{2}}{v_{\max }} t_{f}=t_{2}+\frac{v_{\max }-v_{f}}{a_{m} a x}
\end{array}\right.
$$

Proposed analytical solution allows constructing the fastest trapezoidal velocity profile without usage of complicated solvers, however in the general case constraints of the problem as well as the objective function are not linear.

\subsection{Communication}

The graphical user interface communicates with the robot through the CERN network, which allows to establish a connection with any device connected through ethernet, WiFi and GSM/UMTS inside the CERN area. Especially, CERN provides an internal full 4G coverage (Table 3.4 shows the specifications of the CERN internal network coverage), which must be always available for safety reasons, in order to provide a worker with the possibility to call using his/her mobile phone in case of emergency. In this way, all the devices connected 
to the CERN network are reachable from any other device. Taking advantage of this network infrastructure, it is possible to control any active robot on the CERN area using a standard $\mathrm{PC}$. The robot, then, can be connected to the network using $\mathrm{WiFi}$, if available, or $4 \mathrm{G}$. The Graphical User Interface contains the list of all the controllable robots, together with the robot configuration (e.g. manipulator structure, number of cameras, position of cameras, robot 3D model, platform type and dimensions etc.). In the connection phase then, the GUI shows to the operator the list of available robots and their position. When clicking on a robot, the hardware configuration of the robot is shown, providing information about the connectivity and availability of the robot. When the operator presses Connect the GUI establishes the communication with the robot, adapting itself to the stored robot configuration. At the connection, different network sockets are opened:

- a TCP Service socket on which service messages are sent;

- a TCP Control socket for each controllable device (i.e. platform, arm and PTZ camera);

- a RTP Camera socket for each camera feed. Apart from the Service socket all the unused sockets are paused by the GUI in order to not occupy bandwidth (e.g. a not used camera, or the platform Control socket while using the arm).

\begin{tabular}{|c|c|c|c|}
\hline $\begin{array}{l}\text { Available } \\
\text { Network }\end{array}$ & Bandwidth & Delay & Offered Services \\
\hline 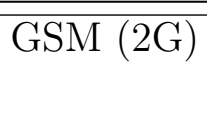 & less than $50 \mathrm{kbit} / \mathrm{s}$ & 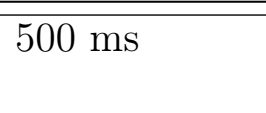 & $\begin{array}{l}\text { SMS, text and picture messages, } \\
\text { MMS, and Voice communication. }\end{array}$ \\
\hline $\begin{array}{l}\text { UMTS } \\
(3 \mathrm{G})\end{array}$ & $\begin{array}{l}10 \quad \text { Mbps } \\
\text { Mbps up }\end{array}$ & $200 \mathrm{~ms}$ & Data transmission \\
\hline LTE (4G) & 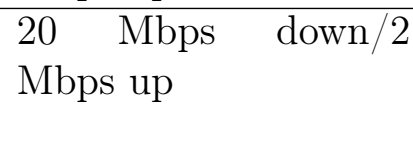 & $\begin{array}{l}40 \mathrm{~ms} \text { with } \\
\text { peaks of } 250 \\
\text { ms }-300 \mathrm{~ms}\end{array}$ & Data transmission \\
\hline
\end{tabular}

Table 3.4: Available networks in the underground accelerator

Using internet-based protocols could create problems in terms of communication delays, which are mainly related to the congestion of the network and the distance in terms of network nodes between the operator and the robot. For this reason, the header of a robot application protocol packet contains a timestamp in milliseconds indicating when the data contained in the packet was produced. Hence, the timestamps of all the connected nodes must be syncronized in order to react properly to communication delays.

The synchronization is based on the standard four-timestamp mechanism of the Network Time Protocol (NTP). This commonly used method measures the transmission round-trip 
time between connected nodes and uses this to estimate the offset between their respective clocks. The protocol relies on the assumption that, during the synchronization, the communication delay is symmetric.

The protocol works as follows:

1. The HRI sends a packet containing the current HRI timestamp to the robot at time $T_{s}^{h}$.

2. The robot receives the packet at time $T_{r}^{r}$ and immediately sends back the current robot timestamp at time $T_{s}^{r}$.

3. The HRI receives the packet containing the robot timestamp at time $T_{r}^{h}$.

In the previous notation, in $T_{s}^{h}$ and $T_{r}^{h}$ the $h$ indicates the time relative to the HRI reference, while in $T_{r}^{r}$ and $T_{s}^{r}$ the $r$ indicates the time relative to the robot reference. The relationship between $T^{r}$ and $T^{h}$ is the following:

$$
T^{h}-T^{r}=O
$$

where $O$ is the offset between the two timestamps taken by the two agents at the same moment and the value estimated by the process.

As previously stated, the method is sensitive to asymmetry in communication delay. Unfortunately, it is not possible to measure communication asymmetries in the network with only two agents. Therefore, the method will estimate $\widetilde{O}$, which will be only an approximation of the real $O$.

In order to estimate more accurate $\widetilde{O}$, two conditions can be imposed. First, it is safe to assume that the offset between the local timestamp of the robot and the local timestamp of the HRI $O$, and therefore its estimation $\widetilde{O}$, is constant or slowly changing during the entire intervention time. The unavoidable fluctuations of the clocks between the two agents which will make $O$ vary in time are negligible. Thus, it is possible to create a moving average of $\widetilde{O}$ in order to filter rapid changes coming from the estimation process, which are more probably generated by communication asymmetries.

Furthermore, during the computation of the communication delay $d$ for a specific packet $P$, this delay $d_{P}$ can not be lower than 0 (this would mean that one agent received a packet before it was transmitted). However, this situation happens frequently when dealing with highly asymmetric delays. Therefore, the estimated offset $\widetilde{O}$ is, at least, lower estimated by a value $d_{P}$, which provides, then, a lower-bound for $\widetilde{O}$.

Table 3.5 shows the network offset calculation with different network delays and comparing them with vanilla four-timestamps mechanism of the NTP protocol. Network delays were simulated using Clumsy, an open source software for simulating network lags on Windows computers, and $t c$, a linux standard application for traffic control. These programs were set to simulate a network delay with a $10 \%$ variation on their average value. The asymmetry in the network delay was guaranteed by using the traffic control programs independently on both machines, only for outbound packets. For calculating the ground truth offset, standard four-timestamps mechanism of NTP has been used without any traffic control program 


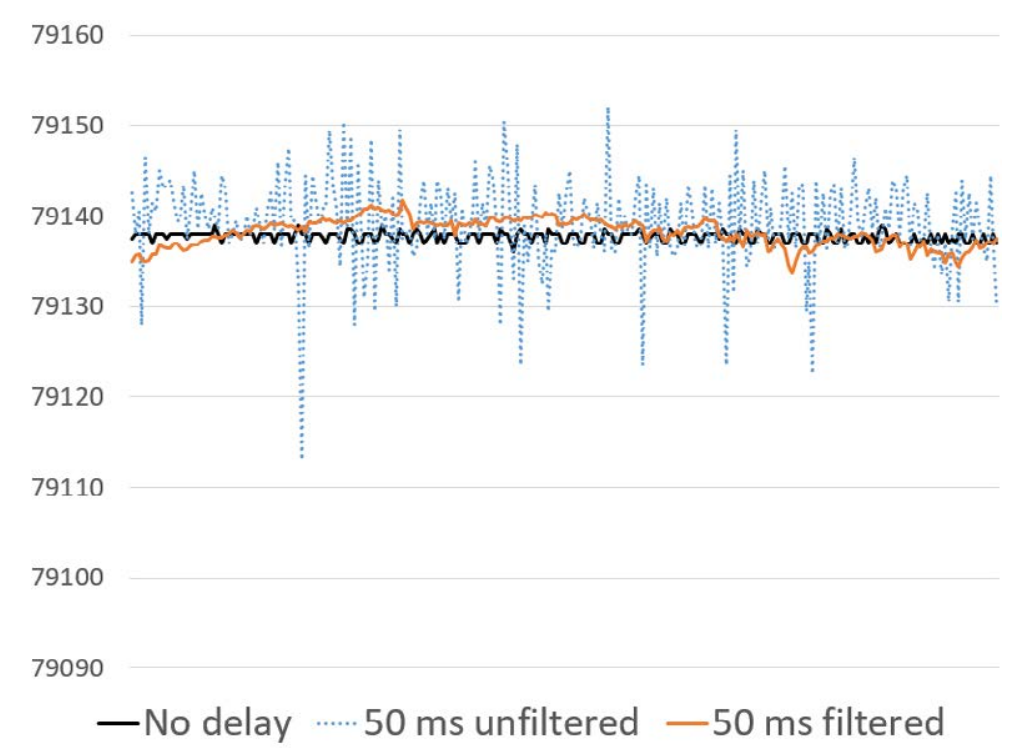

Figure 3.17: The timestamp offset calculation for $50 \mathrm{~ms}$ simulated delay. The black line represents the ground truth, the blue dashed line represents the four-timestamps mechanism of NTP and the orange line the estimated offset.

running. It can be noticed how the offset estimation using the proposed filtering (both the moving average and the positive delay condition), provide better results than the standard four-timestamps mechanism of NTP (see Figure 3.17 and 3.18).

\begin{tabular}{|l|l|l|}
\hline Known delay & Offset average & Offset standard deviation \\
\hline \hline 0ms (Direct LAN) & $79137.7 \mathrm{~ms}$ & $0.53 \mathrm{~ms}$ \\
\hline $50 \mathrm{~ms}$ (NTP) & $79139.3 \mathrm{~ms}$ & $5.01 \mathrm{~ms}$ \\
\hline $50 \mathrm{~ms}$ (filtered) & $79138.1 \mathrm{~ms}$ & $1.53 \mathrm{~ms}$ \\
\hline $100 \mathrm{~ms}$ (filtered) & $79136.9 \mathrm{~ms}$ & $1.15 \mathrm{~ms}$ \\
\hline
\end{tabular}

Table 3.5: Network offset estimation using different network delays. It is evident how the proposed filtering using a moving average on the offset and compensating negative delays, gives better results than then standard NTP protocol. 


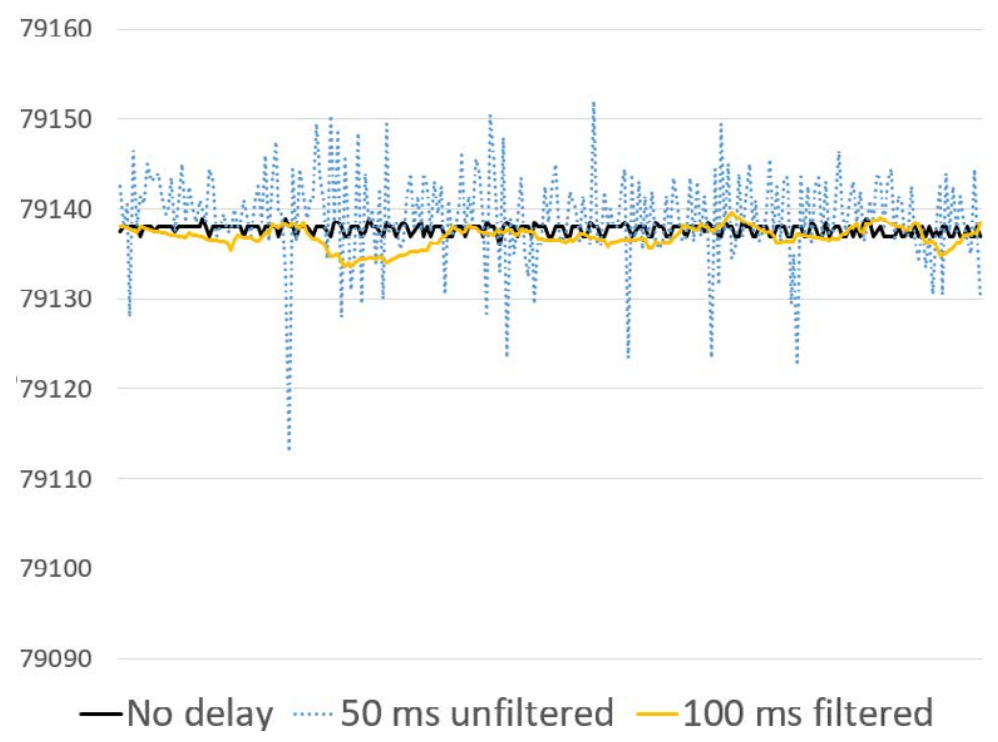

Figure 3.18: The timestamp offset calculation for $100 \mathrm{~ms}$ simulated delay. The black line represents the ground truth, the blue dashed line represents the four-timestamps mechanism of NTP and the yellow line the estimated offset. 


\section{Chapter 4}

\section{Key Functionalities for Advanced Remote Handling Performances}

\subsection{Environmenthal Perception and Measurements}

To circumvent a majority of the constrains presented in hazardous and cluttered environment, perception and visual awareness have become essential for the development of robotic systems involving autonomous surveying or telemanipulation. To increase this autonomy an environmental reconstruction is necessary. To achieve this the $6 \mathrm{D}$ pose estimation of different objects is crucial. Another way to achieve this reconstruction is using algorithms to merge different point clouds taken by a RGBD Camera.

To merge point clouds to create a model of the environment, algorithms like Kinfu [226], Kinect Fusion [227] or Elastic Fusion [228], could be used (see Figure 4.1). This kind of algorithms do a real-time dense visual SLAM system to capture comprehensive globally dense maps of room scale environments explored using an RGB-D camera.

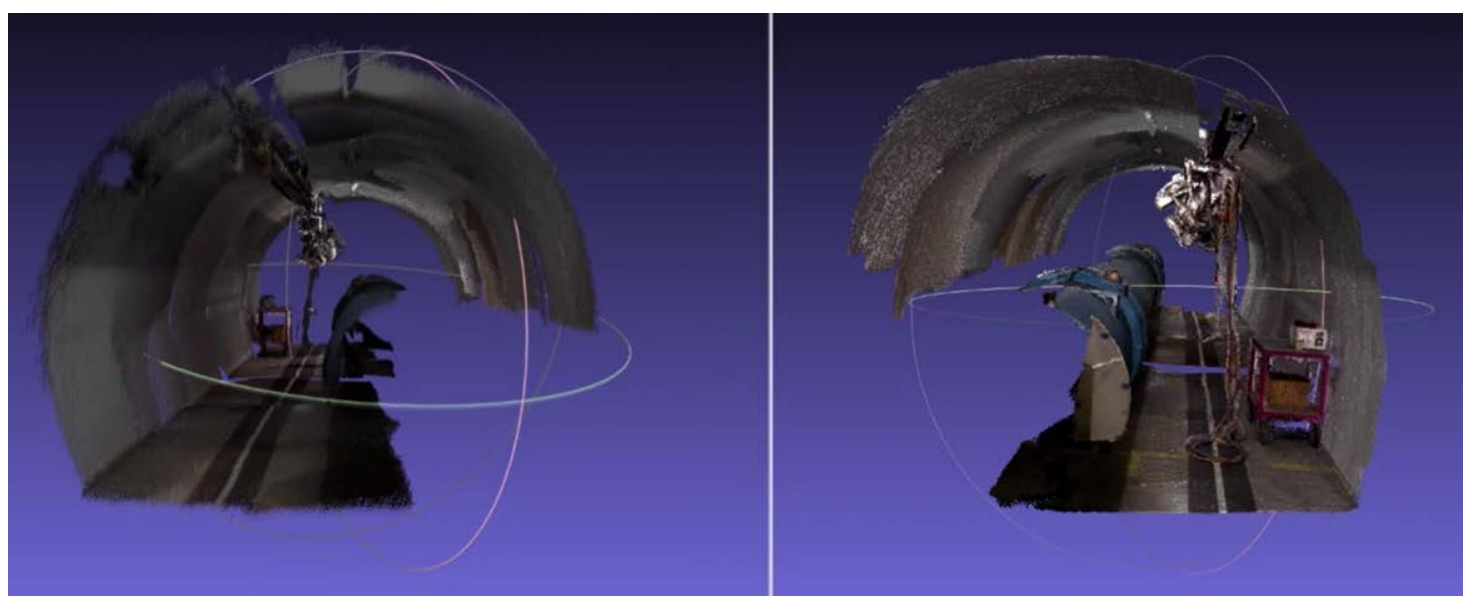

Figure 4.1: Images taken from point cloud reconstructions of the LHC mockup at CERN using Elastic Fusion based environmental reconstruction techniques [228] 
Several works for 6D pose estimation, such as LINEMOD [229], PWP3D [230], Sliding Shapes [231], allow to find the exact position of an object. For the CERNTAURO framework, a new algorithm has been developed [196] for 6D pose estimation using a RGBD Camera to compensate the problems of previous works on reconstruction due to presence of metallic objects. And considering recent advances in the deep learning community regarding $6 \mathrm{D}$ pose estimation [232, 233], it has been aimed to fuse the pose estimation with object detection in the scene [234] (see Figure 4.2).

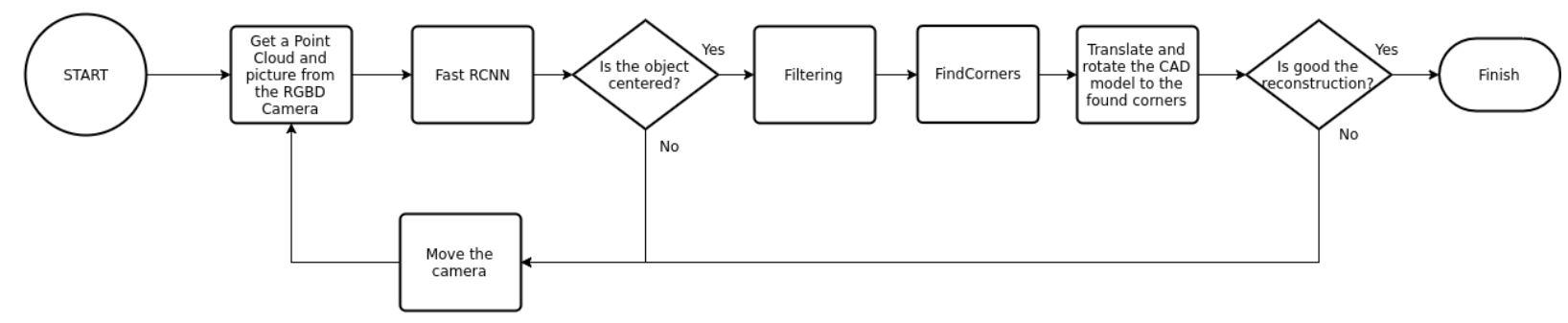

Figure 4.2: Pipeline of the novel proposed algorithm[234]. After the reconstruction of the object, machine learning is applied to identify the location of the elements on the object. After, filtering and segmentation techniques are applied and a verification is launched if the element is found.

The main challenge of handling metallic objects is that these objects produce reflections, potentially leading to incomplete point clouds. This cannot be avoided by modifying the lights, as RGB-D cameras work with their own light projector. Therefore, the algorithm must work with both incomplete information on the depth field and high noise.

The CERNTAURO framework is composed also by a novel developed modules named MORPC (Metalli cObjects Robotic Perception at CERN) that reconstructs and recognizes objects while the robot is performing its tasks.

The novel proposed algorithm is organized into two parts, one based on the the use of Faster-RCNN [110] for object detection and classification, and another one based on the use of clustering and segmentation to find a robust point versus changes in the position of the camera. The first part use Faster-RCNN to reduce the complexity of the filtering pipeline of the previous algorithm, because it targets only specific areas and thereby needs to filter less points.

The best results were achieved training Faster-RCNN with a Resnet101 model using COCO [235] pre-trained values. In total, 500 pictures of 6 different objects of interest were used to train the method along 100.000 steps. However, the loss function already converges for classification and box estimation in step 30.000, as seen in Figure 4.3.

When testing a trained Faster-RCNN model, a bounding box around the object of interest in the image is retrieved. From this information, all the points of the point cloud that correspond with the pixels in this image are selected, extracting only the relevant object from the scene (see Figure 4.4).

After the point cloud inside of the bounding box is recovered, the algorithm uses clustering algorithms and segmentation to find a robust point $P_{r}$, i.e. a point with unique features 


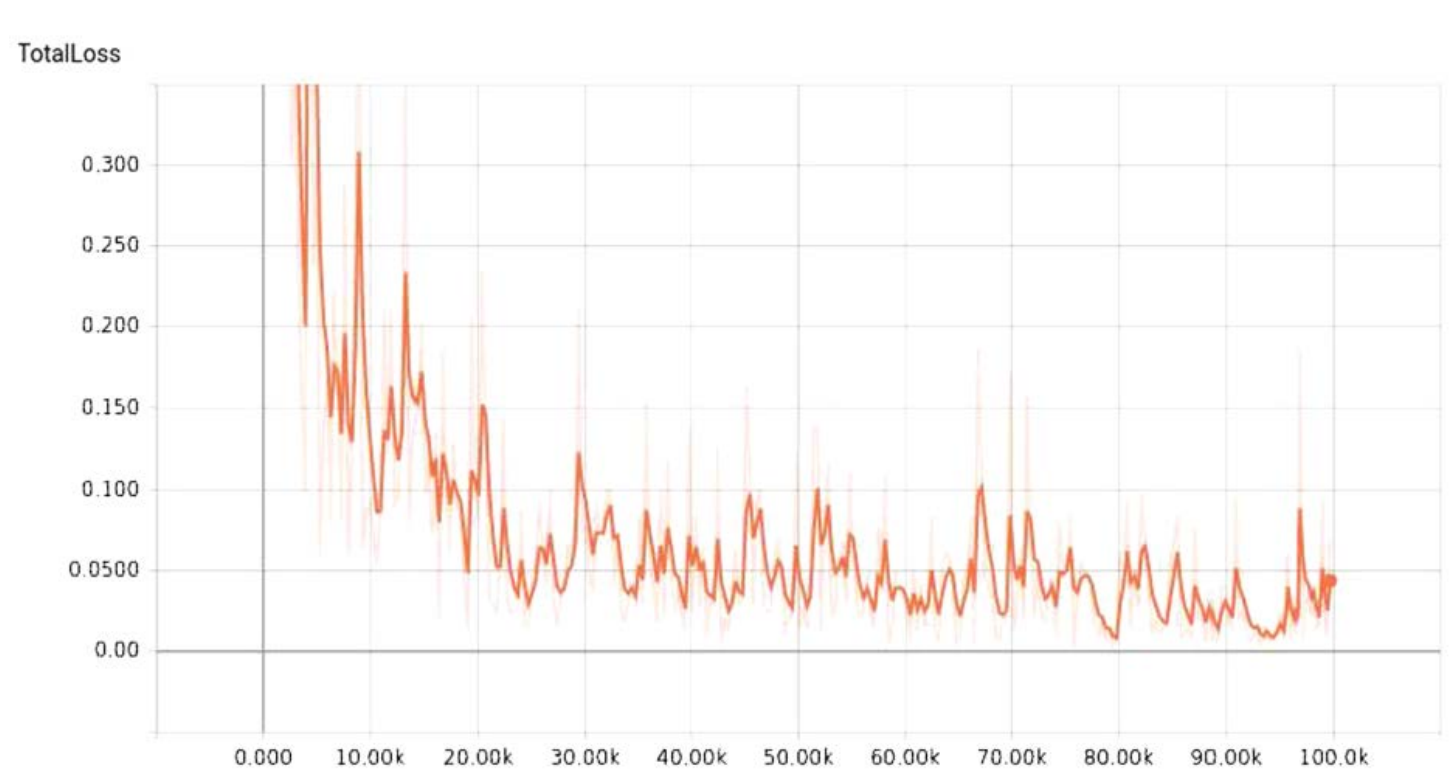

Figure 4.3: Total loss during all training steps of Faster-RCNN with Resnet101.

DETECT OBJECT AND CREATE BOUNDING BOX

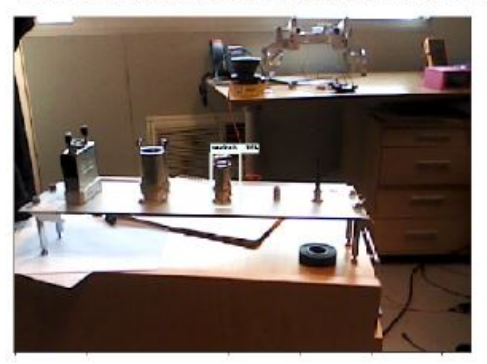

GET POINT CLOUD
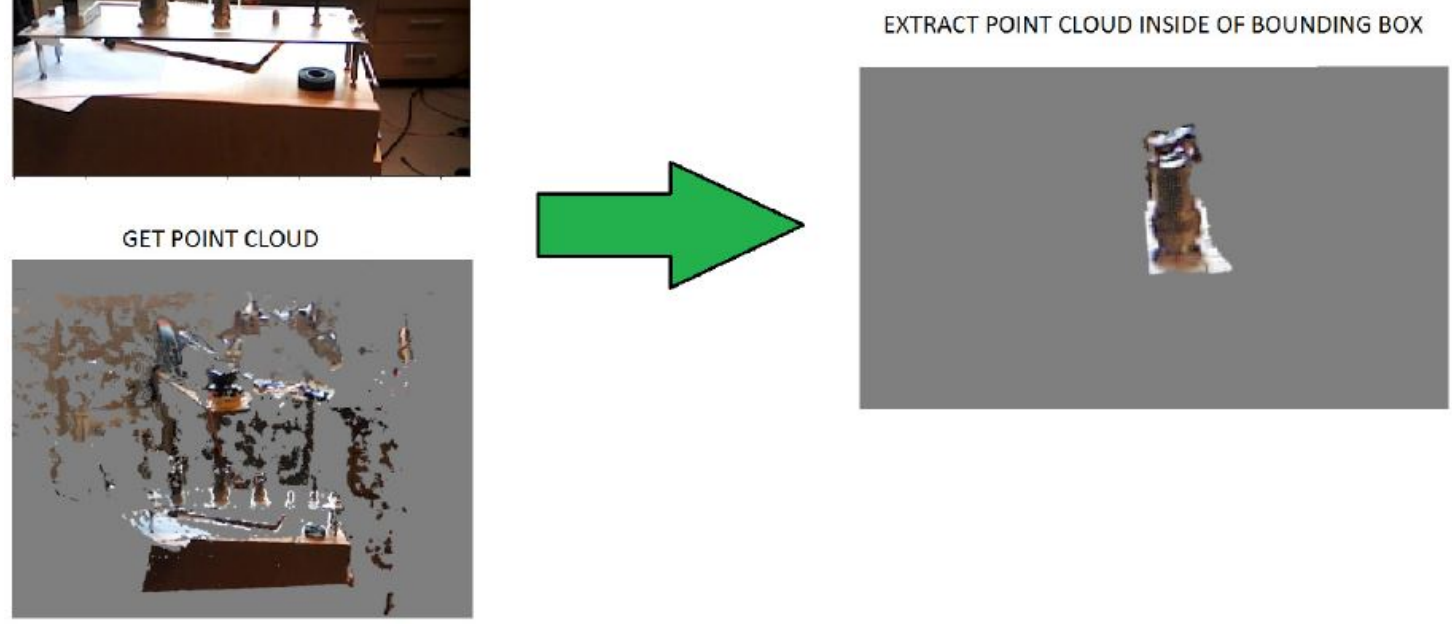

Figure 4.4: Extraction from the complete point cloud using the bounding box from FastRCNN [234].

invariant to camera perspective (see Figure 4.5).

Finally a point cloud from the CAD model of the object is matched with the coordinate of $P_{r}$, translating the 3D object to its respective position. In this new reference frame, the position of the object is clear for subsequent $3 \mathrm{D}$ reconstruction. The parts of the target, 


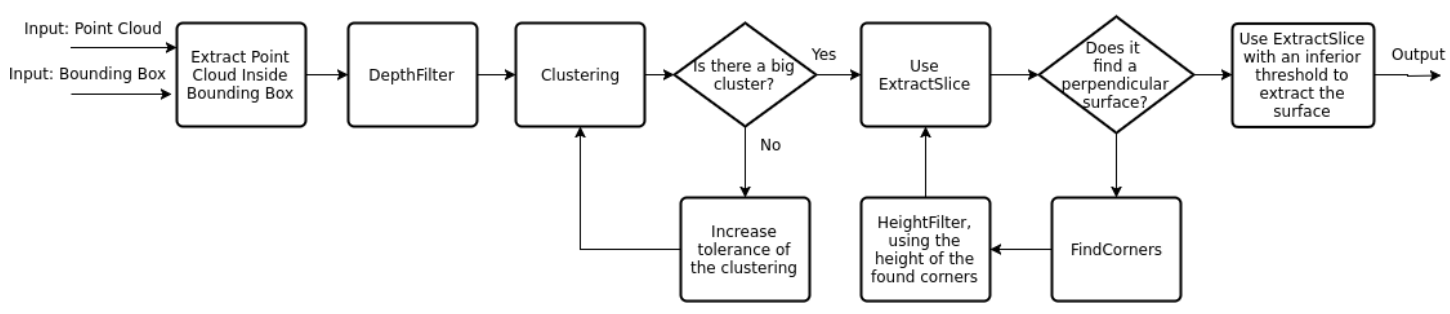

Figure 4.5: Flow chart of the filtering box in Figure 4.2 [234].

which are lost or occluded, appear reconstructed in the point cloud (see Figure 4.6). In case the matching fails the robot moves the camera to another position and try again.

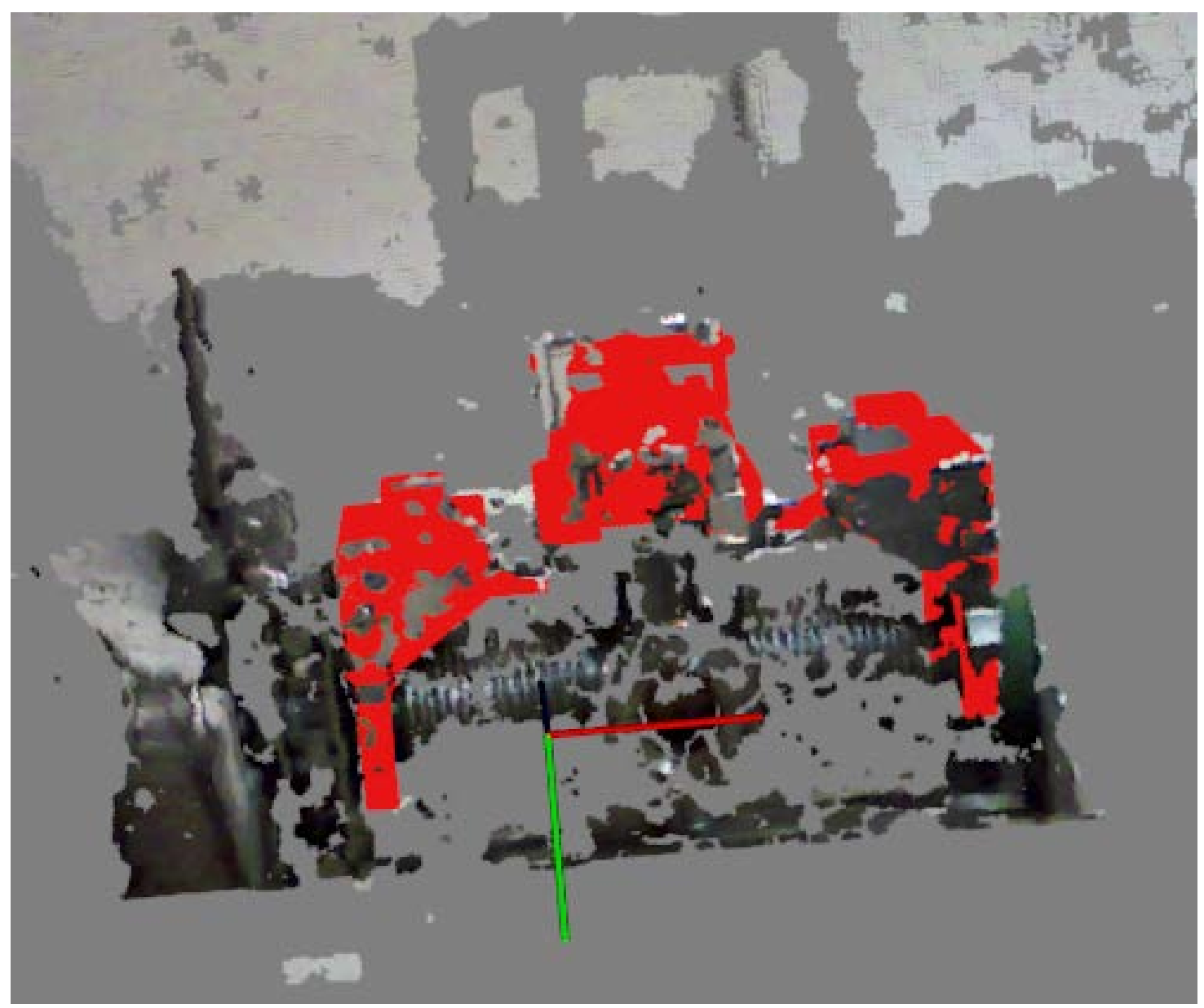

Figure 4.6: Three-dimensional reconstruction of the object.

The following submodules of the filtering pipeline have been developed:

BoundingBox , after the Faster-RCNN method returns the bounding box of the different objects detected in an image, it extracts all the points of the point cloud that corre- 
spond to the pixels inside of the box. This is done in the Extract Point Cloud Inside of the Bounding Box block of the flow chart.

DepthFilter, the target objects must be close to the camera, so distant points can be filtered more easily.

HeightFilter, this method has the same purpose of DepthFilter, removing points of the target object far from the plane of interest.

Clustering, this methods filters parts of less interest of the point cloud by clustering the main parts based on a nearest neighbors approach.

ExtractSlice, as the main part of the filtering module, this method recovers the plane in 3D Euclidean space, iteratively searching planes with a certain normal between intervals. We apply this twice, once to detect a thick plane, filtering a lot of points that could lead to a plane misdetection, and another time to extract the surface of the detected thick plane.

VFindCorners, once the desired plane is found, it is necessary to locate the corners, usually searching for the object's top corners.

\section{HRRS : a Human Reconnaissance System}

The most important goal of disaster robotics is to search for victim presence in catastrophe environments. Currently accepted practice is to have a human operator for each rescue robot to control its movements, evaluate received data from on-board sensors and perform actions. However, rapid algorithm and sensor development have attracted a lot of researcher attention to create autonomous robots for various tasks such as mapping, rubble removal, structural inspection, logistics, object detection and recognition, signal amplification and medical assessment. Practical use of autonomous reconnaissance robots for victim localization could be very useful extension for every Search And Rescue (SAR) team. After the position of a victim has been estimated, robot could activate its on-board sensors for victim status monitoring and automatically send data (e.g. respiration rate, temperature distribution) to the command center for evaluation.

Despite the numerous attempts by many researchers, autonomous human seeking disaster robots remain as prototypes in laboratories. Various senors types are used (e.g. RGB-D sensors, thermal cameras, radars, $\mathrm{CO} 2$ analyzers, microphone arrays, etc.) to perform the task of autonomous human detection and respiration activity acquisition. However, most important shortcomings according to explored literature are the following:

1. Limited battery capacity makes the use of powerful and highly accurate equipment impossible.

2. Restricted amount of computational resources on board prohibits using sophisticated detection algorithms and many layer neural networks. 
3. Researchers tend to focus on solutions for particular disaster scenarios, which is not the case in real life with constantly changing conditions.

4. Absence of sizable, publicly accessible training data taken in real disaster environments prevents researchers to deliver more robust solutions.

According to [236], approximately $80 \%$ of victims after major disasters, such as earthquakes, are relatively easily distinguishable from surroundings (i.e. located on the rubble). Considering previously mentioned shortcomings, main priority of rescue robots should be to focus and report on easily distinguishable victims over the whole area of disaster as fast as possible, while leaving the challenging tasks to human rescue team.

Every potentially usable sensor technology is designed to measure a specific phenomena and has its own drawbacks. Furthermore, their use in mobile applications comes with a performance drop due to restricted on-board computational power. In this study, both issues are addressed - we present a work-flow for ground robots to perform computationally efficient autonomous human detection and remote vital parameter acquisition using a combination of commercially available visual sensor and radar. Such system could be extendable for implementation on aerial units, however it would require a set of customized sensors and algorithms to tackle the issue with aircraft movement. A prototype of proposed system is built to demonstrate its ability to work on mobile platforms with computational, size and energy restrictions by combining the best features from two different worlds - visual sensors and radars. The contributions of this work include:

1. A work-flow for autonomous human detection and remote vital sign acquisition using a combination of visual sensor and UWB radar.

2. System Performance evaluation using a demonstration prototype for different human postures, clothing and distances.

Different sensors and detection algorithms have been used in human detection studies, where the most prominent are visual sensors, RGB-Depth sensors, infrared cameras and radars.

Vision based human detection by far is the most advanced technique and is extensively used for autonomous victim detection. In [237] authors propose using Unmanned Aerial Vehicles (UAV's) to perform autonomous exploration of disaster sites. The received drone images were processed by combination of two Histogram of Oriented Gradients based detectors [238], where the first was trained on full body recognition and the other was trained to detect upper bodies of people. An important outcome of experiment was, that a combination of given detectors performed better than each of them separately. HOG detectors are not the first choice for object detection tasks anymore, because neural network based object detectors have proven to be much more accurate for this task. Nevertheless, an important advantage of HOG detector is real-time performance with limited computational resources, relatively low amount of necessary training data and simplicity, which is the reason why they still get some interest. 
Another successful detector type is based on cascade of Haar features [239] and is commonly used for face recognition in visual as well as thermal images. The detector showed a good performance in [240], however in order to be useful for rescue robotics, it needs to be combined with other detectors, for example, HOG, skin colour based techniques, etc.

A group of researchers proposed an approach for autonomous ground robots equipped with RGB-Depth camera to separate background (floor) from the Region Of Interest (ROI), i.e. victim body and performed template matching [241], [242] for detection.

After presentation of AlexNet [243], Convolutional Neural Networks (ConvNets) became an important tool for victim detection. A successful attempt to detect a person in disaster scenarios only by visible body parts with ConvNets is reported in [244]. The worst detection rate was for the legs with $64.91 \%$ and higher (up to $80.56 \%$ ) for other body parts. A small database "Freiburg people in disaster" was introduced for testing purposes, however the ConvNet was trained using PASCAL Parts data-set, because there are no existing datasets to use for ConvNet training in victim detection. Due to the time constraint in search and rescue applications, only computationally efficient techniques are admissible, therefore dimensions of neural network is an important parameter.

Thermal imaging enables human detection also in zero visibility conditions, i.e. complete darkness or smoke, therefore infrared cameras are often used by firefighters and rescuers to perform navigation and search for survivors in disaster scenarios. Most of reviewed human detection methods with infrared cameras use traditional detectors with manually picked features, like, Haar feature cascade [245], HOG detector [246] and also in combination with neural networks [247]. Temperature distribution over a specific region is a unique feature important for victim detection [248].

High performance thermal cameras are available in the market, but still very expensive, therefore researchers mostly work with low resolution cameras (less than 480x320p). This is also one of reasons why traditional detectors are still used, sometimes the only usable feature is the silhouette of person, which is were HOG detectors are performing very well. The approach works very good with pedestrian detection [247], however has limited use in search and rescue scenarios due to high variety of postures the victim may have. Another more recent study used Haar features [245] with Adaboost machine learning algorithm. Problems with detection were reported in cases when human is not frontally facing the camera. Results were also highly dependent on distance to the target. Fake detections were reported to originate from surface reflections (i.e. glass) as well as from objects resembling human shape.

Thermal imaging is an interesting technique for search and rescue missions due to capability to perform simple diagnostics. Analysis of human body in thermal image provides useful information about temperature distribution across it. It is even possible to record temperature changes of human body across time in order to detect vital signal presence, such as respiratory rate [249].

Various work works [237], [250], [240] identify the problems with visual as well as thermal sensors and encourage to perform sensor and data fusion as one of the solutions to increase robustness of victim detection.

[251] and [252] have reported the Colorbot - mobile security robot, equipped with UWB 
radar, having 1 TX and 2 RX custom made directional Vivaldi antennas, for super-resolution indoor positioning and localization applications. Authors successfully performed navigation towards a lying victim and detected respiration signs inside an obstacle area filled with smoke. The experiment showed a disadvantage of radar usage for human detection systems - the robot had to stop completely in order to sense the environment and localize victim.

In another experimental study done by Nezirovic et al. [253], researchers used UWB radar consisting of two directional antennas to perform person presence detection at $1.5 \mathrm{~m}$, based on respiration activity and recorded it. Furthermore, respiratory data were compared for different person postures. Best respiration signal was acquired when human was sitting with chest towards radar. In another similar study [254], the researchers used Continuous Wave (CW) Ka-band (26.5-40 GHz) radar to record heartbeat of a person, sitting $2 \mathrm{~m}$ away in changing directions. They concluded, that best acquired signal was achieved when person turned back to the radar due to minimized harmonics interference. In both cases results showed, that human detection is feasible with radar, however, the quality of vital signal acquisition is highly dependent on posture of person. An excellent review on vital sign detection with radars is provided by [255].

Another radar based detection system is reported in [256], which could detect small amplitude variations due to heartbeat and lung activity at a distance more than $30 \mathrm{~m}$ across free space using $\mathrm{CW}$ radar at $10 \mathrm{GHz}$ microwave radiation frequency. The system could also detect person behind a cinder block wall over $3 \mathrm{~m}$ away, which underlines a significant advantage of radar - possibility to see through obstacles. A major drawback of CW architecture, however, is inability to detect distance to the object, and simultaneous multiple target acquisition requires signal processing.

In [257], researchers performed more complex Micro-Doppler signal processing, that can be used for target classification with machine learning algorithms. A significant radar disadvantage is the necessity for a long measurement time (up to couple of seconds) in order to perform the Doppler signal analysis, therefore many research efforts are directed to overcome this limitation [258], [259], [260], [261].

A very detailed review of unobtrusive cardiorespiratory monitoring techniques is given in [262]. Radar-based lung and heart activity monitoring methods have advanced, simple, low cost respiration sensors [263] are already available in market. Nevertheless, they are not medically approved, which leads to conclusion, that their performance is still limited.

Alternative remote vital sign acquisition techniques consist of thermography and Videobased motion analysis. The first method acquires data about blood and respiration flow by observing frequency of temperature oscillation around regions of interest, e.g. neck and nose whereas the video based motion analysis monitors the same phenomena as radars - body vibration due to respiration or hearbeat, however measures it with visual sensor. Up to our best knowledge there are no products available in the market for any of both techniques.

Figure 4.7 illustrates the work-flow of the proposed method, which can be subdivided in two tasks: human detection and respiration monitoring.

The HRRS system decets detects victims using light-weight ConvNets. Convolutional neural network was chosen for real-time human detection due to its superior results over the rest machine learning-based methods in image processing and classification tasks [243]. 


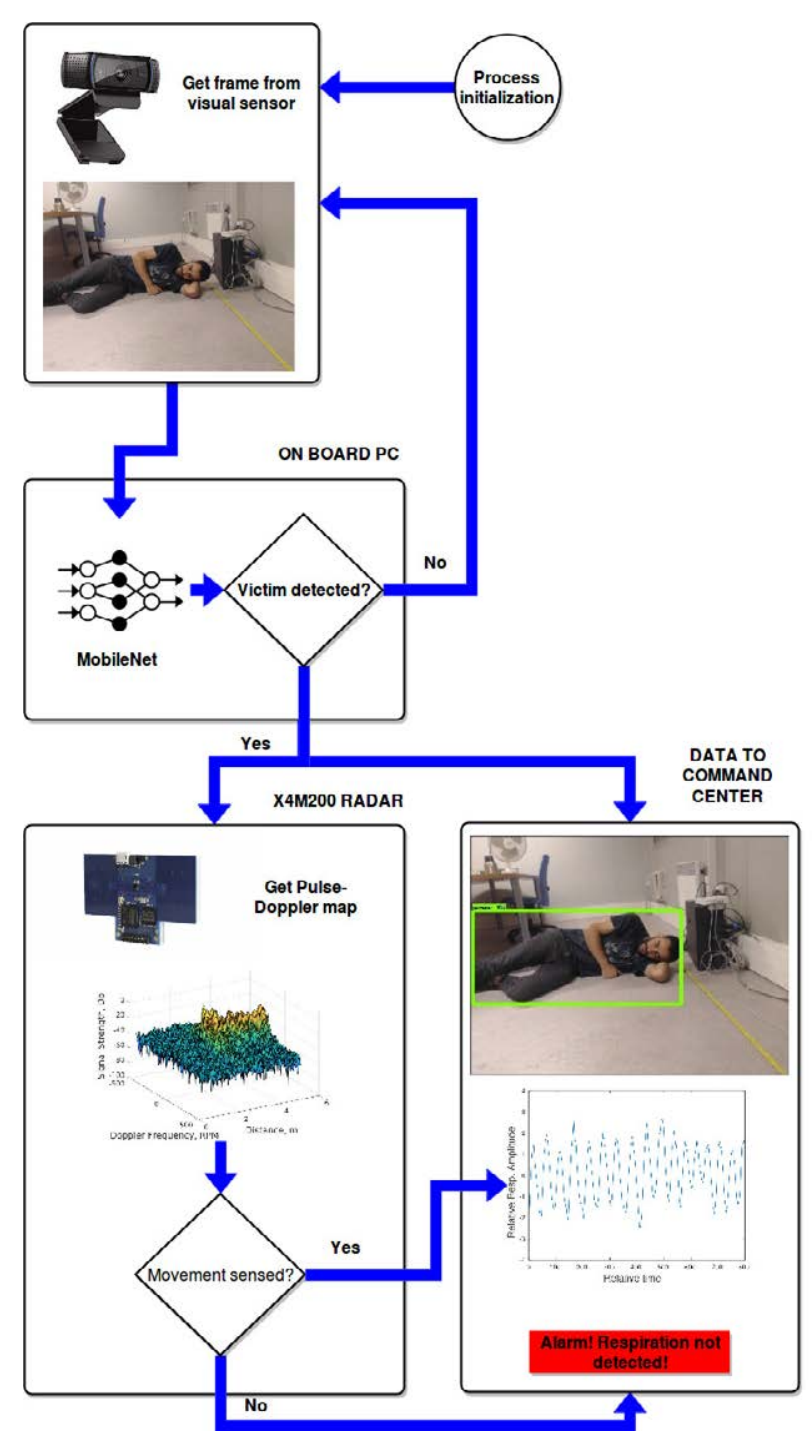

Figure 4.7: Work-flow of the proposed method. After an initial image acquisition, the captured frame is passed to a trained neural network to recognize a person. Once the person has been recognized, the radar will monitor the respiration rate.

In general, CNN-based detectors require much more computational resources than traditional ones, which is a major limitation for their use in mobile applications. Our approach is to use small, low-latency and low-power model, which is suitable for platforms with limited computational power, e.g. MobileNet [264]. Tensorflow repository [265] offers a variety of MobileNet models, which have been trained on ILSVRC-2012-CLS [266] image classification dataset. The models vary in size and accuracy. For validation purposes, the MobileNet_v1_1.0_224 was implemented on prototype.

As soon as person is detected, system initializes radar to perform a room scan, as shown in Figure 4.7. Mostly used radar architectures for respiration monitoring are $\mathrm{CW}$ and 


\begin{tabular}{|c|c|}
\hline \multicolumn{2}{|c|}{ X4M200 Respiration Sensor } \\
\hline Architecture & UWB, System on Chip \\
\hline Operational frequency & $6.0-8.5 \mathrm{GHz}$ \\
\hline Bandwidth & $2.5 \mathrm{GHz}$ \\
\hline Detection distance & up to $5 \mathrm{~m}$ \\
\hline Antenna amount & $1 \mathrm{TX}, 1 \mathrm{RX}$ \\
\hline Antenna type & Non-directional, $90^{\circ}$ Elevation, $110^{0}$ Azimuth \\
\hline
\end{tabular}

Table 4.1: Technical specifications of X4M200 respiration sensor currently used by the CERNTAURO framework.

UWB. We propose to use the latter, due to its ability to deliver not only complex signal phase movements, but also the distance to the oscillating object. For method evaluation we took a commercial low-cost System on Chip (SoC) sensor from Xethru with existing algorithm for human respiration detection, which makes it suitable for getting started with UWB radars, exploring the possibilities of technology and understanding shortcomings as well as easy integration into already existing systems. Table 4.1 refers to more detailed specifications of radar [263].

\section{Objects tracking using vision}

In order to perform safe robotic interventions in harsh environments it is necessary to help the robotic operator with a Human-Robot Interface that provides multimodal interactions, from low level interaction methods to bilateral teleoperation with force feedback. These interaction modalities, though, rely purely on the operator's skills. With the objective of providing a safer system, higher-level applications can be integrated in the interface in order to provide some help to the operator, like objects tracking and obstacle avoidance systems, without relying uniquely on his/her capacities. Laser-based [267] generate a collision avoidance wrapper against the environment obstacles. Nevertheless, if the task is to reach a specific goal, it is mandatory to bring this information from a higher-level feedback. Tracking algorithms make use of different kind of features, which allow them to track the region of interest (ROI) set it up for that purpose, either chosen by an operator, by some feature extractor/detection algorithms, by deep learning, etc. It is very important to keep in mind the scope where the robot has to work, since the performance of each algorithms will depend on where its features come from. With that in mind, it was necessary to discard all those HAAR-based algorithms [268, 269, 270], due to the fact that are based on the pixels light intensity, and opting for those that are using Histogram of Oriented Gradients (HOG)[271, 272], since the environment where the most of interventions have to be carried out is metallic, reflecting randomly the light.

Taking all of this into account, this work presents a novel object recognition and tracking system which runs in real-time on the robot while the operator is operating it. The tracking system enters in the teleoperation loop and helps the operator to achieve the requested 


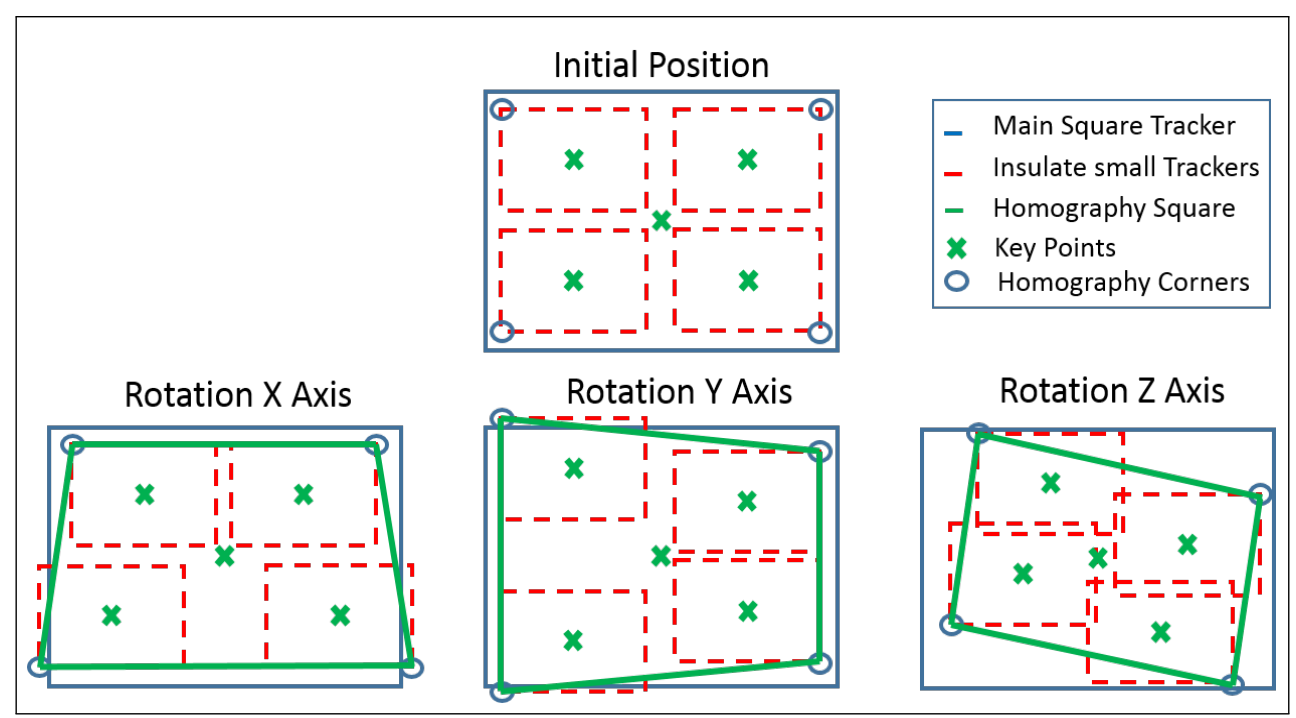

Figure 4.8: Solution for rotation and partial occlusion invariance that overtakes the limitation of standard tracking systems. The schemes explains the novelty of the proposed system that integrates different tracking regions in one main ROI.

goals. The system is optimized to track featureless objects such as metallic plates, metallic connectors and monochromatic objects. Moreover, the algorithm provides improvements with respect to previous tracking experiments, including depth estimation in order to better interact with the velocity control of the robotic arm when approaching the target, as well as high reliability with partial occlusions.

The CENTAURO's tracking system deployed is based on Kernelized Correlation Filters (KCF) algorithm [273], which fulfils most of our necessities in contrast to others algorithms such as:

- Boosting [274], the faster performance against the lower accuracy.

- Multiple Instance Learning (MIL) [275], highest precision penalizing in computational time.

- Tracking-Learning-Detection (TLD) [276], which although to be able to work under temporally occlusions, it presents a high number of miss-detections.

The KCF method presented two sharp weakness, the not invariance to partial occlusions and the rotations, troubles that was fixed splitting the ROI in 4 sub-regions capable to track its own region in insulated way (see in Figure 4.8). Figure 4.9 and Figure 4.10 show the performance of the solution implemented against these issues.

The movement of the five squares (the main one and the new four small ROIs) are related through Euclidean distance [277] with an adaptive threshold. This gives the possibility to detect if some of these ROIs it are losing their tracked because of the featureless area. 

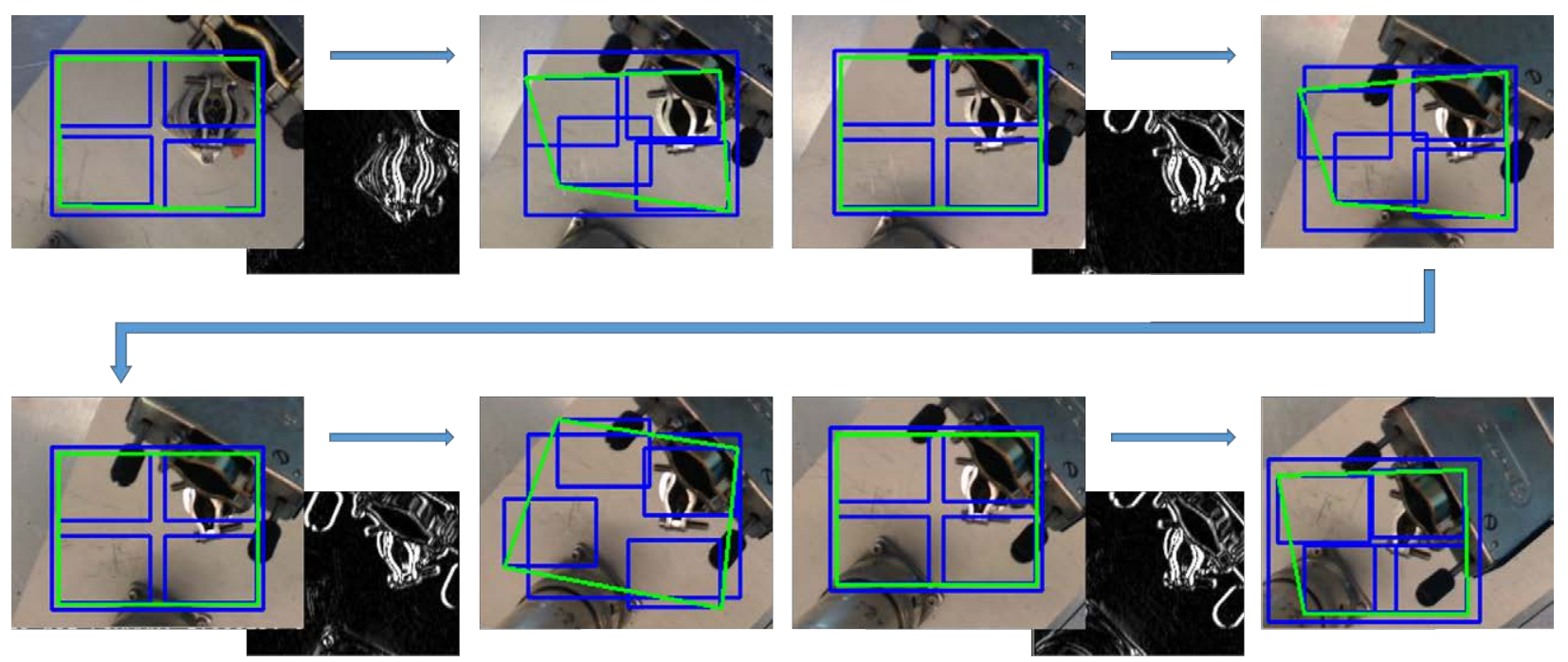

Figure 4.9: Visual explanation for the partial occlusion problem present in standard tracking systems. From the image flows (left to right), it is evident that, when a region of interest (ROI) is occluded, the system in real-time uses the SURF algorithm taking the current pattern (in black) to recalculate the new ROI.

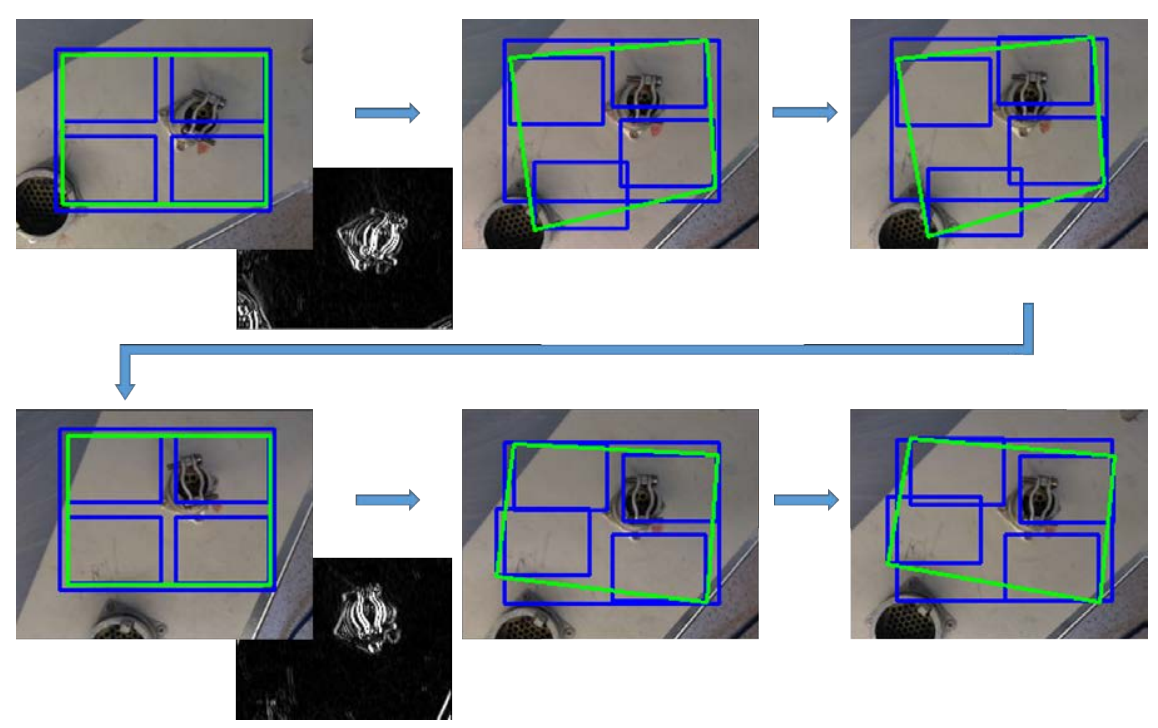

Figure 4.10: Visual explanation for the image rotation problem present in standard tracking systems. From the image flows (left to right), it is evident that, when a region of interest (ROI) is not anymore tracked because of image rotations, the system in real-time uses the SURF algorithm taking the current pattern (in black) to recalculate the new ROI. 


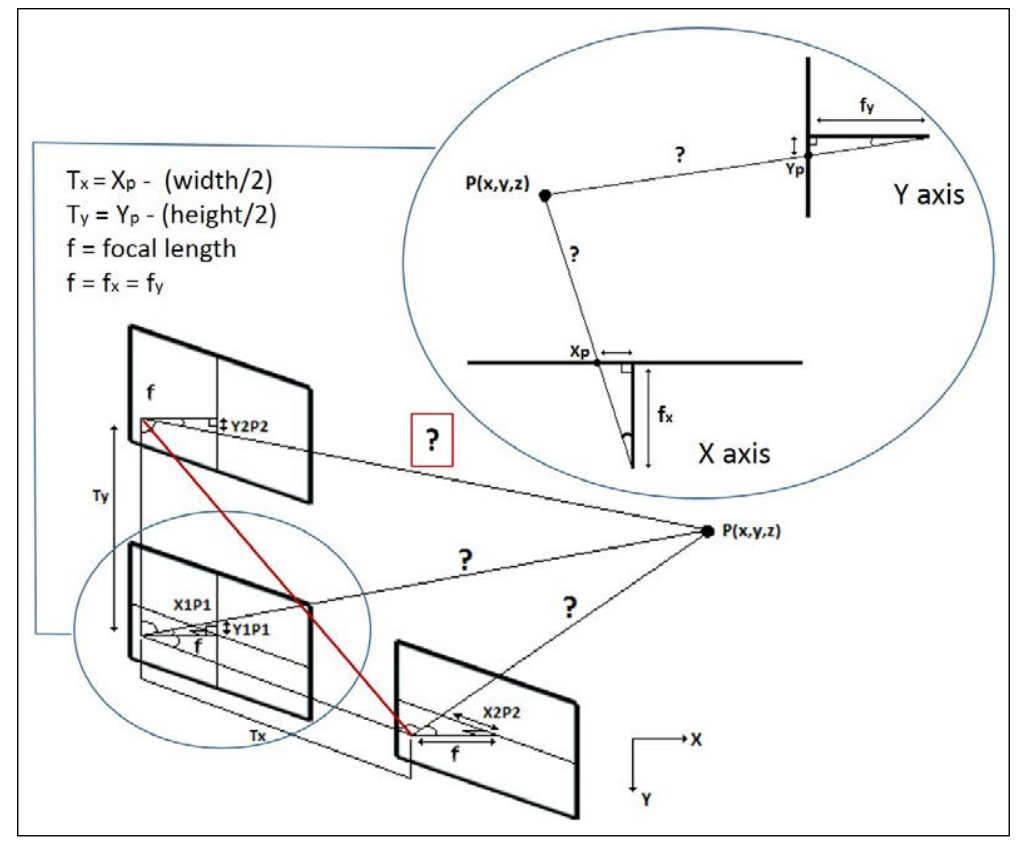

Figure 4.11: Triangulation proposal in $\mathrm{X}$ and $\mathrm{Y}$ axis. This graphical solution explains the triangulation estimation chosen by the proposed system. Normally this solution is used by epipolar geometry only in $\mathrm{x}$ axis. This has been extend in $\mathrm{y}$ axis also thanks to the new architecture.

Then, extending the Epipolar Geometry [278], which works exclusively on the X-axis, it endows of 2D freedom movement to the system (either rotations and translations), and using the matrix of the robot and a set of key-points, the system takes advantage of the camera and robot movements to get the correlation of these key-point, estimating the distance to the target from the select area in the ROI chosen as shown in Figure 4.11. This information will be given by augmented reality to the operator and it will adapt the robot velocity regarding to this distance.

In addition, the CENTAURO's tracking system is capable to recover by itself. The ROI of the first frame in each restarting is going to be stored as a contingence pattern, which will be used in case of the tracking lose by Speeded-Up Robust Features (SURF) algorithm [279]. If the area disappears from the field of view, the tracking stops and turns SURF on automatically. If the area is found, the tracking will restart (see Figure 4.12). In order to provide the user with a higher level of interaction with the system, a deep learning object recognition module has been added (see Figure 4.13), which enables robust recognition of metallic objects (e.g. connectors and patch panels) with lack of texture features. The module is based on Faster-RCNN [280]. The neural model that offered better accuracy for this technique, detecting a higher number of metallic objects, is the ResNet-101 [281], which scored a total losses under $0.05 \%$ instead of other models such as Inception-v2 [282] and ResNet-50, whose score was over $0.1 \%$. To train the neural network was used a set over two hundred pictures and six different categories. This solution can detect objects in the 


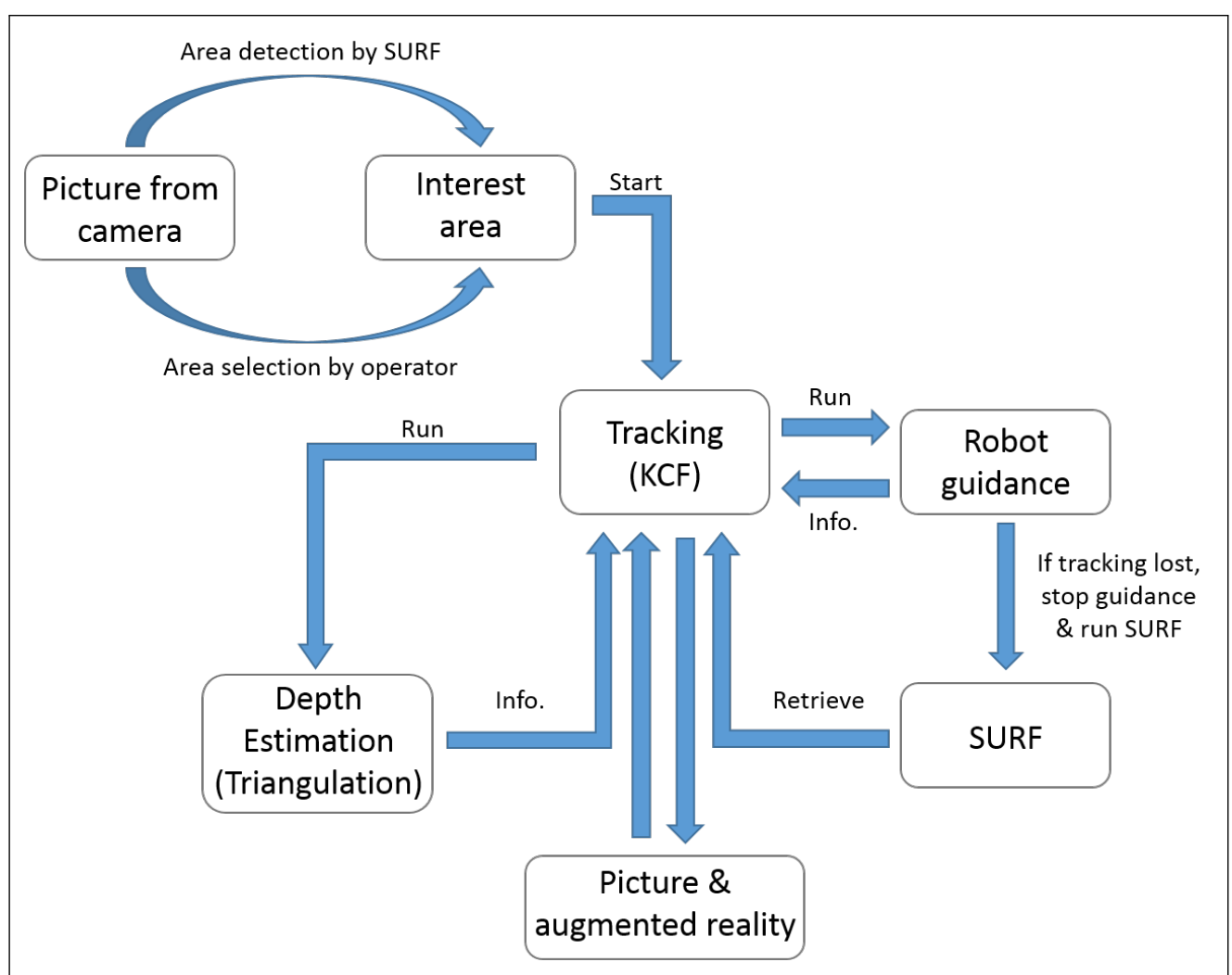

Figure 4.12: Tracking system diagram. The operator or the system/HRI selects the region of interest to be tracked. The tracking algorithm drives the control of the arm, communicates with the dept estimation algorithm and generates the augmented reality features.

remote robotic site in less than 1 second, providing a bounding box of each object detected. Then, the operator can directly choose the object to track, which starts the depth estimation procedure.

\section{Collision avoidance}

The CERNTAURO framework requires a robust collision avoidance [283, 284, 285] system that prevents any harm between the robot manipulators and other components, as the robot base. Since any manipulator might be placed on the CERNTAURO, the collision avoidance system needs also to provide adaptability. Therefore a reconfigurable system that prevents any impact between multiple manipulators and fixed objects is desired.

In harsh and hazardous environments like CERN accelerators complexes, robustness and reliability of the system are of great significance. Surrounding equipment must be protected, avoiding any accident heavier than the problem trying to solve, since collision must be prevented by any means. Consequently, modelling a robot by virtual objects that envelope the real one presents a trustworthy solution. 


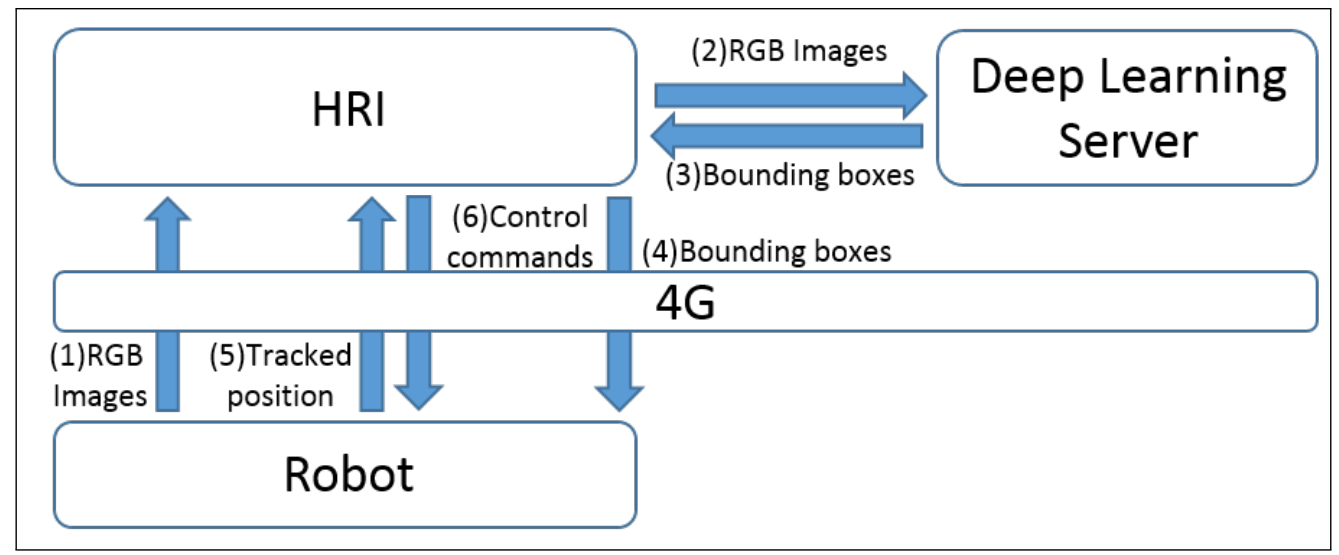

Figure 4.13: Deep Learning integration diagram. The artificial intelligence module has been added to automatically detect an object without the user interaction.

The chosen representation for the robot are virtual objects $[286,287,288]$ that cover its body. The 3D volumes that have been selected to envelope the robot are basic geometric shapes: spheres, boxes and cylinders. The reason is the low time consumption compared to the use of AABBs, OBBs, RSSs or kDOPs [289]. Each of the virtual objects require the position and orientation of the link or joint associated. Links location is found in the middle point between the two joints that connect them.

Modularity in this system has been implemented in order to provide reconfigurability and versatility. The different modules:

Robot Parser, module in charge of reading all of the features defined in the configuration files(see Figure 4.14).

Robot Definition, provides the kinematics and virtual objects computation for any robotic arm.

Collision Detection, module is responsible for checking if the diverse virtual objects are in collision.

Virtual Visualizer, make use of visualization tools so as to represent the virtual definition of the diverse robots.

The Collision Detection module works as follows. First, the module receives as input the allocated name and configuration of the manipulators to be loaded and modeled. Then, a vector containing all the desired manipulators is created. In order to check the collision between the manipulators and the external fixed objects, the Robot Parser is called to create the virtual objects. Then, a text for reporting which objects have virtually collided is generated in line with the number of virtual joints, links and external objects.

Every time that the an action is commanded to the robot, the Collision Detection module needs to be called. This runs the Collision Check algorithm, (see Figure G.1), that is made up to four functionalities: 


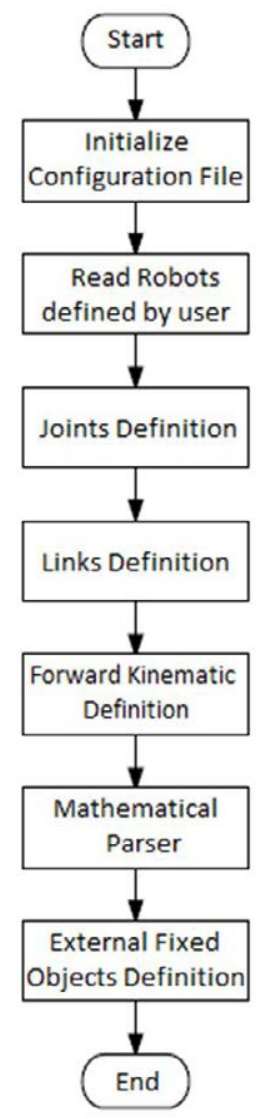

Figure 4.14: Collision avoidance test done by the robot parser, that is in charge of reading all of the features defined in the configuration files.

1. Check Self-Collision, self-collision of the manipulator is tested. It computes the distance among the defined virtual objects of the manipulator.

2. Check Robots Distance, a distance query is carried out between the distinct manipulators. It returns whether the distance between all the manipulators is lower than the fixed thresholds or not.

3. Check External Objects, manipulators and external fixed objects are checked for collision.

4. Check Environment Collision, a distance query is performed between the manipulators and the unknown surrounding environment.

Then each robot's pose is updated. The different functionalities can be activated/deactivated as desired, improving the modularity of the system.

The proposed solution allows also to avoid collisions in between the operator an the robotic arms, when as master device a robotic arm is used, option that is provided by the 


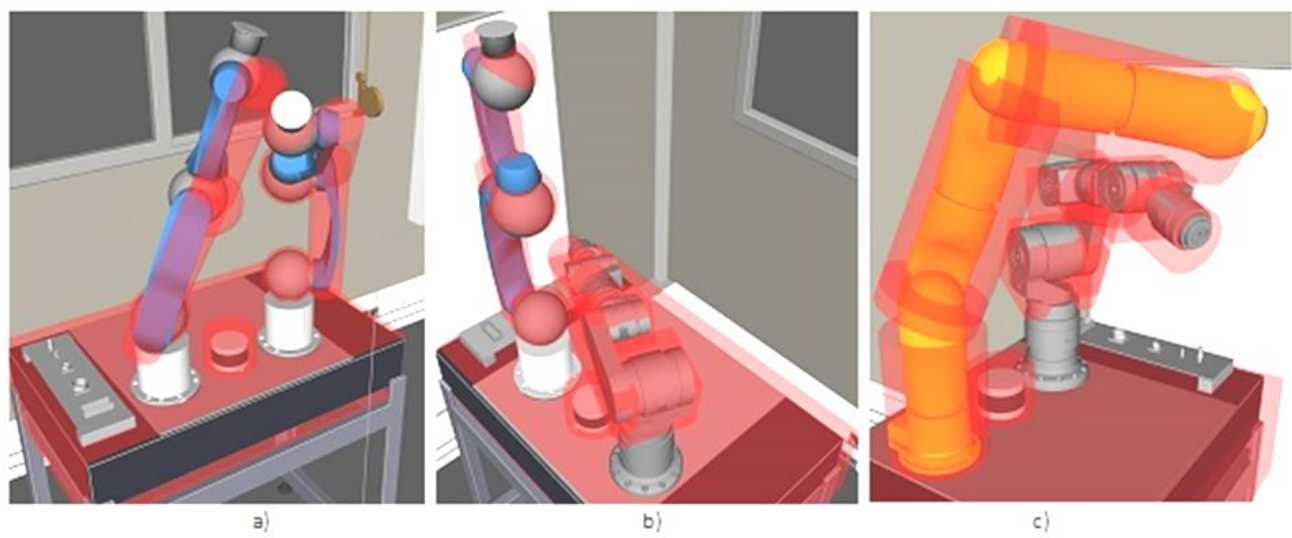

Figure 4.15: Simulation of the virtual constraints to avoid collisions in between different arms and in between arms and environmental fixed objects.

proposed framework (see Chapter ). This unique feature is fundamental to allow a safe use of a robotic arm as master device in teleoperation.

The proposed Collision Avoidance System has been properly implemented and tested in the CERNTAURO framework, see Figure 4.15. In these tests, two robotic arms were used, the Schunk LWA4P and the Schunk PowerCube. The collision reaction integrated was the stop of the robot if the next commanded action had moved the robot below a threshold. An example is shown in Figure 4.16, in which the Schunk LWA4P was stopped when finding a collision between its last joint and one of the motors of the Schunk PowerCube. In these tests, the danger zone was set to 4 centimetres and the collision distance was reduced to 2 centimetres, letting the robots come closer but still avoiding the impact.

For one of the test to reconstruct an environment a Kinect camera has been used. Thus, the proposed system has been tested in unstructured environments recognizing the collision between the virtual robots and the real-time virtual 3D reconstruction.

\section{TInspect : Vision Based Change Detection System for Inspection}

During tunnel inspections, personnel may have to enter hazardous environments. CERN has more than $50 \mathrm{~km}$ of tunnels, hosting machinery used for different experiments in difficult environments. One of these, is a tunnel hosting the Large Hadron Collider (LHC). To ensure safety, the $27 \mathrm{~km}$ long tunnel structure, together with the devices within it, need to be regularly monitored. In order to access the LHC tunnel, personnel require personal protective equipment (PPE) [290]. Access is strict and needs to be coordinated with the CERN Control Center (CCC) beforehand. Furthermore, when personnel enter the tunnel they might be exposed to risks from the presence of radiation, and need to abide by certain rules [291] to keep the dose levels according to the 'As Low As Reasonably Achievable' (ALARA) principle adopted at CERN. 

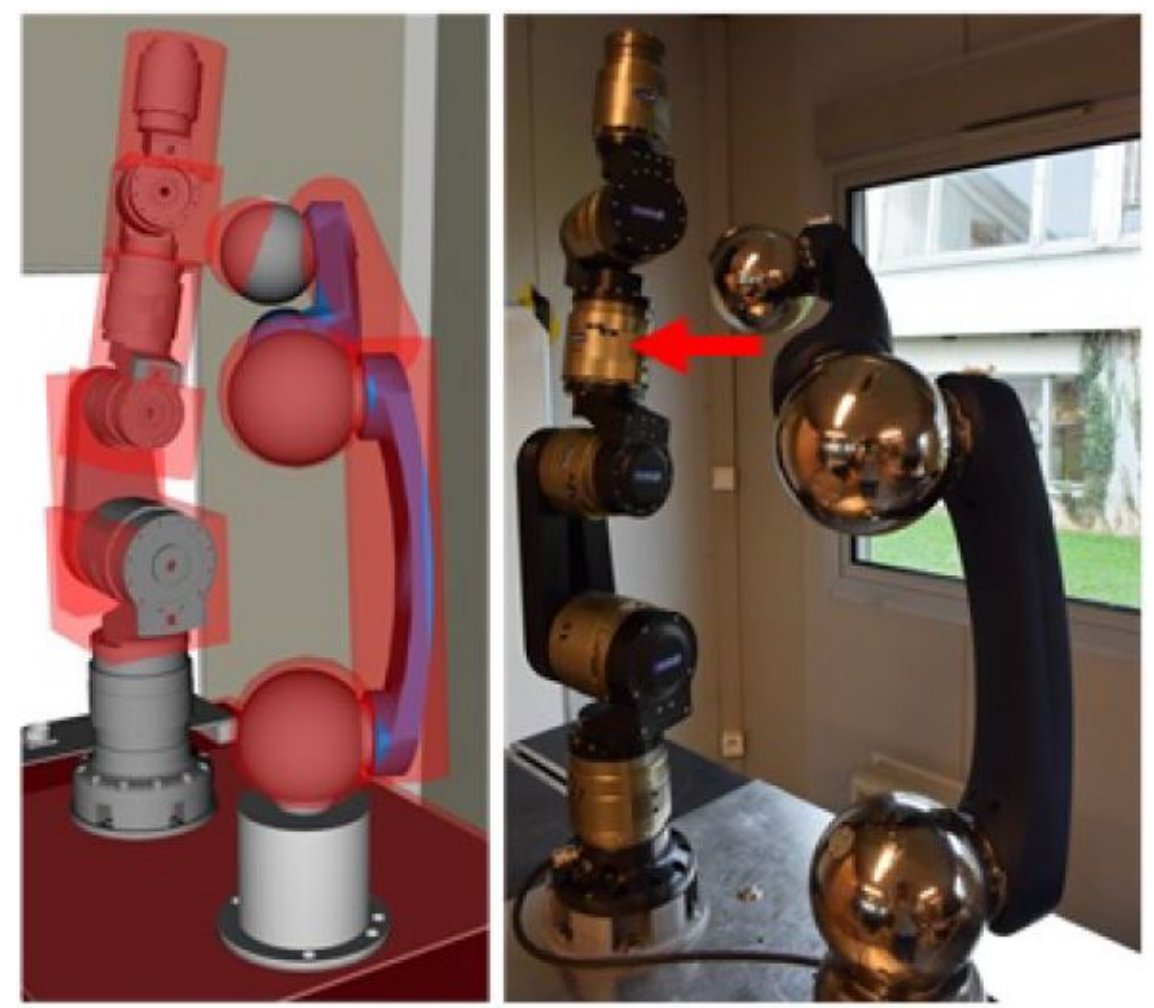

Figure 4.16: Pictures of the collision avoidance test.

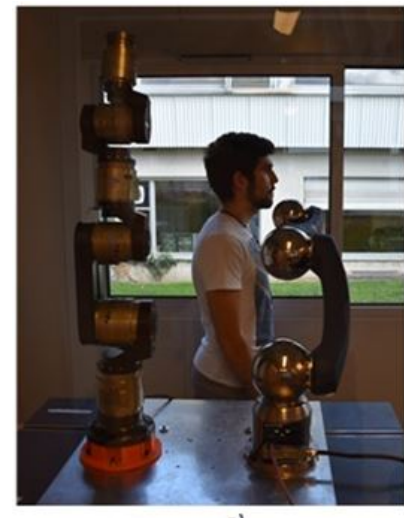

a)

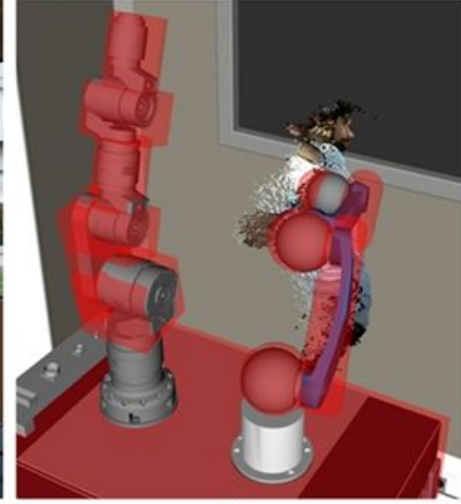

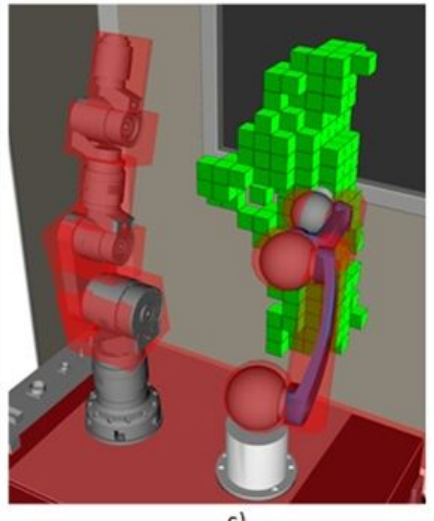

Figure 4.17: Simulation of the virtual constraints to avoid collisions in between different arms, in between arms and environmental fixed objects and in between arms and person.

Over time, tunnel infrastructure shows signs of deterioration due to aging and stresses which may lead to problems in structural integrity. Consequently, to ensure safety in concrete tunnels, periodic inspections have to be conducted. The CERNTAURO framework is endorsed with the TInspect [292] system that in an autonomous way can monitor the health status of the tunnel lining using a vision-based change detection system. Such changes are 
identified through image processing and photogrammetry based techniques and include detecting the presence of cracks on walls or anomalies like water or smoke presence.

While automatic tunnel inspections able to detect and classify wall defects and cracks have been recorded in the literature, change monitoring systems are still lacking. Contrastingly, TInspect offers a new means of automatically detecting changes on tunnel wall linings over time. It corrects for position offset between temporal images resulting from image capture position variances due to the moving platform using an alternative approach, that of image mosaicing with binary edge detection as a high level feature for registration. This is followed by a new hybrid change detection algorithm using neighbourhood image differencing, binary image pixel comparison and optical flow. The system achieves both a high sensitivity of $83.5 \%$ and $82.8 \%$ precision, as well as an average accuracy of $81.4 \%$. This provides a reliable tunnel wall change monitoring framework which is able to detect changes with a resolution of around $10 \mathrm{~cm}$ in width or height.

Tunnel structures made of concrete need to be inspected for cracks, leakages and other issues from time to time. To monitor the structure, automatic inspection systems are advisable to avoid human detection errors, damage or crack misidentification, data management inefficiency, subjective survey reports and personnel presence in harsh environments. Thus various tunnel inspection systems have been proposed in the literature.

A tunnel scanner to capture high-precision panoramic images of the surface of a tunnel lining was developed in [293]. An image mosaicing algorithm was used to splice adjacent images while the gradient of the luminance along line edges was utilized for the detection of cracks. A low cost robotic system was designed in [294] to capture images, process them and then produce maps of tunnel linings for manual detailed inspection afterwards. Crack detection and measurement were achieved in [295] using a mobile robot system consisting of optical, mechanical and data storage devices. Edge detection and neighbour region linking were used for crack detection. An automatic crack detection and classification method for subway tunnel safety monitoring was proposed in [296]. The system developed in [297], uses the Otsu method for leakage recognition and an algorithm based on the features of the local image grid is used to recognize cracks. A general survey on existing robotic tunnel inspection systems can be found in [298].

Detection of cracks using image processing has been explored by many researchers, however literature on comprehensive frameworks to monitor the health of tunnel linings over time using image processing is still lacking. Such a framework should include a complete solution involving two main modules: Image Acquisition and Change Detection. The following sub-sections discuss previous work in the field of each of these modules separately.

\section{Image Data Acquisition}

Inspection systems need to be ideally unmanned such as in [295]. The latter system uses a line-scanning camera for crack detection. The system proposed in [293], uses combined arrays of line-scanning camera arrays and powerful lighting. Charge-couple device (CCD) line-scan cameras were used in systems [299] and [300]. While line-scanning cameras acquire data quickly, due to their limited field of view, image distortion can be quite severe and 
hence the robot motion relative to the target must be carefully controlled. A fisheye camera together with structured light were used for inspection and to determine the robot's location via visual odometry in [301].

The potential of using a simple DSLR (Digital Single Lens Reflex) for image mosaicing is shown in [302]. This technique is mostly manual, requiring known reference points or laser markers and a precise geometry is assumed. A similar system was presented in [303] using a rig of DSLR cameras, however the capture process is manual. The system in [294] also uses multiple DSLR cameras in combination with a polarised LED array for lighting. Another project is that of Tunnelings [304]. The tunnel inspection system, developed by Euroconsult and Pavemetrics, consists of cameras and laser sensors, scanning tunnel wall linings at speeds of up to $30 \mathrm{~km} / \mathrm{h}$. Such robotic systems can complete the inspection process with objective results and high efficiency.

\section{Change Detection}

The majority of currently existing tunnel inspection systems detect cracks and deformities as well as the presence of water along the tunnel linings. However, a rather more useful and informative survey should include regular tunnel inspection to monitor the health of tunnel linings over time. The ability to detect changes that quantify temporal effects using multi-temporal imagery provides a fundamental image analysis tool in diverse applications, thus a considerable amount of research has been done in this field.

For a reliable comparison, the images must be taken from exactly the same point. This is quite a difficult task when the cameras are mounted on a moveable platform. In order to counteract this, images need to be registered first, such that they are spatially aligned. Once this is done, image comparison can be done through simple image differencing, ratioing or various other algorithms.

\section{Image Stitching and mosaicing}

In our scenario, images will be continuously captured while the TIM is moving and therefore the exact capture position is almost impossible to match the previous survey. For this reason, a position offset correction method had to be implemented to have a reliable comparison for change monitoring. The use of image mosaicing is proposed for this offset correction, and therefore a brief analysis of the state of the art in image stitching and mosaicing is provided below.

Image stitching algorithms take a multiple number of images as input and combine them to form a larger single image. Applications, include multiple image super-resolution, video stitching, aerial land surveys and medical imaging. Image stitching algorithms are commonly bundled with most digital cameras, smart-phones and tablets to create wideangle panoramas. Recent surveys of existing image stitching algorithms can be found in [305], [306] and [307].

A mosaic is generated by stitching multiple rows of photos that were taken without rotating the camera around a single point as in panoramas, but by keeping the camera perpendicular to the subject. Image mosaicing techniques have been reviewed in various 
publications including [308] and [309].

Image mosaicing applications share a common pipeline consisting of two stages: Image Registration and Image Compositing. Registration is the process of spatially aligning two or more images of the same scene but taken from different viewpoints, using multiple sensors or even at different dates (due to difference in lighting or other physical conditions). Detailed surveys of image registration techniques can be found in [310], [311] and [312]. Also, a study of image registration in the context of image stitching can be found in [313].

After aligning the images, the remaining task is to stitch these images to form a single image. Seams along the overlapping area should be as imperceptible as possible and exposure differences between the images to be stitched should be adjusted to avoid false positives. One method is gain compensation as used in [314]. A number of effects such as, vignetting, registration errors and parallax effects may cause some image edges to be still visible even after such a compensation. Consequently, a blending strategy should be adopted such that the value of each pixel in the overlapping area of the input images are combined using a specific function to produce the value of the pixel in the output image. In this way, the content presents a smooth transition between the images in the final mosaic. Rankov et al [315] use alpha blending, where proportions of the corresponding image values are taken depending on the distance of the pixel from the image edge. Brown and Lowe [314] use the multiband blending technique which was originally proposed in [316]. The idea of multiband blending is to blend low frequencies over a large spatial range and high frequencies over a short range using blending weights.

\section{Image comparison}

Vision-based change inspection detection involves identifying the differences between two images of the same scene as observed at different times. Image comparison techniques are applied to the images to be able to detect any changes. Pixel-based methods include image differencing, image ratioing and image regression amongst others. Transformation-based approaches such as Fourier Transform involve converting image intensity data to another domain and then comparing the resulting data. A reasonable study which demonstrates various aspects of different change detection techniques is provided in [317]. The effectiveness of various change detection approaches for identifying land cover changes, as implemented in the MATLAB environment was investigated in [318].

\section{System Overview}

The proposed TInspect system consists of four core components as portrayed by the block diagram in Figure 4.18. The shading correction applied by the Photo Module at the preprocessing stage enhances the image brightness and eliminates shadows. Since images are to be captured while the TIM is running, images compared over time are from different locations, raising the need for position offset correction. Our algorithm corrects this offset using image mosaicing. Once the two closest reference images are stitched by the Mosaic Engine, the Survey Mapper locates the survey image in the mosaic image. Finally, the Change Detector uses a sequential combination of image differencing, binary image comparison and 
optical flow analysis to detect any changes occurring from one inspection to another. The Photo Module uses the position of an input image, it retrieves the closest two images from a previous survey and then pre-processes all the 3 images, enhancing their brightness and eliminates shadows Photo Module, Mosaic Engine, Survey Mapper and Change Detector and their corresponding inputs and outputs at the top and bottom respectively. The Mosaic Engine takes the reference images from the previous survey and stitch them together, to provide a larger reference image, including the whole area covered by the survey image. The survey Mapper performs image registration such that it locates the survey image withing the mosaic reference image. The Change Detector compares the registered images using a sequential combination of image differencing, binary image comparison and optical flow analysis to detect changes occurring from one inspection to another. Each of these modules is described in detail in Appendix F.

\subsection{Autonomous Navigation for Workplace Approach}

Autonomous navigation in unknown unstructured environments is still an open issue, although there has been a lot of effort in the last decades in this research field. Nowadays, research focuses on the development of self-driving vehicles, tackling a lot of fields like safe navigation, human-robot collaboration etc [319]. However, In the matter of this work, one of the most important features among the autonomous navigation field is the robot localization. Robot localization is fundamental during accurate environmental data collection in the accelerator's environment. Nevertheless, an accelerator's tunnel appears very difficult in the matter of precise localization without the use of artificial features (such as beacons) for localization, due to the similarity of different places, lack of stable features and symmetries of the tunnel.

The ability to acquire in real-time a 3D model of the environment in order to navigate safely in it and accomplish complex tasks is called Simultaneous Localization and Mapping (SLAM) [320]. In the absence of global positioning information like in GPS-denied environment, the robot's state (the robot's pose and map) is essential to obtain a safe and reliable autonomous navigation. Obtaining large and accurate maps of generic unknown environments is still an open problem, especially when performed in real-time.

SLAM algorithm compute data coming from various sensors needed for the autonomous navigation. More in mathematical terms, the goal of a SLAM algorithm is the maximization of the following likelihood:

$$
p\left(x_{t}, m_{t} \mid x_{t-1}, m_{t-1}, z_{t}, u_{t}\right)
$$

where, $x_{t}$ is the 6 DoF pose of the robot, $m_{t}$ is the representation of the environment, $z_{t}$ are the sensorial data and $u_{t}$ are the controls.

In order to properly estimate the $6 \mathrm{D}$ pose of the robot, the CERN Robotic Framework fuses together various onboard sensors: the motor encoders, an IMU, a 2D LIDAR and a monocular camera. The implemented SLAM method uses various filters to accurately 


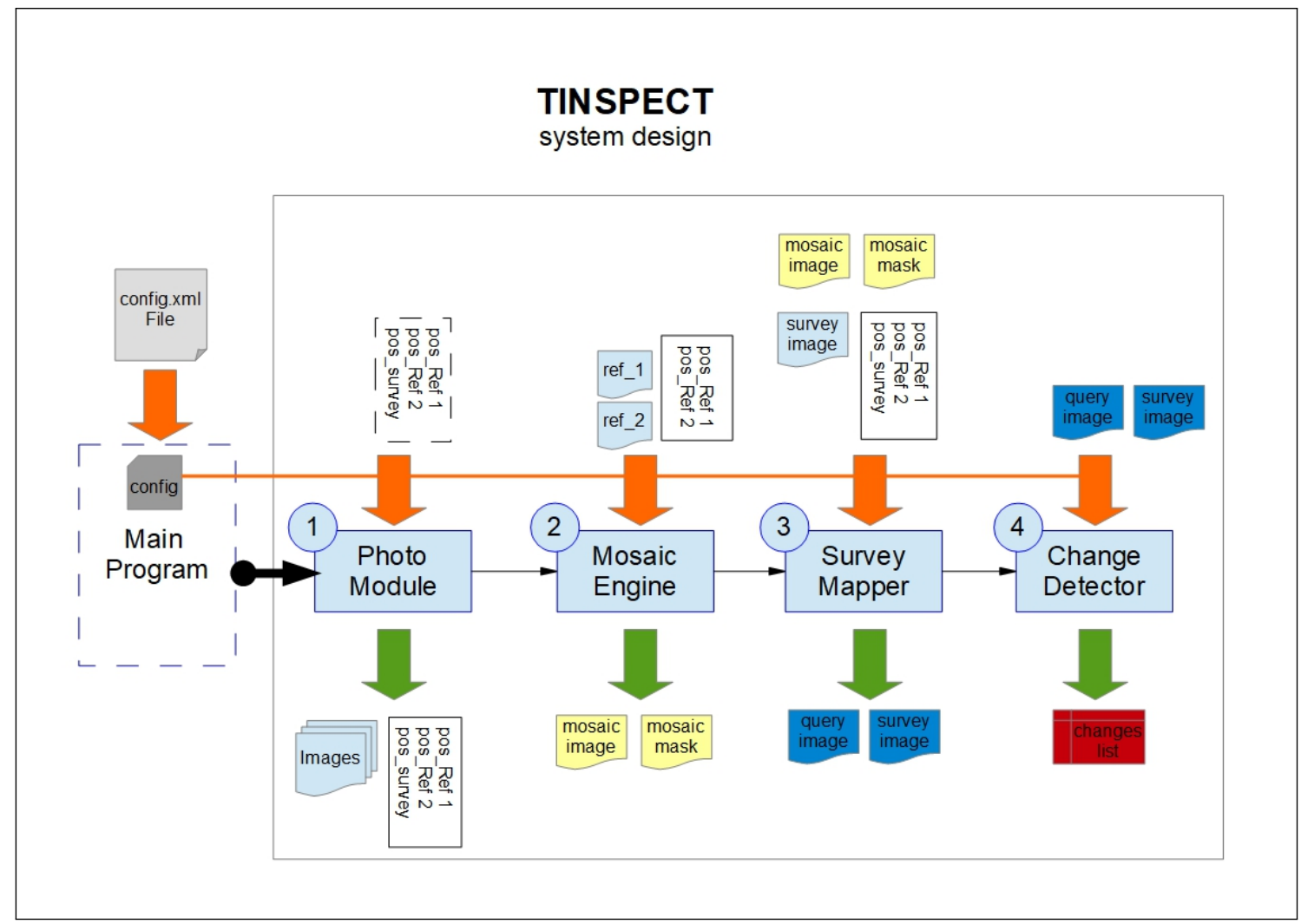

Figure 4.18: System block diagram with four modules. The proposed system consists of four core components as portrayed here. The shading correction applied by the Photo Module at the pre-processing stage enhances the image brightness and eliminates shadows. The algorithm then corrects for position offset between different capturing times using image mosaicing. Once the two closest reference images are stitched by the Mosaic Engine, the Survey Mapper locates the survey image in the mosaic image. Finally, the Change Detector uses a sequential combination of image differencing,binary image comparison and optical flow analysis to detect any changes occurring from one inspection to another.

estimate the odometry steps of the robot and fuses together the outputs of such filters in a lightweight particle filter.

More in details, at first the robot odometry is estimated by fusing the motor encoders with the IMU output (accelerometers and gyroscopes) through an Unscented Kalman Filter. The output of this filter provides a first estimation of the movement of the robot. However, this estimation is affected by wheels slippage on the floor, which usually offers little grip in accelerator's tunnel, and IMU drifts.

This first estimation is used by a visual odometry system. The visual odometry system, explained in details in the following, uses a monocular camera installed in front of the robot and a 2D LIDAR to compute a more accurate estimation of the movement performed by the robot. The 2D LIDAR, in this case, is used to compute the factor between the local scaling typical of any Visual Odometry algorithm and the real environmental scale. The 
output of the visual odometry is considered, then, the best estimation of the odometry step performed by the robot.

Once the odometry step is computed, data from sensors are fused by the SLAM algorithm for obtaining a global localization. The SLAM algorithm implements a particle filter to estimate the global 6D pose of the robot. Differently from standard particle filter SLAM methods, the presented approach is more suitable for realtime. Other approaches like Hector SLAM [321] provide a 6 DoF state estimation but a 2D map. The approach proposed in this paper thesis provides a full 3D map, more suitable for autonomous navigation. The proposed method is suitable both for UAVs and AGVs. The AGVs can provide also wheels odometry information that can replace the UAV's IMU.

More in details, the SLAM module maximizes the probability given in the equation 4.1 by analysing the input processed in the previous modules. The main idea follows the particle filter SLAM implementation using a Montecarlo localization based approach [198]. Initially, as illustrated in Figure 4.19, given the estimation of the pose of the robot $\widetilde{x_{t}}$ coming from the odometry system, the previous calculated map $m_{t-1}$, the proposed algorithm performs multiple ray casting [322] operations extracting the distance $z_{t}^{k *}$ from the first filled voxel on the map $m_{t-1}$ at the position $\widetilde{x_{t}}$. Using a specific laser sensor model, the algorithm weights the measured distance, given by the laser, returning the probability to have the measured distance given the position $\widetilde{x_{t}}$ the map $m_{t-1}\left(p\left(z_{t} \mid \widetilde{x_{t}}, m_{t-1}\right)\right.$. Following, the algorithm repeats the previous weights operation on several $\widetilde{x_{t}}$ generated according to the motion model of the robot returning a set of weighted poses. Unlike particle filter SLAM, instead of keeping every particle with its own weight and its own map for all the execution time, in the new proposed method, only the position that has the highest weight is kept. This operation is performed for all the ranges at different angles of the 2D laser scanner and could be used also on 1D or 3D laser scanners. Standard Particle Filter SLAM algorithms, running on embedded platforms, use around 10 particles during their execution having 10 consistent maps but only using 10 samples of the robot motion model at each step. The new proposed approach, running on embedded platform, uses up to 100 samples at each step. In this way, the space around $\widetilde{x_{t}}$ is sampled with more points providing much more accurate maps. Moreover, Standard Particle Filter SLAM methods need to bring always the same number of particles during the whole algorithm process, while the new proposed approach is flexible and, not being linked to previously maps, it can use different particles number according to the map error estimation and needs. The algorithm is fast enough allowing two consecutive calculated points to be very close reducing the map errors that could come from the deletion of all previous calculated maps. We can consider that at every step, the algorithm does an error correction of the estimated pose $\widetilde{x_{t}}$. Furthermore, referring always to a previous calculated map, the proposed algorithm keeps a global consistency, assuming that the map gathers together all the previous measurements.

Once the occupancy grid map is generated, it is possible to apply on top of it state-ofthe art path planning algorithm. The most common algorithm available in literature and therefore applied in this framework is RRT* [323]. Nevertheless, applying path planning algorithms directly to the spatial occupancy grid map could generate an unsafe path for the robot. The behaviour of the robot while navigating in harsh environment could be affected 


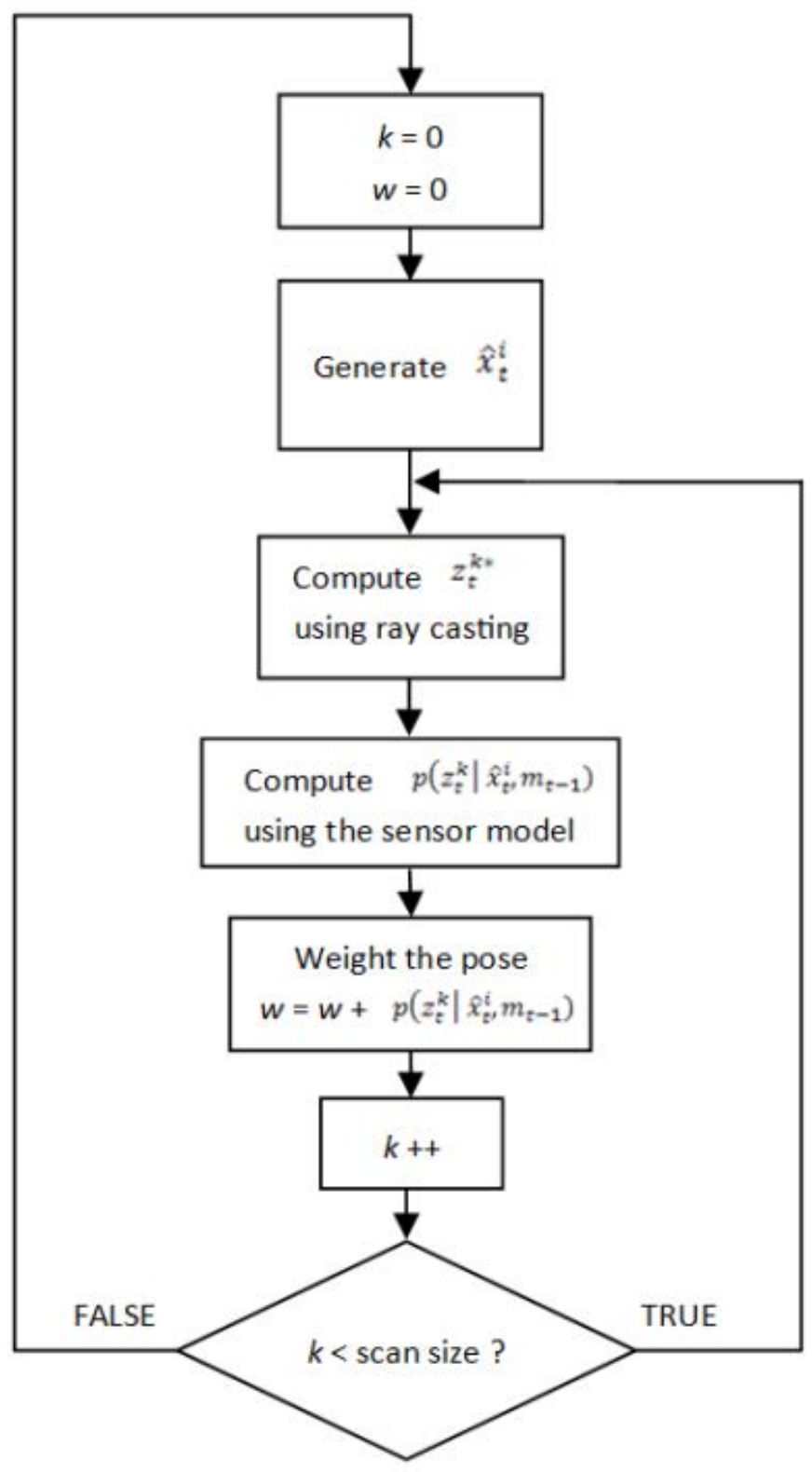

Figure 4.19: Description of the sequential flowchart of the proposed SLAM module. Initial parameters assumption are introduced to the ray casting module and then the probabilistic approach is computed using the sensor model. After the robot's pose is estimated before closing the step for the next loop. 


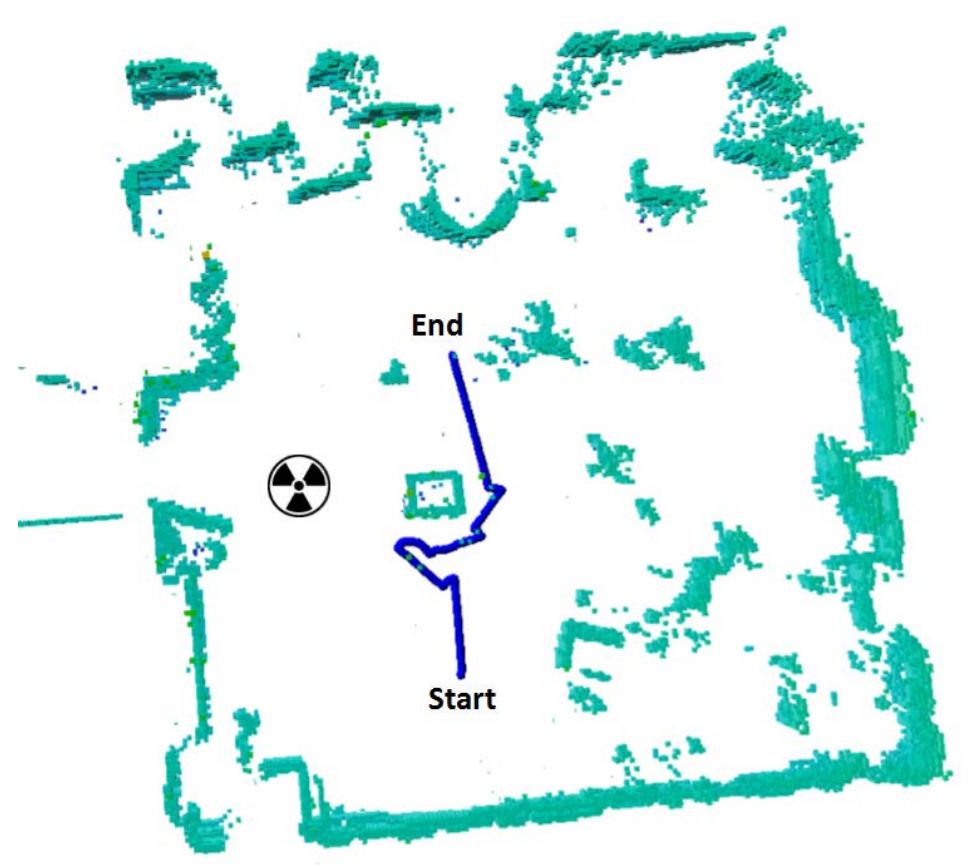

Figure 4.20: The occupancy grid map collected in real-time by the robot with the covered path. To notice the change of path when the environmental radioactivity was considered too high for the robot.

by high radiation, high magnetic fields, high temperatures. Therefore, it is important that during the autonomous navigation, the robot takes into account these environmental factor in order to protect itself from possible damages. This is achieved by running the RRT* algorithm not on the map generated by the SLAM algorithm, but on an "augmented" map, which contains virtual obstacles generated during the navigation according to the environmental sensorial data measured by robot (see Figure 4.20).

\section{Real-Time Visual Positioning System}

The SLAM algorithm presented before is endorsed with a Direct Sparse Odometry (DSO) based visual odometry method coupled with a 2D LIDAR to increase the accuracy on the positioning of the robot in environments with little stable visual features, where classical algorithms are prone to significant drift error. Camera and laser data are synchronously acquired in order to be processed by the point cloud module. The output of this odometry system is then provided to the SLAM system for the global positioning computation.

Most of the odometry systems developed by now rely on a sparse set of keypoints extracted from an acquired image, mostly based on simple geometric features such as corners, edges with a large image gradient. However, these algorithms not only require a highresolution camera and distinct characteristics in each image for robust feature extraction, weak intensities and smooth gradients are generally ignored. In keeping with this, CERN's facilities do not guarantee robust image features, mainly due to metallic reflections, large 
spaces, and repetitive environments.

In contrast, direct camera tracking makes use of pixel intensities directly, omitting the cost-expensive feature extraction, description, and matching steps. More information from the image can be used, guaranteeing increased precision and robustness. The direct odometry method finds the relative 6 DOF camera pose transformation $T_{i, j}$ from an image $I_{i}$ to $I_{j}$ that minimizes the per-pixel intensity difference (photometric error):

$$
T_{i, j}=\arg \min _{T} \sum_{k}\left\|I_{j}\left[p_{k}^{\prime}\right]-I_{i}\left[p_{k}\right]\right\|_{\sigma}^{2}
$$

where

$$
p_{k}^{\prime}=\prod_{c}\left(T *\left(\prod_{c}^{-1}\left(p_{k}\right)\right) * d_{p_{k}}\right)
$$

describes the projected point position of $p_{k}$ with inverse depth $d_{p_{k}}$. We denote the projection from world coordinates to image coordinates as $\prod_{c}: \mathbb{R}^{3} \rightarrow \Omega$ and $\prod_{c}^{-1}: \Omega \times \mathbb{R} \rightarrow$ $\mathbb{R}^{3}$ as the corresponding back-projection, where c represents the intrinsic camera parameters for the camera model. More specifically, we can express the relative transformation $T_{i, j}$ as

$$
T_{i, j}=T_{j} T_{i}^{-1}=\left[\begin{array}{cc}
R & t \\
0 & 1
\end{array}\right]
$$

where $T_{j}$ describes the transformation from the coordinate origin to frame $\mathrm{j}$. This notation will be used in the future. The photometric error minimization problem formulated in Equation 4.2 is usually solved with nonlinear optimization methods [324].

Sparsity Odometry systems can be classified as dense or sparse, depending on the amount of an acquired image that is further processed. In particular, dense systems tend to use most or all of the pixels in each frame, exploiting the connectedness of the image region to formulate a geometry prior that typically favors smoothness. This prior then appears in the log-likelihood as an additional regularization term for global optimization [325]. While this smoothness may increase the map attractiveness, it can act as a bias that distorts the pose estimation and unnecessarily increases the scale drift. Moreover, dense reconstruction methods are known to be computationally expensive and generally require GPUs to run in real-time. Sparsity, i.e. extracting only a small selected subset of pixels and mapping a coarse scene representation, drastically reduces computational cost and prioritizes localization tracking over point cloud reconstruction.

Sensor Fusion for Global Scaling In monocular odometry systems, motion scale is generally unsolvable without resorting to aid from coupled sensors, dynamic motion assumption models, or the installation of markers with a priori known size such as April tags [326]. Moreover, pure vision often suffers in scenes with low texture, high dynamic range, and high speed motion. Solutions to this problem include the use of stereo cameras, iner- 


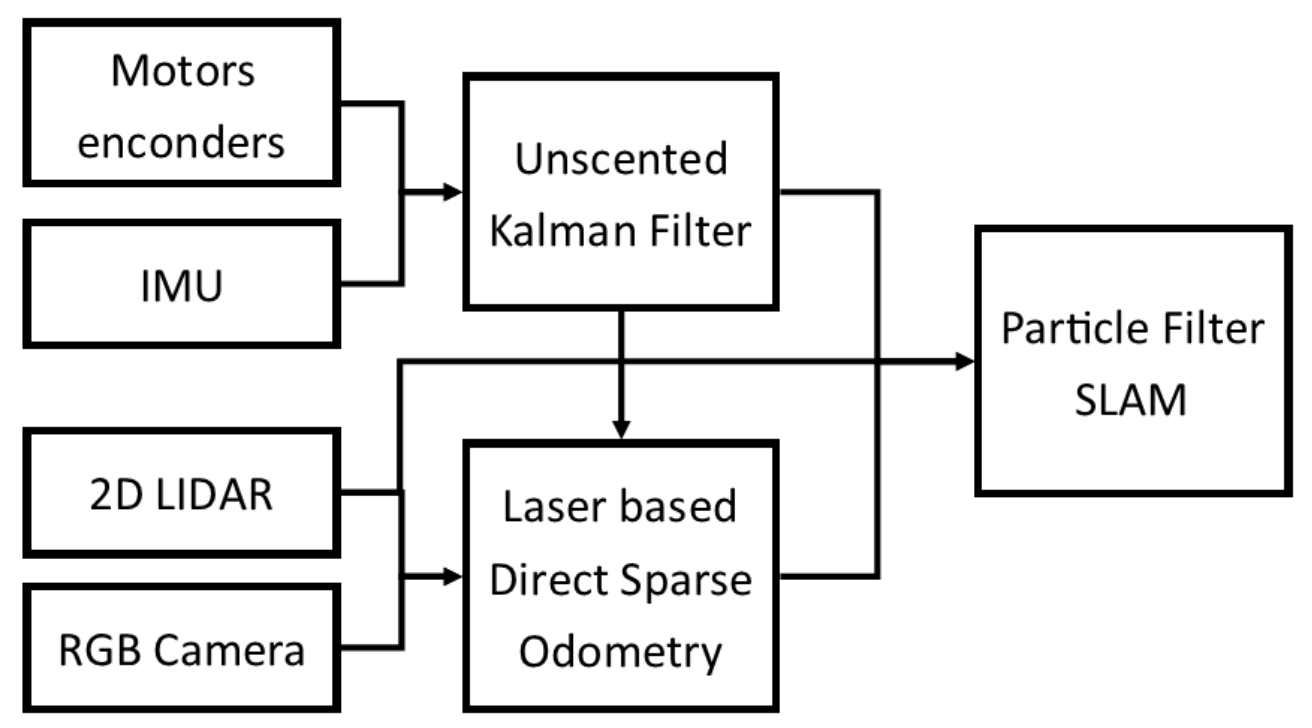

Figure 4.21: The SLAM architecture of the CERNTAURO framework. From left to right the flow goes in a first step from the hardware read-out and data fusion to kalman filter and direct sparse odometry as second step. In parallel the particle filter SLAM computes the localizaion and mapping.

tial measurement units (IMUs), Global Positioning Systems (GPS), RGB-D cameras or any combination of these to retain scaled depth values in a reconstructed scene.

In the autonomous navigation community, visual systems are frequently coupled with lidar systems, as they are suitable in computing the surrounding depth for long ranges. Zhang et al., for example, uses scan matching-based lidar odometry to refine the motion estimation and point cloud registration simultaneously with a monocular camera and a 3D laser scanner, from which also the state can be estimated [327]. Droeschel et al. integrates a multi-camera visual odometry into a scan-matching method based on a multi-resolution point cloud representation [328]. the proposed method method only uses one camera and a static 2D scanner in the system, mainly focusing on high speed.

For example, Tong et al. employs feature matching from intensity maps generated by stacking scans of 2-axis lidars, modeling the motion with constant velocity and Gaussian processes [329]. This is computationally expensive, however, as dense point clouds are required for feature extraction merely from laser scans. Moreover, Bosse and Zlot propose a method for mapping an underground mine with the help of a hand-held device attaching a 2D laser scanner and an IMU with a spring [330]. The sensor trajectory is computed by a batch optimization process with certain boundary constraints, however, this is more suitable for offline surveys. In our case of teleoperated assembly in one of the tunnels, the robot is required to provide live location data. Moreover, IMUs are not suitable for our application due to excessive vibration of the robotic base during surveys. Filtering noise through a low-pass filter did not guarantee robust measurements.

The full pipeline of the novel proposed system is visualized in Figure 4.21. 
The novel proposed visual odometry algorithm operates directly with pixel irradiance, thereby omitting the feature description and descriptor matching. It is based on the nonlinear optimization of a photometric error function describing a sparse set of pixels sampled over a residual pattern. Optimization is performed in a sliding window, where camera poses and points leaving the camera's field of view are marginalized. In its essence, it can be seen as the photometric equivalent of windowed sparse bundle adjustment.

1. Photometric Camera Calibration: In the following, the method is formulated for a pinhole camera model for the sake of simplicity, assuming geometrically undistorted images. The image formation model from [325] is used, accounting for a non-linear response function $G: \mathbb{R} \rightarrow[0,255]$ and a lens attenuation (vignetting) $V: \Omega \rightarrow[0,1]$. In effect, the model is given by Equation 4.5, where Bi and Ii are the irradiance and the observed pixel intensity in Frame $i$, and ti is the corresponding exposure time. Thus, the photometrically correcting all frames in a pre-processing step is done by Equation 4.6.

$$
\begin{gathered}
I_{i}(x)=G\left(t_{i} V(x) B_{i}(x)\right) \\
I_{i}^{\prime}(x):=t_{i} B_{i}(x)=\frac{G^{-1}\left(I_{i}(x)\right)}{V(x)}
\end{gathered}
$$

From this point, we will refer to the photometrically undistorted image as $I_{i}$.

For this purpose, a pipeline to recover $\mathrm{G}, \mathrm{V}$, and B rapidly for any webcam based has been integrated into CERN's robotic framework. The inputs needed are (1) calibration images of a chessboard for the estimation of intrinsic camera parameters, (2) pictures of a static scene at different exposures for estimation of the response function $\mathrm{G}$, and (3) a sequence of images of an Aruco marker from different angles for the computation of the lens attenuation $\mathrm{V}$ across the sensor.

2. Model Formulation: We use the same notation the full photometric error over all active key-frames points is formulated as Equation 4.7 where i runs over all frames F, p over all points $\mathrm{Pi}$ in frame $\mathrm{i}$, and $\mathrm{j}$ over all frames obs(p) in which the point $\mathrm{p}$ is visible. Epj denotes the photometric error of a point $\mathrm{p}$ evaluated on a residual pattern, formulated as a weighted SSD function in Equation 4.8 where $t_{i}, t_{j}$ denote the exposure times in frames $I_{i}, I_{j}$, and $\|\cdot\| \gamma$ the Huber norm [331].

$$
\begin{gathered}
E_{\text {photo }}=\sum_{i \in F} \sum_{p \in P_{i}} \sum_{j \in o b s(p)} E_{p_{j}} \\
E_{p_{j}}=\sum_{p \in N_{p}} \omega_{p}\left\|\left(I_{j}\left[p^{\prime}\right]-b_{j}\right)-\frac{t_{j} e^{a_{j}}}{t_{i} e^{a_{i}}}\left(I_{i}[p]-b_{i}\right)\right\|_{\gamma}
\end{gathered}
$$


In addition to the Huber penalties, a gradient-dependent weighting $\omega_{p}$ is applied equation 4.9 down-weighting pixels with high gradient with some scalar constant c.

$$
\omega_{p}:=\frac{c^{2}}{c^{2}+\left\|\nabla I_{i}(p)\right\|_{2}^{2}}
$$

In summary, the photometric error depends on (1) the inverse depth $d_{p}$ of a point $\mathbf{p}$, (2) the camera intrinsics $\mathbf{c},(3)$ the poses of the involved frames $T_{i}, T_{j}$, as well as (4) their brightness transfer function parameters $a_{i}, b_{i}, a_{j}, b_{j}$. Note the similarity to the photometric error term from Equation 4.2, with the difference of weight multiplication and constant subtraction.

3. Frontend Visual Odometry: The visual odometry front-end determines (1) which points and frames are used, (2) in which frames a point is visible, (3) which points are outliers and if there are occlusions, provides (4) the initialization for new parameters in the energy function, and finally (5) determines when a point or frame should be marginalized.

Frame Management: Vanilla Direct Sparse Odometry (DSO) keeps a fixed size sliding window $N_{f}$ of active keyframes. Every new frame is initially tracked with respect to the newest keyframe, creating a semi-dense depth map. All subsequent frames inside the sliding window are tracked with respect to this keyframe. By using conventional direct two-image alignment in combination with a coarse-to-fine pyramid method, the pose estimation relies on a constant motion model. Subsequently, DSO determines whether the frame is discarded or selected as a new keyframe if too much movement or camera exposure. The photometric error previously described is then optimized, before the marginalization takes place.

Point Management: DSO always keeps a fixed number of active points in the joint optimization, equally distributed across space and active frames. In a first step, the candidate points in each new keyframe are identified - however not directly added to the optimization - and tracked with the help of a coarse depth value for initialization. Candidate points are then activated to be added into the optimization, when new points are needed.

\section{Laser Fusion}

1. Scan Acquisition: A memory-mapped file (MMAP) as a segment of virtual memory is used as the IPC method between CPU and laser. While this file is not associated with a file on a disk, it is suitable for creating shared memory for the communication. In a process separate from the odometry, the writer publishes laser measurements to the MMAP, while a reader iteratively checks if a measurement has been made. If this is the case and the scan at an arbitrary time $t$ is successful, the writer publishes a packet with $\mathrm{n}$ vectors (see Equation4.10), containing each $x$ and $z$ coordinates with respect to the laser origin on the plane of the scanner (see Figure 4.22). We can simply save these coordinates into a $2 \mathrm{D}$ point cloud $\left(Q_{l}\right)$. 


$$
L^{(t)}=\left\{\left[\begin{array}{c}
x_{1, l}^{(t)} \\
z_{1, l}^{(t)}
\end{array}\right], \ldots,\left[\begin{array}{c}
x_{i, l}^{(t)} \\
z_{i, l}^{(t(}
\end{array}\right], \ldots,\left[\begin{array}{c}
x_{n, l}^{(t)} \\
z_{n, l}^{(t)}
\end{array}\right]\right\}
$$

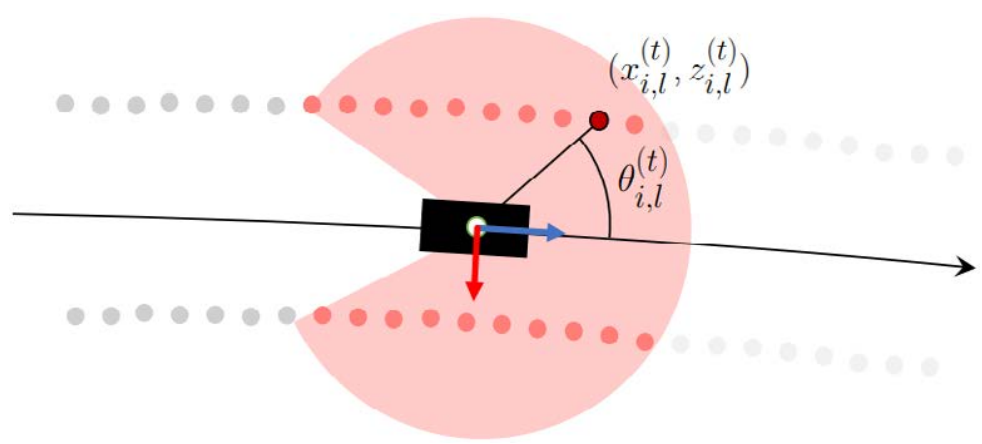

Figure 4.22: Schematic of the laser data acquisition. Laser points are gathered on the fixed $\mathrm{x}$-z-plane and scanned almost $240^{\circ}$ with $0.36^{\circ}$ angular resolution, due to a blind spot in the rear containing the angle $\theta$.

In our method, we save each scan associated with a POSIX time-stamp as the file-name in separate txt-files in a designated temporary folder. This prevents the memory from filling up and slowing down the odometry. Only the files that are needed for fusion can then be accessed from this folder.

2. Time Synchronization: Due to the nature of any visual odometry system assuming the alignment of camera direction and robot motion, reconstructed points appear not in the immediate vicinity of the pose, but rather near future poses. The correlation between current pose and these future poses strongly depends on the frame acquisition frequency and the robot velocity. Therefore, a fixed frame rate and motion are essential in recovering repetitive and accurate results.

As soon as the visual odometry is initialized, we start accumulating point clouds over a fixed number of keyframes $N_{f}$. If a new keyframe is created while the Hokuyo and L-DSO threads run in parallel, we pull the current laser scan from the MMAP. Meanwhile, in the thread of the output wrapper, a keyframe is published and checked whether new points have been marginalized. If this is the case, a new cloud is generated in a txt-file and the associated POSIX timestamp is pushed to a vector that is checked by the visual odometry thread.

In a subsequent step, we check whether the processed number of key-frames is above $\left(N_{f}\right)$. If yes, we initialize the fusion process. If not, we keep appending the current point cloud until these conditions are met.

In a general case, we append the point clouds generated in the $m$ previous key-frames by the visual odometry (see eq. 4.11) until the number of points in $Q_{v}$ surpasses a threshold $\left(n_{p}\right)$. If this is the case, we set $\left(Q_{v}^{*}:=Q_{v}\right)$. To prevent a point cloud too sparse stretched across dozens of keyframes, $\left(m=m\left(n_{p}\right)\right)$ must stay below a second 
threshold $\left(n_{m}\right)$. If both of these conditions are met, fusion can occur in equation 4.12 where $|\cdot|$ describes the cardinality of a set and c denotes the current step. Note that $\left|Q_{v}^{*}\right| \neq \sum_{i=1}^{m}\left|P^{(c-i)}\right|$, as $Q_{v}^{*}$ is a set of point sets. In this case we set $m\left(n_{p}\right)=3$ to make use of $Q_{v}^{*}=\left\{P^{c-3} ; P^{c-2} ; P^{c-1}\right\}$ for subsequent steps. It is possible to visualize this problem setting in Figure 4.23. Since each keyframe object in DSO parents a POSIX timestamp in milliseconds, we can save $Q_{v}^{*}$ correlated to each fusion keyframe $K F_{c}$ in a similar way as described in the previous section to prevent trashing the memory.

$$
\begin{aligned}
& Q_{v}=\left\{P^{(c-m)} ; \ldots ; P^{(c-1)}\right\} \\
& \left|P^{(c-m)}+\ldots+\right| P^{(c-1)}\left|=\sum_{i=1}^{m}\right| P^{(c-i)}\left|\leq n_{p} \wedge m=\right| Q_{v}^{*} \mid \leq n_{m}
\end{aligned}
$$

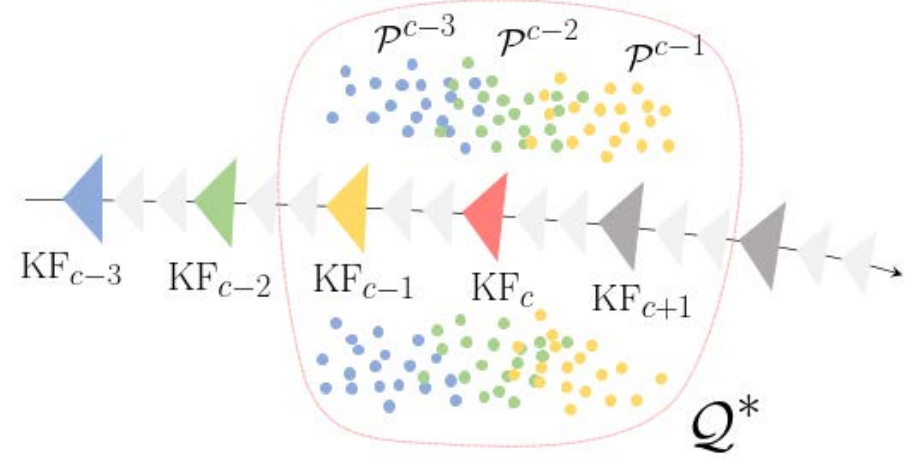

Figure 4.23: Point cloud cumulation over $\mathrm{m}$ keyframes (here $\mathrm{m}=3$ ). The previous three keyframes $(\mathrm{KFc} 3, \mathrm{KFc} 2, \mathrm{KFc} 1)$ generate a point cloud about the position of the current keyframe KFc. Each partial point cloud is characterized by the color of the respective keyframe. We assume that adding Pc would not meet the condition $|Q v| \leq \eta p$.

One of the challenges for sensor fusion - time synchronization of measurements appears due to frequency discrepancy and temporal misalignment of initial measurements. For example, a camera operating at $24 \mathrm{~Hz}$ coupled with a laser at $7 \mathrm{~Hz}$ will not have a modulus for which measurements with identical timestamps can be compared. Therefore, only measurements with the closest timestamps must be taken. While the laser data acquisition iteratively writes txt-files, the scan associated with the closest timestamp to the keyframe timestamp is taken (see Figurw 4.24). This is done with a forward iterator with complexity $O\left(\log _{2}\left(m_{l}\right)+1\right)$ where $\mathrm{ml}$ is the number of laser scans acquired so far. In summary, a cumulative point cloud over $m$ keyframes (from e.g. $t_{1}^{v}=t_{K F_{1}}, t_{5}^{v}=t_{K F_{2}}, \ldots$, and $\left.t_{k-4}^{v}=t_{K F_{m-1}}\right)$ is generated at time $t_{k}^{v}=t_{K F_{m}}$. Subsequently, the closest laser scan timestamp is found $\left(t_{m_{l}-1}^{l}\right)$ and set as the time of fusion $t_{0}^{*}$. The laser point cloud corresponding to this timestamp $Q_{l}^{*}$ is extracted from the memory and prepared for fusion. 


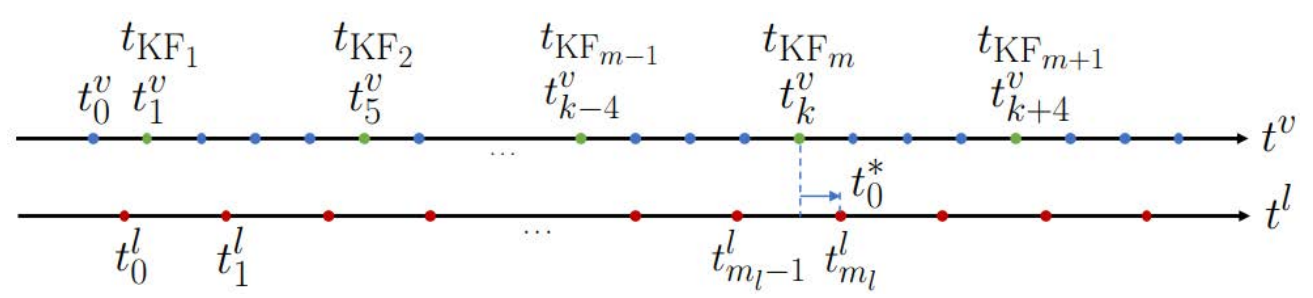

Figure 4.24: Vision-laser time synchronization. For the sake of simplicity in this figure, a keyframe is generated every four frames. In reality, this might heavily deviate, spanning from 5 to 20 frames per keyframe, depending on the marginalization rate (see Section 2)

3. Point Cloud Extraction: At time of fusion $t^{*}$, we go through several processing steps for $Q_{v}^{*}$, namely (1) slicing the point cloud according to the floor normal within a threshold, (2) projecting points in the vicinity of the floor plane on one plane, (3) filtering points according to their distribution in the cloud, and finally (4) applying a box filter through the tunnel corridor to ensure the elimination of all points that could corrupt the fusion quality.

Slicing: In a first pre-processing step of handling $Q_{v}^{*}$, we estimate the $x_{c}-z_{c}$-plane by computing the parametric form. Its normal vector corresponds to the second column of the current pose rotation embedded in $T_{0, c}$, namely $\overrightarrow{r_{2}}=\left[t_{1,2}, t_{2,2}, t_{3,2}\right]$, the direction of yc in Figure 4.25. Its reference point equates to the origin of the camera coordinate system $\overrightarrow{p_{c}}=\left[t_{1,4}, t_{2,4}, t_{3,4}\right]$, where $t_{i, j}$ represents an entry in the ith row and the jth column in $T_{0, c}$ (see Equation 4.13 and 4.14 ).

$$
\begin{gathered}
k_{c}: \overrightarrow{r_{2}} \cdot\left(\vec{p}-\overrightarrow{p_{c}}\right)=0 \Leftrightarrow\left[\begin{array}{l}
t_{1,2} \\
t_{2,2} \\
t_{3,2}
\end{array}\right] \cdot\left[\begin{array}{l}
x-t_{1,4} \\
y-t_{2,4} \\
z-t_{3,4}
\end{array}\right]=0 \\
k_{c}: t_{1,2}\left(x-t_{1,4}\right)+t_{2,2}\left(y-t_{2,4}\right)+t_{3,2}\left(z-t_{3,4}\right)=0
\end{gathered}
$$

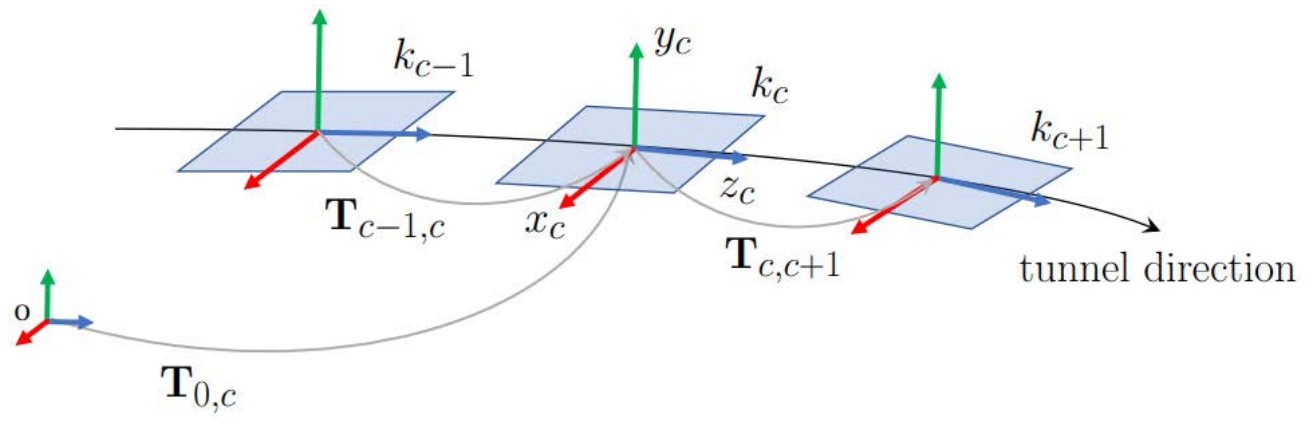

Figure 4.25: Inter-frame transformations and planes. At each keyframe instant, we extract the $y_{c}$-direction from the current pose denoted by $T_{0, c}$ and subsequently span a plane with $y_{c}$ as the normal. 
This parametric model is used to distinguish whether a point in $Q_{v}^{*}$ is close to the height of the LIDAR scan. For ease of notation, we will use $a=t_{1,2}, b=t_{2,2}, c=t_{3,2}$ and $d=-t_{1,2} t_{1,4}-t_{2,2} t_{2,4}-t_{3,2} t_{3,4}$ to arrive at Equation 4.15.

$$
k_{c}: a x+b y+c z+d=0
$$

For this purpose, we check the absolute point-to-plane distance of any point $\overrightarrow{p_{0}}=$ $\left[x_{0}, y_{0}, z_{0}\right]$ by projecting the difference vector between $k_{c}$ and point $-\left(\vec{p}-\overrightarrow{p_{0}}\right)$ onto the normal vector $\overrightarrow{r_{2}}$ as can be seen in the equations below:

$$
\begin{gathered}
d\left(x_{0}, y_{0}, z_{0}\right)=\left|\operatorname{proj}_{\vec{r}_{2}}-\left(\vec{p}-\vec{p}_{0}\right)\right| \\
=\frac{\left|-\vec{r}_{2} \cdot\left(\vec{p}-\overrightarrow{p_{0}}\right)\right|}{\left|\vec{r}_{2}\right|} \\
=\frac{\left|a x_{0}+b y_{0}+c z_{0}+d\right|}{\sqrt{a^{2}+b^{2}+c^{2}}}
\end{gathered}
$$

Plane Projection: Furthermore, all inlier points are now projected onto the plane by strictly enforcing Equation 4.15. The points are moved by their shortest distance to the plane by a mere projection.

Statistical Outlier Removal: The triangulation of landmarks involves not only regions of varying point densities, but also a fair amount of sparse outliers, drastically corrupting the results. Next to complicating the estimation of a clear cloud structure comprised of clean surfaces and curvatures, it might cause point cloud registration failures. For these reasons, a sparse outlier removal based on a k nearest neighbor approach, assuming a resulting Gaussian distribution, eliminates all points whose average distances are outside an interval defined by the mean of the standard deviation.

Box Filter: Moreover, we make use of a box filter to eliminate those points that represent the tunnel floor, i.e. all points that fulfill the following condition:

$$
x_{i, v}^{\left(t^{v}\right)} \geq x_{\max } \wedge x_{i, v}^{\left(t^{v}\right)} \leq x_{\min }
$$

The final point cloud extracted from the vision thread and ready for fusion should contain solely points of the outskirts of the accelerator environments on the $x_{c}-$ $z_{c}$-plane.

4. Scale Inference: Finally, both point clouds $Q_{v}^{*}$ and $Q_{l}^{*}$ can be compared for inferring a trajectory scale. This is done by sweeping through vision points and comparing the angle of each point from the same vantage point (the camera pose) with the points of the laser, computing the weighted average of the quotient of laser and vision distance to infer an ultimate scale. 


\subsection{Cooperative Multi-Agents Scenarios}

One of the main limitations regarding the communication is that most of the robots require a point-to-point radio or wired communication. This can put the robot operator in a safer position with respect to the robot, but still in a position that could be dangerous (e.g. not more than $200 \mathrm{~m}$ from it). The use of wired communications on movable robots in harsh environment has been proved not to be reliable, for example using fibre optic cables that are susceptible to breakage [332], thus limiting robots movements.

It has been developed a system for autonomous relay robot (see Figure 4.26) to allow a good communication between the robots and the teleoperator. This system allows to have a series of moving stations in order to extend the communication range between the robot and the operator.

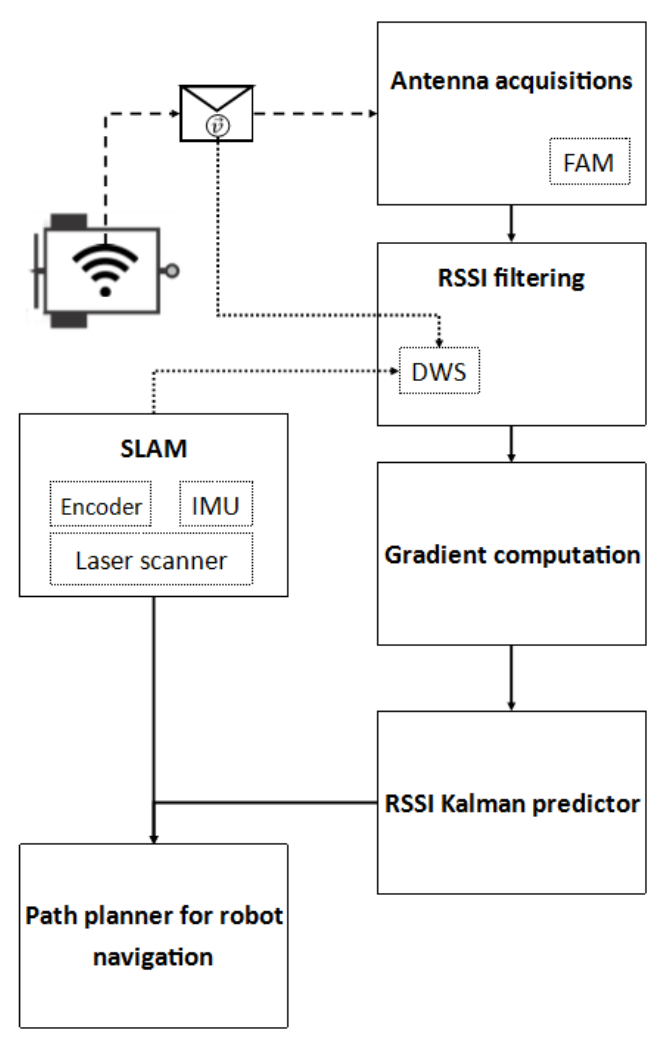

Figure 4.26: Schematic block of the proposed system. The Fast Antenna Measurement (FAM) system is implemented on Linux low level APIs and allows acquiring the RSSI measurements for every single acquired packet by accessing to the 802.11 packets. The filtering module receives as input the four RSSI values of the received packet as well as the velocity of the relay node platform coming from the robot odometry, or the SLAM. The gradient computation module determines the future direction of the robot. The RSSI Kalman predictor is used to predict the RSSI in few meters, which allows to better determine the movement of the robot as well as to avoid black spots. The trajectory planning system, finds the best position of the robot and its navigation path, avoiding black spots and obstacles. 
Wireless communication solves cables disadvantages, but it is still challenging to build up a reliable communication channel in hostile environments such as tunnels and mines[333, 334, 335]. To increase Wi-Fi communication robustness, the introduction of several intermediate relays nodes has been previously proposed[336, 337, 338, 339, 340].

The developed system is a novel multidimensional RSSI based framework for relay robots autonomous navigation in unstructured and harsh environments. This system allows the relay robot to sense, in real time, the Wi-Fi signal strength and to localize multiple Wi$\mathrm{Fi}$ access points. Using these features the robot can autonomously and safely navigate in environments to reach the best area where the Wi-Fi links of all the considered access points is stronger. Moreover, a single robot could also work as a Wi-Fi access point (AP) and, in combination with other relay robots, can work as an extension of the Wi-Fi coverage in the area that was previously absent.

It has been designed a novel antenna data acquisition system and several filtering techniques, including Kalman based ones, to smooth the RSSI acquisitions, which can be highly noisy in indoor environments (see Figure 4.27).
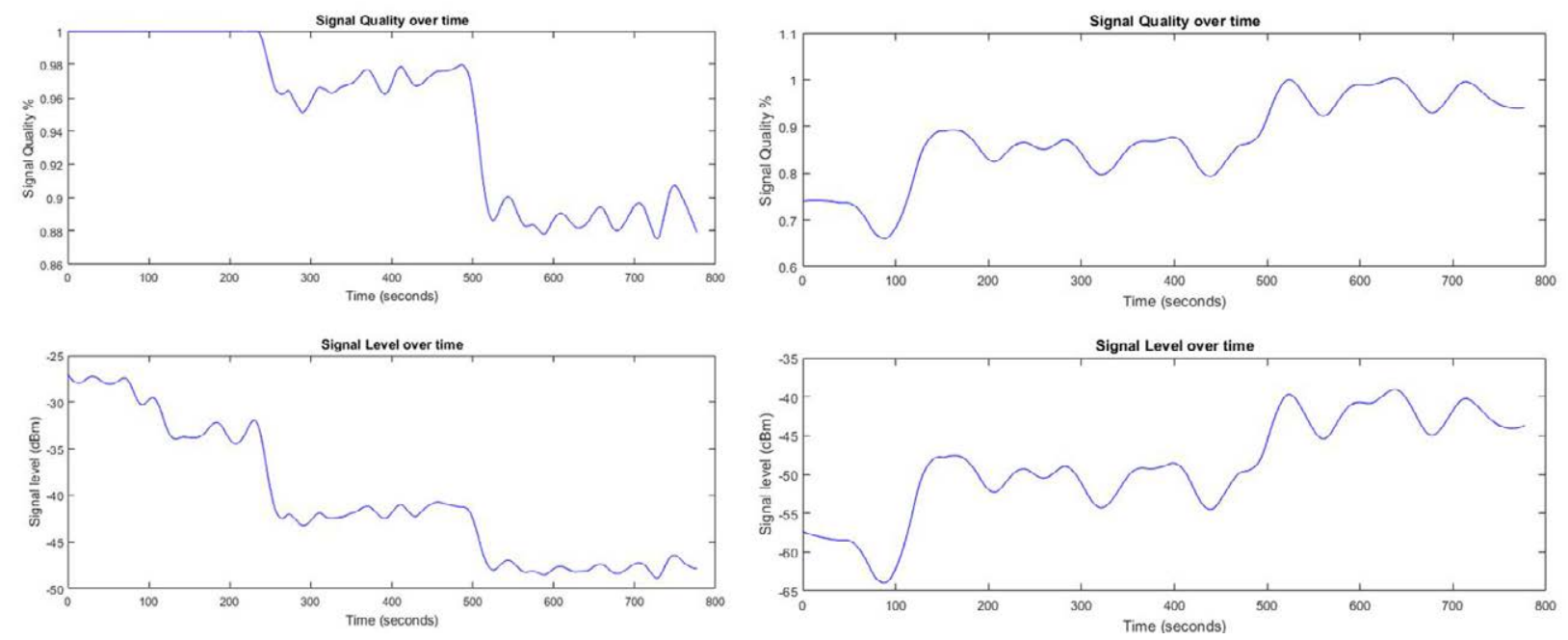

Figure 4.27: Filtered signal quality $(\%)$ and strength $(\mathrm{dBm})$ in $(\mathrm{A})$ Wi-Fi Relay 1 robot and (B) Target Relay2 robot. When the Relay 1 approximates to the Relay 2 the signal strength is increased and more stable when filtered, which grants a better performance for the different tasks the robots are assigned to.

Usually the teleoperator area is a safe zone with the robot out of the sight, and non-well specified physical obstacles can be found between the operator and the robot. It is known that multiple mobile robots (relay nodes) can be used to extend the communication capabilities by tethering the wireless signals [18]. Due to the uncertainty about the environment, the signal can have unexpected behaviours (e.g. attenuations, reflections etc.). The signal strength measurement requires a good filtering in order to be used by the relay node. The architectural overview of the relay robot is shown in Figure 4.28. A scenario using different relay nodes is shown in Figure 4.29. 


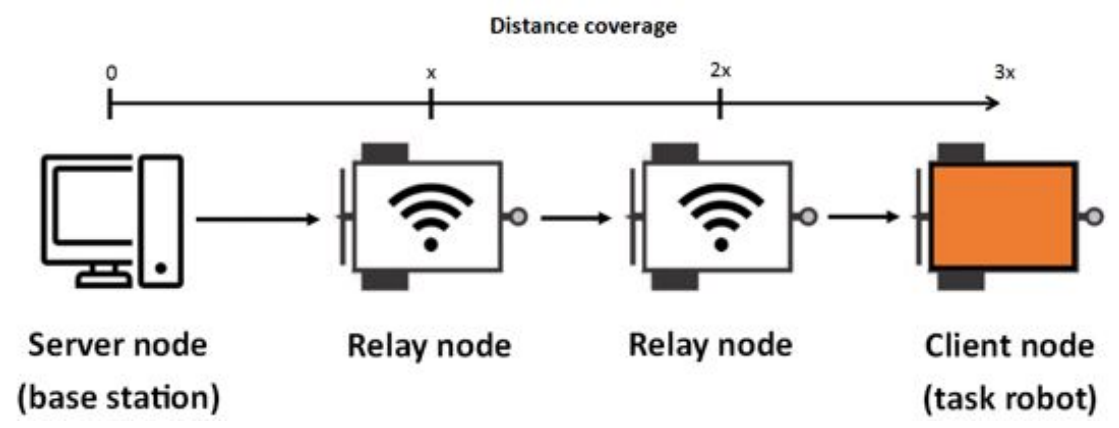

Figure 4.28: Architectural overview of the relay robot use case. The relay nodes are used to extend the network coverage.

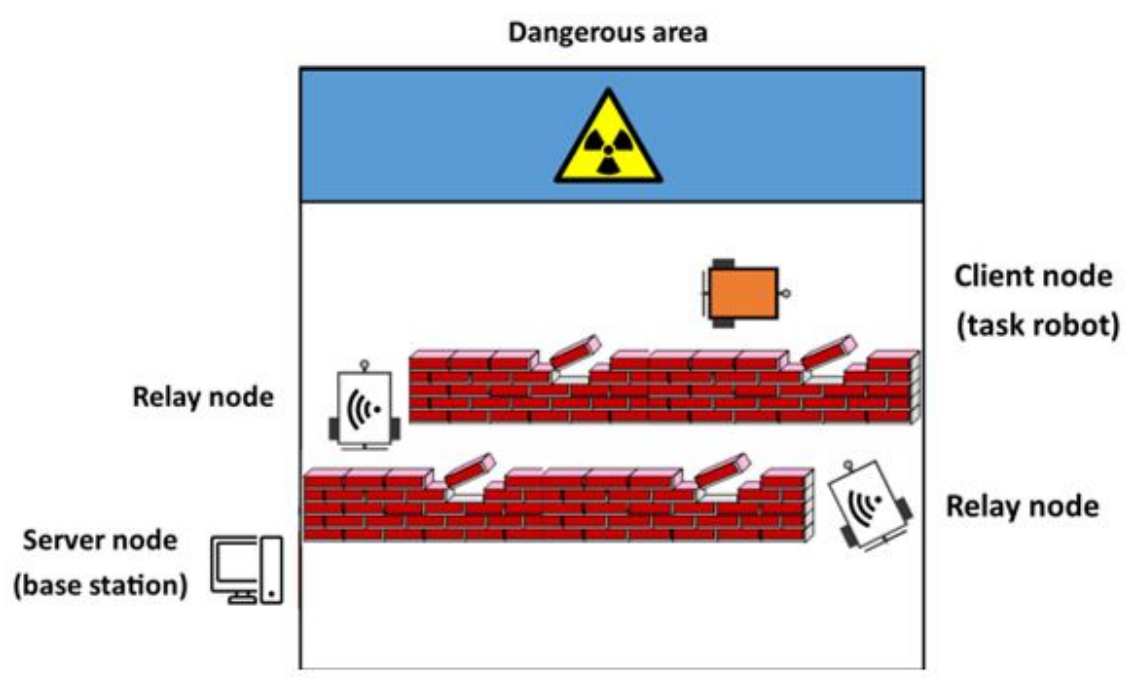

Figure 4.29: A scenario of several robots working together for a given teleoperation task. The relay robots ensure a higher operational distance range even without line-of-sight.

In each node multiple wireless devices are used as sensors to enhance reliability and redundancy in wireless gradients. In the current implementation, four Wi-Fi transceiver are used to sense the RSSI. In addition, having a minimum of two antennas on-board, allows obtaining the gradient orientation to the source of the RSSI.

The first main advantage relies on the fact that the antennas acquisition system provides RSSI measurements allowing the relays robot to still move in the environment while do the signal measurements, and to react quickly in case of fast RSSI changes. The second main advantage is that all the antennas acquire the same packet and measure the RSSI relative to it. In this way, the new proposed filtering algorithms can rely on a high number of packets leading to a more accurate RSSI measurement.

The relative velocity between the robot and the Wi-Fi source is computed as a difference between the robot velocity and the source one that is included in the packet. Using this the relay robots can be able to predict in advance communication black spots and plan its motion 
accordingly. Predicting the wireless signal strength in temporal and spatial domain has been deeply investigated [341], with satisfactory results obtained with a Kalman predictor[342].
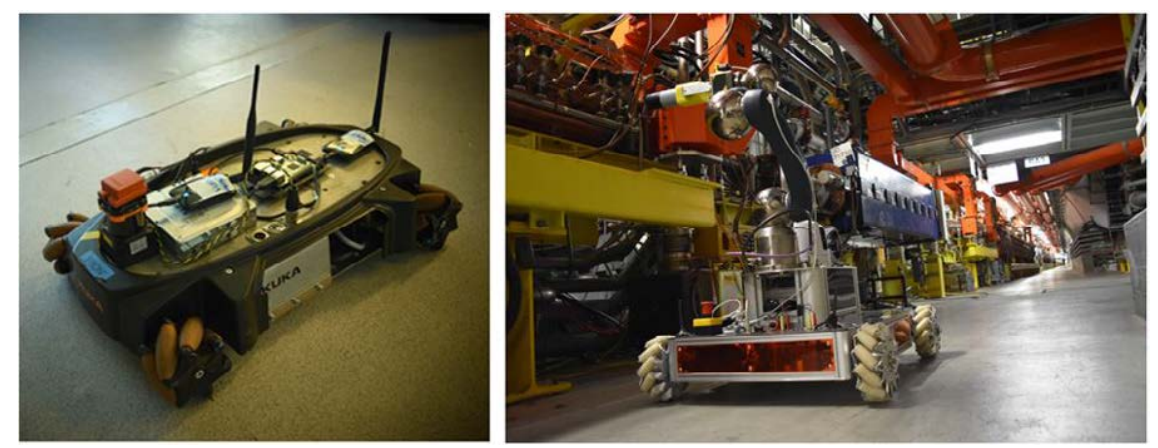

Figure 4.30: YouBot (left) and CERNbot (right) integrating the Wi-Fi source and the SLAM sensors.

The proposed system has been tested in real conditions at CERN accelerators using 2 robots (see Figure 4.30). At first, the robot Relay 2 is performing a SLAM until the Wi-Fi signal drops over a threshold. When this happens, the Relay 1, that is placed near to a WiFi source, starts moving so as to increase the signal received by the Relay 2 . And when the Relay 1 approximates to the Relay 2 the signal strength is increased and more stable when filtered, which grants a better performance for the different tasks the robots are assigned to.

Complex tasks could require multi-robot cooperation to be completed. Transportation of long or heavy objects or cutting an object in multiple parts might require different robotic arms or even different platforms. The synchronization of the movements of the various agents, in such scenarios, is critical in order to complete safely the operation. Having multiple operators controlling single agents is not ideal as the lack of synchronization of their action or miscommunication between them could lead to errors. Therefore, the HRI implements a live scripting system in order to generate custom control strategies on the fly. This allows a unique operator to control multiple agents while guaranteeing a synchronization of the actions and their safety. The HRI provides, through live scripting interface, all the sensorial data of the robot (position of the platform, of the arms, camera images, sensors etc.) and the interface for sending commands to the robot. The operator can implement two types of script: one-stage scripts which take inputs from the operator, modify them and send them to the proper agents (used for example for synchronizing the actions between two agents) or scripts-in-the-loop which run in background as a parallel loop with respect to the operator's inputs (for example in an autonomous leader-follower configuration). The scripts can be programmed on-the-fly during the intervention or in advance, before the intervention. Afterwards, the HRI provides a simple interface for activating and deactivating the scripts according to the stage of the intervention (see Figure 4.31). Then, it is possible, or example, to have a robotic expert who pre-programs an intervention strategy and provides to the operator, possibly expert of the equipment, the interface which will be already prepared to execute the correct scripts in the correct order. 


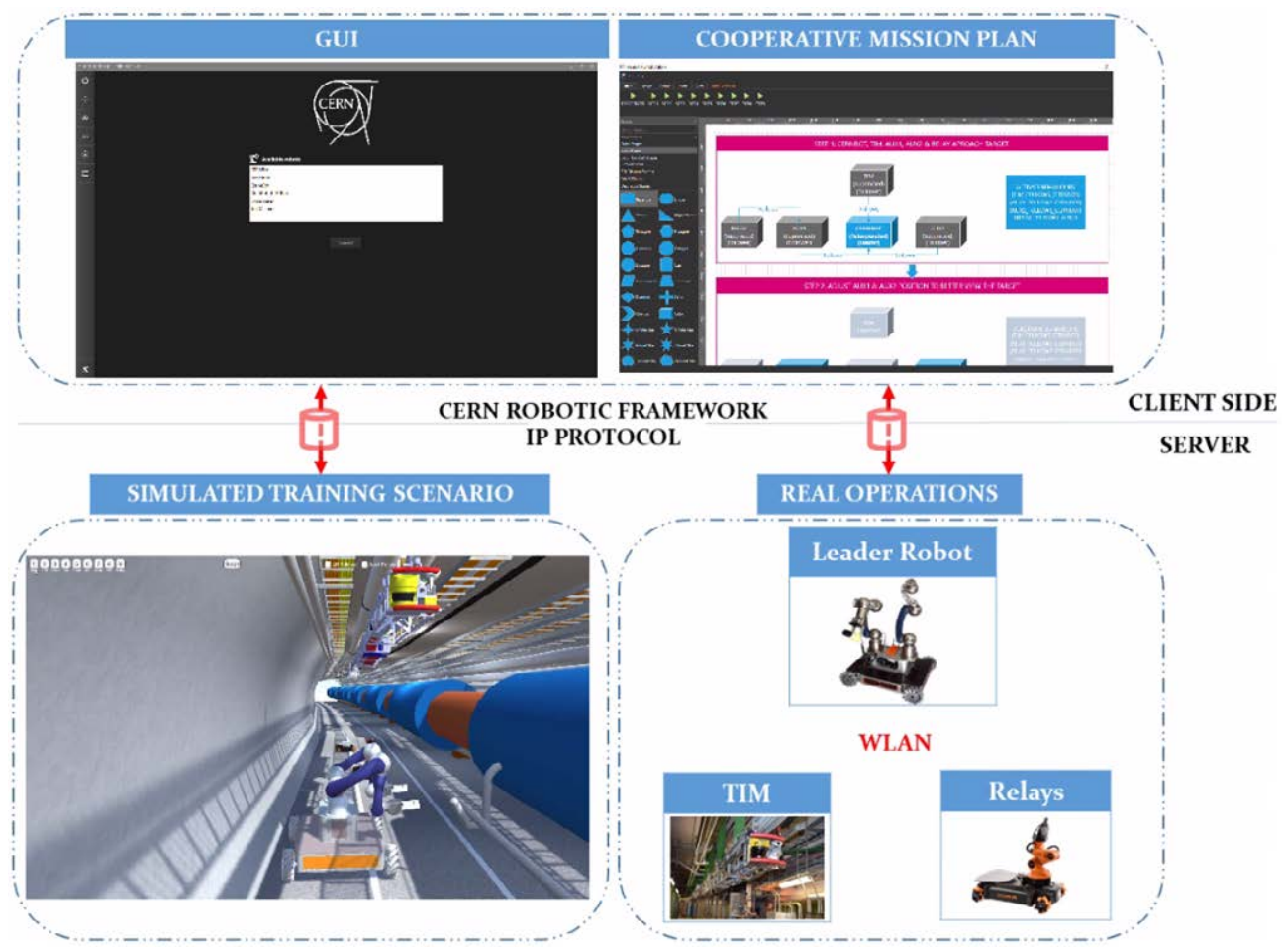

Figure 4.31: The cooperative mission plan scheme. The same operation can be performed and tested in simulation and then applied on the real robots seamlessly.

As the HRI provides to the scripting interface all the data available on the robot, there is no theoretical limit to the complexity of the scripts and any control strategy can be implemented. Nevertheless, as the communication network is unreliable and unstable, it is not possible to achieve soft real-time, or even close to real-time, control methods such as SLAM, obstacle avoidance or other critical functionalities. Therefore, a series of more complex functionality are available on the robot as well. Such functionalities, some presented as well in this work, can be activated and deactivated by the operator, but not modified nor reprogrammed on-the-fly. However, as they are executed on the robot, they are safe with respect to communication loss or instability. 


\section{Chapter 5}

\section{Operators Behaviour and Performance Metrics}

\subsection{Human-Robot Interface}

Human-Robot Interfaces have a key-role in the design of secure and efficient robotic systems. Great effort has been put during the past decades on the design of advanced interfaces for domestic and industrial robots. However, robots for intervention in unplanned and hazardous scenarios still need further research, specially when the mission requires the use of a robotic team, to obtain an acceptable level of usability and safety of such robotic systems. In order to merge together all the work previously explained and to allow efficient HumanRobot Interaction, a unified Human-Robot Interface (HRI) has been developed (see Figure 5.1). The main requirement in the development of the interface has been the possibility to provide a unique system capable of controlling all the robotic platforms developed at CERN with high usability and high learnability, in order to allow even entry-level operators to operate them without constant training.

The HRI is a complete environment, which allows not only the control of a heterogeneous pool of robots, but also the intervention preparation, the training and the post data analysis. The HRI integrates all the available sensors as well as all the high-level applications which are available on the robotic system such as autonomous navigation and SLAM, assisted telemanipulation, object recognition, environmental reconstruction and others.

The HRI offers a multimodal architecture which allows to interact both with the HRI and with the robot by means of different interaction devices (see Figure 5.2). Furthermore, devices dedicated to the interaction with the HRI can't be used for the interaction with the robot, in order to provide a clear mental break to the operator between the type of action. Therefore, when using for example a mouse or vocal commands to interact with the interface, the operator is sure that the robot will not move. This reduces greatly the occurrence of slipses and lapses, which are failure in the mental process which can be caused by failure in the short or long term memory or attention loss, which are more recurrent when dealing with stressful situations such as a telerobotic operation. Among the interaction 


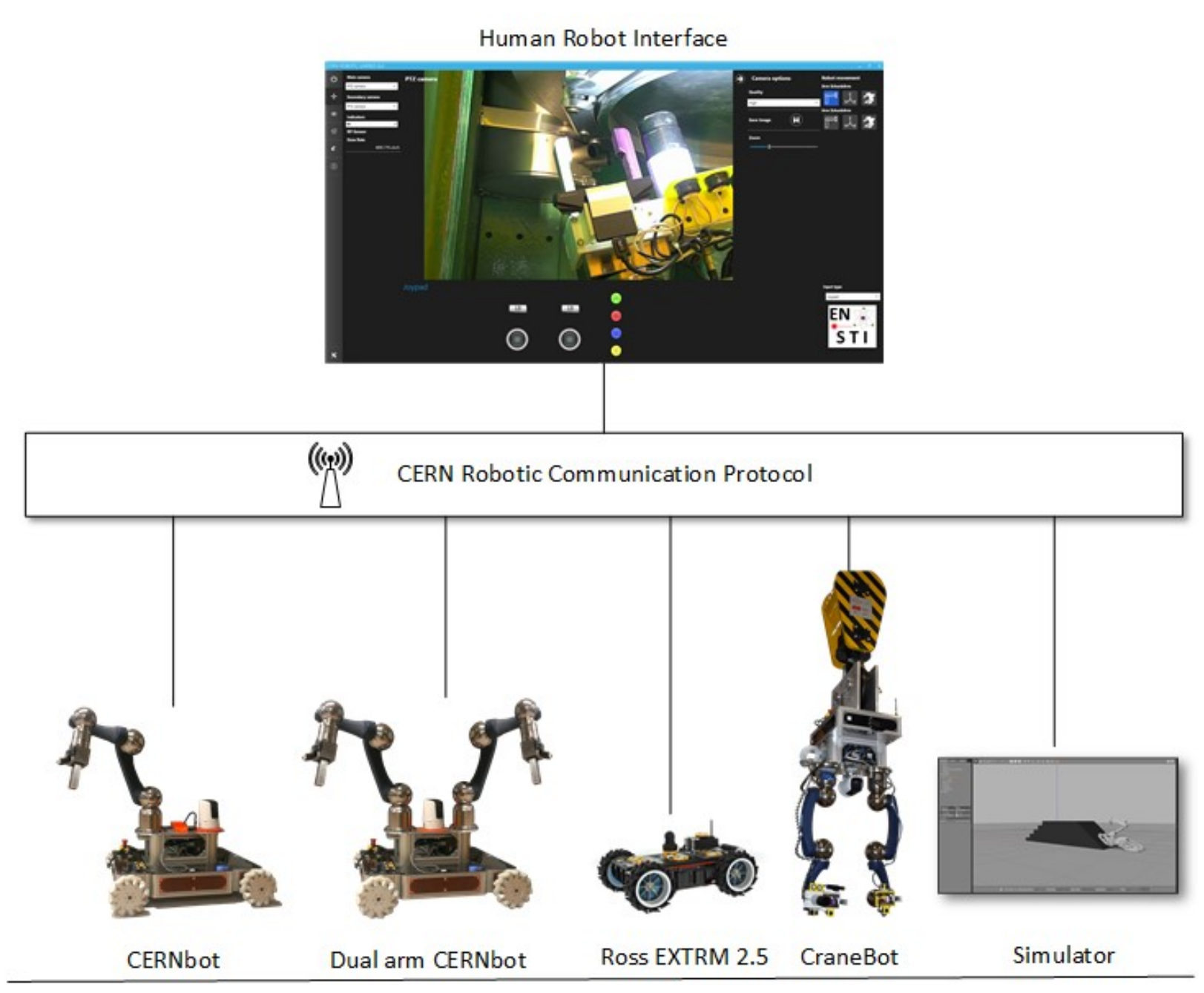

Figure 5.1: The HRI automatically adapts to the robot configuration in order to control in the same modality all CERN's robots pool. 


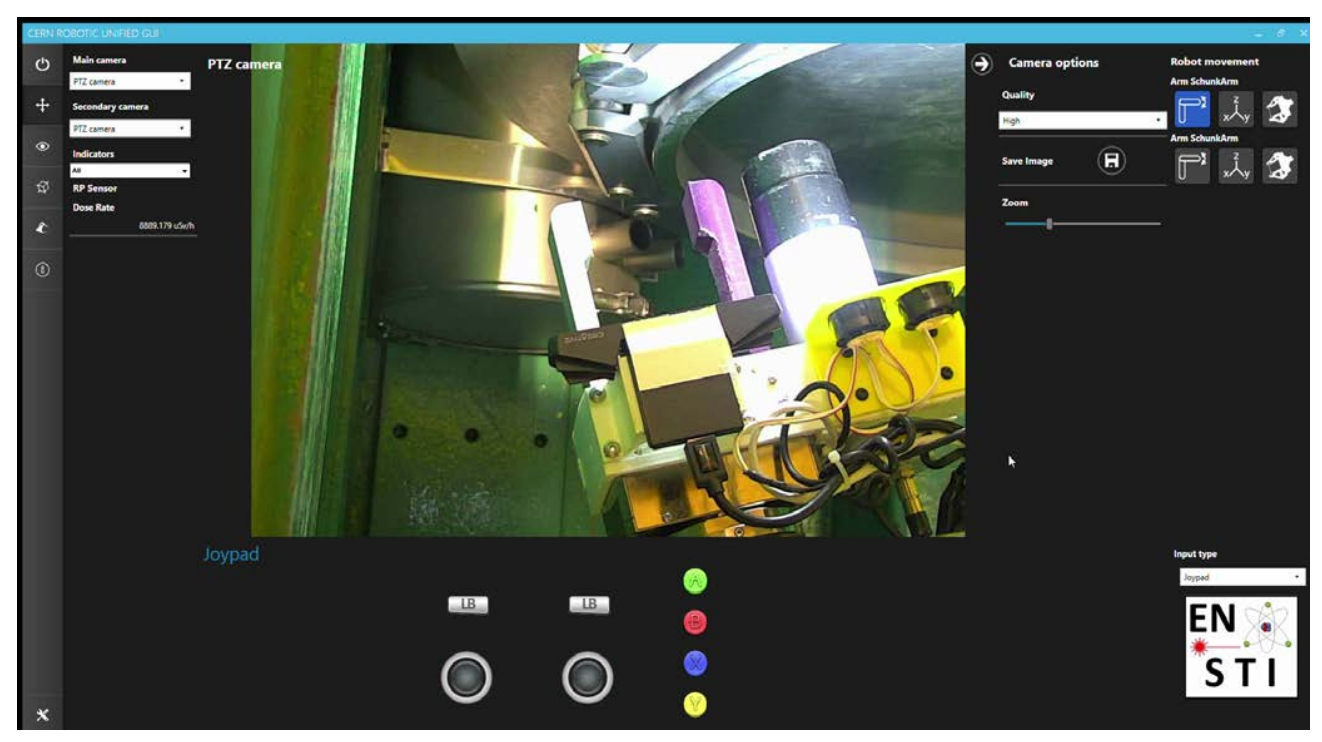

Figure 5.2: The control view of the HRI, reserved to the operator. Different views are available for cameras, 3D reconstruction, statistics etc.

with the robot capabilities, the most suitable and usable during teleoperation task is the master-slave control with force-feedback passivation, which allows a one-to-one workspace representation with the master arm with respect to the remote slave robotic arm. The position error/force feedback allows accurate haptic and safe operation ensuring as well overall system stability when dealing with high network delays. Since the communication with the robot relies always with internet-like network, it is important that the HRI is always aware of the communication delay between the operator and the robot. A huge effort has been done in order to reduce the visualization latency of the HRI: this means that as soon as sensorial feedbacks from the robot are available on the HRI (such as camera images, force feedbacks etc.), they are provided to the operator with the minimal latency possible. Furthermore, network delays are precisely computing using a local timestamp synchronization using a NTP-like four-timestamp mechanism. This method is a commonly used method for timestamp synchronization, but assumes symmetric delays in the network. Therefore the HRI synchronizes its local timestamp recurrently during the operation in order to provide lowerbounds to the timestamp offset between the two agents. The HRI is fully integrated with the Virtual Reality framework for 3D visualization and robotic simulation. The 3D visualization, in particular, includes an accurate environmental reconstruction by means of the lidar sensors and RGB-D cameras installed on the robot. This environmental reconstruction is shown to the operator in order to facilitate the navigation and the interaction with the environment, as well as for gathering data about the environment precisely localized in the space (see Figure 5.3). Therefore, it is possible to create environmental maps containing information such as radiation, temperature, magnetic field, which can be used afterwards offline to better plan a human or robotic intervention.

The integration with the Virtual Reality framework allows also accurate robotic simu- 


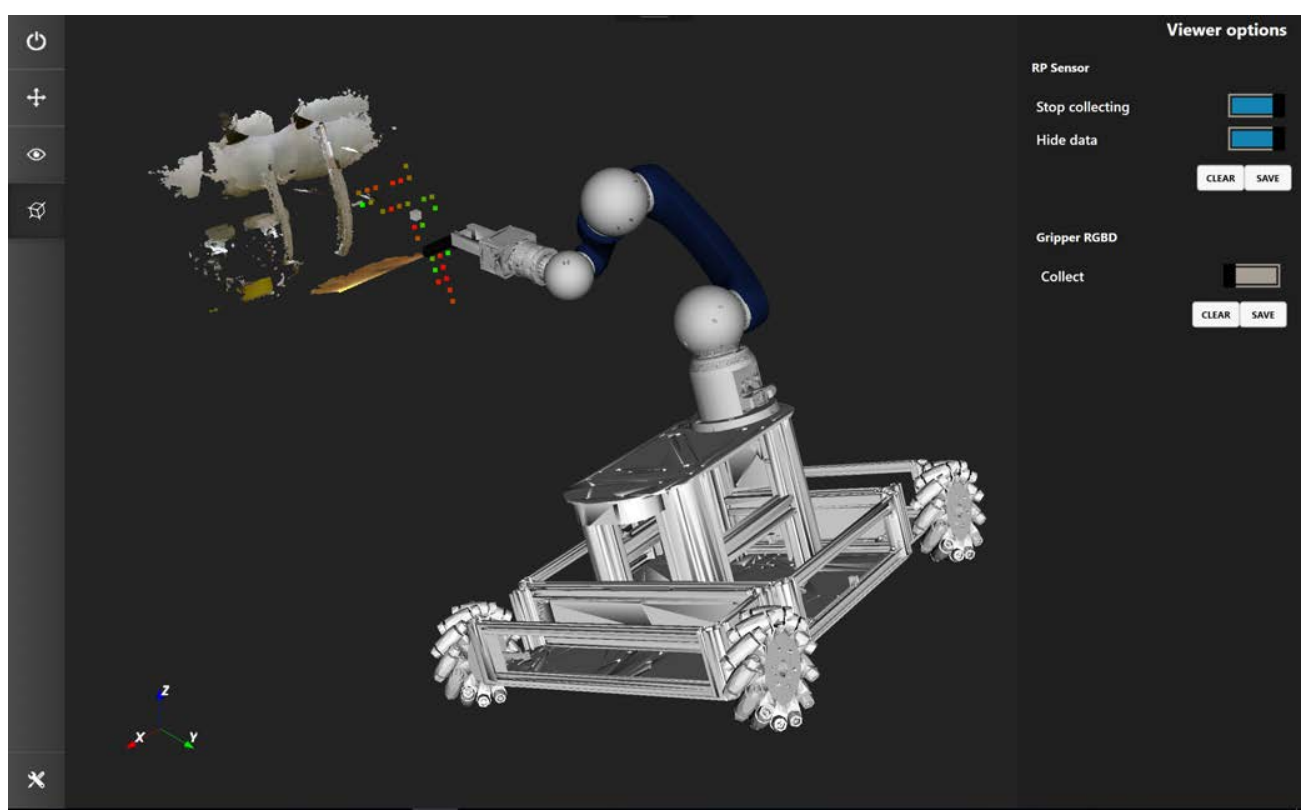

Figure 5.3: A 3D reconstruction of a collimator with the radioactivity values collected in real-time

lation thanks to its physics engine. By using exactly the same interface used for operating real robotic system, an operator can train in a simulated environment to perform complex tasks, to validate intervention procedure and to asses related risks during the operation. This training allows to greatly speed up the real intervention and to increase its safety.

In order to guarantee the proper separation between data and its representation, the HRI is designed according to the well-known Model-View Controller (MVC) pattern. MVC is a software architectural pattern, today standard de-facto with its variants, for the development of Graphical User Interfaces in particular for web applications [343].

The pattern splits the structure of the interface in three separate and interconnected parts in order to provide a separation between data and its representation. It is used often to enhance parallel development and code reuse, but in the context of this project, it appeared particularly effective for enhancing the adaptability of the interface to different robot configurations. In fact, the HRI can provide different representations of the same data but can also provide the same representation of different data. For example, the actual position of a robotic manipulator can be shown to the operator in different ways (3D model, sliders indicating the joints position with respect to their limits, 2D sketches, etc.). In this case a unique model takes care of collecting the actual position of the manipulator from the remote controller, and multiple views are available to display it. At the opposite, another example is the visualization of a camera: a camera is always shown to the operator as an image on the screen (unique view); nevertheless, the HRI supports several cameras such as network cameras, USB cameras, thermal cameras, PTZ cameras or RGB-D cameras. Therefore, this design pattern enhances the multi-modality of the interface. The developed HRI makes of its modularity one of its most important requirements. The modularity 


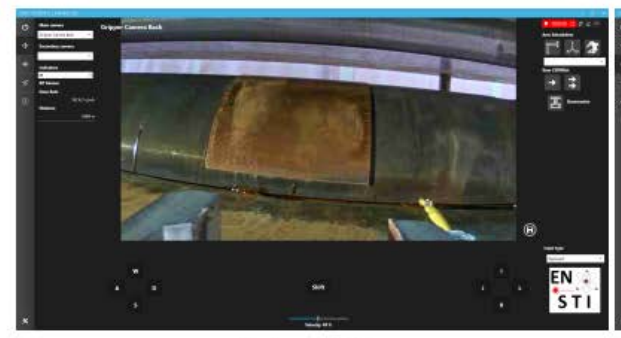

(a) Control View

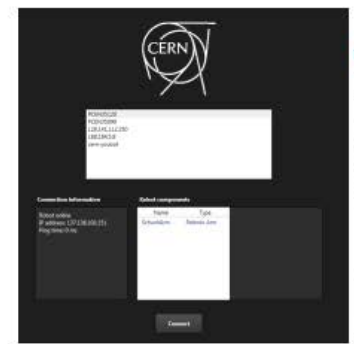

(c) Connection View

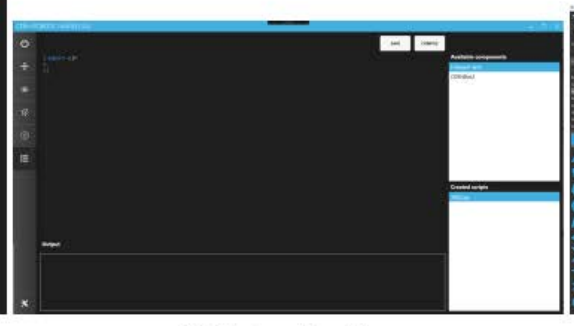

(d) Script editor View

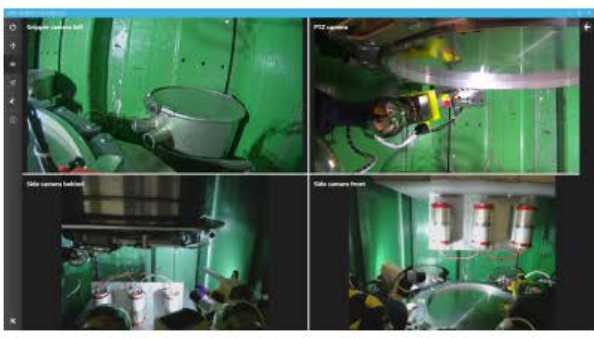

(b) Cameras View

Figure 5.4: CERNTAURO Human Robot Interface Views

appears in every part of the MVC architecture, but gives its greatest results in the model representation of the robot. The HRI adapts its view according to the robot configuration which is defined in the robot configuration file. During the parsing of the robot configuration file, the robot topology is built as well. The robot topology defines a tree-like structure in order to maintain a continuous localization of each robot part in the environment. The robot configuration file is implemented using the XML markup language, which naturally embeds a tree-like structure during its definition. The HRI is composed of several views, each one semantically different from the others (see Figure 5.4).

All the control of the robot is included in the control view: this is to strengthen the separation between the interaction with the HRI and the interaction with the robot. From any other view is possible to send commands to the robot. It can also display a main camera, which occupies most of the screen, a secondary camera, displayed smaller in a corner, and live sensor readings. From this view, the operator can select the control mode and the type of input devices he or she wishes to use. The HRI implements as well a 3D visualization tool, which displays in real-time the robot configuration and the reconstructed environment, including live environmental measurements if provided. At startup the HRI is built according to the robot configuration inside a unique window, and the operator can navigate between views using a menu positioned on the right side. Nevertheless, each view can be detached by the main window and open in a secondary window. According to its definition, a multimodal interface provides different modalities for user interaction (i.e. inputs and outputs). Above that, in this context, it is necessary to make a distinction between two types of interaction: the interaction domain of the user (i.e. the operator) with the interface and the interaction domain of the user with the robot. Although in the second case the user is still interacting with the robot through the interface, according to 


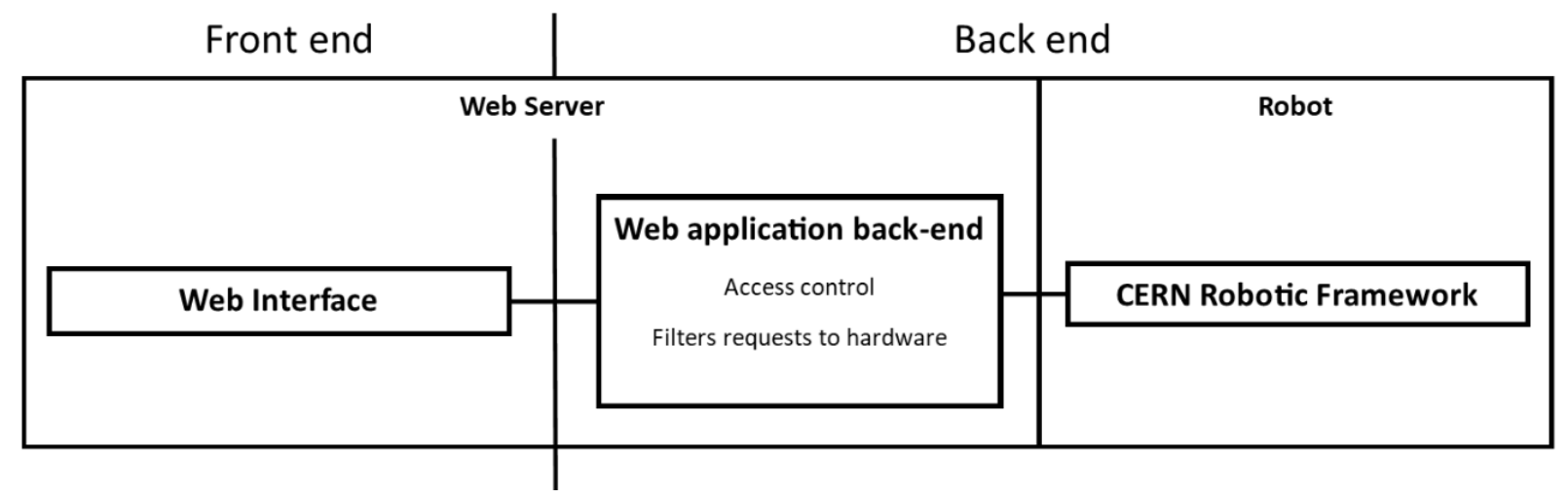

Figure 5.5: The CERNTAURO scheme of the communication via web with the robots.

the principle of telepresence, the user should feel a complete transparency between his/her actions and the robot's response. Furthermore, it is extremely important for the operator to understand which modalities are related to the interaction with the robot and which modalities are related to the interaction with the interface, to reduce the frequencies of slips and lapses. For this reason, an input device is never shared between the two domains of interaction (e.g. the mouse can only be used to interact with the interface, while the keyboard can only be used to interact with the robot). With this distinction, the operator can feel confident that the interaction with the interface will not modify the state of the robot, with the possibility of compromising the safety of the operation. Furthermore, the operator can always drop the robot interaction device in use, knowing that the robot will not move. It is important as well that the passage between the two domains of interaction is smooth, in order to not increase the stress of the operator, but it must be well defined and clear, in order to generate a break between the two different mental processes.

A dedicated web monitoring system for the CERNTAURO framework has been developed allowing the follow-up of the robots and their measurements during the full filling of the demanded tasks. The scheme of the communication via web with the robots operating on he field is shown in Figure 5.5, while Figure 5.6 presents the web page and its mobile version.

\subsection{Teleoperation Factors}

Different factors, listed and described below, influence the behaviour of a teleoperation systems.

\section{Tele Proprioception}

Proprioception (or kinesthesia) is the sense through which we perceive the position and movement of our body. The brain integrates information from proprioception and from the vestibular system into its overall sense of body position, movement, and acceleration. The word kinesthesia or kinæsthesia (kinesthetic sense) strictly means movement sense, 

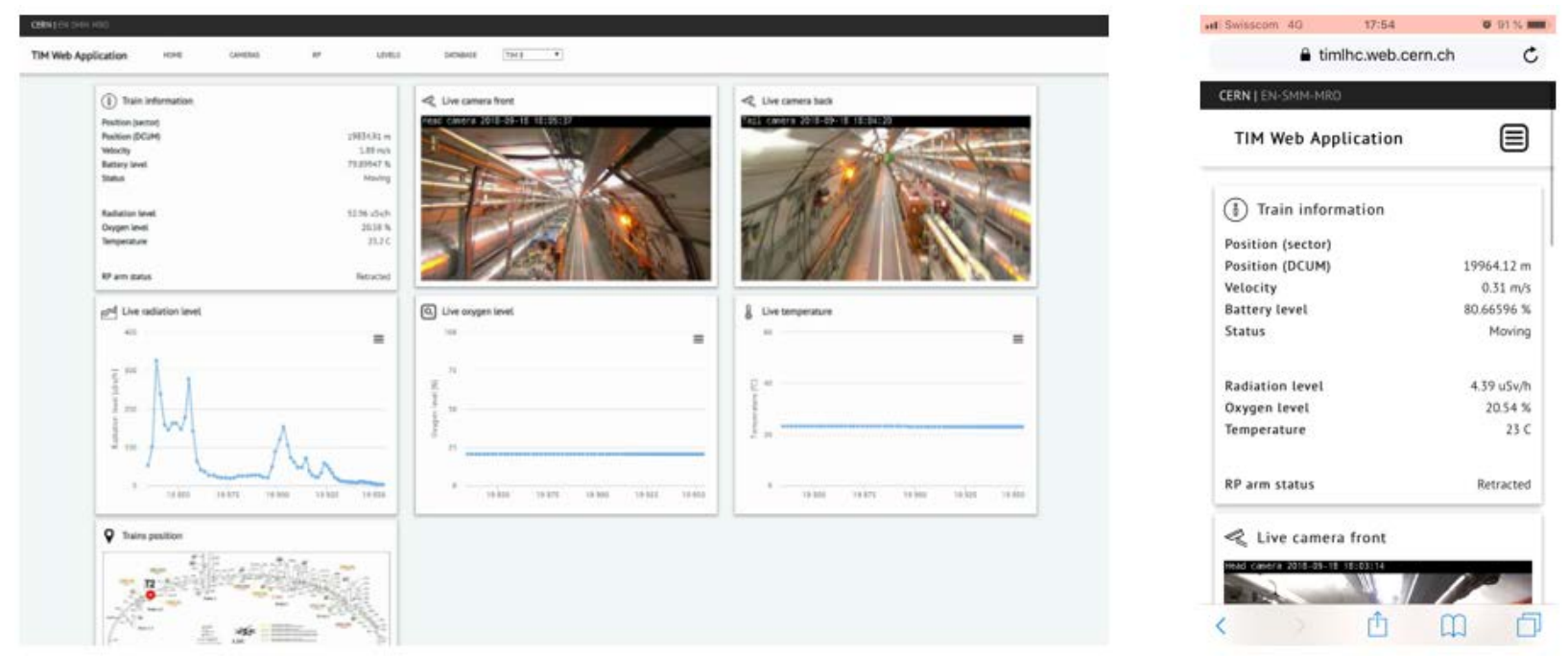

Figure 5.6: CERNTAURO web pages to monitor the robots and their measurements. The mobile version is shown on the right.

but has been used inconsistently to refer either to proprioception alone or to the brain's integration of proprioceptive and vestibular inputs. The Tele proprioception is the capacity that maintains an operator using a telemanipulation system to associate the position of the manipulator with respect to the environment of the point of view. Wrong adjustments during operation can be mechanical, geometrical or observational (see Figure 5.7)

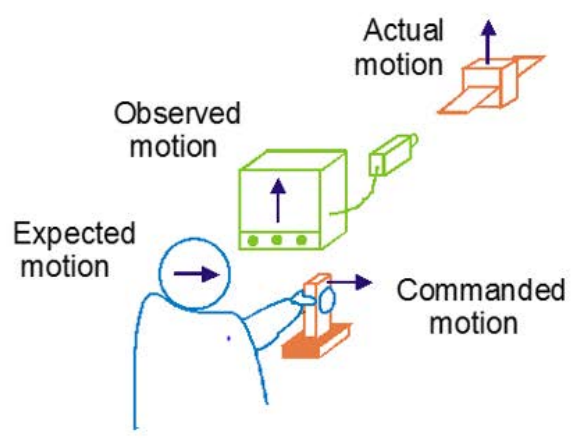

Geometrical wrong adjustment

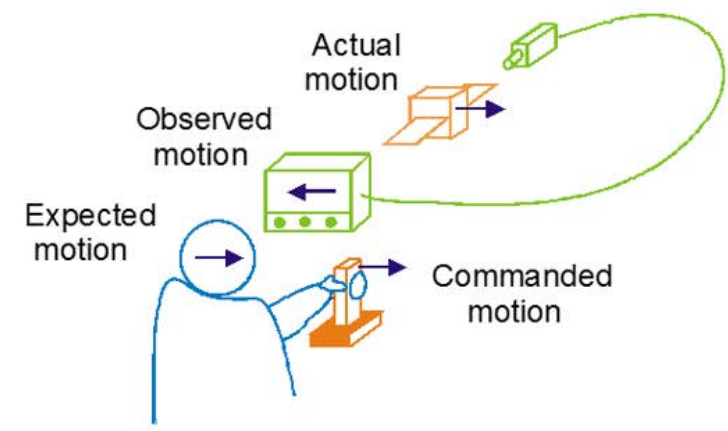

Observational wrong adjustment

Figure 5.7: Example of geometrical and observational wrong prospective.

To avoid these wrong prospective, the CERNTAURO framework automatically adjust the picture orientation given to the operator to increase the tele proprioception and to increase the transparency of the teleoperation system. One simple example of wrong prospective appears during the master-slave telemanipulation task. Usually, the slave robot arm is equipped with a RGB camera close to the arm end-effector. The camera will rotate according to the configuration of the entire arm, changing the point of view of the object with 
respect to the arm. However, the reference frame of the operator is always referred to the base frame of the robot, which is not anymore aligned in terms of rotation to the reference frame of the displayed camera. In order to compensate this effect, the camera image is displayed to the operator rotated, in order to maintain the visualized reference frame of the camera jointed with the robot arm reference frame and compensate their misalignment (see Figure 5.8). This CERNTAURO feature has the main goal to make the operator, the robot and the interface like one operation entity.

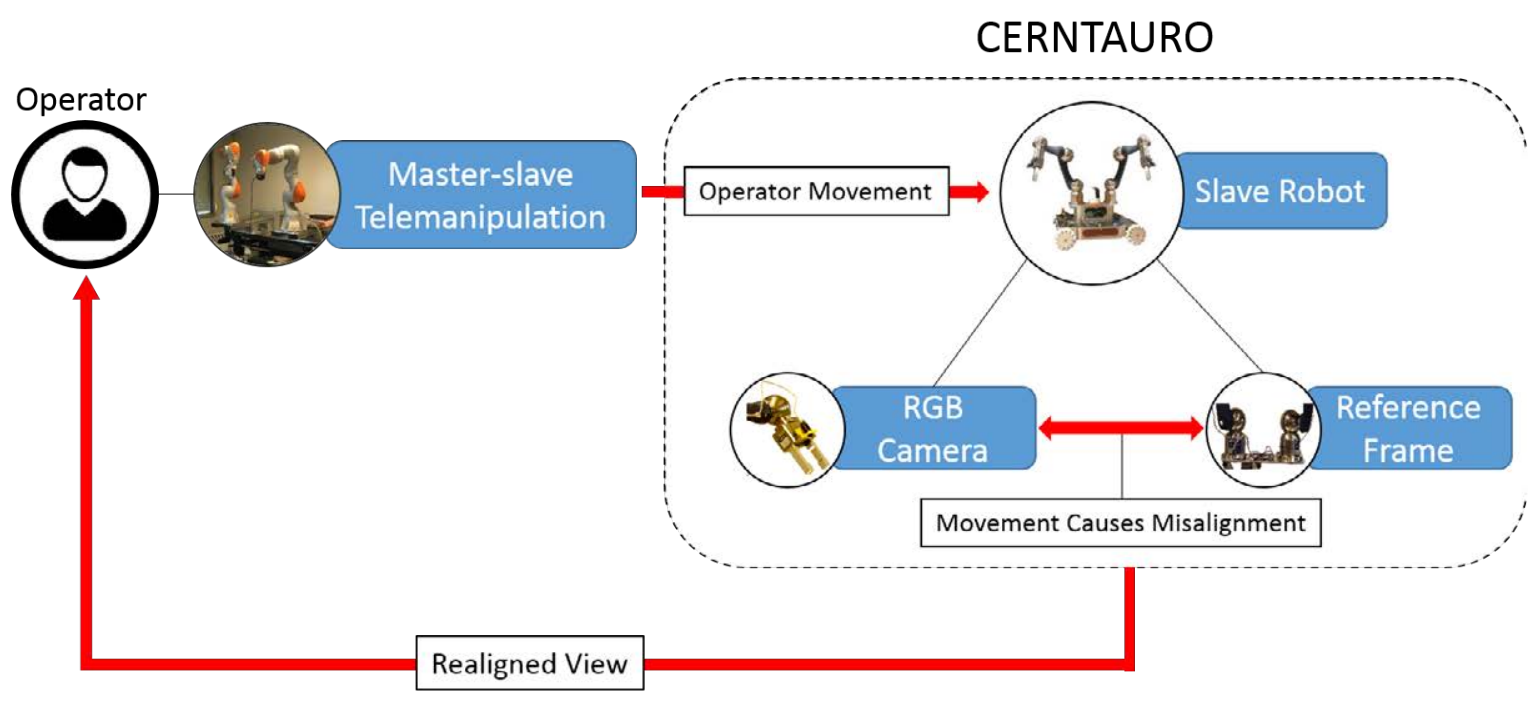

Figure 5.8: Overview of the proposed CERNTAURO system that is used to maintain the visualized reference frame of the camera jointed with the robot arm reference frame and compensate their misalignment

\section{Type of Control}

Teleoperated robots are used in a wide variety of fields. They are mostly used in scenarios where the environment is too dangerous for humans, or simply inaccessible. The human interface can be as simple as a keyboard, mouse for input and a computer screen for output. A bit more ergonomic option is to use joysticks or console controllers. These methods are relatively cheap and can be used to control many types of robots. The main drawback of them is that the operator has to learn which action corresponds to which behaviour of the robot. For example pushing the button " $K$ " will land the airplane. Since there is no similarity between the movement of the input device and robot, operators can easily make mistakes. On the other hand the screen as the single output device also poses limitations. The operator might not be able to perfectly judge the situation and capabilities of the robot. From a screen it is hard to know how much more torque can the drone output, in order to determine how hard can it fly against the wind. Similarly on an underwater robot, even if the pressure is highlighted as number, it is hard for the operator to judge how much deeper it can descend.

The above mentioned limitations can be eased if the controller and output device is 
structurally similar to the actual robot. During master-slave control the controller itself is also a robot. It does not have to be the same model, for example the controller can be a simplified, smaller, cheaper version of the actual working robot. Master-slave control is more intuitive, the operator needs a fraction of time for getting familiar with it, and also makes it easy to judge for example the joint limits of the robot. The master-slave system integrated in the CERNTAURO framework is composed by a direct interaction between the human operator and the robot for teleoperation in harsh and radioactive environment using a Cartesian Space Approach. The control could be in position or in velocity. The position control is suggested when the master and slave kinematics are similar, there are no velocity limitation in the slave, not much telepresence is requested and complex operation with various degrees of freedom are needed. The velocity control is suitable when there is a big kinematic difference in between master and slave, the slave motion is slow and not too many degrees of freedom are needed. The CERNTAURO framework uses the position control coupled with force feedbacks for complicated teleoperation tasks, for some more simple it could use a velocity control. when working with big workspaces, the CERNTAURO framework has the possibility of fusing velocity and position control. The CERNTAURO bilateral architecture control developed (see Figure 5.9) arises from the most general 4- Channel architecture [344], in which master and slaves can exchange forces and positions/velocities signals. The architecture in the framework is composed of three channels: two are used for shared position/velocity information between master and slave and a third used to replace the environment interaction forces from the slave to the master. The specific chosen architecture is known as "Environmental Force Compensated (EFC)" whose stability and transparency have been well discussed [345], also in presence of time delay [346]. In the proposed solution, the operator feels two different kinds of force feedback, a direct one directly from the force sensor, and an indirect one caused by position or velocity error in between the master and the slave positions. 


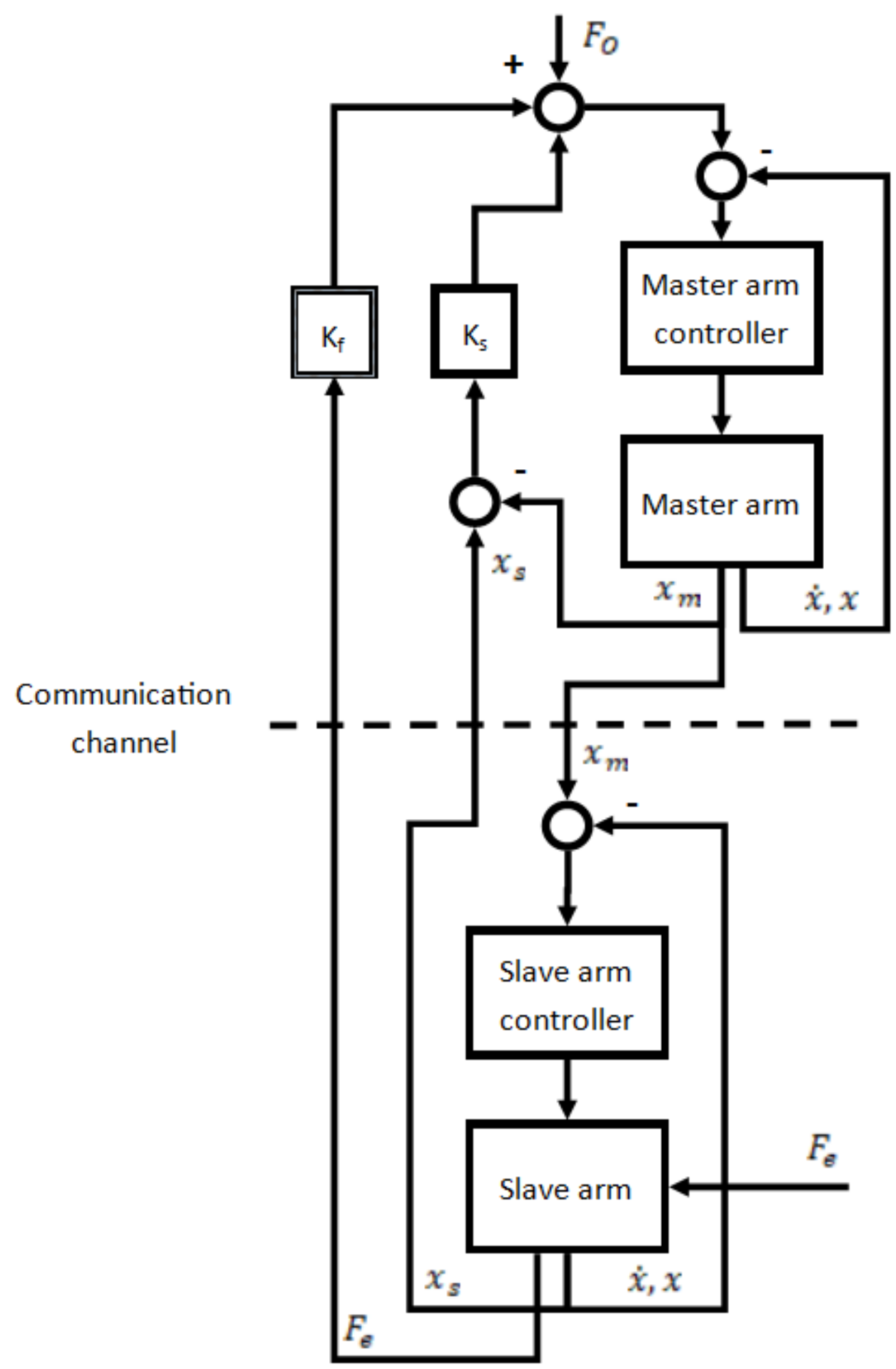

Figure 5.9: Bilateral control system using the master-slave interaction to control a robotic arm. $F_{O}$ is the force applied to the master arm by the operator, $F_{e}$ is the environmental force applied to the slave arm, $x_{m}$ is the position of the master arm, $x_{s}$ is the position of the slave arm, $K_{f}$ is the gain factor for the environmental force feedback and $K_{s}$ is the gain factor for the position-error feedback. 
The most important component of a master-slave controller is the mapping between the movement of the two robots. The mapping should be done between the end-effectors of the two robots. When the end of the master moves the slave should mirror its movement.

Judging the velocity of a moving object from a $2 \mathrm{D}$ video feed is really hard. Our perception of it depends on the camera position and in some cases the camera might be moving at the same time. When the connection is not perfect, packet drops and jitter can be expected. The control of the robotic arm based on velocity becomes dangerous. If for example the connection is dropped suddenly, the robot might keep following the last command and damage itself. This is why mapping the positions instead of the velocities of the two end-effectors is the safer approach.

The mapping is done trough position inverse kinematics. It is able to generalise to any choice of the master-slave robot. This is needed in order to make the system more flexible by making it possible to exchange which robotic hands are used for the master or the slave. The bilateral control system is designed in a way to be easily extendable to enable the operator to use a virtual reality headset instead of a monitor for visual interface. In this case the operator does not see the master robot in front, but is rather put into the viewpoint of the slave robot for more precise manipulation. If on the headset only the end-effector of the master can be seen, the operator should still be able to accomplish his task.

By far the most utilised sense we have is vision [347]. According to studies up to $50 \%$ of the neural context is involved with processing the vision stimuli [348]. In order to create a crude teleoperation system visual feedback should be enough. We can judge the position, velocity and size of objects with our eyes. We can also guess some of their properties, such as their weight, surface properties, friction and acceleration. These parameters we can not directly infer from images, but only based on our previous experiences of manipulating objects. On the other hand for manipulation we utilise highly our sense of touch from a young age. With our perceptive hands we are capable of measuring weight, inertia and acceleration [349]. When children learn how to manipulate objects around them, they touch anything that surrounds them in order to learn their dynamical properties. In the area where maintenance is carried out by members of the Robotics Team many unusual objects can be encountered. It is not possible to judge for example how the magnets and collimators react when they are touched, how heavy they are, since the operator most likely never encountered anything similar to them before.

There is another key difference between manipulating with human hands and through teleoperation. When we manipulate with our hands, we are perfectly used to our limbs and fingers. We know how much force we can exert, and how far we can reach. During teleoperation an untrained operator might misjudge the strength and reach of the robotic arm, since they are not so accustomed to them. If the operator underestimates the exerted force he can easily damage the surrounding environment, if he overestimates it he might break the robotic hand. With the help of force-feedback the operators can easier predict the dynamical properties.

In order to be able to create a force-feedback signal on the master side, the slave robot has to be able to measure the forces and torques applied to its end effector. There are three main ways of measuring forces with a robotic manipulator: 
- Calculating forces from the joint currents

- Measuring torques in each joint with joint torque sensors

- Measuring with 6 DOF force-torque sensor attached to the end-effector

In the general case it can not be expected that the force-torque values can be obtained from the robot directly. The most general way of sensing is by the usage of an external sensor. Most of the industrial robotic arms have directly usable packages for force-torque sensing. In order for the signal to be usable filtering must be applied to the raw measurements. The readings must be translated to the world coordinate frame of the robot and gravity compensation must be applied to it. The Cerntauro framework has built-in filtering, gravity and bias compensation algorithms.

To counterbalance the effect of the noise a filter must be applied to the data measurements. For the Cerntauro project the Kalman-filter was used. The Kalman filter is an ideal linear state estimator. It is fast enough to be run on a low power controller at real-time speeds. The controller needs only the estimation of the measurement and process noises. The measurement noise can be easily obtained by measuring the static values of the readings and calculating their standard deviation. The process noise can be hand tuned the reach the desired phase response.

For the gravity and bias compensation a method was implemented based on least-squares optimisation method. The calibration procedure was developed in a way to be able to quickly performed before an intervention. The method takes 52 seconds to perform, and has precision of $0.1 \mathrm{~N}$. The gravity compensation is done in real-time. It uses the estimates of the mass and inertia matrix of the end-effector obtained trough the calibration.

In order to create the force-feedback in the CERNTAURO framework impedance control with virtual springs was implemented. Virtual springs are a way to create force-feedback between the user in a safe-way. The virtual spring acts as an imaginary spring between a point located on the robot and a point is space. The virtual spring creates a force at the end-effector according to Hooke's Law. The force is based on the distance between the two end points of the spring, and the spring stiffness.

The advantage of using virtual springs is their safe operation even in the close proximity of an operator. When the user modifies the path of the end-effector the end-effector applies linearly rising force-feedback. This way the impulse and the acceleration of the end-effector stays low, it can not harm the operator.

\section{Master Handler}

To make easier the tele-manipulation task, a precise mechanical and electronic system handler has been designed and developed (C). This tool is a human-to-machine interface that allows the user to interact with the KUKA robotic arm during tele-operation. The minimum information that the interface has to provide to the robot are:

- Gripper opening / closing in a friendly way.

- If the operator is handling the robot or not, allowing to release the tool as required. 


\section{Time delay in comunication}

Time-delay passivation is a technique for reducing the energy of the control set point according to the delay between the operator and the robot. Several formulas have been proposed during the years, both in the case of constant time delay and time varying delay [350]. When dealing with an internet-based infrastructure, the delay in the network is influenced by several factors, specially the network load and the $4 \mathrm{G}$ signal strength [351].

The time-delay passivation problem has been studied during the years as a stabilization method for force reflection in master-slave control. In this context, though, the operator can choose between different control devices, which not always provide a force feedback. However, it is important to take care of the time delay and react accordingly.

The definition of the delay is important. In this case, the control loop must be seen from the perspective of the robot. Considering the simplest scenario of an operator using a joypad as control device and using a standard camera as feedback, from the perspective of the robot, the overall delay of the set point provided by the operator is the sum between the transmission delay of the camera image and the transmission delay of the control command. This because the operator sends a specific command to the robot according to the image that is showed by the HRI in that moment, but that it was produced, elaborated and transmitted by the robot some time in the past.

Therefore, the command delay can be defined like in the following equation (see Equation 5.1):

$$
d_{c}=t_{r c}-t_{i}
$$

where $t_{r c}$ is the time when the robot received the actuation command from the network and $t_{i}$ is the time when the image was produced.

Studies showed that people are generally able to detect latency as low as 10-20 ms [352]. Another research, instead, has shown that, when system latency is more than 1 second [353], operators begin to switch their control strategy to move-and-wait instead of continuous commands to compensate for the delay. CERN 4G network present an average delay of $40 \mathrm{~ms}$, with peaks of 250-350 ms. Therefore, the delay is perceivable by the operator, but is not high enough to change the control strategy. Other studies showed that delays above $300 \mathrm{~ms}$ would make the operator decouple the commands from the robot's feedback [354]. Furthermore, as already stated, $4 \mathrm{G}$ latency is not constant, and it is demonstrated that movement times increase by $64 \%$ and error rates by $214 \%$ when latency increases from $8.3 \mathrm{~ms}$ to $225 \mathrm{~ms}[355]$.

Once the delay is defined, actions can be taken to mitigate its effects. The most direct solution would be to create a formula that reduces the overall control energy (in most of the cases the speed set-point for the robot) according to the delay. Nevertheless, this solution can generate confusion on the operator. This because the operator has already the possibility to limit the control energy of the command through a slider available on the control view of the HRI. Therefore, if the operator already limits the control energy to be more accurate in the movements and the energy is reduced even more on the robot side due to the delay, the output energy will be very low and the operator will tend to increase the control energy back 
on the HRI to obtain the desired movement. If in this moment the delay suddenly reduces, the robot accelerates unexpectedly. Therefore, the output energy must be a function of both the delay and the control energy. Using this polynomial function, the output energy is computed as in the following Equation:

$$
\begin{array}{r}
E_{o}\left(d_{c}, E_{c}\right)=p_{00}+p_{10} E_{c}+p_{01} d_{c}+p_{11} E_{c} d_{c}+p_{20} E_{c}^{2}+ \\
+p_{02} d_{c}^{2}+p_{21} E_{c}^{2} d_{c}^{2}+p_{12} d_{c}^{2}+p_{03} d_{c}^{3}
\end{array}
$$

, where $E_{c} \in[0 ; 1]$.

The values of the parameters must be computed according to the type of delay that the communication network presents. Considering the studies mentioned before, at the characteristic of the CERN 4G latency, we can assume that $d_{c} \in[0 ; 500 \mathrm{~ms}]$. If the delay is higher than $500 \mathrm{~ms}$, the output energy is nulled. Moreover, if the delay is lower than $20 \mathrm{~ms}$, the control energy is sent to the actuators unaltered. With these considerations, the values of the parameters presented in Table 5.1 are computed through experimental validation with the current network configuration.

\begin{tabular}{|l|l|}
\hline Parameter & $\begin{array}{l}\text { Value used experimen- } \\
\text { tally }\end{array}$ \\
\hline$p_{00}$ & -0.01152 \\
\hline$p_{10}$ & 1.127 \\
\hline$p_{01}$ & 0.0002581 \\
\hline$p_{11}$ & -0.002793 \\
\hline$p_{20}$ & -0.1152 \\
\hline$p_{02}$ & $-5.591 \mathrm{e}-07$ \\
\hline$p_{21}$ & $-7.57 \mathrm{e}-05$ \\
\hline$p_{12}$ & $1.658 \mathrm{e}-06$ \\
\hline$p_{03}$ & $7.318 \mathrm{e}-11$ \\
\hline
\end{tabular}

Table 5.1: Network Latency Passivation Parameters

\section{Master-Slave relation}

the three main factors that influence the master-slave relation, are the kinematics, the degrees of freedom and the work spaces. The different kinematics in between the master and the slave can be mitigated if the master and the slave are the same or if the master drives the slaves only in the cartesian space. It can be a problem because master and slave can have different singurality points and different joint angles limitations. The CERNTAURO bilateral control systems takes all these aspects into account passivating the master and the slave if these drawback points are reached. The HMI notifies the operator that has to drive the master into another spatial configuration to continue the teleoperation. 


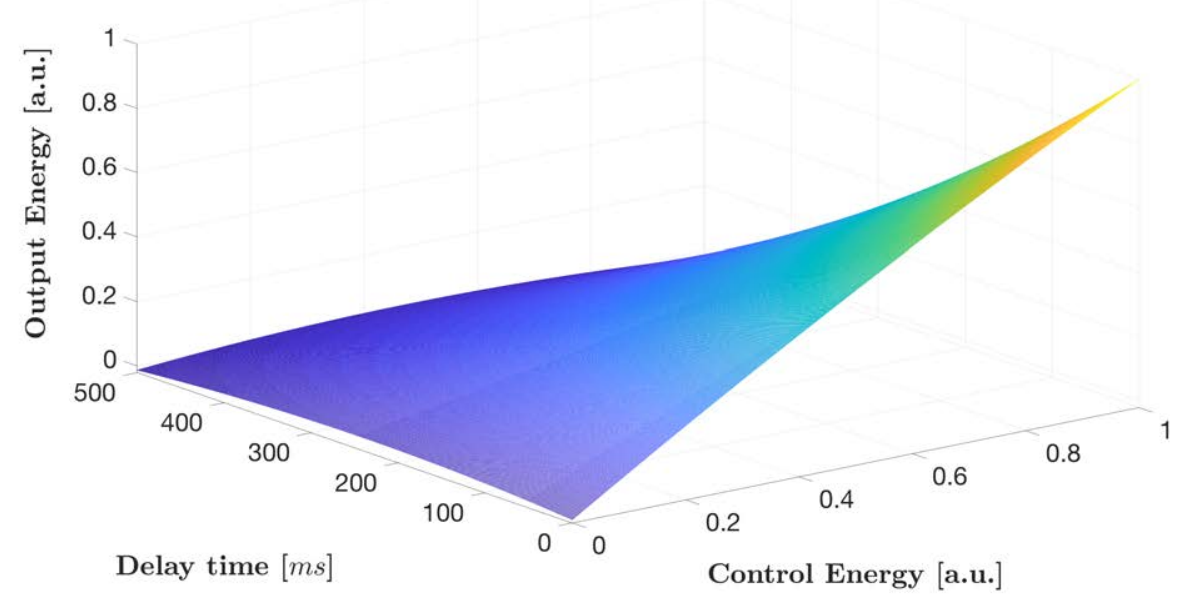

Figure 5.10: Plot of the time-delay passivation function using the parameters defined in Table 5.1. On the $\mathrm{x}$ axis, the control energy is represented in the range $[0 ; 1]$. The y axis shows the time delay in seconds in the range $[0 ; 500]$. Finally, the $\mathrm{z}$ axis represents the output energy in the range $[0 ; 1]$

\section{Image super resolution for operator perception enhancement}

During robotic intervention supervision or teleoperation, is important to have high quality images for feedbacks and visual checks. This is not always possible when limited communication channels are in place, like at CERN. Normally, when communication bandwidths are limited, low resolution images are sent from the workplace to the supervision system location. In addition, having a computer on board enables image vision and compression before transmitting the information (e.g. compressed images or semantic data). The CERNTAURO framework is endorsed with a single image super resolution system that donates to the robotic supervisor and/or operators in surface high quality images that are extracted from low quality images that come from the workplaces.

Single image super-resolution (SISR) is a classical problem in computer vision that aims at generating a high-resolution (HR) image from a single low-resolution (LR) image, thereby increasing the high frequency details and removing the degradations [356]. Image resolution describes the components contained in an image, which means higher resolution has more components about the original scene. Therefore, HR image forms an important part to improve the pictorial information for human interpretation and also help representation for automatic machine perception [357]. For CERN application, all the robots are controlled through $3 \mathrm{G} / 4 \mathrm{G}$ network, thus is needed to avoid to occupy the entire bandwidth. SISR allows to send LR images through $4 \mathrm{G}$ network, while display to the operator HR images. However, SISR is an inherently ill-posed problem, due to multiple solutions for any given low-resolution pixel. In other words, it has non-trivial solution. To solve such a problem, various SISR algorithms have been recently proposed. 
Traditional SISR algorithms have four categories: example-based methods, image statistical methods, edge-based methods and prediction methods [357]. Among them, the example-based strategy outperforms others. However, with the development of artificial intelligence, deep learning is currently active and showing significant performances on SISR tasks, such as Super Resolution Convolutional Neural Network (SRCNN) [358], Deeply Recursive Convolutional Network for Image Super Resolution (DRCN) [359], Deep Residual Learning (VDSR) [360], and Very Deep Residual Encoder-Decoder Network (RED) [361]. These deep learning-based algorithms achieve state-of-the-art performance and reflect the trend of "the Deeper the Better". Additionally, deep learning-based methods do not require prior information, reducing the computing complexity.

To fulfill teleoperation requirements including accuracy and real-time, we firstly apply the residual deep CNN network (DnCNN) [362] to the LR images, which can remove most artifacts from JPEG compression. As observed in Figure 5.11, DnCNN is a kind of Convolutional Neural Network modified from VGG network, and its core idea is residual learning which means the noisy part is predicted and then subtracted from the input. Finally, the direct result is the clean image. Furthermore, DnCNN can be easily extended to handle any JPEG compression quality, achieving better performance in different noise levels. For DnCNN, the size of convolutional filters is set to be $3^{*} 3$, and the depth is 20 . The averaged mean squared error between the desired residual images and estimated ones from noisy input works as the loss function to learn the trainable parameters.

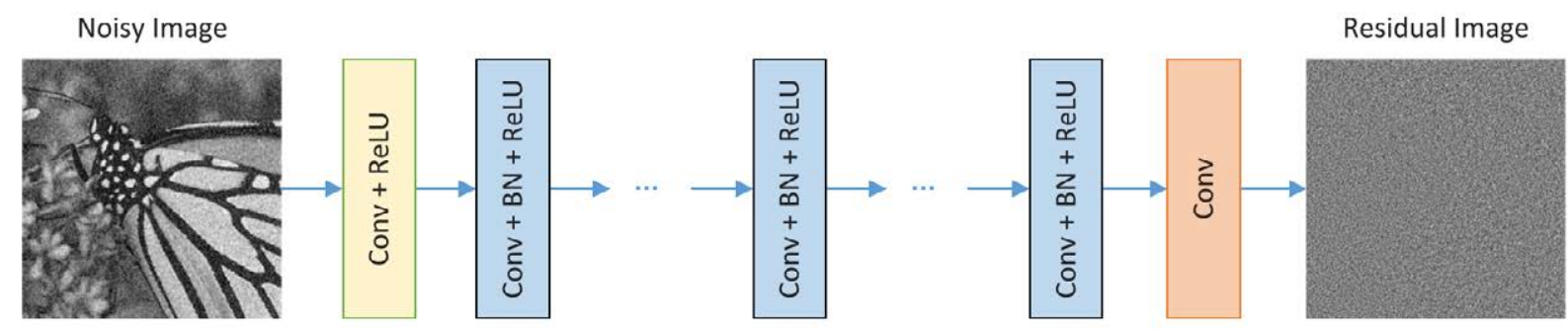

Figure 5.11: The architecture of DnCNN network

For example, Figure 5.12 shows the image with 50\% JPEG compression and the recovered image. The numbers in parentheses represents the peak signal-to-noise ratio (PSNR) and the structural similarity (SSIM), respectively. Compared to the compressed image, the recovered image has higher PSNR and SSIM, which indicates our network removed the artifacts and improved the image quality well.

After removing JPEG compression artifacts, the SISR network can be adopted to map LR images into HR space. ESPCN, efficient sub-pixel convolutional neural network, can provide a real-time speed with quite good accuracy (see Figure 5.13) [363]. In ESPCN, 3 convolutional layers are applied for feature maps extraction, and one sub-pixel convolution layer is used to upscale the low resolution input. Moreover, with ESPCN, we can implement multi-scale super resolution, such as 2X, 3X, 4X, and 8X [363].

Figure 5.14 gives an example of the CENRTAURO super resolution system result. The 


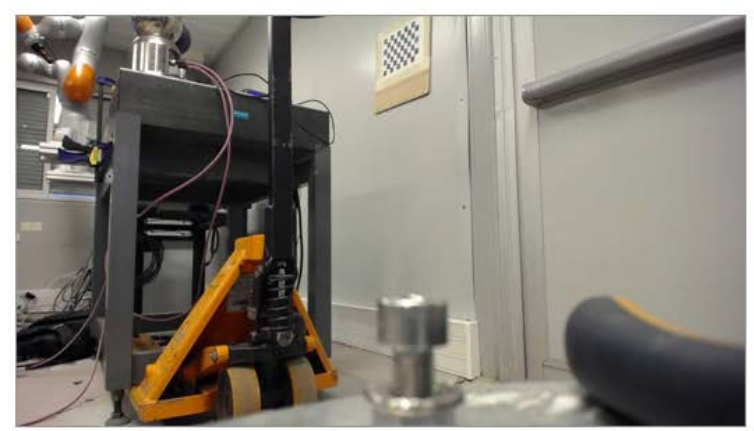

Compression (42.38/0.9810)

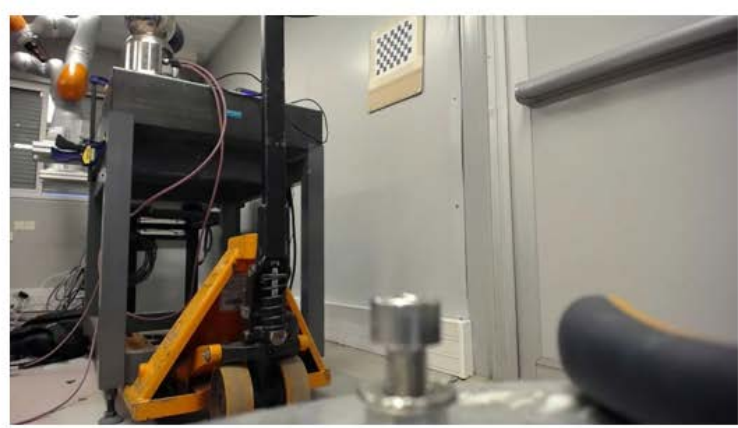

Recover (43.12/0.9849)

Figure 5.12: The compressed image with 50\% JPEG compression and the recovered image via $\mathrm{DnCNN}$. e numbers in parentheses represent the peak signal-to-noise ratio (PSNR) and the structural similarity (SSIM), respectively.

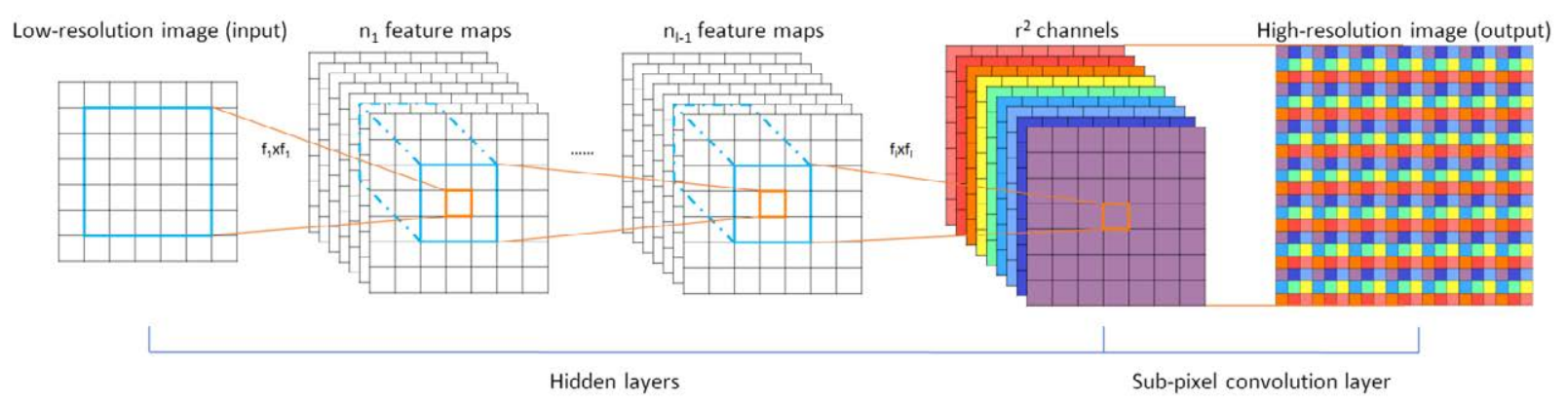

Figure 5.13: The architecture of ESPCN network.

size of the groundtruth image is $282 \mathrm{~KB}$, while the size is reduced to $14 \mathrm{~KB}$ with $4 \mathrm{X}$ downsampling and 50\% JPEG compression. Compared to $282 \mathrm{~KB}, 14 \mathrm{~KB}$ is definitely easier for transmission with a small bandwidth. Then after applying DnCNN and ESPCN networks, both PSNR and SSIM have an increase in the recovered image, which indicates that this system is reasonable for SISR recovery.

\section{Learning by demonstration}

For specific and delicate tasks, techniques like learning by demonstration could be used to teach robots to do actions, having the operators acting as supervisors during the tasks executions. The proposed framework integrates a learning technique based on dynamic movement primitives. Learning motions patterns is the equivalent to performing a specific action that the pattern can relate to. This process begins with an acquisition of a demonstration. To obtain data is the essence of capturing a desired skill to be learned. Using supervised learning methods is useful since demonstrations will come from an expert on the task at hand. The pattern can be extracted from this information, so the robot can then replicate the learned dynamics. With regression methods, the input and output relationship 


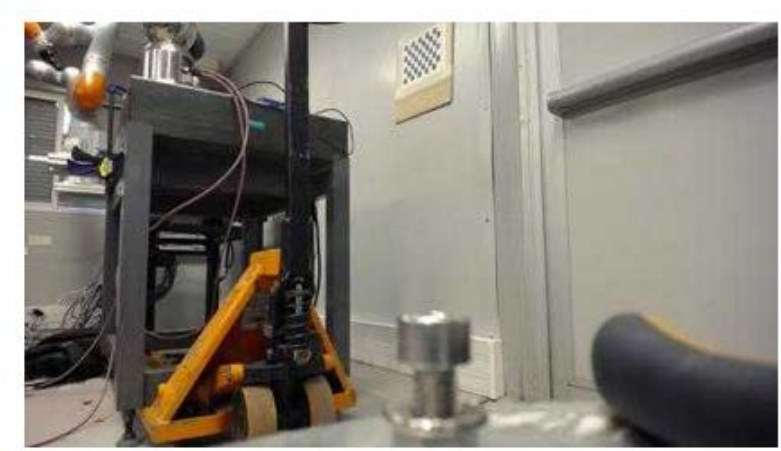

$50 \%$ compression $+4 \mathrm{X}(35.24 / 0.9589)$

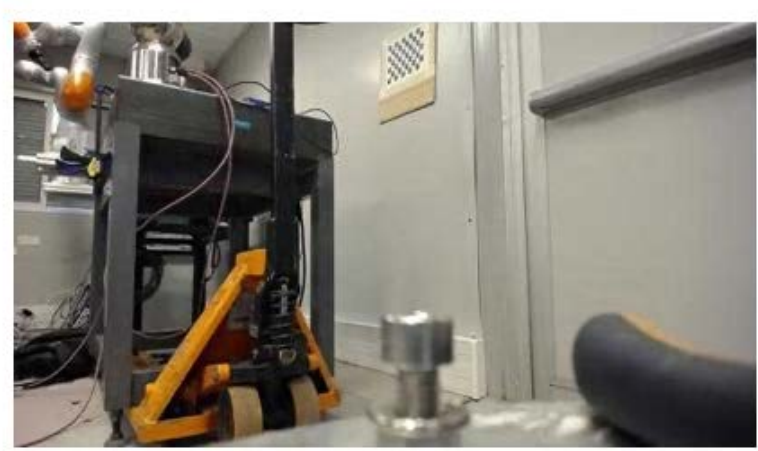

Recovered (35.48/0.9661)

Figure 5.14: The 4X down-sampled image with 50\% JPEG compression and the recovered image via DnCNN and ESPCN. The numbers in parentheses represent the peak signal-tonoise ratio (PSNR) and the structural similarity (SSIM), respectively.

is established by a function which takes an input and links it directly to an output. This relationship is not necessarily linear and it is continuous in space. Therefore, regression algorithms are ideal candidates to receive information from a learned behaviour and use it to feed the control system in this work. The dynamic movement primitives (DMP) is a generic model system that uses multidimensional systems of weakly non-linear equations to generate patterns according to the parameters that are defined in its core equations. Its base functionality is based on the creation of a landscape using the commonly known point attractor spring-damper dynamic equations, which can be used for accomplishing a task in a more complex system [364].

Dynamic movement primitives are originally based on differential equations which are defined as the transformation system. It is a fundamental base where all motion will be generated from. The formula presented in Equation 5.2 is used to represent the dynamics [365].

$$
\tau \frac{d^{2} x}{d t^{2}}=K(g-x)-D \frac{d x}{d t}-K\left(g-x_{0}\right)+K f
$$

Based on the spring-damper dynamic equations, the parameters of $K$ and $D$ represent the stiffness and damping respectively. The $f$ term is the non-linear forcing function that defines the system's pattern to reach the goal position. This function will provide the property of changing the point attractor landscape, a desirable feature for performing complex paths.

Furthermore, the DMP framework introduces a new differential equation known as the canonical system which can be defined with Equation 5.3. [366].

$$
\tau \frac{d s}{d t}=-\alpha_{s} s
$$

The main purpose of using this formulation is to change the time dependency of the DMP, in particular, of the forcing term $f$. By replacing the time $(t)$ with the new state of 


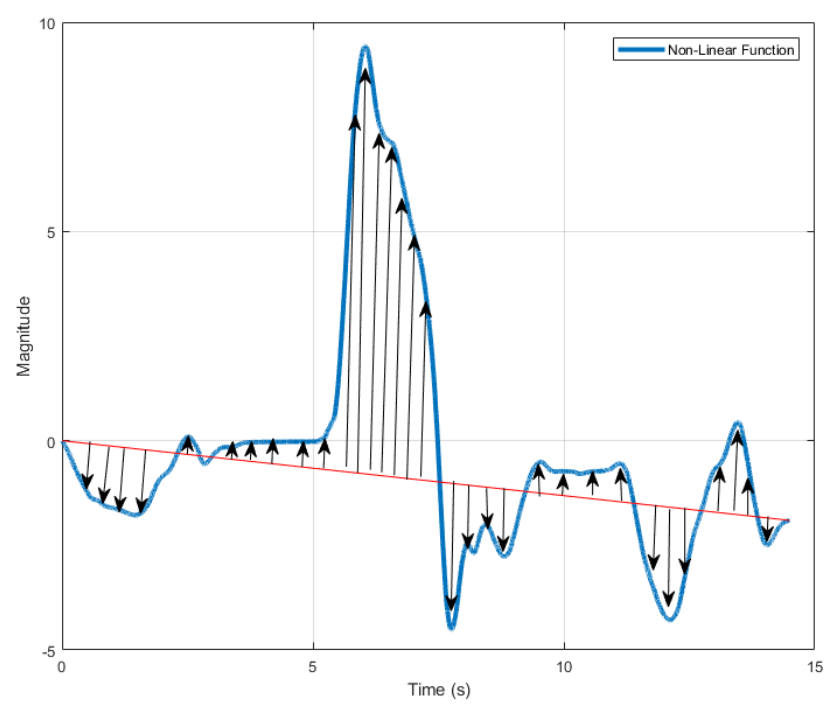

Figure 5.15: Landscape field flow representation for non-linear trajectory

the canonical system it is possible to create an autonomous system which will not directly depend of the time state.

In this new system, the state variable is defined as $s$ and the parameters are $\alpha_{s}$ represent the canonical decaying constant and $\tau$ the temporal scaling parameter.

Notice in Figure 5.15, the arrows represent a forcing value that change the shape of a non-linear function. The red line identifies the linear direction of the system as if there was no forcing term involved.

In order to achieve this feature, the base of forcing term function is chosen as non-linear defined by the Equation 5.4 [366].

$$
f(s)=\frac{\sum_{i=1}^{N} \psi(s) w_{i} s}{\sum_{i=1}^{N} \psi(s)}
$$

It is based on the normalization of linear combinations of a fixed basis function $\psi$, also known as the kernel function, which is selected as a squared exponential. The $w_{i}$ are adjustable weights that can be learned through different methods of machine learning and $s$ is the state variable of the canonical system.

To compute the weight parameters of the non-linear forcing term in the DMP formulation, the method of locally weighted regression is used. Locally weighted regression is a non-parametric statistic algorithm useful for learning complex behaviours in autonomous robotics systems. It uses several local models and finds the line of best fit. It creates the best function that can represent a relationship between the input and output variables [366] [365] [367].

The non-linearities are represented through the activation of several kernel functions $\psi$. For each one of them, the value of $w_{i}$ is calculated by minimizing the locally weighted 
quadratic error presented in Equation 5.5.

$$
J_{i}=\sum_{t=1}^{P} \psi(t)\left(f(t)-w_{i} \xi(t)\right)^{2}
$$

The normal equation used to obtain the weight parameters and is based on the paper presented by [366] shown in the Equation 5.6.

$$
w_{i}=\frac{S^{T} \Gamma_{i} f_{\text {target }}}{S^{T} \Gamma_{i} S}
$$

Where the parameters are defined as follows.

$$
S=\left[\begin{array}{c}
\xi(1) \\
\xi(2) \\
\vdots \\
\xi(P)
\end{array}\right] \Gamma_{i}=\left[\begin{array}{ccc}
\psi_{i}(1) & \ldots & 0 \\
\vdots & \ddots & \vdots \\
0 & \ldots & \psi_{i}(P)
\end{array}\right] f_{\text {target }}=\left[\begin{array}{c}
f_{\text {target }}(1) \\
f_{\text {target }}(2) \\
\vdots \\
f_{\text {target }}(P)
\end{array}\right]
$$

$\mathrm{P}$ denotes the index of the last point of data taken.

One of the greatest benefits of using differential equations is the capability of functionality expansion through coupling terms. This is further extended in the DMP framework at the cost of modifying the dynamic's parameters.

This means it is possible to incorporate new features in the trajectory generation algorithm to improve the system's performance in a simple manner and achieve more complex behaviours. In this case, the modification of the decay rate from a set of parameters and state variables as presented in the Equation 5.8.

$$
\alpha_{s}=-A \log \left(B(\tilde{x}-x)^{2}+C\right)
$$

Where the the values of $A, B$ and $C$ can be restricted in the range presented next.

$$
A<=1.0 ; B>0 ; C \approx 0.1
$$

By modifying values of the decay rate directly of the canonical system, we can change the speed generation of data. This is especially useful in the detection of an obstacle in the system's path. When the difference, or error, between the expected and actual values is too much the system can halt automatically.

The work-flow overview shown in Figure 5.17 presents the phases named as learning and control respectively. In the learning phase, the system will learn the DMP parameters through a demonstration done by a teacher in Hand-Guidance Mode. These learnt DMPs will be stored in a primitive motion library. Afterwards, the control phase comes into play. The system, supervised by the user, will select the DMP from the library according to the desired task. Thereupon, an autonomous mode will use the chosen primitives and the robot control system altogether with the path generator is executed.

The hand guidance mode's basic principle is to use an impedance control to move the 


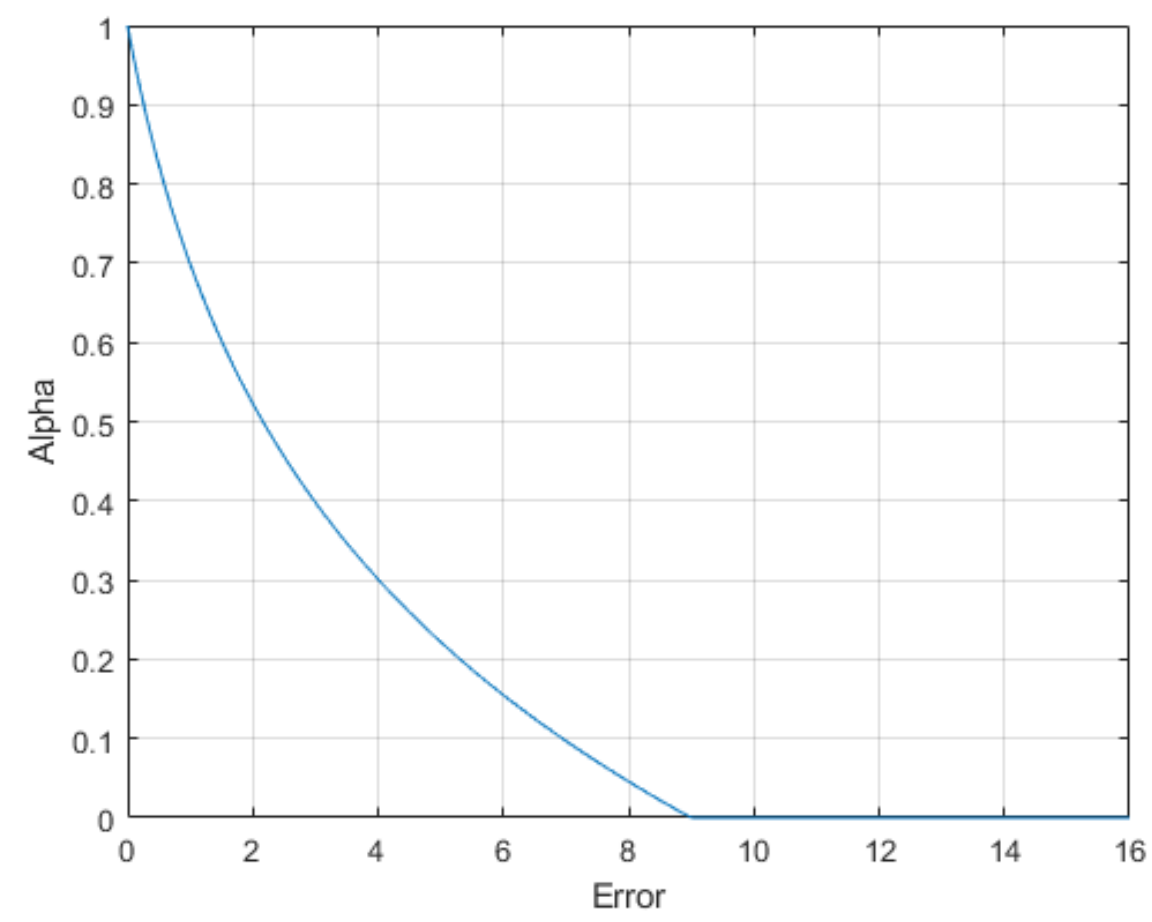

Figure 5.16: Dynamic change of canonical system's parameter $\alpha_{s}$

Learning Phase

Control Phase
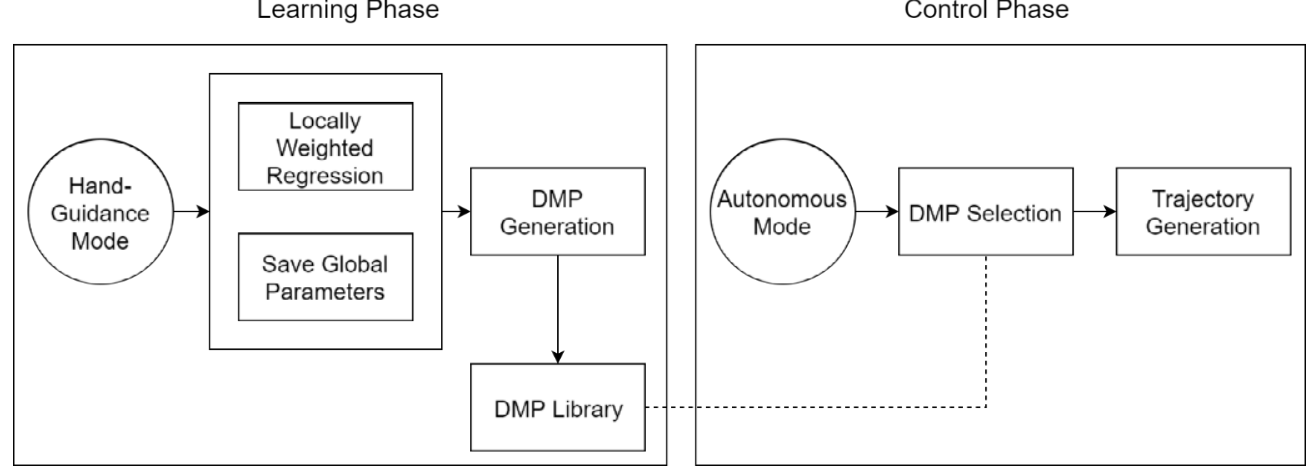

Figure 5.17: Learning workflow diagram 


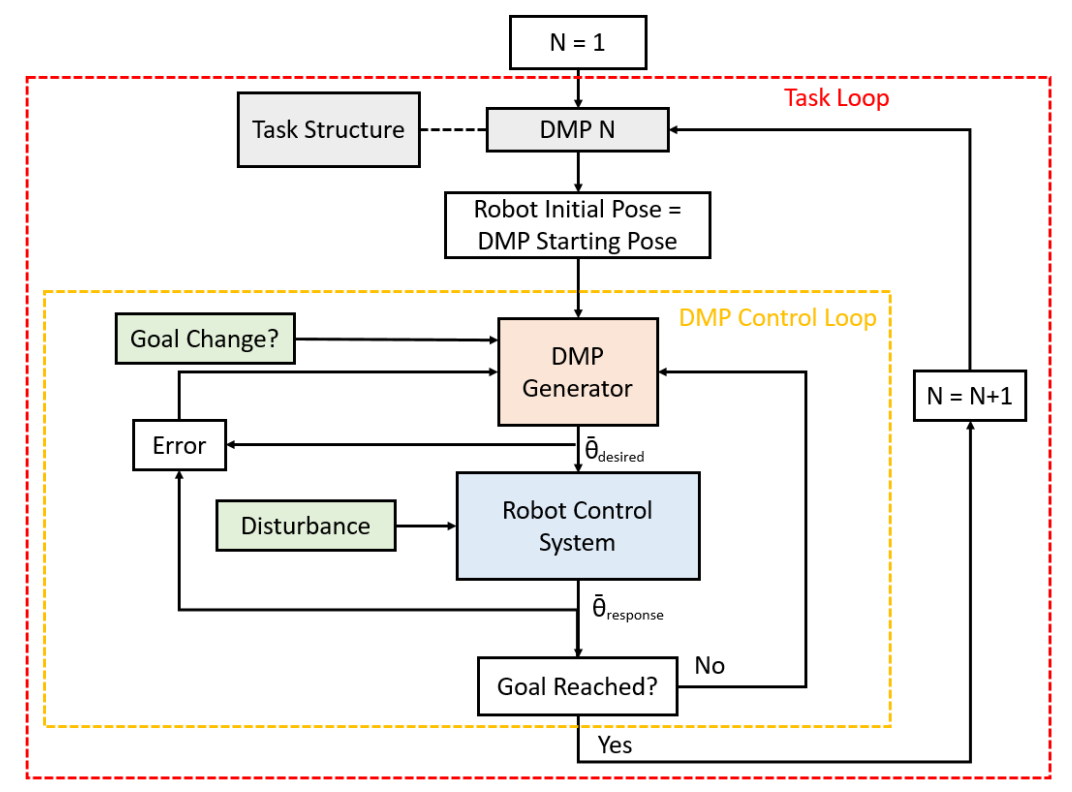

Figure 5.18: Robot's learned dynamics control scheme

robot through different motions using the teacher's guidance. The teacher's hand will be used to generate robot motion into its body and therefore the name of hand-guidance mode.

For accomplishing a task in the current proposed system, first a task must be set and defined also using libraries of DMPs to perform a particular task. This means, that several primitives must be developed first and then used accordingly to achieve a goal. To do so, the learning phase is used as necessary to get the needed primitives to perform the desired activity. By reaching the final goal the task can be considered finalized.

Once the task structure has been defined, the program can take this data and start the robot's control scheme, as shown in Figure 5.18. The system is initialized in the task loop, from here the parameters of the corresponding DMP will be acquired and then the initial pose of the robot will be checked with if its equal to the current selected DMP. If it is different then the robot will move to the initial position. After the check is done, the system enters in the DMP control loop which will take the acquired parameters and use the DMP generator to create the commands from the transformation system in and feed it to the robot control system.

The control system is done through an impedance controller to add a compliant behaviour when there is some sort of disturbance coming from the environment. Furthermore, the error coming from the expected position and the real position is to be used into the canonical coupling term altogether. Through this implementation it is possible to minimize any kind of damage it might come from an obstacle contact that might not be initially thought of in the robot learning. Additionally, the goal position can change during the execution of the task in which the DMP generator will adapt automatically to the change and create a new path from the updated information. Once the current primitive in use has reached the expected goal, the system will exit the DMP control loop and follow into the next DMP set 
in the task structure.

\subsection{VERO: Virtual Simulation Environments for Opera- tor Training}

To operate operate robots in harsh environment or in place where there are difficulties for human access, there is a strong need of intervention simulators for safety, time and costs related reasons. Mock-ups and bench test rigs are a key part of the process for remote operations development and task validation. Virtual mock-ups are used for task procedure development by default, physical mock-ups are used for testing and validation where the tasks are either novel and challenging or involve physics characteristics which cannot be reliably represented in a Virtual mock-up.

Part of the CERNTAURO framework is the Robot Operator Training (ROOT) module which, according to the difficulty of the mission and the dexterity demanded, suggests the type of training and learning, the operators and the robots must undertake. During the offline training, time and operator stress are monitored. One of the main requirements during the development of this system was to create an environment which is usable for different types of operators. Therefore a classification of the operators was done and different categories of operators were defined [186] according with their robotic operation skills. The development of the system then took into account these categories, resulting in a system that is usable and learnable for entry-level operators but also provides all the advanced features required by an expert operator. From the low-level robot control to the HumanRobot Interface, the entire development was done in such a way as to provide a complete and involving experience to any type of operator.

A novel training simulator, being part of the CERNTAURO framework, for the teleoperated robot at CERN has been developed. The robot are modelled using a robot description format and spawned in the robotic simulator Gazebo. Control of the robot base is also modelled. A graphical user interface is used in order to interface with the simulation, and control the robot with the help of live images coming from the robot's on-board cameras.

The generic robotic simulator, is used to simulate the physics and the visuals of the realistic and functional custom-made model of the robots. Thus, it can provide all the sensors' states, including the state of all the joints declared in the model of the robot, as well as information on the cameras that the robot is equipped with, since these are also modelled and simulated. On the other hand, CERNTAURO control is used to actuate the robot via the available control library, get the required actuators' data from the controllers developed in the mentioned library, and send it to the VERO simulator to actuate the joints. The user can operate the simulator using either a keyboard or a specifically designed GUI that runs on Windows. The output of virtual cameras is also streamed on a web server using CERNTAURO libraries. This allows the user to access the cameras' data and have it displayed in real-time via the GUI. For the simulator to be as realistic as possible, both the mathematical and visual models of the robot needed to be as faithful to the real robot as possible. The mathematical model of the robot was integrated and tested. This was 
followed by the visual modelling of the robot using a robot description format. Furthermore, a simple test environment was also modelled for the robot to operate in. The first step in obtaining the kinematic model of the robotic arm, was to determine the Denavit-Hartenberg parameters. In this manner the reference frames could be attached properly to the links of the robot's arm. This was done by accurately sketching the robotic arm's configuration, to clearly show all the joints and their frames as depicted in Figure 5.19.

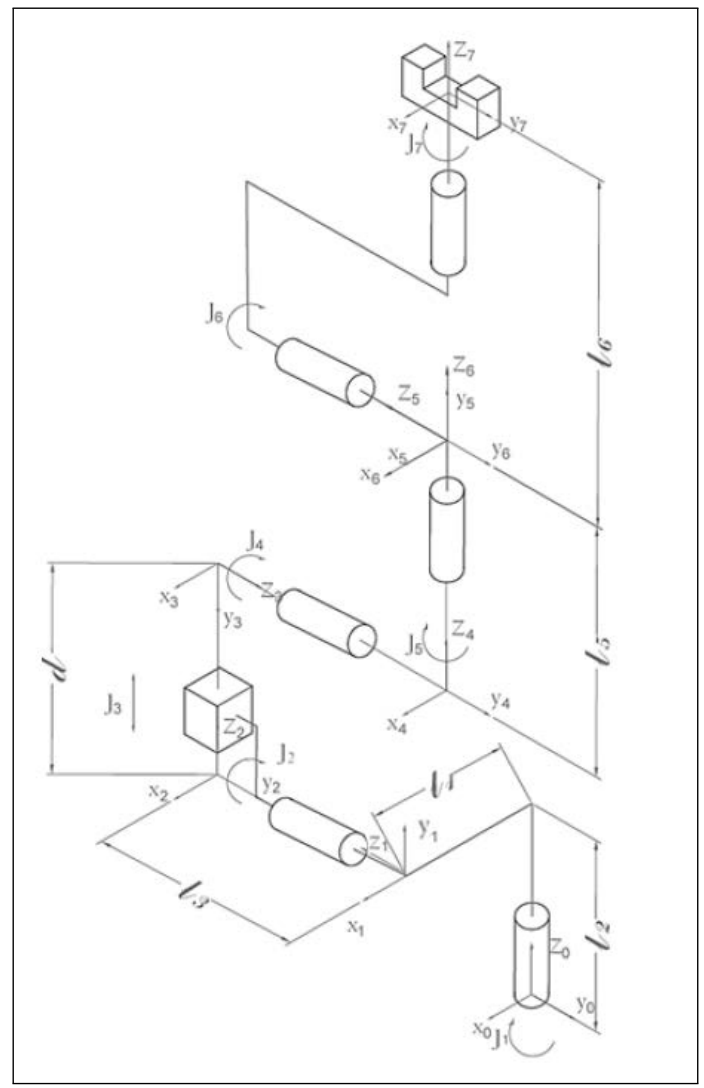

Figure 5.19: The joints and links comprising a robotic arm example (Telemax)

Then, the Denavit-Hartenberg convention [57] was used to generate the transformation matrix of each link with respect to the parent link. At the end, the transformation matrix between the base reference frame and the end-effector was found by multiplying all transformation matrices. The robotic arm can be controlled in two modes, either in a joint-by-joint control mode or in operational-space control mode. In the former the user controls each joint individually. In the operational-space control mode, the user specifies the required end-effector position and orientation in operational space (also referred to as Cartesian space). Then, a controller is used to find the required joint variables, $\mathbf{q}_{\mathbf{d}}$, so that the manipulator reaches the reference position and orientation in $3 \mathrm{D}$ space.

Figure 5.20 shows the adopted joint-by-joint control scheme. Every time the user controls a joint, the current actual joint angle (in case of revolute joints) or joint position (in case of prismatic joints) $\mathbf{q}_{\mathbf{a}}$ is read from VERO and increased (or decreased) by adding (or 


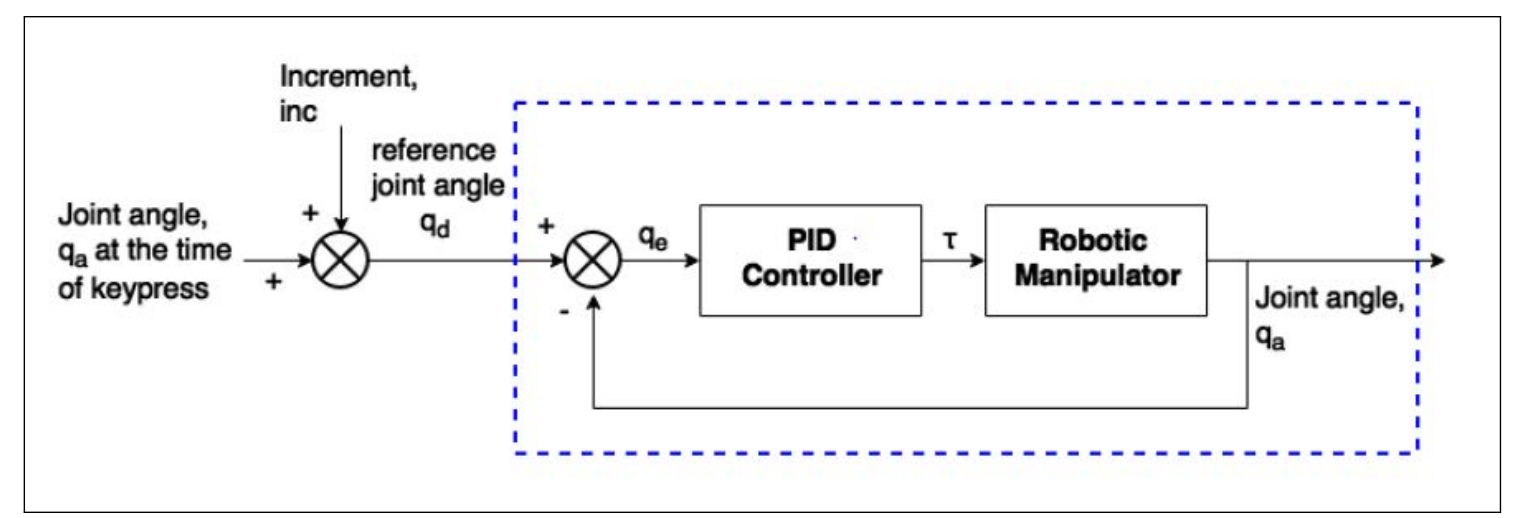

Figure 5.20: Joint-by-joint control scheme.

subtracting) the constant value, hightlight inc properly. This constant is set according to the speed predefined by the user. Consequently, the desired joint angle, $\mathbf{q}_{\mathbf{d}}$, is computed. This value is then sent to the joint angle controller that uses an internal feedback mechanism to calculate the torque required to actuate the joints according to the PID control algorithm. The controller developed in CERNTAURO is enclosed in the blue dotted box (see Figure 5.20) and allows only the reference joint position as an input. The operational-space control scheme is shown in Figure 5.21. Once the user activates the operational-space control mode, the actual joint angles $\mathbf{q}_{\mathbf{a}}$ are used to find the matrix $\mathbf{x}_{\mathbf{a}}$, containing the orientation $\mathbf{R}\left(\mathbf{q}_{\mathbf{a}}\right)$ and the position $\mathbf{P}\left(\mathbf{q}_{\mathbf{a}}\right)$ (see Figure 5.10) of the end-effector, by performing the forward kinematics.

$$
X_{a}=\left|\begin{array}{cc}
R\left(q_{a}\right) & P\left(q_{a}\right) \\
0 & 1
\end{array}\right|
$$

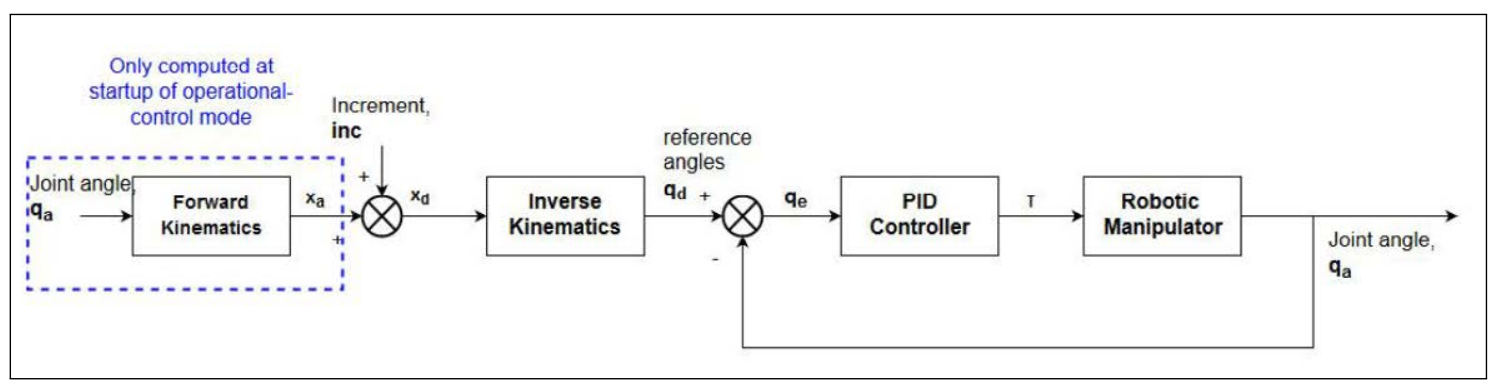

Figure 5.21: Operational-space control scheme.

Then, each time the user needs to change the position/orientation of the end-effector, the new desired rotational matrix hightlights xd properly, which is a function of the desired joint angles $\mathbf{q}_{\mathbf{d}}$, that is found using the incrementing vector, inc. This vector contains six constants, three that give an increment in the $\mathrm{x}, \mathrm{y}$, and $\mathrm{z}$ axis of the end-effector (inc $\mathrm{x}_{\mathrm{x}}$, inc $_{\mathrm{y}}$, and inc $_{\mathrm{z}}$ ), and three that give a rotation around the $\mathrm{x}$ (yaw), $\mathrm{y}$ (pitch), and $\mathrm{z}$ (roll) axis $(\operatorname{inc} \psi$, inc $\theta$, and $\operatorname{inc} \phi)$. To find the new position of the end-effector, the first three 
constants of inc are added with the current $\mathrm{x}, \mathrm{y}$, and $\mathrm{z}$ position $\left(\mathrm{x}_{\mathrm{x}}, \mathrm{x}_{\mathrm{y}}\right.$, and $\left.\mathrm{x}_{\mathrm{z}}\right)$ as follows Equation 5.11 shows.

$$
P\left(q_{d}\right)=\left|\begin{array}{ll}
X_{x} & +i n c_{x} \\
X_{y}+i n c_{y} \\
X_{z}+i n c_{z}
\end{array}\right|
$$

To find the orientation of the new frame, $\mathbf{R}\left(\mathbf{q}_{\mathbf{d}}\right)$, as denoted by [57], the composition of successive rotations with respect to a fixed frame is obtained by premultiplying of the single rotation matrices in the order of the given sequence of rotations. Hence, Equation 5.13, where, $\mathbf{R}(\phi)$ is the orientation matrix as a function of the desired increment angles (inc $\psi$, inc $\theta$, and $\operatorname{inc} \phi$ ). Hence, this matrix gives the desired rotation transformation. In [368], the authors also show that the orientation matrix $\mathrm{R} \phi$ is given by Equation 5.14. In this equation, $\mathrm{C}$ and $\mathrm{S}$ denote the cosine and sine trigonometric functions of the subscript angle respectively.

Taking the set of angles in Equation 5.12, these are computed in Equation 5.14, and the orientation matrix that the actual coordinate frame needs to be rotated with, is found. In order to find the final desired orientation matrix, $\mathbf{R}\left(\mathbf{q}_{\mathbf{d}}\right), \mathbf{R}\left(\mathbf{q}_{\mathbf{a}}\right)$ and $\mathbf{R}(\phi)$ are multiplied together as in Equation 5.13. Once the required rotation matrix $\mathbf{x}_{\mathbf{d}}$, is found, one can move on to change from the coordinate space to the joint space. To find the joint variables required for the robotic arm to move to the desired position and orientation, the inverse kinematics are computed on the desired frame. Following this, the newly computed reference joints are passed through the joint angle controller that is the same as that used in the joint-by-joint control.

$$
\left|\begin{array}{l}
\psi \\
\theta \\
\phi
\end{array}\right|=\left|\begin{array}{l}
i n c_{\psi} \\
i n c_{\theta} \\
i n c_{\phi}
\end{array}\right|
$$

For the computation of the kinematics that were used in the operational-space control, the Kinematics and Dynamics Library (KDL) [369] by the Orocos Project [370] was used. It provides generic forward and inverse kinematic solvers by the use of numerical solutions. The forward kinematic solver of KDL is used in real time to calculate the forward kinematics of the manipulator. To calculate the inverse kinematics, the solver TRAC-IK [371] was used. TRAC-IK gives solution in a relatively low computational time whilst taking in consideration the joint limits. The gripper of the robotic arm was also modelled and controlled in a similar way to the joint-by-joint control scheme. The gripper consists of six revolute joints which are moved simultaneously in order to open and close its claws.

$$
\begin{gathered}
R\left(q_{d}\right)=R\left(q_{a}\right) R(\phi) \\
R(\phi)=\left|\begin{array}{ccc}
C_{\phi} C_{\theta} & C_{\phi} S_{\theta} S_{\psi}-S_{\phi} C_{\psi} & C_{\phi} S_{\theta} C_{\psi}+S_{\phi} C_{\psi} \\
S_{\phi} C_{\theta} & S_{\phi} S_{\theta} S_{\psi}+C_{\phi} C_{\psi} & S_{\phi} S_{\theta} C_{\psi}+C_{\phi} C_{\psi} \\
-S_{\theta} & C_{\theta} S_{\psi} & C_{\theta} C_{\psi}
\end{array}\right|
\end{gathered}
$$


figure 5.22 shows a testing exercise of the simulated robotic base. In this test the operator drove the robot around a maze and made it climb different obstacles. By controlling the flippers of the robot, the robot was able to successfully climb up a maximum height of 50 $\mathrm{cm}$.

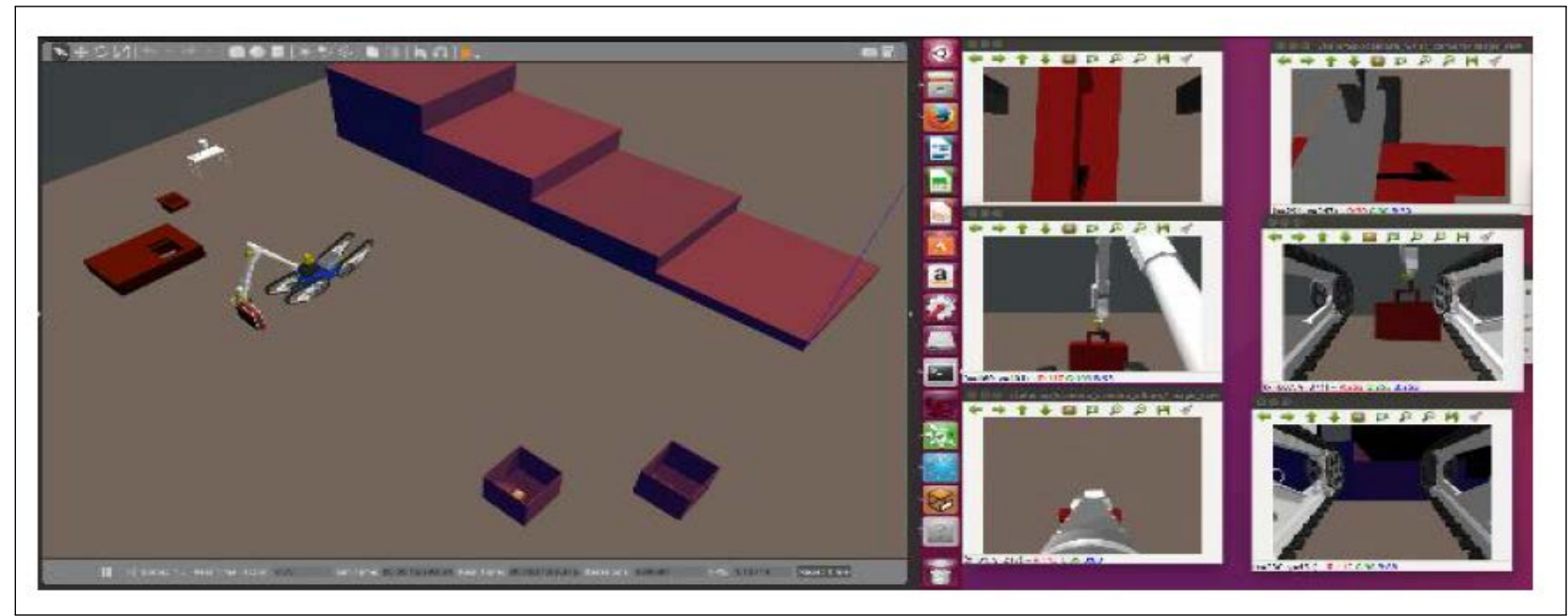

Figure 5.22: Robotic operation simulation using visual feedback from the on-board cameras.

The user can interface with the simulator via the CERNTAURO HRI, by which the user can switch from one mode to another and move the robotic arm, or the robot base, using the assigned control (see Figure 5.23).

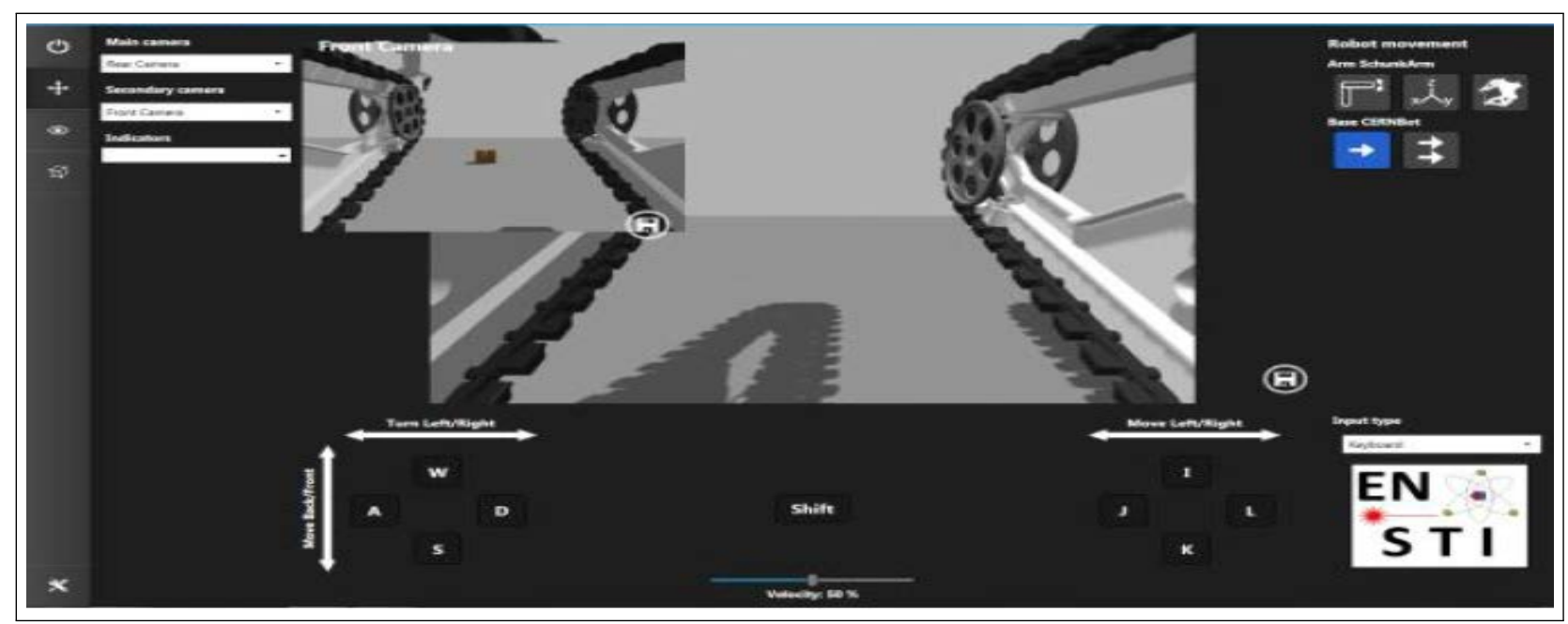

Figure 5.23: The GUI used with the simulator.

The above described robot simulator is part of the novel CERNTAURO's module VERO (Virtual Environment for intelligent Robotic Operation) which final scope is the intervention 
preparation. This module is fundamental for the design of the robotic procedures, tools and recovery scenarios that are vital in harsh and hazardous environments.

Virtual Reality (VR) is a simulation of an immersive environment produced by a computer. For this immersion the user can use different systems to replace the real world sensations by the computer generated ones. For example, for visual the user can use a headset, for audio a headphones, for tactile sensation different haptic devices, etc.

The immersive technology as virtual reality offers an wide variety solutions in different fields. It allows to incorporate large scale volumetric data of any complex $3 \mathrm{~d}$ structure for visualisation, design and training propose [372] [373]. The head-mounted display (HMD) gives the possibility to have a wide field of view of the virtual environment. Furthermore, the low latency tracking and the high-resolution displays make the experience more realistic. This technology is being used also in education to bring more intense experiences to students. For example, in the research [374], a educational game for History subject is developed for students in order to learn and develop historical thinking in a authentic, interactive and explorative experiences in the virtual environment. Researches prove that this technology improve the interest and the perform of the students [375] [376] [377].

Virtual reality is also been considering in medicine as a successful simulator for training propose and survey planing [378] [379]. Althought the major aim of VR in medicine is survey practice, it is also used in medical care with multiple professionals in a shared virtual environment for share decision making for an actual surgical intervention [380] or trauma decision-making [381].

In robotic field, virtual reality has been used for teleoperation in risky place as Tokamaks [372] or JET [382]. In both research, VR is used as a tool in Remote Handling (RH) for design robotic arm's operation and training. Applying the imitation leaning [147] the robot is able to learn and perform complex tasks or solve the complex dexterous manipulation problem [143]. By using external controller or human body tracking, bimanual robot is easily controlled [383]|384] [385]; this last one was presented in DARPA Robotics Chalenge [386] performing complex manipulation tasks. Moreover, it is possible to add real pictures features as texture of the virtual environment [387] and other feedback source in order to increase of realism, help to go out from the gaming effect and decrease the fatigue and stress while using VR [388].[389] [390].

A VR system can be resumed as Figure 5.24 shows. Among different components of the VR system, input and output devices are crucial in this system. They are in charge of immerse the user in the virtual environment. With input devices, the user can interact with virtual scene. Output devices bring the sense and different feelings to the user. 


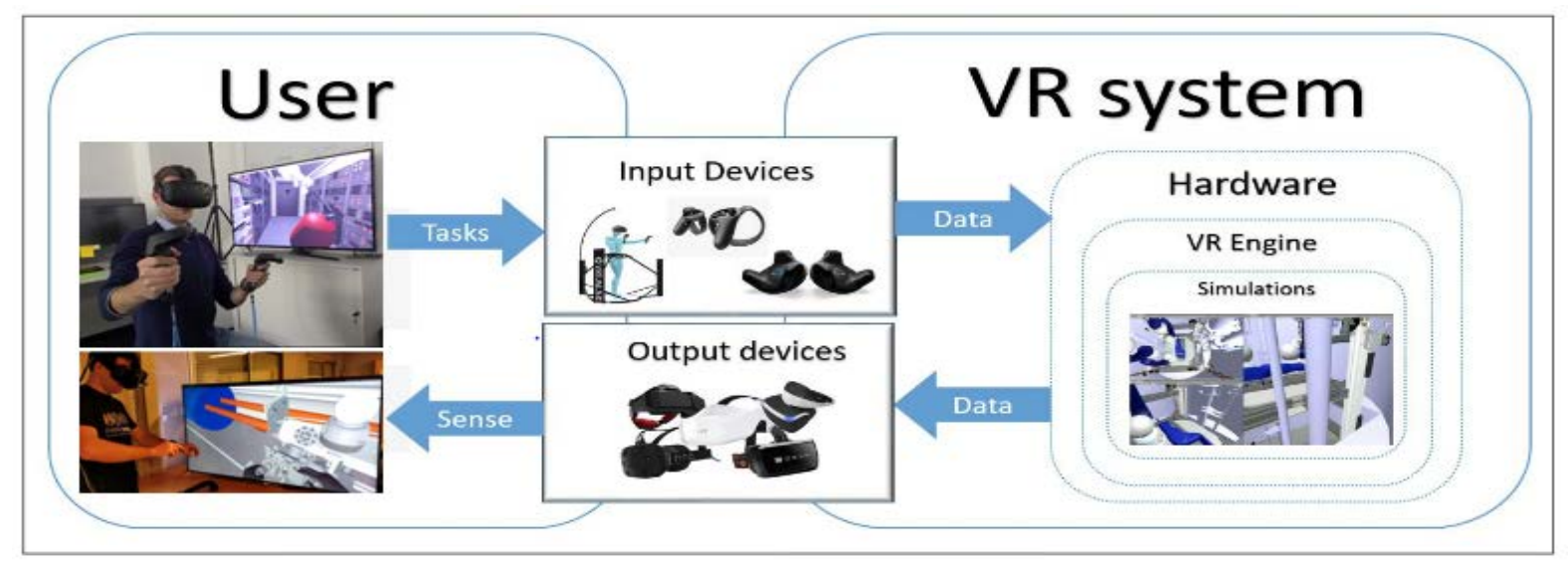

Figure 5.24: Virtual reality system

The basic input devices are used to track the position of the user together with the head orientation; normally these features are incorporated in the HMD. To capture the actions of users, different devices can be used. For example a treadmill for tracking the movement as walking or running; externals trackers for body tracking or controllers that allow user interact with objects or do different actions. Several input and output devices are presented in the Figure 5.25. A comparison of two most common known HMD is showed in the Table 5.2 and Table 5.3.

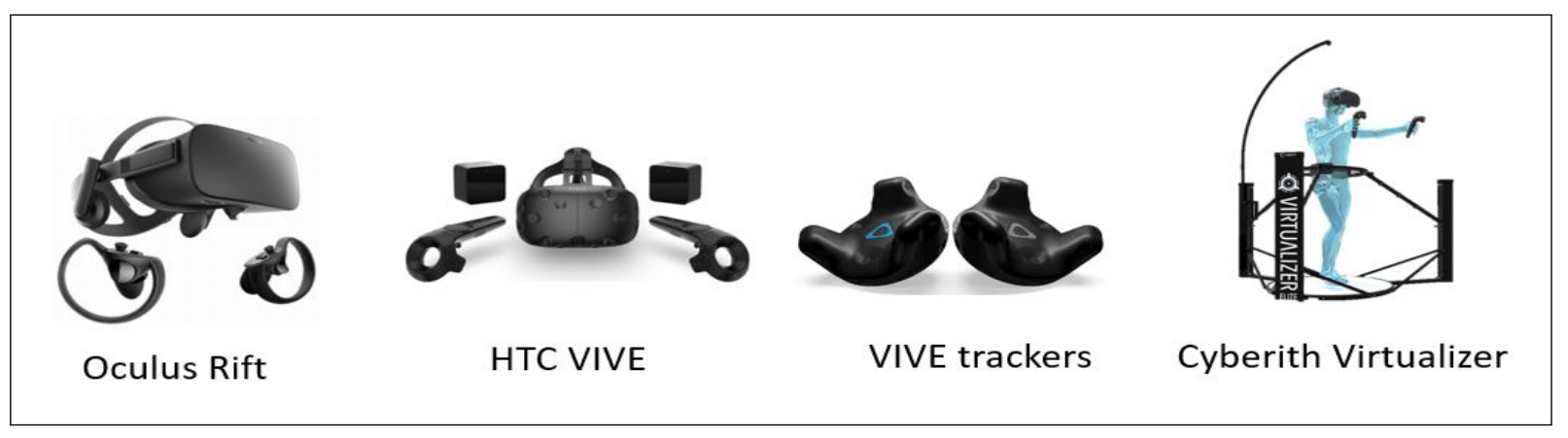

Figure 5.25: Input and output devices 


\begin{tabular}{|l|l|}
\hline Display Type \& Size & Dual low-persistence Samsung AMOLED \\
\hline Display Size & $90 \mathrm{~mm} \times 2,456 \mathrm{ppi}$ \\
\hline Resolution & $1200 \tilde{\mathrm{A}}-1080$ (per eye) \\
\hline Refresh Rate & $90 \mathrm{~Hz}$ \\
\hline Field of View & $94 \mathrm{H} \times$ 93V-degrees at optimal 12 mm lens-to-eye distance \\
\hline Sensors & Accelerometer, gyroscope, magnetometer \\
\hline Tracking Technology & 6 DOF Constellation camera optical 360-degree IR LED tracking \\
\hline Audio & Microphone, integrated supra-aural 3D spatial audio headphones \\
\hline Dimensions & $171 \times 102 \mathrm{~mm}$ \\
\hline Weight & $470 \mathrm{~g}$ \\
\hline
\end{tabular}

Table 5.2: Specifications of Oculus Rift

\begin{tabular}{|l|l|}
\hline Display Type \& Size & Dual low-persistence Samsung AMOLED \\
\hline Display Size & $91.9 \mathrm{~mm} \times 2,447 \mathrm{ppi}$ \\
\hline Resolution & $1200 \tilde{\mathrm{A}}-1080$ (per eye) \\
\hline Refresh Rate & $90 \mathrm{~Hz}$ \\
\hline Field of View & $110 \mathrm{H} \times 113 \mathrm{~V}$-degrees at optimal 8 mm lens-to-eye distance \\
\hline Sensors & Accelerometer, gyroscope \\
\hline Tracking Technology & 6 DOF IR Laser-based 360-degree tracking using "Lighthouse" \\
\hline Audio & Mase Stations \\
\hline Dimensions & $190 \mathrm{~mm} \times 127 \mathrm{~mm}$ \\
\hline Weight & $563 \mathrm{~g}$ \\
\hline
\end{tabular}

Table 5.3: Specifications of HTC Vive

VERO is based on a powerful graphics engine and a physical simulator that make possible to users feel the physical presence in the virtual environment. Furthermore, this module is practical for acquainting with the environment in order to have better planning for the interventions. In addition, with VERO, the operator can train the operations before the interventions. In this way, using this system will save time, avoid unnecessary steps or overexpose to radiation, and foresee possible dangers.

In nuclear plants and in general in hazardous environments, it is mandatory to do an analysis of the environment before starting an operation. For example, at CERN, there are several challenges to face when is working with tele-operated or semi-autonomous systems due to the complicate environments and the conditions as high radiation and temperature. This situation will be more complicate if the operation needs to be done by human.

Due to the differences challenges described above for hazardous areas, the main aim of this module is build a virtual environment as similar as possible to the real environment. For this reason, the input data are the different components of the environment. This input 
data can be sketches, CAD models, photogrammetry or laser scanning or 2D plans of the environment, robots and tools (see Figure 5.26).

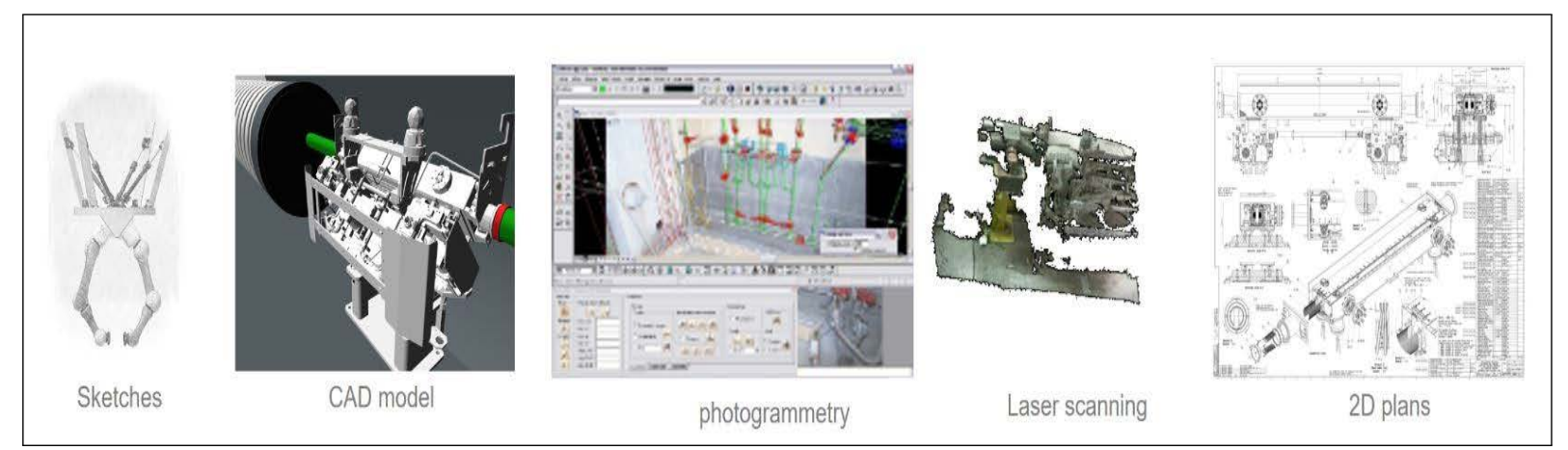

Figure 5.26: Type of input data of VERO

VERO system is designed to be modular; each input is exported as an independent 3D model. In addition, different actions or interactions with the environment are developed as different modules, each module has a specific function. Depending on the requirement of the intervention, different 3D model and function modules will be added to the virtual scene. In this way, repetitive actions can be easy imported to different simulations.

The flowchart from receiving a requirement to having the plan for intervention is shown in Figure 5.27.

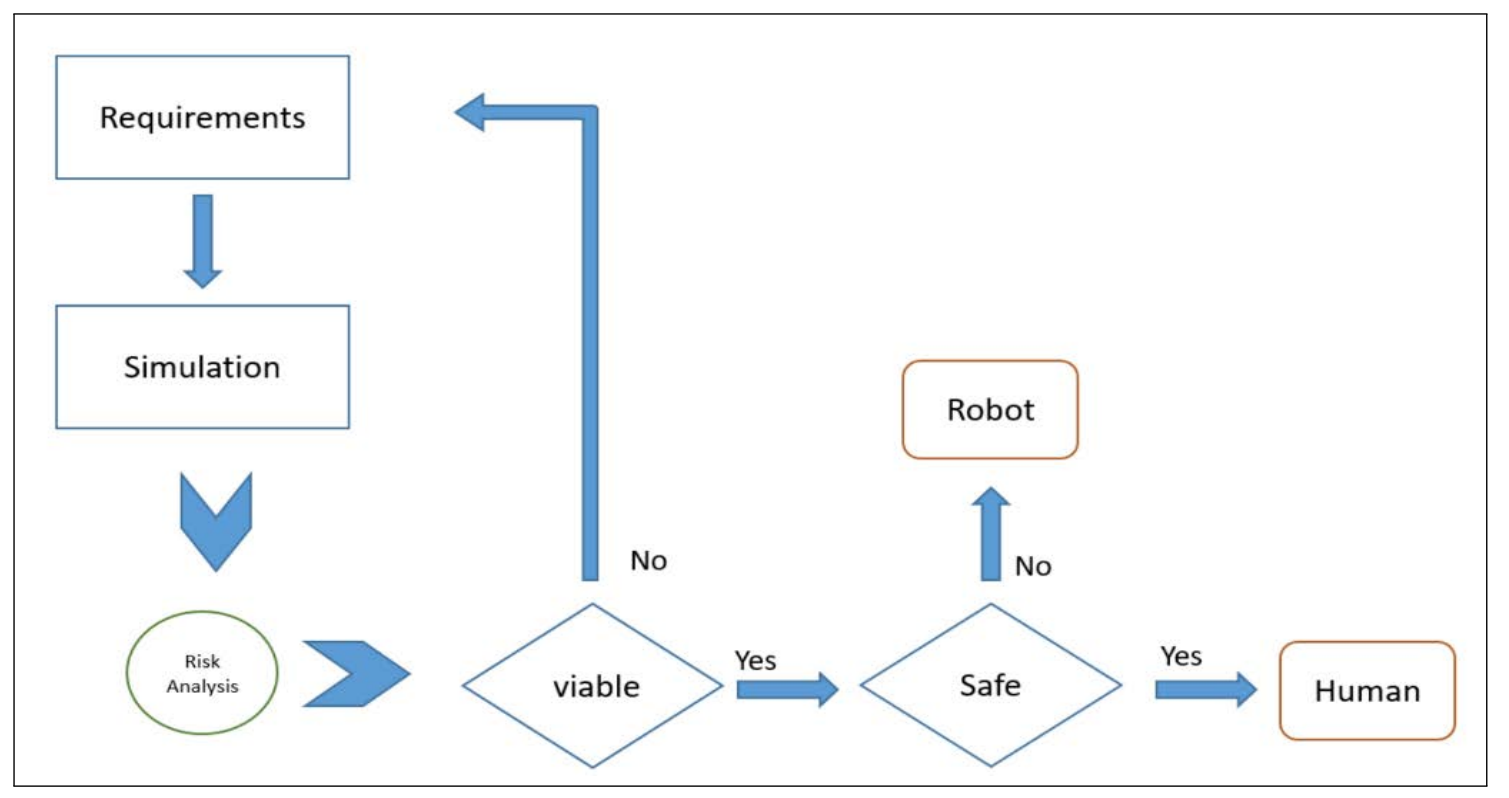

Figure 5.27: Flowchart interventions planning with VERO

- Requirements: First, a requirement is received. The requirement needs to be most detailed as possible, describing the goal of intervention, all the possible risk and giving the necessary input data model of the environment. 
- Simulation: After understanding the requirement, it is necessary to create the virtual environment for simulation. Each requirement needs to be studied in different simulators. This simulation includes the 3D model of the environment and all the modules necessaries for the intervention. For example, in some cases it is necessary unscrew, then the module of screw/unscrew is needed to be incorporated in the virtual environment.

- Risk Analysis: The simulator is crucial in the sense that with this is possible to do a risk analysis and to know if the plan scheduled is viable or not. In addition, it is very important because in this step the radiation dose that will receive the human or robot during the intervention is calculated. Depend on the result of this analysis, the operation will be done by operator or robot.

As the result of this system, a safety and viable plan is obtained. This plan will be the most efficient for both robot and human. The head mounted display (HMD) system used are the Oculus RIFT [391] with only one base station which offers 70 degree of field of view (FoV) and HTC VIVE [392] with 2 base stations which allow $120^{\circ} \mathrm{FoV}$. The first one allows only player in sit mode. By using the second one, the operator can move around the maximum play area of $3 \times 4$ meters. Although using HTC Vive, the play area is incomparable with the size of the environments need to explore. Moreover, the environments may have several floors. In some case, the access from one floor to another can be the stairs, in another case can be the lift or even can be a vertical hole. For these reasons, a flexible navigation in the whole navigation is primordial. VERO framework also incorporates a virtual interface apart from the different modules preview mentioned. The interface offers more possible of interaction with the environments. For example, two different way of navigation around the scene, laser pointer for extra information or move object, or general location map among others functions (see Figure 5.28).

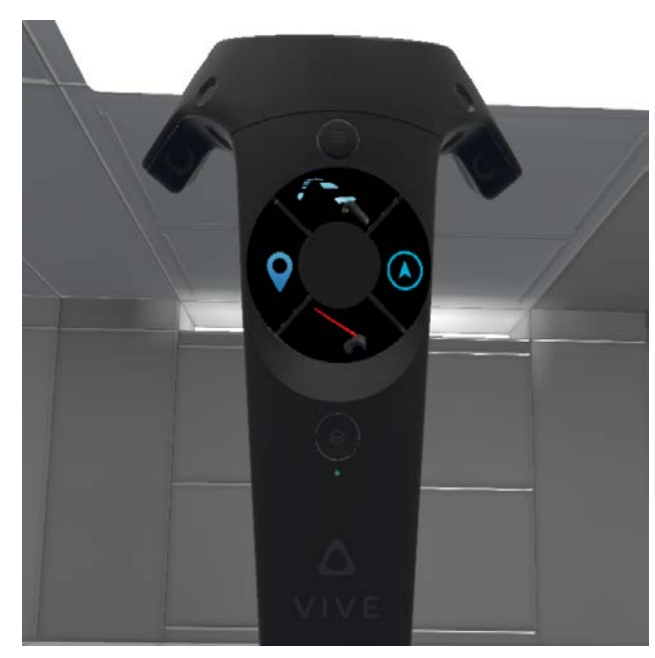

Figure 5.28: Four functions displayed in HTC VIVE controller. Teleport(Up), Map(Left), Navigation(Right) and laser(Down). 


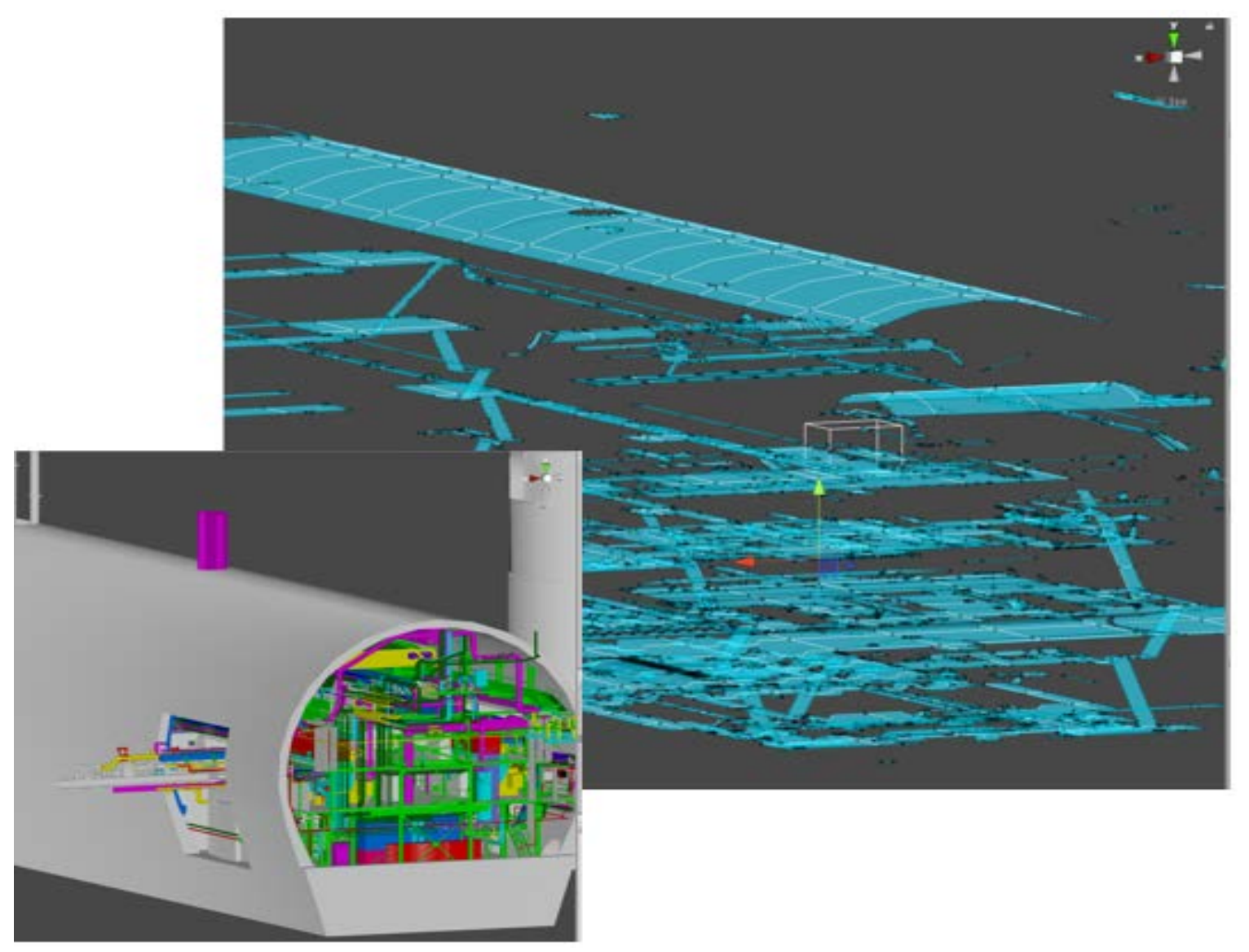

Figure 5.29: Navigation map

In order to know which points of the environment are navigable and which are not, VERO is able to create automatically a continue navigation map removing all the obstacles and joining different levels of the environment. In this way, a blue circle will appear when the user choose a navigable point and a red circle will appear if the point it is navigable or unreachable. An example of navigation map is showed in Figure 5.29.

For the purpose of reach all the points of the environment, free movement navigation mode is developed. With using this option, the navigation will follow the gaze of the user. In this way, it is possible to pass through walls or obstacles easily. Furthermore, it is very useful for exploring inside of a big object or machine. Another option to be highlighted is the laser pointer. Through this option the user will be able to move objects of the scene or for example pieces of a big component to look into the inside composition. Moreover, by using the laser, it is possible to select different object of the scene to have an extra information. 


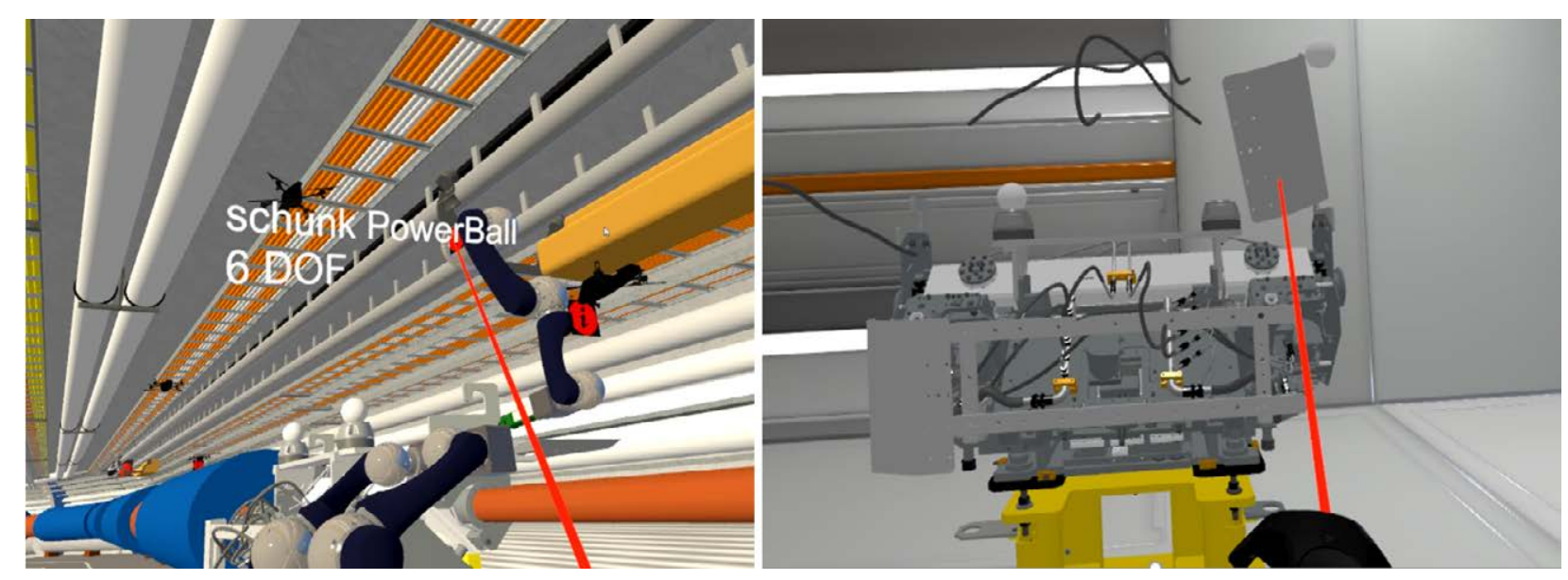

Figure 5.30: Example of laser in virtual environment

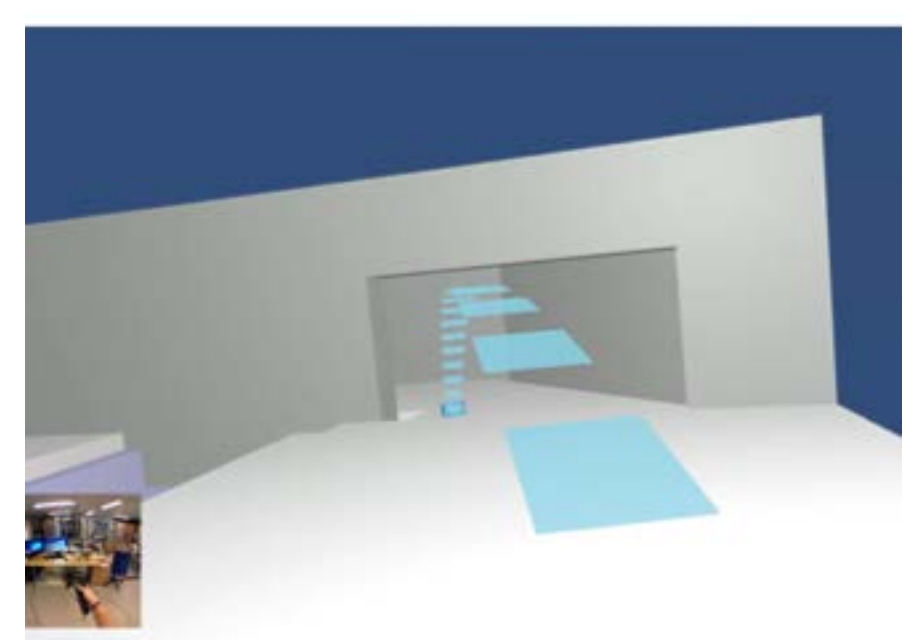

Figure 5.31: Example of selecting a navigation point.

The most common actions are screw/unscrew and cut objects as pipes. For these actions, two tools are created in order to interact with the environment (see Figure 5.32). Both tools are animated as a real one. Furthermore, sound effect and haptic feedback are added to improve the realism.

Each tools is attached by its scripts for animation controlling or action performing. With the purpose to just drag these modules and use, it is necessary to set up some parameters in the virtual environment, tag, collider and Rigidbody component.

A tag [393] is a reference word which can be assigned to one or more objects of the virtual environment. It is very useful to identify or to call objects for scripting purposes. For the case of driller, it is important to set the tag of the 3D models as "screw". In this way, these objects with the tag will be distinguished from the rest components of the scene. The same way, tag "cut" should be added to all the objects that can be cut. 


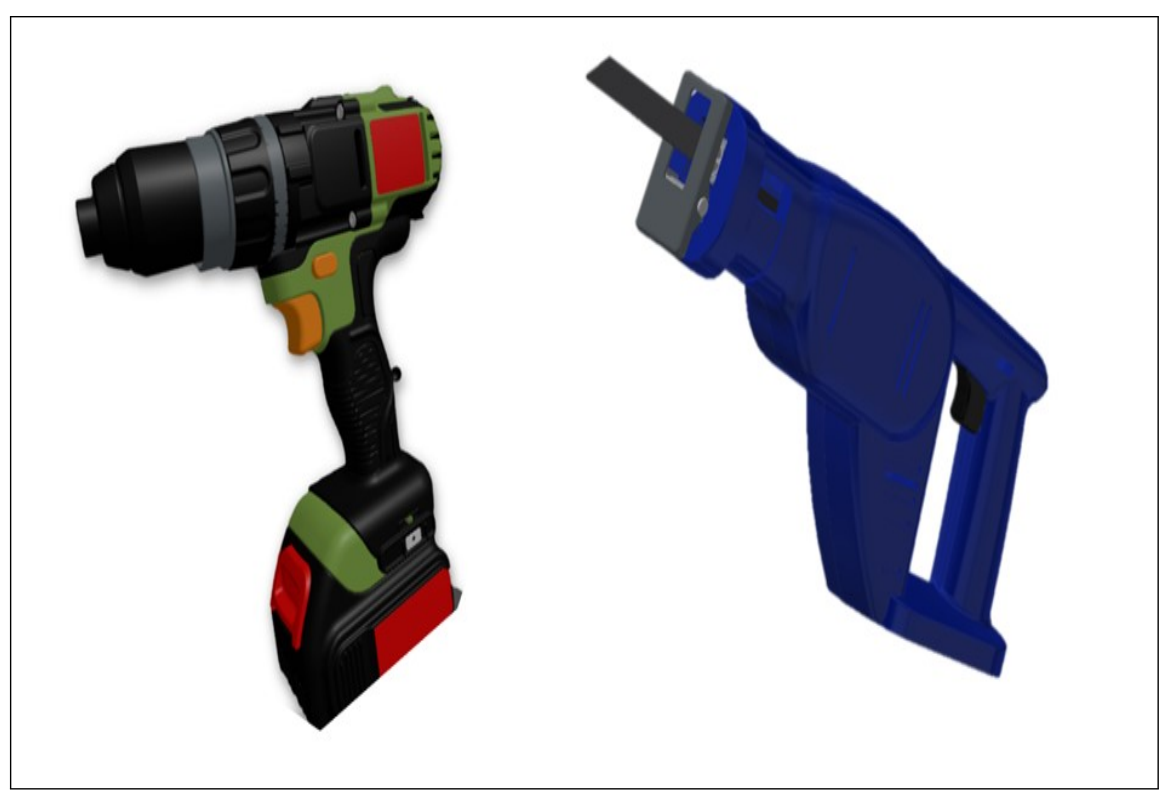

Figure 5.32: Electrical driller (left) and electrical handsaw (right) models imported to Unity

A collider [394] is an invisible mesh which define the shape of an object for physical collisions purposes. This invisible shape does not need to be exactly the same shape as the object; normally an approximation is more efficient in computation time. There are three primitive collider types, Box Collider, Sphere Collider and Capsule Collider as shown in Figure 5.33. These simplest colliders are less processor intensive because of the number of vertices of the mesh, making the colliders less complex. If more precision is needed, then mesh collider it is more appropriate (see Figure 5.34).

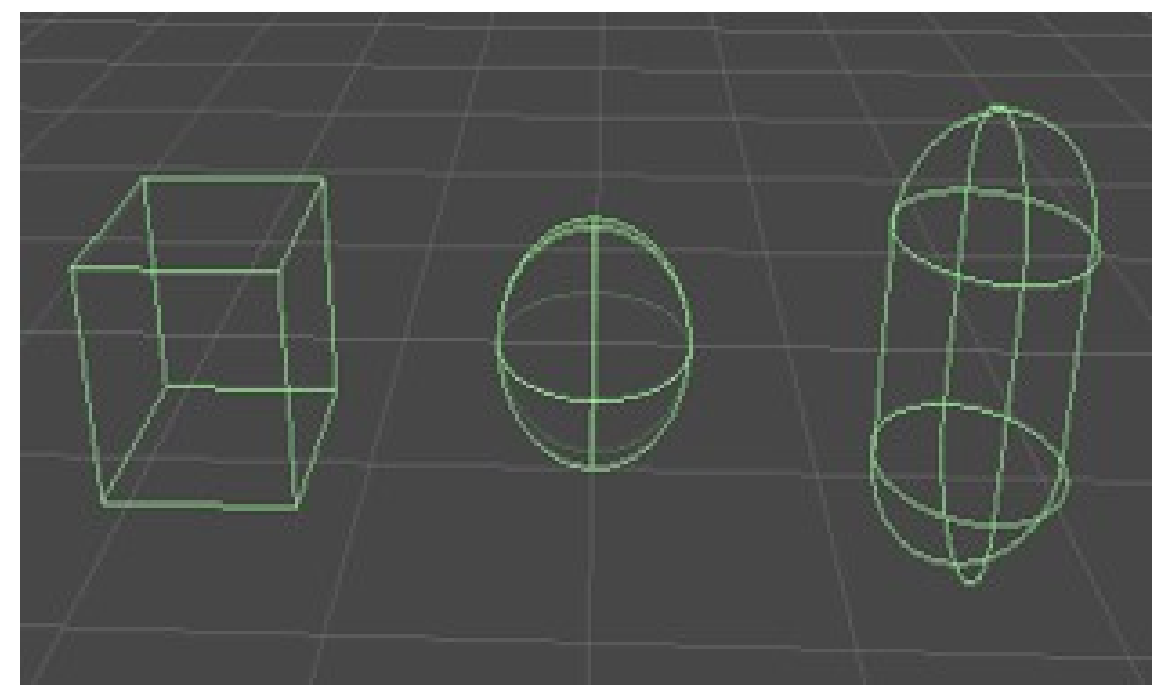

Figure 5.33: Primitive collider. From left to right, box collider, sphere collider and capsule collider 


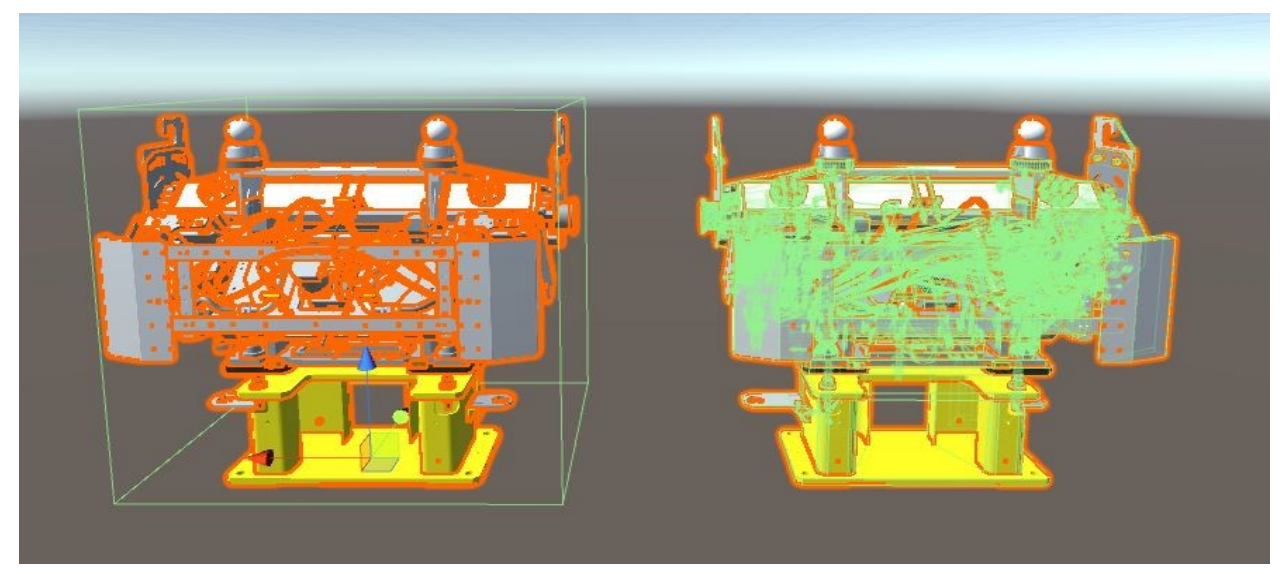

Figure 5.34: Comparison between box collider with mesh collider

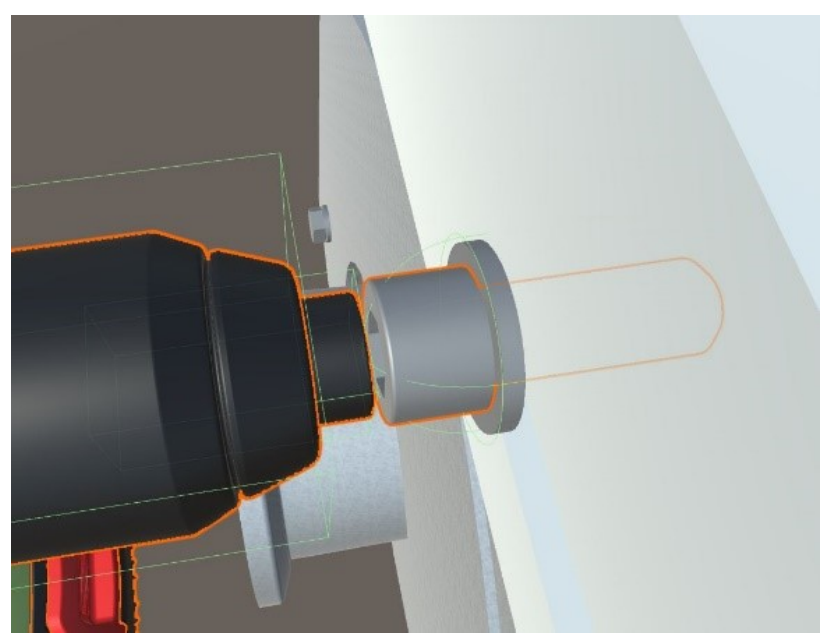

Figure 5.35: Box collider added to the end of the driller and capsule collider added to the screw

By adding rigid body (known as rigid-body [395]) to an object, it is possible to make the object acts under physics control. This property can receive forces and torques to make the object move in a realistic way, such as gravity influence. In this case, it is very important the mass, the option gravity and is Kinematic. The mass by default it is defined in kilograms. Enabling the gravity option, the object will be affected by the gravity. For example, when the screw is not hold, it will fall. The last one, Is Kinematic, if it is enableed, the physic engine will not drive the object, and it needs to be manipulated by its Transform via scripting. Another module what should be highlighted it is the danger estimation module. It is a fundamental module for planning operations in given environments. With this module, it is possible to do a risk analysis of an operation and review the plan or changes.

This system uses an animated human body in the virtual environment to make the movement more real and improve the currency of estimation. To calculate the radiation 
dose received by the human, the radiation map provided by FLUKA [396] is incorporated. This radiation map is showed in virtual environment using different colours. Each colour represent an amount of radiation dose. Moreover, the system can show three important values, the current radiation dose that is receiving the operator, the accumulated dose received since the operation started and a timer to know how long the operation will take (see Figure 5.36).
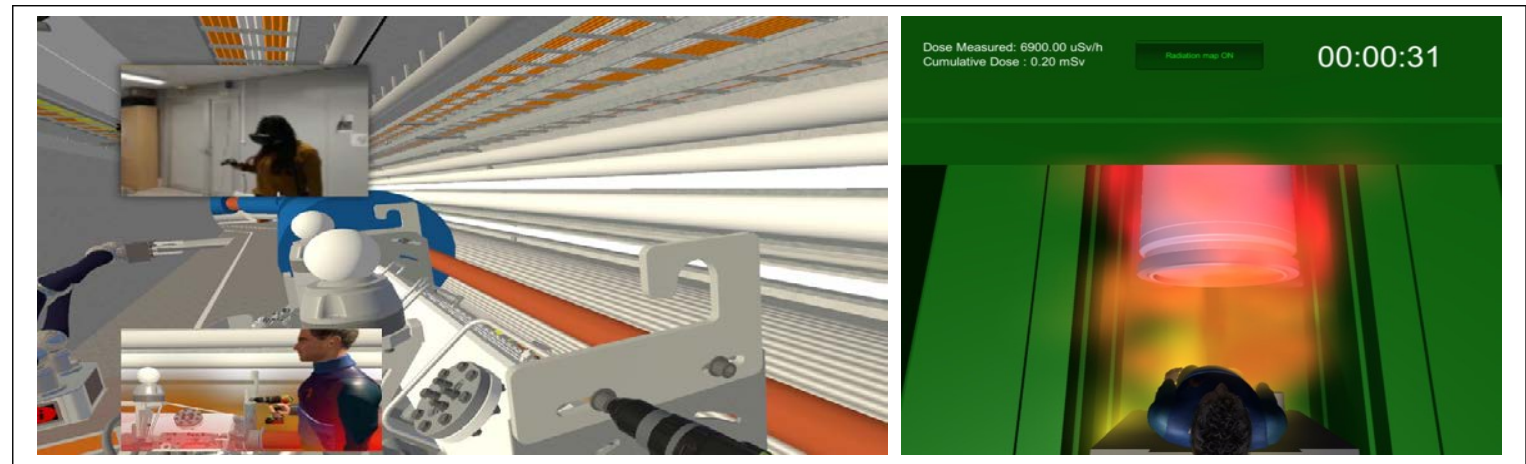

Figure 5.36: Operator tanning in simulated LHC tunnel; arm movement tracking incorporated and reproduced in the animated human body model (Left). Danger estimation system incorporated in TDE maintenance planning; radiation map and animated body are incorporated (Right).

With the purpose of operator training with the robots, also module of robot controller and master - slave training are developed (see in Figure 5.37). Through the module of the robot controller, the operator can move the robotic arm join by join and move the robot base around the scene. In this way, it is possible to simulate the operation more realistic and check if the robot will reach the target. In addition, with this tool it is able to analyze the viability of the robot in small area. For the same reason, the master-slave simulation in virtual reality helps the operator to get confidence with the operation and the environment among the others functions.
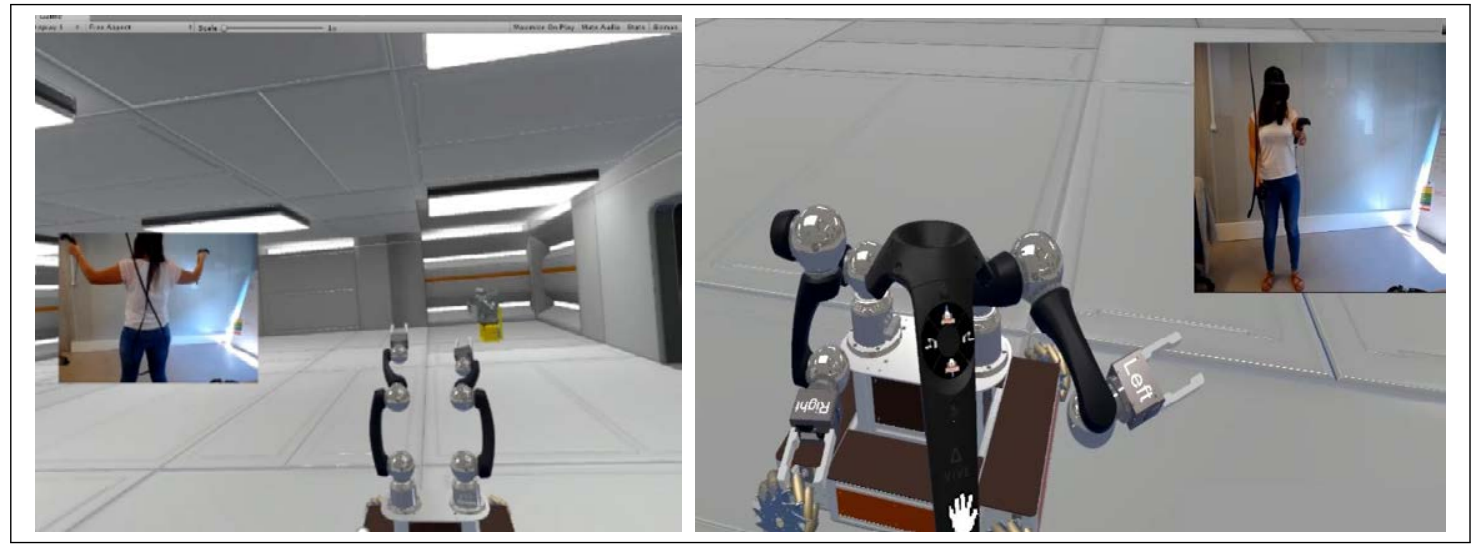

Figure 5.37: Module master-slave (left). Module robot controller (Right). 


\subsection{Comparative Analysis and System Usability Tests}

The overall framework has been validated several times in the latest year through real interventions. Figure 5.38 shows the number of interventions performed from September 2014 until January 2019. The vertical red line indicates the beginning of the development and application of the CERNTAURO framework, with the first intervention performed with CERNBot. It is clearly visible the inversion of tendency for which more and more interventions have been performed. Although difficult to measure through mathematical models, the difficulty of the interventions has increased as well, passing from simple visual inspection tasks to real manipulation intervention with a deep interaction with the environment (see Figure 5.39).

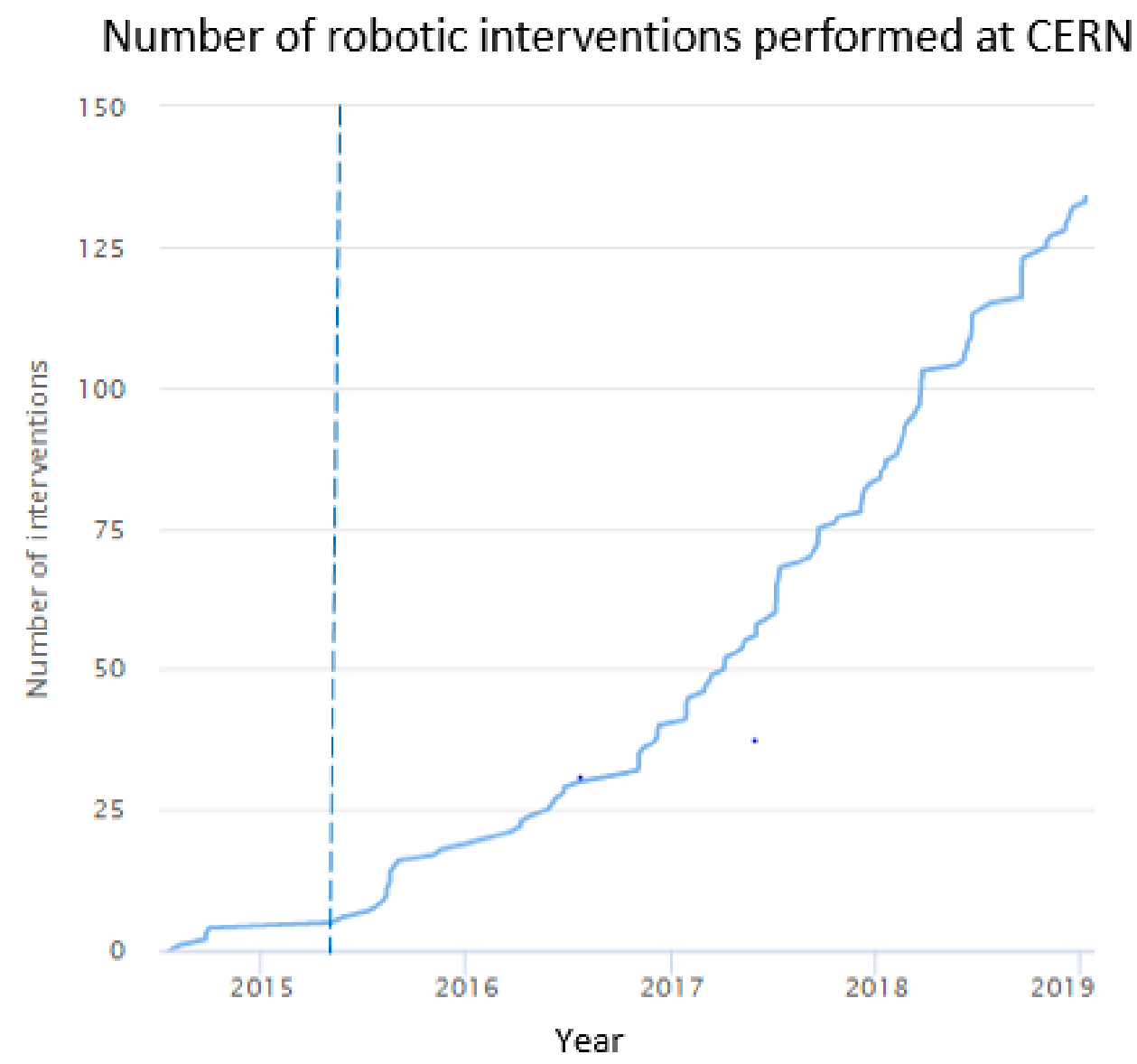

Figure 5.38: Number of interventions performed from September 2014 until January 2019. The dashed line represents the period since when CERNTAURO solutions were applied.

Radiation surveys play a central role during robotic interventions in CERN accelerators complex, allowing accurate planning through the virtual reality framework, training, and decisions between human intervention and robot interventions. This allows also to estimate 
Training and tests: $4.1 \%$

Radiation survey: $34.5 \%$

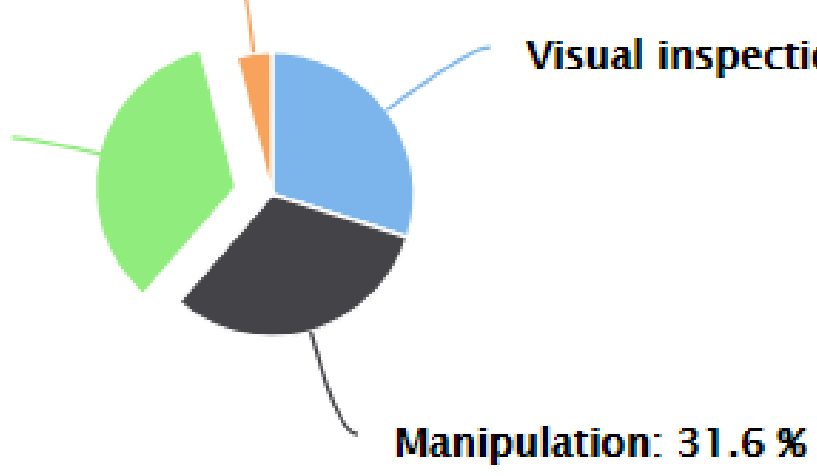

Figure 5.39: Types of interventions performed from September 2014 until January 2019.

the dose saved to humans during the intervention when it is performed using robots (see Figure 5.40). The risk of exposure to radiation of an operator could be computed simulating the human operation in the VERO framework using real radiation values collected (if available) or simulated.

Figure 5.41 shows the distribution of the usage of the various robots for the interventions. It is noticeable how the CERNTAURO robots are used for almost $60 \%$ of the total interventions. Furthermore, this percentage is destined to raise as industrial robots, like Telemax, were was mainly used during the first year and a half of interventions before the construction of CERNBot. However, Teodor, with its huge payload, is still of primary need for all those interventions in which great strength is needed.

Additional consideration should be done; custom CERN made robots allowed operation which could not be performed with standard commercial robots (such as vacuum sniffing, metal cutting, resin pouring etc.) as the integration of additional sensors and actuators is not possible. Furthermore, the use of $4 \mathrm{G}$ as a communication channel allowed CERN robots to arrive in locations which were not possible before due to the range limitation of standard radio channels of commercial robots.

In order to demonstrate the effective user-friendliness of the system developed, several tests have been carried out. The validation phase comprises two main experiments:

\section{Telemanipulation experiment Nr. 1}

This experiments consists of 2 tasks:

1. The first one consists in approaching and grasping an object inside a box and moving it into another box. 


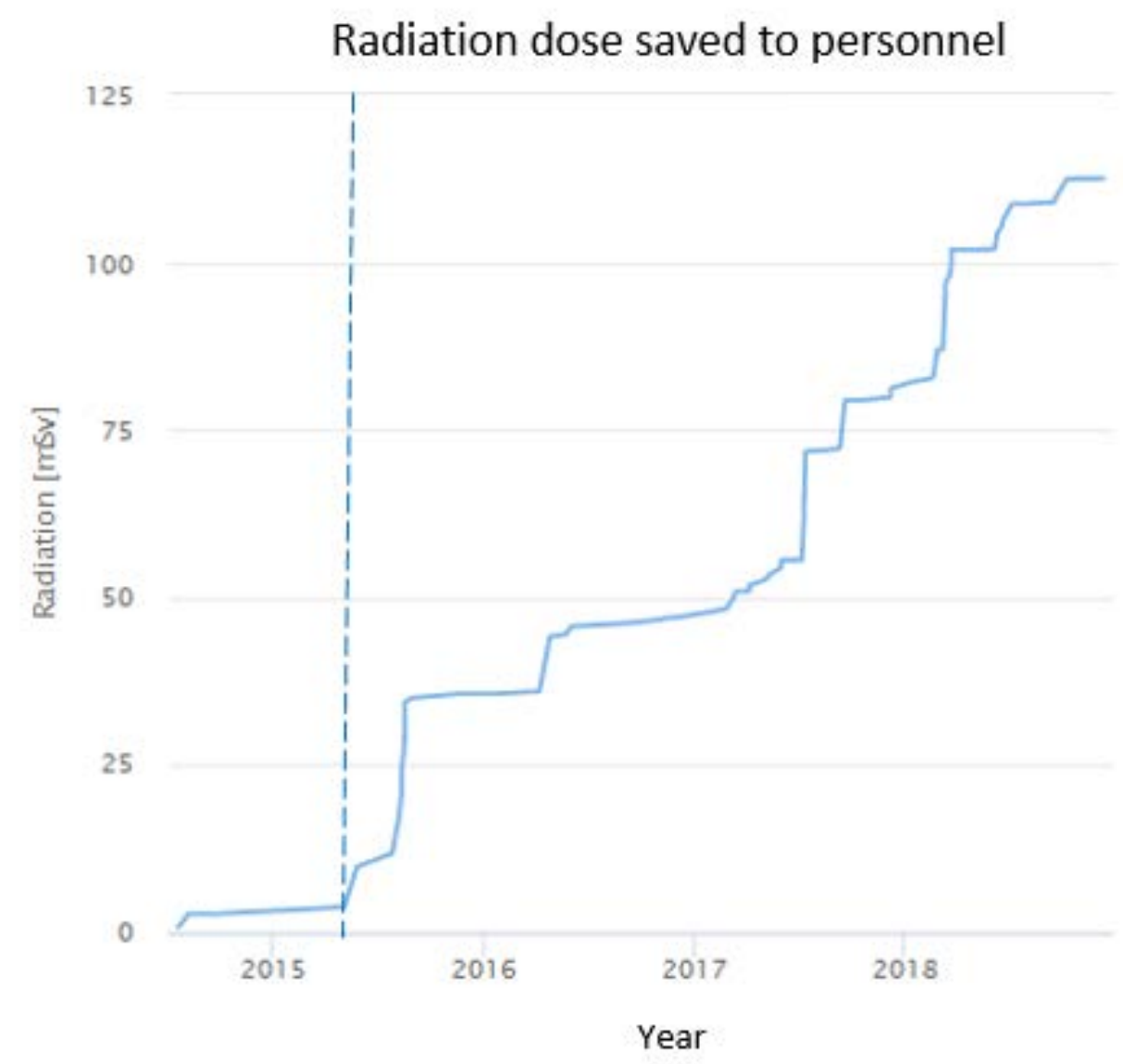

Figure 5.40: Dose saved to personnel from September 2014 until January 2019. The dashed line represents the period since when CERNTAURO solutions were applied.

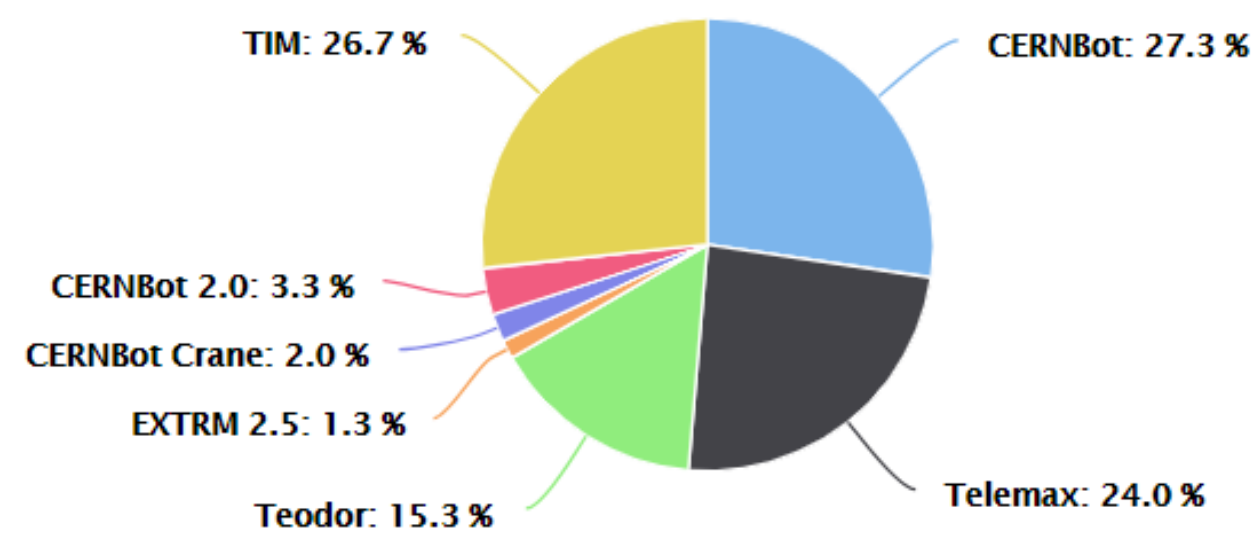

Figure 5.41: Robots used for interventions from September 2014 until January 2019. 


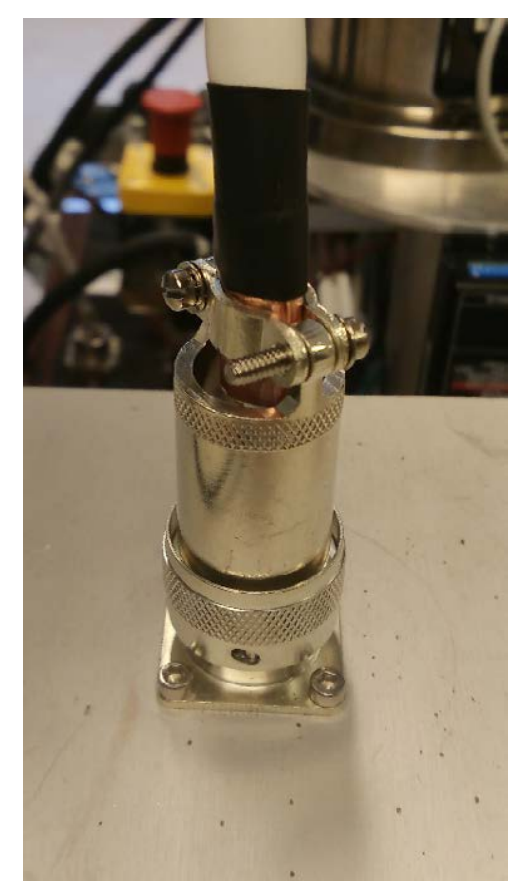

Figure 5.42: A burndy connector used for the test

2. The second consists in an operative task of approaching, grasping and unscrewing a cylindrical electrical burndy connector (see Figure 5.42)

The actual time for the accomplishment of the tasks assigned to the un-trained operators set has been measured and the results have been compared with the ones of the expert users set. 10 operators compose a set. During the operation, the heartbeat of each operator has been monitored.

Table 5.4 and Table 5.5 show the performances achieved during the fulfilling of the first task with both systems. The untrained users, in both cases, were able to fulfil the task, without failing, but the time taken to complete the assignment with the proposed system is much lower than the time taken to complete the same task using the actual system. The same kind of performance's improvement could be noticed for the well-trained users. In particular they felt more comfortable controlling the robot manually than using a console. Moreover, using the proposed master-slave robotic system, the heartbeat increase during the operation is much lower comparing to the one monitored during the use of the industrial robotic system.

Table 5.6 and 5.7, instead, show the performances achieved during the fulfilling of the second task. This task is a real operative and more complex one which lead to a completely fail from the un-trained users using the industrial robot. Their main difficulties were not the approach, but essentially the finding of the good robotic arm configuration to grasp the object. Inevitably, this produced an increase of the heartbeat and stress conditions for the operator. With the proposed system, all of the un-trained operators were able to fulfil the assigned task with success. They declared how the force feedback has played a key role 
in positioning the robot on the best configuration for unscrew the bolt and the increase of the heartbeat is negligible. In addition, a significant time performance increase has been observed also by the trained operators in using the proposed system. This demonstrates how the proposed system could be an important added value in the field of teleoperation.

\begin{tabular}{||c|c|c||}
\hline & Un-trained users set[s] & Well-trained users set[s] \\
\hline Proposed system & $109 \pm 4$ & $52 \pm 2$ \\
\hline Robotic Industrial system & $216 \pm 32$ & $71 \pm 3$ \\
\hline
\end{tabular}

Table 5.4: Results obtained during the fulfilling of the first task

\begin{tabular}{||c|c|c||}
\hline & Un-trained users set[s] & Well-trained users set[s] \\
\hline Proposed system & $412 \pm 9$ & $114 \pm 2$ \\
\hline Robotic Industrial system & Failed & $213 \pm 31$ \\
\hline
\end{tabular}

Table 5.5: Results obtained during the fulfilling of the second task

\begin{tabular}{||c|c|c||}
\hline & Un-trained users set[s] & Well-trained users set[s] \\
\hline Proposed system & $20 \%$ & $10 \%$ \\
\hline Robotic Industrial system & $30 \%$ & $12 \%$ \\
\hline
\end{tabular}

Table 5.6: Hearthbeat percentage increase during the fulfilling of the first task

\begin{tabular}{||c|c|c||}
\hline & Un-trained users set[s] & Well-trained users set[s] \\
\hline Proposed system & $25 \%$ & $10 \%$ \\
\hline Robotic Industrial system & $50 \%$ & $15 \%$ \\
\hline
\end{tabular}

Table 5.7: Hearthbeat percentage increase during the fulfilling of the first task

\section{Telemanipulation experiment Nr. 2}

This experiments consists of approaching and grasping a male connector inside a box and screwing it on a female one (see Figure 5.43) using the master-slave teleoperation system of the proposed CERNTAURO framework.

We have compared the time for executing successfully these 2 tasks by using the Telemax commercial robotic system from Telerob, equipped with one robotivc arm, and the masterslave system of the presented work in single and dual arm configurations. Figure 5.44 shows the performance comparison between non-experts and experts operators using the Telemax commercial robotic system from Telerob. The operators were using all the Telemax cameras, including a pan-tilt-zoom type located on the base of the robot. 

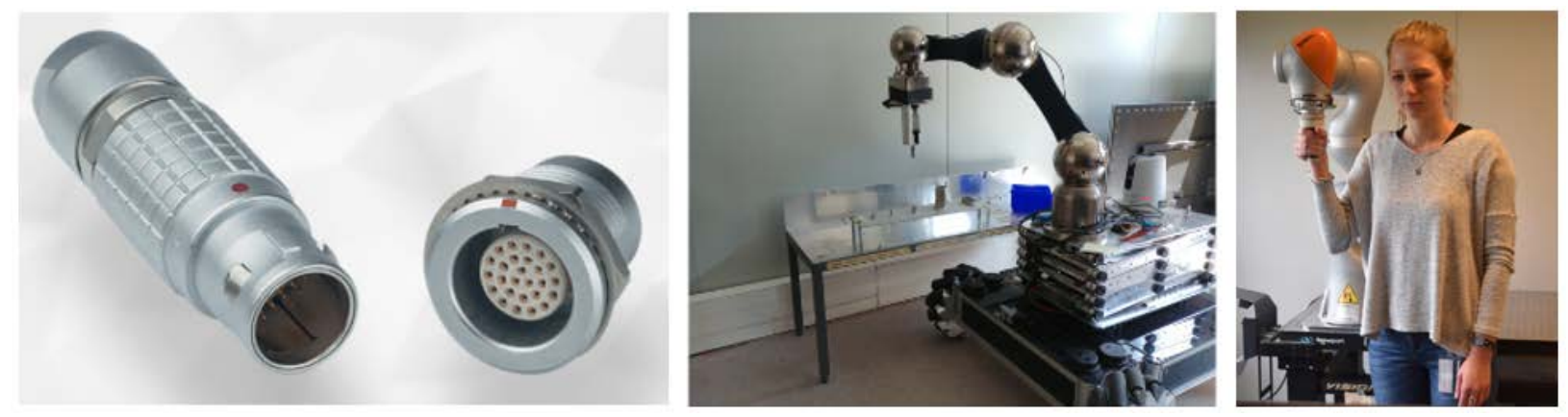

Figure 5.43: Picture of the Lemo B-type standard self-latching multipole connectors, with alignment key, used for the test (left), the connection task being performed by CERNbot in single arm configuration (center) and an operator at the master station (right).

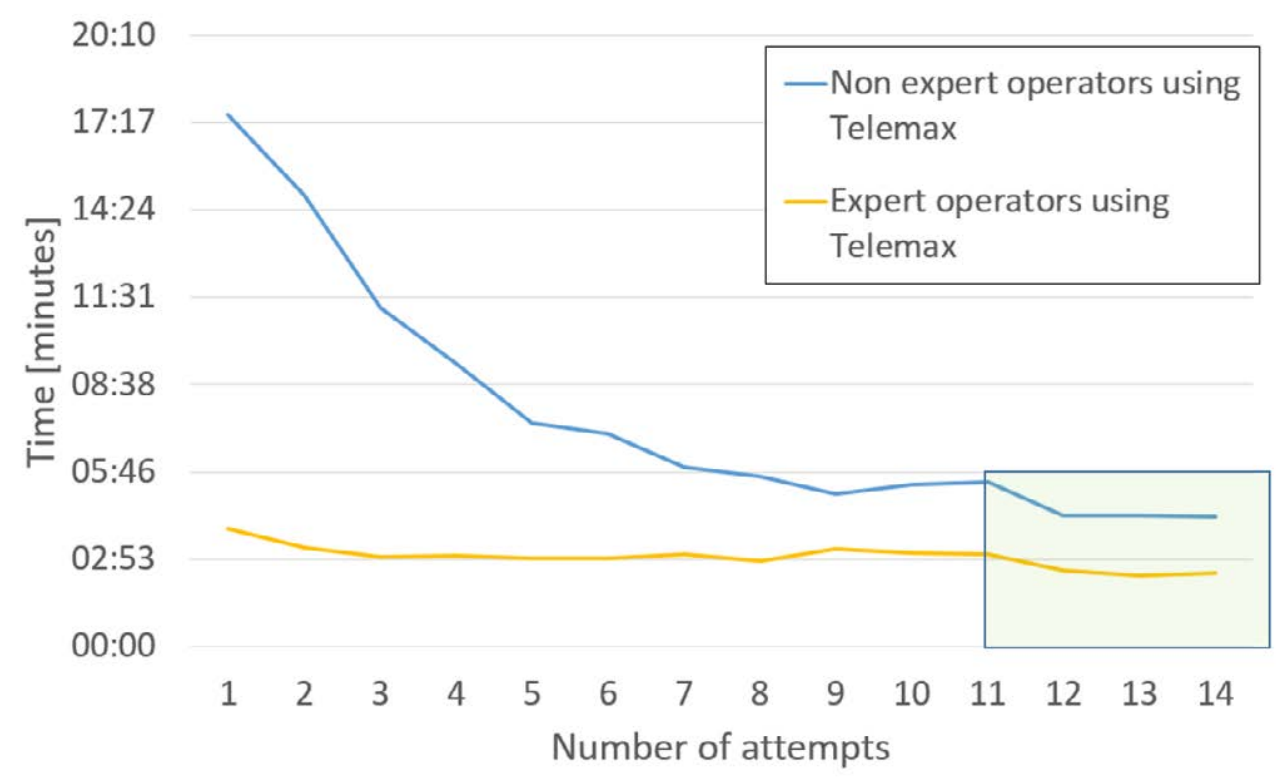

Figure 5.44: Time performance comparison between non-experts and experts operators using the Telemax robot in doing the Lemo connection task. Each attempt shown is a time average taken from 5 different operators' attempts. The highlighted final parts in green contains the tentative done with the addition of an environmental camera that helped to improve the operational time performances stepping-out from the steady-state conditions reached beforehand. 
Figure 5.45 shows the performance comparison between non-experts and experts operators using CERNBbot in single arm configuration. The operators were using an in-hand camera and a pan-tilt-zoom type located on the base of the robot (see Figure 5.43). Figure 5.46 shows a comparison in doing the same task by using Telemax and CERNbot in single arm configuration. It is evident that the user-friendliness of the proposed novel masterthesis telemanipulation system allows the operators to operate and learn faster with respect to the industrial system. In addition, even after several tests and trials, the time performance and the robustness of the task executions using the CERNTAURO system are better that the Telemax industrial system. The user friendly and usability of the CERNTAURO telemanipulation system allows non expert operators to reach similar time performances in comparison with expert operators using Telemax robot, after only few trials. Also for the specific case of CERN expert robotic operators, the CERNTAURO is an evident added value for the time performance of the robotic task.

The dual arm configuration foreseen on the CERNTAURO robots gives the possibility to use one arm equipped with a camera as an additional prospective view. The same task described above was performed also by different expert CERN robotic operators using CERNbot in dual arm configuration having one arm equipped with a camera for an additional prospective view (see Figure 5.47). In this case, the time execution has been lowered by $10 \%$. 


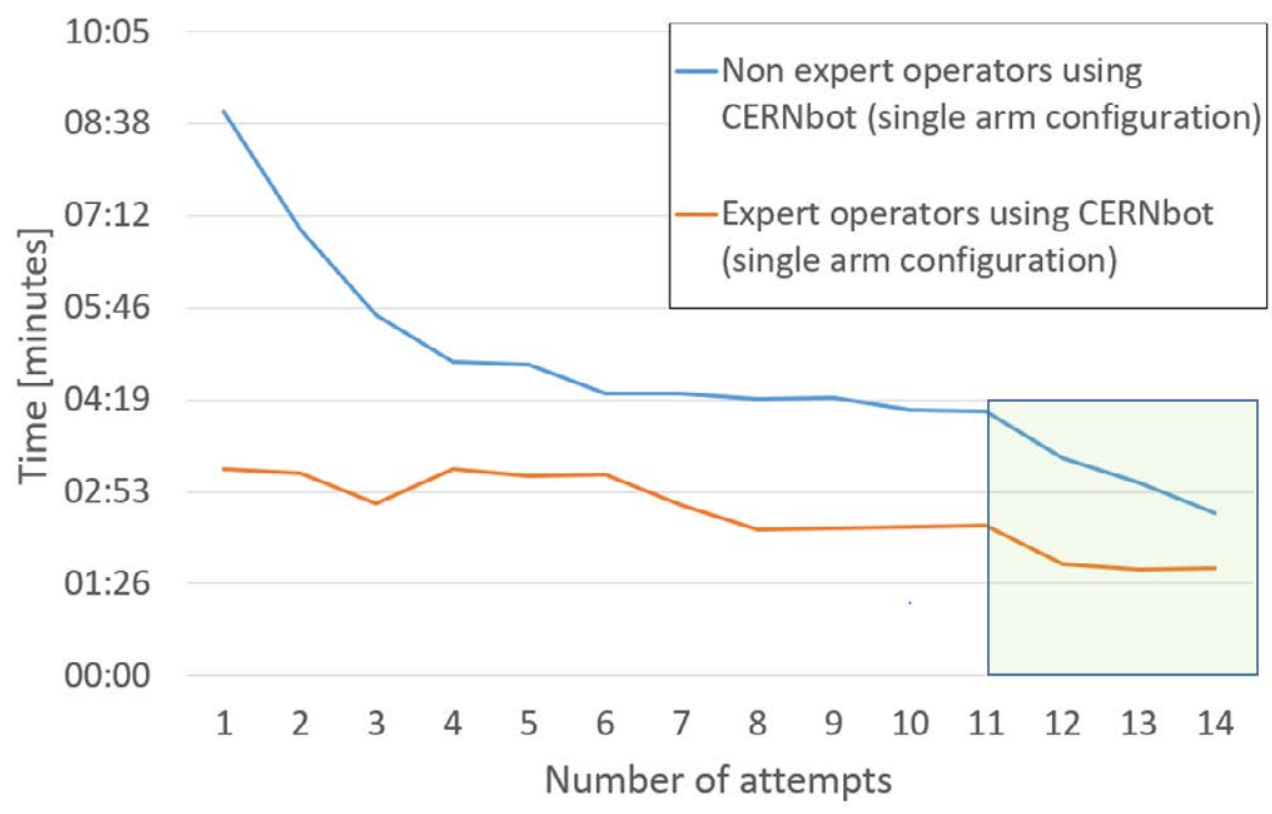

Figure 5.45: Time performance comparison between non-experts and experts operators using the CERNbot robot in doing the Lemo connection task. Each attempt shown is a time average taken from 5 different operators' attempts. The highlighted final parts in green contains the tentative done with the addition of an environmental camera that helped to improve the operational time performances stepping-out from the steady-state conditions reached beforehand. 


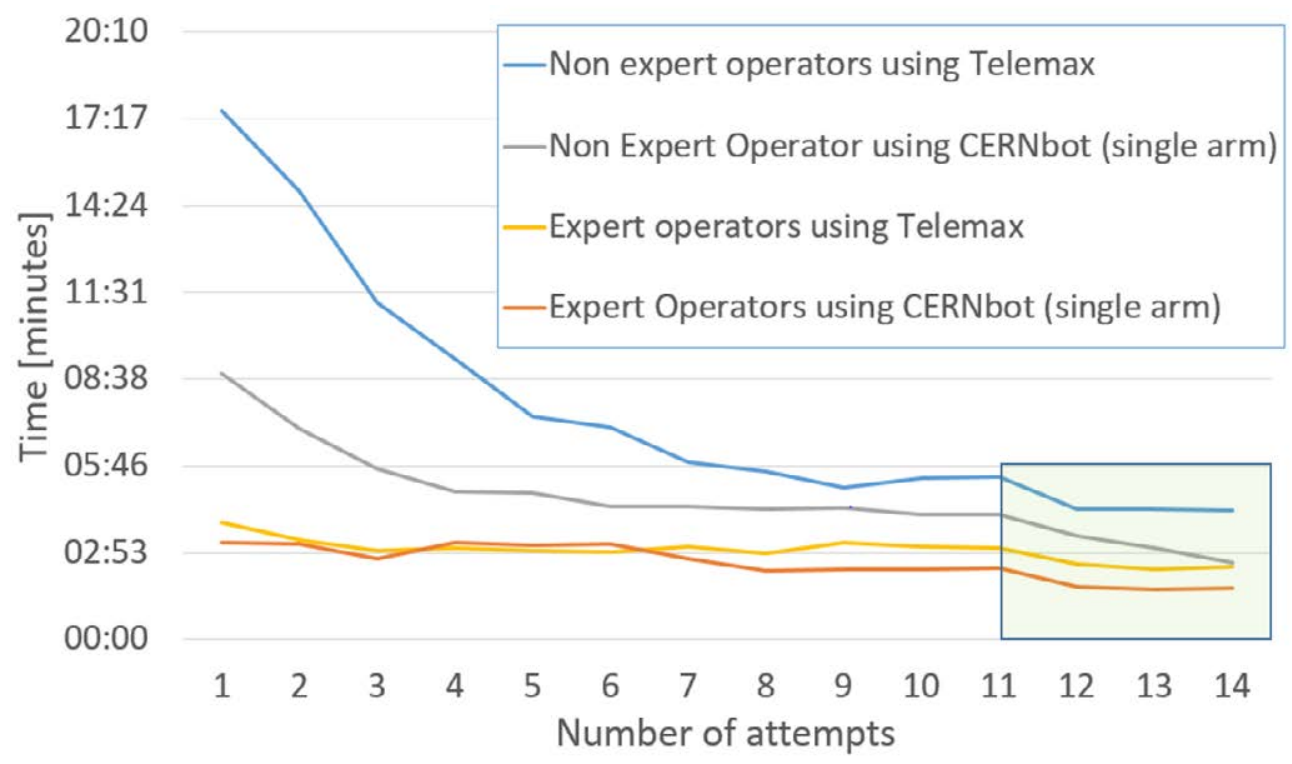

Figure 5.46: Time performance comparison between non-experts and experts operators using the Telemax and the CERNbot robot in doing the Lemo connection task. Each attempt shown is a time average taken from 5 different operators' attempts. The highlighted final parts in green contains the tentative done with the addition of an environmental camera that helped to improve the operational time performances stepping-out from the steady-state conditions reached. It is evident that the user-friendliness of the proposed novel masterthesis telemanipulation system allows the operators to operate and learn faster with respect to the Telemax industrial system. Non expert operators using the CERNTAURO system reach similar time performances in comparison with expert operators using Telemax robot, after only few trials 

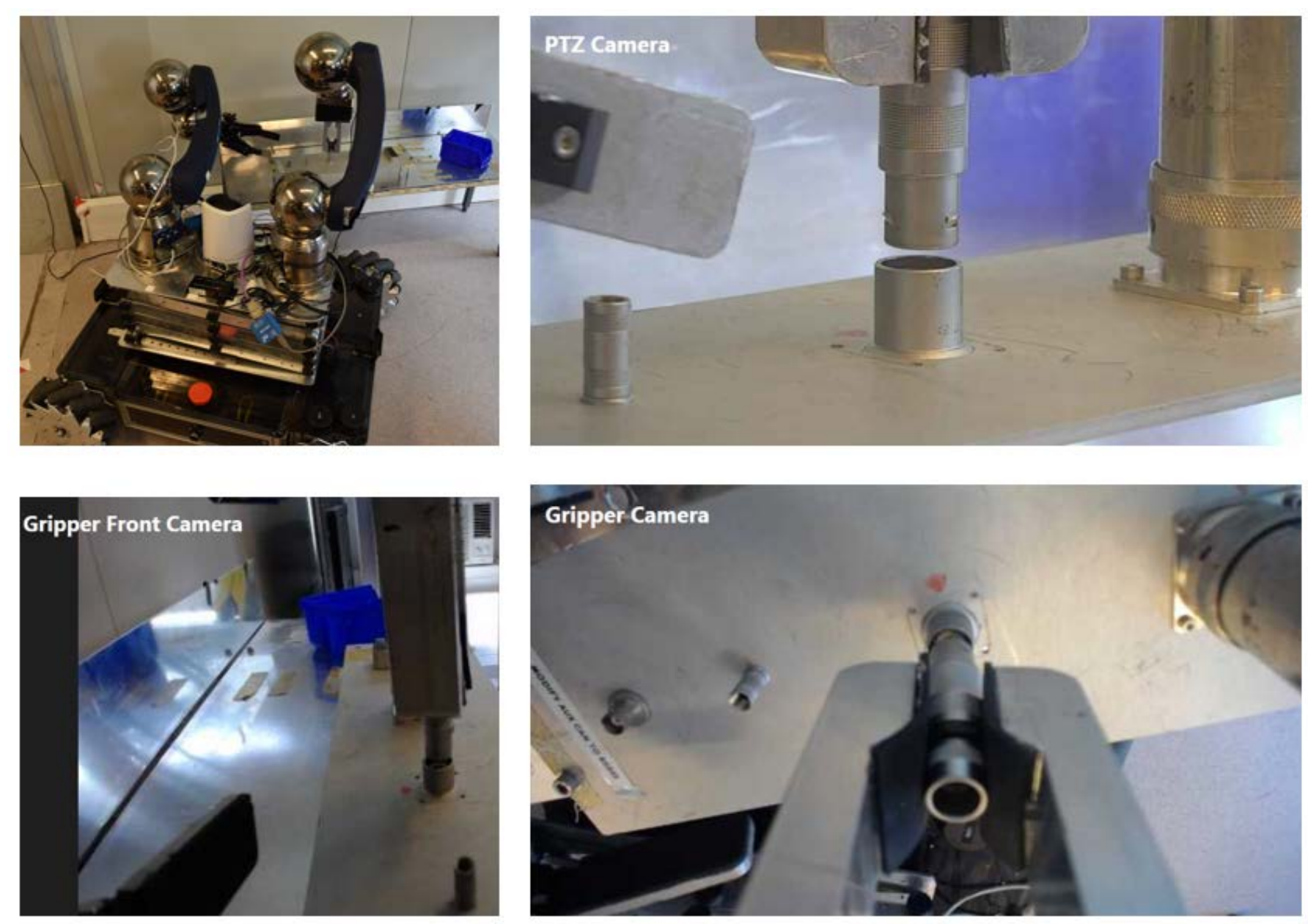

Figure 5.47: Picture of the tasks being performed by CERNbot in dual arms configuration. Overview of the scene (top left) and the different views seen by the 3 main CERNbot cameras. In-hand left arm camera (bottom left), main base pan-tilt-zoom (PTZ) camera (top right), in-hand right arm camera (bottom right). 


\section{Chapter 6}

\section{Results and System validation}

\subsection{Autonomous Navigation and Inspections in CERN Accelerators Area}

The autonomous navigation framework has been validated in different CERN's restricted areas. The goal of such tests was to evaluate the accuracy of the localization and mapping system, as well as to evaluate the performance of the autonomous navigation method. A first test has been done for mapping an unstructured environment with high accuracy in order to produce the best occupancy grid map possible. For this test, CERNBot 1.0 has been used equipped with omnidirectional wheels, a 2D LIDAR and 3-axis AHRS (Attitude and Heading Reference System) and a radiation sensor. CERNBot autonomously explored the environment (see Figure 6.1) without hitting any object and producing a high quality occupancy grid map which can be used afterwards for environmental study. The resulting occupancy grid map is shown in Figure 6.2.

After the mapping of the environment finished, the robot has been asked to approach the most radioactive object found in the area. Using the map built so far, containing as well the radiation values collected, $\mathrm{RRT}^{*}$ is applied in order to compute the path to object (see Figure 6.3).

\subsection{Tracking-based Depth Estimation System}

The depth estimation module performance was tested in real robotic interventions managing a high robust results, providing depth estimation and tracking of key-points correlation in real time. The system is integrated in a human-supervised user interface, and achieves more than $90 \%$ of accuracy in the depth estimation measurements (see Figure 6.4), with an error of less than one centimeter.

In Figure 6.5, the behaviour of the proposed algorithm is shown. The algorithm uses as basis the SURF and KCF estimators, which differ in terms of stability (i.e. percentage of frames that calculate successfully the depth), although each solution provides an accuracy 


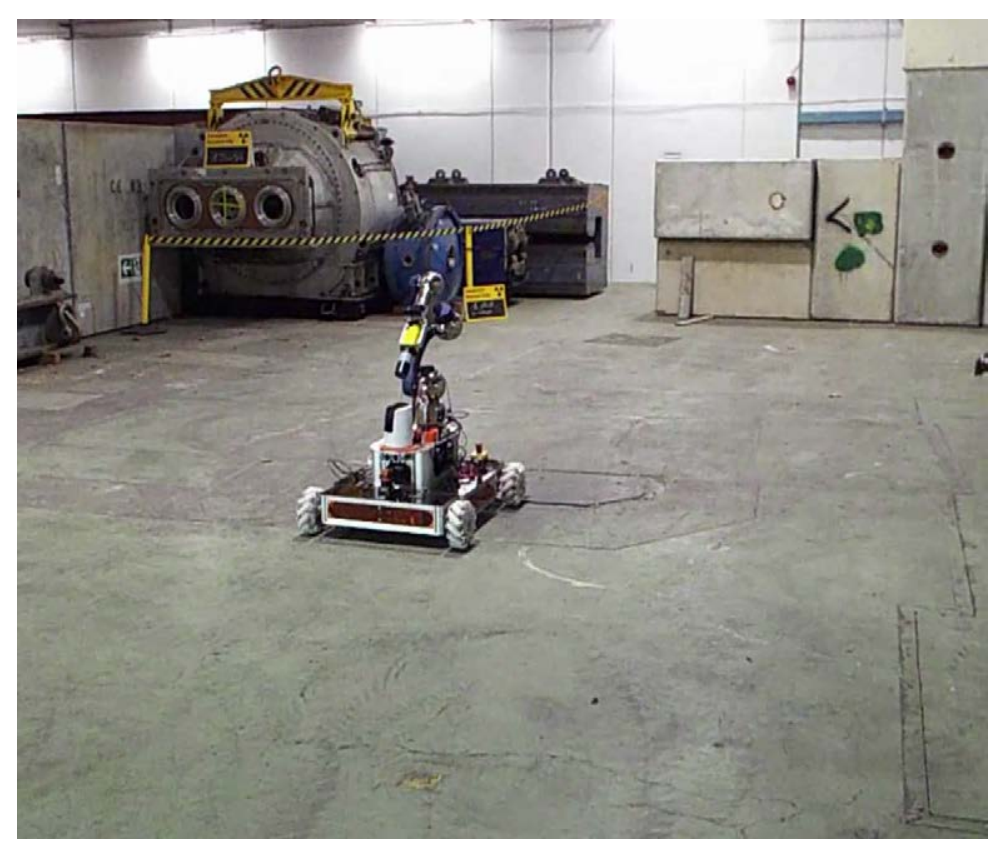

Figure 6.1: CERNBot 1.0 while navigating autonomously in the environment

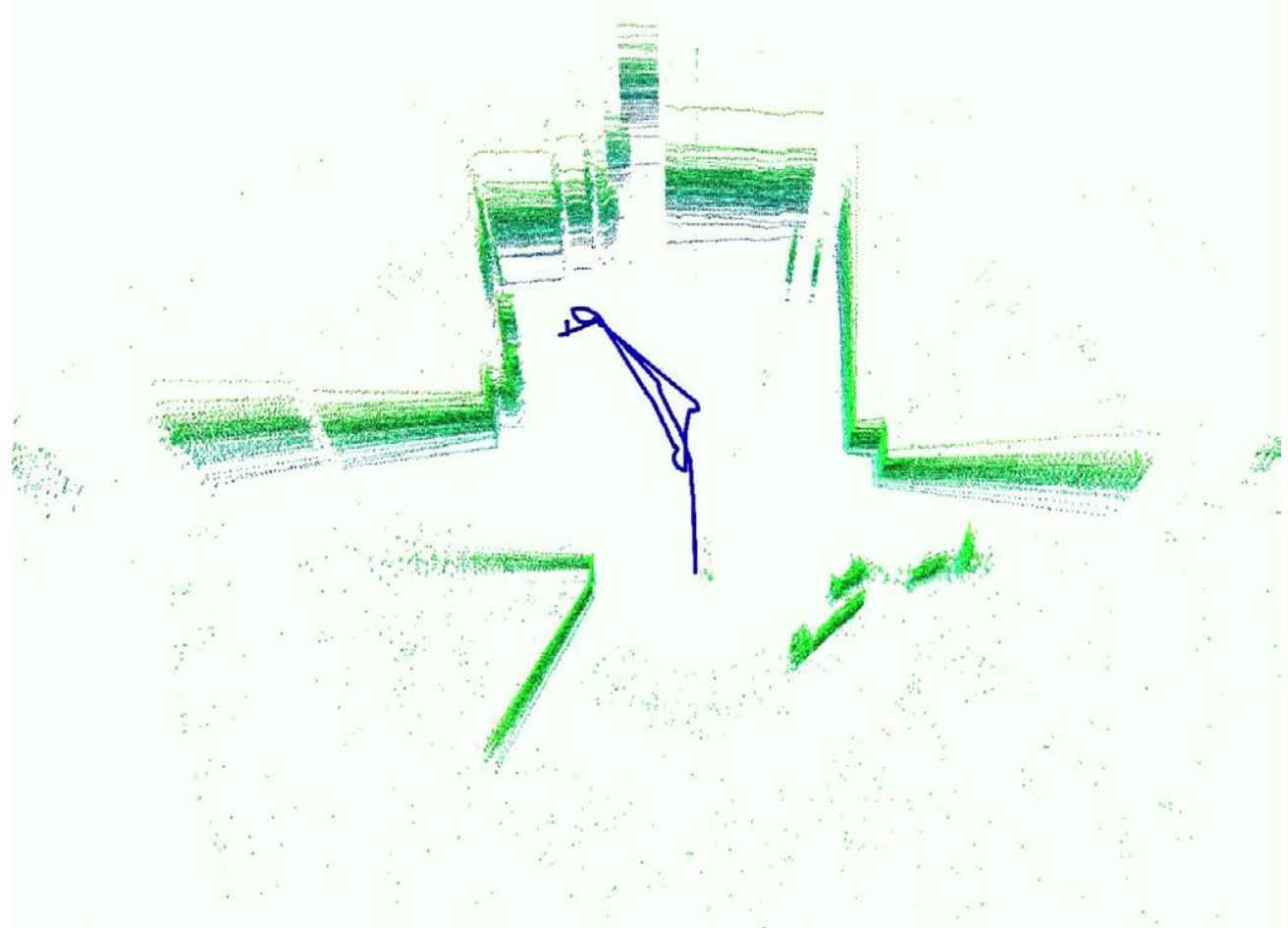

Figure 6.2: The occupancy grid map of the reconstructed environment 


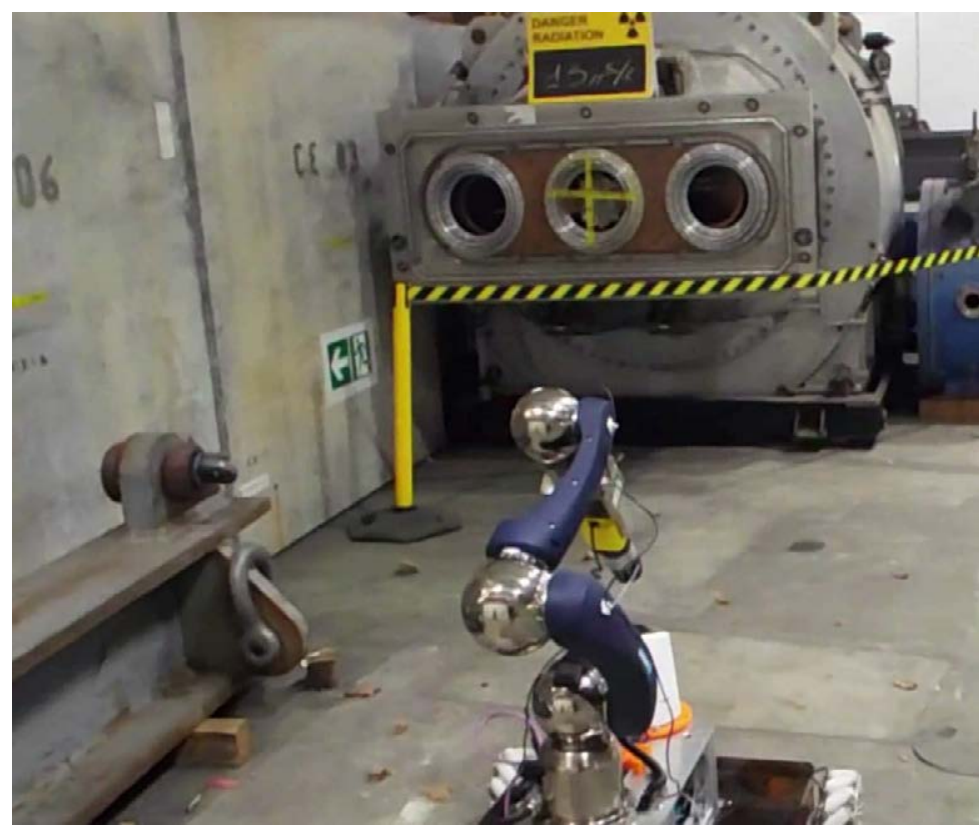

Figure 6.3: CERNBot 1.0 while approaching the radioactive object discovered in the area

error below one centimeter (see TABLE 6.1), the KCF-based gives a stability over $94 \%$.

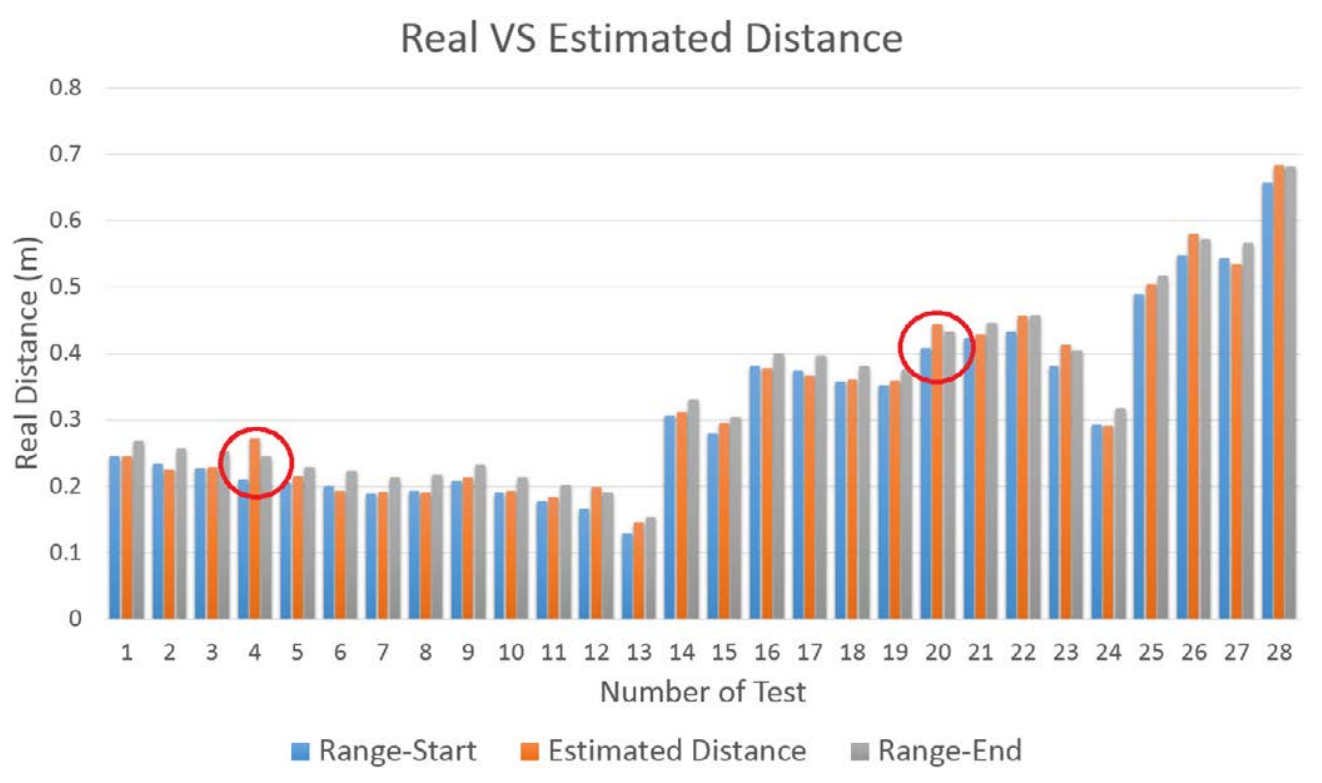

Figure 6.4: Accuracy test. Blue and gray columns are the range of the real distance and orange one the estimation. Red circles show the two errors obtained over $1 \mathrm{~cm}$. but always under $2 \mathrm{~cm}$. 


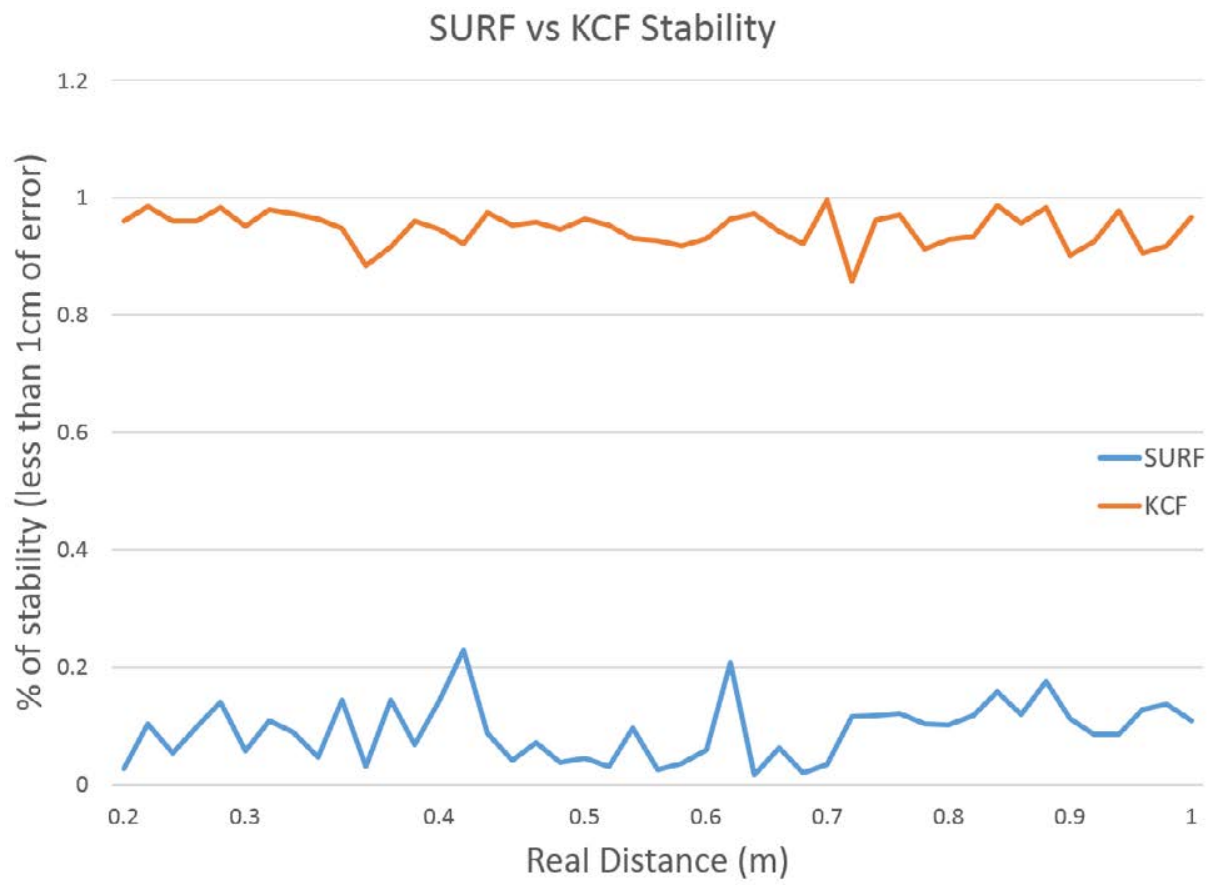

Figure 6.5: Stability comparative between SURF and KCF based solutions proposed: X axis shows the distance to the target, and $\mathrm{Y}$ axis represents the percentage of frames that provide the correct measurement.

Table 6.1: Accuracy and stability of SURF and KCF based solutions

\begin{tabular}{|l|l|l|l|l|}
\hline $\begin{array}{l}\text { Real } \\
\text { Dist. }\end{array}$ & SURF-Error & KCF-Error & $\begin{array}{l}\text { SURF- } \\
\text { Stability-\% }\end{array}$ & KCF-Stability-\% \\
\hline $\mathbf{0 . 2 0} \mathbf{m .}$ & 0.0022346 & 0.0047224 & 0.08395766 & 0.970001464 \\
\hline $\mathbf{0 . 3 0} \mathbf{m .}$ & 0.0046036 & 0.0043484 & 0.08859312 & 0.963883933 \\
\hline $\mathbf{0 . 4 0} \mathbf{m}$. & 0.0020228 & 0.0055516 & 0.12287338 & 0.92588872 \\
\hline $\mathbf{0 . 5 0} \mathbf{m .}$ & 0.0038266 & 0.0033858 & 0.05564268 & 0.959756087 \\
\hline $\mathbf{0 . 6 0} \mathbf{m}$. & 0.0033424 & 0.0044288 & 0.04943245 & 0.932125796 \\
\hline $\mathbf{0 . 7 0} \mathbf{m}$. & 0.003555 & 0.0051822 & 0.06802373 & 0.959685824 \\
\hline $\mathbf{0 . 8 0} \mathbf{m .}$ & 0.0041976 & 0.0060142 & 0.11116344 & 0.926650095 \\
\hline $\mathbf{0 . 9 0} \mathbf{m .}$ & 0.002221 & 0.0045714 & 0.1359251 & 0.952898557 \\
\hline $\mathbf{1 . 0 0} \mathbf{m .}$ & 0.0020276 & 0.0026512 & 0.10816896 & 0.939239064 \\
\hline Average & 0.00311458 & 0.0045396 & 0.09153117 & 0.947792171 \\
\hline
\end{tabular}


To supply the best data possible to the triangulation, a set of Euclidean-distance-based thresholds have been established, avoiding the wrong behavior of the squares, removing the outliers key-points to not disturbing the estimation. Once the triangulation is done, a normal distribution is used to estimate the depth under an error previously established $(5 \%$ by default) by the operator. Thus, a robust solution has been managed, using a HOG-based tracking algorithm, to depth estimation, with a fixed set of key-points, invariant to rotations [397] and partial occlusions, and providing high accuracy and stability.

Besides this, the algorithm keeps a fast performance, endowing maximum freedom movement to the robotic arm, translating and rotating the camera either in $\mathrm{X}$ and/or $\mathrm{Y}$ axis. Due to this, sometimes translation has to be increased in order to compensate the rotation done, since the rotation can be excessive for the triangulation, keeping the key-points outside of the scene.

In Figure 6.6 the relationship between the rotation and the translation in the proposed system is presented.

Unlike the stereo systems performance, the distance estimated is regarding to the current position of the camera in the world, instead of the center of the system created by both images. Therefore, it gives an valid estimation in the current moment.

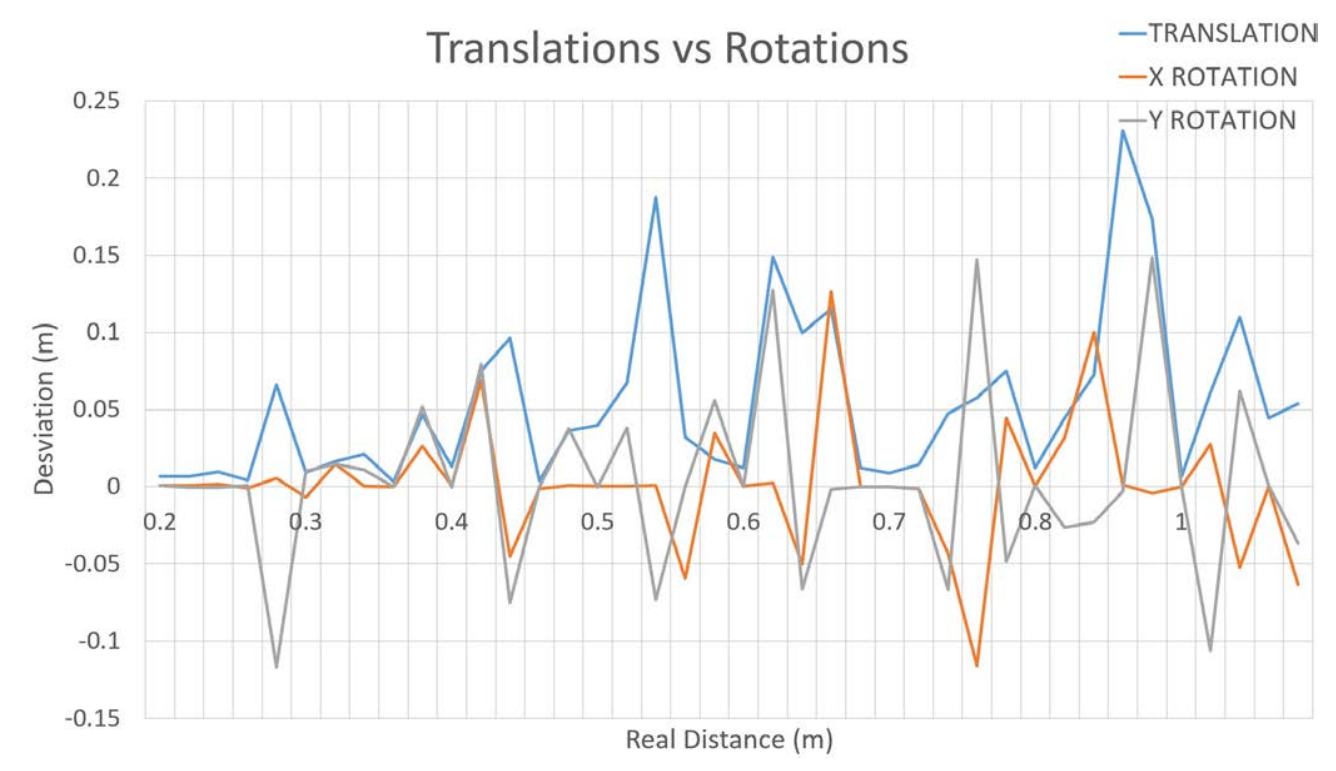

Figure 6.6: Relationship among translation and rotation to reach triangulation.

\subsection{Structural Monitoring of Tunnel Areas}

While the new structure to hold the camera on the robotic arm extending from the TIM is still a work in progress at CERN, the algorithms discussed in this paper were tested using the same mirror-less interchangeable lens camera to be used on the TIM but placed on a tripod. For each capture, the tripod was placed approximately at the same distance from the wall as the TIM so as to mimic the operational scenario. The distance $d$ of the camera 
from the wall, which would eventually be measured automatically using a laser distance meter, was noted manually. The value of $d$ had a range of 1-1.2 $\mathrm{m}$ over all the testing datasets. The spacing $x$ between each capture position was noted as the distance along the tunnel which ultimately would be measured by the TIM's position encoder.

Datasets have varying image content such as grooves on the wall, fire extinguishers, markings etc. To mimic changes, both pen/pencil markings and real scratches of varying dimensions were made on the walls. Table 6.2 lists the different aspects of each dataset.

\begin{tabular}{|c|c|c|c|c|c|c|c|}
\hline \multirow{2}{*}{ Set } & \multicolumn{3}{|c|}{ Exposure Settings } & \multicolumn{2}{c|}{ Change Type } & \multirow{2}{*}{$\begin{array}{c}\text { Number } \\
\text { of images }\end{array}$} \\
\hline & A & S(s) & ISO & P & M & S & of \\
\hline 1 & $\mathrm{f} / 4.5$ & $1 / 60$ & 1600 & & $\checkmark$ & $\checkmark$ & 51 \\
\hline 2 & $\mathrm{f} / 4.5$ & $1 / 100$ & 1600 & & $\checkmark$ & & 15 \\
\hline 3 & $\mathrm{f} / 4.5$ & $1 / 60$ & 1600 & $\checkmark$ & & $\checkmark$ & 47 \\
\hline
\end{tabular}

Table 6.2: Dataset information, including camera exposure settings: A-Aperture, S-Shutter speed and ISO and the type of the manually created change: P-Pencil, M-Marker, SScratches

For each testing dataset, three sets of images were captured. Primarily, a REFERENCE $(R)$ set of reference images were shot. Afterwards, changes on the wall involving scratches and markings to mimic cracks were made. Alongside each 'change', a piece of tape was placed on the wall to obtain the GROUND TRUTH $(G T)$ set. Subsequently, the tape from the wall was removed and the final SURVEY $(S)$ set of images was taken. Images from $S$ were then compared to images in $R$ for change detection. The sizes of the changes required to be detected are set through the configuration file.

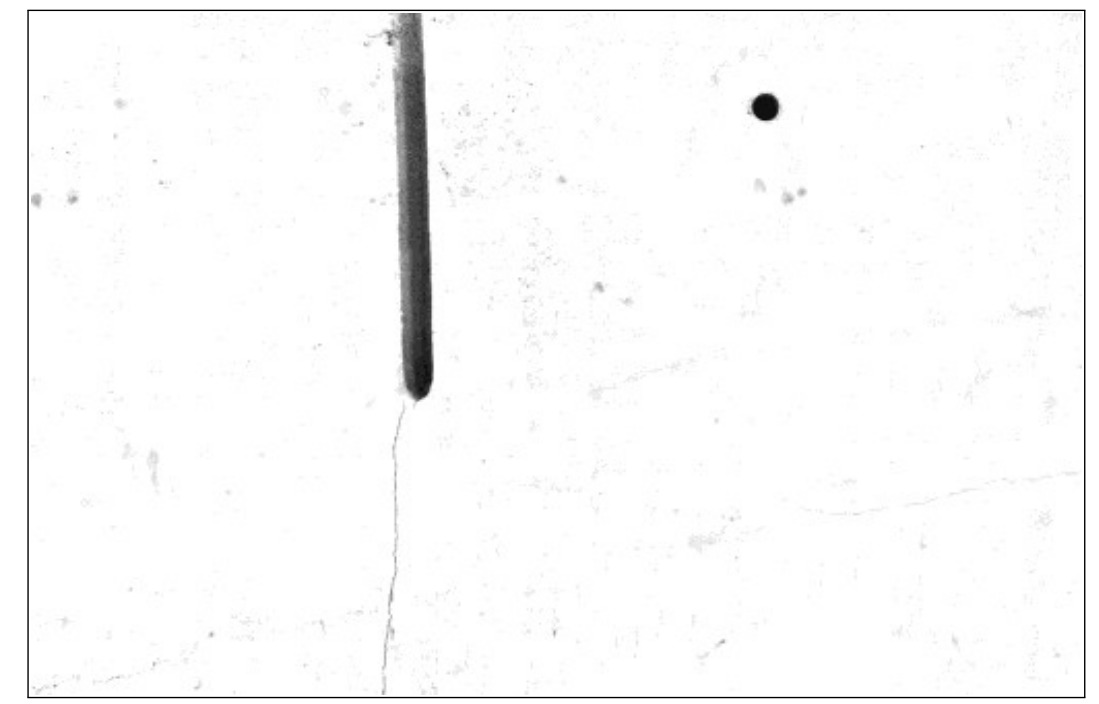

Figure 6.7: A sample image from the Query dataset at time $t_{1}$ 


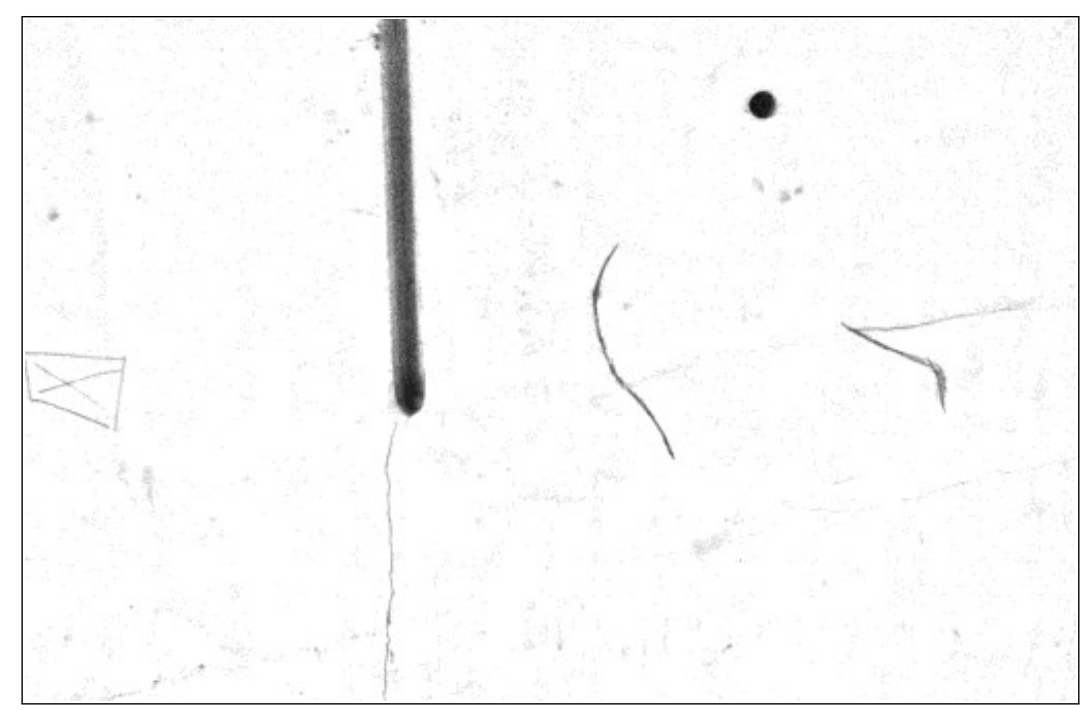

Figure 6.8: A sample image from the Survey dataset at time $t_{2}$

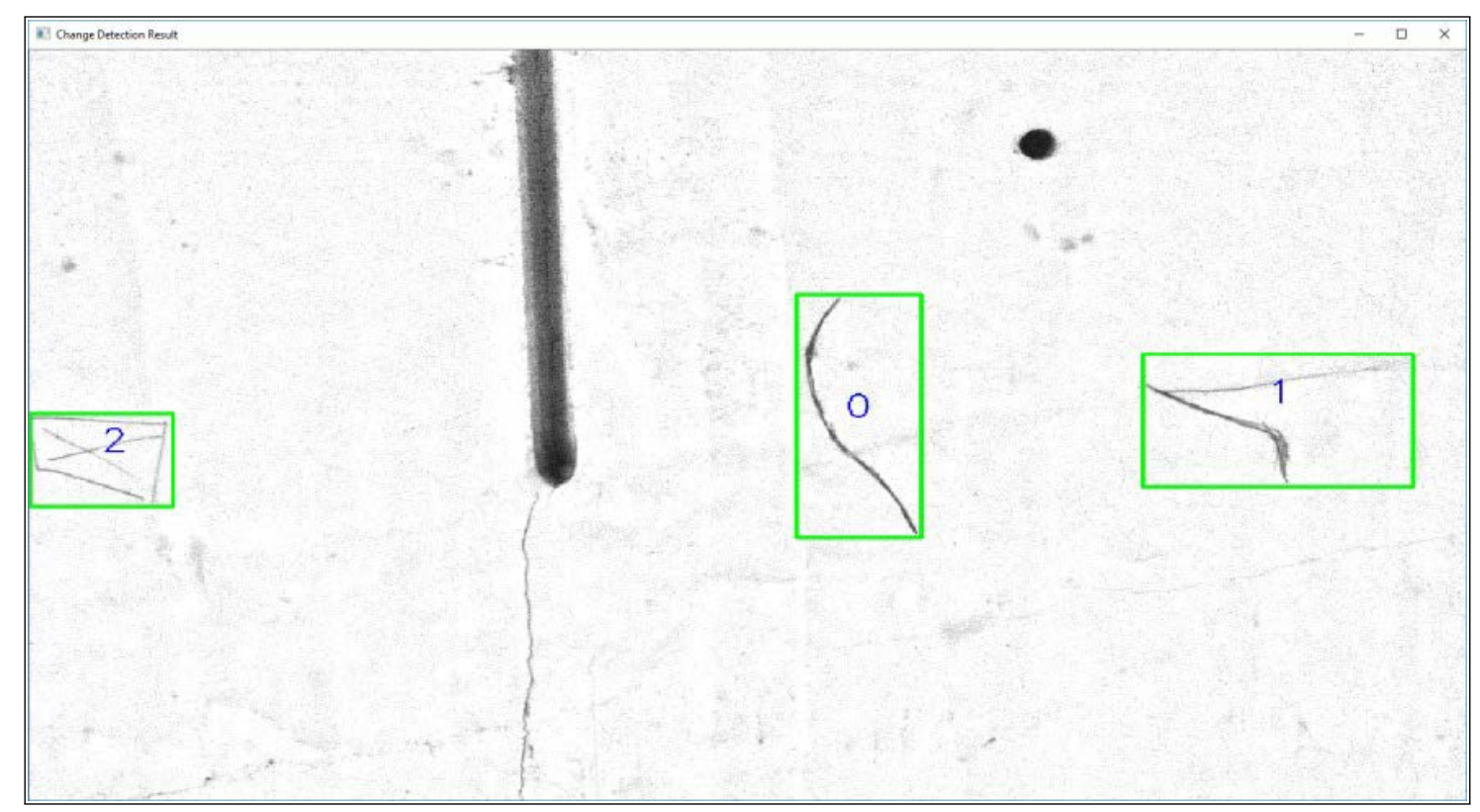

Figure 6.9: This screenshot shows the change detection result from the sample set of two temporal images, query image in Figure 6.7 and survey image in Figure 6.8. The bounding boxes envelope the areas detected as change. 


\begin{tabular}{|c|c|c|c|}
\hline \multirow{2}{*}{ Change } & $\begin{array}{c}\text { Centroid Location } \\
(\mathrm{x}, \mathrm{y})\end{array}$ & \multicolumn{2}{|c|}{ Change Dimensions } \\
\cline { 3 - 4 } & $(603,331)$ & width $(\mathrm{mm})$ & height $(\mathrm{mm})$ \\
\hline 0 & $(908,335)$ & 197 & 220 \\
\hline 1 & $(52,371)$ & 104 & 120 \\
\hline 2 & & \\
\hline
\end{tabular}

Table 6.3: Attributes of the detected 'change areas' including location and description information. The location of the center of the bounding box as well as its width and height are recorded.

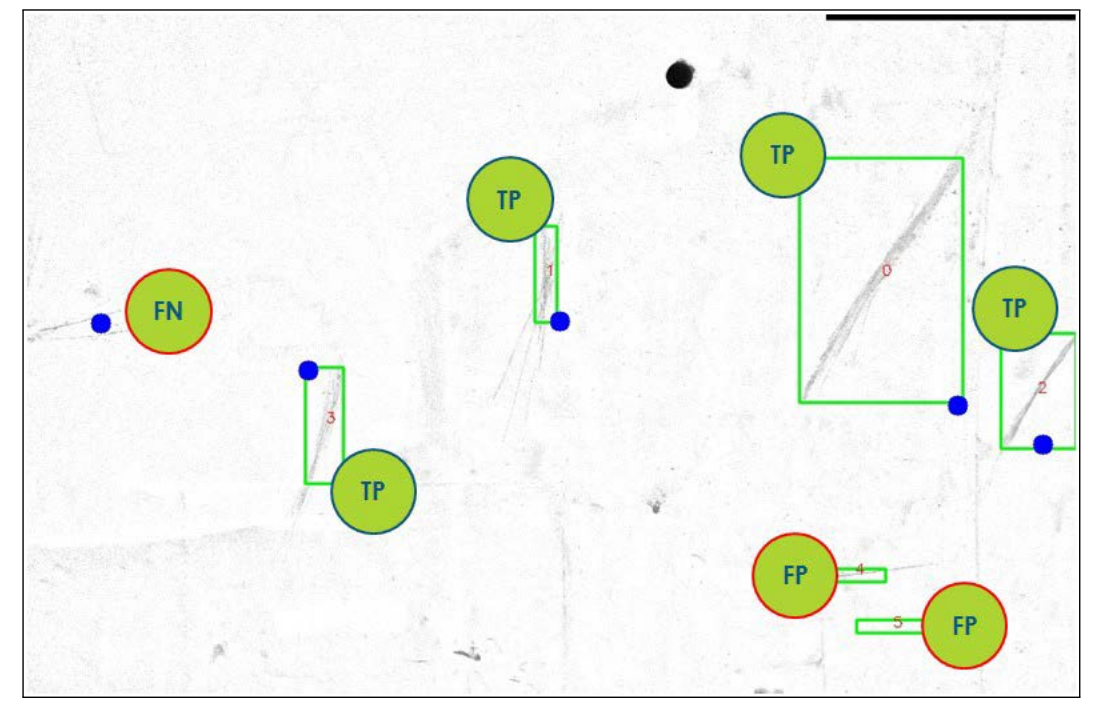

Figure 6.10: Manually marked TP showing a correctly identified change, FP marking an area incorrectly detected as a change and FN marking missed changes.

\begin{tabular}{|c|c|c|}
\hline Set & Average TPR (\%) & Average PDR (\%) \\
\hline 1 & 71.9 & 77.3 \\
\hline 2 & 86.4 & 90.8 \\
\hline 3 & 90.1 & 82.1 \\
\hline
\end{tabular}

Table 6.4: Average TPR and PDR ratios for each of the datasets that were used for testing

The change detection output obtained from the Change Detector module was manually compared to the corresponding marked image in the GT set as shown in Fig. 6.10. For each test scenario, an area which was correctly identified as change was recorded as a True Positive $(T P)$. Each 'change' area in GT which was not detected by the system was listed as a False Negative $(F N)$. On the other hand, an area which was detected as a change but did not correspond to any of the marked changes in the GT image, was noted as a False Positive $(F P)$. 
The sensitivity of the proposed system was calculated using the True Positive Rate $(T P R)$. This is the ratio of changes that are correctly identified. The TPR is given by:

$$
T P R=\left(\frac{T P}{T P+F N}\right) \times 100
$$

where $T P$ is the number of true positives and $F N$ is the number of false negatives.

The precision was calculated using the Positive Detection Rate $(P D R)$, which is the ratio of the 'true changes' detected to the total number of changes identified. The PDR is defined by:

$$
P D R=\left(\frac{T P}{T P+F P}\right) \times 100
$$

where $T P$ is the number of true positives and $F P$ is the number of false positives.

For each of the mentioned datasets, the average values for TPR and PDR were calculated and listed in Table 6.4. In addition to this, the overall average ratios were calculated on all the images obtained. The system achieved an average of $83.5 \%$ TPR and $82.8 \%$ PDR. The overall accuracy of the system was calculated using the harmonic mean of precision and sensitivity:

$$
\text { Accuracy }=\left(\frac{2 T P}{2 T P+F P+F N}\right) \times 100
$$

where $T P$ is the number of true positives, $F P$ is the number of false positives and $F N$ is the number of false negatives.

This results in an overall average accuracy of $81.4 \%$. The system was able to detect changes as small as $10 \mathrm{~cm}$ in any dimension.

\subsection{Human Detection and Remote Respiration Monitor- ing}

Human detection in search and rescue scenarios using robots is needed to reduce risks for the human intervention team and to endorse the search and rescue capabilities of a specific intervention. For this, the robot that intervenes in a disaster zone must be able to detect possible victims in the area and passing their vital parameters to the human intervention team.

The results of the novel proposed human reconnaissance system of the CERNTAURO framework is described in this section.

Figure 6.11 shows the proposed prototype, which is used for experiments, that could be integrated on intervention robots. The system consists of three elements: Logitech C920 web-cam, X4M200 respiration sensor and a 12V light source for operating in dark environments. 


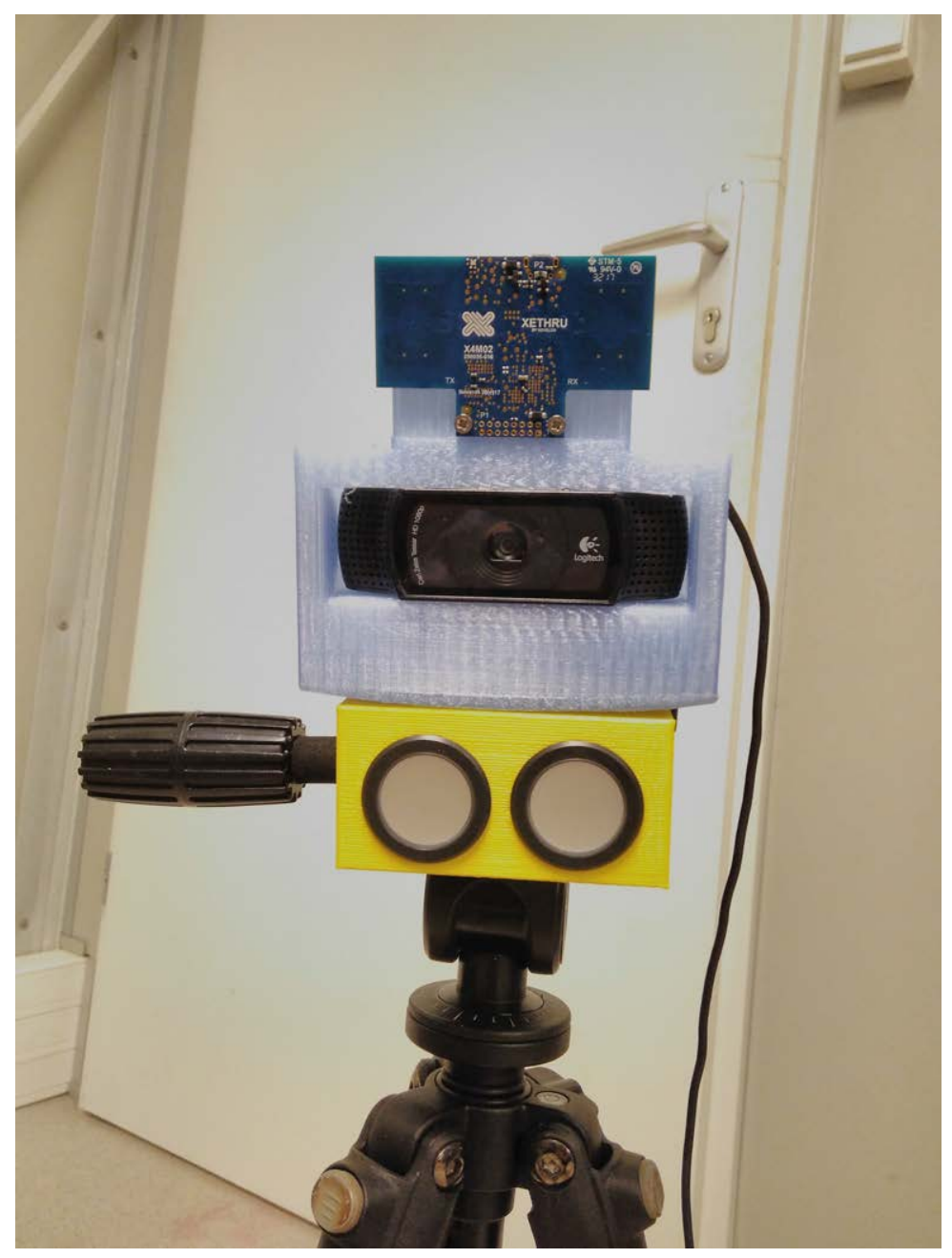

Figure 6.11: Prototype system for test. Above installed X4M200 respiration sensor, in the middle Logitech C920 web-cam, below 12V light source.

A set of experiments was conducted in office environment with a person laying on floor to simulate an unconscious victim in a disaster scenario indoors. The volunteer was instructed to lay in different positions, which can be classified into five large groups - on spine, on side towards the camera, on side away from it, with head towards camera and then with legs towards. The performance of prototype was evaluated not only by posture, but also in relation to distances to the sensor system. Impact of clothing was also explored for respiration detection. Furthermore, performance of prototype was tested with multiple victims in the field of view. Sensor system was installed on a tripod at $60 \mathrm{~cm}$ height to simulate the view from a ground robot. Before experiments started, X4M200 sensor was left inside the empty room to generate a noise map to achieve the best possible performance. Initialization takes approximately 120 seconds. Sensitivity of sensor was put to maximum. Figure 6.12 shows the five positions of victim with bounding boxes generated by MobileNet together with acquired respiration graphs and pulse-doppler maps. 

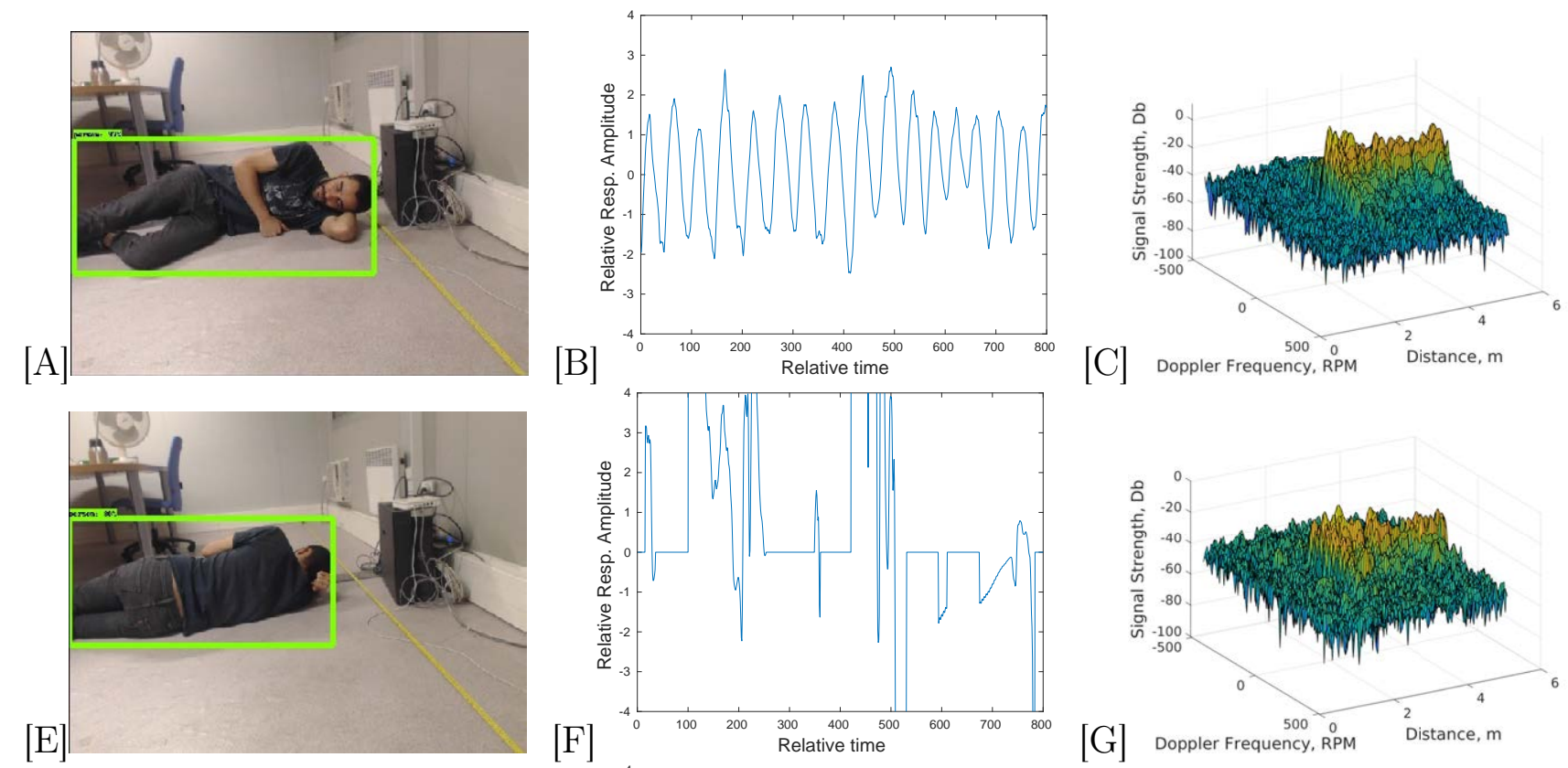

$[\mathrm{C}]$
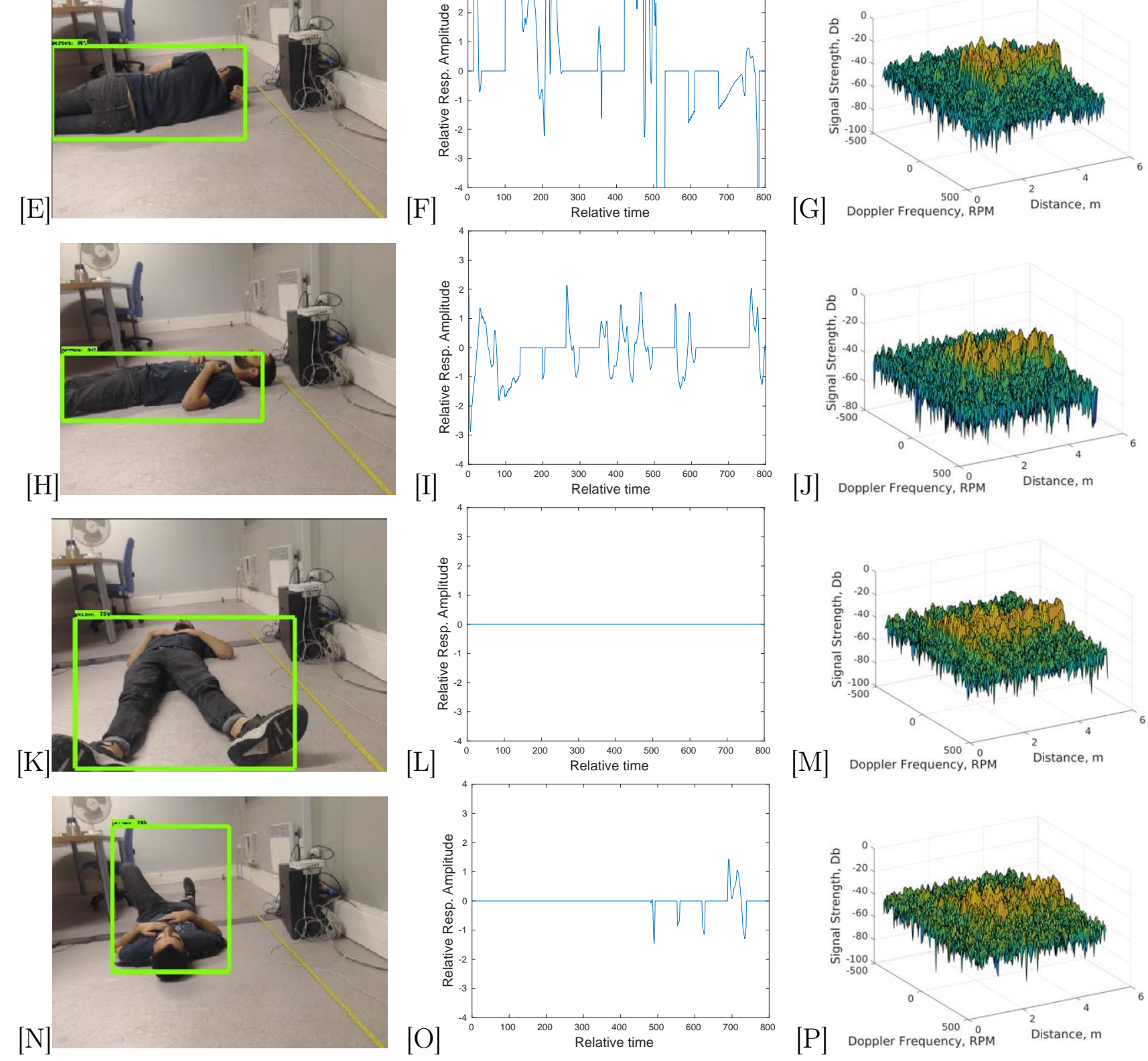

Figure 6.12: System performance at $2 \mathrm{~m}$ distance. A,E,H,K,N represent five postures and bounding boxes around detected victims, generated by MobileNet. B, F, I, L, O show acquired respiration data (subject was breathing normally at all times). C, G, J, M, P are pulse-doppler maps for each posture. 

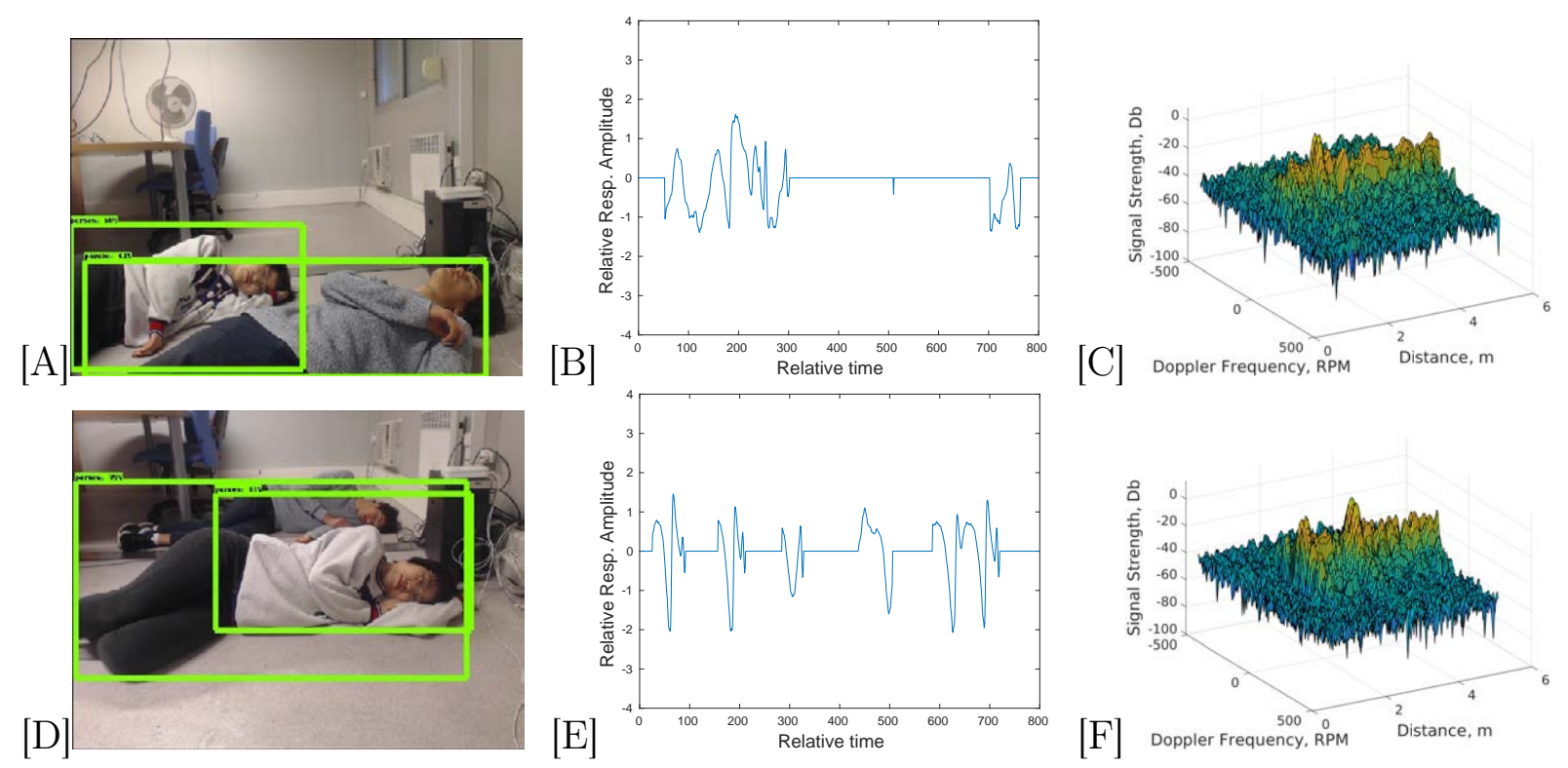

Figure 6.13: Multi-person view

Figures 6.14 and 6.12 show the performance of MobileNet for lying person recognition. Region of detected person was marked with a green bounding box. Only the detections, which are above $50 \%$ threshold were counted as positive recognitions. Each position is evaluated separately for corresponding distance to victim. Results confirm, that MobileNet peforms well (detection rate up to $90 \%$ ) for detection of persons lying on floor in different positions. The effective recognition distance (detection rate higher than $50 \%$ ) is 4 meters. Some positions were less favourable, i.e., there were no reliable detections with the head facing towards the camera. The best performing positions were with person lying on either of his sides.

MobileNet also performed well when camera captured only a part of body (due to close distance) as shown in Figure 6.16, and in presence of irregularities such as shadow on the leg domain and chair blocking the view on lower part of body (see Figure 6.15). In case of multiple persons inside the field of view, as shown in Figure 6.13, the detector had some trouble with adjusting bounding boxes properly, however the number of detected persons is correct.

Series of tests were conducted in order to evaluate performance of the commercially available respiration sensor X4M200. According to manufacturer [263], the maximal respiration sensor detection distance is 5 meters. To evaluate the effective respiration detection range, we gathered data from radar across varying distance (from 1 to 5 meters with step of 1 meter) between device and human. Table 6.5 shows the achieved respiration signal detection rate of a person wearing a T-shirt over experiment time for all positions in all distances measured. Gathered data clearly show, that sensor performance is strongly dependent on the posture of victim. The experiments were conducted again, but this time with the same person wearing several layers of clothing (shirt and a jacket) to compare the performance of radar. However, the acquired results did not show any significant impact of clothing on 
experiment results (see Table 6.6).

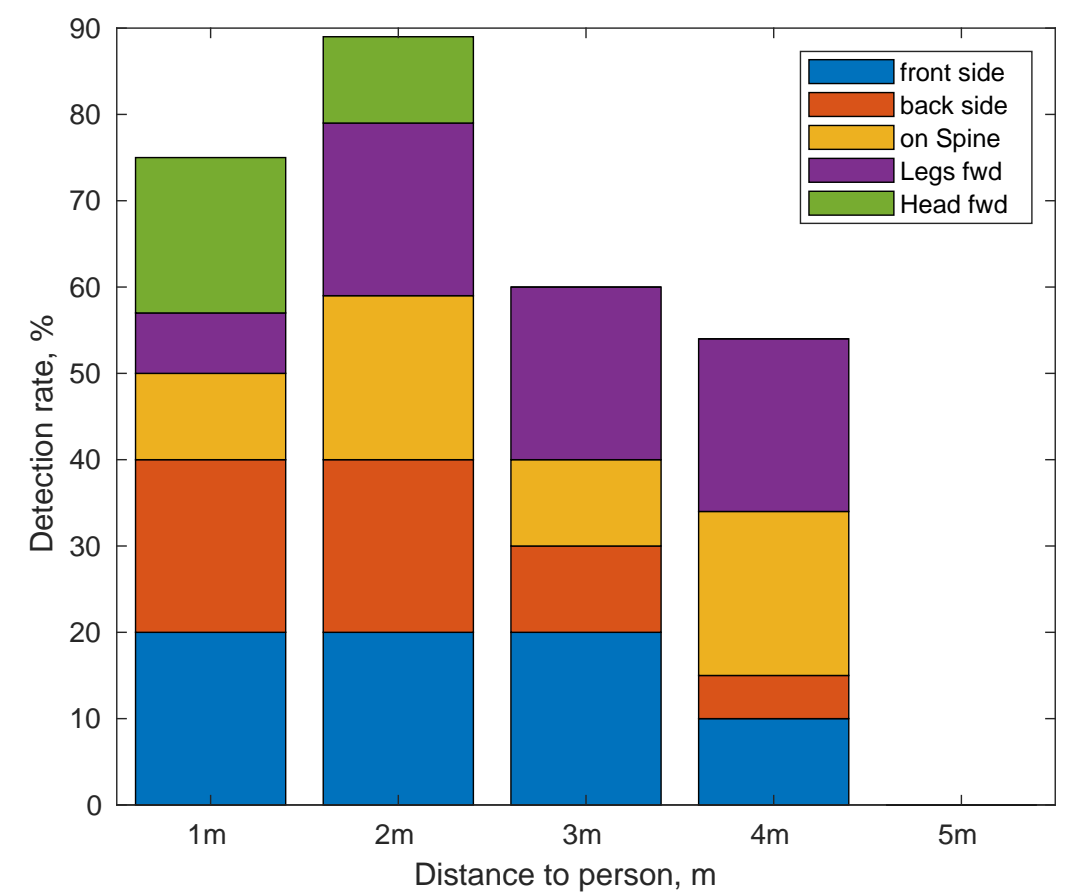

Figure 6.14: Detection performance of MobileNet at various postures and corresponding distances 


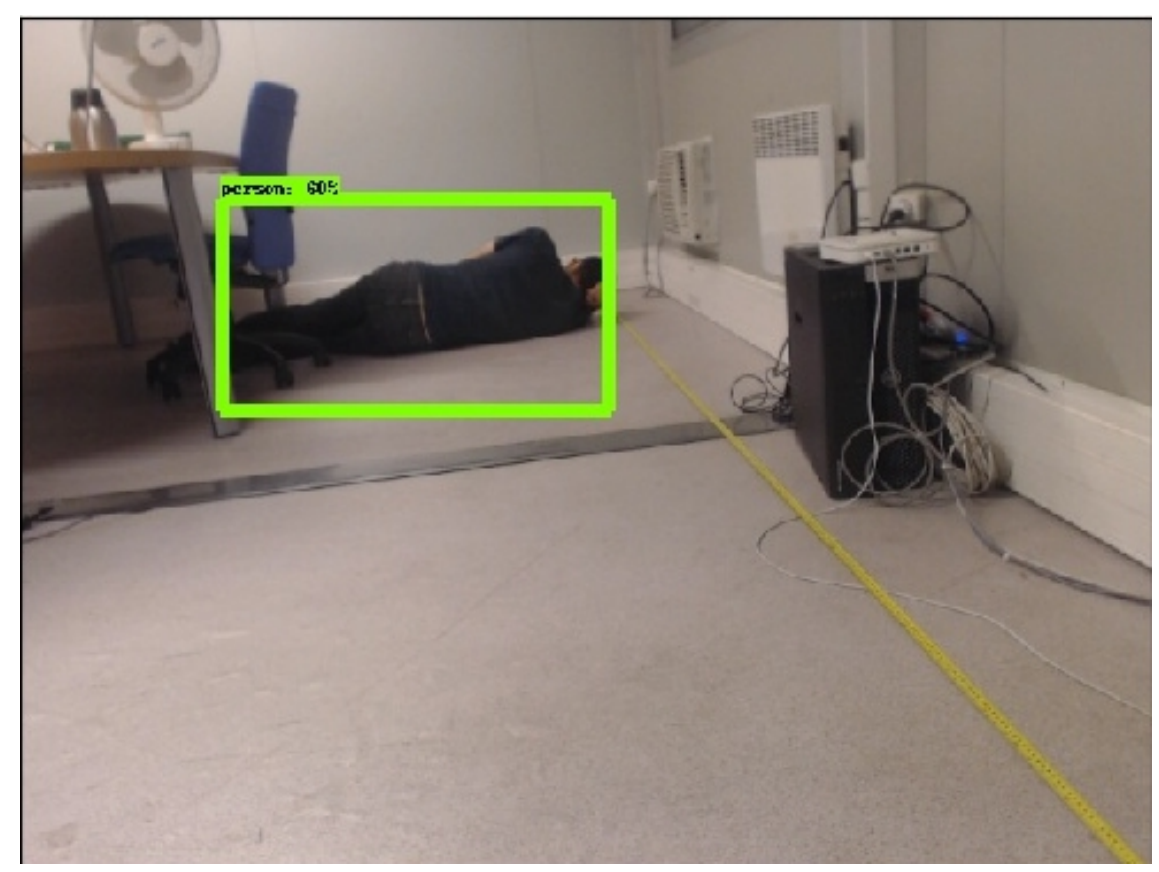

Figure 6.15: Person on side turned away from camera at 4m. Green bounding box generated by MobileNet.

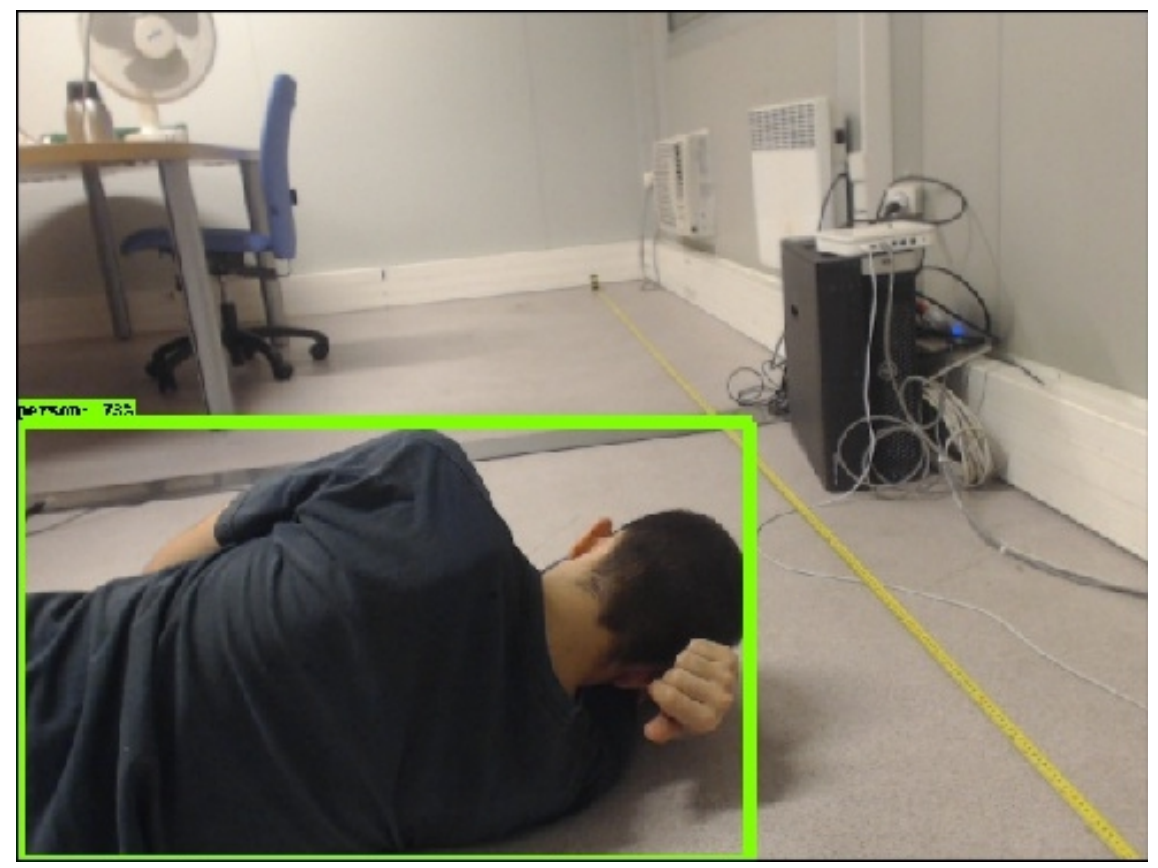

Figure 6.16: Person on side, turned away from camera at $1 \mathrm{~m}$.

The most favourable position to measure respiration activity is, when lung movement is directed perpendicularly towards the radar. Pulse-doppler maps from Figure 6.12, confirm, 
that in case of person lying perpendicularly with chest towards radar (C), the signal strength is much higher than surrounding noise, and a peak at $2 \mathrm{~m}$ distance (which is distance to subject's chest) can be easily spotted, whereas coming signal from the back $(\mathrm{G})$ is just a bit higher than noise level. This can be explained by comparatively small back movements due to tight muscles in the spine. Regarding the other positions ( $\mathrm{J}, \mathrm{M}$ and $\mathrm{P})$, it is seen, that signal gets distributed over multiple range bins according to length of the body. This is the most unfavourable situation for radar, because no definite peak in pulse-doppler map can be distinguished, which makes radar scan between multiple range bins.

Even though the UWB radar could not always accurately detect respiration rate of a person, it could clearly detect small movement presence and predict the distance to victim. In case of multiple persons, located at different distances from radar in the field of view, as shown in Figure 6.13 case D, the used algorithm for respiration detection should monitor breathing of the closest subject. Device could not perform reliable respiration monitoring, even though position was the most favourable. On the other hand, pulse-doppler map shows, that radar could distinguish two peaks, i.e. persons about 1 meter away from each other. Performance of X4M200 was also tested, when subjects were almost next to each other, as in case As from Figure 6.13. Here, radar could only detect movement without distinguishing two separate subjects.

\begin{tabular}{|c|r|r|r|r|r|}
\hline & $\mathbf{1 m}$ & $\mathbf{2 m}$ & $\mathbf{3 m}$ & $\mathbf{4 m}$ & $\mathbf{5 m}$ \\
\hline back side & $25.9 \%$ & $31.3 \%$ & $28.7 \%$ & $4.2 \%$ & $17.4 \%$ \\
\hline front side & $70.1 \%$ & $91.5 \%$ & $48.9 \%$ & $60.8 \%$ & $0 \%$ \\
\hline on spine & $8.0 \%$ & $42.7 \%$ & $10.2 \%$ & $0 \%$ & $0 \%$ \\
\hline legs fwd & $0 \%$ & $0 \%$ & $0 \%$ & $0 \%$ & $0 \%$ \\
\hline head fwd & $31.3 \%$ & $12.9 \%$ & $0 \%$ & $0 \%$ & $0 \%$ \\
\hline
\end{tabular}

Table 6.5: Respiration detection rate. Male in t-shirt.

\begin{tabular}{|l|r|r|r|r|r|}
\hline & $\mathbf{1 m}$ & $\mathbf{2 m}$ & $\mathbf{3 m}$ & $\mathbf{4 m}$ & $\mathbf{5 m}$ \\
\hline back side & $22.3 \%$ & $15.8 \%$ & $84.9 \%$ & $0 \%$ & $0 \%$ \\
\hline front side & $74.6 \%$ & $80.2 \%$ & $52.5 \%$ & $90.8 \%$ & $0 \%$ \\
\hline on spine & $74.6 \%$ & $38.9 \%$ & $2.0 \%$ & $0 \%$ & $1.6 \%$ \\
\hline legs fwd & $6.5 \%$ & $0 \%$ & $0 \%$ & $0 \%$ & $0 \%$ \\
\hline head fwd & $0 \%$ & $6.6 \%$ & $19.9 \%$ & $0 \%$ & $0 \%$ \\
\hline
\end{tabular}

Table 6.6: Respiration detection rate. Male in thick clothing.

Respiration signal acquisition is dependent on victim's posture, therefore a solution for this problem to use visual sensor for human posture recognition and automatically relocate the radar to a more favourable position for reliable monitoring. Radar takes a significant amount of time to accumulate and process pulse-doppler data after it can declare a positive detection, which is even slower with multiple victims in the field of view. This shortcoming 
could be overcome by fusion with data acquired from the much faster camera detection. On the other hand, radar can help to avoid miss-detections in environments, where visual sensor has low performance. This leads to a conclusion, that both sensors have their own advantages, and, as already stated by multiple researchers, combinations of microwave technologies with other sensors, such as optical or infrared, can provide robust capabilities by performing sensor fusion. No single detection method or system can perform perfectly in all conditions, and for search and rescue scenarios, where miss-detections are very costly, the combination of sensors holds a significant potential.

In the future we are planning to extend the existing prototype with a thermal camera and focus on data fusion of multiple sensor types for robust real-time human detection and medical field diagnostics in harsh environments. In addition, techniques like adaptive filters, patter recognition and descriptors will endorse the system robustness and precision.

\subsection{Learning by Demonstration Results}

The following data is taken using hand-guidance mode that uses the robot's end-effector frame based on the robot base reference. To evaluate performance of algorithm, the learned path coming from the DMP generator is compared with the initial data. Visually, it can be seen two independent axes in (Figures 6.17 for X-Axis and Figure 6.18 for Alpha-Axis) that the demonstrated signals have small oscillating motion in some areas due to the operator's hand movement. It is an expected behaviour due to using a kinesthetic learning method through a low stiffness robot which will be sensitive to the mismatch between the teacher's and robot arm's kinematics. Nevertheless, these oscillations are not noticeable in the predictions since the predicted signals are all smoothed out. As a result of the dynamic movement primitives' use of kernel functions (LWR) and differential equations (Transformation system). Furthermore, the canonical system links all degrees of freedom and each signals reaches their destination. 


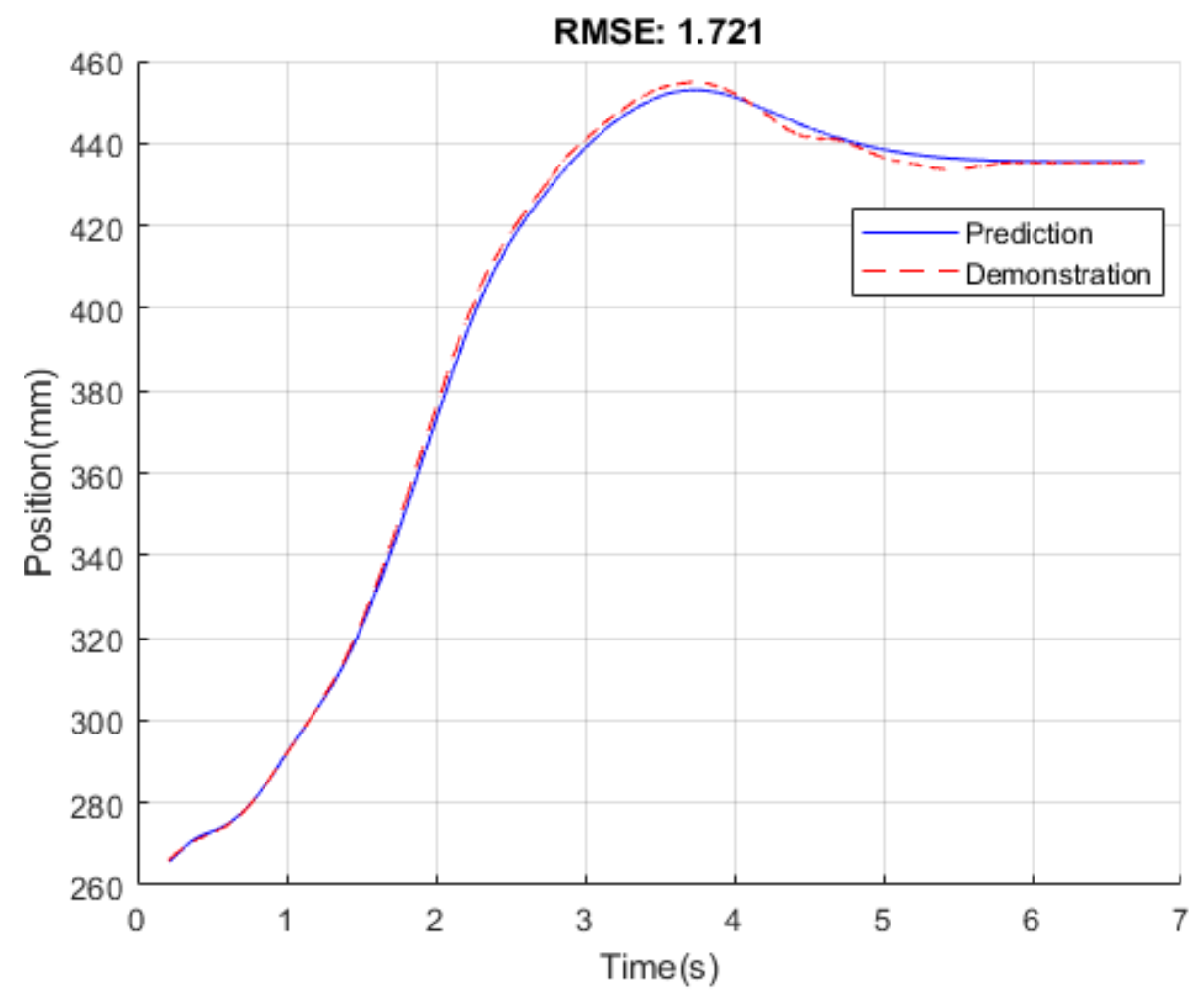

Figure 6.17: TCP's X position prediction

Due to the impedance controller, there is an error in the position that needs to be considered by the systems performance. This error lets the robot have some leeway in the positioning response while achieving also some level of compliance. As a result, the RMSE will have a direct relationship between the DMP performance's and the robot arm's imprecision given by the impedance controller. If the impedance controller's stiffness were to increase then the position control can achieve higher precision at the cost of reducing the robot arm's compliance, therefore a middle ground is achieved empirically from the compliance desired towards the external environment.

In Table 6.7, the robot's position and the DMP's RMSE was computed in each axis given by the end-effector's frame in XYZ and quaternion space while preforming a singular primitive. Notice how the $\mathrm{Z}$ axis' RMSE is higher in comparison to the $\mathrm{X}$ and $\mathrm{Y}$ axis. This is the result of the before-mentioned impedance controllers imprecision and the lack of a perfect gravity compensation in the robot control. The weight of the end-effector is increased by a gripper attached in the robot arm and needs to be perfectly calibrated to have the best results. Since this is not the case, the robot arm is pulled slightly downwards due to the weight parameter imprecision as seen in the results. 


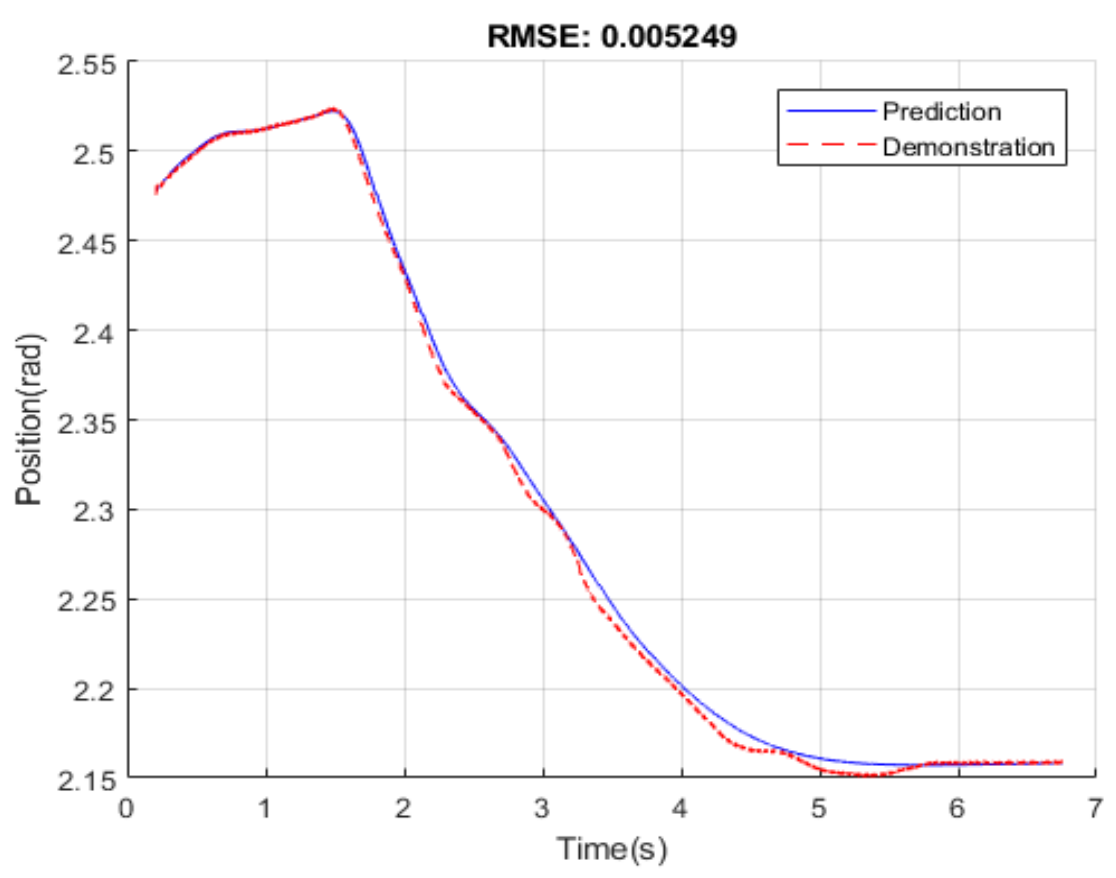

Figure 6.18: TCP's Alpha orientation prediction

\begin{tabular}{|c|c|}
\hline State Variable & RMSE \\
\hline$X$ & 0.9849 \\
\hline$Y$ & 1.4880 \\
\hline$Z$ & 5.3165 \\
\hline$q_{w}$ & 0.0046 \\
\hline$q_{x}$ & 0.0172 \\
\hline$q_{y}$ & 0.0094 \\
\hline$q_{z}$ & 0.0061 \\
\hline
\end{tabular}

Table 6.7: RMSE of Dynamic Movement Primitive and Robot's end-effector real position

\section{Unscrewing of Burndy Connector with DMP library}

The desired task to learn is unscrewing a burndy connector with the robot arm. The endeffector will have a griper to pick the burndy connector and unscrew it with a twisting motion. In order to accomplish this task, initially three primitives will be learned by the system. They are going to be stored in the library and will be chosen to perform this task. For the first part of the task, that is to reach the objects area, the primitive will be defined as "reach-object-vicinity". To test the dynamic adaptability of the framework, this particular primitive, will be taught with a different goal location and later the goal position will be changed according to the specific situation in which the robot arm needs to perform. The action, for this primitive, will be to reach the area in which the connector is but is not precise enough to need to know where it is located exactly (see Figure 6.19). 


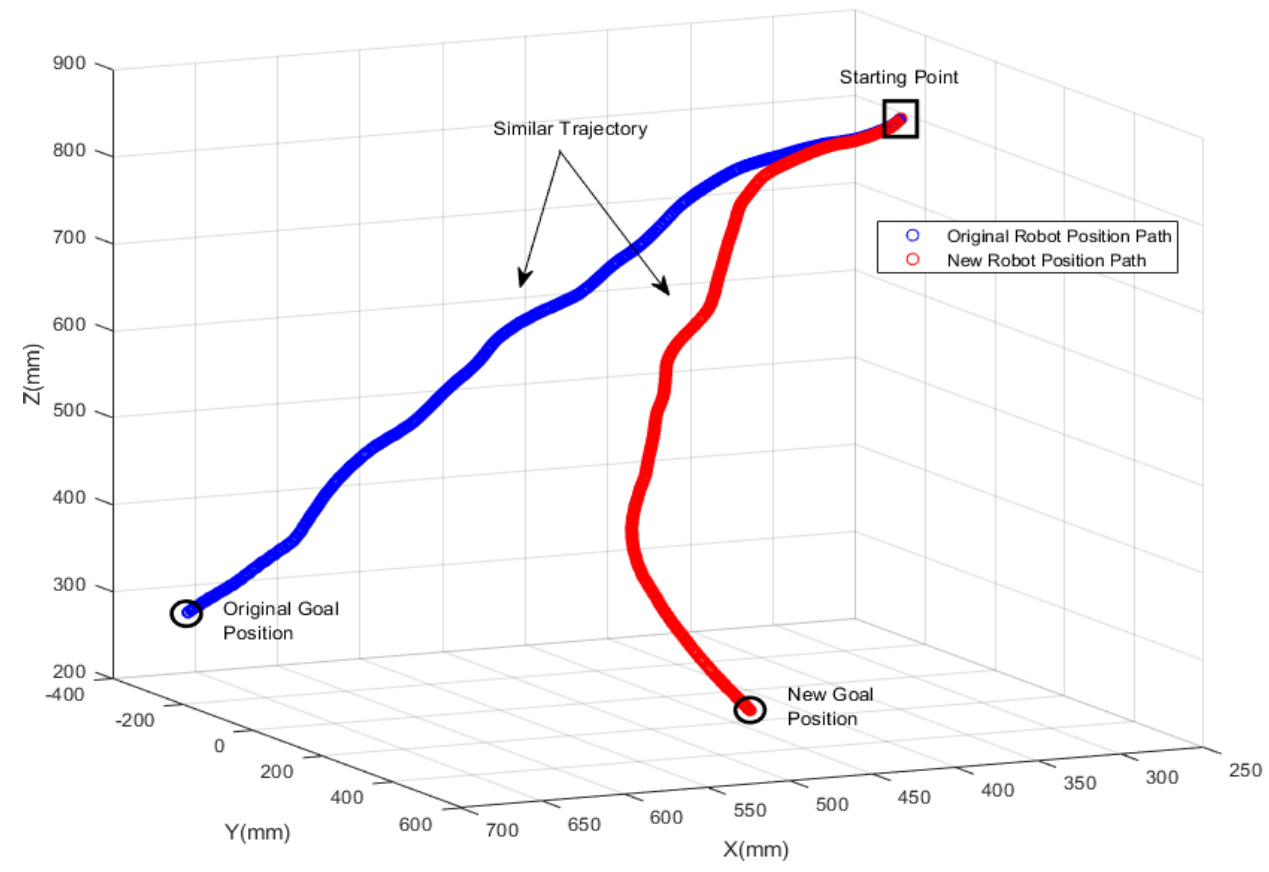

Figure 6.19: Kuka robot finishing Reach-Object-Vicinity Primitive

A second primitive will be named as "go-down-extended-pose". The robot will normally be extended in this primitive, but not enough to be near the singularity zone. Then the end-effector will move slowly towards the burndy connector and be in position to grasp it with the tool. In this primitive a more precise location of the connector needs to be defined as this motion will allow to put the robot arm in the correct initial pose to start unscrewing the connector. Finally, the third primitive will be the "turn-and-pull" action. In this part, the end-effector will slowly turn to the desired direction to unscrew the connector, and pull once the rotation is finished. The full task performance can be observed in Figure 6.20.

The completed task's duration took approximately 45 seconds, time which includes the grasping of the object by the gripper and pulling from the table. The longest primitive's duration was on the turn-and-pull action, due to the level of carefulness that the teacher presents the primitive to the robot. The interaction between teacher and robot arm needs to be done slowly in order perform correctly this part of the task. Finally, as a result, the task was successfully completed and the burndy connector was unscrewed by using all the before-mentioned primitives in the correct sequence.

\subsection{Metallic Objects Detection and 6D Pose Estimation}

To validate the proposed solution for metallic objects detection and 6D pose estimation, for the first experiments the Orbbec Astra Pro [398] performance was used due to its low-level of noise in the point cloud that it generates. The integration of the object detection module and its resulting camera centering into the pipeline, however, often avoided the reflection of 


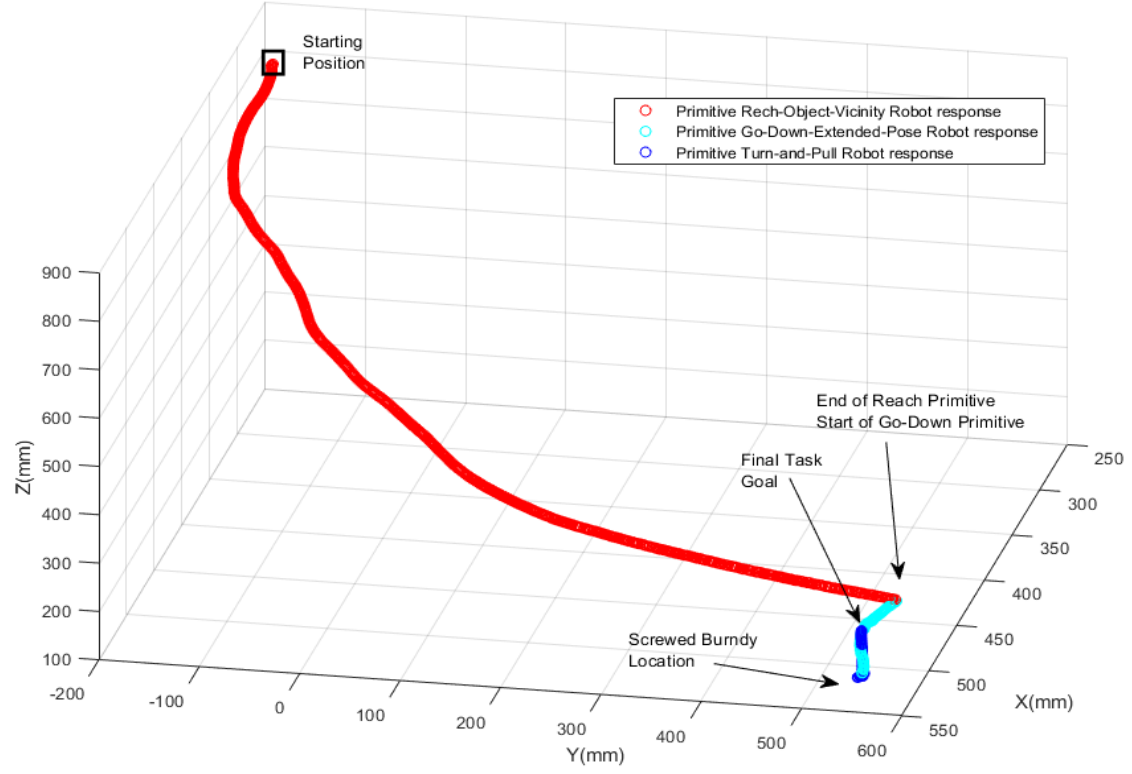

Figure 6.20: Full path of TCP performed by KUKA robot with 3 primitives for task completion

metallic objects, thereby enabling the use of smaller cameras, as RealSense R200 or SR300 [399]. Even though these cameras are slightly noisier, minimizing the size is substantial to enhance the robot arm's capability to incorporate sensors for surveying tasks.

An Intel Core i7-3630QM, 4/8 cores at 2.4 Ghz with 76.8 GFlops was used for processing. A PC with an Intel(R) Xeon(R) CPU E5-1630 at 3.70GHz with 32GB of RAM and a Nvidia GTX 1080 graphic card was used for the deep learning training. For the deployment of the deep learning part in the robot a Nvidia Jetson TX2 board has been used. Furthermore if the wireless communication is stable, the robot can exchange the data with a server with a Nvidia GTX 1080 using the ZeroMQ middleware. This middleware was selected due to positive prior experience [400] at CERN using ZeroMQ in its Controls Middleware [401].

The vision system has been integrated on a CERNbot equipped with a Schunk Arm Powerball.

The objects of interest trained and tested are all part of the collimator system at CERN [402], as visualized in Figure 6.21. Collimators are highly relevant for the correct operation of the Large Hadron Collider (LHC) and therefore of high importance in technical inspections. In addition, they are one of the hot spots of radiation in the LHC, qualifying as an essential target for robotic surveying.

The algorithm works if the setup is subject to the following restrictions:

- The camera is in front of the target. The integration of deep learning into the pose estimation pipeline enables autonomous camera centering. 


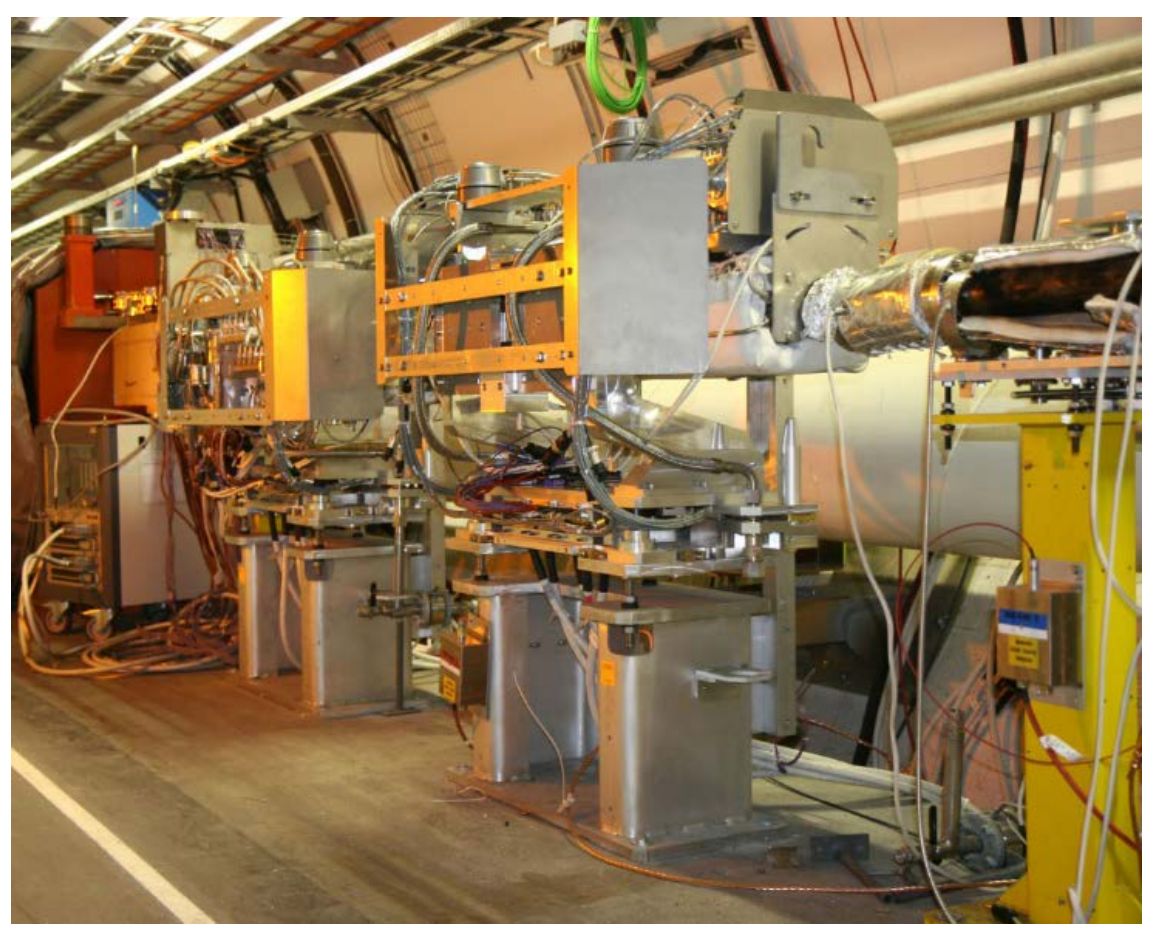

Figure 6.21: A Collimator in the LHC tunnel.

- The targets are straight, i.e. not lying on their side.

- The targets are rigid. Usually the objects of interest are metallic.

The test were made at distances to the target between 50 and $136 \mathrm{~cm}$, the maximum distance in the tunnel, (see Figure 6.22). The tests evaluated with the current algorithm exhibit position errors with less than $2 \mathrm{~cm}$ for the collimator. It was found that the pose estimation error directly correlates to the distances of the camera to the target. In this case, the distance between camera and collimator was measured at around $65-75 \mathrm{~cm}$, due to the restricted dimensions and protective measures in the LHC tunnel. This, however, can be avoided by specifically targeting small parts of the collimator and subsequently inferring the global pose estimation from these.

It has been verified that the processing time of the algorithm is less than 5 seconds, thanks to the centering with the help of Faster-RCNN, facilitating the main pipeline to find the planes. Moreover, the centering reduces the runtime of the segmentation operation, eliminating several iterations to find the matching plane.

This solution has achieved two main goals:

- increasing the level of autonomous operation of the robots with the use of deep learning to detect objects in images and

- estimating the $6 \mathrm{D}$ pose of the objects of interest with a 3D reconstruction of the target object 
Being of special interest for teleoperated tasks in hazardous and unstructured environments, it allows the operator to better estimate the distances and to visualize the reconstructed parts the sensors cannot reach.

\subsection{Radiation Dose Measurements with i-TIM}

The two novel i-TIM units, described in Appendix A and installed in the LHC, have performed several successful environmental inspections completely autonomously, with a total of more than $600 \mathrm{~km}$ ran inside the tunnel without failure. Radiation dose measurements taken by TIM are validated by comparing the data obtained with the expected radiation dose in each position. Since data are taken along with the position in the LHC tunnel in cm, the dedicated TIM website displays the data and compares the position with the LHC layout database, adding the possibility to match the radiation dose peaks with the corresponding LHC machine (see Figure 6.23).

The reproducibility of these measurements is crucial to validate the performance of iTIM as an LHC inspector, therefore the data taken in the different missions during 2018 are compared in Figure 6.24. As it can be observed, the peaks in the plot match for every different mission, which means that the measurements are reproducible in terms of position, thanks to the precise positioning system of i-TIM inside the LHC tunnel. In order to guarantee the reliability and the repeatability of the measurements and as well to allow the robotic system to be fully autonomous, a precise positioning system has been developed for i-TIM. The system uses a fail-safe Programmable Logic Controller (PLC) based encoder odometer to compute the position of the train. The possible odometer error due to frictions and slipping of the traction system, is then eventually corrected with barcodes installed on the tunnel monorail every 60 to 100 meters. These barcodes are also necessary to correct the irregularities of the monorail. The resulting positioning accuracy is lower than 1 centimeters along the 27 kilometers of the LHC tunnel.

\subsection{Challenging Teleoperated Tasks in Harsh Environ- ments}

In the latest years, several robotic interventions have been performed in CERN's accelerators environment, resulting in a great reduction of exposure of personnel to radiation hazards. In this section three challenging succesful interventions are presented in order to show how the presented framework can be applied to extremely complex tasks.

One of the different successful mission that the CERNTAURO framework has done is an in-situ repair of a water leak present in a water-cooling pipe serving a proton beam collimator in the North Experimental area [403] at CERN. The location of the broken pipe below the collimator was challenging, in addition to the high radiation and fragility of the equipment (see Figure 6.25 and Figure 6.26). The in-situ tasks were mainly to free the area around the leak location cutting different metal sheets using an ad-hoc tool and 

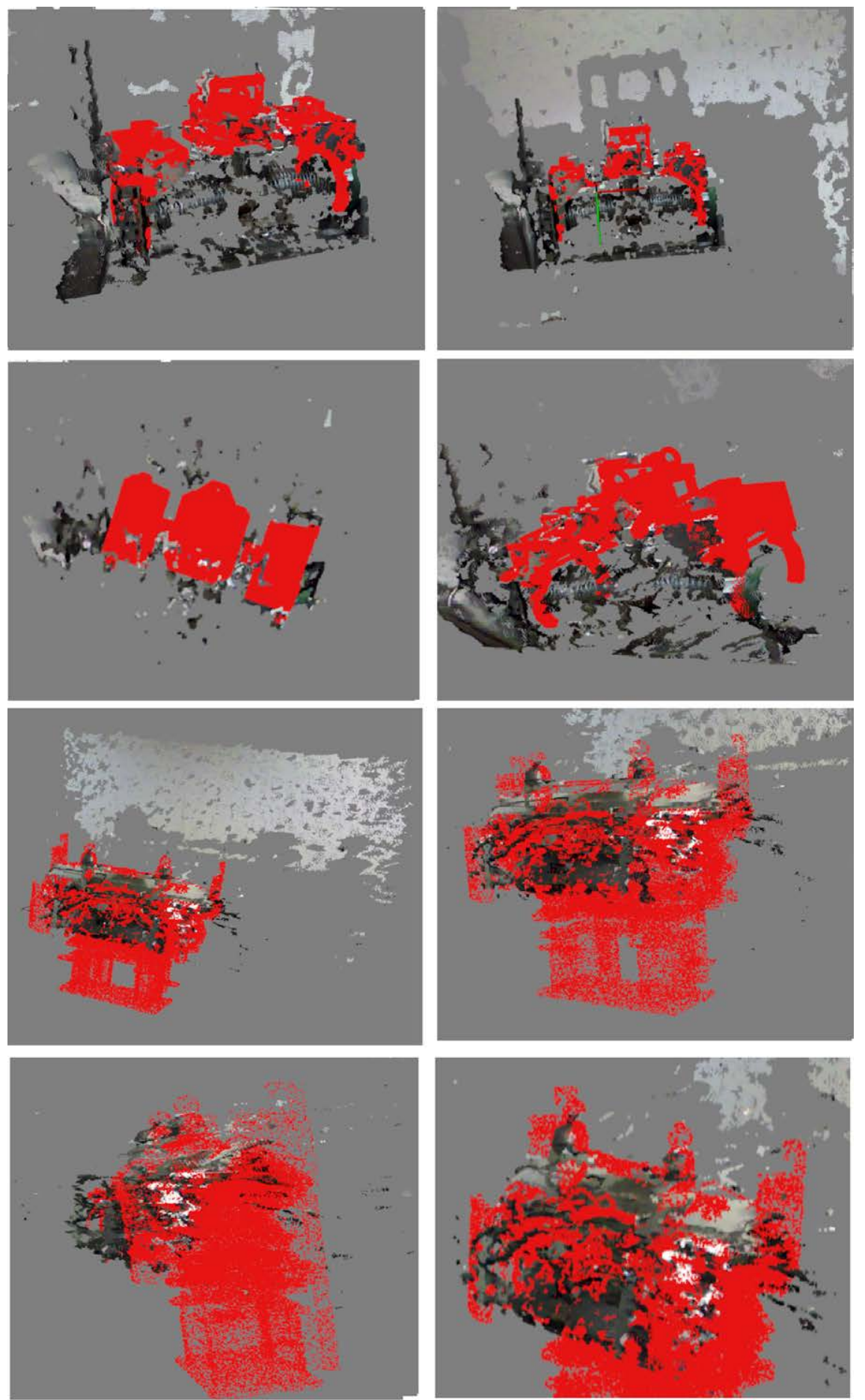

Figure 6.22: Reconstructions with different views of different point clouds of the separator (first and second row) and the collimator (third and forth row).

enclosing the broken pipe by building a container around the broken spot. The pipe's 


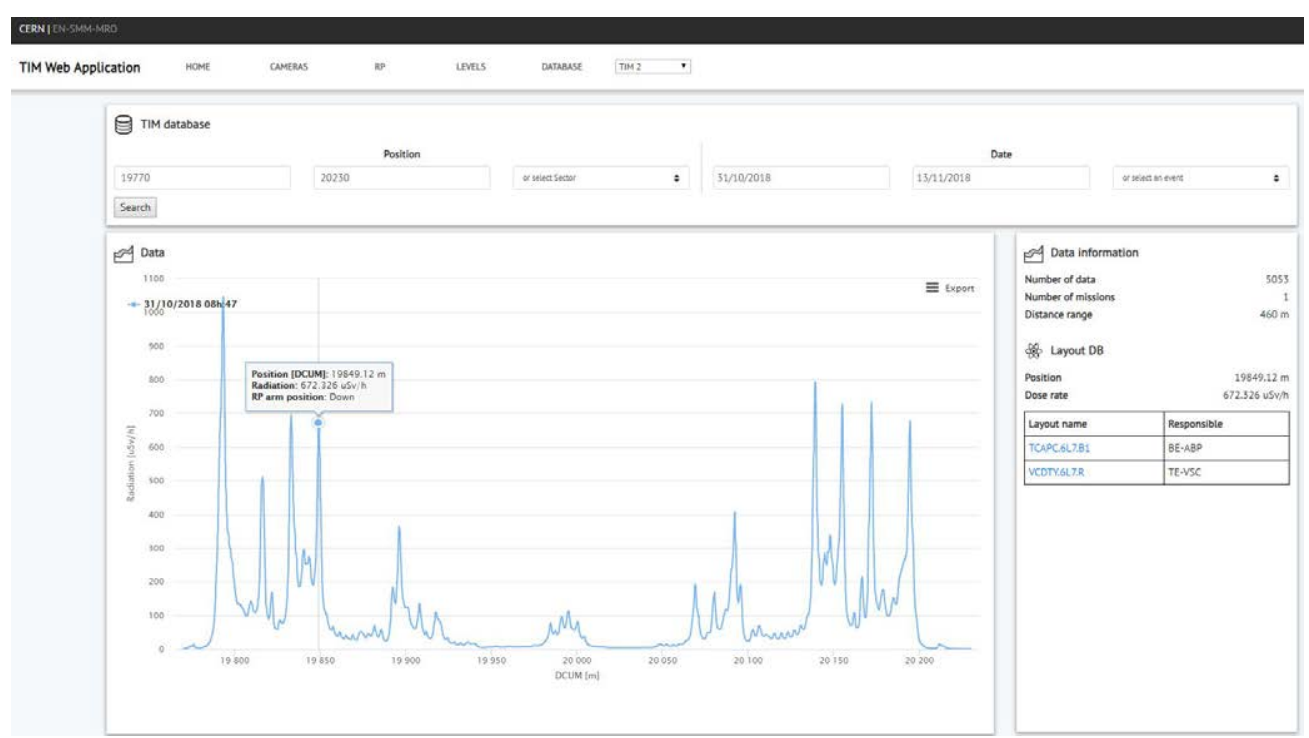

Figure 6.23: i-TIM website data display showing the corresponding LHC machine on the right side

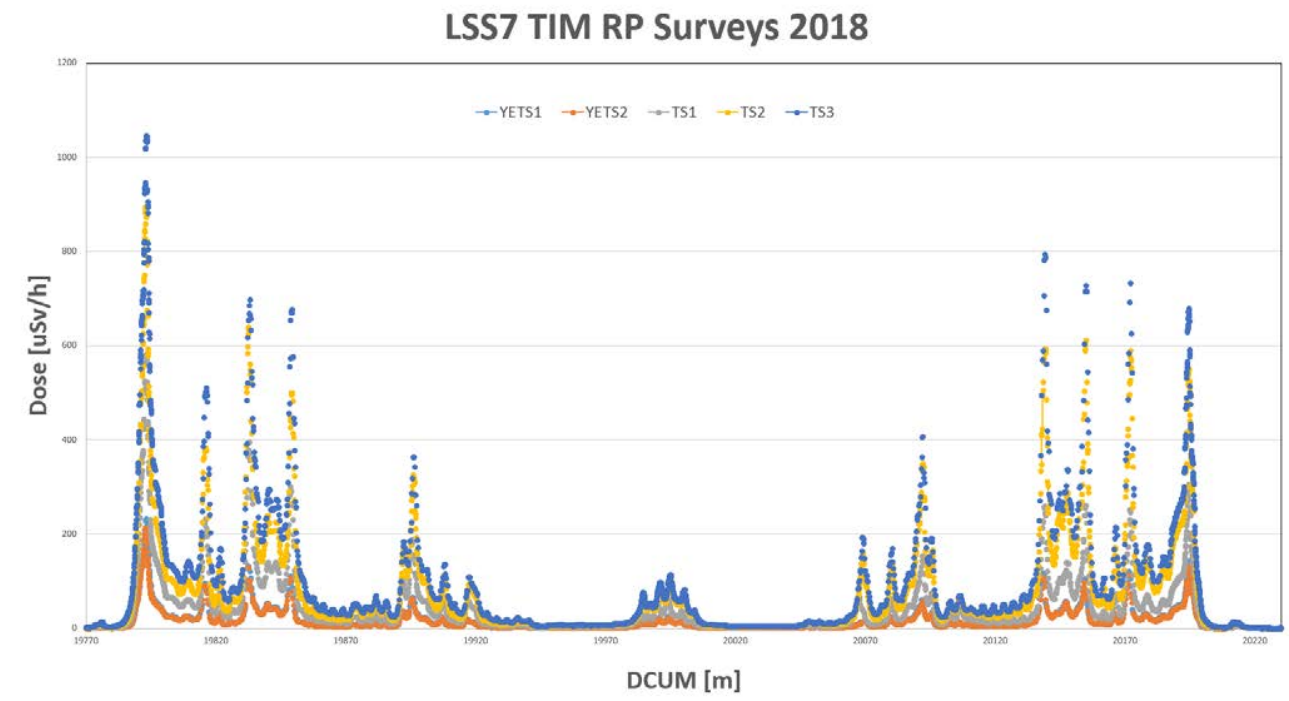

Figure 6.24: Data comparison between the different i-TIM radiation inspection missions in the Long Straight Section of Point 7 of the LHC, during 2018 


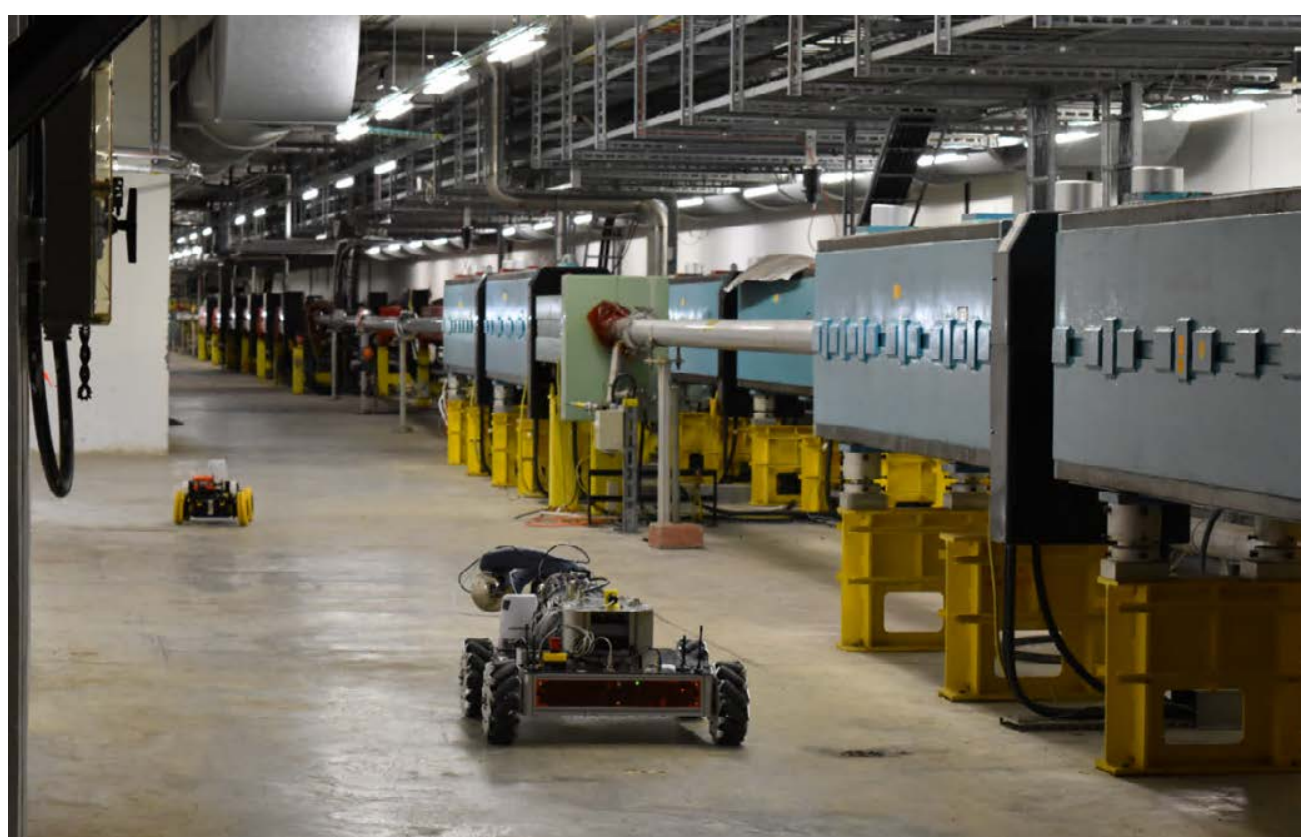

Figure 6.25: CERNBot v1.0 and EXTRM in North Area beamline

leak location has been enclosed and filled with special radiation hard resin to seal the leak (see Figure 6.27). The intervention pipeline followed all the CERNTAURO framework steps. The compliant master slave control of the CERNTAURO framework demonstrated its versatility for different delicate tasks like precisely injecting the resin and for more harsh tasks like cutting $5 \mathrm{~mm}$ metal sheets using ad-hoc tools.

Another successful mission that the CERNTAURO framework has done is an in-situ survey and filling of 25 oil recipients on the magnetic beam kickers of the Super Proton Synchrotron accelerator [404] at CERN (see Figure 6.28). In the past years this task has been accomplished using industrial robots and industrial controls [44]. By using the CERNTAURO framework, the mission time has been reduced increasing the machine availability and reducing human exposure to radiation because the operators are operating outside of the tunnel and far from hazards, as was not the case in the past years where standard industrial solutions were used.

Another success has been the handling of a radioactive iridium source. The radioactive source had a length of $20 \mathrm{~mm}$ and had to be removed from a radioactive transport container, taken out from its support, then being integrated into another support and the whole assembly was put in an experimental container that is irradiated by the Proton Synchrotron beam in the frame of the Antimatter Production facility [405] at CERN. For this intervention, CERNbot running CERNTAURO framework and equipped with an elevating stage and dual robotic arm system (a Kinova for fine telemanipulation and Schunk Powerball for high payload tasks) was used (see Figure 6.29). Specific tools and procedures were built following the proposed framework approach. 


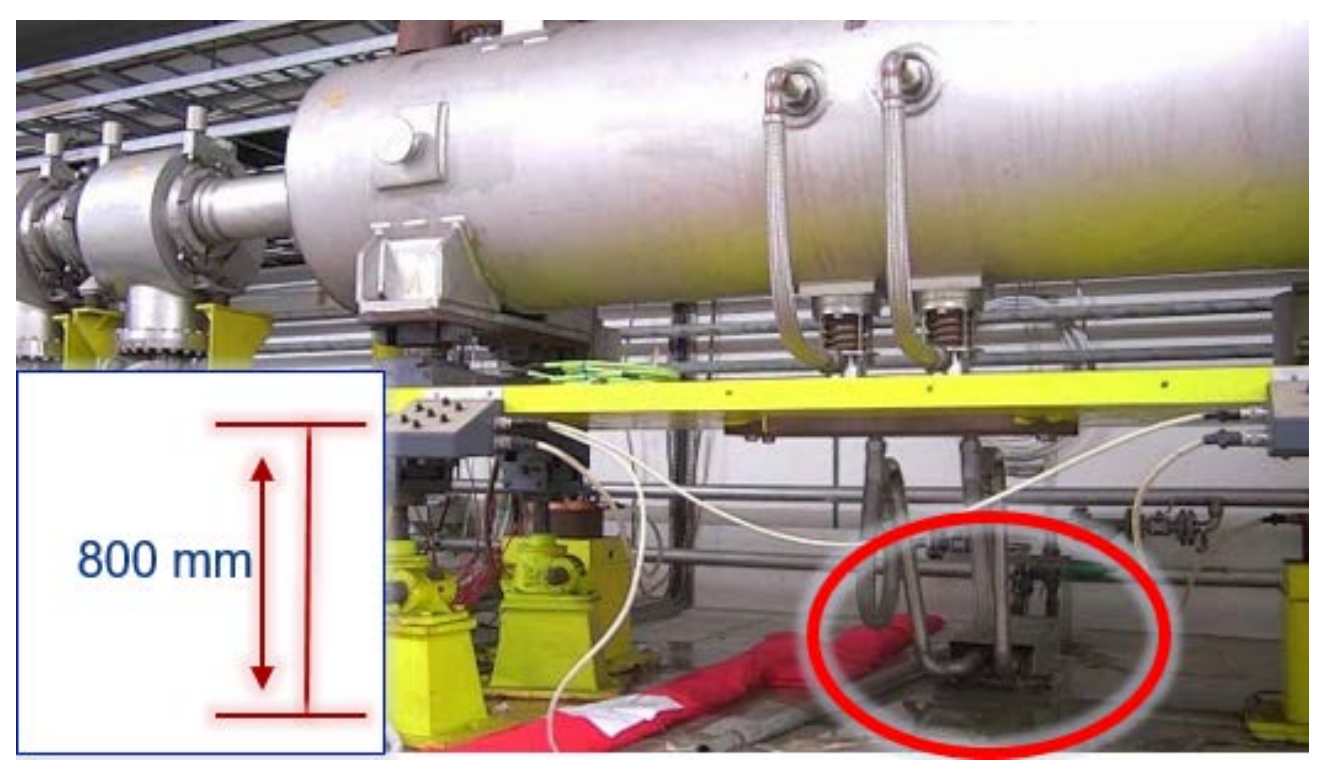

Figure 6.26: Location of the water leak
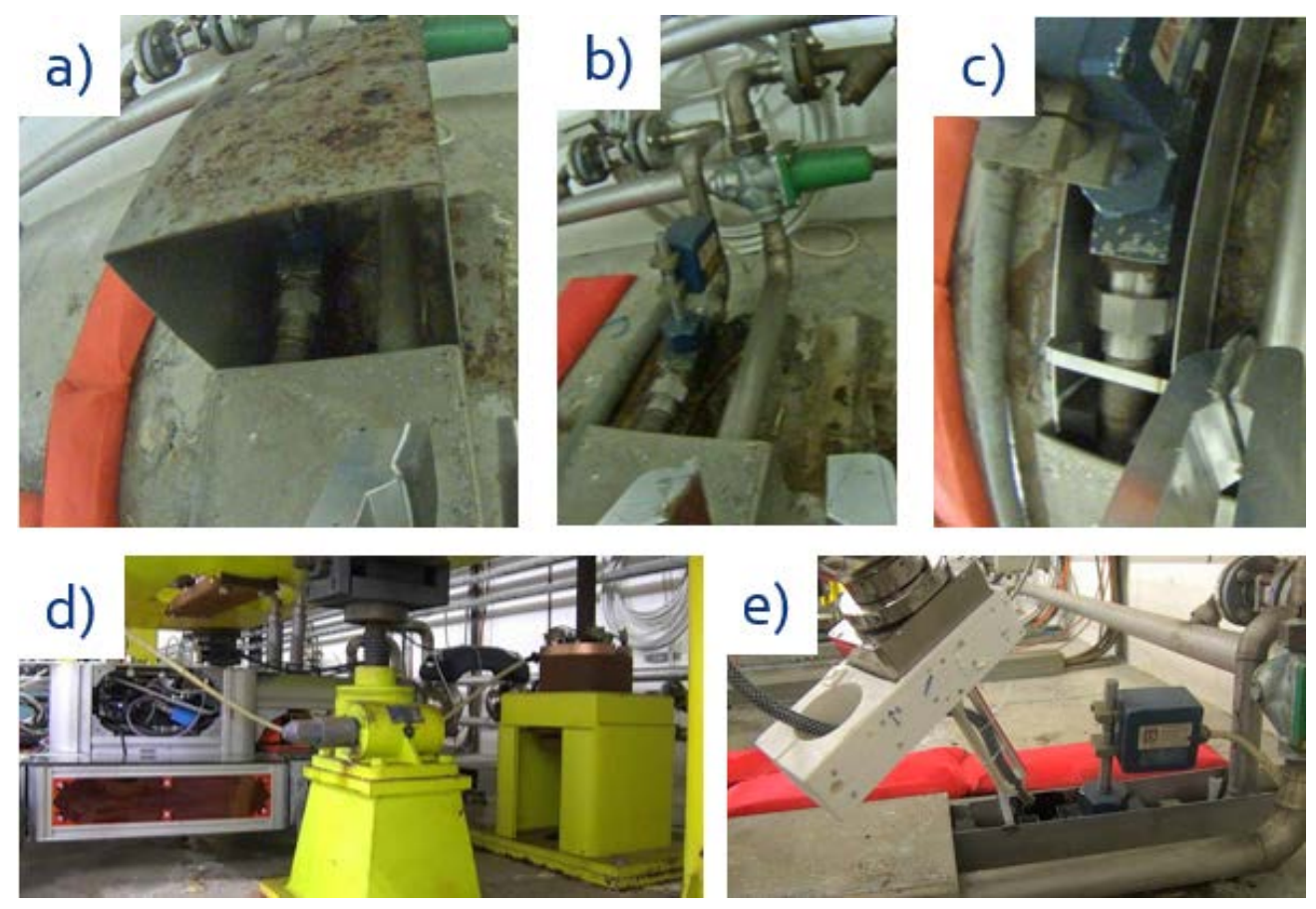

Figure 6.27: Water leak area before (a) and after the intervention with the resin injected (e). Metal cover cut (b). Building the recipient to confine the leak area (c). CERNbot running CERNTAURO framework below the collimator. 

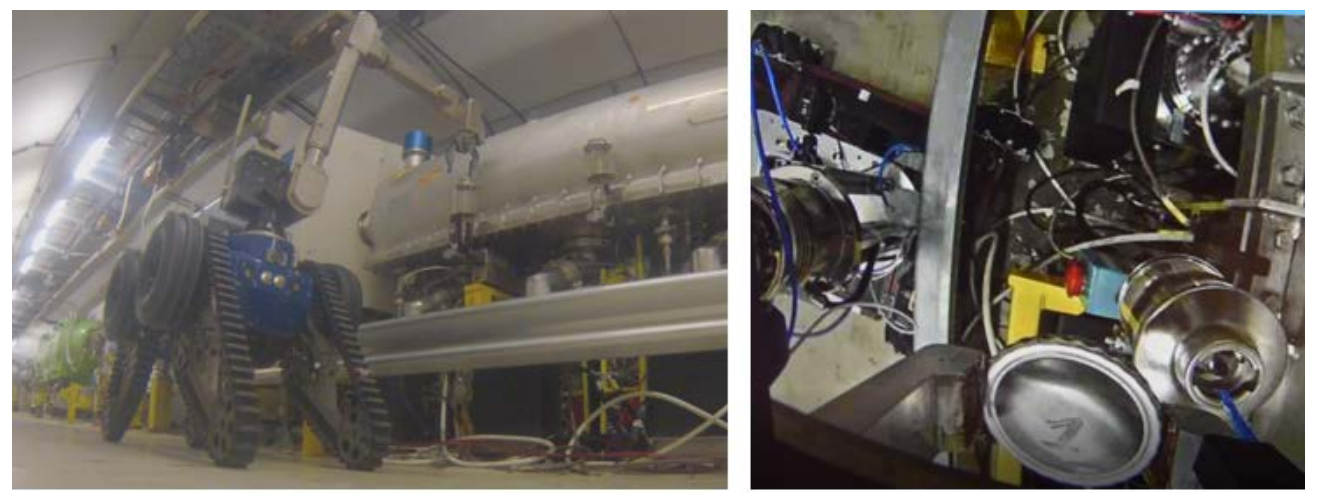

Figure 6.28: Filling of oil recipient done using industrial solution (left) and custom made solution using CERNTAURO framework (right).
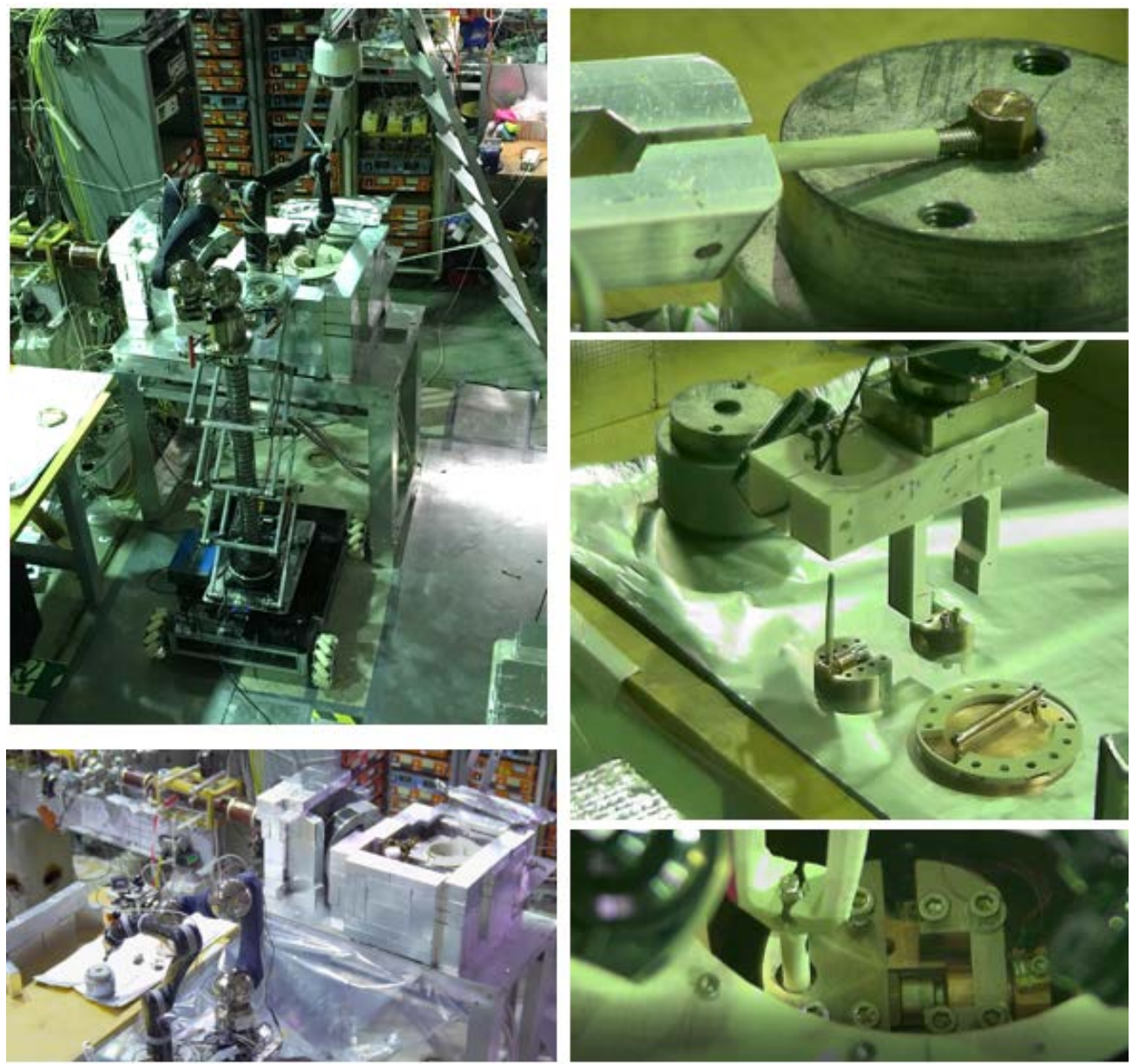

Figure 6.29: CERNbot in dual arm configuration (left pictures) and the iridium radioactive source handling (right pictures) 


\section{Chapter 7}

\section{Conclusions and Future Lines of Research}

In the last years, highly sophisticated algorithms and techniques like deep and broad learning have increased robot perception capabilities, setting the base for future self-sustainable robots and opening discussions on ethical aspects briefly discussed in this work. From the mechatronic point of view, physics is the only limits to human creations but on the contrary, considering modern machine learning techniques, the technological limits are not yet well defined. The motivation of the proposed work is that robots must improve the quality of our lives and work by taking over dangerous, tedious, and dirty jobs which are not possible or safe for humans to perform. Robots can learn and reproduce what humans program but they will never be fully safe, bug-free or have ethical behavior. For this reason, autonomous robots are not currently suitable to intervene for the maintenance of expensive and delicate machines and tele-operation is currently the only solution. A user-friendly robot human interface coupled with artificial means (e.g. learning, virtual and augmented reality) are essential to help the operator perform robotic tasks in a comfortable way, increasing the success rate and safety, and decreasing the intervention time. The presence of variable timedelays and the singularities of the scenes in semi-structured environment have highlighted the needs for the development of a new telerobotic system.

A novel robotic tele-manipulation framework for harsh and hazardous environments was developed, commissioned and is currently in operation in the CERN accelerator complexes. The new framework, based on core-periphery model, is modular and transportable on different robots thanks to configuration layers, which takes several factors into account such as the type of hardware, communication layer, and operator needs. The novel system is adaptable on different robots and can perform unmanned tasks in hazardous and semi-structured environments. Many advanced with respect to the state of the art have been made thanks to this work, like for example the introduction of operational modular robotic controls and the user adaptability. In addition, based on the numerous robotic operations performed at CERN during the testing and commissioning phases of the proposed framework, a best practice work flow for remote intervention has been created allowing robotic operators and intervention supervisors to generate a safe and efficient intervention pipeline including com- 
mon tools to be used. Thanks to the continuous development model adopted, different robots running the CERNTAURO framework have been designed with different mechanical architecture and structure to overcome the challenges and to satisfy the high demands for remote inspection and in-situ maintenance at CERN. In addition, a novel human-robot interface operating several different robots on the field has been developed. A novel object recognition and tracking system which runs in real-time on the robot while the operator is operating it, has been developed. The tracking system enters in the teleoperation loop and helps the operator to achieve the requested goals. For extended plants, novel computer vision system, TInspect, that uses a robust hybrid change detection algorithm to monitor changes on tunnel linings is, presented. The presented framework is composed also by artificial intelligence features, like for example visual based machine learning algorithms and learning by demonstration techniques, that are able to give a robust level of autonomy to the controlled robots, that are able for example to navigate autonomously in unstructured environments.

The solutions provided by CERNTAURO are not only based on the software, but also on electrical engineering, electronics, and mechanical engineering.

The main contributions of this work are detailed in the following, involving designs, robotic controls and mechanical systems realization, experimental setups and real field applications:

- Requirements: the first part of the presented work has been the retrieval of the whole set of requirements for remote inspection and maintenance in hazardous and semistructure environments based on the experience gathered doing these tasks, in the CERN accelerator complexes, using commercial robots, during the first part of the described thesis. The main challenges to accomplish remote intervention in hazardous and semi-structured environments like CERN are the presence of radiation, lack of communication and proper light, time pressure, unknown and cluttered environments, as well as the needs to have robust recovery plans and fail-safe scenarios.

- State of the art: the presented work introduces the current technologies used in application of robotic systems in industry, particle accelerators, power plants and nuclear facilities. Aspects like tele-manipulation, haptic tele-presence, preparation for robotic intervention and operators training have been presented, as well as a small dissertation of ethical aspects in the use or robots in our society,

- Modular robotic framework: the motivation of the presented work have the needs for a novel robotic control system architecture that has been developed, which allows a safe and robust robots control in hazardous and semi-structured environments. The novel control system can run in real time and has different added values with respect to the state of the art of common robotic operating system used. The framework focuses on safety, rapid adaptation of software and hardware to the specific mission, as well as the possibility to control robot teams from a single operator. In addition, the objective is to be used by expert and non-expert users, being easy to plug and play. The proposed control system architecture is endorsed with several modules like 
for example autonomous navigation, relays robots, collision avoidance, environmental reconstruction and objects detection, energy management, communication, learning by demonstration and artificial intelligent technologies.

- Methods for multirobots operations: based on the numerous robotic operations performed at CERN during the testing and commissioning phases of the proposed framework, a best practice work flow for remote intervention has been created allowing robotic operators and intervention supervisors to generate a safe and efficient intervention pipeline including common tools to be used. A novel architecture for cooperative multi-agents scenarios has been developed and several modular and multipurpose remote handling tools used in robotic intervention have been presented.

- Design: different robots have been designed with different mechanical architecture and structure to overcome the challenges and to satisfy the high demands. The Design of the robot has been focused on robustness, availability, modularity and reconfigurability allowing adaptability of robotic systems according to the mission plans and tasks. The robots have been first validated in several mock-ups designed and developed and afterwards used in real field application saving human exposure to dangers at CERN.

- Sharing operational aspects: the above tools and methods have been tested in several field interventions and the operational aspect results are described as well as examples of real robotic interventions at CERN.

- Performance metrix: a novel human-robot interface operating several different robots on the field has been developed. A novel framework based on virtual reality technologies that allows integration, training and safety simulation has been designed and commissioned. The novel interface allows different human-robot interactions, from manual to semi-autonomous. Comparative results in using industrial adapted solution and novel custom solutions, presented in this work, have been shown.

- Robots for hazardous environments and results sharing: A full solution for remote inspection and teleoperation in hazardous environments not compatible with remote handling compatibility has been designed and commissioned. The results and the feedbacks of applying the proposed solution in challenging environments have been presented.

- Object detection and tracking of metallic pieces: With the objective of providing a safer system, higher-level applications can be integrated in the interface in order to provide some help to the operator, without relying uniquely on his/her capacities. This work presents a novel object recognition and tracking system which runs in realtime on the robot while the operator is operating it. The tracking system enters in the teleoperation loop and helps the operator to achieve the requested goals. The system is optimized to track featureless objects such as metallic plates, metallic connectors and monochromatic objects. 
- Plants structural monitoring: a novel computer vision system, TInspect, that uses a robust hybrid change detection algorithm to monitor changes on tunnel linings is presented. The system achieves a high sensitivity and accuracy. The proposed system is also congurable through dierent parameters to adapt todierent scenarios, making it useable in differel tunnel environments and plants.

The main added value with respect to the state of the art in robotic applications present in other particle accelerators and power plants, are summarized in Table 7.1

Table 7.1: Main added values of the CERNTAURO framework with respect to other particle accelerators and power plants.

\begin{tabular}{||c|c|c|c|c|c||}
\hline & CERN - CERNTAURO & DESY & SNS & JET & ITER \\
\hline Manual robot control & YES & NO & YES & YES & YES \\
\hline Supervised control & YES & NO & NO & NO & YES \\
\hline Autonomous behaviours & YES & NO & NO & NO & NO \\
\hline Multimodal controls & YES & NO & NO & NO & NO \\
\hline Modular robots & YES & NO & NO & NO & NO \\
\hline Cooperative robots & YES & NO & NO & NO & NO \\
\hline Passivity controls & YES & NO & NO & NO & NO \\
\hline Challenges for communication & YES & YES & NO & NO & NO \\
\hline
\end{tabular}

In literature and in real application, robotic control systems that covers all the aspects of the robotic interventions, from its preparation until the realization and the robots operation including recovery scenarios, do not exist. Consequently, thanks to the various novelties and added values of the CERNTAURO system with respect to the state of the art, the presented framework could be considered as benchmark for robotic control systems in harsh and semi-structured environments.

The CERNTAURO architecture has demonstrated to be very efficient in terms of reliability and safety, according to the results obtained from more than 80 real interventions. A future line of research could be the preparation of the architecture for cooperative multirobot interventions, such as grasping and transporting objects (e.g pipes), that nowadays are being performed by using two operators, with also two mobile manipulators, in addition to experiments to localize the robot using low radio frequencies. As can be appreciated in Figure 3.3, the enhanced architecture for cooperative multi-robot interventions is prepared to deploy a Wifi Local Area Network on the robotic side, having a Leader as the main mobile platform, where the data distribution unit module is running, acting also as router from the Wifi LAN Robotic team and the external 3G/4G network. Also, from the user interface side, in order to enhance the safety of the operation, is agreed to have an expert operator per mobile manipulator, having a leader operator that has access to the Cooperative Planner user interface module, where responsibilities can be shared with the assistant operator, in 
terms of supervising a given mobile manipulator during the intervention phase. Also, the leader operator will assign the roles of robotic relays and autonomous robots, which will for example follow a particular leader to give a specific external point of view of the target during intervention. 


\section{Appendix A}

\section{Novel Robots Developed}

*The Intelligent Train Inspection Monorail for the LHC

The Intelligent Train Inspection Monorail (I-TIM) [406] is a robotic system completely designed and developed for CERN's specific needs in order to provide unmanned actions in tunnel areas, such as the Large Hadron Collider (LHC) tunnel. I-TIM is a monorail robot running on the tunnel's ceiling able to perform several missions, some completely autonomously, such as reconnaissance, radiation surveys, visual inspections, civil infrastructure monitoring, remote helium leak detection, fire detection and survey alignment measurements of accelerator devices.

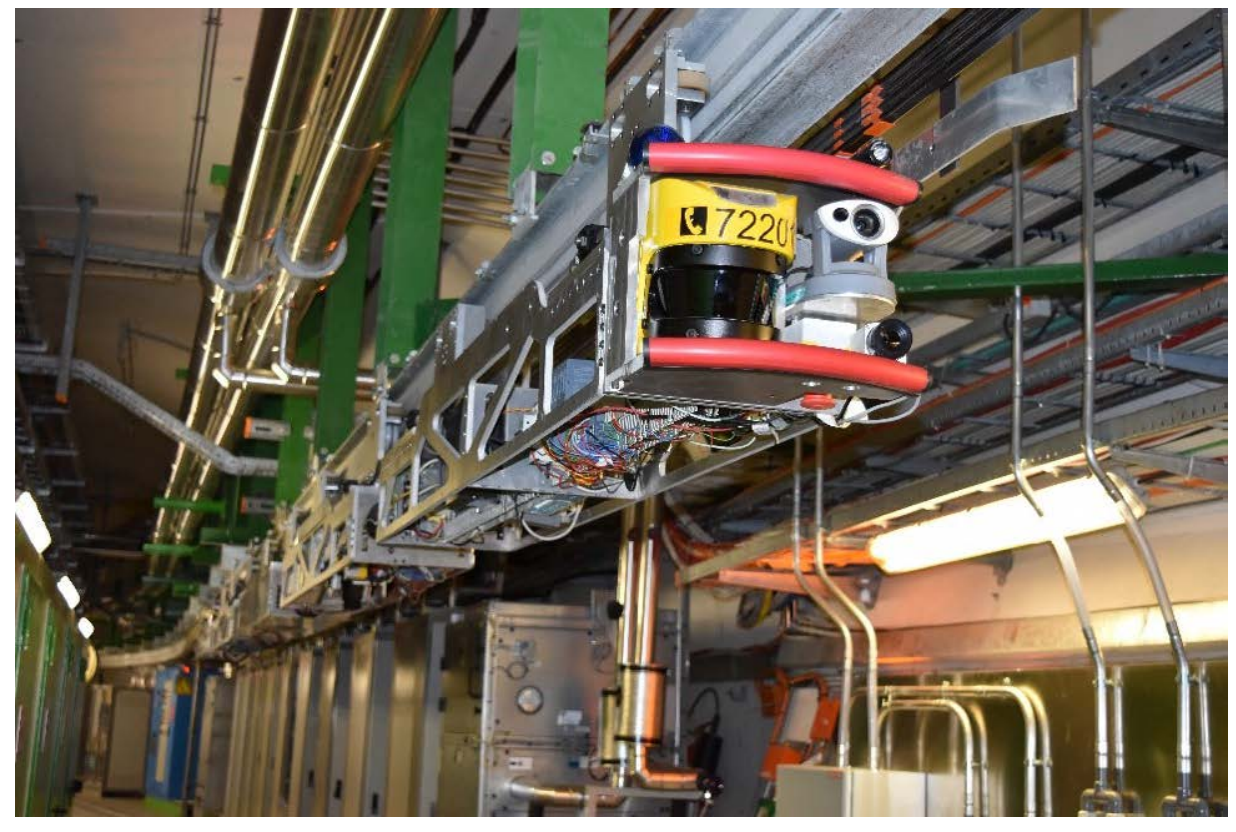

Figure A.1: The Intelligent Train Inspection Monorail (I-TIM)

I-TIM consists of a battery-powered vehicle running on a monorail with an I-beam profile linked to a tunnel ceiling. Even though i-TIM was specifically designed for the LHC tunnel 
at CERN, its mechanics can be adapted to any kind of I-beam profile monorail, making it suitable for running in any kind of underground tunnel equipped with a monorail. I-TIM is composed of different wagons (see Figure A.3), each one designated for different tasks. Different configurations are possible by adding or replacing wagons, while the three main wagons remain the same in all configurations. The first wagon is the control wagon, containing the main electronic controller, cameras and safety anti-collision system. The control wagon is followed by the battery wagon, containing two $12 \mathrm{~V}$ lead acid batteries connected in series for powering the complete system, and featuring as well a robotic charging system for connecting to the available power rail next to the monorail all through the LHC tunnel. This way, I-TIM can charge its batteries anytime and anywhere, by simply connecting itself, when not moving, with the robotic charging arm to the power rail. Following the battery wagon, the motor wagon contains the main traction of the I-TIM system. Composed by a DC motor and a motor driver, the motor wagon is the core of I-TIM. Whichever is the last wagon of the system, it must replicate the safety and anti-collision system of the control wagon. In between the motor and the last wagons, I-TIM can be equipped with several different payload wagons, according to the needs of the mission to be performed, and its modular design allows new types of wagons to be easily added in the future, without major changed to be implemented in the existing system. An example of a I-TIM payload wagon is the radio protection (RP) wagon, which is featured with a robotic arm carrying a radiation probe, that is deployed from the wagon in order to measure the radiation of the LHC accelerator equipment at the beam height (see Figure A.2). Payload wagons may contain different sensors and mechatronic systems, according to the needs of the operation. I-TIM's mechanics are as well designed with the maximum possible dimension, 30 by $30 \mathrm{~cm}$, that allows the robot to pass the sector and ventilation doors of the LHC. The length of each wagon is of $180 \mathrm{~cm}$ (see Figure A.4). 

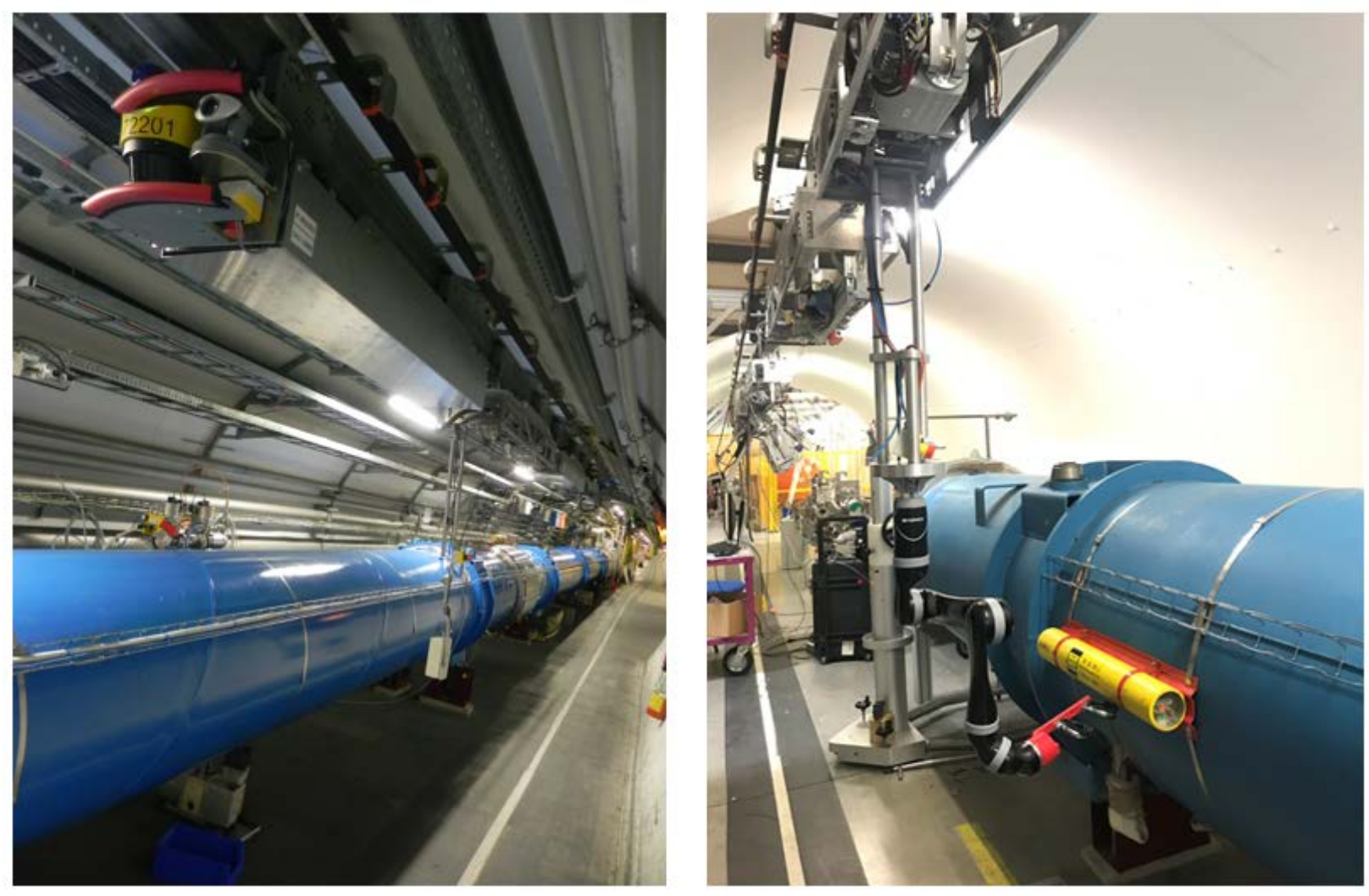

Figure A.2: The robotic arms integrated on the i-TIM robot. A one degree of freedom arm has been integrated for the first robot version (left). A six degrees of freedom arm is integrated in the current robot version (right).

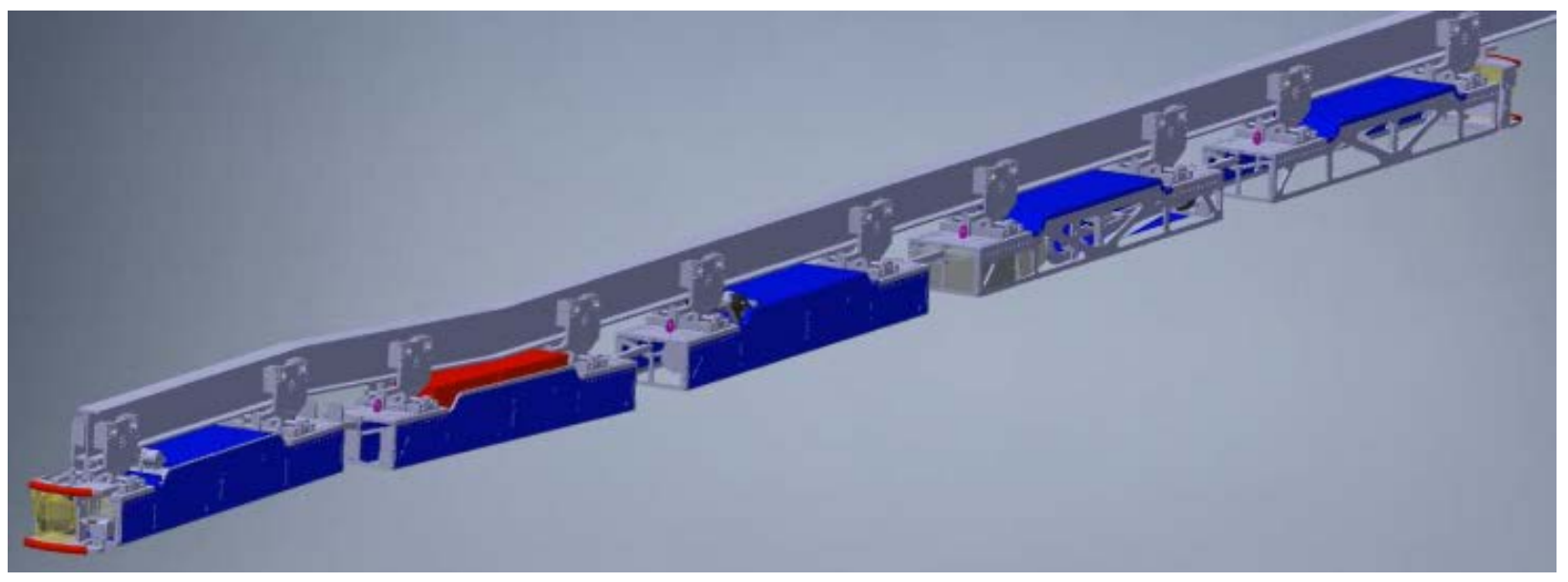

Figure A.3: 3D model of I-TIM mechanical configuration 

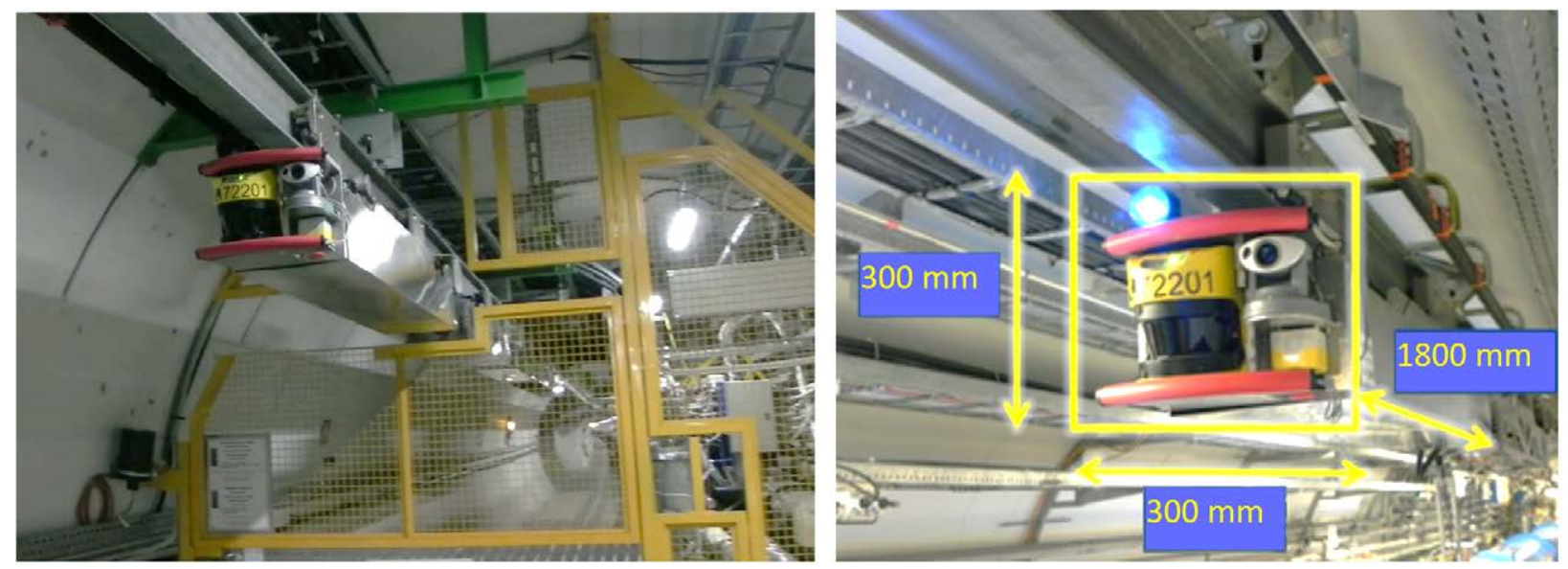

Figure A.4: I-TIM passing a sector door in the LHC and dimensions of one wagon

\section{I-TIM electronics and control system}

In order to guarantee the modularity of I-TIM when adding or replacing a wagon, the electrical design has to be adaptable. For this, the connection in between wagons is standardized with a modular connector system, which allows the different types of signals to pass from one wagon to another. By designing the new wagon compatible with these signals, the compatibility is guaranteed. The I-TIM embedded control is based on a fail-safe PLC for the low-level safety and a NUC running Linux for high-level control purposes. The control system is designed in layers (see Figure A.5), in which the main parts are the hardware abstraction layer (HAL) and the supervision layers. Composed by sub-layers, the two main control layers guarantee modularity, reusability and portability [407]. This is part of the CERNTAURO framework. The lowest sub-layer of the HAL contains the PLC and the Safety PLC inputs and outputs, where the system communicates directly with all sensors and actuators of the system at the lowest level. Then, the hardware abstraction layer contains the low-level functionalities of the system, such as the positioning, motion, vision and communication. In the supervision layer, the managers are the functions in charge of the main functionalities. The application framework layer contains the autonomous missions, recovery scenarios and artificial intelligence from the highest level of the on-board control. The interface with users and operators is done through the graphical user interface, e-mail notification and SMS control in case of emergency. This architecture allows changes and additions in the very low level that will never affect the rest of the system, as well as changes and additions of different payloads. All the data, obtained from a survey with I-TIM, is collected on board along with the Timestamp and their precise position in the order of 1 $\mathrm{cm}$. 


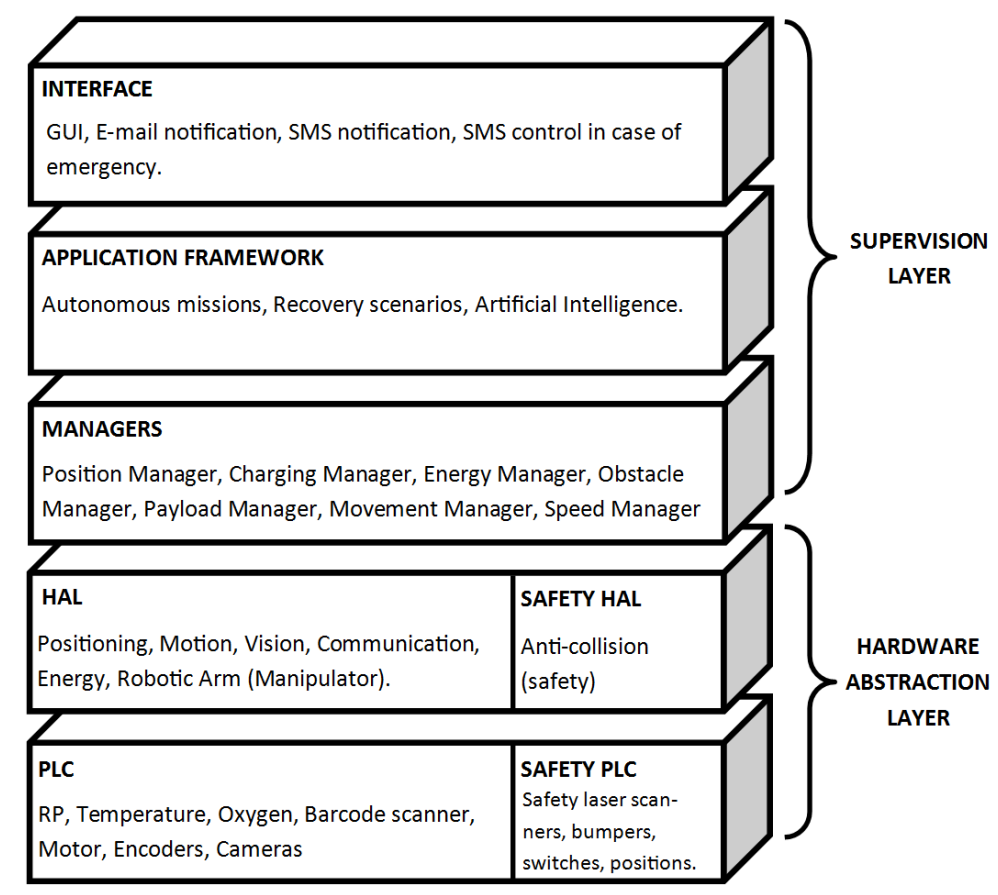

Figure A.5: I-TIM control architecture in layers

\section{i-TIM safety}

Several i-TIM features guarantee the fail-safe system, such as the PLC safety program in LAD programming logic. The safety laser scanners installed at both ends of i-TIM are connected to the speed encoder and the PLC controller, so they can regulate the maximum allowed speed of i-TIM in case of obstacles in the field of view. The safety bumpers on both ends of i-TIM are connected directly to the fail-safe PLC inputs and included in the safety program. In order to guarantee human safety operating nearby and around i-TIM, safety volumes are created around all the deployed mechatronic systems, especially the robotic arms, though the installation of a series of distance measurement sensors allocated both at the wagon structure and along the mechatronic system [408]. This avoids collisions with the accelerator's devices and ensures safe human operation around the robot. Figure A.6 shows a possible scenario where an extended robotic arm is in risk of collision. The threshold distance is set so the arm can safely overtake Obstacle A. However, the arm is in risk with collision with Obstacle B, as it violates the threshold distance, therefore, when detected, I-TIM will stop the movement and safely retract the robotic arm in order to avoid the collision. Figure A.7 then illustrates the front part of the wagon carrying the robotic arm where 8 sensors evenly spaced in between them are installed. By installing 8 sensors, the minimum threshold is significantly decreased (reducing the blind areas) in comparison with a configuration including less sensors. 


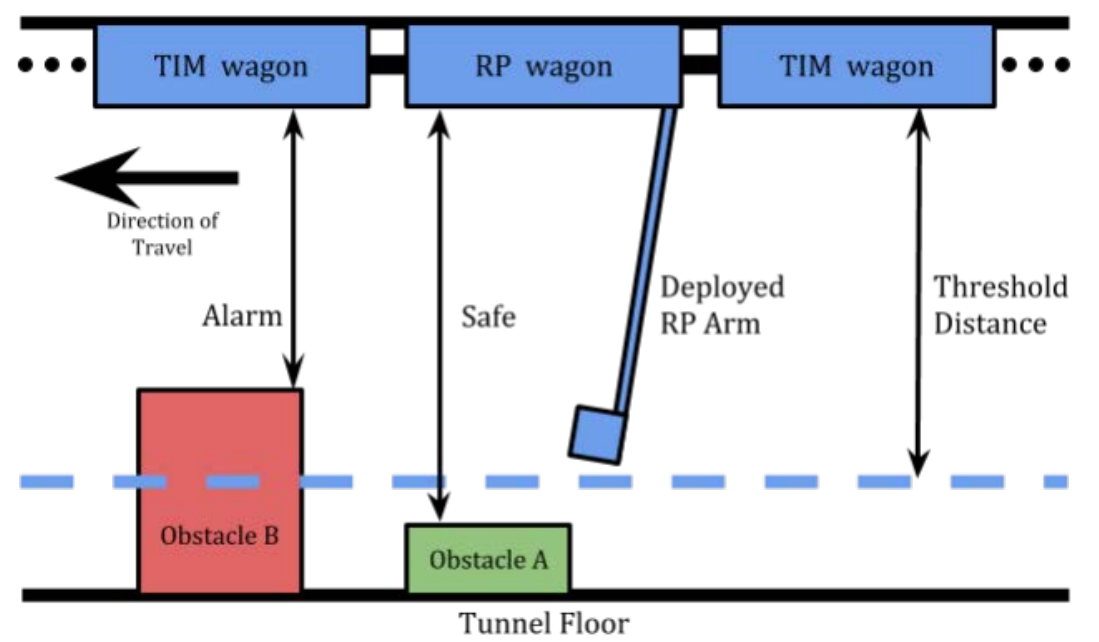

Figure A.6: Possible operational scenario with a robotic arm deployed from I-TIM

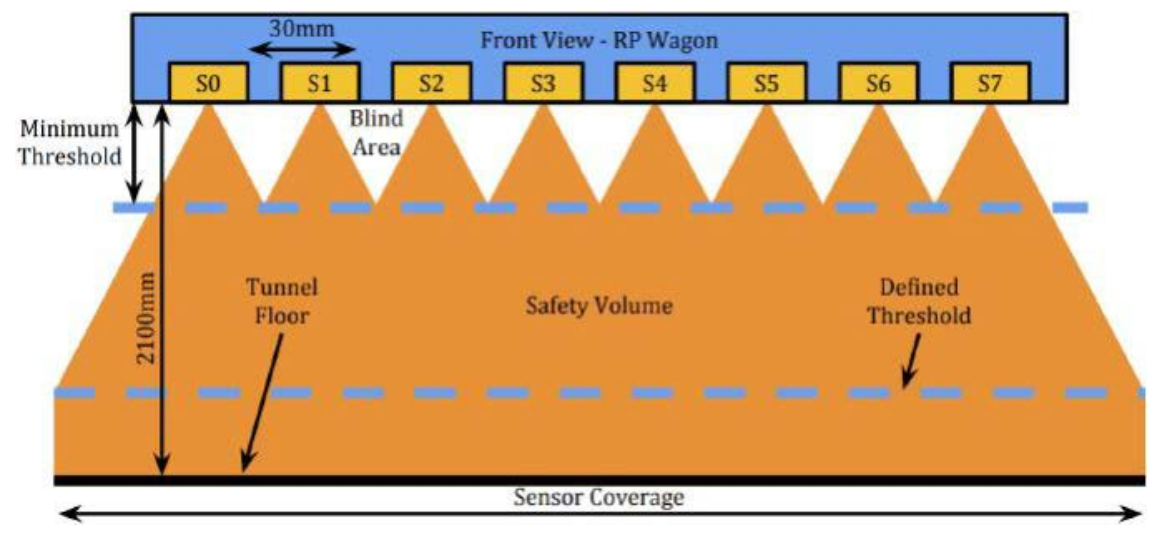

Figure A.7: Coverage provided by 8 sensors installed on a i-TIM wagon

\section{I-TIM payloads and configurations}

The actual existing payloads for I-TIM are listed below:

- Radiological measurement (RP): this payload wagon includes a six-axis robotic arm that places a radiation probe at the beam height for measurements along the LHC machine.

- Tunnel structural monitoring: a photogrammetry system, consisting of a camera taking high definition pictures of the tunnel while I-TIM runs and an on-board data processing system comparing current and previous images, in order to detect tunnel degradation signs like walls cracks and structure deformations.

- Multi-axis robotic arm: a novel wagon designed for featuring one multi-axis robotic arm that can move in space using precise position control, placing sensors or cameras 
in a desired position with respect to the LHC equipment to inspect. Moreover, these wagons are used for tele-manipulation with single or multiple robotic arms.

- Collimator alignment survey: consisting of two wagons, it includes the sensors, cameras and mechatronic systems for detecting collimator misalignments in the LHC using photogrammetry.

- Vacuum leak detection: a wagon equipped with a multi-axis robotic arm that activates a nozzle connected to a helium bottle to flush helium on equipment under ultra high vacuum searching for vacuum leaks.

\section{I-TIM operation}

The Human Robot Interface (HRI) of I-TIM is used from the surface by the operators, who can set up the type of operation or mission to perform and follow its progress live (see Figure A.8). Visual inspections are performed through the HRI's displays of all the on-board cameras (thermal camera, PTZ cameras, 360 cameras). I-TIM is able to perform complete inspection missions, like radiation measurements and safely recover to its garage position afterwards, without need of human intervention during the operation, so the operator configures the mission by selecting the accelerator tunnel sectors to inspect, the type of mission and the option of self-recovery after it has finished inspecting. Then, I-TIM autonomously moves to the mission start position, deploys all mechatronic systems and sensors needed, and starts moving towards the end position while taking the measurement data. The operator can follow the mission at all times through the HRI's indicators and camera feedbacks. Once the mission has finished, the data is published on a dedicated website and sent to the operator and the LHC equipment responsible by certified e-mail.

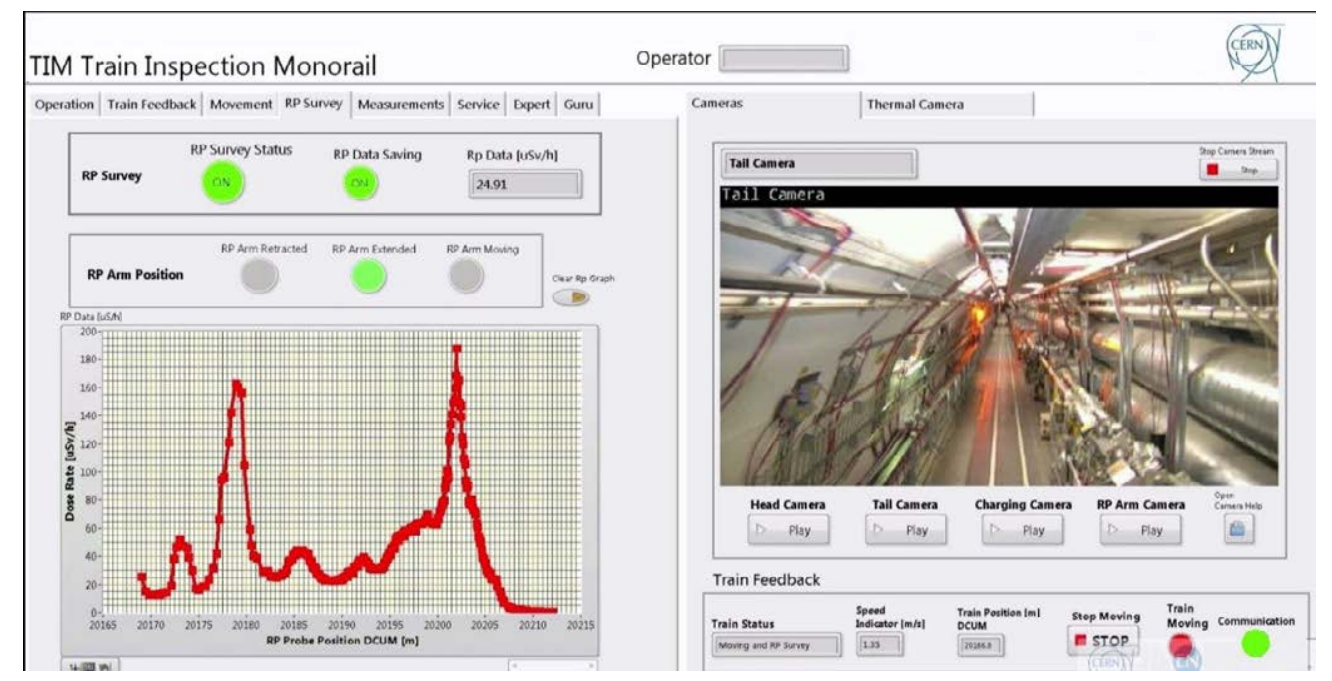

Figure A.8: I-TIM Human Robot Interface 


\section{CERNbot}

CERNbot is a novel modular robotic base system [409] (see Figure A.9), designed and built at CERN within the work of the CERNTAURO framework, with the goal of guaranteeing autonomous inspection and supervised telemanipulation in accelerator and experimental areas. The robot was designed to operate in hazardous and unstructured environments and to guarantee maximum flexibility in terms of mechanics and electronics. With a structure divided in subsystems, each subsystem can be modified separately and adapt to the general functioning of the robotic platform. This allows multiple configurations of the robot according to the specific needs of the intervention to perform.

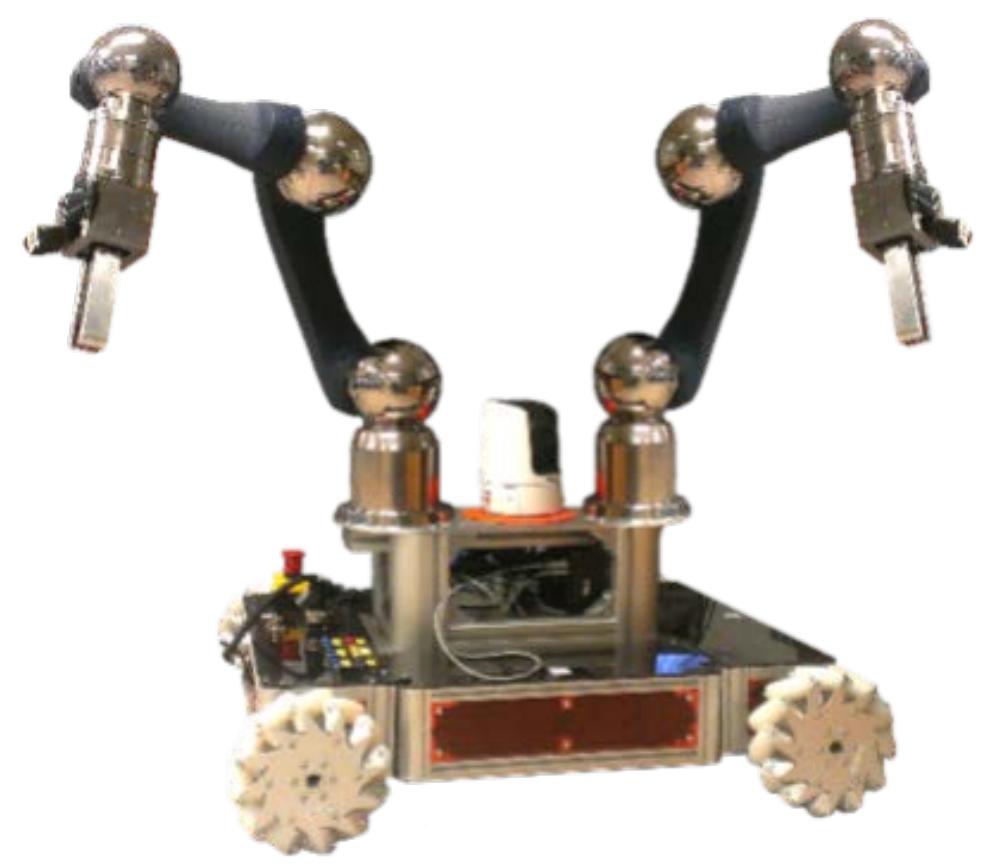

Figure A.9: Multi-functional reconfigurable CERNbot platform

\section{CERNbot mechanics and modularity}

During the preparation period of an intervention, the mechanical CERNbot configuration is adapted to the new operation requirements, as well as the CERN Robotic Framework software configuration, which dynamically adapts according to the connected sensors and manipulators. The structure of CERNbot is divided in subsystems as it is depicted in Figure A.10. 


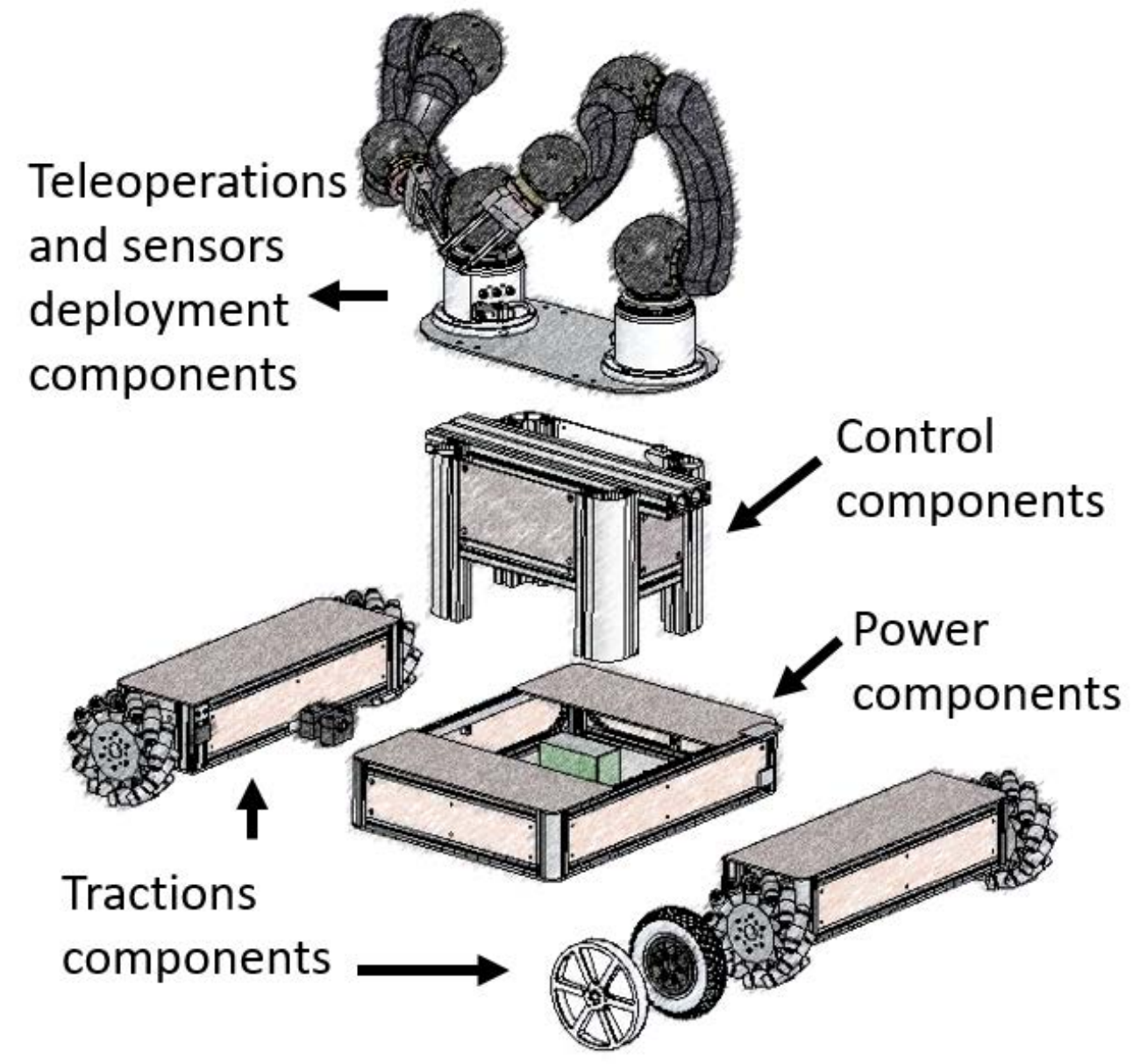

Figure A.10: CERNbot modular architecture

The traction part has a symmetrical design in order to facilitate maintenance and spare parts. Different kinds of wheels can be accommodated on the robot, including omnidirectional mecanum wheels, mainly used for teleoperation tasks, and pneumatic wheels, used when there is the need of covering long distances during inspection and reconnaissance missions. The traction module guarantees the CERNbot a maximum speed of $6 \mathrm{~km} / \mathrm{h}$, in a condition of maximum load of $160 \mathrm{~kg}$. CERNbot is designed to overcome obstacles, such as cables with a maximum diameter of $40 \mathrm{~mm}$. This feature results very efficient in real interventions in accelerator areas when approaching difficult targets for which cables and pipes must be overcome. A passive joint is integrated in the middle of the robotic base, in order to reduce mechanical frictions and to be able to move the traction block in an orthogonal way with respect to the robotic base, which gives the possibility of lifting one wheel with respect to the others, giving stability and elasticity to CERNbot. The central part of the robotic platform is dedicated to the power storage and management, which allows an autonomy of 6 hours. The batteries are located in the lateral part of the module and at the lowest point. This guarantees the mechanical stability of the platform. Regarding the type 
of operation, different robotic arms can be mounted on CERNbot. The core of the system can as well be connected to a crane in cases in which accessibility from the ground can be a problem (Fig. A.11).
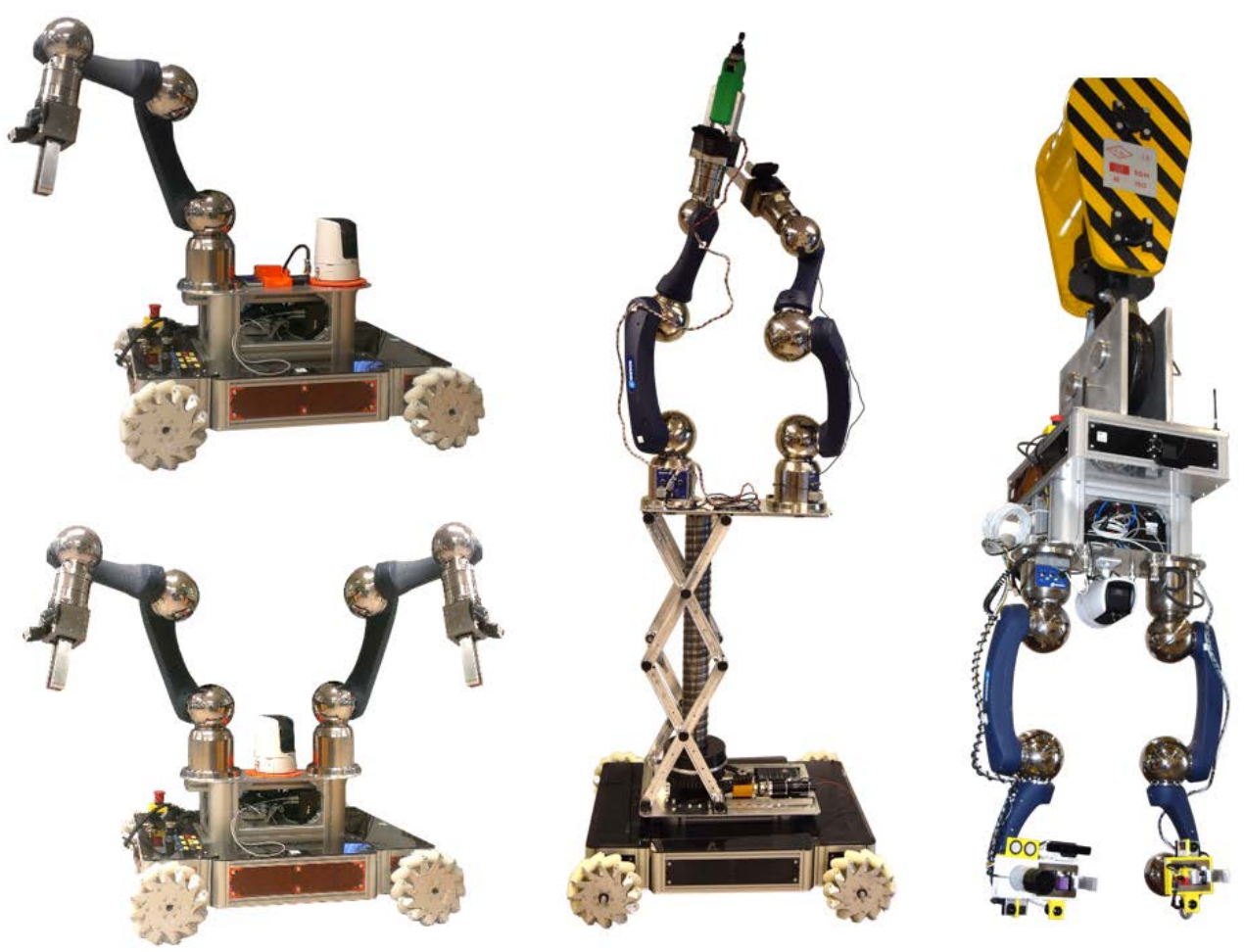

Figure A.11: CERNbot in different configurations

In cases in which the robotic arms have to reach a very high target and the height of the base is not enough, a second CERNbot platform is available featuring a mechanical lifting stage. The lifting stage height is adjustable, and it can bring the top platform of CERNbot up a maximum height of $160 \mathrm{~cm}$. The maximum load of the lifting stage is 400 $\mathrm{kg}$, which allows the possibility to install any kind of robotic arm or arms, and a huge variety of cameras and sensors. The batteries of CERNbot provide $24 \mathrm{~V}$ to the system. The motors are connected to the motor drivers, which are directly connected to the batteries. A micro-controller at the battery output takes care of computing the state of charge of the batteries, the charging manager and the safety. The control board of CERNbot needs an input voltage of $19 \mathrm{~V}$, therefore, a DC/DC converter is connected between the batteries and the board. Other DC/DC converters are needed for powering the different sensors and arms for each CERNbot configuration. Different power outputs are available through the chassis of the platform, so any kind of device needed can be immediately connected. There is as well a LAN connection available and a $4 \mathrm{G}$ modem, in order to connect any $\mathrm{PC}$ to the robot LAN through tunneling. In the following, the specific different configurations of the CERNbot are descibed in details. 


\section{CERNbot different setups}

CERNTAURO looks for adaptability to every constraint could appear in the infinity range of different interventions request from along of CERN's facilities. In the following the different CERNbot setups and versions are listed:

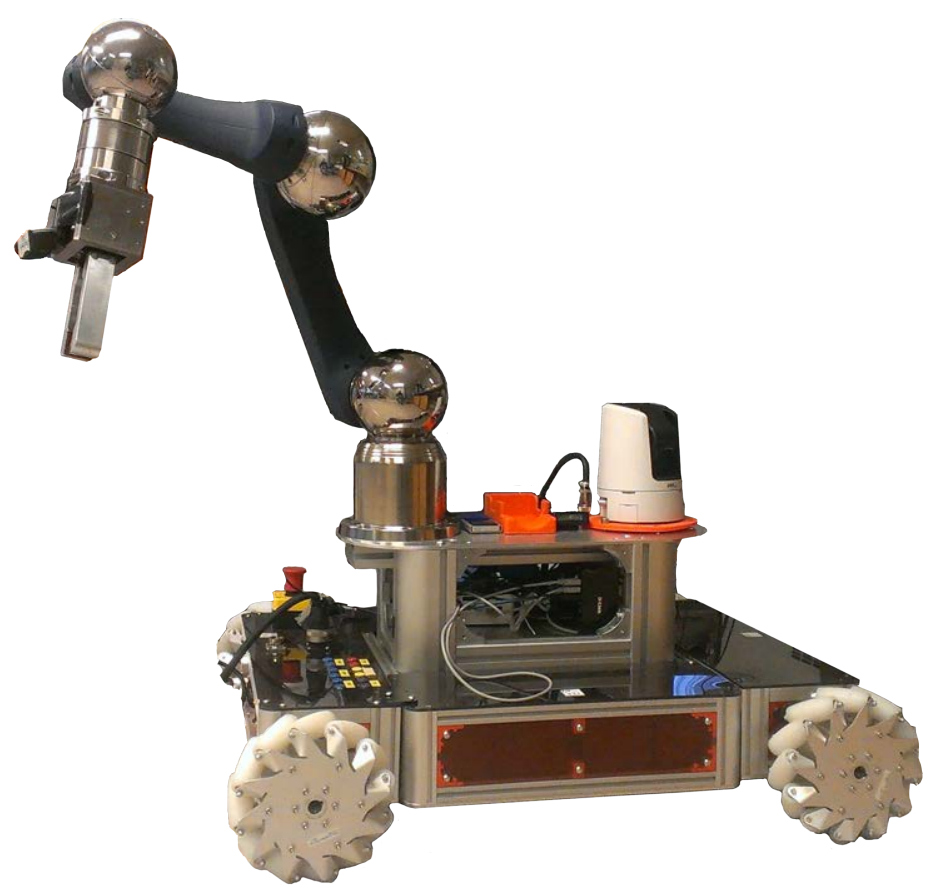

Figure A.12: The CERNBot1 with one robot arm.

- CERNBot1, shown in the Figure A.12 on its normal configuration (one robotic arm and a Pan-Til-Zoom (PTZ) camera). It is the standard version, the base from the different setups implemented derive with the aim to fulfil the necessities of each task and are listed below:

- Dual-arm configuration. In Figure A.9, it can be seen how the PTZ-Camera is relocated on the centre of the stage, leaving the two extremes free to attach a robotic arm on each. Then the collision avoidance systems (see Section4.1) is switched on allowing the two arms working simultaneously fearless of crash one to each other (including the PTZ-Camera).

- Scorpion. Nuclear facilities needs to perform high safety measurements in order to avoid radiation leaks. Thus, interventions that require to cut something, has to pay special attention on the possible items that might be generated (as for example dust). That means the majority of the time, the usage of electronic tools such as saws can not be used for the purpose. It is because of this (see Figure A.13) a special cutter was set it up, which was really useful to cut pipes tasks. 


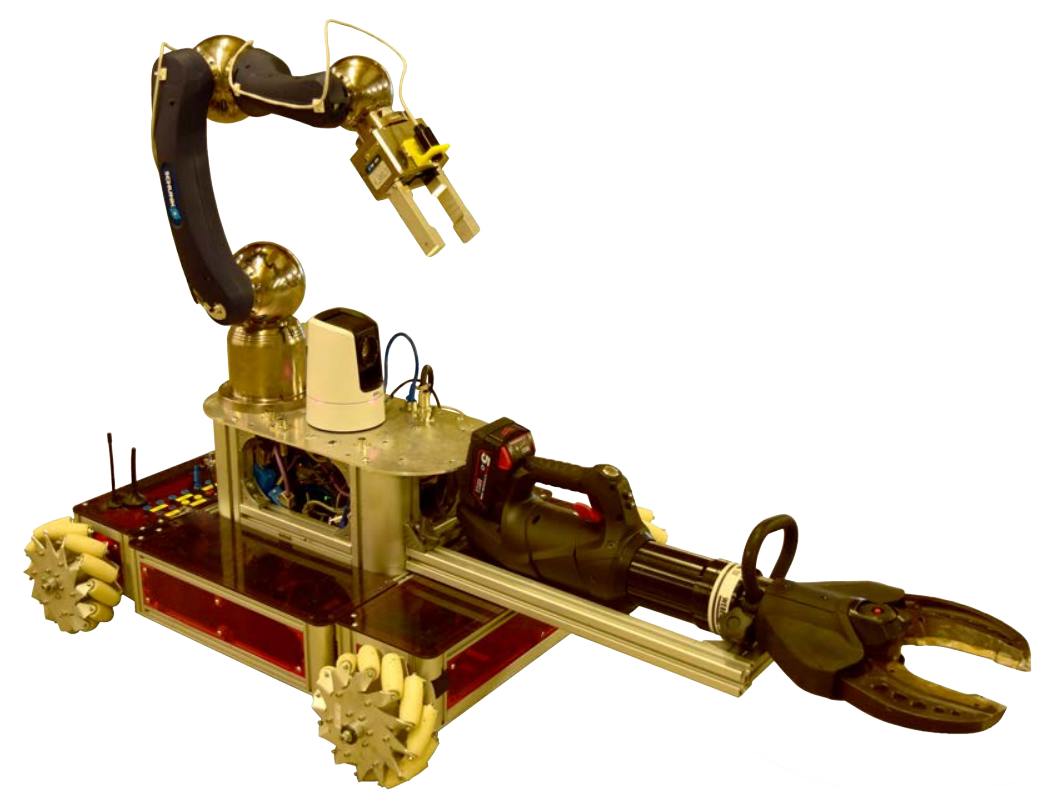

Figure A.13: The CERNBot1 with cutter.

- Comodo Dragon (see Figure A.14). New position, orientation, and extension for the robotic arm. In addition the PTZ-Camera has had to be relocated because of this, thus the end-effector can be always tracking by the operator as a second view (critical for a proper execution task). Now, our CERNBot is capable to fit in a farther and lower scenarios.

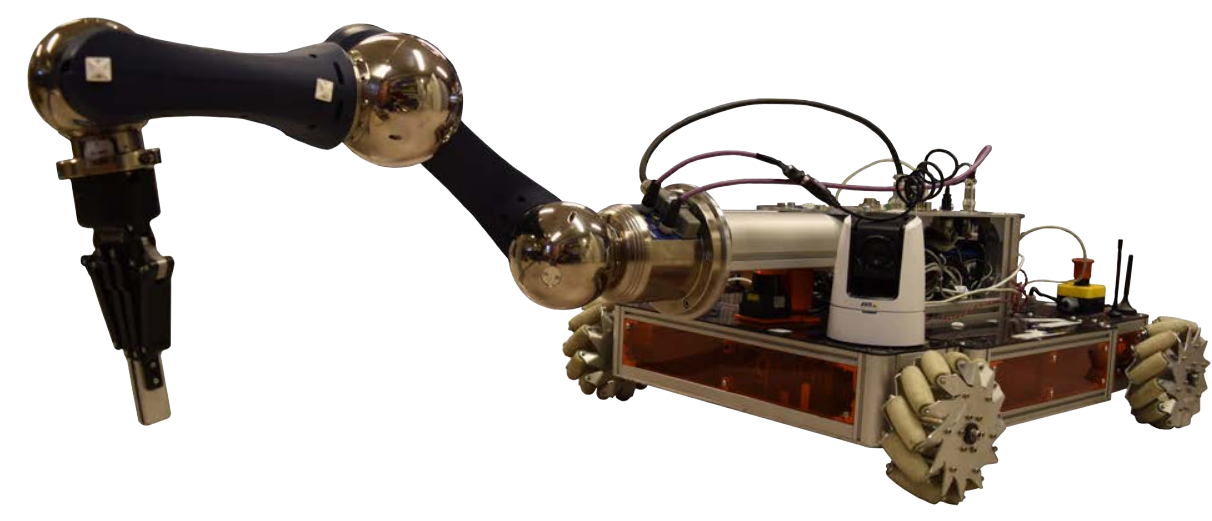

Figure A.14: The CERNBot1 with the robotic arm in front position.

- Turtle. As the setup explained just above, this configuration try to pull down the height of the robot. It is more comfortable from the operator side, since allow to open the field of view due to the fact the area where the robot arm is attached is 
bigger than the front or rear part, instead of the Comodo Dragon that close this field of view.

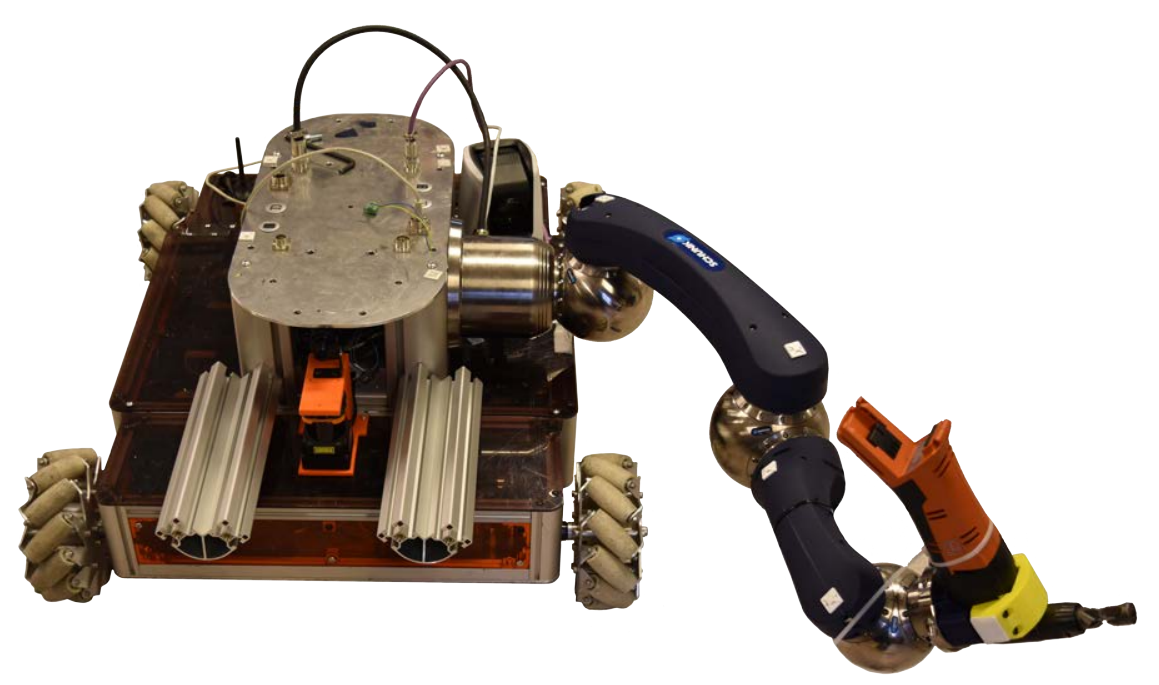

Figure A.15: The CERNBot1 with the robotic arm in one side.

- Camera prolongation. Figure A.16 shows one of the setups that were used to perform this kind of tasks regarding to the necessities.

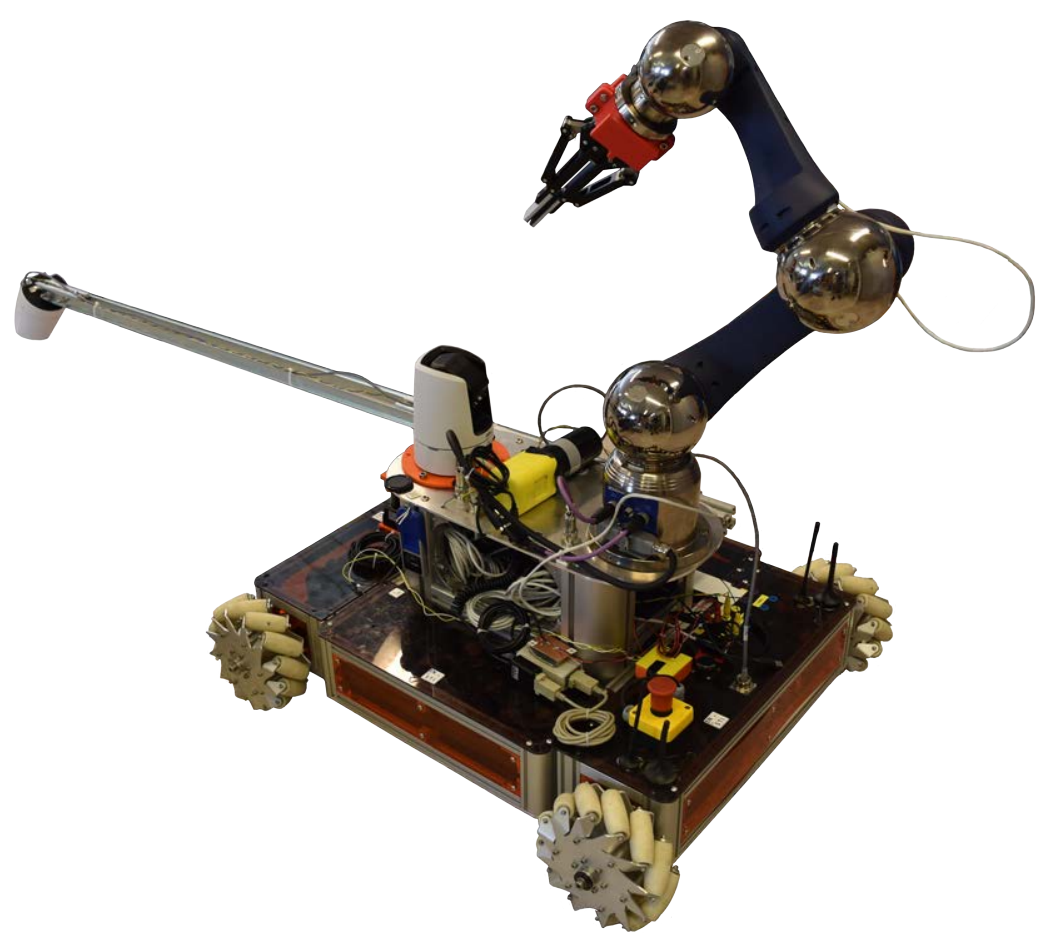

Figure A.16: The CERNBot1 ready for inspection. 
- Helium injector. As it can be seen in Figure A.17, the structure of the CERNBot is a good host for any kind of guest. Adding a few spares pieces, any object (in this matter a helium bottle) shall be perfectly held. Furthermore, for this occasion, the robotic arm was pulled up to reach the target regarding the specifications got before the intervention.

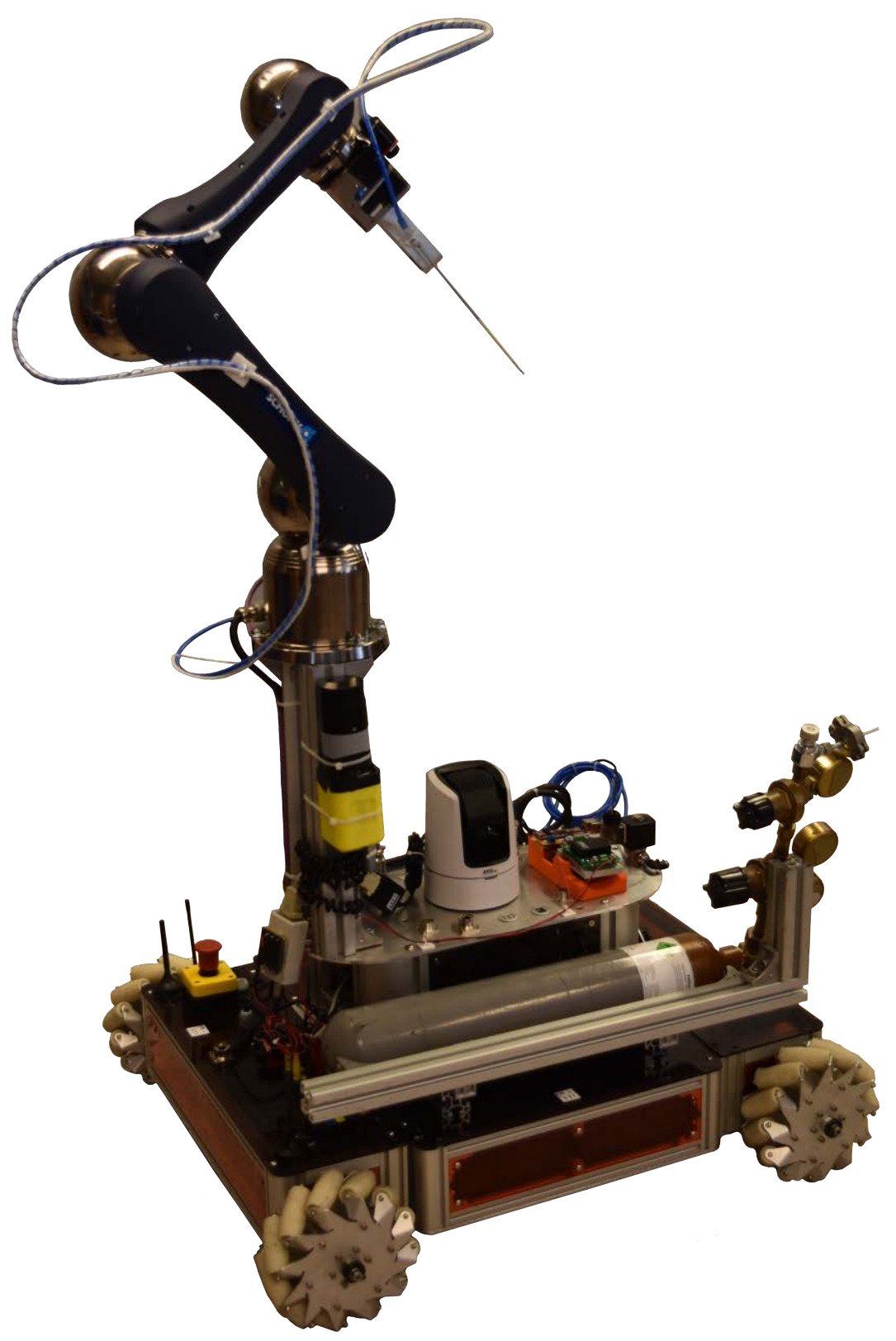

Figure A.17: The CERNBot1 with helium injector system attached. 
- InspectionBot (see Figure A.18), due to the fact the inspection of the target area is usually the first step to carry out the interventions in the most suitable way, a hundred percent robust and friendly use robot has been develop to make easier the inspections tasks, with very slim size (50\% of the CERNBot1 dimensions) and, fitted with an extendable camera, that even could be setup to carry two Schunk robotic arms.

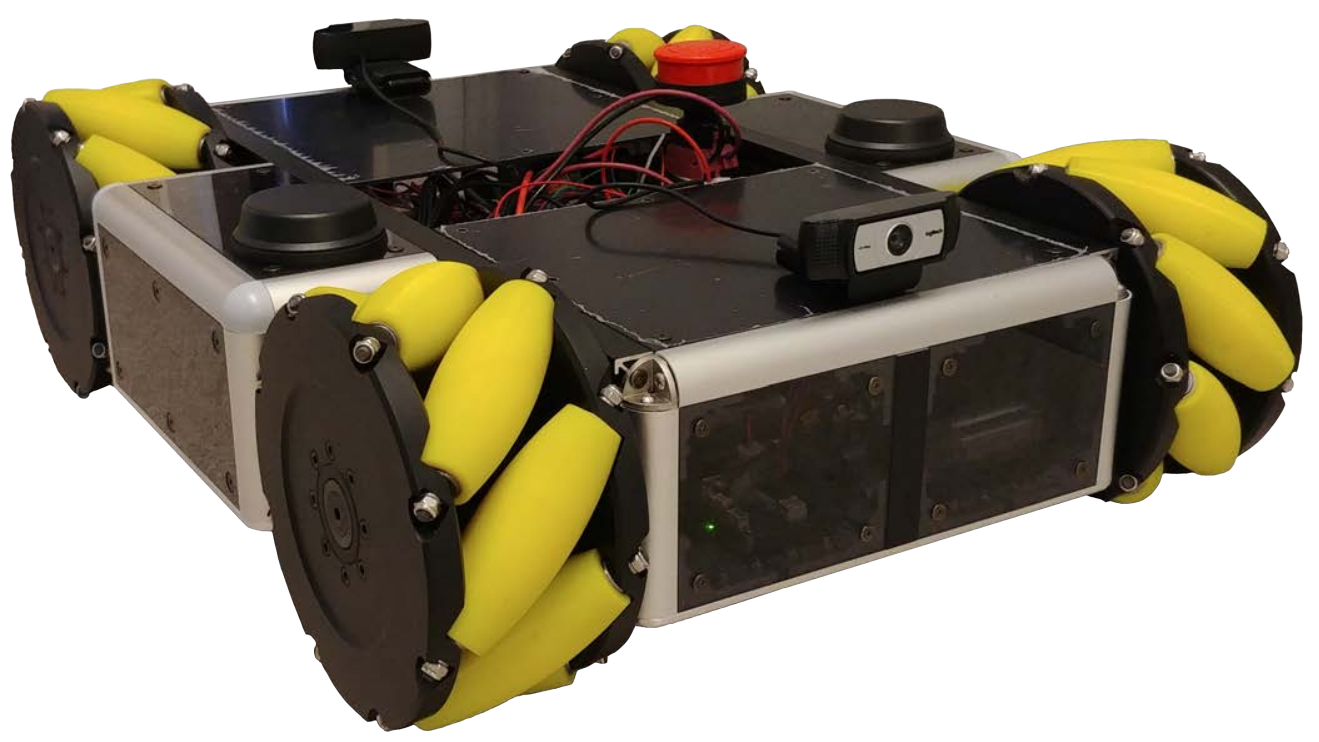

Figure A.18: The CERNBot release for visual inspection.

- CERNBot2. The most important update comes with the aim to overcome the limitations that CERNBot1 platform got by the time to carry out task in a great height scenarios, so it was seek the right way to improve adaptability to such environments. Thus, a new module to pull-up the CERNTAURO's base-line has become upon essential piece, a lifting stage capable to reach up to to $1.6 \mathrm{~m}$ from the initial $20 \mathrm{~cm}$ height (a $800 \%$ of its origin height), with $400 \mathrm{~kg}$ of payload by a spiral lift, achieving a full electric system development without hydraulic component. Then, the solution was a structure where the spiral lift hold all the vertical forces and, thanks to the external skeleton, the torsional stress is managed (see Figure A.19). 


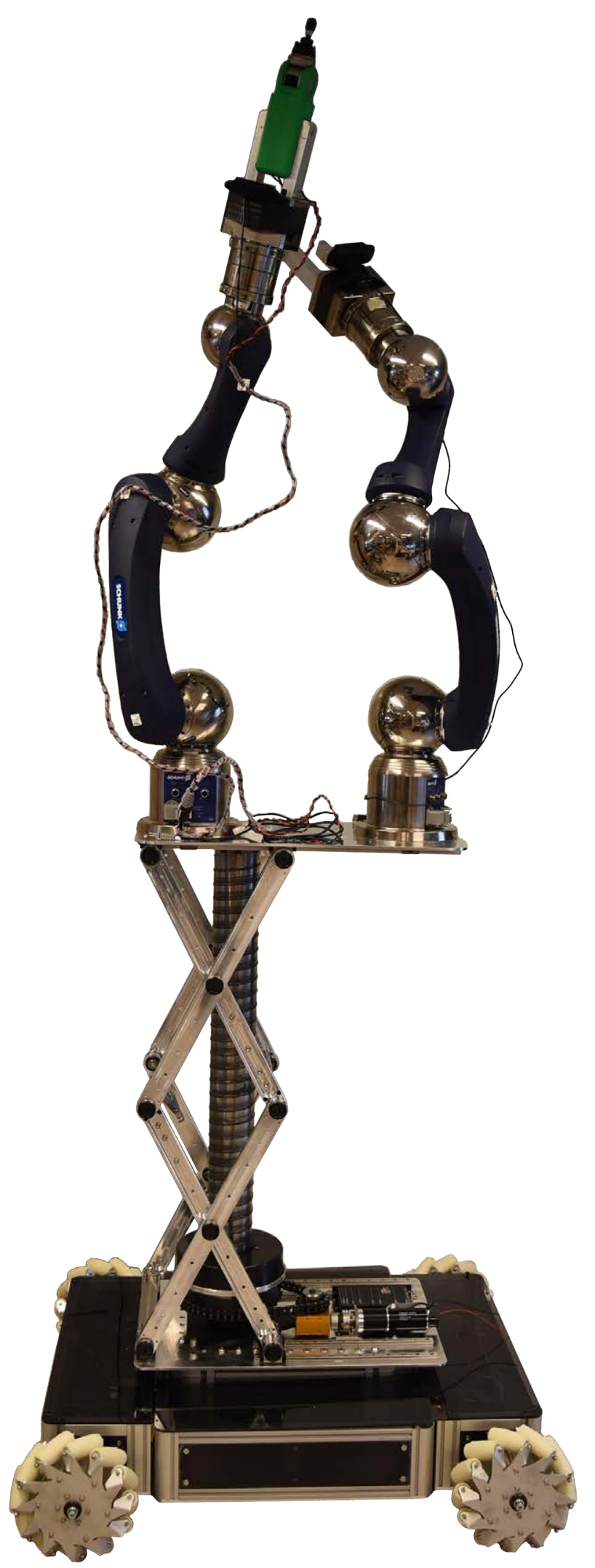

Figure A.19: The CERNBot2 with the stage up carrying dual robotic arms. 
- CraneBot (see Figure A.20), is the release developed to reach to inaccessible locations for ground robots, where only by crane it is possible to arrive.

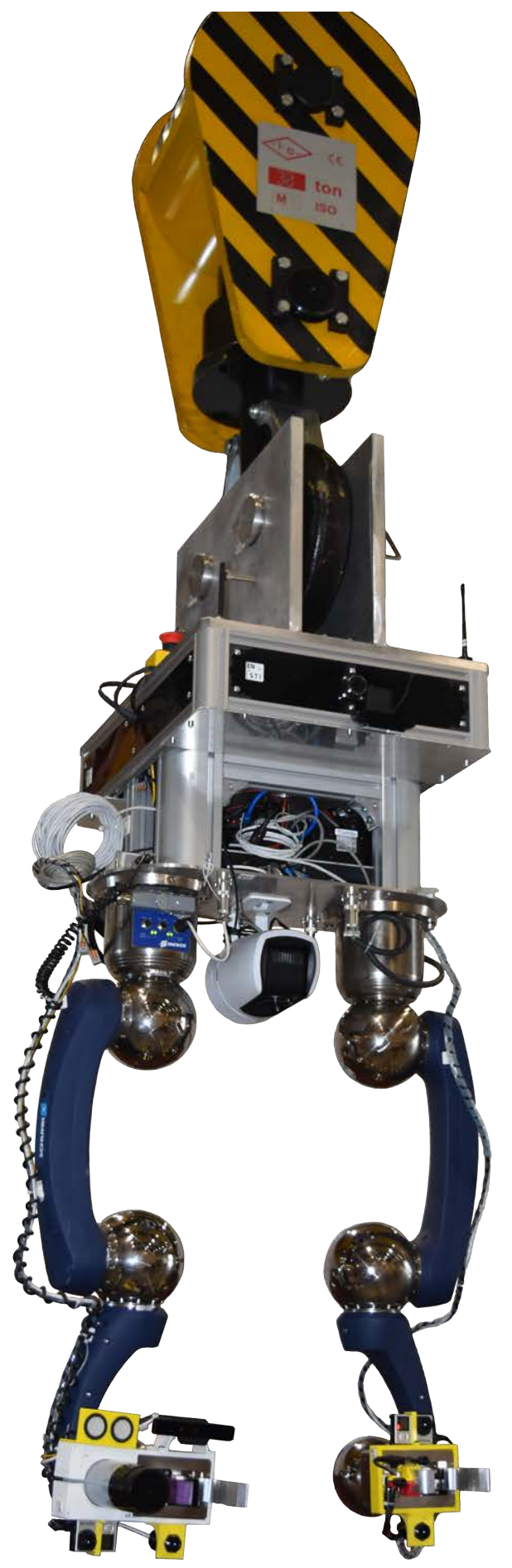

Figure A.20: The CRANEBot carrying dual robotic arms. 


\section{Appendix B}

\section{Experimental Setups}

Several experimental setups are available for the testing of the new features developed for the CERN Robotic Framework. The Large Hadron Collider mock-up (see Figure B.1 and Figure B.2) represents a piece of 30 meters of length of the LHC tunnel. With a size scaled 1:1 with the real LHC tunnel, the mock-up includes a dipole magnet and a collimator for testing purposes. The monorail and power rail are also available at the ceiling of the mockup, which allows it as well to be the commissioning and testing area of the TIM train. Objects like ladders, safety signs, cable trays and cables can be added in order to make the environment more similar to the real LHC tunnel, which is very useful when testing, for example, vision algorithms to detect certain parts of accelerator equipment.

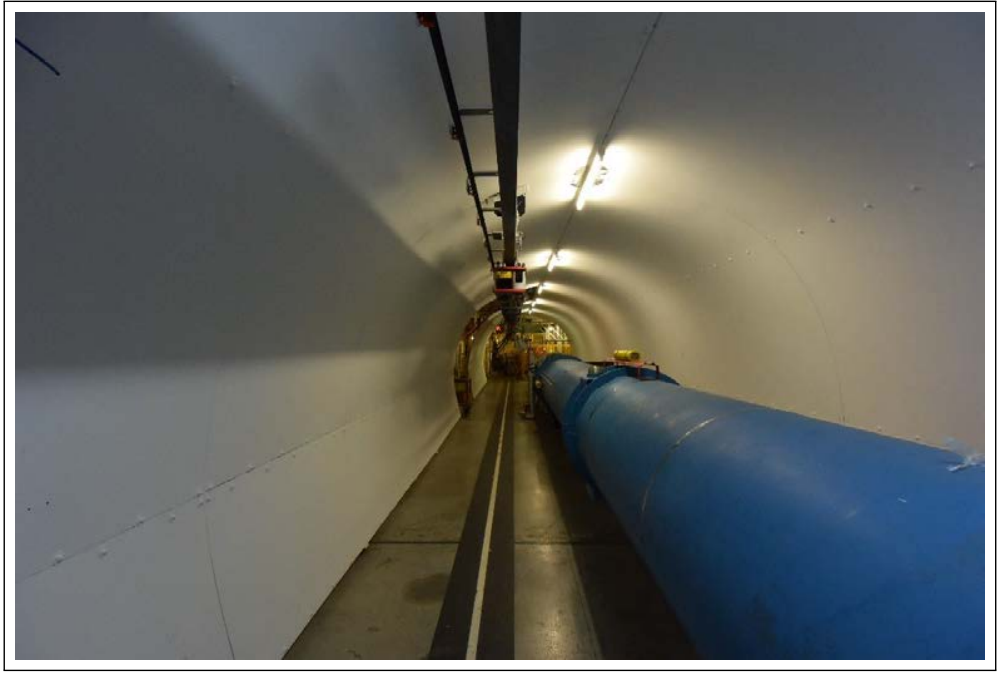

(a) TIM in tunnel mock-up

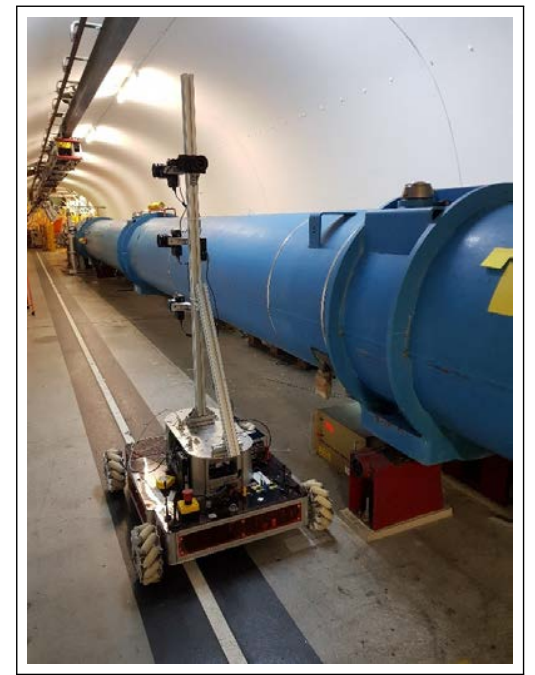

(b) CERNbot in tunnel mock-up

Figure B.1: LHC tunnel mock-up with TIM on monorail and CERNbot. 


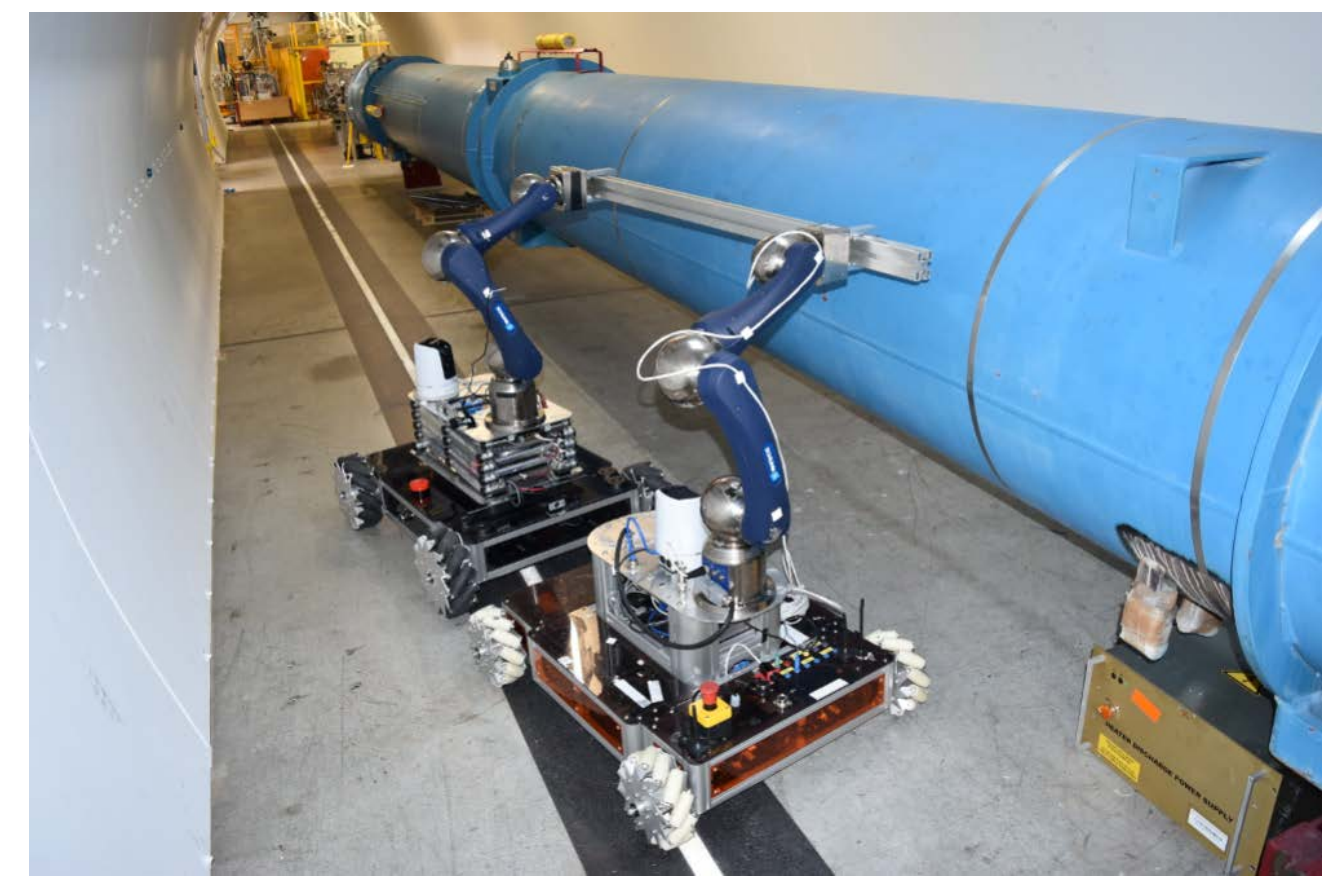

Figure B.2: Two CERNbot units in LHC mock-up doing collaborative tasks.

To test the bilateral master-slave teleoperation system a dual arm setup was constructed. Two arms were mounted on top of a mobile base. These arms would function as the slaves in the master-slave system. The master arms were mounted on an ergonomically designed platform in a laboratory. The choice of the slave robotic arm is constrained by its size, weight and power consumption (see Figure B.3). Most industrial arms are built for factories, they are big and they are plated with metal, which makes them too heavy to be carried by the CERNBot. Usually robotic arms are not constrained by their power consumption, but CERNBot draws its power from batteries, so the less power the manipulator needs the more time can be spent on operation without recharging the robot. An additional requirement is for the robot to be freely programmable, and not need specialized controller, so the control logic of the base and the robotic arm can be handled by the same computer on-board the platform. Unfortunately, there is only a few possible candidates on the market. The possibilities are the Kinova Jaco and the Schunk Powerball.

Kinova Jaco is an ultra lightweight robotic arm. It is capable of 6 DOF movement. It weighs only $4.4 \mathrm{~kg}$ and can lift $2.6 \mathrm{~kg}$. It has absolute encoders in each joint, so the movement of joints are not limited by joint limits. Each joint also has built in torque sensors. The company has a very open approach towards researchers and users of their products. The robotic arm can be easily programmed through $\mathrm{C}++$, Python or ROS. The disadvantage of this robot arm is that in most situations its payload is simply not enough for the operation. It can only be used for handling tools such as screwdrivers. Additionally one of the links in the arm meets in 45 degrees with the next joints instead of 90 . This unusual link configuration makes it harder to map from Joint to Cartesian space. The Kinova Jaco has a very limited choice of end-effectors, only 2 types of grippers are available as of the 


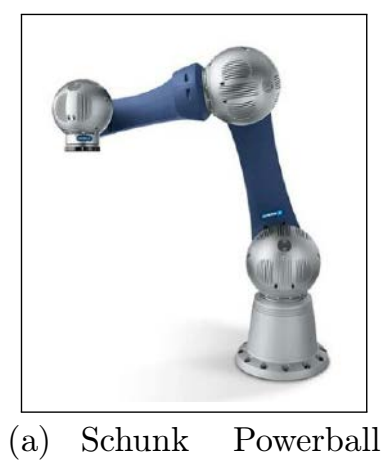
Arm

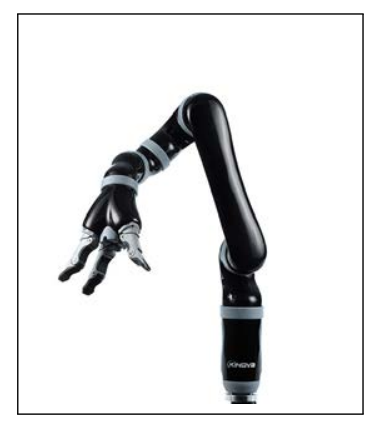

(b) Kinova Jac

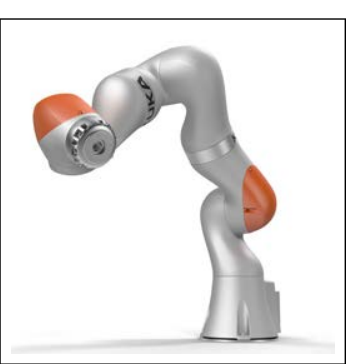

(c) Kuka iiwa LBR

Figure B.3: Robotic arms used and integrated by the CERTAURO framework.

writing of this thesis.

The Schunk Powerball robotic arm is the mainly used manipulator in the CERNBot plafform. This is the slave part of the master-slave teleoperation. It has 6 DOF. Three balls contain 2 motors each. This construction makes the robot very space efficient and robust. It has low power consumption, and operates at $24 \mathrm{~V}$ DC. The joints operate with joint limits of \pm 160 degrees, and they do not contain torque sensors. The robot is able to exert forces well over $100 \mathrm{~N}$. The manipulator has several extensions which can be purchased from Schunk $\mathrm{Ag}$. It can be fitted with force-torque sensors and there are dozens of different end-effector types available. It is possible to control it through CAN commands, so the programming is possible in $\mathrm{C}++$. The main reason I chose it as my platform was that it is able to lift much heavier payload, and its mechanical setup is much more traditional. The laboratory has already had several force-torque sensors and grippers for this robotic arm.

The master robot has to satisfy very different requirements. Since the master-slave teleoperation is carried out from the laboratory the robotic arm is stationary. The size, the power consumption and even the payload is not a limiting factor. The arm will be handguided. Hand-guidance can be done through impedance control. In this control mode the robotic arm senses the forces exerted by the operator on its joints or end-effector. It complies with the forces and moves even with the slightest push. In order to perform teleoperation the most important is its precise movement and high precision impedance control with high speed. There are only a few robots available today which have sufficiently advanced impedance control. We chose the Kuka iiwa LBR robot as the master controller.

The Kuka iiwa LBR is the first series produced sensitive robot. It was developed to work in a human-robot cooperation. The impedance control mode was created so the human workers who do their job in the same workspace are always in safety. The robot has built-in high precision force-torque sensors. When it senses that outside forces are exerted on the joints, such as during a collision, it can stop and move along them. The iiwa LBR has the same precision and durability as any other manufacturing robot. It has a hard real-time controller which can accept commands with a $20 \mathrm{~ms}$ delay. It has an impedance mode with which the robot end effector can be hand guided, this mode already includes gravity 
compensation. The end-effector can be modified with the flange, which can be calibrated according to the load. The robot can be programmed only through the Kuka SunriseOS. This is a closed source programming Api, which can be accessed in Java.

To allow for safer teleoperation, and to enable the control of the slave robot gripper a specialised teleoperation end-effector was created. The teleoperation end-effector is connected to the bottom of the flange. It was designed to resemble as a commercial joystick. It can be held by one hand, the operator should hand guide the master robot through it. Its length was determined so that it would fit right into an average hand.

The upper part of the end-effector is a ring. Inside the ring there are 2 mechanical switches. The user can create two different signals depending on turning the signal left or right. With the signal it is possible to control the gripper located on the slave robot. The signal can be used as boolean, to the left it opens the gripper, to the right it closes it. On the other hand during test I have decided to instead use it to set the direction of the velocity of the gripper. When the user turns the ring, the gripper on the slave slowly starts to close. This decision makes the gripper control much more flexible. It allows the user to pick up fragile items, which would otherwise just be crashed by the gripper.

With the end-effector it was possible to add another safety layer to the teleoperation. The joystick was covered with piezoresistive force sensors. The sensor used was the Flexiforce A301. It is a very small and thin plastic sensor. Inside the sensor the semiconductor changes its resistance when force is applied on it. By changing the resistance the analog output voltage of the sensor changes. This change is then measured by an Arduino Micro which is located inside the joystick. The measurements values are sent through the flange to the robot controller. I have used this signal to dynamically enable and disable of the master robot's movement. The robot can only move while someone is holding the handle.

If something unexpected happens during an intervention simply letting go of the controller will stop teleoperation. It also allows the operator to take short breaks, without the need to start up the system again. When the robot movement is disabled a virtual spring is put at the end-effector with very high stiffness. It allows the user to only change its position by a few millimetres. This method of stopping the arm is better compared to applying the brakes in the joint. If the arm is stopped by the brakes it stops very abruptly. By stopping it using the virtual springs it stops very smoothly. This smooth motion is more comfortable both for the user and the robot. Enabling and disabling the teleoperation can be done consecutively, changing state takes less then $100 \mathrm{~ms}$. 


\section{Appendix C}

\section{Master Handler}

A haptic handler (see Figure C.1) was designed and developed to be attached on the operator side within the CENTAURO's master-slave system (see Figure C.2). This is a friendly and intuitive tool, which releases the brakes of the robot arm when the operator squeezes the handler and lock them when he/she releases it (see Section 5.2), thus the operator might stop the task whenever feels tired. In addition, it is made up with a ring on the top to open/close the gripper in the slave side avoiding having to care about to check if the rotation of the end-effector changed some button position.

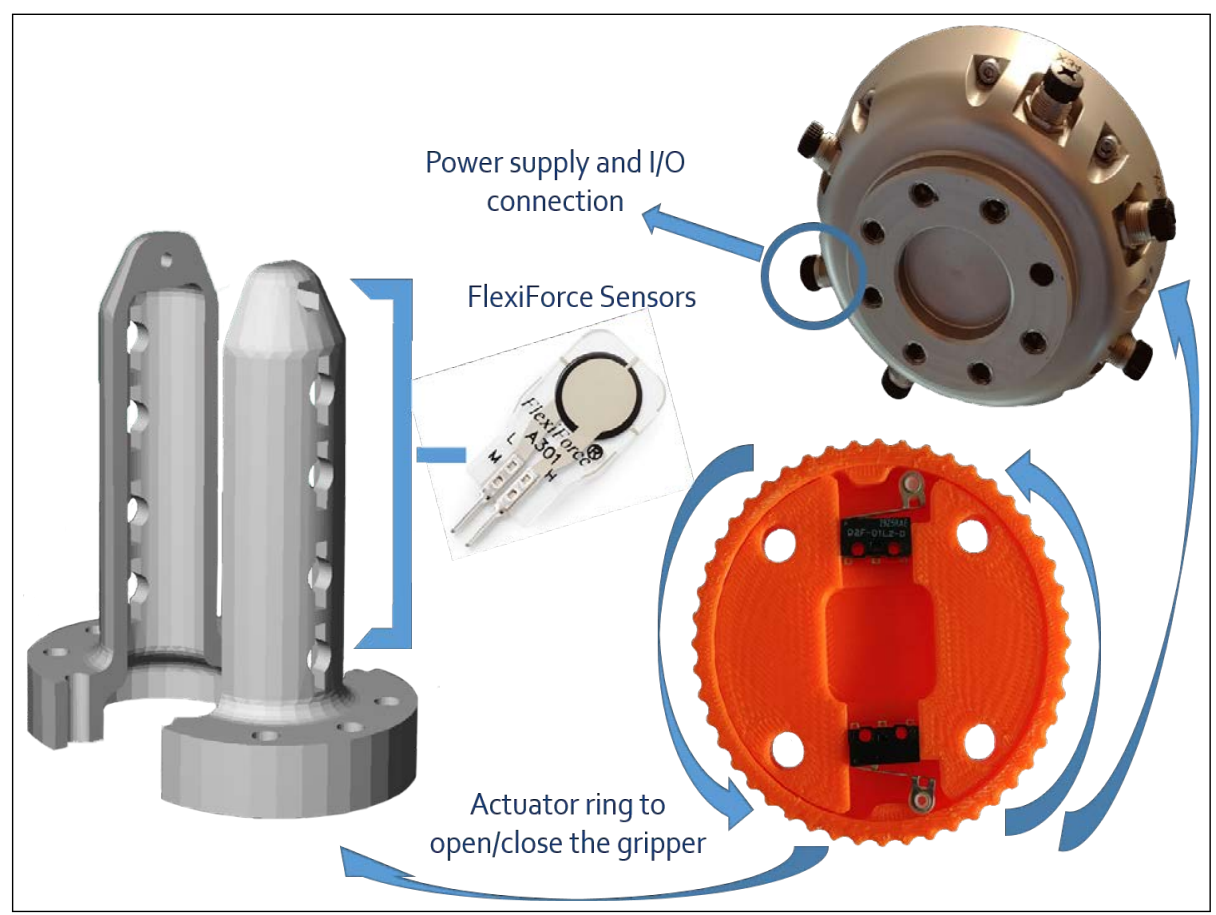

Figure C.1: Haptic friendly handler for master side arm.

The mechanical structure of the handler is shown in Figure C.3. The force sensors are placed through the whole body of the device in order to detect the hand pressure and 


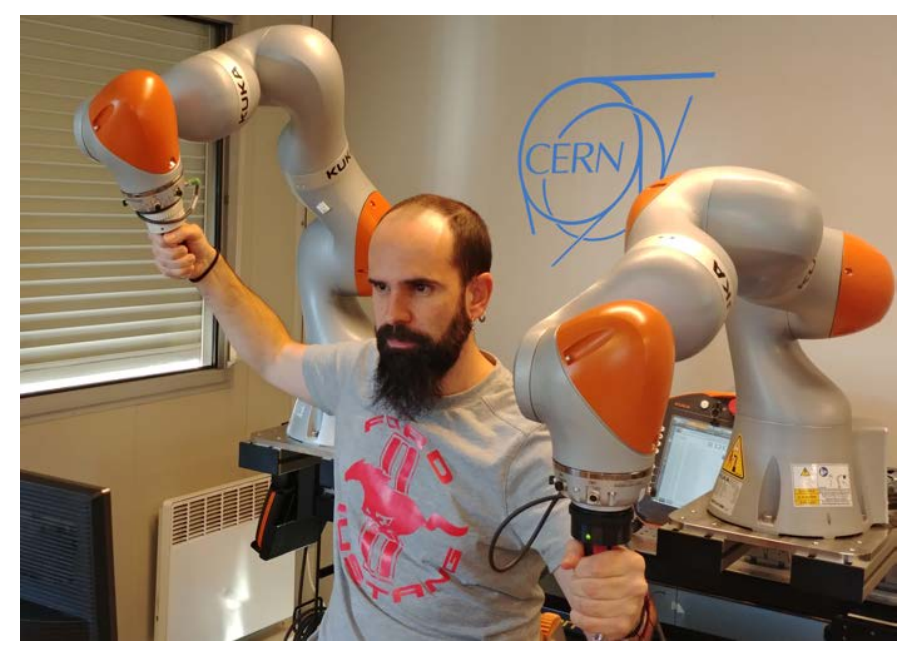

Figure C.2: Operator performing an intervention by the Master-Slave system with dual arms.

determine whether the operator is using the robot or not. There are two switches on the top of the handler in two opposite positions that can be activated by a ring.

- If the ring is turned right then the gripper will open.

- If the ring is turned left then the gripper will close.

- Otherwise the gripper maintains its position.

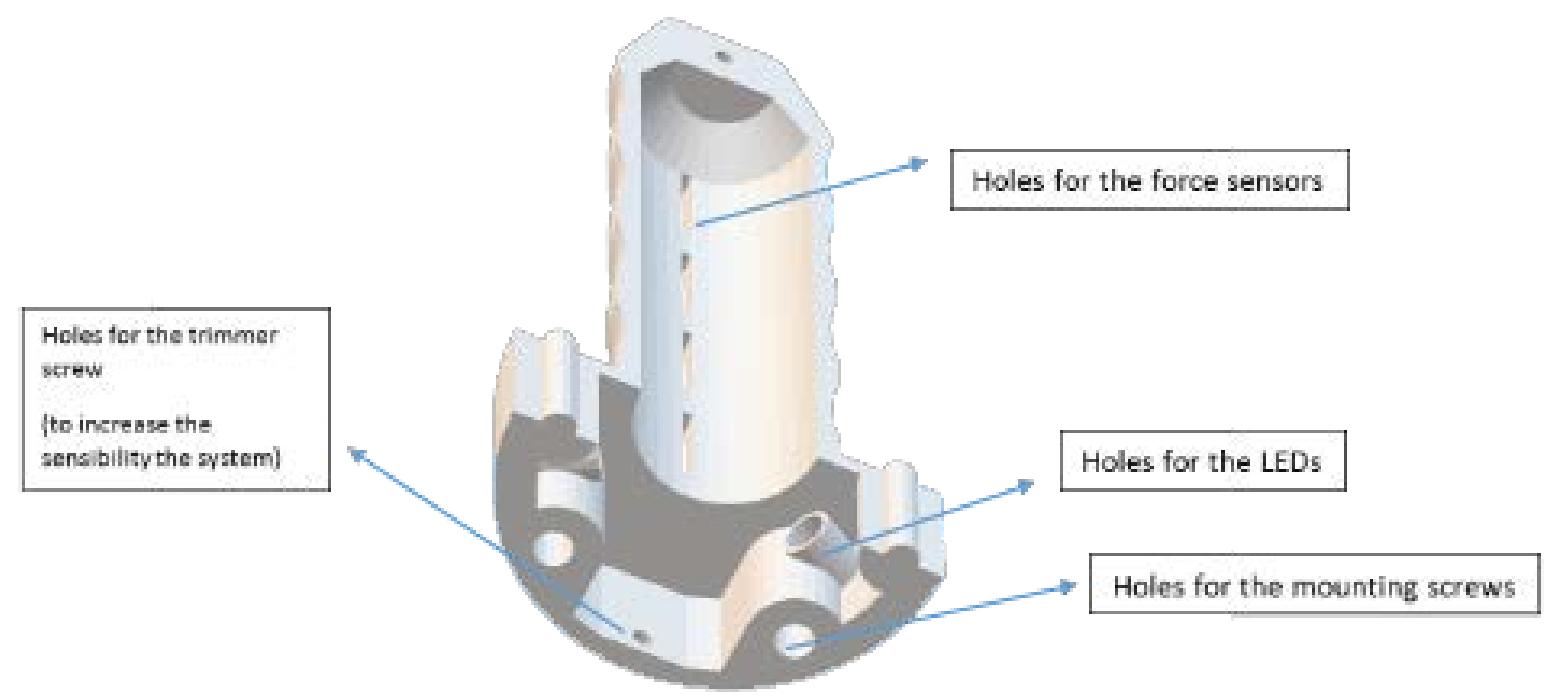

Figure C.3: Cat-design of the handler 


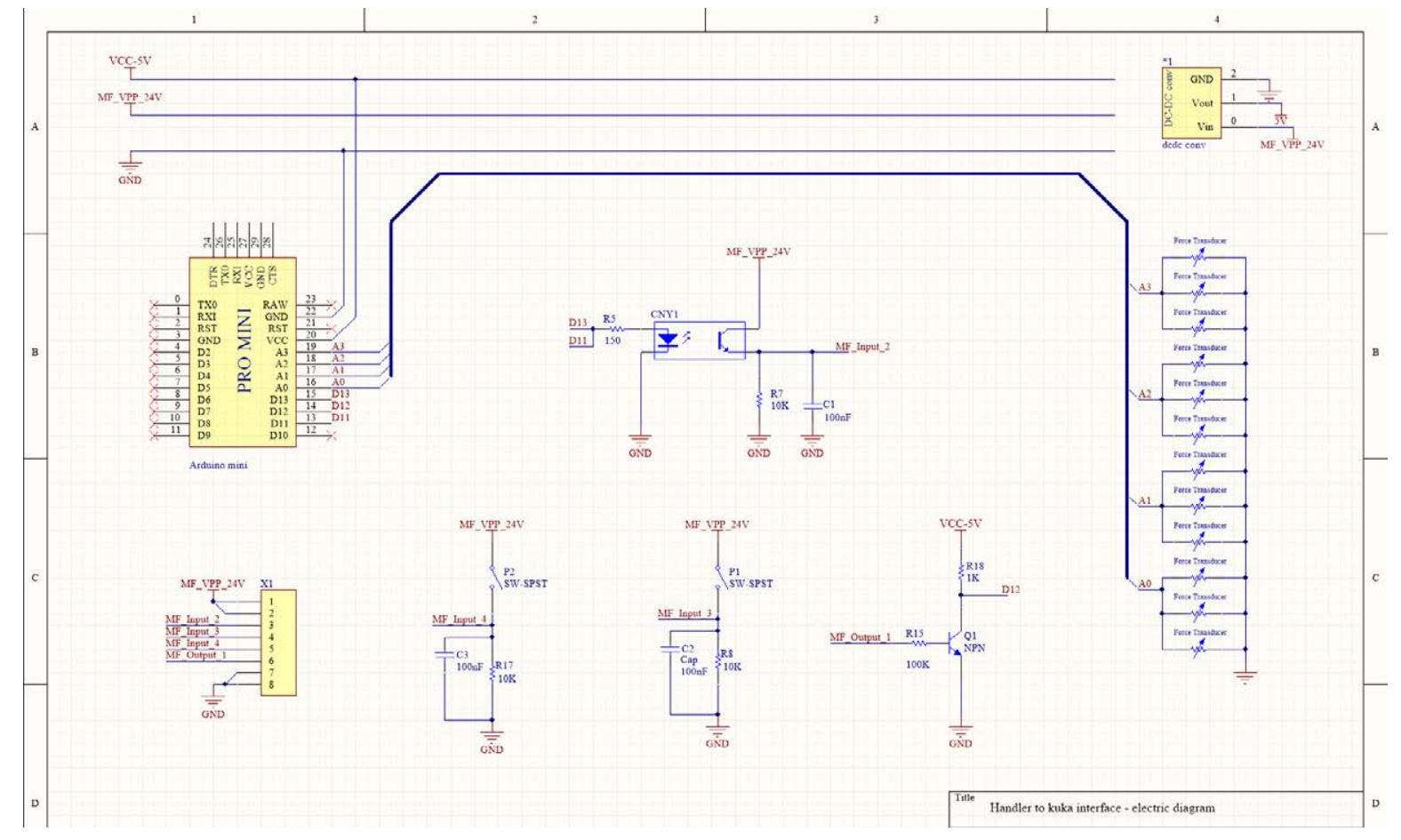

Figure C.4: Electric diagram of the first release

Electric Diagrams There were two different solutions in the process of design and manufacturing the tool. The first one is shown in the Fig. above and the explanation is given below:

- This solution was adopted in the first place, the analog inputs of the microcontroller are all set in pull-up mode, in order to create a voltage divider with the force sensors and get a signal changing according to the pressure applied.

- The circuit interface between the microcontroller and the KUKA robot is made with an optocoupler (Uc - > KUKA) and a NPN bjt (KUKA -> Uc).

- For the ring system (opening/closing of the gripper) two switches have been used in pull-down configuration, with additional debouncing capacitors.

- The whole system is supplied by the KUKA 24V, so a 24 to $5 \mathrm{~V}$ DCDC converter was needed to supply the microcontroller.

The use of a microcontroller and the integration of a DCDC converter was causing some problems for what concerns the available space inside the device, for this reason another version of the system was developed. 


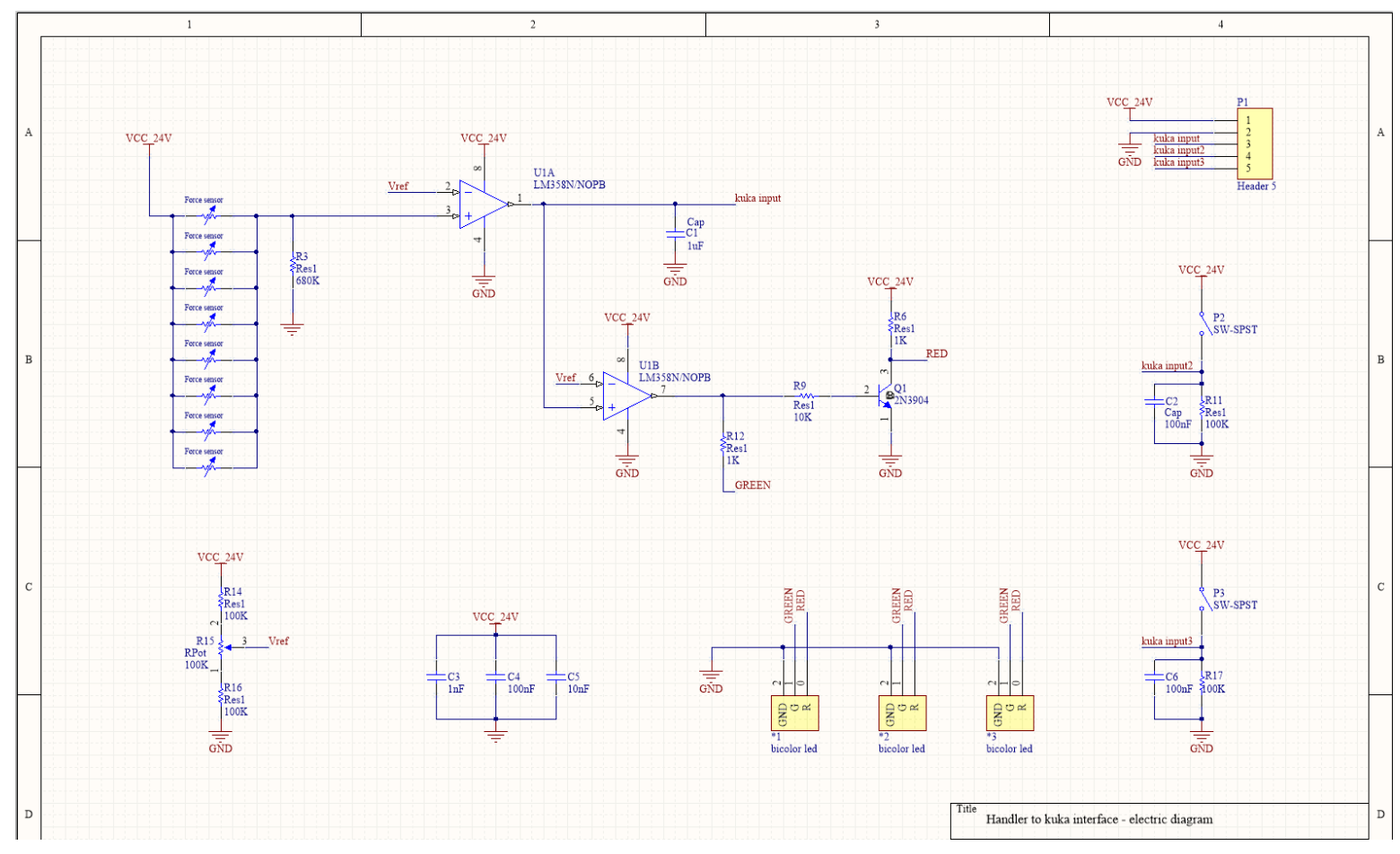

Figure C.5: Electric diagram of the current release

Due to the mechanical constraints, the space for the PCB layout was limited, and for this reason the microcontroller solution was temporarily discarded in order to save as much space as possible.

For this reason a full analog $24 \mathrm{~V}$ electronic system was implemented, avoiding big components and sub-circuits like the $24 \mathrm{v}$-to- $5 \mathrm{~V}$ DCDC converter, a microcontroller, auto-reset circuit, (optional) oscillation circuit (see Figure C.5):

- The $24 \mathrm{~V}$ power supply of the circuit comes from the KUKA robot, the circuit is designed so that all configurations and the components (such as the operational amplifier) are $24 \mathrm{~V}$ tolerant and single supply.

- C3, C4 , C5 are bypass capacitors for the 24V KUKA power supply.

- The force sensor are resistive sensors (Flexiforce) and they vary their output from 10 $\mathrm{M} \Omega$ (not pressed) to $100 \mathrm{k} \omega$ (pressed). They are all put in parallel, so that the input to the robot is given if at least one of them is pressed.

- The signal conditioning circuit for the sensors is a simple voltage divider between the force sensors and the resistor R3, which has been determined empirically, since the resistive output of the sensors applied to the handler was still unpredictable (they are bended when applied). Using a voltage divider (and not a Wheastone bridge), the output of the signal conditioning circuit is not linear, but for this application linearity was not necessary, since this signal is used for a comparator. 
- The signal Vref is set by a voltage divider (R14,R15,R16) and it can be modified by a trimmer accessible from outside of the handler.

- The comparator U1A takes as input Vref (the threshold trigger voltage) and the signal from the force sensors. If the value from the transducers is greater then Vref (pressed condition) the output of the comparator (that is the input of the KUKA robot) will be high $(24 \mathrm{~V})$ and viceversa.

- The PULL-DOWN configuration (P3, C6, R17) (P2, R11, C2) directly connects to the KUKA inputs and $\mathrm{C} 6$ and $\mathrm{C} 2$ are necessary for filtering the mechanical bounce of the switches.

- U1B is the second operational amplifier in the LM358 IC and it basically replicates the output of the other amplifier U1A, it is used as a buffer for the LEDs driver (only one OP AMP couldn't provide enough current for both the KUKA input and the led driver).

- The LED driver circuit is based on a NPN bjt:

- When the output from U1B is HIGH (pressed) the green LEDs turn on, saturating the Bjt and turning off the red LEDs.

- When the output from U1B is LOW (not pressed) the green LEDs turn off, the BJT is interdicted and the red LEDs turn on.

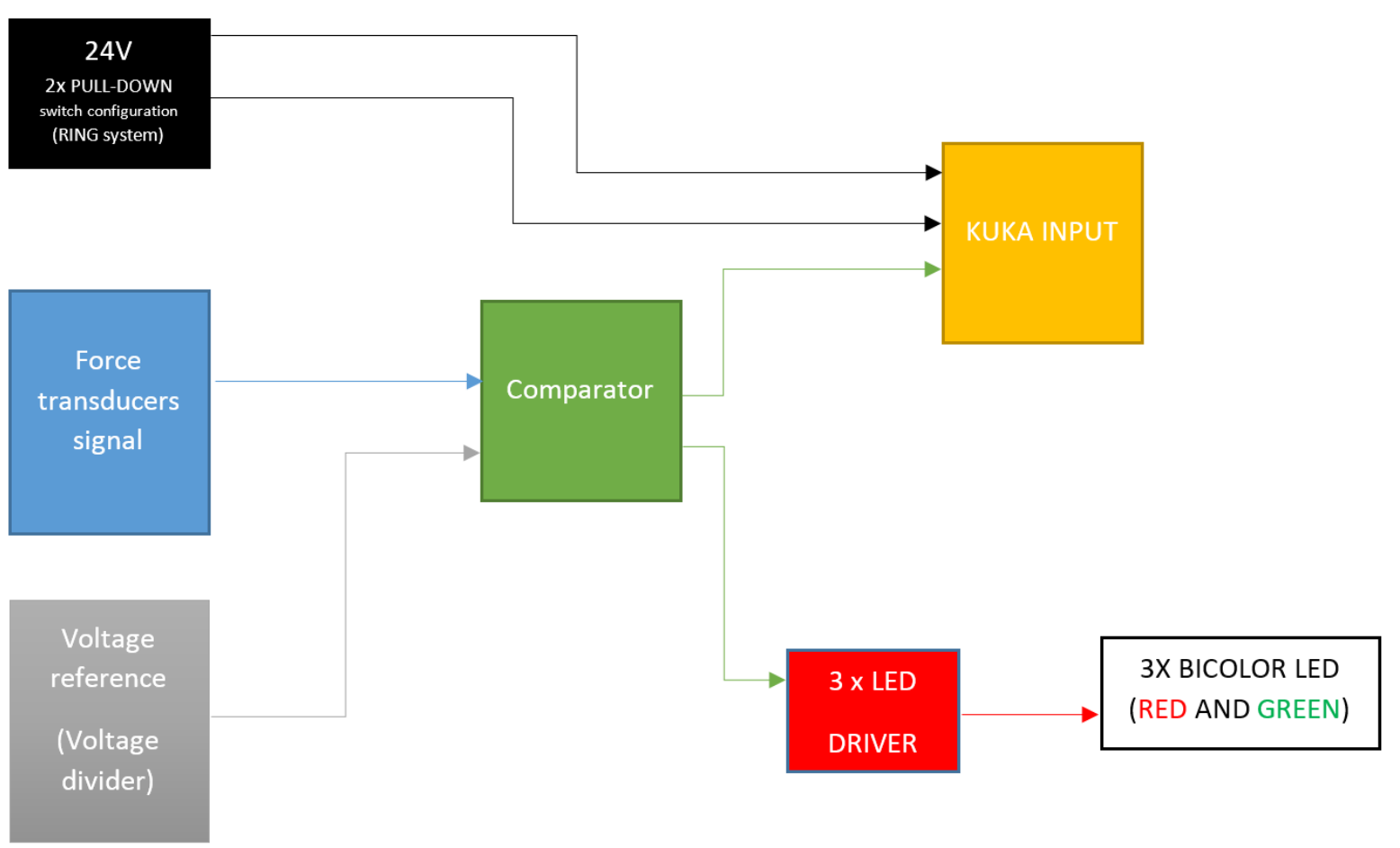

Figure C.6: Box-diagram of handler behaviour 
The final PCB layout used in the current version is explained below:

- The PCB layout and board shape(marked in purple) was modelled according to the mechanical constraints, for this reason the $2 \mathrm{~d}$ cad design of the top of the handler (marked in green) was imported in the pcb design tool and used as a base.

- The screw of the trimmer $\mathrm{r} 5$ is accessible from the outside thanks to a hole in the $3 \mathrm{~d}$ model, so that the sensibility of the device can be changed at anytime.

- Also a test point was placed in order to measure Vref if necessary.

- The LEDs are soldered to the board and inserted in small holes, so that they are visible from the outside.

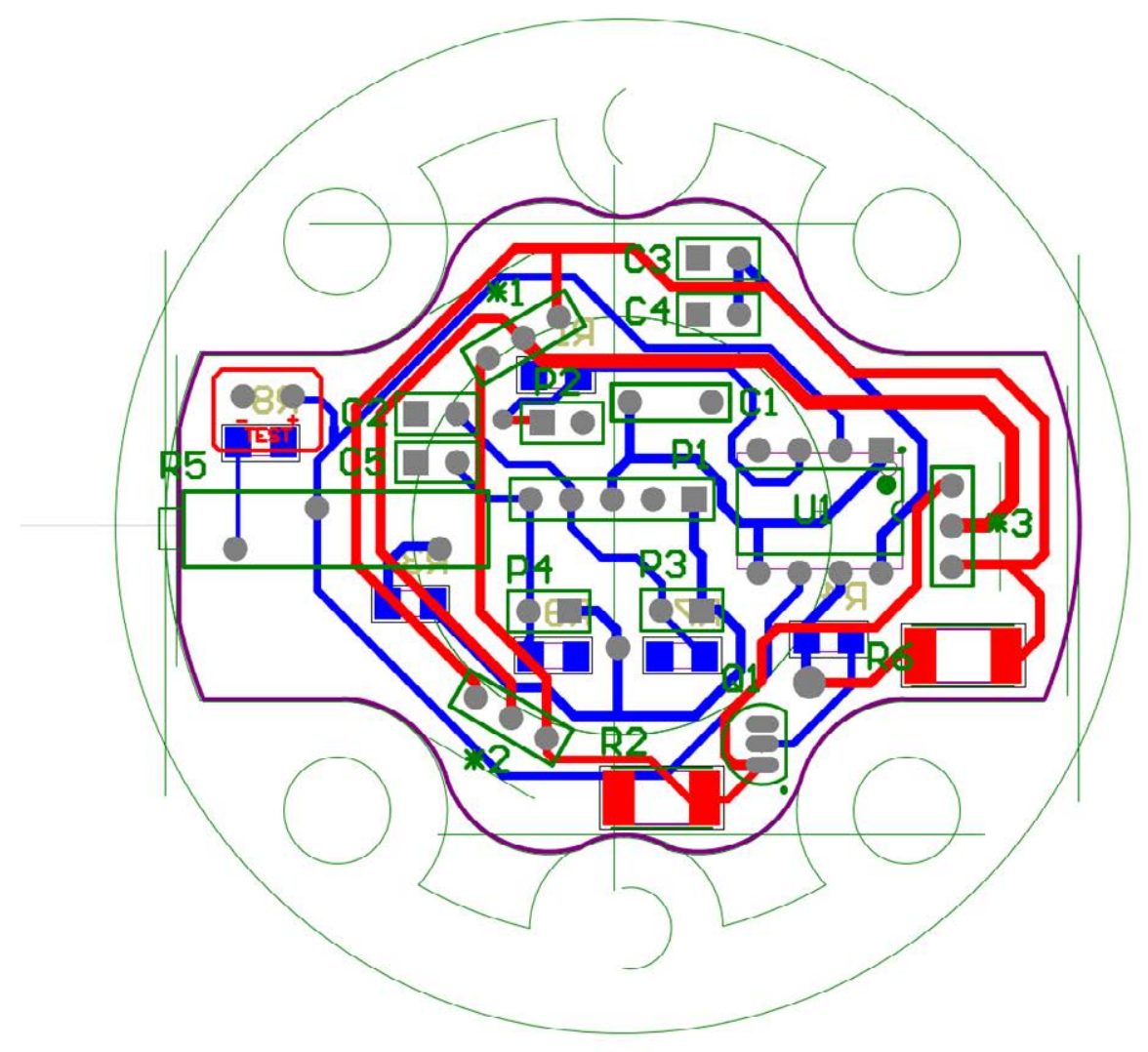

Figure C.7: Final PCB layout

In order to allow the shortest return path and the lowest impedance, and also creating minimal loop current (which reduces emi) power planes were used.

VCC on the top and GND on the bottom, adding small bypass capacitance for the power supply (see Fig. C.8 and Fig. C.9). 


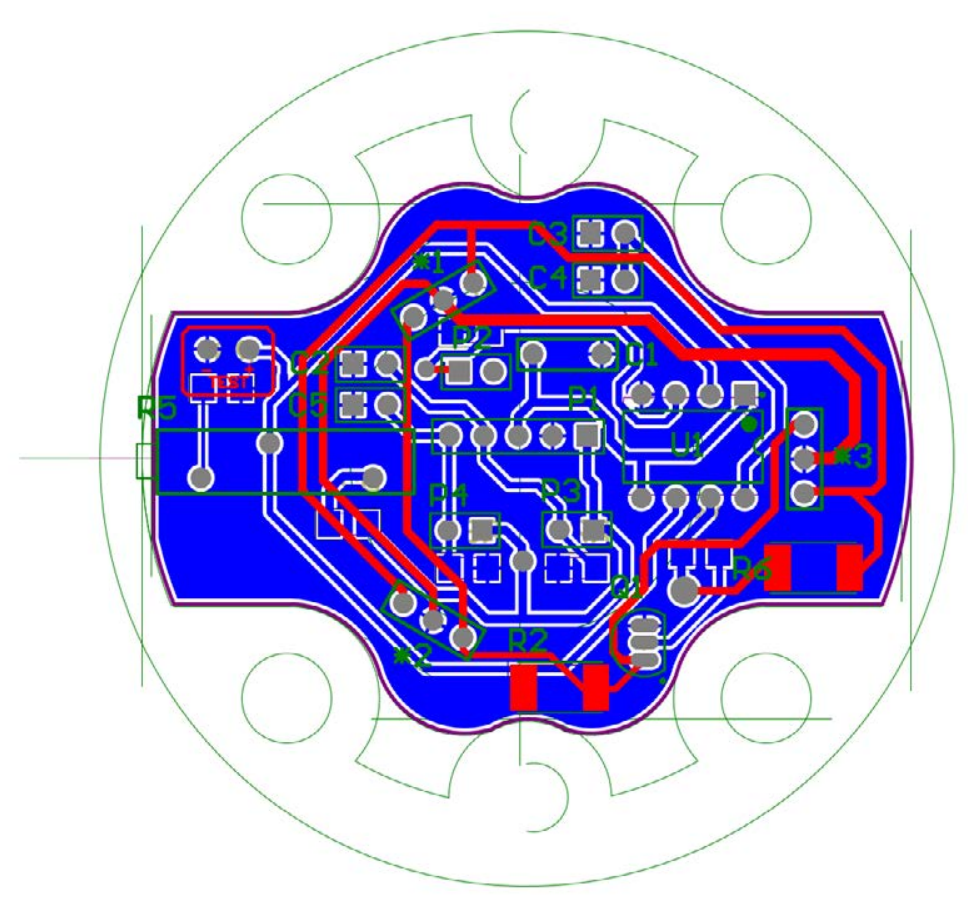

Figure C.8: Bottom polygon pour net GND

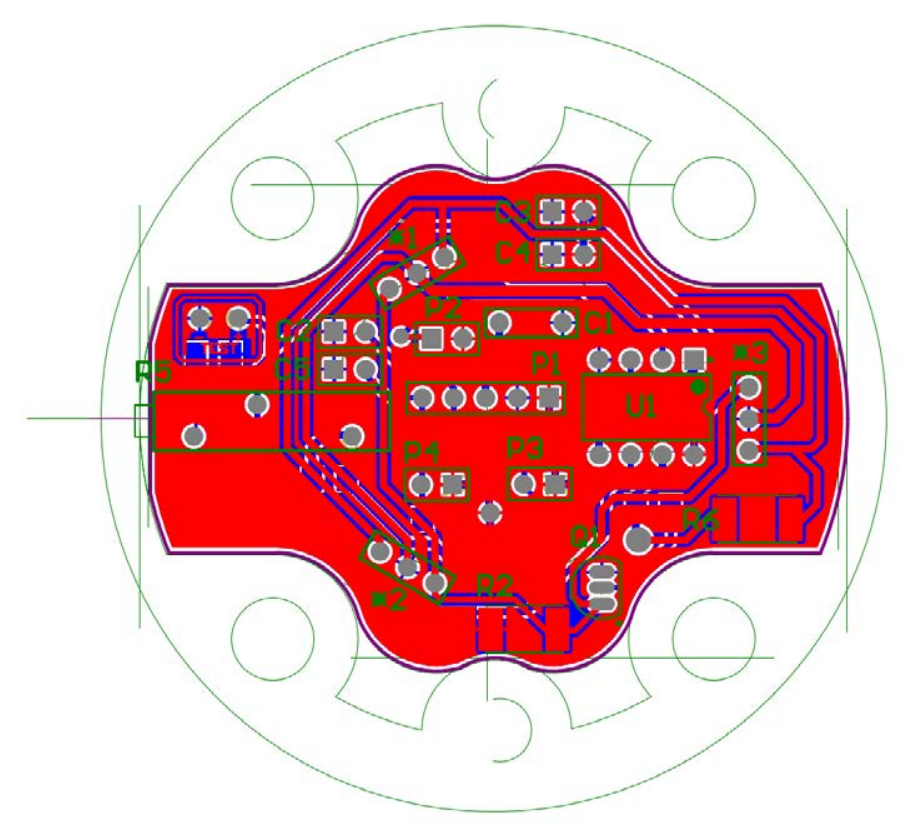

Figure C.9: Top polygon pour net VCC 


\section{Appendix D}

\section{Advanced Tools}

During the design and development phases of the CERNTAURO framework, a variety of tools to perform remote tasks were adapted to different robots and/or produced and integrated on the robots (see Table D.1). The continuous integration development model of the CERNTAURO framework gives the possibility to include further tools on the robots in operation.

\begin{tabular}{|c|c|c|c|}
\hline TOOL & FUNCTION & РНОТО & $\begin{array}{c}\text { EQUIPMENT } \\
\text { CONFIGURATION }\end{array}$ \\
\hline $\begin{array}{l}\text { Electrical } \\
\text { screwdriver }\end{array}$ & $\begin{array}{l}\text { Torque control capabilities } \\
\text { to un/screw bolts }\end{array}$ & & $\begin{array}{c}\text { CERNbot } 1 \\
\text { CERNbot } 2 \\
\text { Cranebot }\end{array}$ \\
\hline $\begin{array}{l}\text { Helium spray } \\
\text { system for } \\
\text { vacuum leak } \\
\text { detection }\end{array}$ & $\begin{array}{l}\text { Helium mass flow } \\
\text { remotely controlled } \\
\text { for leak detection }\end{array}$ & & $\begin{array}{l}\text { CERNbot } 1 \\
\text { CERNbot } 2 \\
\text { Cranebot }\end{array}$ \\
\hline Nibbler & $\begin{array}{l}\text { Tool to cut } \\
\text { metal plate }\end{array}$ & & $\begin{array}{c}\text { CERNbot } 1 \\
\text { CERNbot } 2 \\
\text { Cranebot }\end{array}$ \\
\hline
\end{tabular}




\begin{tabular}{|c|c|c|}
\hline Scissors & $\begin{array}{l}\text { Cut different materials } \\
\text { such as plastic, metal, } \\
\text { paper, etc. }\end{array}$ & $\begin{array}{l}\text { Teodor } \\
\text { Telemax }\end{array}$ \\
\hline Liquid pump & $\begin{array}{l}\text { For filling or emptying } \\
\text { oil or resin }\end{array}$ & $\begin{array}{c}\text { CERNbot } 1 \\
\text { CERNbot } 2 \\
\text { Cranebot }\end{array}$ \\
\hline Cutter & Cut cables & Teodor \\
\hline $\begin{array}{l}\text { Impact } \\
\text { wrench }\end{array}$ & $\begin{array}{l}\text { Deliver high torque } \\
\text { output with minimal } \\
\text { exertion by the robot }\end{array}$ & Teodor \\
\hline Foam pistol & Provide and place foam & $\begin{array}{c}\text { CERNbot } 1 \\
\text { CERNbot } 2 \\
\text { Cranebot }\end{array}$ \\
\hline
\end{tabular}




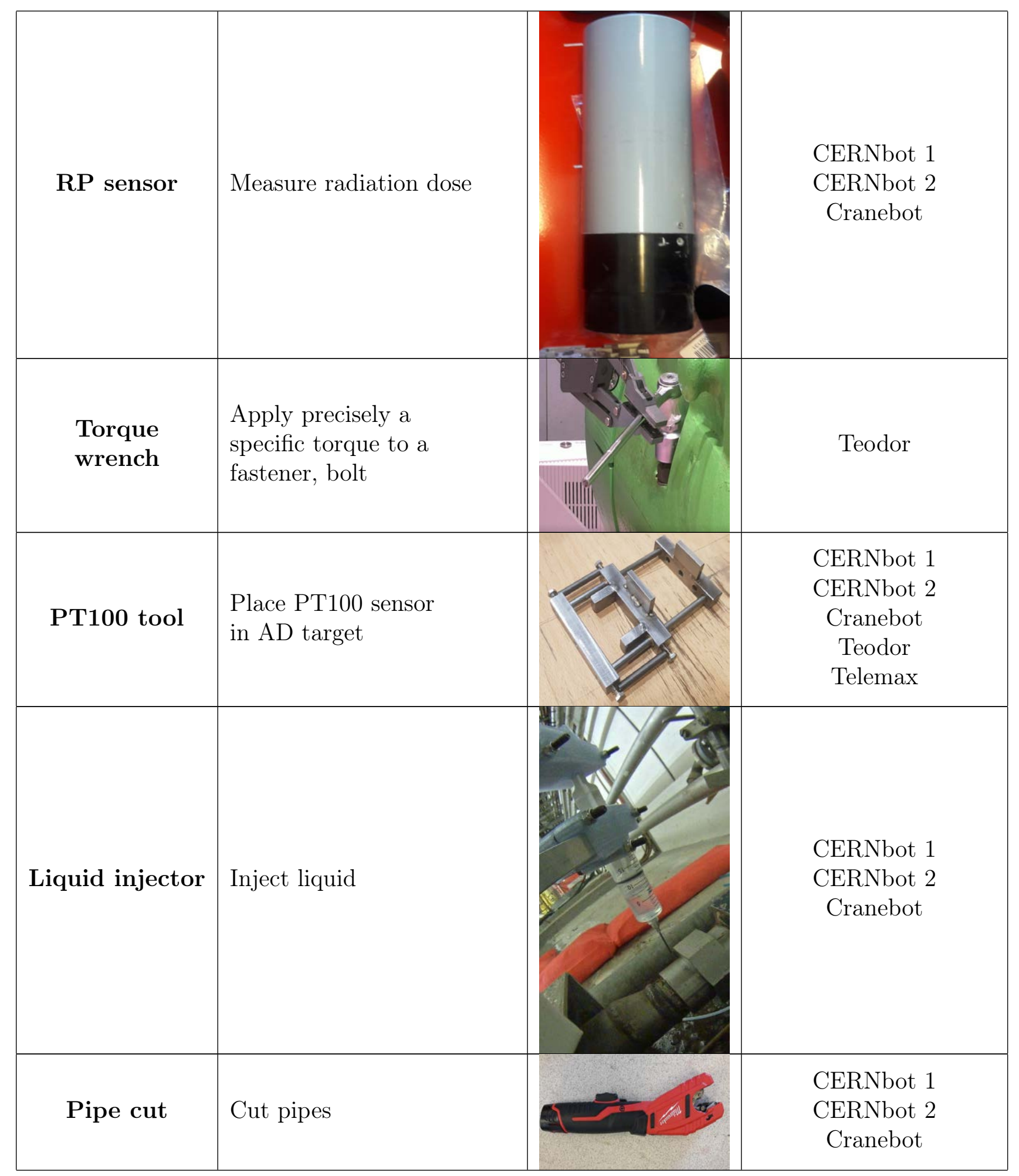




\begin{tabular}{|c|l|c|c|}
\hline $\begin{array}{c}\text { Universal } \\
\text { screwdriver }\end{array}$ & $\begin{array}{l}\text { Tool to engage and } \\
\text { un/screw various types } \\
\text { and sizes of screw } \\
\text { fastener heads }\end{array}$ & $\begin{array}{c}\text { CERNbot 1 } \\
\text { CERNbot } 2 \\
\text { Cranebot } \\
\text { Teodor } \\
\text { Telemax }\end{array}$ \\
\hline Saw & $\begin{array}{l}\text { Cut surfaces such as metal, } \\
\text { aluminium and wood }\end{array}$ & $\begin{array}{c}\text { Teodor } \\
\text { Hook for securing and } \\
\text { moving loads }\end{array}$ & Teodor \\
\hline
\end{tabular}

Table D.1: Tooling pool database 


\section{Appendix E}

\section{CERN Remote Handling Code of Practice}

The CERNRHCOP provides standards on RH compatibility with the plants, procedures, fasteners, electrical and fluids connectors, design of RH equipment and tools, as well as RH operations. The main components and guidelines of the CERNRHCOP are described in the following.

\section{Modularity}

A fundamental aspect for a successful remote intervention is the modularity, that organizes the system into smaller subsystems, where each subsystem is a module. A modular system can provide several advantages for remote operations. However, it is essential to design the modules with determined features for remotely maintainable systems. The advantages, requirements and design practices are explained below:

- Maintenance time: the modules allow better performance during disconnection and transport in maintenance operations. In this way in-site repairs are avoided because modules could be extracted and replaced by new ones.

- Overall cost: the number and complexity of maintenance operation decrease, so the repair time and cost is reduced.

- Spare parts inventory: modularity reduces the spare parts inventory and helps the chance of standardization in the facility.

In addition:

1. Modules should be designed taking into account a remotely maintenance plan where aspects, as for example the necessity to have interfaces to be managed, should be considered.

2. Modules design should use standard or available maintenance equipment and avoid the need of special tools (if is possible). 
3. Provide the dimensions of all modules or replaceable components.

4. Provide the virtual modules, for example CAD.

5. Provide the order and instructions for the replacement of the module, including methods to stop flows or bypass process materials and to drain components before their removal (if required).

6. Specify methods for removal and storage of process or product materials.

7. Avoid space and removal interferences.

8. Mark and label to identify the modules.

9. Mark and label all connecting cables, wires, process lines, etc.

10. The design and associated maintenance time periods should meet operating availability requirements.

11. Modules containing elements of similar lifetime.

\section{Accessibility}

The designers have to ensure that there is an adequate access to the equipment and humans in the work areas. This access also includes space for the maintenance system as well as auxiliary system like a vision camera, wherein visual and space limitation are decisive in remote handling tasks as is explained below.

- Space: the equipment necessary for maintenance must be taken into account for accessibility. This includes cranes, manipulator, robots, robotic arms, cameras etc. The $\mathrm{RH}$ equipment as the human operator should have enough space to long the path to the work space.

- Visual: Components and equipment have to provide enough visibility of the work space from several points of view.

Some general design practices for adequate accessibility can be summarized in:

- Components should be designed to be reached, disconnected, and handled by available remote handling equipment, taking into consideration location in the cell and the relation of other modules and equipment.

- Maintenance procedures should be evaluated to allow enough space for the equipment operation. For example: when a bolt is unbolted to disassembled component.

- Definition of paths for the equipment and the extracted/installed components.

- Components must be designed to be transferred through available and defined path. 
- Avoid the obstructing components.

- Heavy components are usually moved by means of cranes or auxiliary hoists attached to the manipulator. These components should be accessible by an overhead bridge crane or floor path and should be designed to be disconnected and removed with a minimum of disturbance to other equipment.

- Components and equipment should be designed to easily discerned from background equipment and to ensure adequate viewing from the remote TV cameras, windows or periscopes defined for the RH system in the facility.

- The component connections should be clearly identified and visible from multiple locations.

- The equipment should be designed for ease of inspection and maintenance within the surrounding remote-environment space.

\section{Viewing and Visibility}

The visibility of tasks using remote cameras is a crucial consideration. If the task area cannot be seen in the camera views it will be impossible to perform remotely. In general, and whenever possible, the IMS will incorporate a large number of high quality cameras and associated lighting systems. However, inside the torus the number and quality of the camera images will be severely restricted as a result of the combined effects of the very high radiation levels and the very restricted access spaces. It is essential for all remote handling compatible plant that there is as much provision as possible of visual cues. The implementation of visual cues depends significantly on the application. However, the following guidelines should be followed:

- Provide as much high contrast difference between two mating plants.

- Provide physical features that clearly align when correctly assembled.

- Provide colour differences where possible.

- Provide assistance to mechanical alignment features by making use of fixed laser or other light sources projecting onto a viewable target on mating pieces.

- Avoid highly reflective surfaces where possible.

- IMS handling equipment should be visually easily distinguishable from plant being handled.

- All plant items, particularly connectors, wiring and other multiple items must be clearly marked and identified. 
- All plant items which require inspection in service should, wherever possible, incorporate inspection access facilities e.g guide tubes for deployment of viewing probes in difficult areas.

- All plant items which require metrology inspection must incorporate suitable attachment points for targets and datum references.

\section{Simplicity}

Simplicity is essential to increase the availability of the facility. Thereby, the maintenance or replacement of components should be as simple as possible. For this, it is necessary follow some recommendations to simplify the operations in remote interventions:

- Components: the number of components and complexity of the systems should be reduced as much as possible. The use of few, simple and well-verified components are preferable. Moreover, simple and easy handled mechanisms are preferable that complex elements.

- Components design should be adapted to be managed by RH equipment and adequate work area distribution.

- Procedures: the procedures should be minimized and the tasks without added value should be eliminated.

- Natural laws aid: the use of gravity in the operative process and during assembly/disassembling is an advantage because laws never fail, increasing reliability.

\section{Standardization}

Components, equipment and tools should be standard or at least comply with the standards. Using standards have several advantages such as increase the availability, assurance of a level of quality, security, robustness and reliability of components. Moreover, reduces overall design and construction costs of a system, reduces spare parts inventories and fixtures, minimizes operator-training time for maintenance, and decreases downtime. It also avoids high cost due to errors or lack of information since the characteristics are registered and defined. General standardization guidelines for designers include the following recommendations:

- Commercially purchased components, eventually modified, are preferred to specially designed and fabricated components.

- Limit and reduce the number of different sizes for the same type of component or fixture.

- Use the same component and process in different applications in the facility.

- Limit the number of different types of commercially purchased components for a remote handling environment. 
- Limit and reduce the number of different methods and processes in the facility.

- Utilize standard design solutions, wherever possible in preference to creating new solutions.

Also, standardization in equipment design minimizes requirements for special remote tooling. For example, if fasteners, connectors, flanged joint bolts, or any components that have to be handled to perform a certain operation are designed to be of the same size, the same tool can be used for each category of such components.

\section{Reliability}

Components under a high standard of reliability are essential in all accelerator facilities because reliability has a crucial implication in the availability of an activated facility. Since in most cases it is necessary to perform $\mathrm{RH}$ operation and this needs time to execution, which has a cost that increases with the time. For this reason it is essential to avoid failures or maintenance operation with a reliability design of the components in the facility. Design practices for high reliability are essentially:

- Establish working conditions, which have to be below the rated nominal values of the component. For example: maximum mechanical power supplied by a motor, max. Load for a crane, max. working temperature.

- The use of redundant electric drives, electronics and mechanical components.

- Proper work conditions and monitoring: Monitor the working conditions of the components, which have significant probability of failure to achieve confident derating.

- Practise careful design, use commercial components, standards or well proved concepts.

- Extensive tests of the degree of component reliability before final assembly is essential.

- Plan the periodic tests and inspection of the components having unknown lifetime or such that are critical components.

\section{Radiation}

Radioactive environments or activated materials are usually required to be remotely maintainable to minimize exposure to personnel. The designer has to take into account the effects of ionic radiation on components and remote-maintenance system materials. In this way, to avoid frequent fails and remote repairs, the material of components should be resistant to ionizing radiation or protected with local shielding when alternative materials are unavailable. Electronic components and organic materials such as elastomers, oils, and plastics may be severely affected and may have very limited use in areas of high radiation. On the other side metals and ceramics are far more radiation resistant.

However, it depends of the radiation levels in the working areas. The lifetime of the components and RH equipment are defined by many factors: 
1. type of radiation;

2. spectra and intensity of radiation;

3. the exposure time;

4. material of each sub-component of the device.

Some important recommendations for components and equipment located in a radiation environment are:

- Allow enough space for local shielding of components having the shortest lifetime.

- The choice of a shielding material depends on the available space, cost, availability of the material, easy welding and mechanising, type of radiation and other factors.

- Components and modules having similar lifetime for all the subcomponents or submodules.

- Locate rad-sensitive components out-cell such as electronics, because the level of radiation is smaller.

- Cooling could be considered in some parts of the facility where the radiation is intensity high and dangerous for humans.

- Use where possible, rad-hard or radiation compliant electronics and ceramic based detectors for environmental measurements.

\section{Decontamination}

Design for easy decontamination is necessary in any facility with radioactive environment. Nevertheless, the requirements depend importantly on the component itself and the type of facility. Reducing the degree of contamination implies the improvement of processes, systems and several components. So the following design considerations are important for easy decontamination in a radioactive/activated environment:

- Decontaminable surfaces: avoid plastic material.

- Smooth or polished surfaces are favourable. For example, electro-polishing is fast, inexpensive and effective for complex structures.

- Try to avoid holes, deep grooves, discontinuous welds, cracks, crevices, porous materials and joints that trap dust.

- Apply coatings or liners on porous materials, for example concrete.

- The cleaning of the component should be greater when the probability and degree of contamination is higher. 
- Protective covering: joints and mechanisms should be covered in contaminated environments, especially if these will require hands-on maintenance or are difficult to decontaminate. The protective coatings must be removable and replaceable. However, this protective coating do not have to impair the operation.

- Decontamination equipment: the material part not covered and non-easy replaceable should be decontaminated until acceptable levels. Another part such as bearings and connectors are susceptible to damage by decontamination solutions, in this case could be removed and replaced.

- Material equipment: the equipment, not to be reused, may be constructed of lower grade materials not necessarily resistant to the decontamination reagents. However, if require a re-use continues the material, this should be radiation resistant (if possible). In addition, the materials for the equipment that have to be decontaminated, should resist degradation by the decontamination fluids and procedures.

\section{Fasteners}

The fasteners are mechanical elements that are used for a fixation between pieces. Wherein, the bolts or screws can be inserted into holes, through parts of pieces and / or attached to a nut. The insertion or extraction could be through a special tool (i.e.: Allen), wrench or screwdriver. Thereby, this section provide the preferred method and recommendations for mechanical fasteners with the aim to ensure all mechanical fasteners are fully RH compatible for and easy and comfortable handled. In this document the term 'fasteners' includes any type of bolt, nut, screw, zip, clasp, clamp or pin suited to hold two or more elements together. The dimensions, materials and properties of each one depend of different factor that are also explained in the following.

- Bolts: typical Remote Handling bolt is constituted by several parts: head, body and point. The main parts are showed in Figure E.1.

- Head: the most favourable bolt head are the hexagonal head and socket-head. Moreover, a 'cone head' in the top favors the coupling of the tool with the bolt head (see Figure E.2). The preferred style should be socket-head (see Figure E.3). If the use of a socket head fastener is not possible, a standard size external hexagonal bolt head could be used. A lead-in cone may be made to provide easier engagement of the $\mathrm{RH}$ ring spanner (see Figure E.4). An integral shoulder is preferable to maintain engagement of the spanner. This feature is useful especially for pop-up bolts. If the use of a socket head fastener is not possible, a standard Bi-Hex bolt head could be used (see Figure E.5). Bi-Hex heads have a smaller across-flats dimension than standard hex heads and provide the spanner with more precise and better location and security under load. The head design has an integral shoulder to assist the location of the spanner.

- Body: threaded part and a waisted part is common to allow captivation of the bolt. 
- Point: bolt could be constituted by a tapered and cylindrical 'lead-in' for positioning and to avoid or minimize the cross-threading during the starting of engagement. Example of this parts is visualized in Figure E.6.

- Nuts: a nut is a mechanical part with a central hole, this is used to attach a screw, in a fixed or sliding way. The nut allows fastening and fixing joints of removable elements. Sometimes a washer can be added so that the union closes better and remains fixed, but it is not recommended for RH due to the difficulty of handling.

- Captive Bolts: these bolts remain attached to the component after unbolting, allowing greater ease to operate. Two types of captive bolts are presented: 'Free Captive Bolts' and 'Spring-loaded Captive Bolts' can be distinguished (see Figure E.7). In Free Captive Bolts, the screw remains attached to the component after unbolting but the position of the bolt after disengagement is free and unknown. In Spring-loaded Captive Bolts, the screw remains attached to the component after unbolting and the position of the bolt after disengagement is defined and known because means to separate the bolt from the threaded hole are devised. It is recommended to install the bolts preferably in vertical or horizontal positions for faster removal with simple systems. In addition, is recommended to avoid the position of inclined bolts, because it will require more complex trajectories of the tool and more skilled operators. It is more difficult for operators, telerobotic systems or/and mechanical master/slave systems.

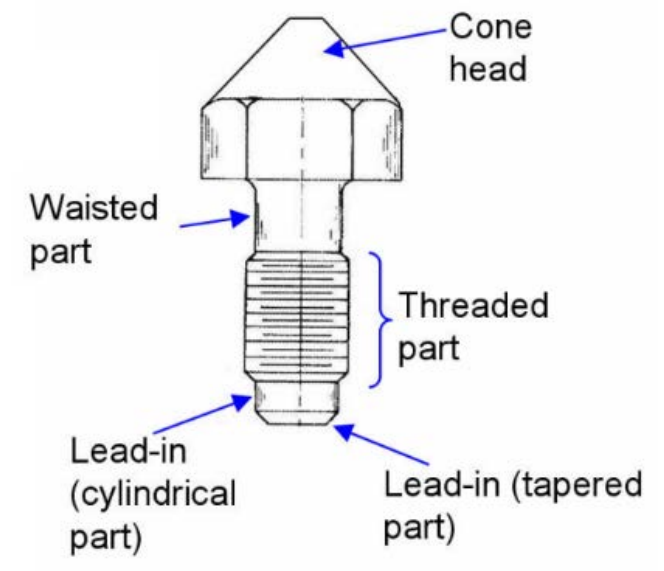

Figure E.1: Main parts of the bolt. 


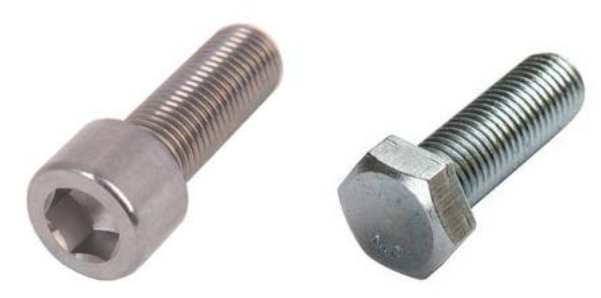

Figure E.2: Socket-head (left), Hexagonal-head (right)

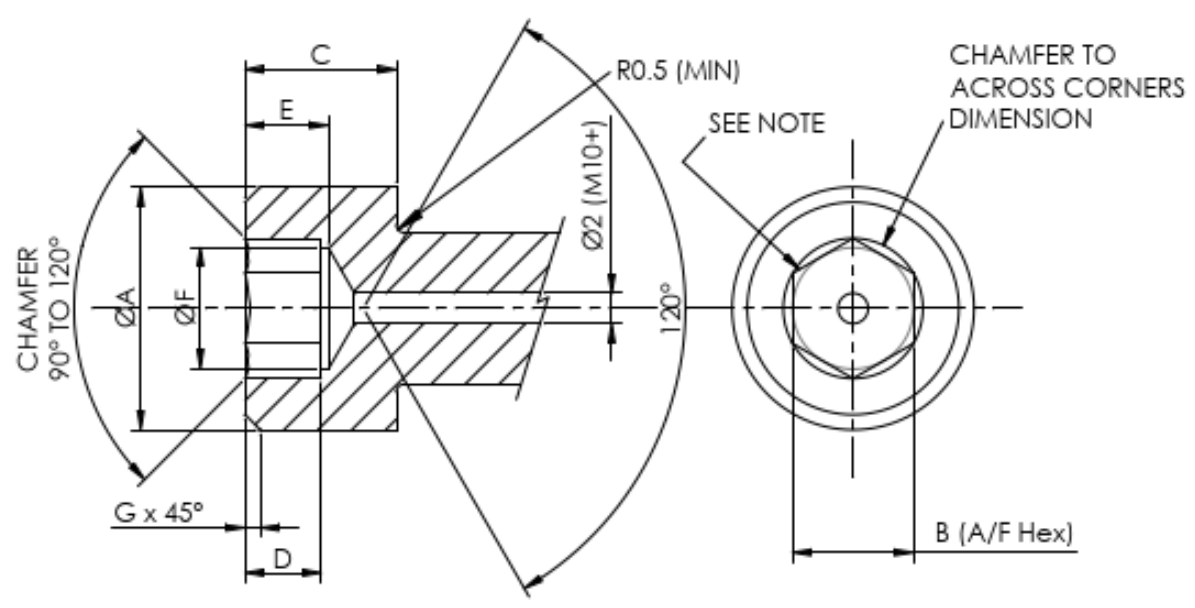

Figure E.3: Socket Head.Opposite flanks of hexagon must be parallel and internal corners must be sharp.

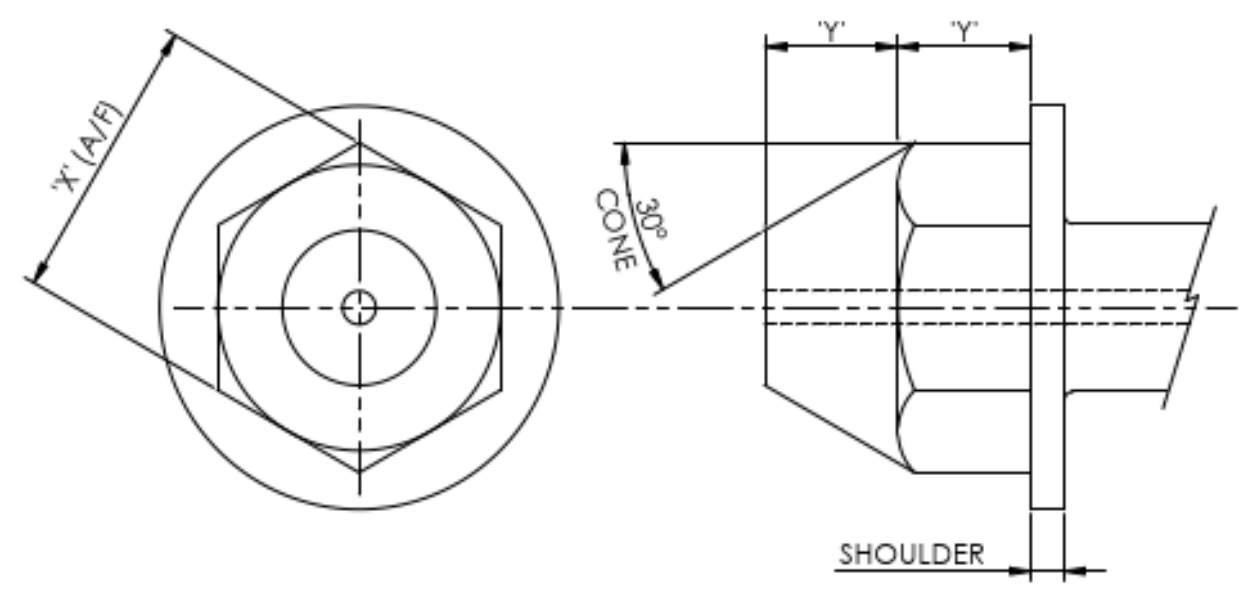

Figure E.4: Hex Head Modifications 

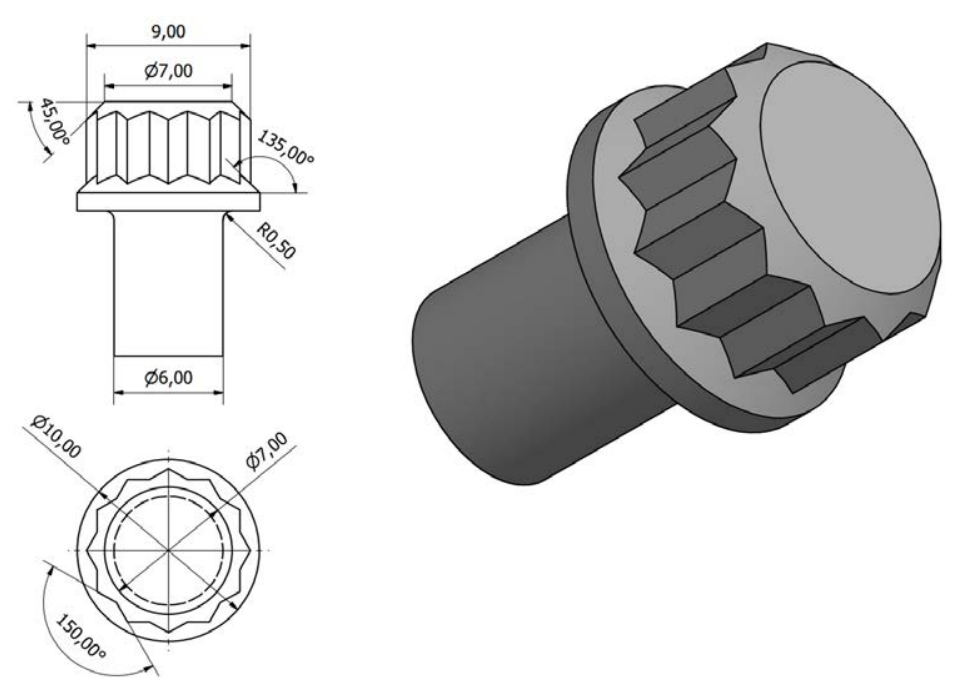

Figure E.5: Bi-Hex Head

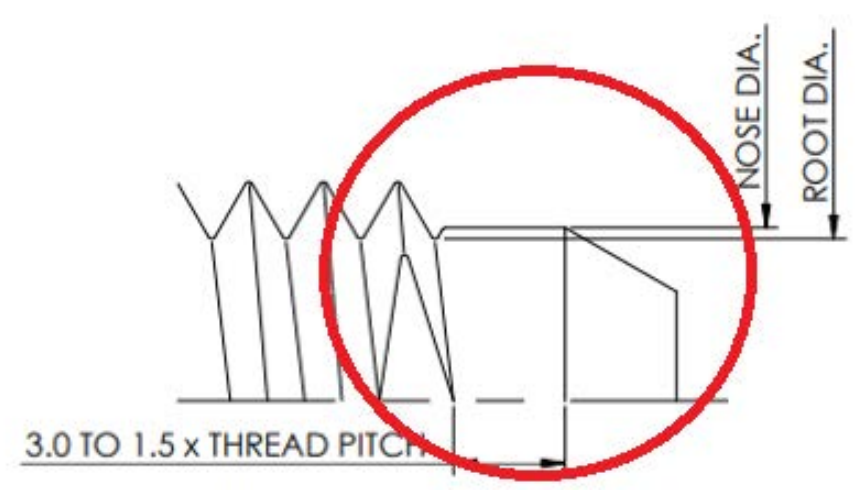

Figure E.6: Example of point to avoid cross-threading. 

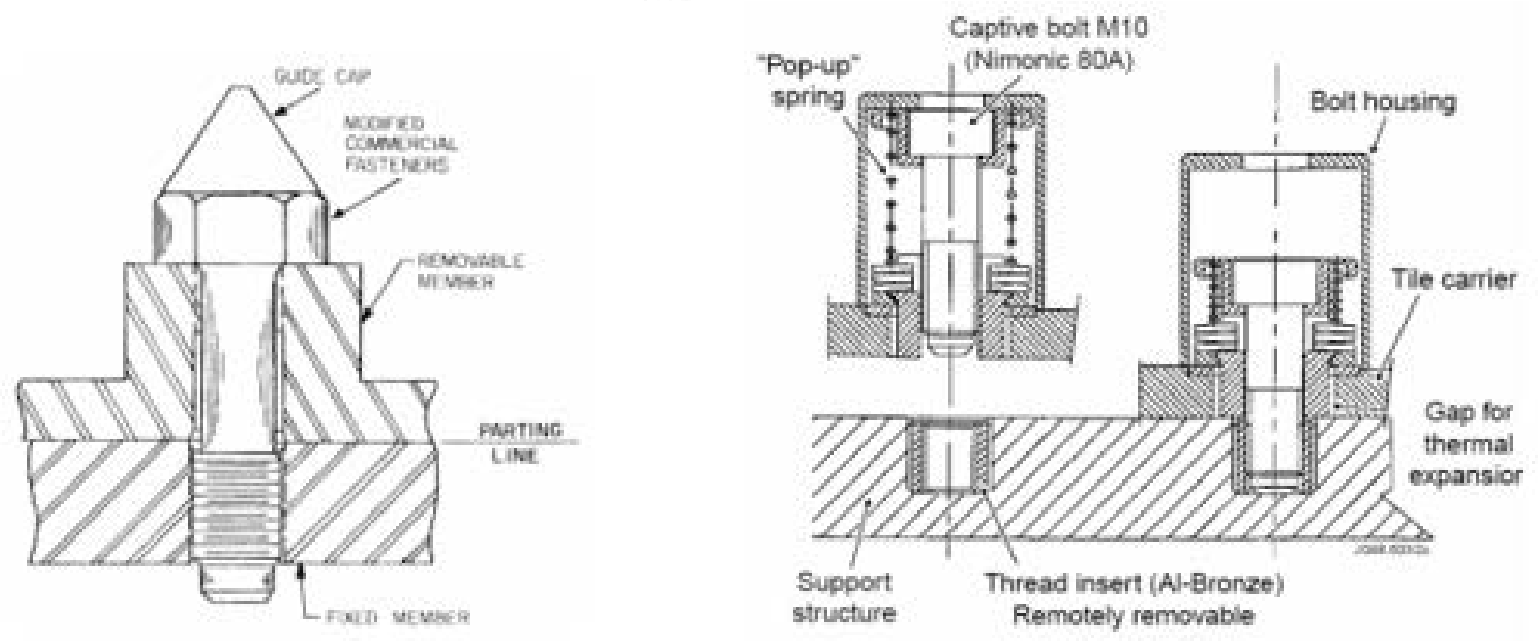

Figure E.7: Free captive bolts (left), close-up view of spring-loaded captive bolt (right).

The correct configuration of the bolts is essential to achieve good result in a short time. Three possible configurations are possible by RH (sorted by preference):

1. Bolt without nut: the screw is inserted into the component hole, but the hole where the screw is inserted should be threaded. This allows an aid to the insertion / removal, fastening and avoid cross-threading.

2. Captive bolt (spring-loaded Captive): the bolt system is more complex, but it allows to avoid re-engagement of the unbolted bolts during loosening of the other bolts on the same component, especially if the bolts are bolted downward and facilitates the joining of the mating components during assembly.

3. Bolt with nuts: this option should be avoided, if it is possible to use the previous options. Because, the nuts cumbersome to handle during insertion or removal operations.

It is recommended to avoid the use of nuts in favour of other solutions like screw the holes of the components or by captive bolts. If this is not possible, it is recommended to use hexagonal nut. It is recommended to use the self-locking a Spiralock $\mathrm{R}$. This thread form provides preload to the threads to resist the effect of loosening self-loocking, radical load distribution and improve the fatigue life.

There are different types of screw head, but for RH it is advisable to use socket- head or hexagonal head. This depends on the size of the screw:

- Hexagonal head (exterior) is recommended for values greater than $17 \mathrm{~mm}$ (M17). Socket-head is recommended for values less than $17 \mathrm{~mm}$ (M17). Acceptable fastener materials for RH equipment are stainless Steel or aluminium Bronze. Moreover, fastener metals require a minimum hardness of Rockwell C45 to resist damage from tools. 
It is recommendable use dissimilar materials (or different hardness). Since, bolts and nuts or screws and threaded seats of dissimilar materials or of dissimilar hardnesses resist galling.

\section{Design considerations}

When the position of a screw or a connector is designed, consideration should be given to what $\mathrm{RH}$ equipment and tooling will be used to perform the task. The orientation of the plant items need to be considered, i.e. installation on the ceiling or on a vertical wall is complicated by the gravity vector. Supporting indicators such as built-in spirit levels should be used to aid plant orientation where possible. Unambiguous sensors/indicators should be used where practicable to show that plant items are fully engaged (e.g.lining up features on mating items). Locations devices should be designed such that it is impossible to mate plant incorrectly (e.g. asymmetric dowel pins prevent the wrong orientation). Visual cues should also be used where practicable to assist correct assembly. Careful consideration should be given to choosing the correct datum and then tolerancing all plant items accordingly with respect to that datum. The design of a location device should take into account the realistic capture range of the mating features consistent with the size and weight of the plant. Edge guides are a simple method of providing plant location. In a single plane three points of contact are used to define the correct position and two perpendicular forces are required to fully constrain the item. Edge guides are particularly appropriate where a clear view of the installation process is required as the supports are external to the located plant item. For future machine design, remote handling compatibility must be taken into account. The life cycle activities to be considered in the design phase are listed in the following:

- Manufacture.

- Assembly.

- Installation.

- Commissioning.

- Maintenance.

- Repair.

- Upgrade

- Removal.

- Disposal.

At each stage, characterization of handling equipment and implications for design requirements must be taken into account. 


\section{Guide Pins}

Guide pins should be used to reduce mechanical misalignment. These pins are useful to provide coarse initial location in reducing the DoF's of a located device item. The guide pin reduces the initial misalignment to bring dowel pins within their capture range which themselves provide accurate final positioning (see Figure E.8).

Guide pins should be mounted on the device, machine or plants that needs to be maintained, it is preferable not to have it on the mating structure, so that the location holes in the structure can be used for visual guidance during assembly. The length of the guide pin is determined during design by the interface and the available visibility during assembly (e.g a long guide pin is required where visual access is weak).
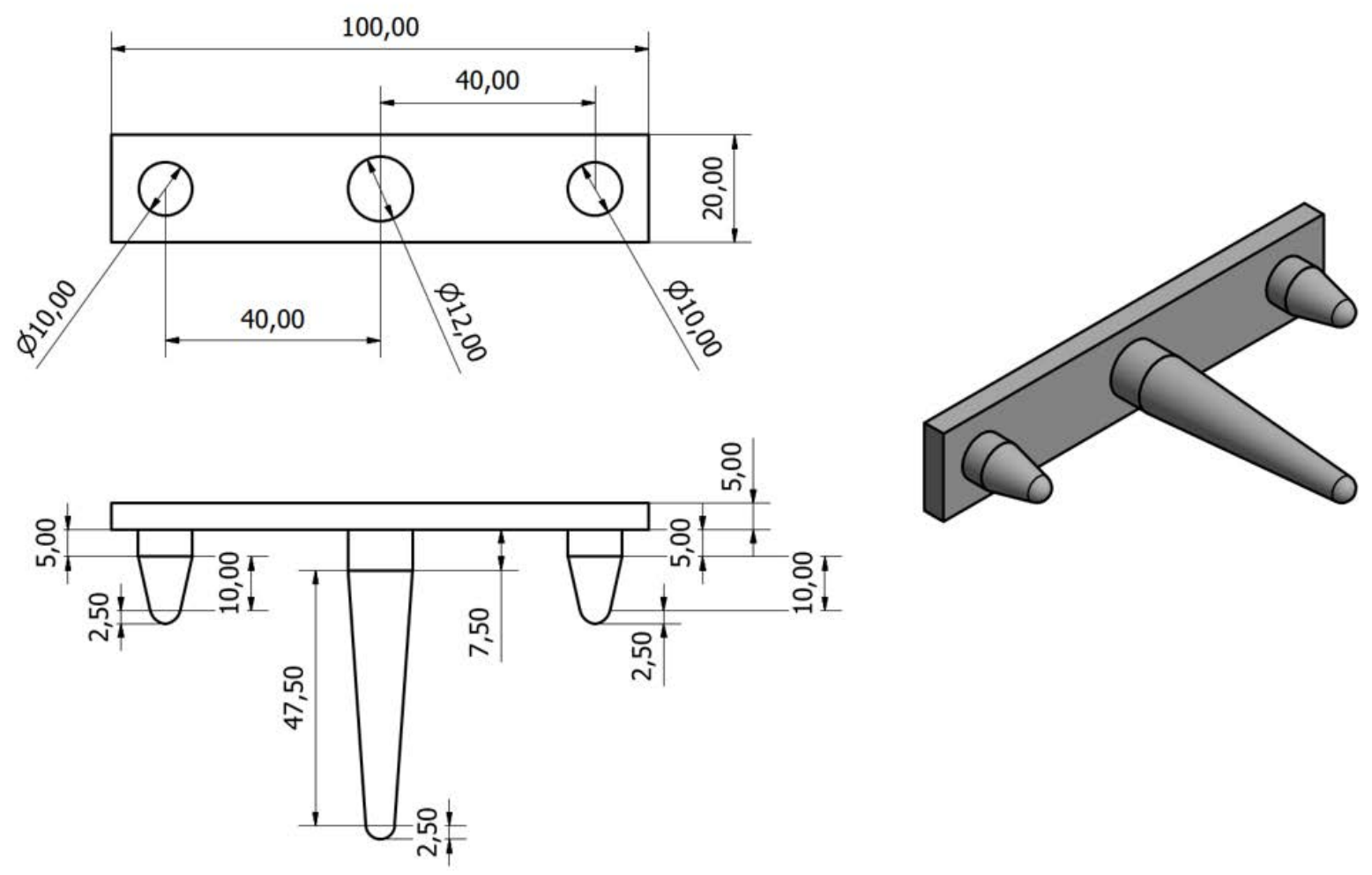

Figure E.8: Guide Pin

\section{Dowelling Arrangement}

Dowels are usefull element to be used when two mating items need to be matched. Two dowels and one contact surface are required to fully constrain an item of plant. The dimensions of the dowel (length and diameter) must be designed appropriately with respect to the weight of the elements and the method of manipulation used. Dowels could also be used to protect against side loads on mechanical fasteners during installation, maintenance and dismantling phase. One limitation of this method of location is that the dowel(s), contained within the body of the plant, are obscured during engagement. This method, 
therefore, relies upon good kinematic constraint and good viewing / sensing. Dowels should be located on the machine to be handled allowing a better visual guidance during assembly. Two mating surfaces are generally mated using parallel dowel pins. One dowel positioned in a fitted hole eliminates two translational DoF's. The second dowel eliminates a rotational DoF (see Figure E.9).

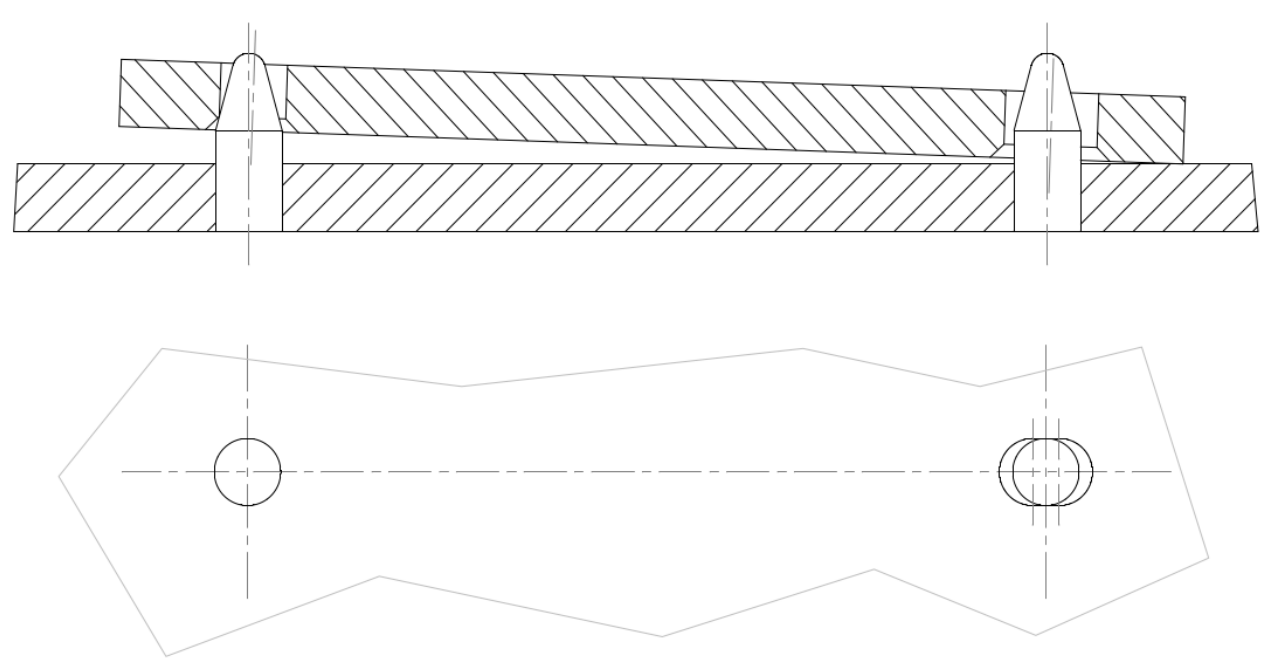

Figure E.9: Parallel Dowel Pin

With the aim of minimizing the risk of jamming, dowels should be as short as possible to provide adequate location and strength. The profile of the nose of the dowel should increase the capture range avoiding jamming. Cylindrical dowel pins should be spaced as far as possible in order to minimize jamming of the pins when angular misalignment of the plate occurs during assembly phases. A ball-ended dowel arrangement example is show in Figure E.10 and details of dimensioning and tolerancing are given in Figure E.11. 


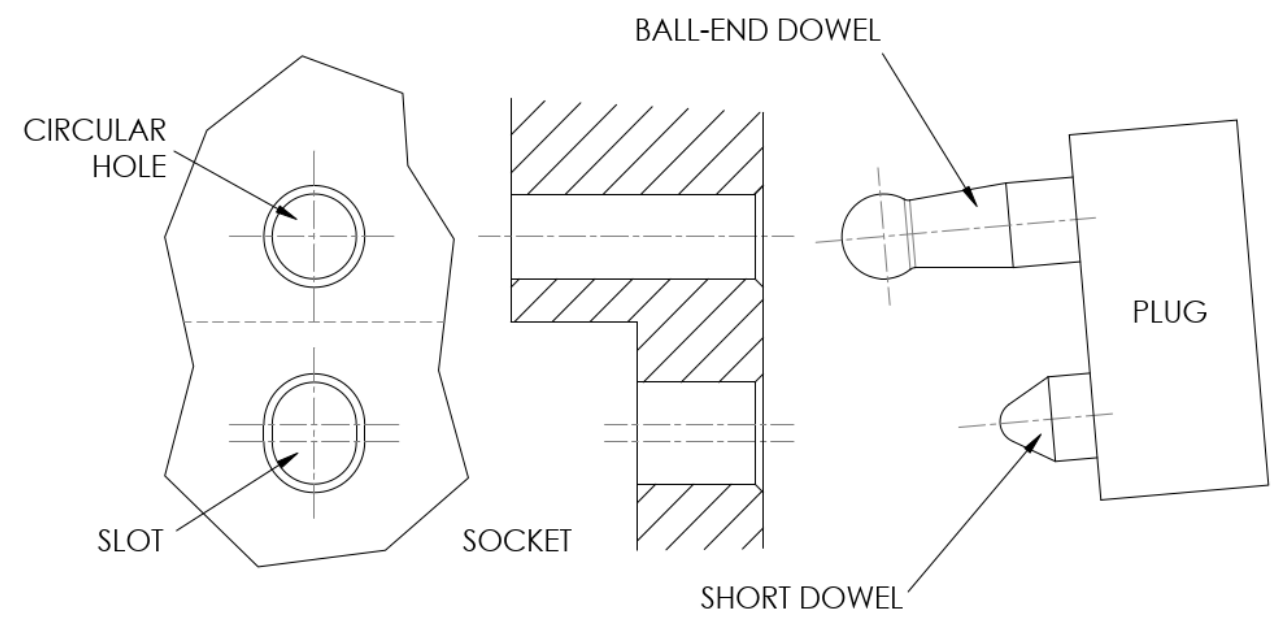

Figure E.10: Ball-End Dowel Arrangement 


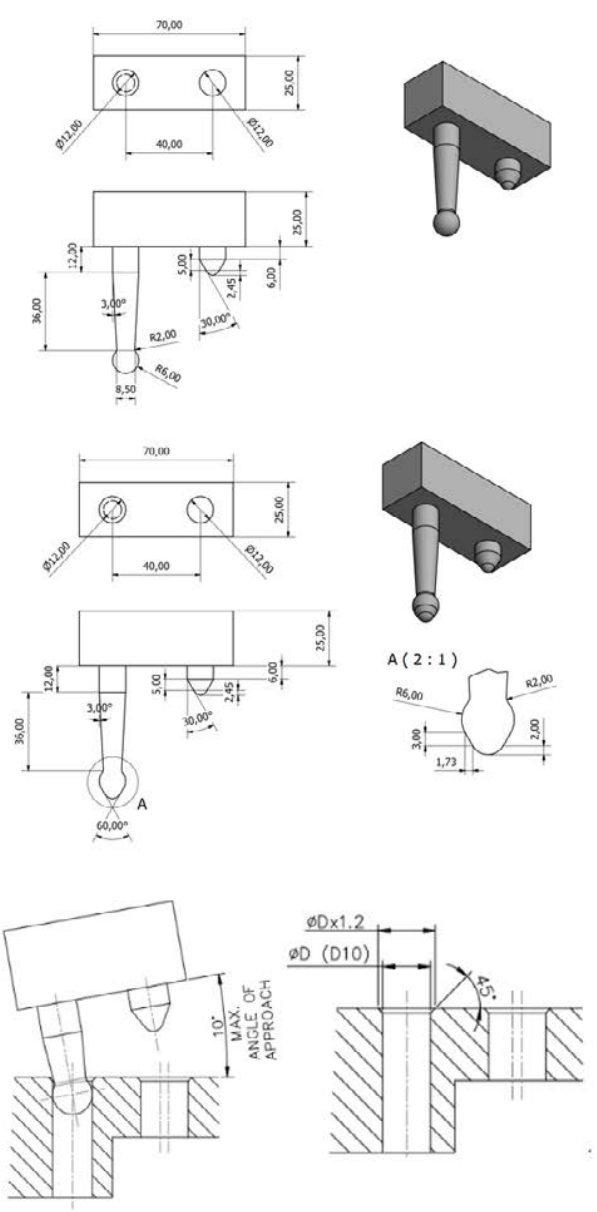

Figure E.11: Dowel Dimensioning and Tolerancing. This design incorporates a long ballended dowel that locates in a circular hole and a short parallel dowel that locates in a short slot. On top, it is shown the round shape design. In the middle an alternative design to help the insertion. On the bottom it is indicated the maximum approach angle.

\section{Electrical Connections}

Electrical cables allow connect electrical devices, junction boxes or exit ports. Their function is to transfer power and signals. they are divided in two categories: cable and connecting cable (connectors). Cable transmits the power and signal to different points. The cable should be selected according to the characteristics of the working environment, in this it influences: the placement, the material the form, the design and other elements that will be explained in the next section. In addition, the cable must provide flexion and accommodate the relative movement between dispositions and connections. The connector allows to connect the end of the cable. It is diveded in two parts, one part located in the cable and the second one located at the end of the connection box, port or the electrical device. Both ends parts should have a connector designed for an easy handling. In this way, the electrical device can be removed with its associated cable, while reducing the likelihood of cable failures occurring between the cable and the device. However, electrical 
connector selection for systems targeted for remote maintenance is highly dependent upon the respective maintenance system and its capabilities (e.g., equipment and tools available to handle the connector).

Push-pull connectors are preferable to that requiring rotary motion for mating. In case of needing rotary movement, minimizing the number of revolutions is recommended. Moreover, the connectors have to be capable of being engaged and disengaged, reliable, accessible, easily hand-held and handled by servo-manipulator or by extension tools.

On the other hand, angled connectors (90 degrees) are preferable to in-line straight connectors. This is because it allows a greater handling and the electrical lines do not interfere with the manipulator, remote viewing and the gripping of the connector. However, rotary locking type connectors are not well suited to angle models.

Commercial and standard solutions are preferable for cables and connectors, this increases their availability, compatibility and decreases the price. However, sometimes specific designs must be ordered depending on the environment.

The position and orientation are essential for the easy handling of the connectors and to avoid interferences. It is important take into account the following recommendations:

- Electrical connectors should be located in positions that do not interfere with the removal of the main components, to avoid collision damage.

- The electrical connector should be mounted with its axis approximately vertical, preferable downwards (cable straight by means of the gravity force). Never at an angle greater than 30 degrees from the vertical. If this disposition is not possible, an extra support is needed to avoid the bending of the cable or pipe at the connector and excessive forces on the connector.

- A connection to the underside of a horizontal face should be avoided, if necessary, favors an in-line connector.

The connectors need a minimum space between them to allow grip, handling, connection and disconnection. The minimum space between connectors should be $40 \mathrm{~mm}$, but it depends on the size of the connector and the force needed for manipulation.

The use of connectors with a suitable gripping interface results in easy and fast (dis)connection operation. Recommendations are exposed below:

- Connector bodies with flat, parallel surfaces are easier to grip with manipulator tongs than are cylindrical bodies.

- Knurling on the gripping surfaces aids operability, especially for rotary motion connectors.

- A connector with a lanyard design introduces a potential tangling hazard, and a gripping surface is still required for re-joining. 
The length of the cable must provide sufficient length to allow the operation of (dis)connection and bending of the cable. However, the length must be sufficient to relieve the cable tension or avoid tangles during the operation or replacement.

The materials depend on the environment (i.e.: temperature, radiation, etc.); these must be compatible with the workplace. Examples of these materials can be:

- Rad-hard materials.

- Austenitic stainless steels for connector bodies.

- Ceramic inserts.

- Noble metal plating on pins and sockets.

- Hermetically sealed units.

- Gold plated pins for the connectors

It is recommended to minimize the number of cables and connectors. It reduces the quantity of cable lines, saving space and simplifying the system. Multi-connectors allow reducing the number of cables, but they increase the cost.

Depending on several factors, the choice between multiconnectors or individual connector could be decisive. The advantages of multiconnectors are that they can include power, multipin signal, and coaxial connectors installed in a sole structure. For this reason, multiconnectors are preferable in work spaces with constrains and to provide remotely maintenance. However, they are specifically designed for each application and so result in a higher cost.

Two general types of electrical connectors are used: those that transmit electrical power and those that transmit data. It is preferable not to mix the power and signal lines in order to avoid noise and electrical interference. However, they are placed in a single tether in some cases, for example:

- Remotely operated vehicles.

- Many signal and power lines use two connectors, one for the different signals and one or two power connectors might be convenient.

- In presence of few power and signal lines, it should be assessed the use of only one connector.

- Servomotor connections

It is necessary to calculate the force that is needed for the (dis) connection of the connectors. Depending on the result, if the servo-manipulator does not have the necessary force, different alternatives could be required: 
- Connection mechanism: It guides or/and adds force during the operation. This is the preferred alternative.

- Connection Tool. It helps the manipulator during the operation of (dis)connection.

It is recommended that the connectors have alignment guides and / or staggered alignment guides. Conventional connectors have some degree of guiding mechanism, but usually not enough. The alignment guides must be visible at all times, otherwise the operation becomes more complicated and damage may occur.

Cable Carriers are needed for all cables and jumpers. Where they should be totally integrated with the design modules. This allows the cables are not loose, which prevents obstacles, interference and unexpected accidents. Cable carriers should be included:

- Cable trays.

- Cable trunking/conduits.

- Cable chains

- Festoons.

Permanent alignment marks are helpful. In case the alignment guides of the connectors are not visible, the marks must be obligatory.

Cables have to pass through the roof, walls or soil. Thereby, the electrical service penetrations should take into account the mode of repair, replacement, and upgrading of the cables. There are different proposals like integrate the cables in a removable shielding plug, remove the cables from the inside of a conduit located in a fixed non-removable structure or remove the cable from the inside of a conduit located in a removable shielding plug. The option depends on the cost, maintenance schedule, feasibility, power involved, probability of failure, etc.

The selection of connectors and cables depends on several factors:

- The capacity of the manipulator for handling (dis) connection of the connector.

- The strength of the manipulator.

- Number of connections.

- Maintenance program.

- Environment.

- Reliability.

- Availability.

- Cost. 


\section{Dowel for Electrical Connections}

The ball-ended dowel design is recommended to be used where an electrical connection needs to be done. It provides high level mechanical guidance over the connector. The electrical contact pins drive the choice of the length of the dowel pins. To prevent damage on the electrical pins and jamming, the ball-ended and short dowel must first be engaged on their parallel shafts before final connection of electrical pins (see figure 3.9). The fastener for a dowelled connector should be located as close as possible to the contact pins to minimize lateral forces on the pins. The recommended solution is with the pins located around a single central fastener.

\section{Pipes and Flanges}

The aim of pipes is to transport fluid from point to point in the work area. Pipes have flanges that provide break points on the pipes. Then, some examples of flanges that allow the possibility of easy handling:

Quick disconnect system of flanges (see Figure E.12) allow an easy and fast (dis)connection of the clamps and flanges. The clamp holds the flange, and a screw allows opening and closing the clamp. It is the most recommended method for remote handling. Various designs of fast disconnection clamps are present in the commercial market. Figure E.12 shows an example of a QDS clamp.
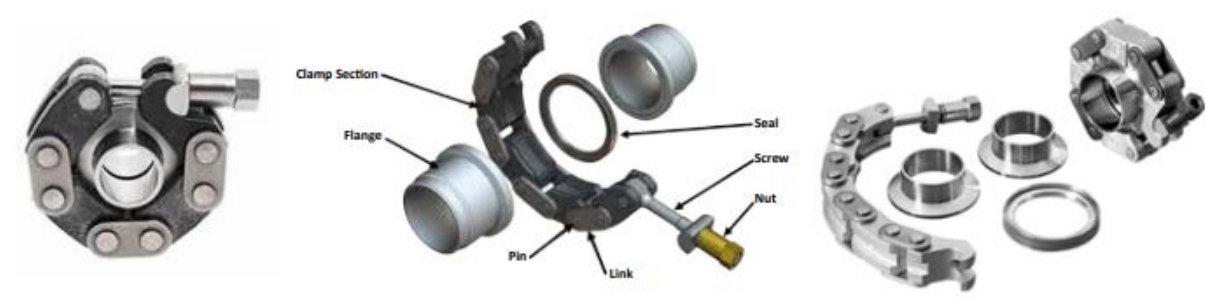

Figure E.12: Quick Disconnect System example from Technetics

In bolted systems, flanges are (dis)connected through screws and nuts (see Figure E.13). This solution could be not adequate from the remote handling point of view. This is because handling the bolt or the nuts could be a difficult operation and could increment the operation time. The use of captive bolts and guiding aids is preferrable. 


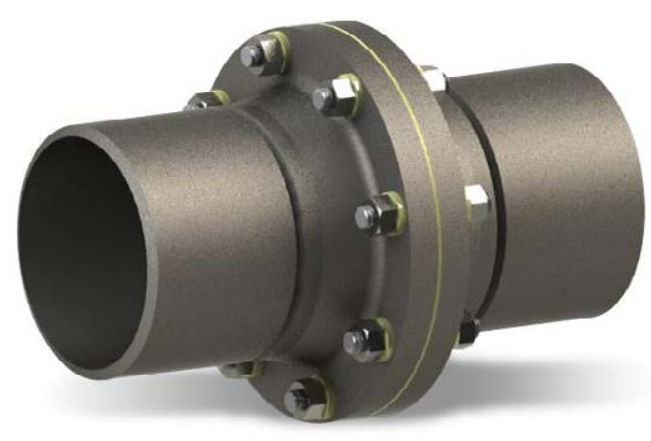

Figure E.13: Example of bolting flanges

The most favorable welded joint for remote handling are lip welds, but weld and sleeve welds. Multicouplig containing several flanges for different pipes. In addition, electric connectors may be included in the same module. Some companies offer commercial semistandar couplings such as STÄUBLI (MCS type multicouplings). The selection of the type of flange depend on the capabilities of maintenance, maintenance scheduled and cost. QDS flanges are a recommendable system due to the easy and fast disconnection. The number of pipes and flanges should be minimized. The proposal to minimize the number of lines consists in grouping a multiple pipe lines in a single flange. The more suitable position to handle the flanges is a vertical axis position. If flanges are in the horizontal axis, it is appropriate to use supports guides and captive bolts. On the other hand, the set pipe + flange should remain stable in position during time operation. Spigots and extra support can be helpful to achieve a stable position. Axial and azimuthal location can be provided by external spigot guides (see Figure E.14). Two such guides are used per flange at 180 degrees. 


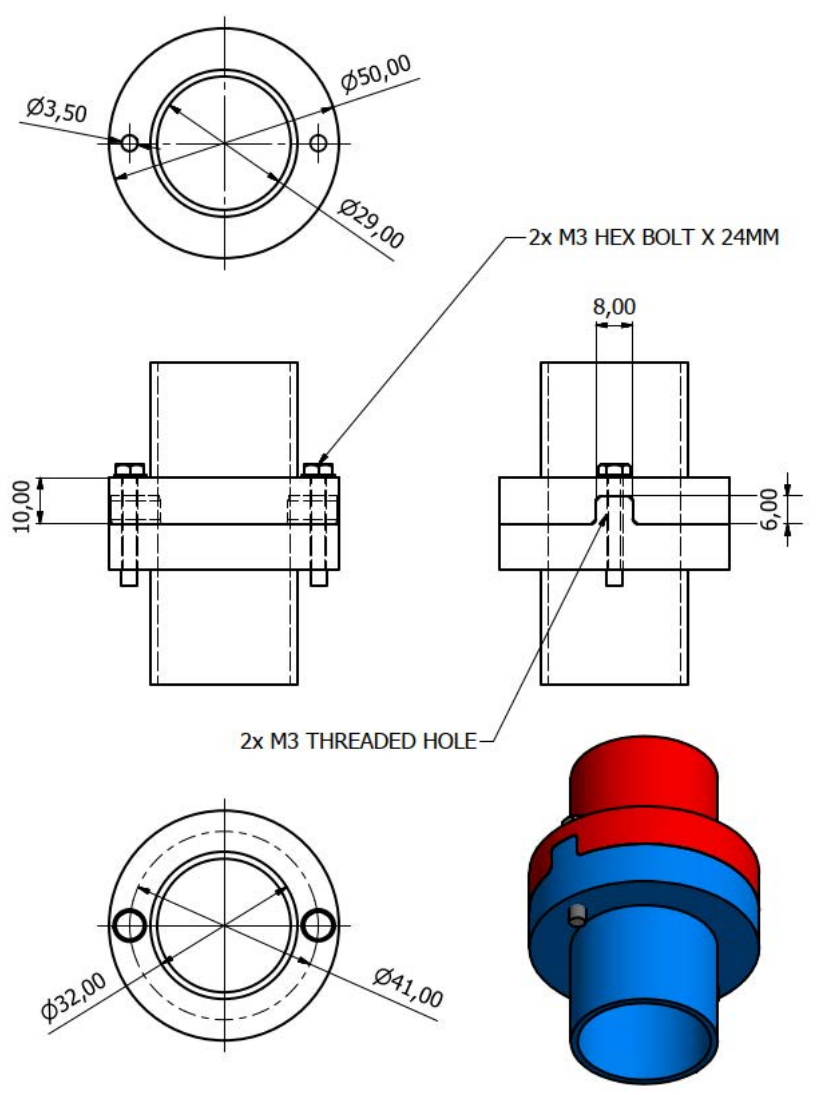

Figure E.14: Axial \& Azimuthal Flange Location

Bolted flanges can be located axially by an internal spigot arrangement. Figure E.15 shows the spigots as used on the Class 300 vacuum flanges.

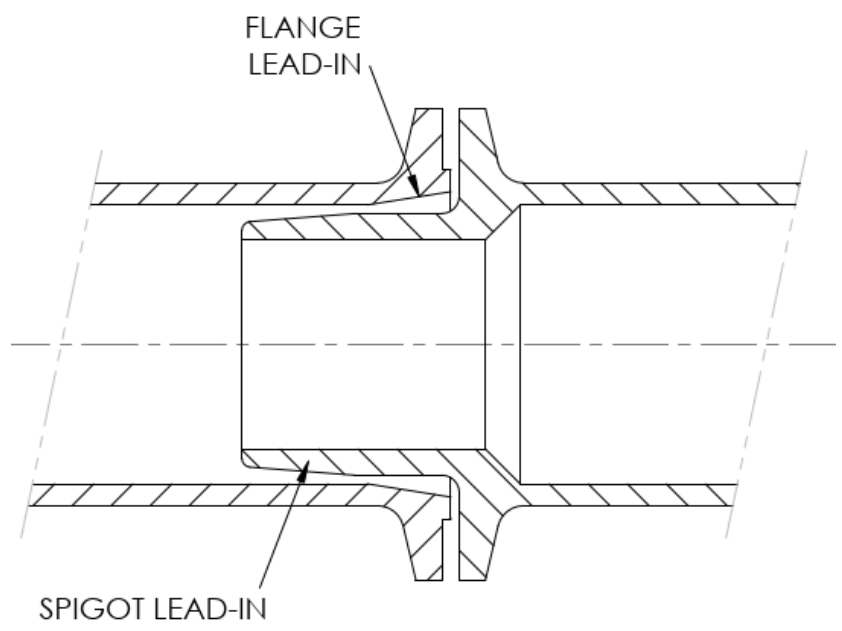

Figure E.15: Flange Spigots 
Space has to be provided for the access of the personnel/staff, tools and equipment required for the removal/installation of each particular flange. Study the time of the flanges operation. The time operation for the bolted flanges increases for this reason. The use of quick disconnecting flanges is recommended.

To assist the (dis)assembling operation, spigots and guide pins are recommended. It is recommendable to use bellows to allow smooth (dis)assembly. The seal rings of the flanges should be supplied with mechanical limits to avoid overcompresion of the seal. Seals to be installed/replaced by RH, it is recommendable to use seal tabs. For example: Garlock Helicoflex@. Captive bolts are recommendable in case of horizontal-axis bolted flanges. Both flanges should be removable in case of replacement or refurbishment.

\section{Reliability}

Components under a high standard of reliability are essential in all accelerator facilities because reliability has a crucial implication in the availability of a radioactive facility. Since in most cases it is necessary to perform RH operation and this needs time to execute, which has a cost that increase with the time. For this reason it is essential to avoid failures or maintenance operation with a reliability design of the components in the facility. Recommended design practices for high reliability are listed in the following.

- Establish working conditions, which have to be below the rated nominal values of the component. For example: maximum mechanical power supplied by a motor, maximum Load for a crane, maximum Working temperature.

- The use of redundant electric drives, electronics and mechanical components.

- Proper work conditions and monitoring: Monitor the working conditions of the components, which have significant probability of failure to achieve confident derating.

- Practise careful design, use commercial components, standards or well proved concepts.

- Extensive tests of the degree of component reliability before final assembly are essential.

- Plan the periodic tests and inspection of the components having unknown lifetime or such that are critical components.

\section{Location of Device and Kinematic Constraint Examples}

Figure E.16 shows an example of a LEMO RH electrical connector. The connector assembly consists of an MSM gripper body which includes a Lemo electrical contact plug and a ballended dowel pin. The location of the long ball-end-dowel on its parallel shaft reduces the degrees of freedom(DoF) to two; one rotation and one translation. The shorter body of the Lemo plug is used as a dowel to constrain the rotational DoF. In addition, the translation DoF, along the axis of the dowel pins, enables full engagement of the Lemo plug without risk of damage to the electrical contact pins. 


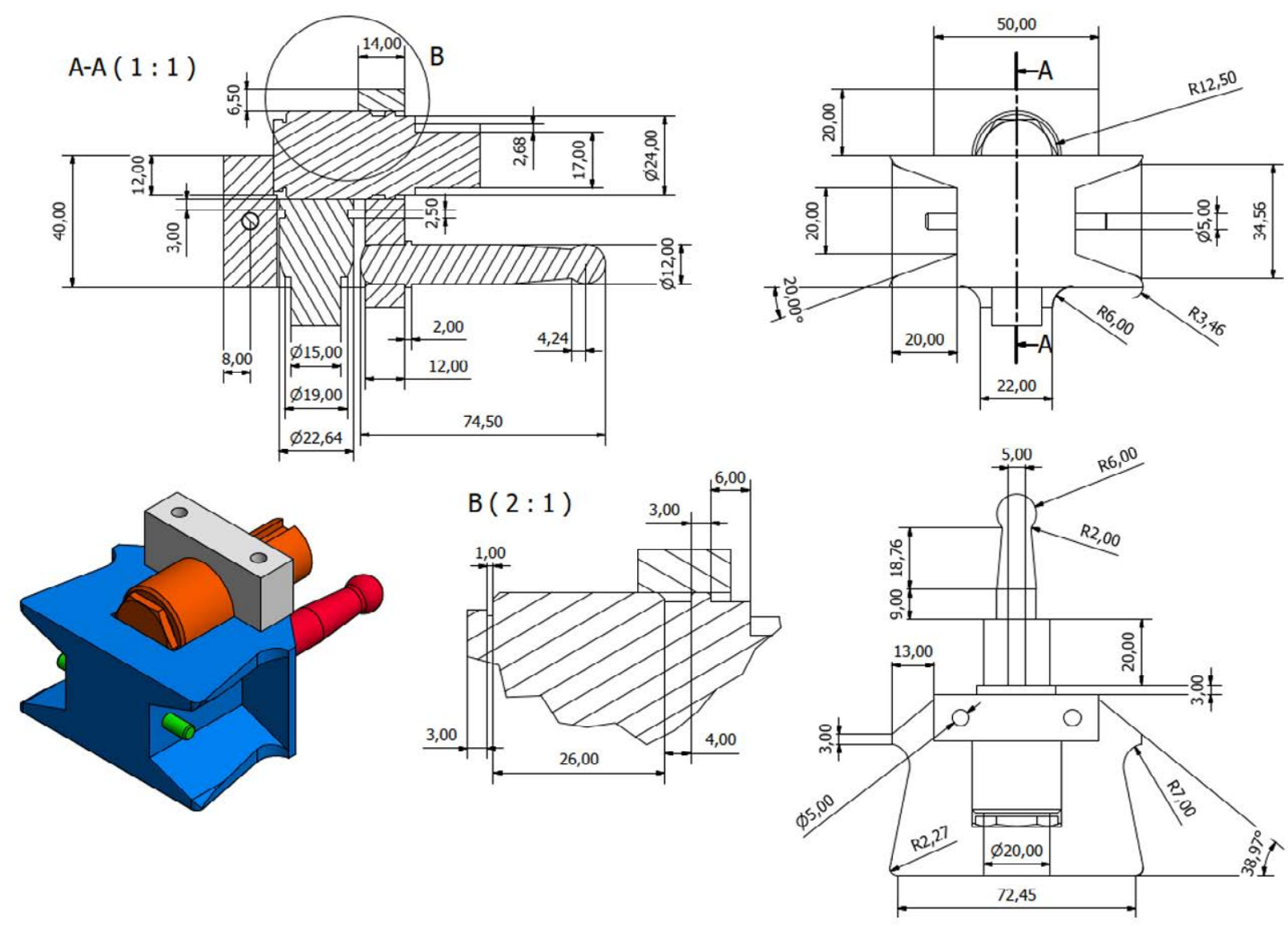

Figure E.16: RH Lemo Connector.

The purpose of the locating device is to progressively reduce the number of degrees of freedom available to the item being handled in order to ensure that the coupling process is carried out in a controlled manner without any damage occurring.

An example of an RH compatible plug connector using the kinematically constrained staged approach is given in Table E.1) and Figure E.17. At each intermediate position, the number of DoF's is reduced until the plant item is fully installed (DoF $=0$ ). This general principle of staged kinematic constraint should be applied when developing an RH location device. For each application, the method of kinematic constraint should be clearly defined. The number of steps required is dependent on the scenario complexity and the required location accuracy.

Figure E.18 shows an example of a CERNTAURO intervention at CERN where custom designed tools, described RH procedures, plug-and-play connectors and quick disconnect systems have been used. 
Table E.1: Staged Kinematic Constraint

\begin{tabular}{||c|c|c||}
\hline step & doF's & Type \\
\hline \hline 1. Plant item held in free space & 6 & $\begin{array}{c}\text { 3 Translation } \\
\text { 3 Rotation }\end{array}$ \\
\hline 2. Plant item located on dowel ball-end & 4 & $\begin{array}{c}\text { 1 Translation } \\
\text { 3 Rotation }\end{array}$ \\
\hline 3. Plant item located on single long ball-ended dowel pin & 2 & $\begin{array}{c}\text { 1 Translation } \\
\text { 1 Rotation }\end{array}$ \\
\hline 4. Plant item located on second short dowel pin & 1 & $\begin{array}{c}\text { 1 Translation } \\
\text { 0 Rotation }\end{array}$ \\
\hline 5. Plant item fully in contact with mating face (Fully Installed) & 0 & $\begin{array}{c}\text { 0 Translation } \\
\text { 0 Rotation }\end{array}$ \\
\hline
\end{tabular}

(1)
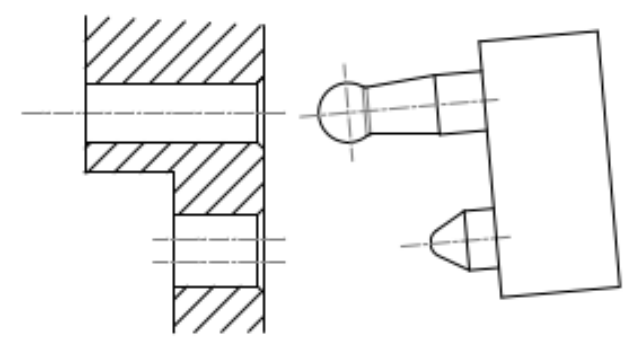

2

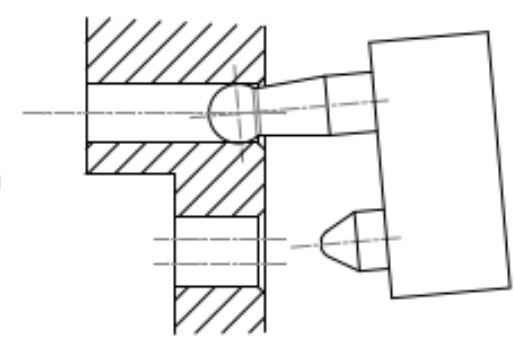

(3)

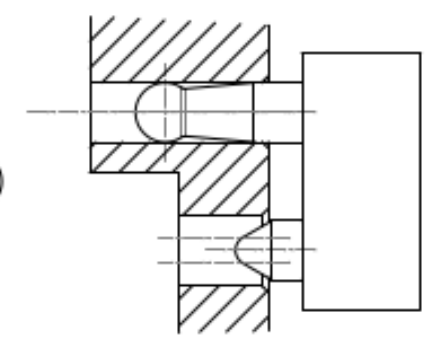

(4)

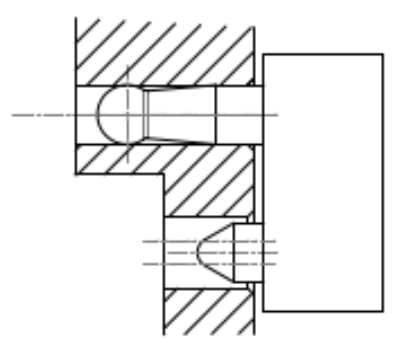

(5)

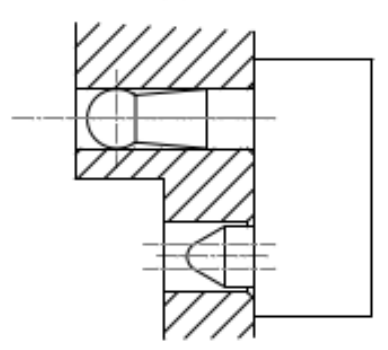

Figure E.17: Kinematic Constraint Stages 

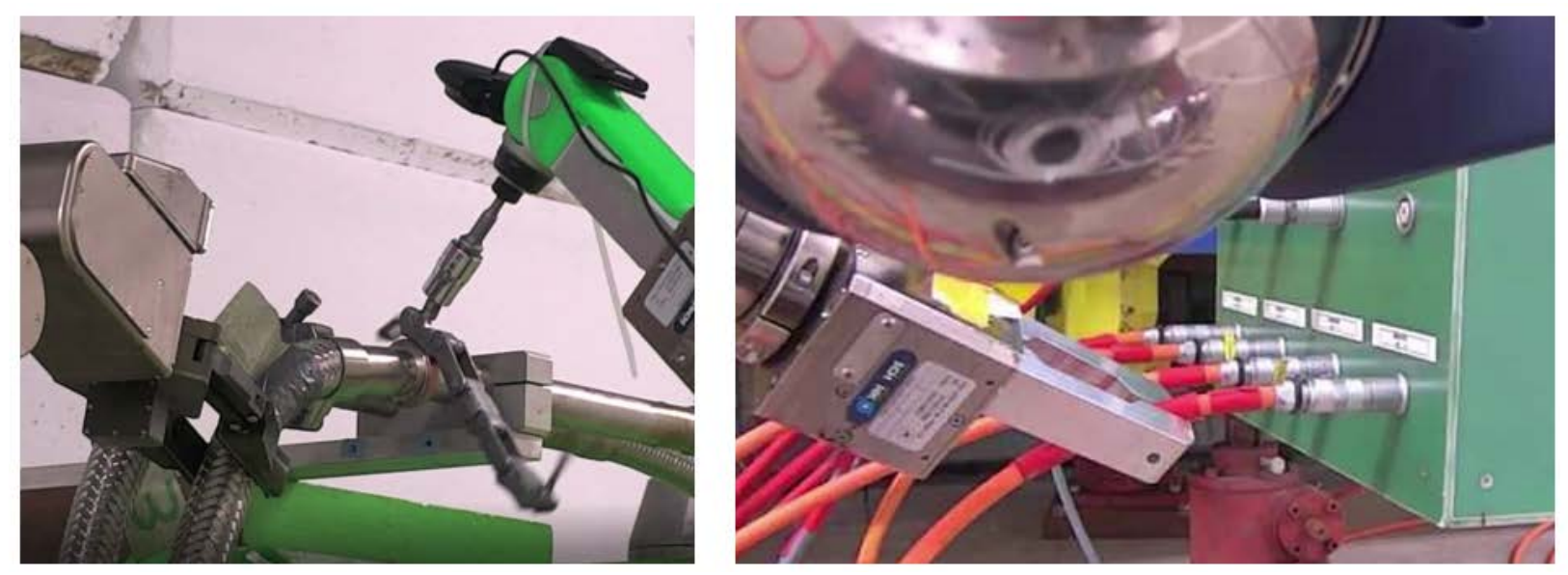

Figure E.18: Example of CERNTAURO interventions at CERN where custom designed tools, described RH procedures, plug-and-play connectors (left) and quick disconnect systems have been used (right).

The CERNTAURO framework uses several tools that have been developed for this thesis, more details in Appendix D. 


\section{Appendix F}

\section{TInspect Modules Description}

This section describes in details the developed modules for the TInspect system.

\section{Image Acquisition}

To keep up with time and space constraints, inspection systems should be easy to set up and not large in size. In our scenario, various other restrictions are imposed by the LHC tunnel environment and the TIM physical attributes. Such constraints affected the choice for the acquisition setup to be used by our system.

\section{LHC Tunnel Environment Constraints}

The LHC tunnel structure consists of eight straight sections connected by eight arcs. The tunnel's cross-section has an internal diameter of $3.76 \mathrm{~m}$ and is virtually divided into two parts as marked in Figure F.1. The TIM monorail track is installed above the passageway and due to the limited space available in this part, the data capturing equipment should be as small as possible. Low-lighting and dust are among other conditions present within its structure. Moreover, the amount of light varies from one part of the tunnel to another. This light variation causes shadows on the wall, as seen in Figure F.2, which need to be handled in the image pre-processing stage. 


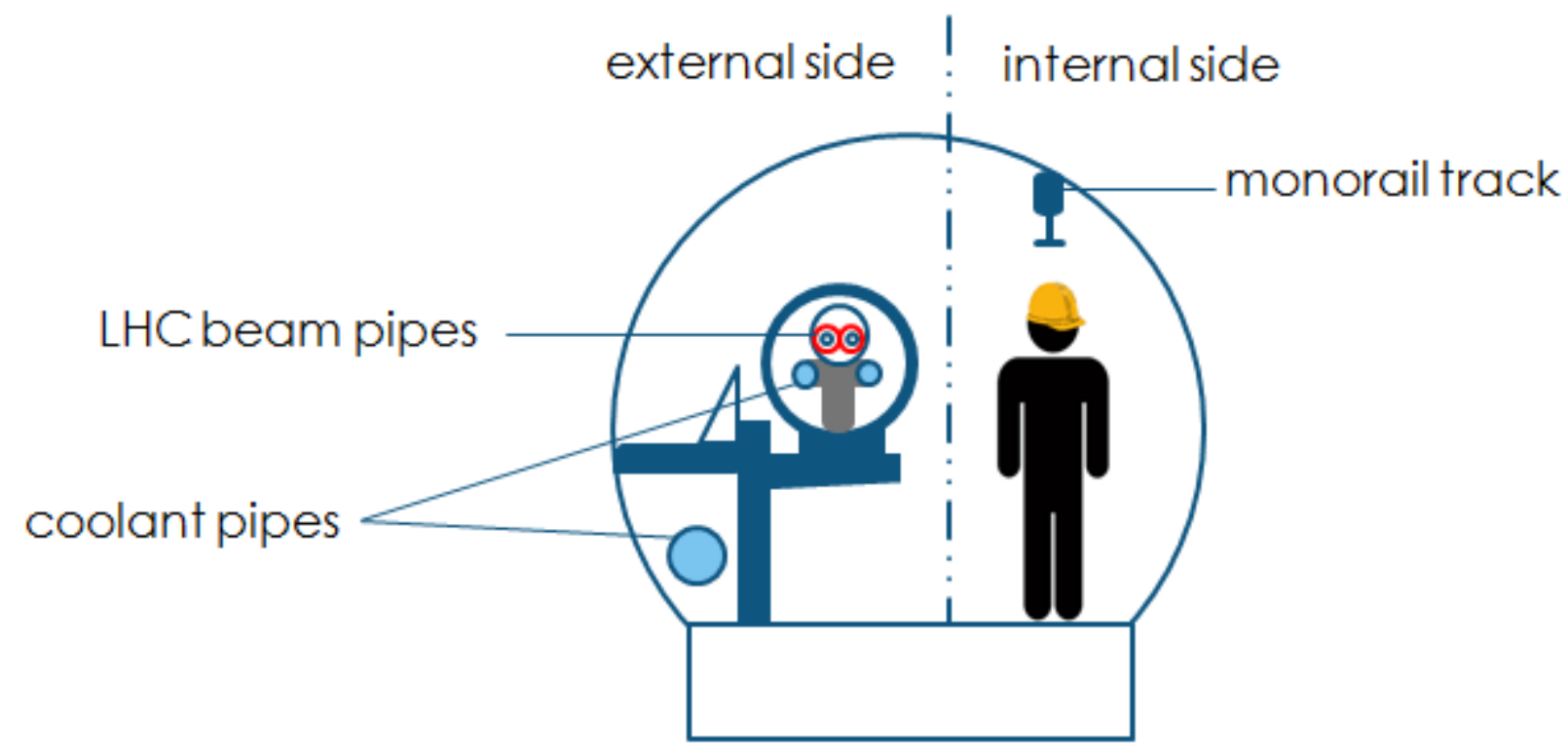

Figure F.1: LHC tunnel's cross-section,, consisting of two parts, the one hosting the beamline and the other that is available for personnel and other machinery to be moved in the tunnel such as the TIM monorail robot on the ceiling.

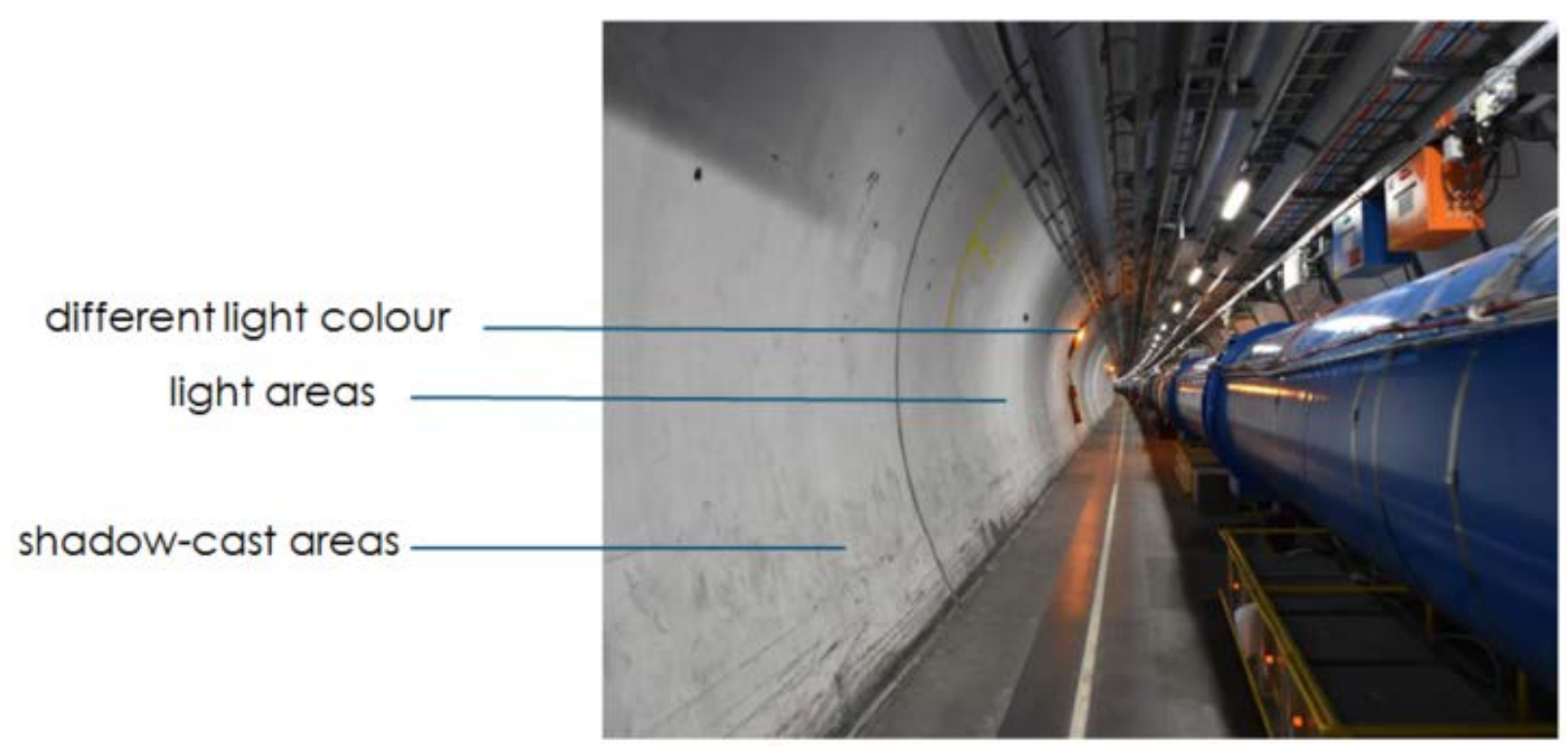

Figure F.2: Light variation in the LHC tunnel, some areas are darker than the others because of no light tubes in the area or because of shadow cast from the existing equipment

The LHC tunnel is divided in several sections which are physically separated by doors in which only a limited passage is available for the train to run through as depicted in Figure 


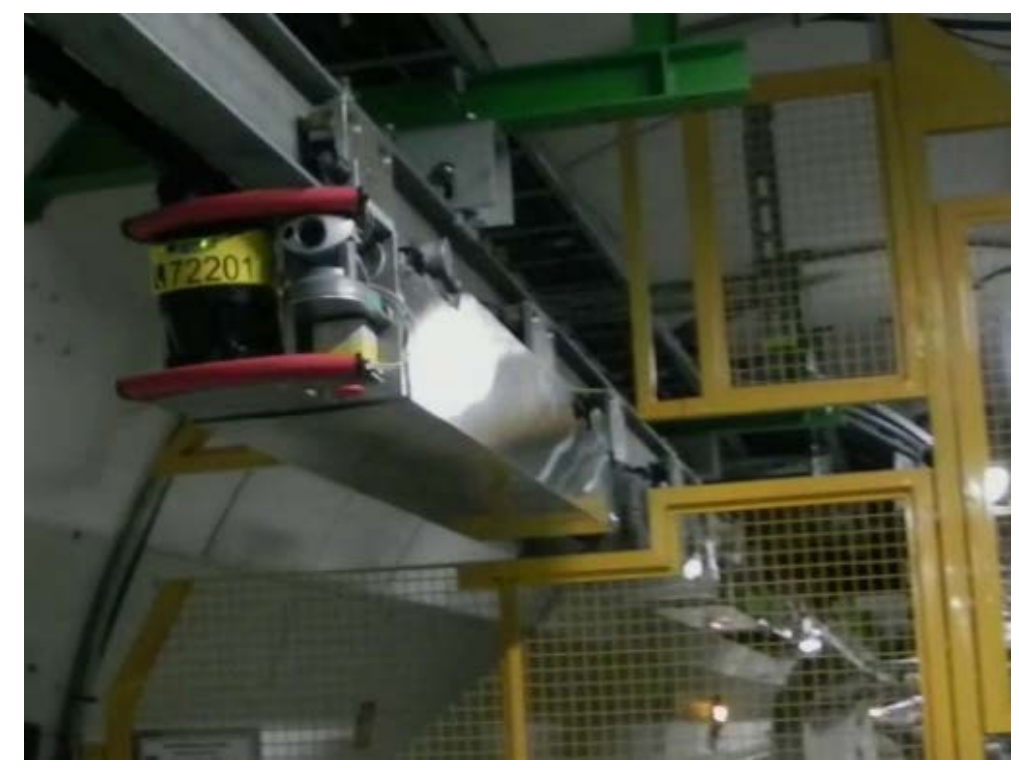

Figure F.3: TIM passing through sector doors passageways.

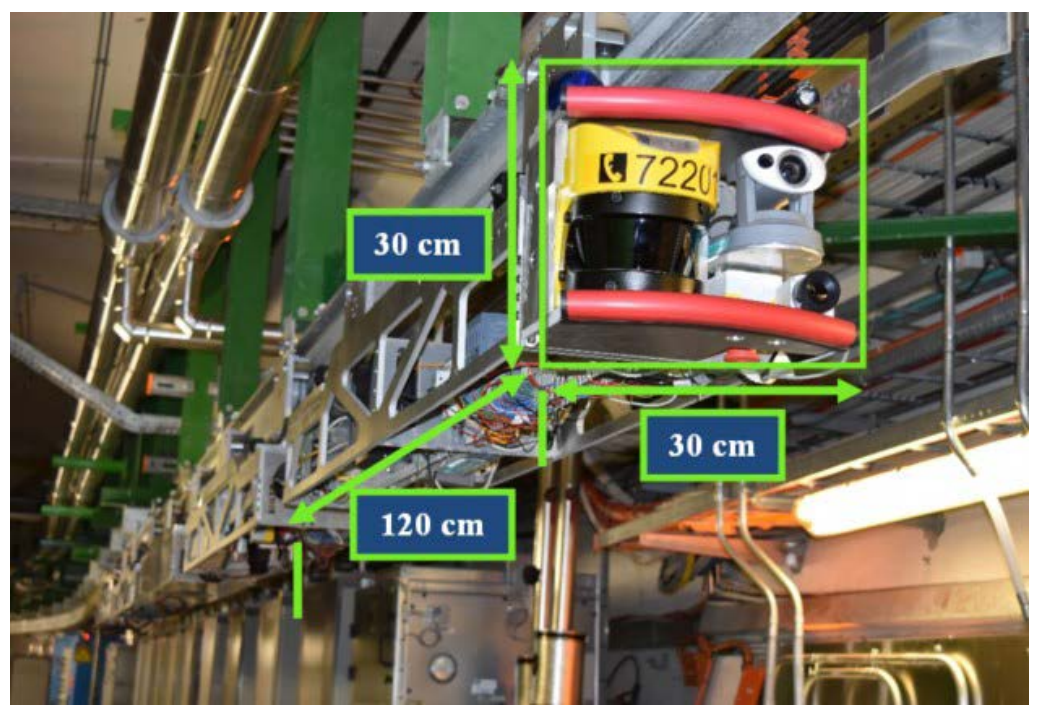

Figure F.4: TIM dimension constraints

F.3. Taking this into consideration, the robotic arm extending from one of the wagons should be retracted upwards before the TIM passes through such gates. Any photographic equipment placed on the arm should fit in the space within the wagon while the arm is not down. The available openings allow wagons of size $30 \mathrm{~cm}$ by $30 \mathrm{~cm}$ (see Figure F.4). Due to the tunnel being circular, to allow for motion around bends, the wagon length can only vary from 100 to $120 \mathrm{~cm}$. Moreover, images will be automatically captured by the camera while the TIM is running, limiting the speed of the exposure, in order to reduce the effect of blurring caused by the train's motion. 


\section{Image Acquisition setup on robots}

A Nikon 1 V3 Mirrorless camera was selected as the image capturing device after comparing different image sensors and optical systems. This was mainly due to the angle of view that can be achieved, compactness and light weight of the camera as well as its simple software integration for automatic image capture. The 10-30 $\mathrm{mm}$ kit lens was used with the focal length set at $10 \mathrm{~mm}$ to get the widest angle of view. The images used for testing were RGB colour images with a resolution of $5432 \times 3488$ pixels.

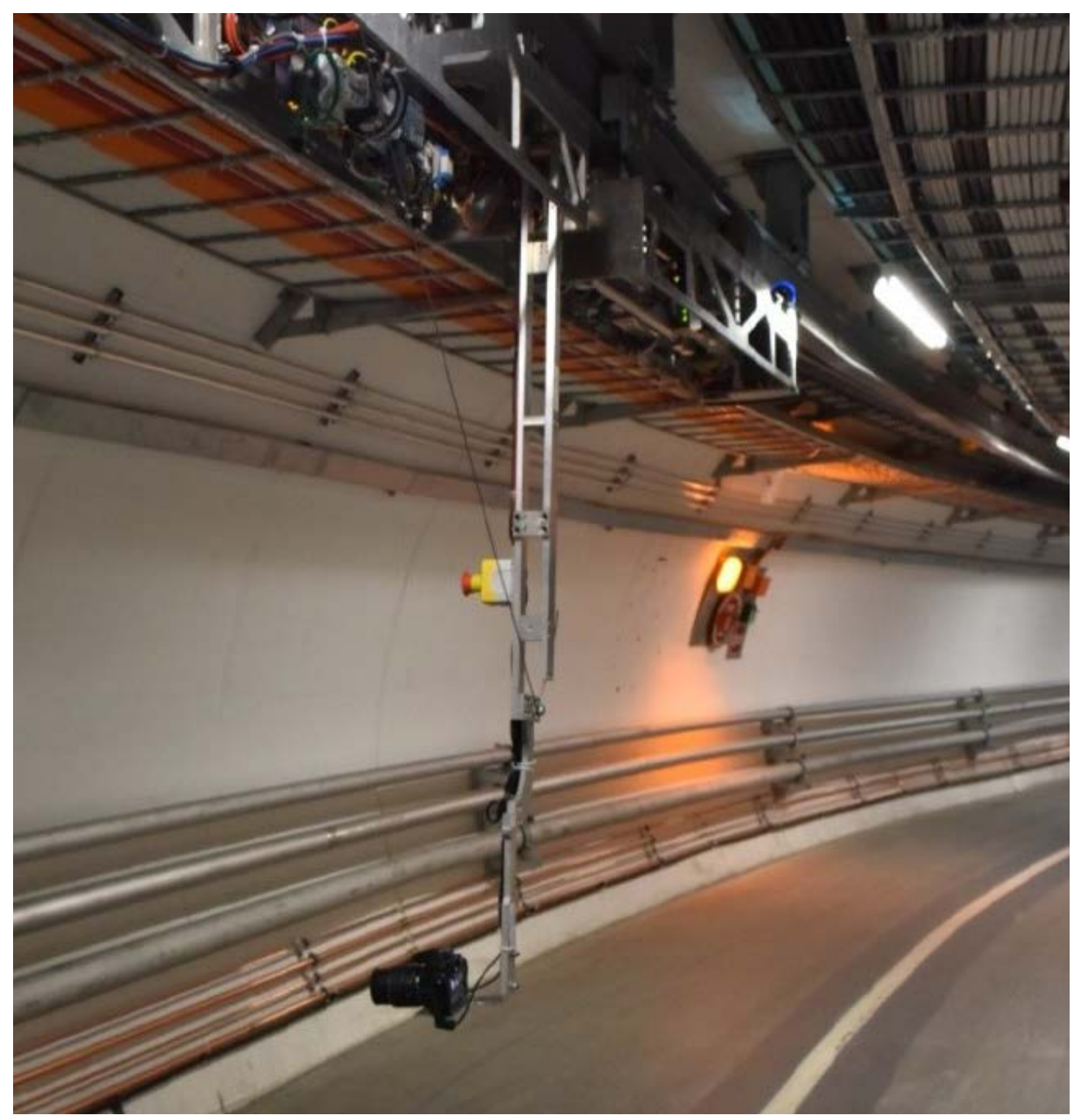

Figure F.5: Camera in the proposed orientation, such that it is facing the wall, capturing images containing a 'plain' background with wall cracks and other defects as foreground.

For the purpose of monitoring change on the tunnel linings, the camera orientation was 
set such that the lens faces the wall as shown in Figure F.5. After investigating several options, for the image capturing device, a mirror-less camera was chosen. Some benefits arising from this choice include the low cost of the equipment, its small size, its light weight and the fact that inner vibrations are eliminated as no mirror is used within the camera housing. In order to be able to operate autonomously, the TIM needs to know its position as it moves around the LHC. It measures the distance travelled using an encoder fitted to the traction wheel. In addition to this, position bar codes have been installed next to the monorail every $100 \mathrm{~m}$ around the ring tunnel to avoid cumulative errors. Therefore at each position, the location within the tunnel referred to as DCUM (Cumulative Distance in metres) is known. The DCUM refers to an existing reference system based on the cumulative distance around the LHC ring. While TIM is running along the tunnel, images will be automatically captured by the camera and saved to a data repository. Each image will have the DCUM as its file name and stored in the respective inspection date folder for easier access. Moreover this position information will be used as another sensor data in combination with the actual image content in the whole system algorithm to improve accuracy.

In general, the aim of pre-processing is to suppress unwanted information from the image data and enhance the desired image features which will be used for further processing. Various pre-processing techniques were implemented as discussed in the following sections.

A smaller image takes less storage space, requires less memory during program runtime and less computation time. Minor details such as random camera noise corrupting the image, are suppressed also through down-sampling, eliminating unwanted information in subsequent image processing steps. As a first pre-processing step, our system downsizes the images by a scale factor of 0.2 of their original dimensions.

Often, images shot in tunnel environments are characterised by low light and low contrast, both of which qualities are present in all the images datasets captured in the LHC Tunnel, as seen in Figure F.6. To cater for this, rather than adding extra lighting to the acquisition setup, our system uses the pre-processing stage to eliminate the effects caused by the latter conditions in the tunnel. We tested two contrast enhancement techniques: global image histogram equalization and CLAHE (contrast limited adaptive histogram equalization) as well as shading correction.

Figure F.7 shows that global histogram equalization improved the contrast of the image at the expense of emphasising the highlights and shadows of the images. CLAHE (see Figure F.8) improved the contrast in the image locally, such that each neighborhood has contrast within itself. However, the overall image did not improve much and features on the wall are still lacking, possibly causing difficulty in subsequent image processing steps. 


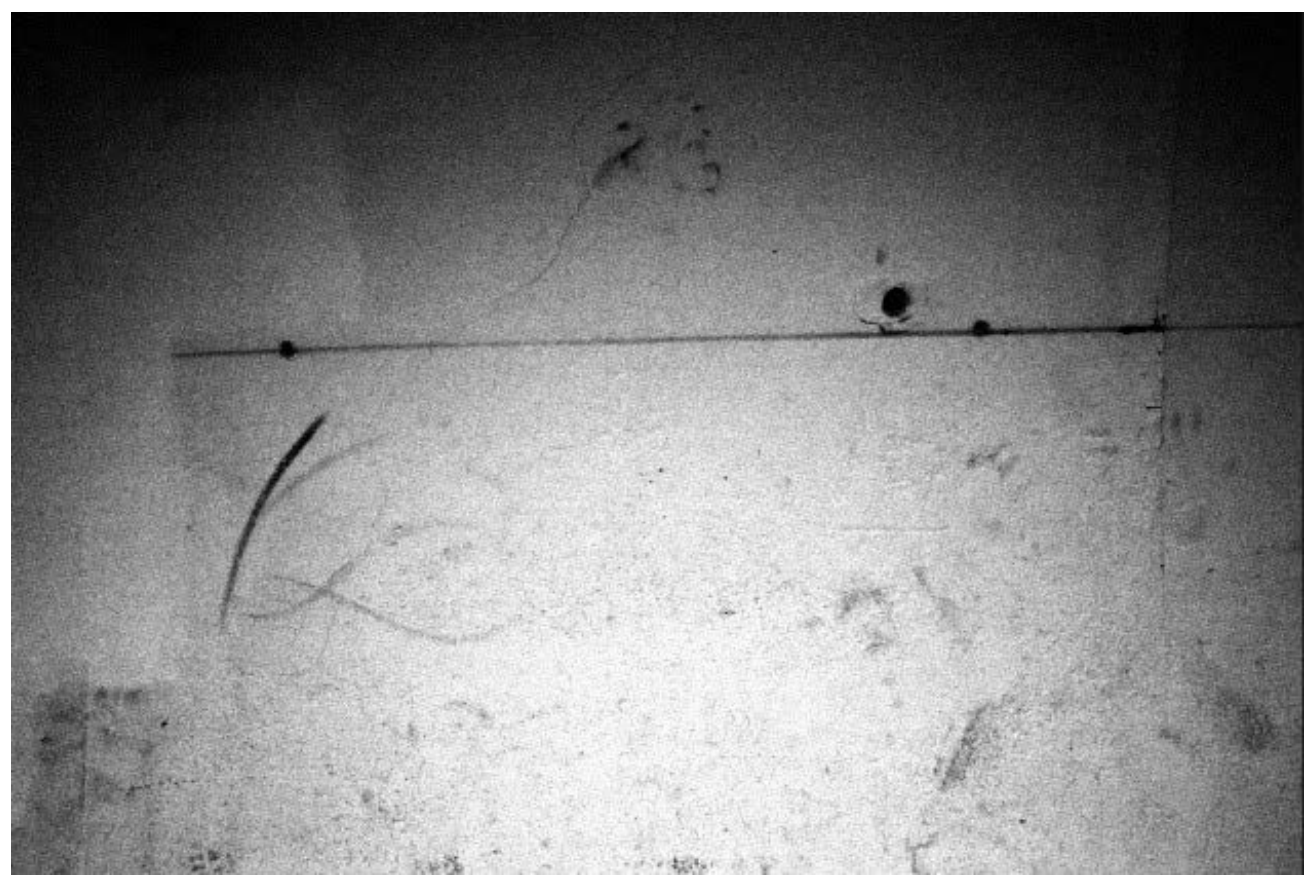

Figure F.7: Image after enhancement using global histogram equalization

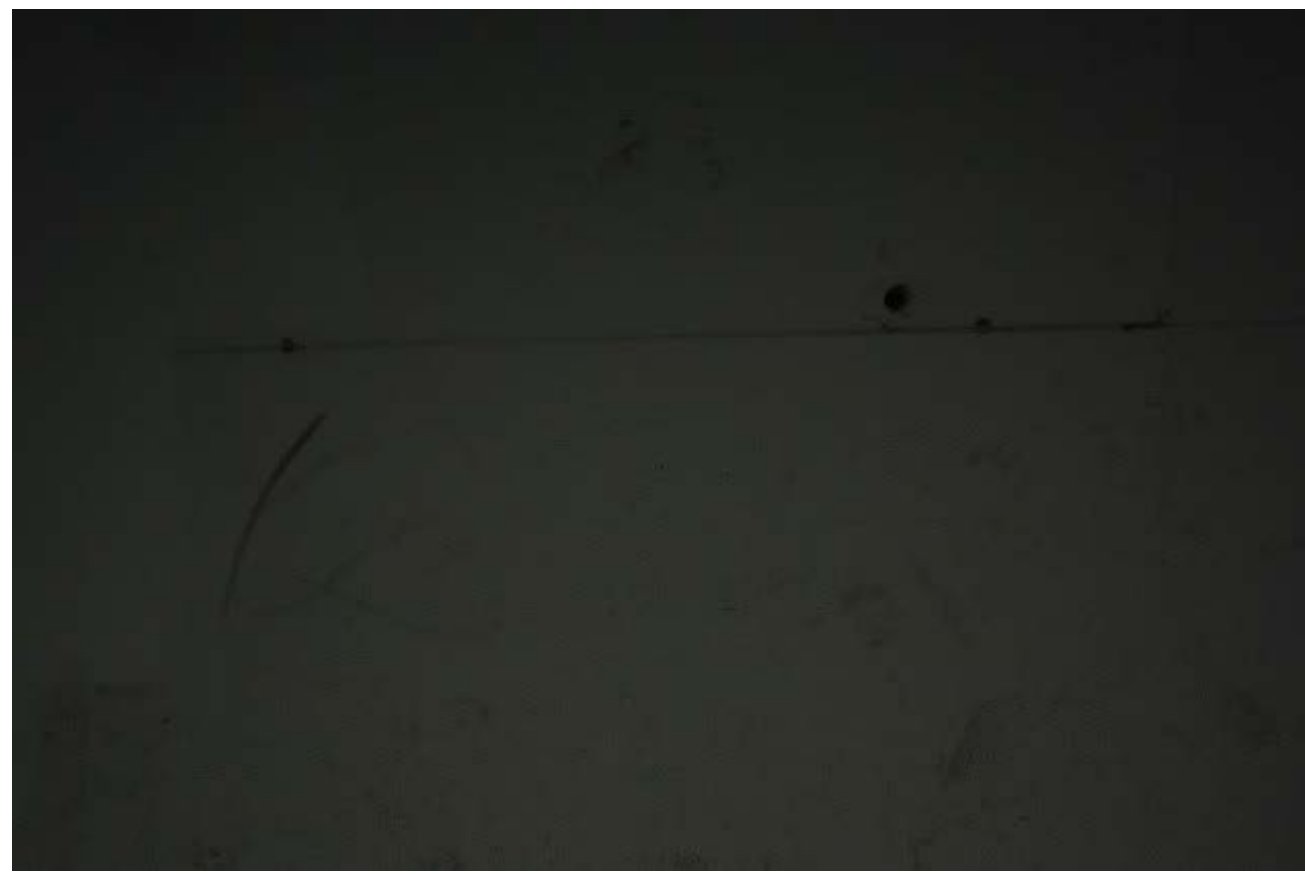

Figure F.6: Original image before pre-processing

The images in our scenario are also affected by uneven illumination which is caused by shadows cast from different light source directions, direct lighting on certain areas only as well as due to vignetting caused by the camera lens. Uneven illumination can be thought 


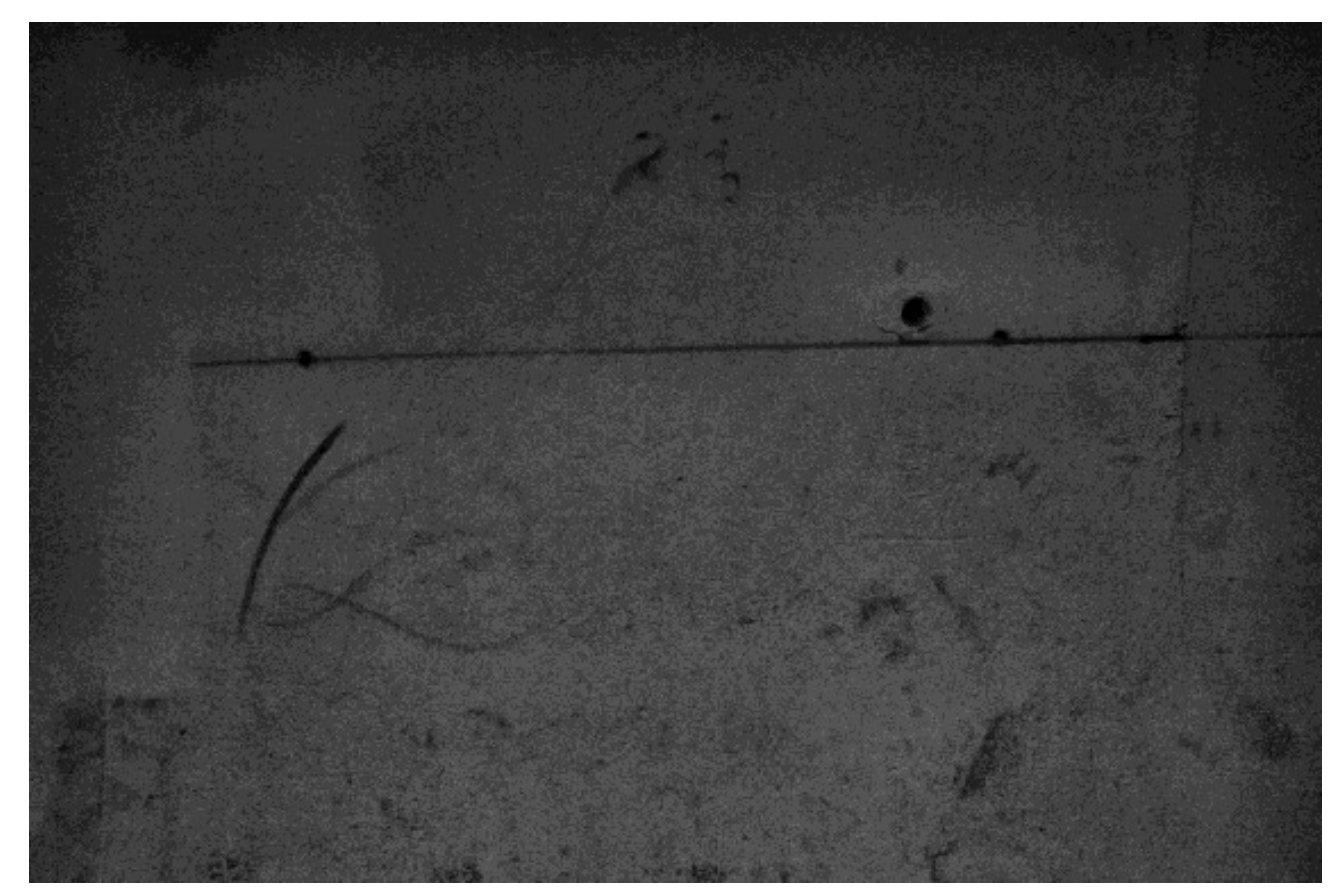

Figure F.8: Image after enhancement using CLAHE

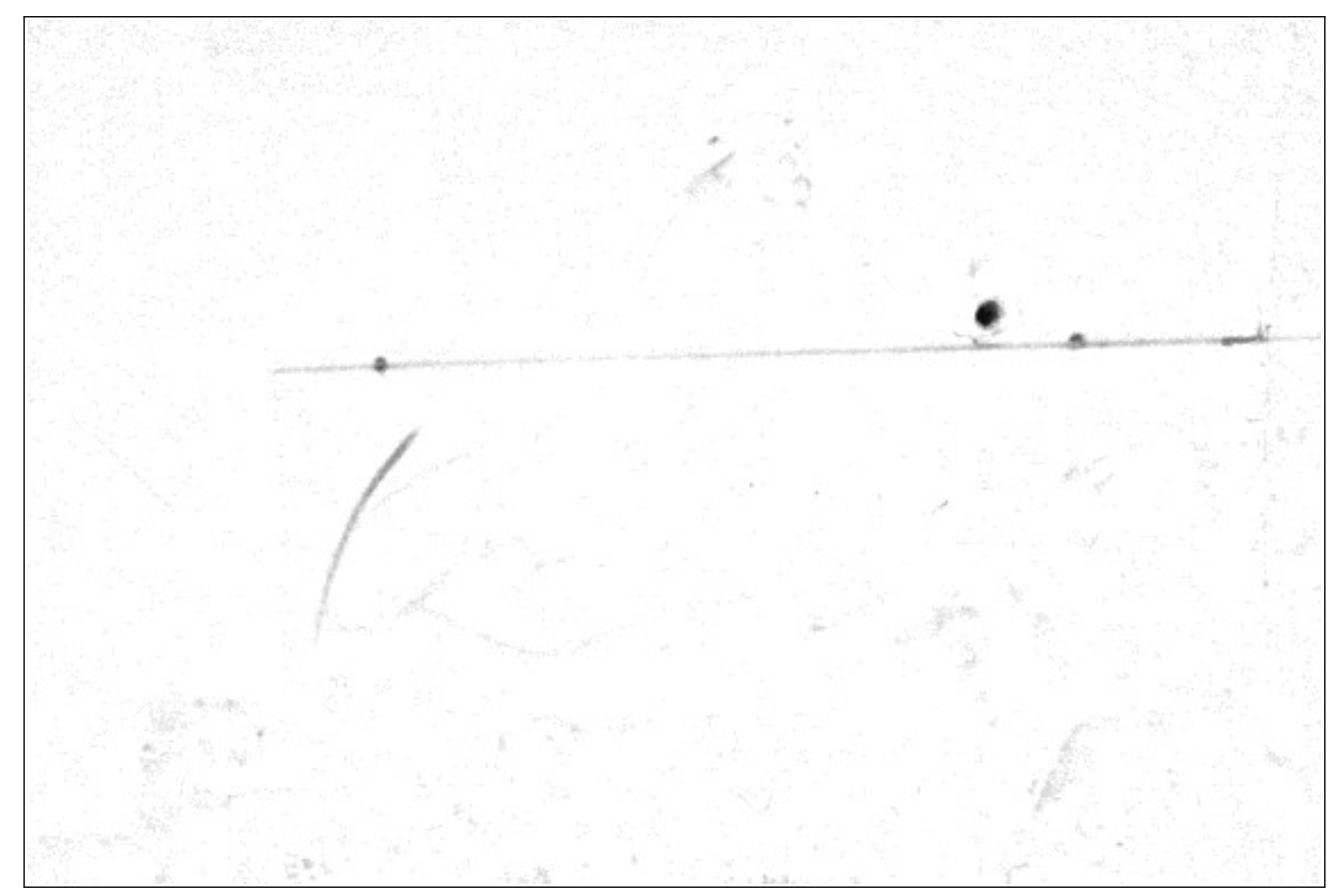

Figure F.9: Image after enhancement using the proposed shading correction 
of as a slowly changing function of the spatial coordinates, that is, consisting only of low frequency content and therefore can be isolated from the 'foreground' objects. This can be considered as the multiplication of the 'perfect image' and a 'shading function':

$$
I(x, y)=I_{\text {ideal }}(x, y) \times S(x, y)
$$

where $I(x, y)$ is the original image with uneven illumination, $I_{\text {ideal }}(x, y)$ is the 'perfect image', and $S(x, y)$ is the shading function.

The original image is low-pass filtered using a median filter with a large kernel to obtain $S(x, y)$. Rearranging Equation F.1, the illumination corrected image $I_{\text {ideal }}$ is obtained by dividing the original image $I(x, y)$ by the low-pass filtered image $S(x, y)$ pixel-wise.

The shading correction method simultaneously eliminates the uneven illumination caused by vignetting and shadow as well as it improves the contrast in the image such that wall 'features' are distinct from the 'white' background as seen in Figure reffig:10. This approach is computationally more expensive due to the filtering step followed by a division operation, however the processing time of the latter step can be easily reduced using parallel programming. Therefore shading correction was chosen as an effective pre-processing method to provide useful images for subsequent processing.

\section{Image Registration}

When images of the same scene need to be compared, ideally their pixel coordinates must correspond in 2D space for reliable matching. When the acquisition module is based on a moveable platform, such a condition is almost impossible to achieve, requiring image registration prior to image comparison. Registering images involves spatially aligning images such that one image is re-mapped to the same coordinate system as the other.

\section{Position offset correction using mosaic}

Each TIM mission results in a set of images which can be used for comparison with images taken on a different date, according to their tunnel DCUM reference. Consider a survey image taken at position $x$ at time $t_{2}$. Given that the platform is in motion, it is highly unlikely that a previous image at exactly the same position $x$ exists. Therefore, the images taken during the previous survey at time $t_{1}$ while at positions $\left(x-d_{1}\right)$ and $\left(x+d_{2}\right)$ can be stitched to form a mosaic such that the resulting image contains the whole image at position $x$. The constraint is that these two images contain enough overlap (ideally $50 \%$ or more) to guarantee continuity of the image. This is explained graphically in Figure F.10.

The field of view (FOV) in an image can be easily calculated using the angle of view of the camera and distance from the wall. Using the FOV of each image and the DCUM position information, the closest two images at time $t_{1}$ that are able to produce a mosaic that will contain the content of the image at $t_{2}$ are retrieved. The latter two images can be directly stitched to form the mosaic using the mentioned calculations, however this would only allow rough stitching, thus we also propose a more reliable approach. 


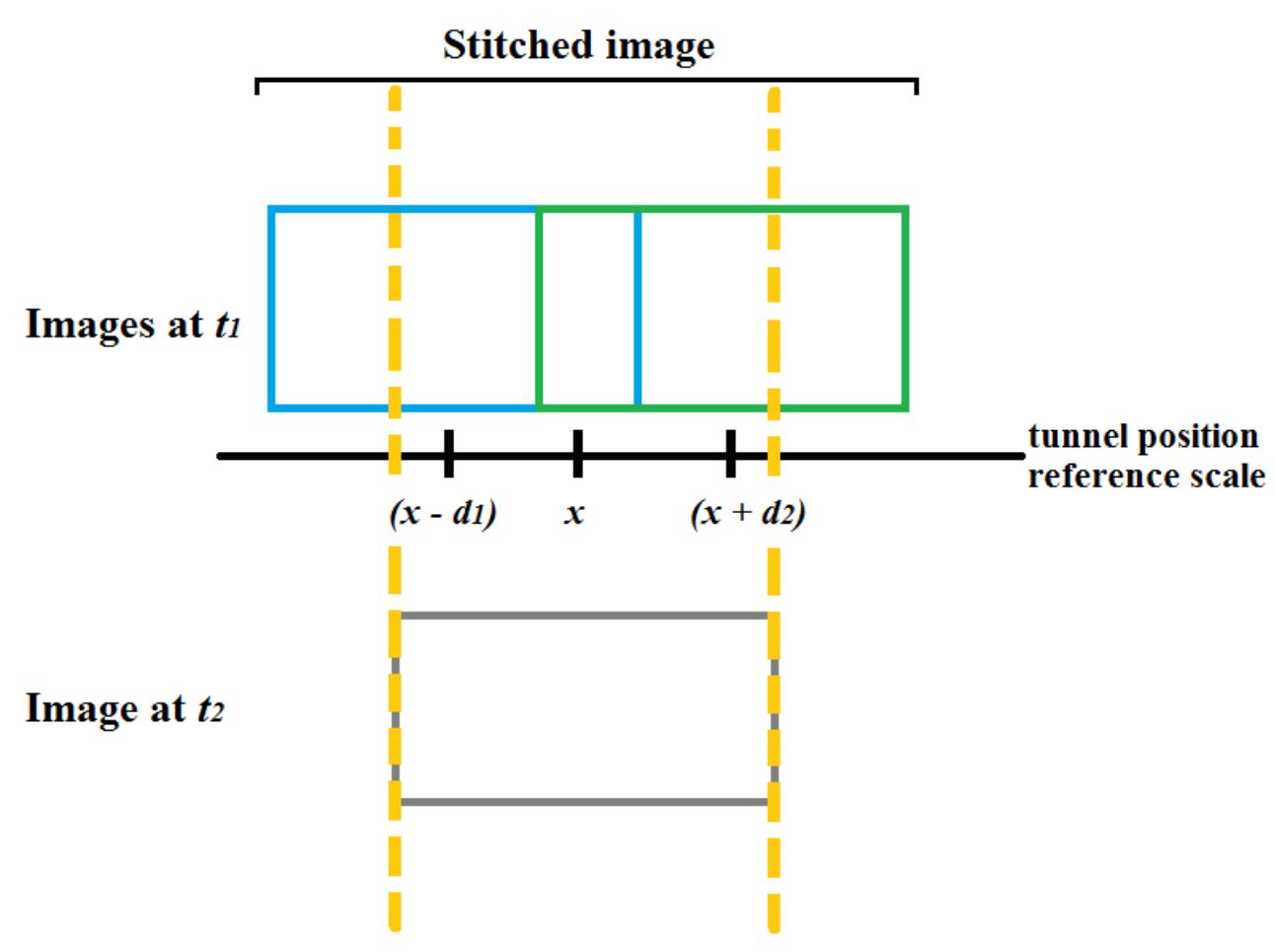

Figure F.10: Position offset correction using mosaic

\section{Image Mosaic using feature-based registration}

Image registration using features such as SURF and ORB was not successful in general due to the lack of keypoints and their limited uniqueness. This led to unreliable feature matching and so, such features could not be used for registration. Inspired by [410], we implemented a mosaic algorithm using binary edge detection as a high-level feature for feature extraction. The images in Figure F.11 will be used as a reference in the next sections to show the image mosaicing stages.

\section{Edge Detection}

In our proposed implementation, we use the Canny edge detector to produce edge maps. The Canny algorithm is slightly more complex, but its performance is superior to other mask operators. By applying the first two steps of Canny edge detection (filtering and Sobel masks) an automatic setting of these thresholds is proposed. The gradient magnitude average $\left(\mu_{m a g}\right)$ is calculated for each image. The low threshold for the Canny edge detection is then automatically set to a value of $1.33 \times \mu_{m a g}$ and the high threshold is set to twice as much.

\section{Correlation Matching}

Similarity correspondence is then applied to find where the images overlap, using normalised cross correlation $(N C C)$ as a cost function. This involves using part of the right image as 


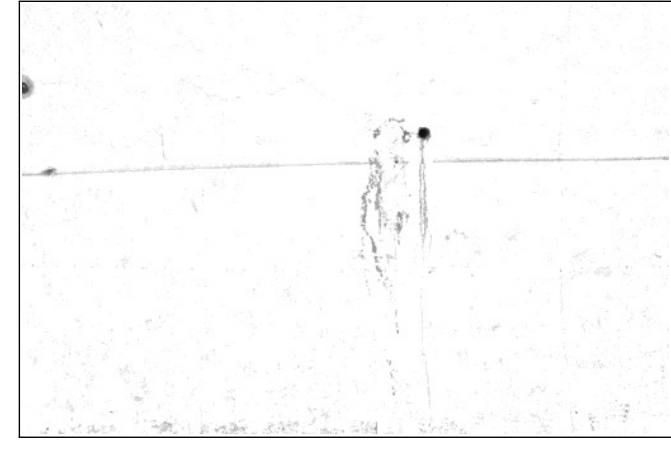

(a) Left image

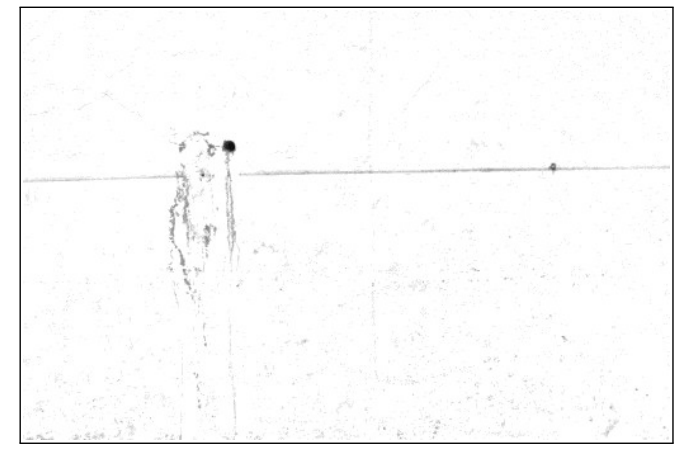

(b) Right image

Figure F.11: Pre-processed images used for mosaicing. Such images are closest in location in relation to the current survey image to be inspected.

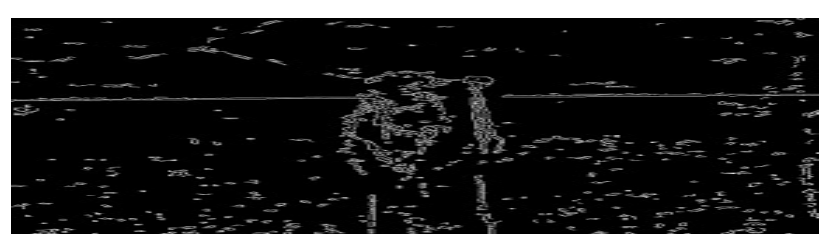

(a) template

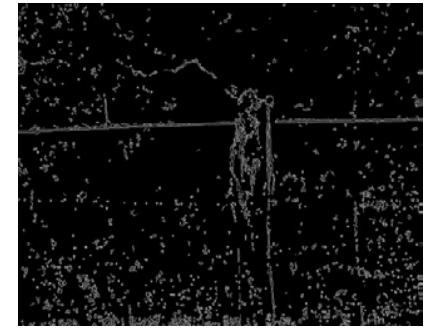

(b) source

Figure F.12: The edge images used in template matching involved in the image registration step, (a) is used as the template and (b) is used as the source

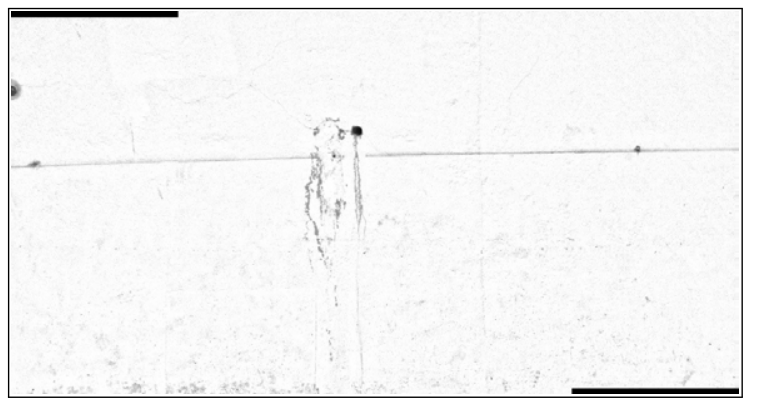

Figure F.13: Mosaic image without blending. 
a template (see Figure F.12(a)) and sliding it onto the left image (see Figure F.12(b)). The point with the highest correlation value is then found, implying the best match, and a translation vector is determined. The latter is compared to the one estimated from the FOV calculations. If the difference along the width of the image exceeds a threshold, the images are stitched using the estimated translation and the calculated best match vector is ignored.

\section{Image composition}

After template matching, the two images are spatially aligned using the translation parameters found such that the right image is shifted according to the translation vector. Both images are now lying on the same canvas area, overlapping each other. Images can be stitched by copying the second image on the first exactly where the overlap starts. If there is a difference in illumination between the two images, then blending is necessary to get rid of the seam at the stitching point. By applying the shading correction at the pre-processing stage, the illumination in both images was very similar resulting in identical results with and without blending; without any seams visible at the overlapping region as seen in Fig. F.13. Therefore the images in Fig. F.11 were directly stitched to each other to form the mosaic image, eliminating the computation time which would be otherwise required by blending.

\section{Survey Image Mapping}

The image mosaic contains the full content of the survey image as well as some extra data on the sides. Thus, the survey image needs to be located within the mosaic image. Using the $F O V$ of each image and the position information of the images, the image at $t_{2}$ can be located within the mosaic image. Using this technique, the location of the survey image is estimated.

The registration method described described above, is used to discover where the survey image content lies within the mosaic image. Taking the survey edge image as the template image and the mosaic edge image as the source, template matching is applied to obtain the point with maximum similarity, determining the location of the survey image (see Figure F.14) in the mosaic image as illustrated in Figure F.15. The two image locations are compared and if the difference is considerable a warning is raised, implying that registration may not be accurate.

\section{Hybrid Change Detection}

Once the survey image is located in the mosaic image, it is then extracted as a separate image referred to as the query image. To find any differences between the query and the survey images, change detection techniques need to be applied. The latter techniques are generally classified in two: Pixel-based (PBCD) and Object-based (OBCD). The query image in Figure F.16 and the survey image in Figure F.17 as located in the mosaic image will be used in the sections that follow to discuss the various change detection methods which we investigated. All query and survey images used in this section have been masked with the mosaic mask produced in the previous step, thus the black regions in the upper 


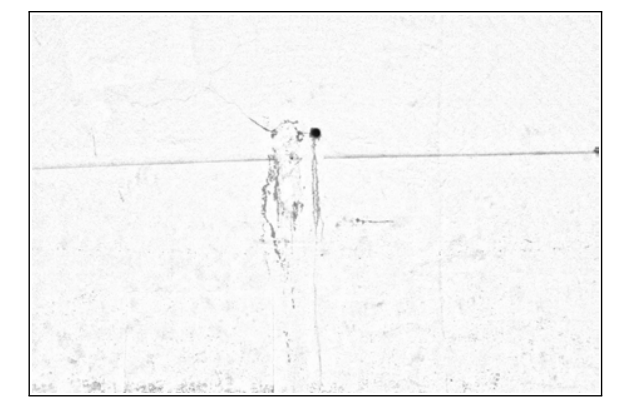

Figure F.14: Survey image

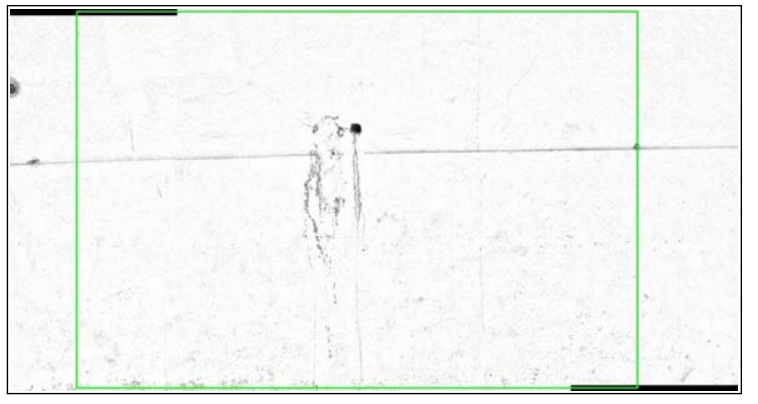

Figure F.15: Edge-based survey image mapping

right and bottom left areas in some of the images.

PBCD methods are simple and require low computation time but require exact registration. On the other hand images in this scenario cannot be easily segmented due to the image content where there are no definite 'objects', making OBCD methods impossible to use exclusively. Analysing the complementary advantages of PBCD and OBCD methods, a hybrid approach making use of variants of both methods is proposed. The process involves a sequential combination of image differencing as a PBCD method and components analysis as an OBCD method. The following sections discuss each step of the hybrid approach separately.

\section{Pixel-based Change Detection}

This type of detection method tries to identify changes in a scene by comparing images pixel

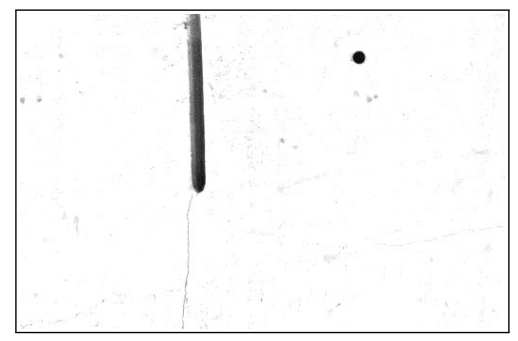

Figure F.16: A sample image from the Query dataset at time $t_{1}$ 


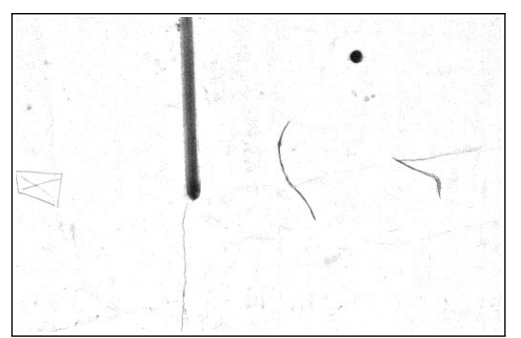

Figure F.17: A sample image from the Survey dataset at time $t_{2}$

by pixel. PBCD is simple and fast however it ignores spatial context and its quality highly depends on the accuracy of the image registration step.

\section{Simple image differencing}

In this approach, the intensity values of the survey and query image are subtracted pixelwise. After the subtraction, the magnitude of the difference value is compared against a threshold $T$. Pixels with difference magnitude exceeding the pre-defined threshold $T$ are classified as 'change' and set to white, otherwise they are noted as 'no change' and set to black, creating the change map $(C M)$, mathematically represented by:

$$
\begin{aligned}
\operatorname{Diff}(x, y) & =\left|I\left(x, y, t_{1}\right)-I\left(x, y, t_{2}\right)\right| \\
C M(x, y) & =\left\{\begin{array}{cl}
255 & \text { if } \operatorname{Diff}(x, y) \geq T \\
0 & \text { if } \operatorname{Diff}(x, y)<T
\end{array}\right.
\end{aligned}
$$

where $I\left(x, y, t_{1}\right)$ and $I\left(x, y, t_{2}\right)$ are images at time $t_{1}$ and $t_{2}$ respectively and $T$ is the threshold.

The effect of changing the value of $T$ is now analysed. As seen in the images of Figure F.18, as the threshold value is increased, the number of white pixels decreases, implying elimination of lower difference magnitudes, thus increasing noise suppression.

\section{Neighbourhood Image Differencing}

Compared to the previous method, neighbourhood image differencing has a slight element of spatial context. The images are first smoothed using a filter kernel such that pixels are more similar to their neighbourhood ones before being subtracted pixel-wise. Consequently, differences caused by misalignment are reduced and image noise is also suppressed.

As shown in Figure F.19, the outcome of this approach varies depending on the value of the pre-defined threshold as in the previous approach. However it can be observed that for the same threshold values, neighbourhood image differencing suppresses alignment errors and image noise better than simple image differencing. On the other hand, when using high threshold values, as shown in Figure F.19(c) and Figure F.19(d) on the left side, some areas of true change may be lost due to the smoothing applied during the first stage. 


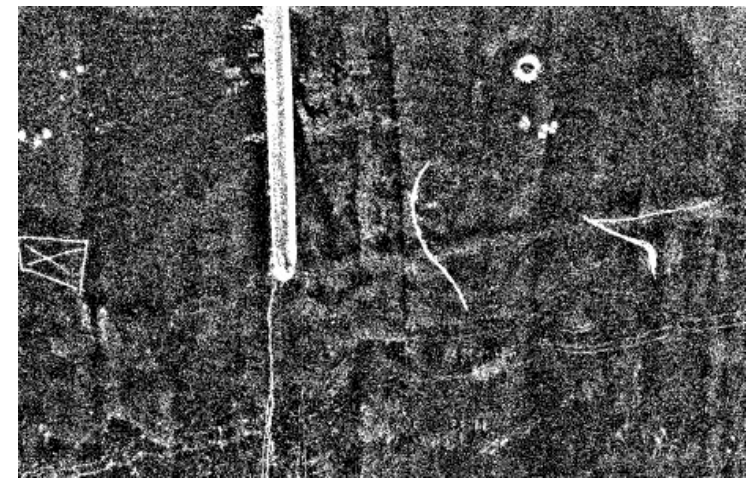

(a) threshold set to 10

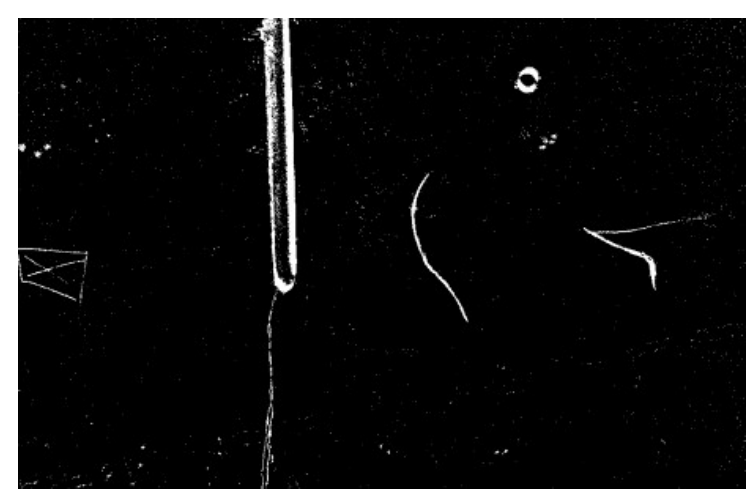

(c) threshold set to 50

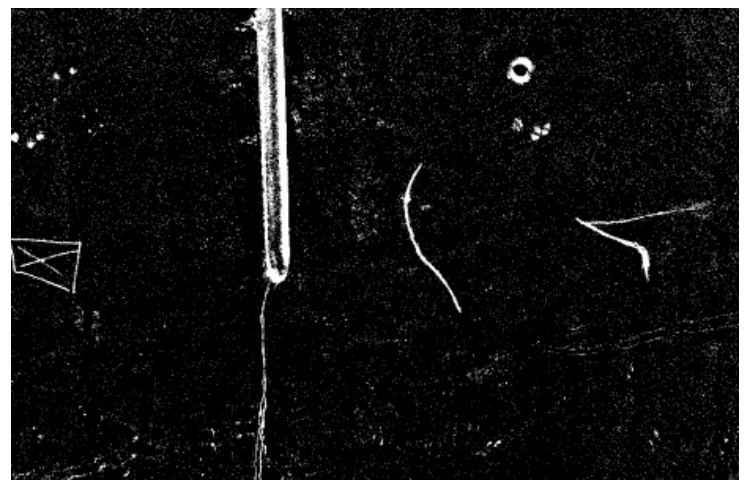

(b) threshold set to 30

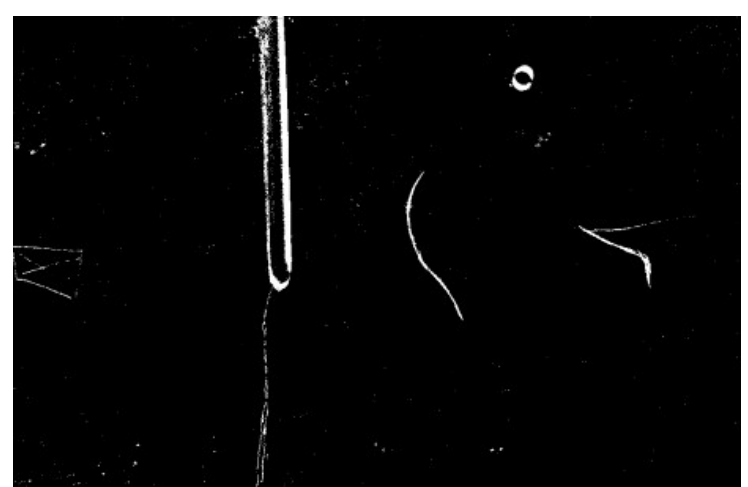

(d) threshold set to 70

Figure F.18: Simple Image Differencing when using different thresholds. When the threshold was set to a low value such as 10 and 30, the difference image contains a lot of 'image noise'. Higher threshold values, suppress such noise as well as alignment errors better. 


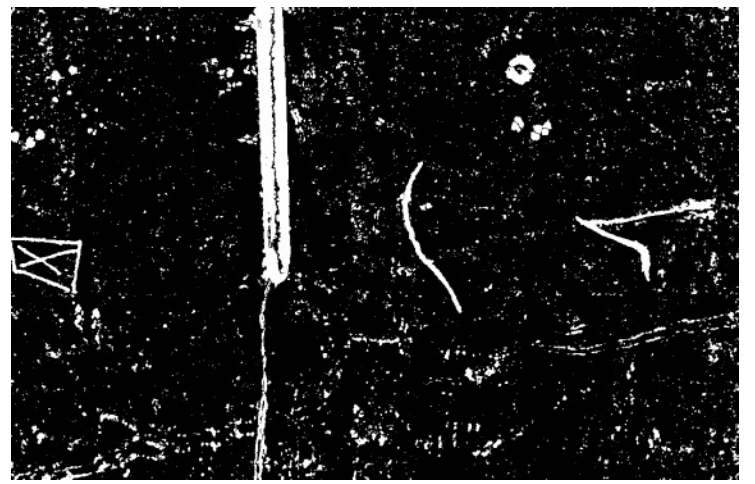

(a) threshold set to 10

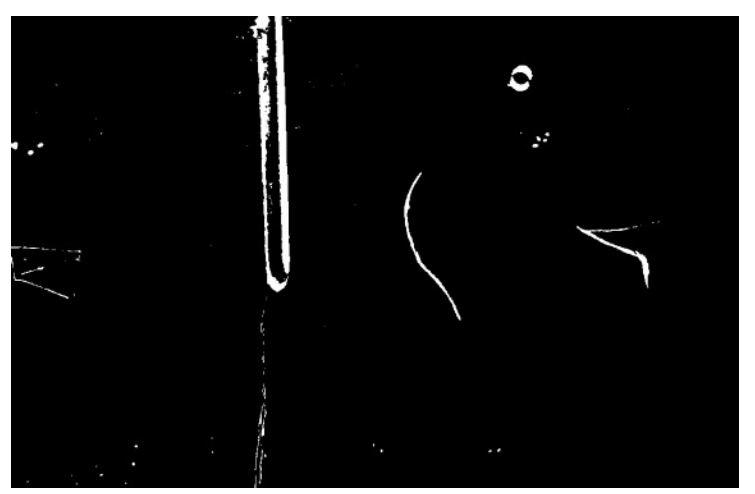

(c) threshold set to 50

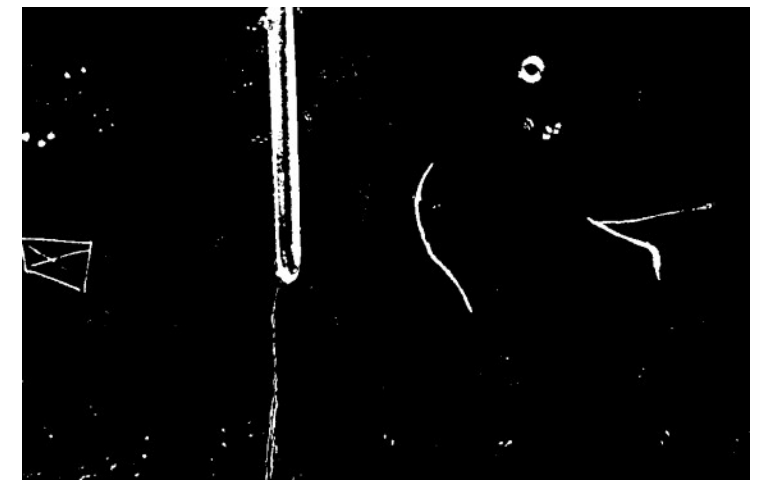

(b) threshold set to 30

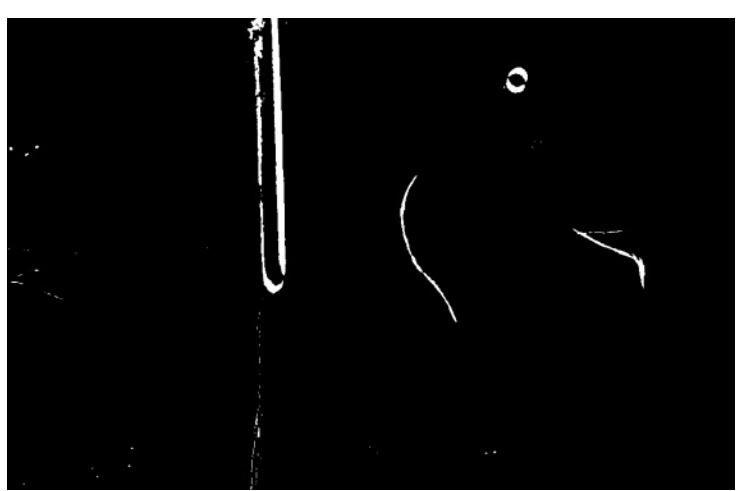

(d) threshold set to 70

Figure F.19: Neighbourhood Image Differencing when using different thresholds showing that for the same threshold values, neighbourhood image differencing suppresses alignment errors and image noise better than simple image differencing. 


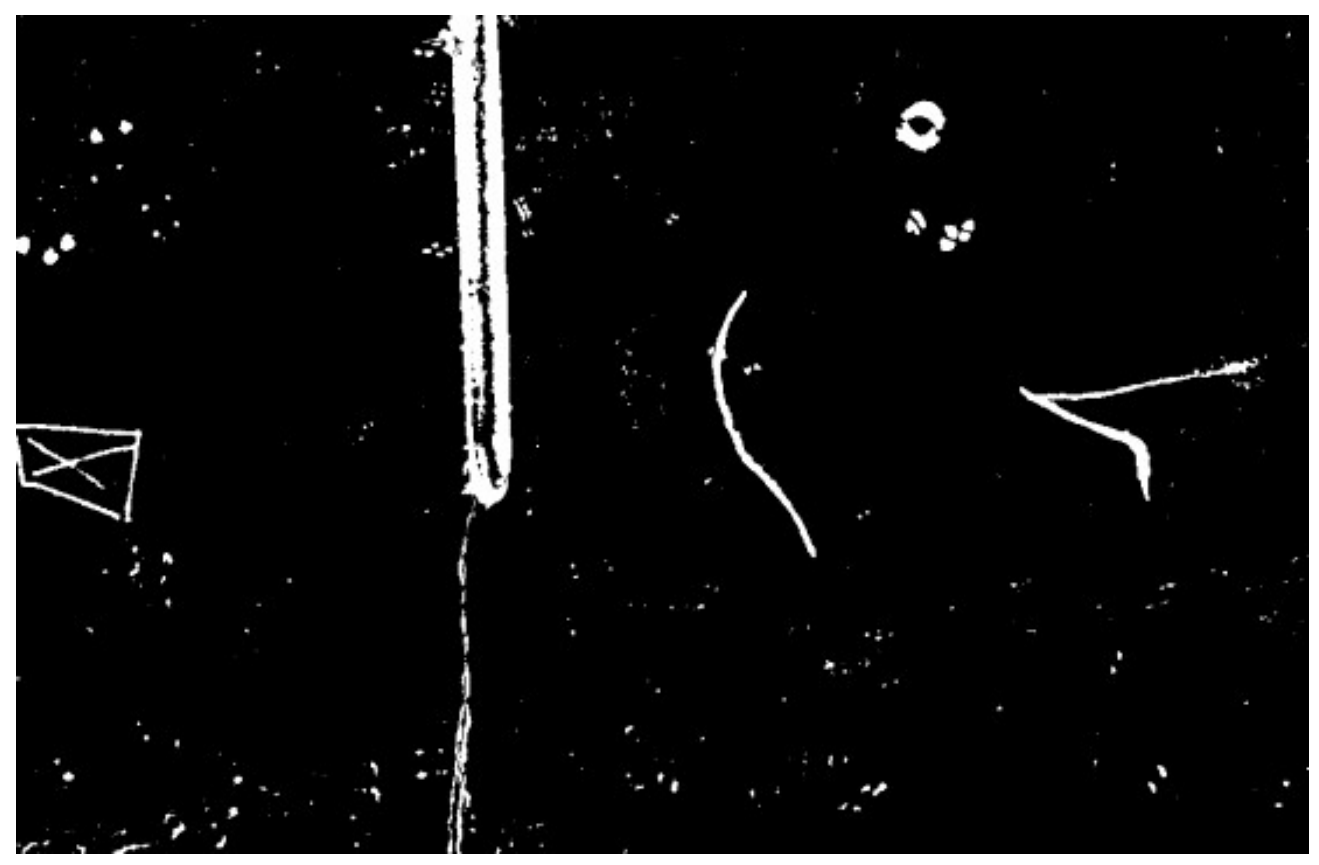

Figure F.20: Neighbourhood image differencing with a low threshold value as used in the algorithm.

Figure F.21: Difference image after closing morphological operation, further suppressing irrelevant changes due to image noise

\section{Coarse Change Detection using Neighbourhood Image Differencing}

Considering the outcomes from the two PBCD methods discussed, neighbourhood image differencing with a low threshold was used. At this stage, a coarse change map is available as shown in Figure F.20. This allows identification of 'probable' change areas. The output difference image has 'definite regions' and can be segmented.

\section{Change Image Enhancement}

The difference image contains some small 'change' areas that should be eliminated. On the other hand, small gaps existing within segments supposed to be complete, should be filled. These can be achieved using erosion and dilation respectively. A closing operation which involves dilation followed by erosion is applied to fill in the 'holes' in the 'change' areas and then remove noise pixels as shown in Figure F.21.

A connected components algorithm using 8-connectivity is applied to the enhanced coarse change map, such that linked pixels are marked as the same 'object', segmenting the image into components. The bounding rectangle enclosing the maximum and minimum positions of pixels within each component was extracted. For each component, if none of its dimensions exceed a pre-defined threshold, the component is eliminated. This further suppresses small false change areas as shown in Figure F.22. 


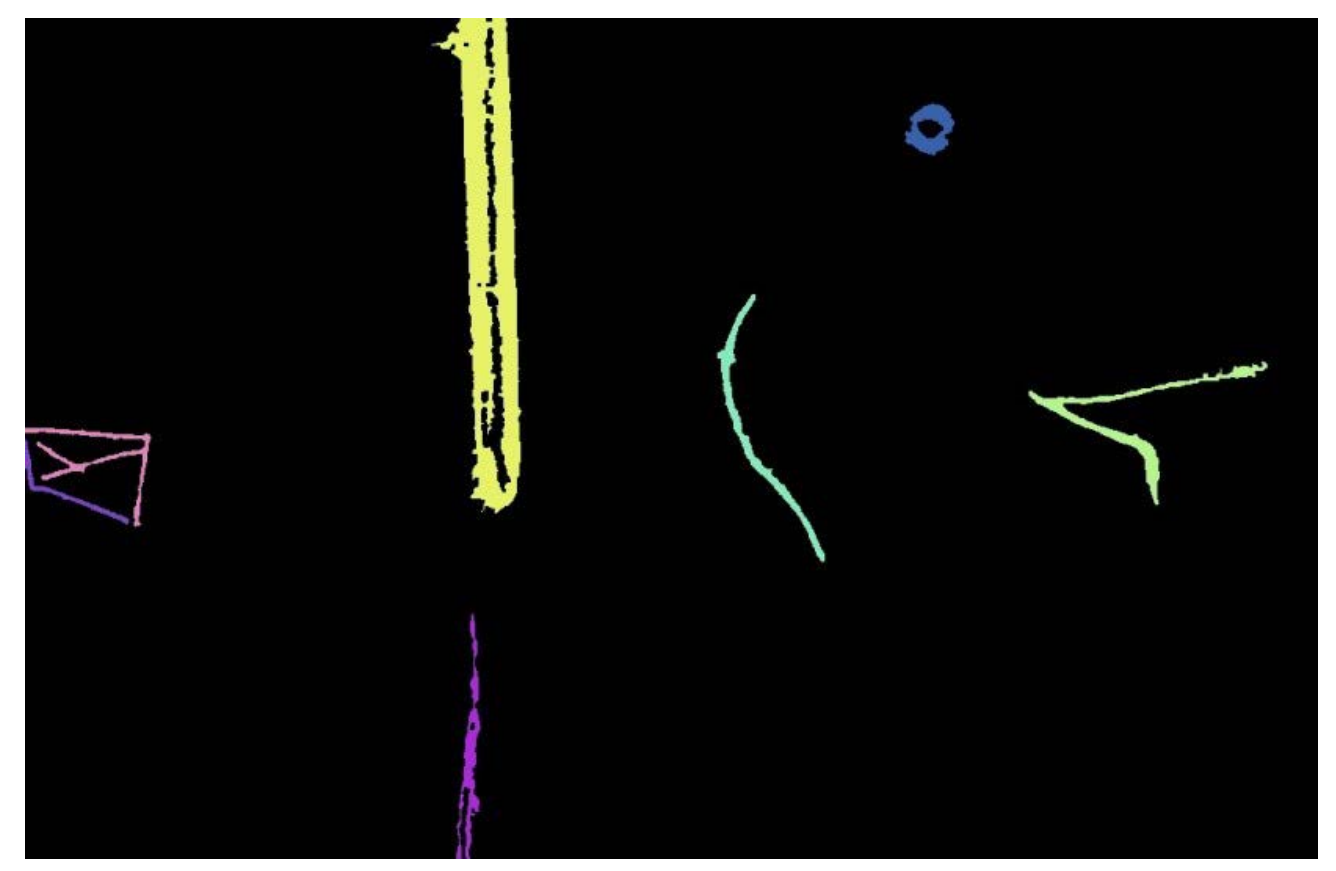

Figure F.22: Connected components labelling whereby neighbouring pixels are grouped and labelled as separate objects.

Some scenarios that have gaps within objects in the difference image, might produce separate labelled components as in Figure F.23(a). To avoid this, a closing operation is applied as in Fig. F.23(b). Also the first round part at the top of the image would have been filtered out due to its dimensions as in Figure F.23(b).

In scenarios similar to the query and survey images in Figure F.24, after morphological operations, some components which should have been a single one might be assigned different labels due to tiny separations existing in between, as shown in Figure F.25. A merging algorithm is proposed to merge any components whose bounding areas overlap. During the merging process, the bounding area is taken as the bounding rectangle plus a few extra pixels in each dimension to allow for registration errors. At this step, very close components are merged into a single one as in Figure F.26.

\section{Object-based Change Detection using Component Analysis}

OBCD techniques are based on image objects, rather than individual pixels as in PBCD. First, a connectivity analysis technique is applied to both images to be compared, such that the outcome is two sets of blobs. Secondly, the blobs in one set are compared to those in the other set, using attributes like the area, perimeter and centroid. If blobs in different sets correspond to each other, then these segments are eliminated, leaving the 'change' components only. OBCD methods are more robust to alignment errors since they use spatial context rather than mere individual pixels. This is done at the expense of being more complex and time-consuming due to image segmentation.

Now, a set of 'candidate change components' and their corresponding bounding rectan- 


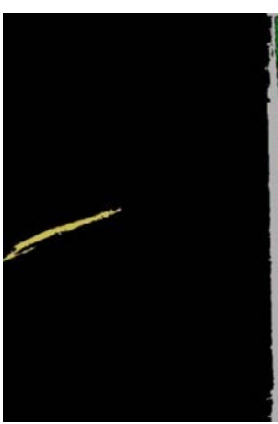

(a) without closing

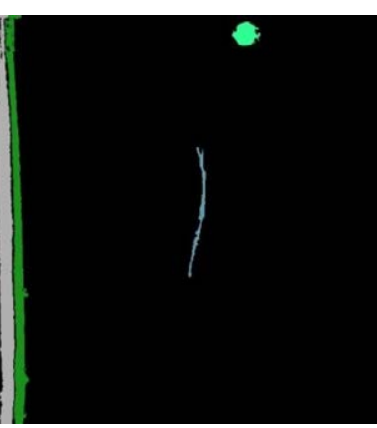

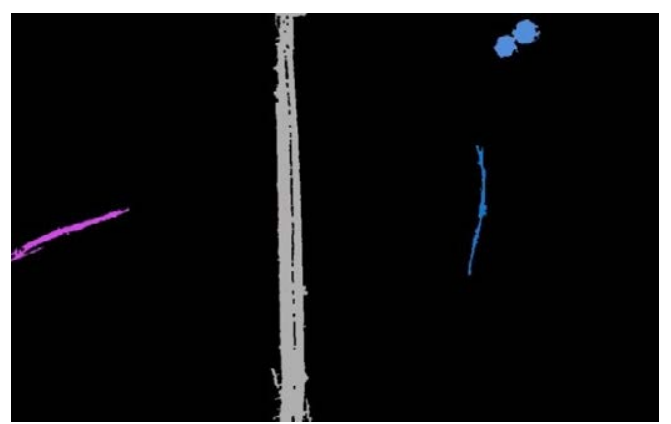

(b) with closing

Figure F.23: Connected components labelling, when applying morphological closing operation and not.

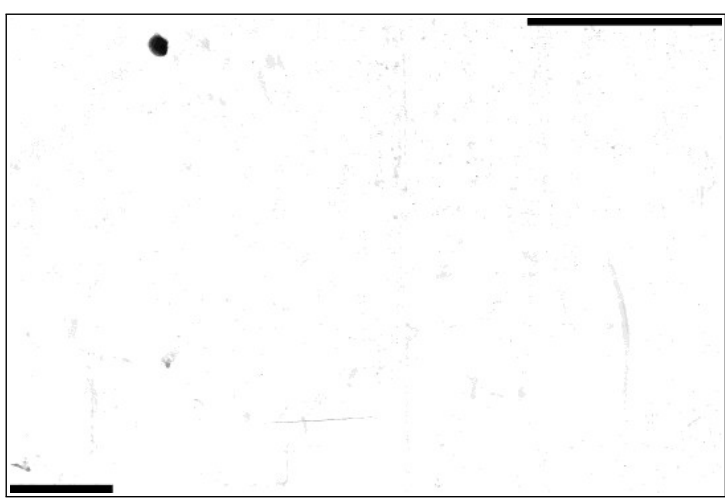

(a) Query image

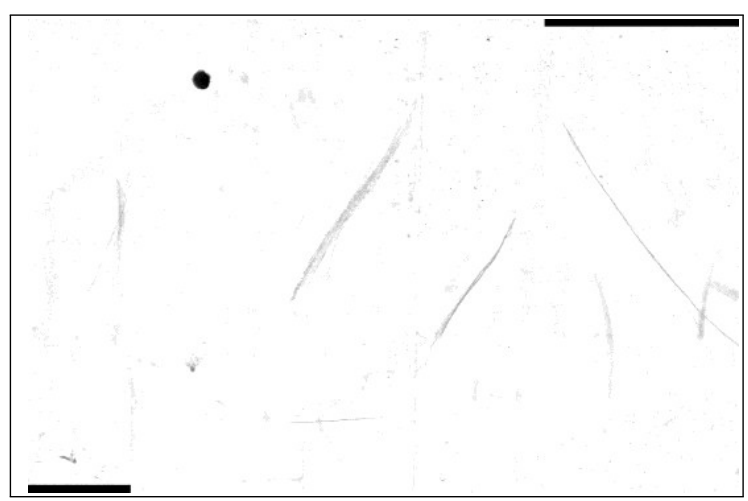

(b) Survey image

Figure F.24: Merging components scenario

gles are available as displayed in Figure F.27. However, superimposing these components in the query (see Figure F.16) and survey (see Figure F.17) image, one realises that only three of these components correspond to 'true changes'. Components $<2>,<3>$ and $<4>$ involve markings and scratches on the wall. On the other hand components $<0>$, $<1>$ and $<5>$ resulted in the difference image due to registration errors. This implies that a further analysis must be made on each of the components extracted from the initial difference image.

\section{Component analysis by binary comparison}

Applying thresholding on the images, a binary version of the query and survey image is obtained. By taking the bounding rectangle of each 'possible change' component with a few extra pixels along each side, the bounding area is masked out of the latter binary images. Comparing the number of white pixels should give a rough idea if the coarse change is a true change or a difference caused by misalignment.

If the number of white pixels in the query binary image is very similar to that in the 


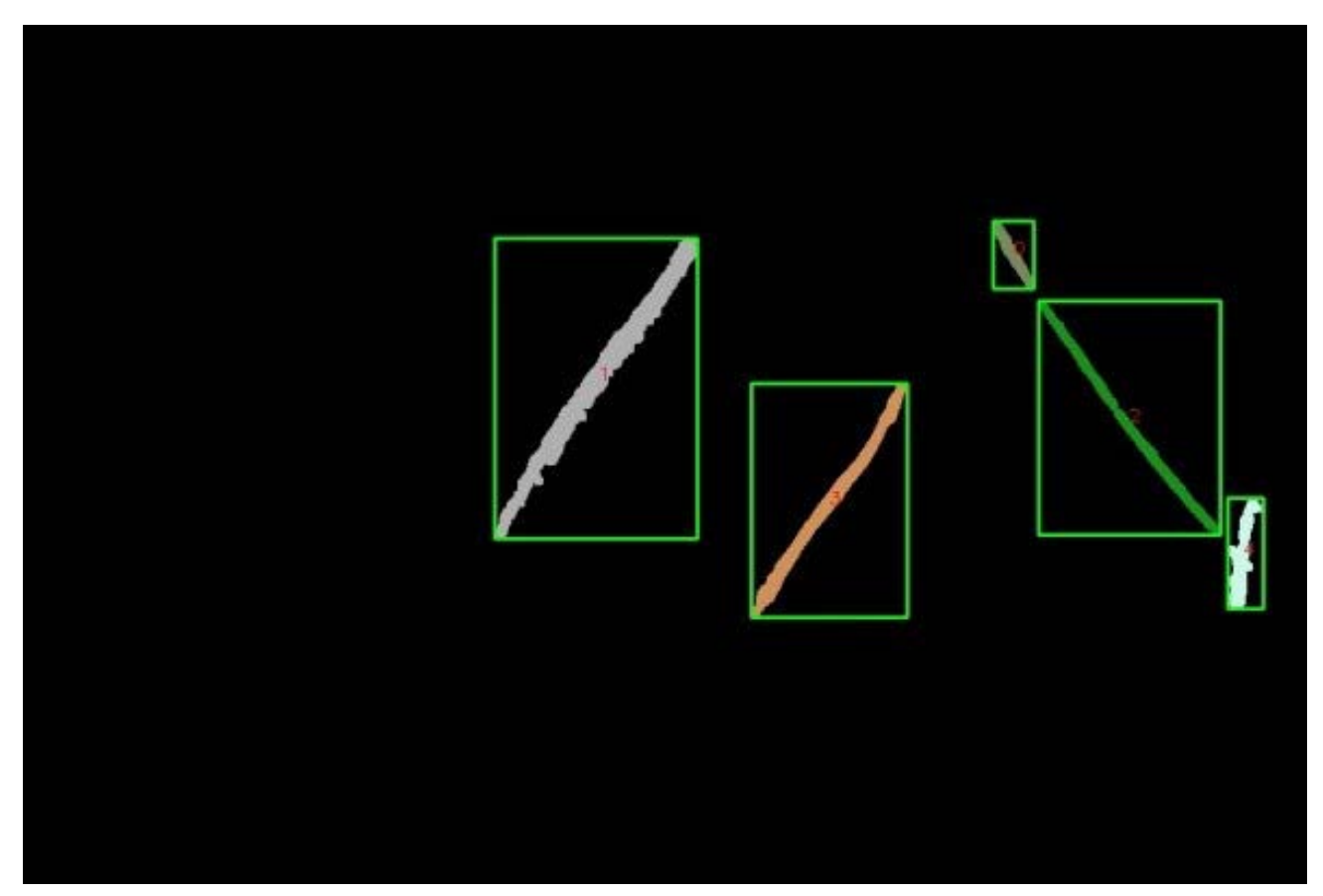

Figure F.25: Bounding boxes of candidate changes before merging

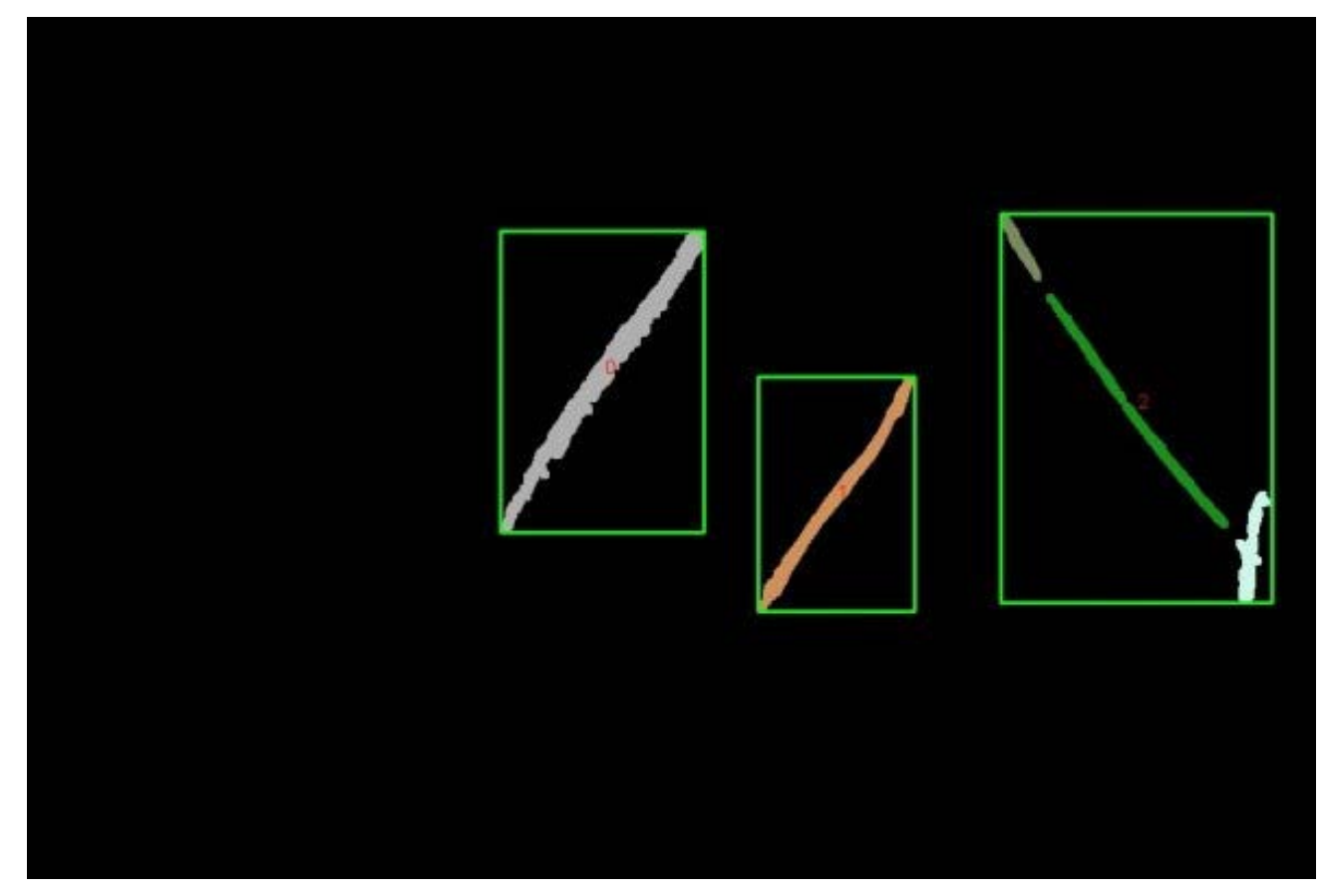

Figure F.26: Bounding boxes of candidate changes after merging 


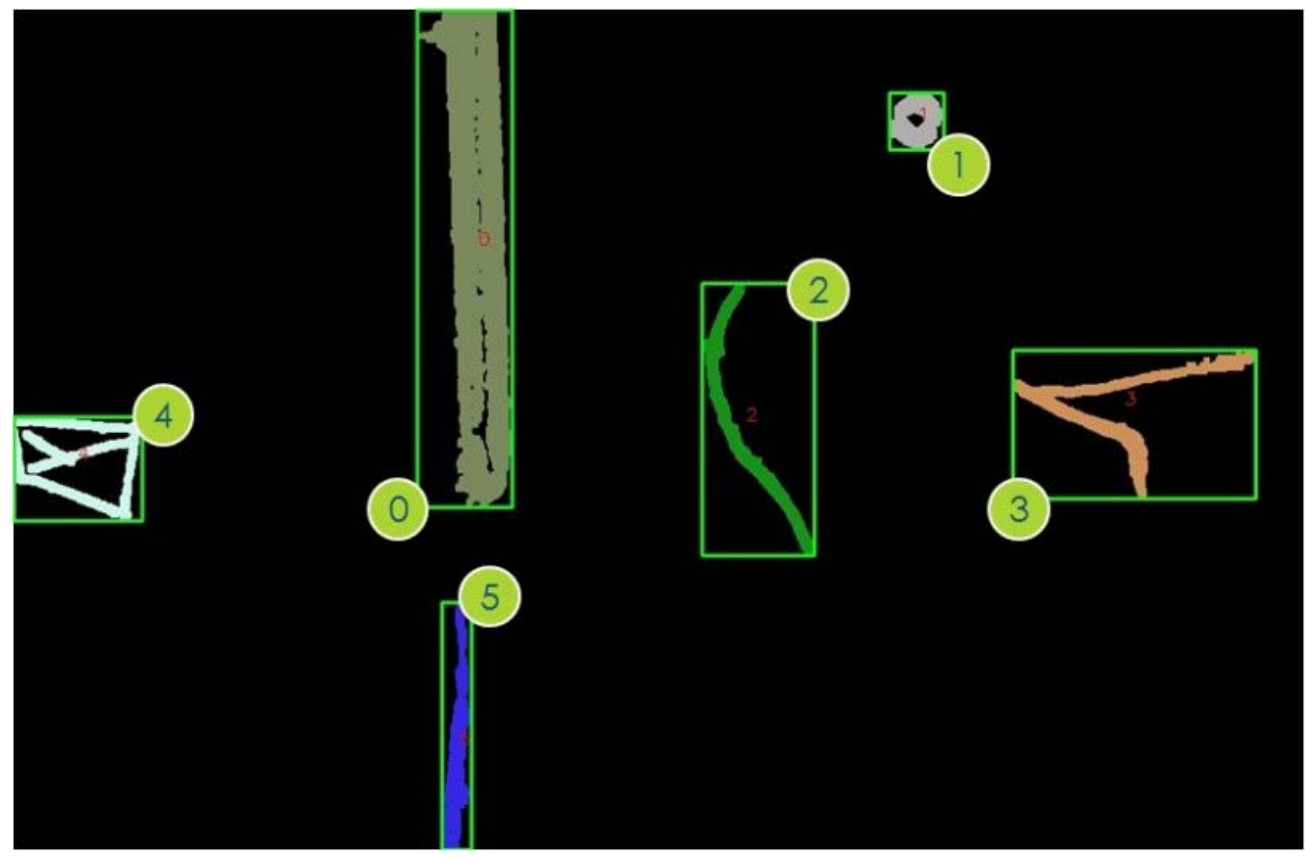

Figure F.27: Possible change components

survey binary image as in Figure F.28, then most probably, it is a false positive. On the other hand, if a change has occurred on the wall, then the number of white pixels will differ substantially as implied in Figure F.29. Two pre-defined thresholds were set to be able to discriminate on this.

\section{Component analysis using Optical Flow}

Optical flow is the distribution of apparent motion of pixels in an image sequence (see Figure F.30). In our scenario, the query and survey image can be considered as a two-frame sequence. A novel approach for change detection using optical flow analysis is proposed in this section.

The proposed method uses Gunnar Farneback's algorithm for dense optical flow, which is explained in [411]. The OpenCV implementation for this algorithm was used to find the motion vectors between the query and survey images. For the purpose of clarity, only the motion vectors at every $p$ pixels were drawn in the following optical flow images.

From Figure F.31, one observes that the majority of optical flow vectors have very low magnitude or even zero. This implies areas of no difference between the query and survey images. In addition to this, there are some regions where the magnitudes and/or the orientations vary substantially in the same confined area. It can be noticed that the latter correspond more or less to the same areas of change obtained using image differencing. In order to compensate for any differences occurring due to registration errors, the modal magnitude value is found and set as a threshold to reduce such vectors to a null vector. This results in the optical flow given in Figure F.32.

Once such filtering is done, the vector magnitudes and orientation are normalised to the 


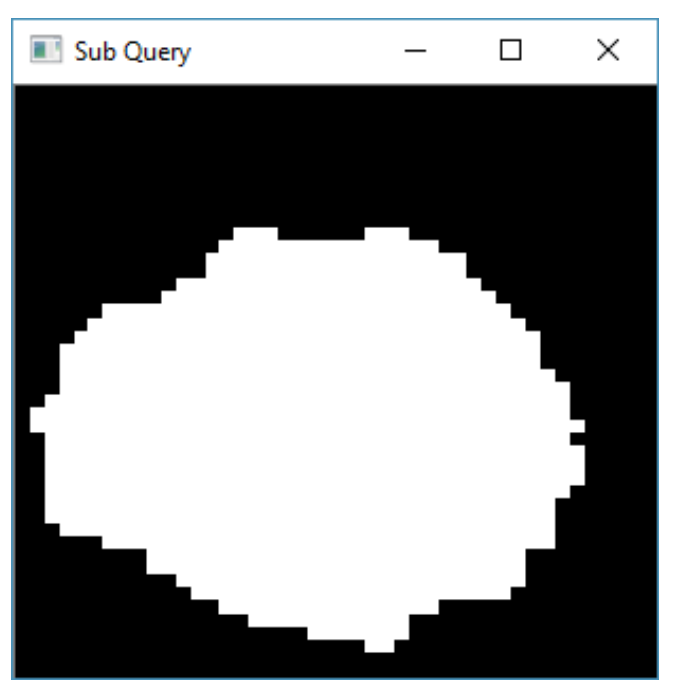

(a) Query $<1>$

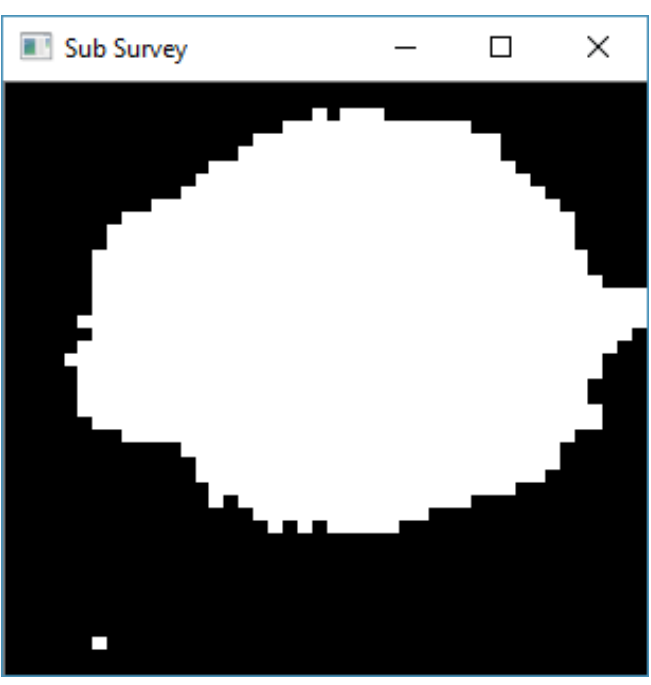

(b) Survey $<1>$

Figure F.28: Binary comparison of sub-image with a registration misalignment (no change)

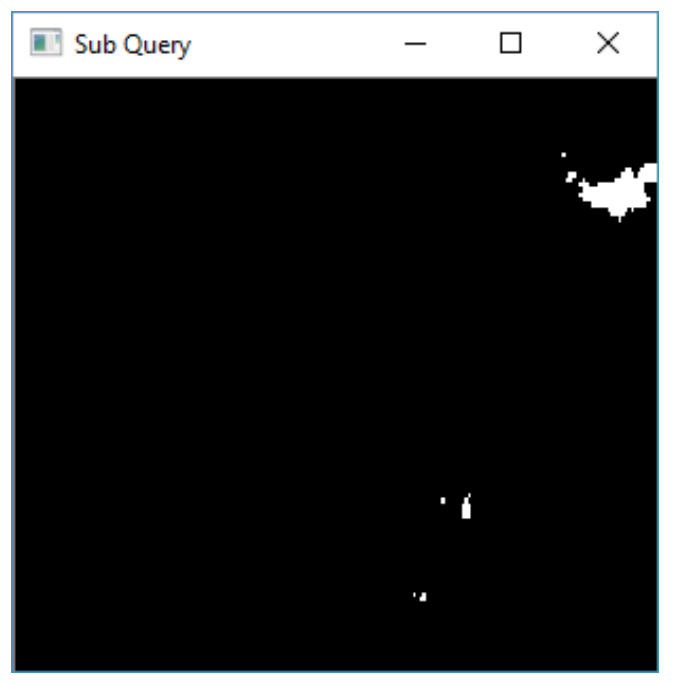

(a) Query $<3>$

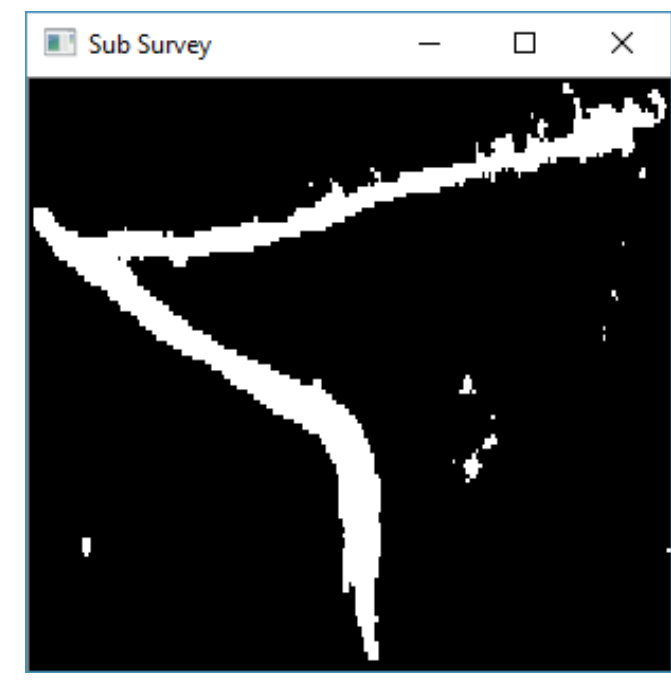

(b) Survey $<3>$

Figure F.29: Binary comparisons of sub-images with a true change 


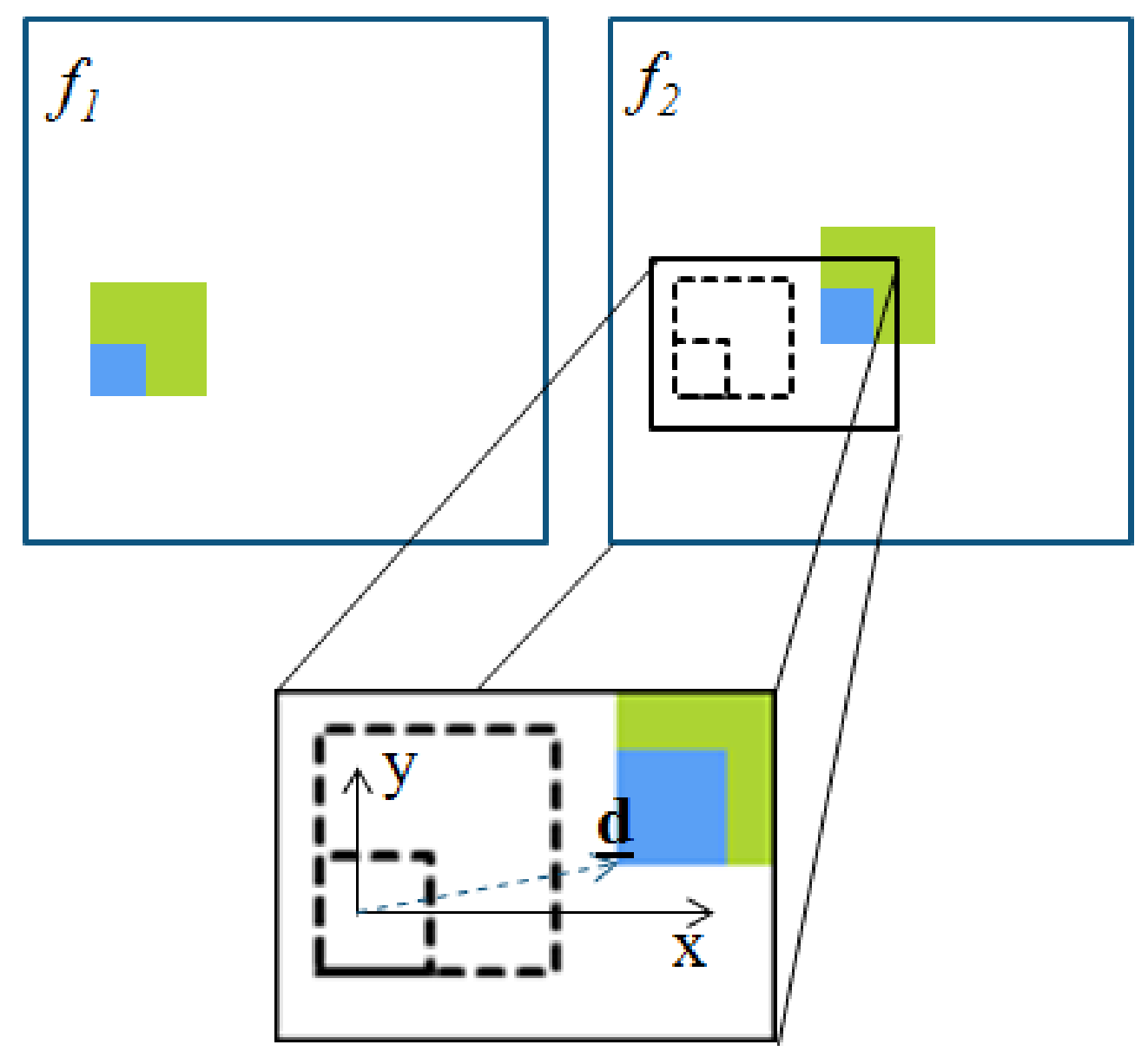

Figure F.30: Optical Flow between two images 


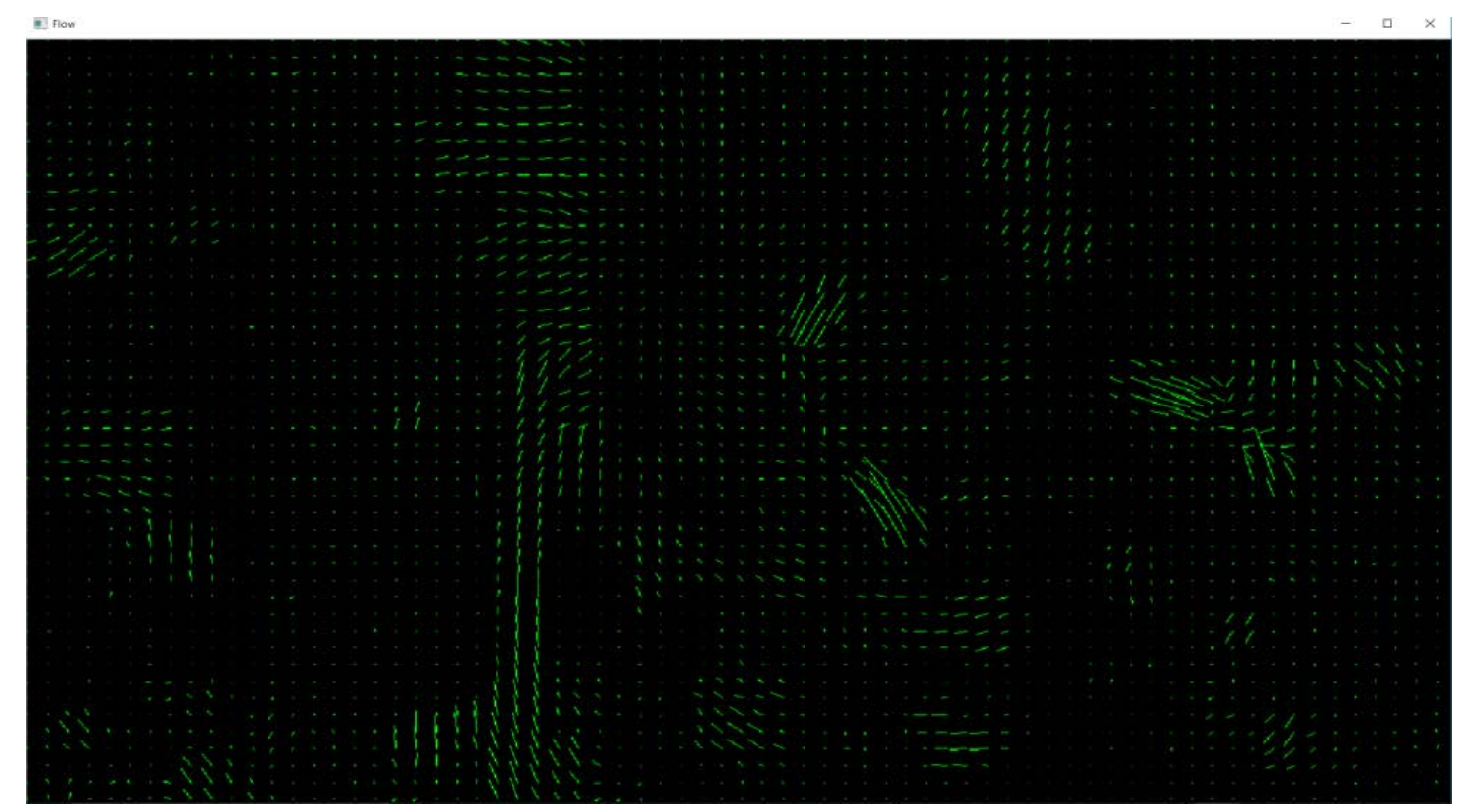

Figure F.31: Result after optical flow calculated from Figure F.16 and Figure F.17

range $[0,1]$. Using colormap overlay, a visual representation of the optical flow is generated. The different colours in the scale represent different values of magnitude/orientation. The 'candidate change' bounding rectangles extracted at the coarse detection stage are superimposed on the variation of magnitude and orientation in Figure F.33 and Figure F.34 respectively.

From experimentation, it was noted that, if the only change in the image is a translation then all vectors have similar orientation, i.e. direction of translation, and magnitude, i.e. number of shifted pixels. This is implied by the uniform-coloured regions. On the other hand, if a change such as a new crack appeared on the wall, then the vectors have varying magnitude and/or orientation. This is implied by the varying colours in the confined areas. Therefore, by analysing the magnitude and orientation of such vectors, the proposed method determines whether a detected difference in the change map is a possible 'true change' or not.

The magnitude and orientation maps are generated for visualisation purposes only. The measure of variability is done through standard deviation which measures the amount of dispersion of a set of data values. Analysis of the variability of the vector magnitude and orientation using standard deviation was carried out and plots of the variability values were drawn. Figure F.31 shows that for magnitude, the majority of the values for 'no change' areas were concentrated in the range of $[0,0.1]$, with only a few outliers lying above the standard deviation value of 0.1 . On the other hand, Figure F.32 indicates that the standard deviation values corresponding to orientation had no definite distinction in their ranges. 


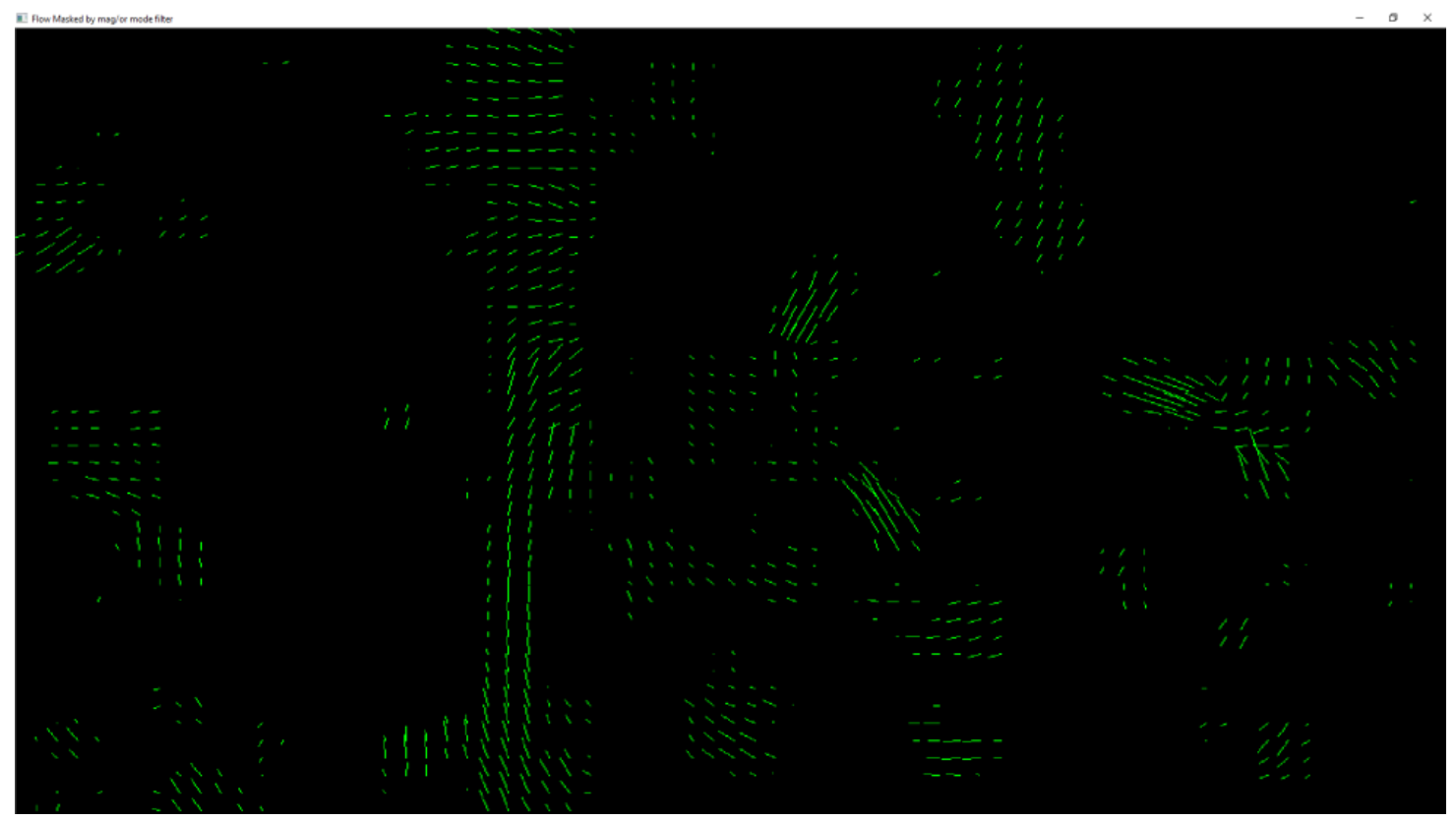

Figure F.32: Filtered optical flow, showing only the significant vectors according to their magnitude

\section{Change Detection using combined weighting}

From the latter observations, none of the implemented change component analysis methods could be used exclusively for change detection. Instead, a collaborative analysis of the difference ratio and optical flow vector variability is used to determine the true nature of the 'possible change' components obtained at the coarse change detection stage. A weighting system is used, whereby each component has a zero opening weight balance. First the component's bounding box is masked with the filtered optical flow, if the area intersects a zero optical flow region, this component is declared as 'no change' and skipped. Using binary comparison, if the difference ratio is lower than $T_{\text {Blow }}$, then the total weight is reduced by $w_{1}$. On the other hand, if the difference ratio is larger than the pre-defined threshold $T_{B h i g h}$, the component is more probable to be a change and so a value $w_{2}$ is added to the total. If the difference ratio lies in between, the total weight is increased by a lower amount $w_{3}$. While the latter step gives a good indication, it is not enough for a reliable decision. Therefore, optical flow vector analysis is then applied to find the variability in flow magnitude and orientation. Considering the results of standard deviation analysis, for magnitude, a value of variability exceeding $T_{m a g}$ is most probably a change and adds $w_{4}$ to the total weight. As for orientation, values are less distinctive and therefore a lower weight $w_{5}$ is added if the standard deviation is more than $T_{o r}$. In addition to this if a 'candidate change' intersects an area of large flow magnitude, a small weight $w_{6}$ is added. If the final total weight exceeds the pre-defined change confidence threshold $T_{w}$ then the area is marked as a 'change', otherwise as a 'no change'.

Using this weighting combination, survey and query images are compared to detect 
changes. The change detection output for the scenario images in Figure F.16 and Figure F.17 is displayed in Figure 6.9. The green bounding boxes enclose the areas in which the change detection module identified a change between the query and survey image. Here one observes that the number of 'candidate change' bounding boxes identified in the initial difference image (see Figure F.27) were reduced to the final 'change areas' marked in Figure 6.9. Each bounding box has its attributes such as the centroid location, width and height, as shown in Table F.1. Such values can then be translated into physical dimensions.

\begin{tabular}{|c|c|c|c|}
\hline \multirow{2}{*}{ Change } & \multirow{2}{*}{$\begin{array}{c}\text { Centroid Location } \\
(\mathrm{x}, \mathrm{y})\end{array}$} & \multicolumn{2}{|c|}{ Change Dimensions } \\
\cline { 3 - 4 } & $(603,331)$ & width $(\mathrm{mm})$ & height $(\mathrm{mm})$ \\
\hline 0 & $(908,335)$ & 197 & 220 \\
\hline 1 & $(52,371)$ & 104 & 120 \\
\hline 2 & &
\end{tabular}

Table F.1: Attributes of the detected 'change areas'

\begin{tabular}{|c|c|c|c|c|c|c|c|}
\hline \multirow{2}{*}{ Set } & \multicolumn{2}{|c|}{ Exposure Settings } & \multicolumn{3}{c|}{ Change Type } & Number \\
& A & S(s) & ISO & P & M & S & of images \\
\hline 1 & $\mathrm{f} / 4.5$ & $1 / 60$ & 1600 & & $\checkmark$ & $\checkmark$ & 51 \\
\hline 2 & $\mathrm{f} / 4.5$ & $1 / 100$ & 1600 & & $\checkmark$ & & 15 \\
\hline 3 & $\mathrm{f} / 4.5$ & $1 / 60$ & 1600 & $\checkmark$ & & $\checkmark$ & 47 \\
\hline
\end{tabular}

Table F.2: Dataset information, including camera exposure settings: A-Aperture, S-Shutter speed and ISO and the type of change: P-Pencil, M-Marker, S-Scratches 


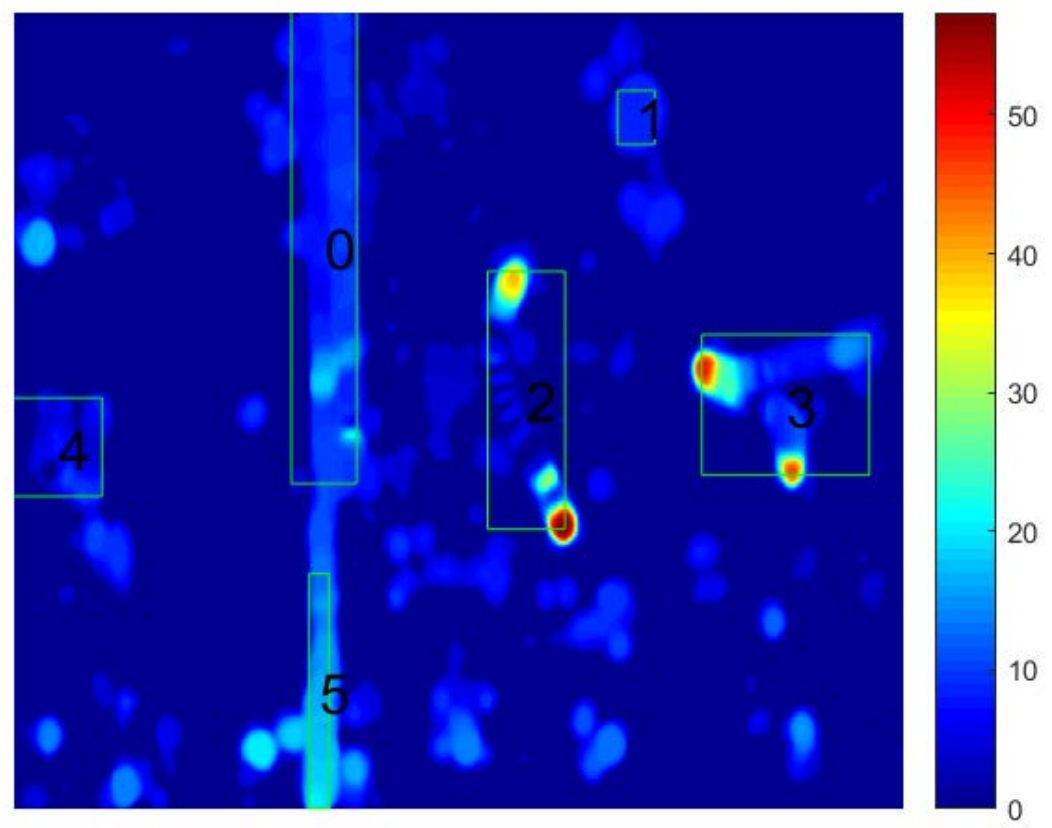

Figure F.33: Optical flow vector magnitude map using the colour scale on the right

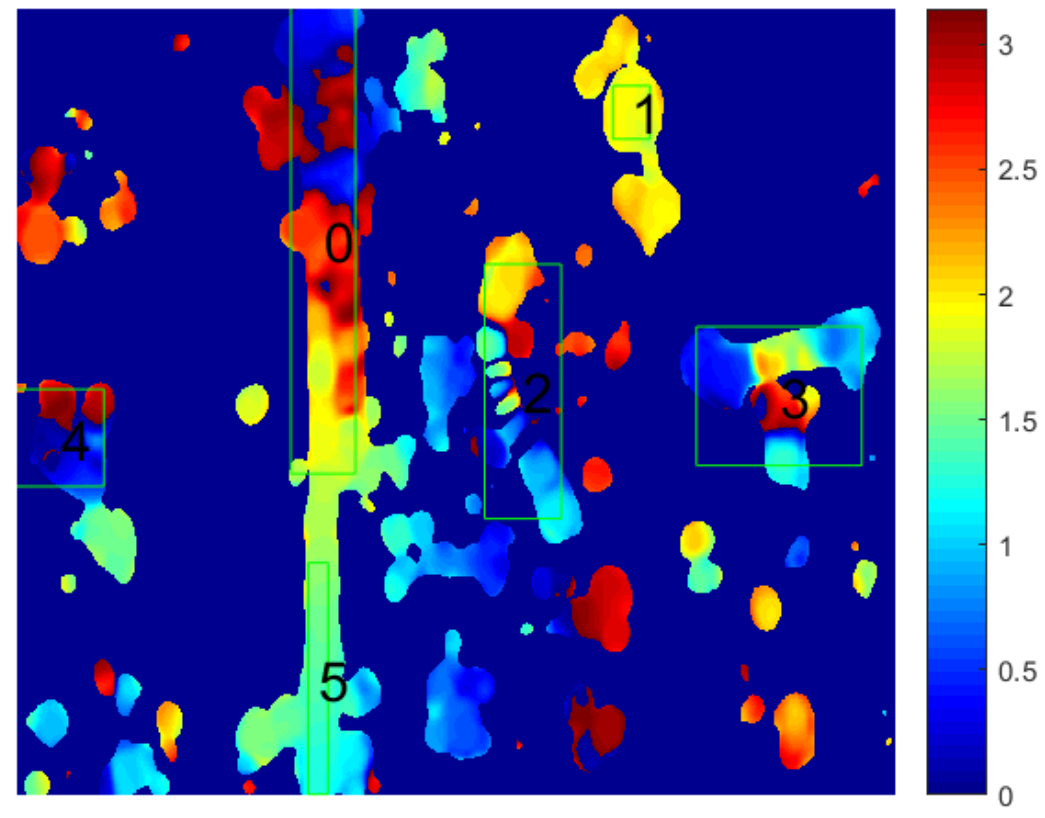

Figure F.34: Optical flow vector orientation map using the colour scale on the right 


\section{Appendix G}

\section{Software Code}

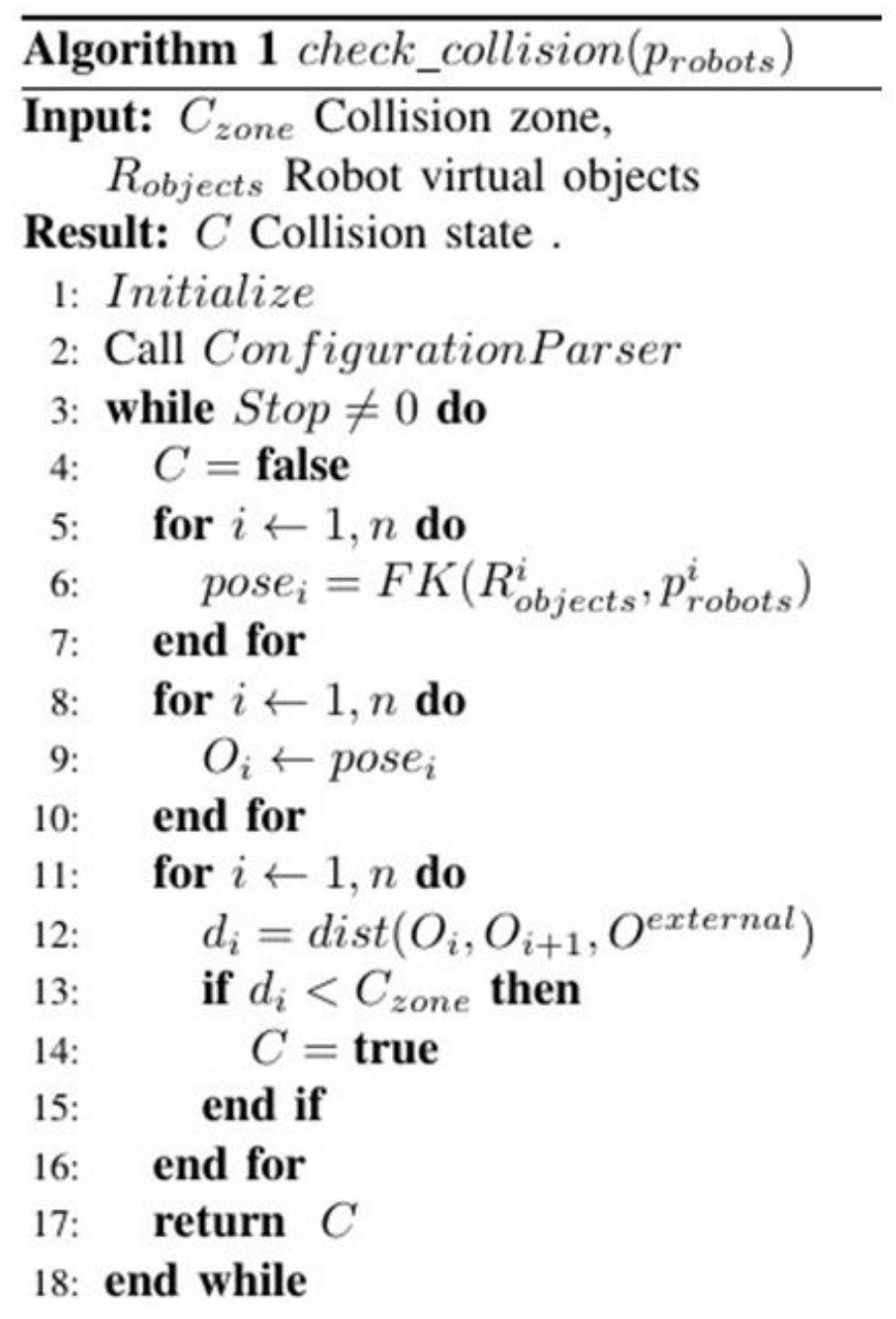

Figure G.1: Check collision algorithm. 


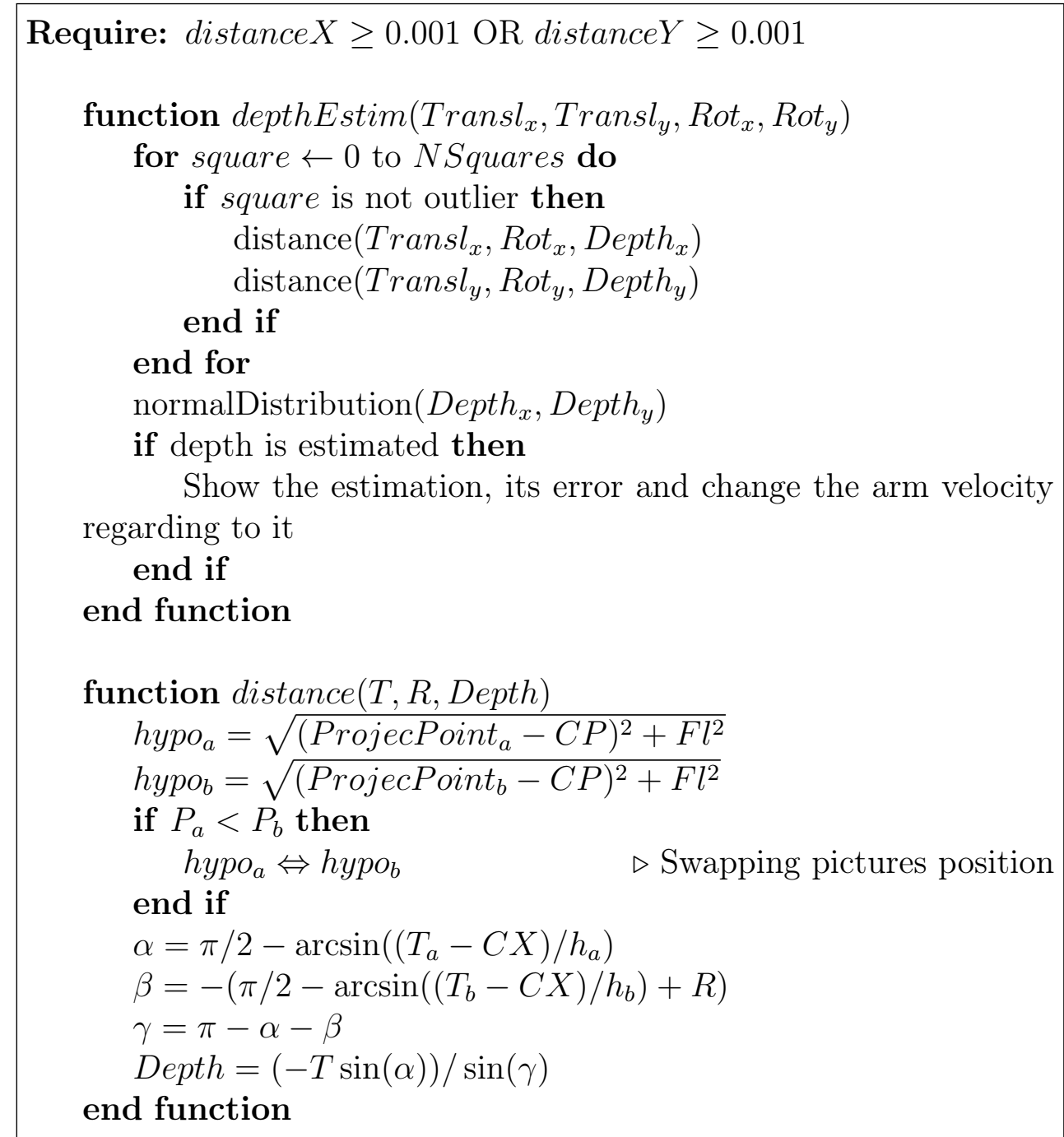

Figure G.2: Tracking-Based Depth Estimation algorithm. 


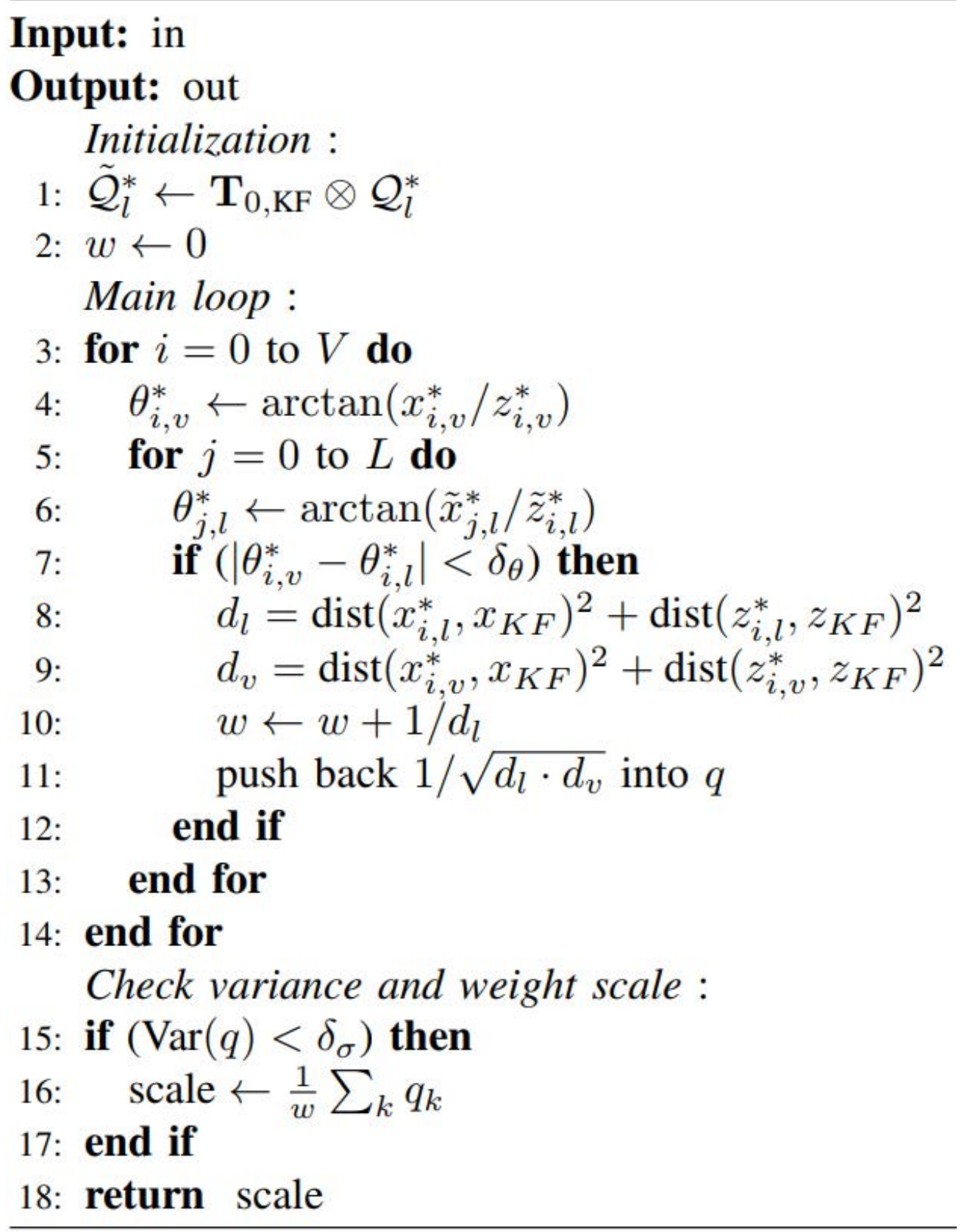

Figure G.3: Scaling Process in L-DSO. V and L are the number of points in $Q_{v}^{*}$ and $Q_{l}^{*}$, respectively. $x_{i, v}^{(t *)}$ and $z_{i, v}^{(t *)}$ are coordinates of $Q_{v}^{*}$ at time $t^{*} . \tilde{x}_{j, l}^{*}$ and $\tilde{z}_{i, l}^{*}$ are coordinates of the transformed point cloud $\tilde{Q}_{l}^{*} . x_{K F}$ and $z_{K F}$ are the translational elements of the transformation from origin to current keyframe camera pose $T_{0, K F} . \mathrm{w}$ is the weight of the scale, the inverse distance of a point to the pose origin. $q$ is the unweighted scale vector of accepted distance quotients. 


\section{List of Figures}

1.1 Kuka Omnirob mobile manipulator (adapted from [55]) . . . . . . . . . . 4

1.2 The Thorium nuclear reactor (left) [64] (adapted from www.newscientist.com) and the Compact Muon Solenoid experiment (right) [65] . . . . . . . . .

2.1 A 3D image of the remote handling system for the ITER divertor on the left and the Divertor cassette placement inside the vacuum vessel by means of the cassette toroidal mover (cable handling device deployed) on the right (Credit: Assystem) . . . . . . . . . . . . . . . . . 13

2.2 JET Torus (left) and remote handling approach using the MASCOT system [80] (Credit: www.euro-fusion.org) . . . . . . . . . . . . . . . . . 14

2.3 Remote handling control room (left) and the Telerob EMSM 2B telemanipulator system in use at SNS (Credit: www.ornl.gov) . . . . . . . . . . . .

2.4 Examples of robotic systems entered into the Fukushima nuclear plant. The irobot (left, credit: www.popularmechanics.com) and the Toshiba Scorpion (right, credit: www.toshiba-energy.com) . . . . . . . . . . .

2.5 Detection of different objects in a LHC collimator[117] using Faster R-CNN[110]. The bounding boxes in the picture highlight the detected specific components on a LHC collimator. . . . . . . . . . . . . . . . . . . . . . . .

2.6 Example of camera and light for an extreme environments from Diakont (left) and a pan, tilt and zoom camera axis-V5915 (right). Both commercial camera systems are integrated into the CERNTAURO framework and are the most used for robotic operations at CERN. . . . . . . . . . . . . . . .

3.1 The continuous integration model used for the CERNTAURO framework implementation. The development phase is deployed in operation once it has been judged to be robust enough to allow a safe and reliable robotic application on the field. Operational feedbacks after each interventions drive future developments towards robustness and safety. . . . . . . . . . . . .

3.2 A high-level block diagram with pictures representation of the Central Operating System (COS) that has been developed following the core-periphery model. Each model interacts with the others always through the core part of the COS. . . . . . . . . . . . . . . . . . . . 
3.3 The overall architecture of the CERNTAURO framework. The robot on the field has an onboard intelligent control featuring capabilities like autonomous navigation and energy management. The comunication in between the field/tunnel and the surface is guaranteed through a virtual private network to allow data encryption and safety. The novel human robot interface developed integrates several features like multi-robots cooperation and connection with different supervision layers including multi-client and web applications. . . . . . . . . . . . . . . . . .

3.4 The CERNTATURO system stack diagram. The bottom layer is composed by fieldbus modules and drivers that comunicate with the hardware and the real-time linux based operating system. On top of this, the low-level control including the networking and the robot intelligence provide robot controls interfacing with the sensors and the actuators. The last layer is composed mainly by the artificial intelligence and comunication modules. . . . . . . . .

3.5 CERNTAURO Architecture for Cooperative Multirobot Interventions. Simulation in virtual reality of collaborative robots running CERNTAURO framework (top-right). The architecture for Cooperative multirobot is based on a Leader robot that on the field distributes and manage the different intervention tasks. The Human Robot Interface manages all the high level cooperation planning using also intervention scripting tools. The human control of the CERNTAURO framework for the robotic interventions consists in a leader/main operator and an assisting operator. . . . . . . . . . . . .

3.6 CERNTAURO control system architecture divided in layers allowing modularity and adaptability of the control system to different hardware. The Hardware Abstraction Layer provides an interface between the system and the hardware. The control layer provides all the control strategies for the actuators. The supervision layer contains all the modules which are responsible for determining the complete state of the system in each moment. The application layer contains all the features that the robot can provide such as autonomous navigation. The communication layer provides all the communication methods with the HRI while the OS layer provides all the functionalities in order to maintain all the modules properly active and interconnected. 34

3.7 Software communication diagram that describes the control system communication in between the different layers described in Figure 3.6 . . . . . . 35

3.8 Pipeline scheme of a CERNTAURO robotic intervention preparation . . . 38

3.9 Dowel for electrical connectors example . . . . . . . . . . . . . . . . 42

3.10 Results comparison of current measurements from LEM and arm sensors . . 44

3.11 Robotic arm black box models . . . . . . . . . . . . . . . . . 45

3.12 Comparison of two different black box models of the arm . . . . . . . . . 46

3.13 Black box motion model for System Identification . . . . . . . . . . . . 46

3.14 Comparison of motion models . . . . . . . . . . . . . . . . . 47

3.15 Trapezoidal velocity profile . . . . . . . . . . . . . . . . . . . 49

3.16 Geometrical approach to search for the $t_{f} \ldots \ldots \ldots \ldots$ 
3.17 The timestamp offset calculation for $50 \mathrm{~ms}$ simulated delay. The black line represents the ground truth, the blue dashed line represents the fourtimestamps mechanism of NTP and the orange line the estimated offset. . .

3.18 The timestamp offset calculation for $100 \mathrm{~ms}$ simulated delay. The black line represents the ground truth, the blue dashed line represents the fourtimestamps mechanism of NTP and the yellow line the estimated offset. . .

4.1 Images taken from point cloud reconstructions of the LHC mockup at CERN using Elastic Fusion based environmental reconstruction techniques [228] . .

4.2 Pipeline of the novel proposed algorithm[234]. After the reconstruction of the object, machine learning is applied to identify the location of the elements on the object. After, filtering and segmentation techniques are applied and a verification is launched if the element is found. . . . . . . . . . . . .

4.3 Total loss during all training steps of Faster-RCNN with Resnet101. . . . . .

4.4 Extraction from the complete point cloud using the bounding box from FastRCNN [234]. . . . . . . . . . . . . . . . . . . . . . . . . . . 57

4.5 Flow chart of the filtering box in Figure $4.2[234] . \ldots . . . . .58$

4.6 Three-dimensional reconstruction of the object. . . . . . . . . . . . 58

4.7 Work-flow of the proposed method. After an initial image acquisition, the captured frame is passed to a trained neural network to recognize a person. Once the person has been recognized, the radar will monitor the respiration rate. . . . . . . . . . . . . . . . . . .

4.8 Solution for rotation and partial occlusion invariance that overtakes the limitation of standard tracking systems. The schemes explains the novelty of the proposed system that integrates different tracking regions in one main ROI. .

4.9 Visual explanation for the partial occlusion problem present in standard tracking systems. From the image flows (left to right), it is evident that, when a region of interest (ROI) is occluded, the system in real-time uses the SURF algorithm taking the current pattern (in black) to recalculate the new ROI. . . . . . . . . . . . . . . . . . . .

4.10 Visual explanation for the image rotation problem present in standard tracking systems. From the image flows (left to right), it is evident that, when a region of interest (ROI) is not anymore tracked because of image rotations, the system in real-time uses the SURF algorithm taking the current pattern (in black) to recalculate the new ROI. . . . . . . . . . . . . . .

4.11 Triangulation proposal in $\mathrm{X}$ and $\mathrm{Y}$ axis. This graphical solution explains the triangulation estimation chosen by the proposed system. Normally this solution is used by epipolar geometry only in $\mathrm{x}$ axis. This has been extend in y axis also thanks to the new architecture. . . . . . . . . . . . .

4.12 Tracking system diagram. The operator or the system/HRI selects the region of interest to be tracked. The tracking algorithm drives the control of the arm, communicates with the dept estimation algorithm and generates the augmented reality features. . . . . . . . . . . . . . . . . . s. 
4.13 Deep Learning integration diagram. The artificial intelligence module has been added to automatically detect an object without the user interaction.

4.14 Collision avoidance test done by the robot parser, that is in charge of reading all of the features defined in the configuration files. . . . . . . . . . . . .

4.15 Simulation of the virtual constraints to avoid collisions in between different arms and in between arms and environmental fixed objects. . . . . . . . . . . 71

4.16 Pictures of the collision avoidance test. . . . . . . . . . . . . . . . . 72

4.17 Simulation of the virtual constraints to avoid collisions in between different arms, in between arms and environmental fixed objects and in between arms and person. . . . . . . . . . . . . . . . . .

4.18 System block diagram with four modules. The proposed system consists of four core components as portrayed here. The shading correction applied by the Photo Module at the pre-processing stage enhances the image brightness and eliminates shadows. The algorithm then corrects for position offset between different capturing times using image mosaicing. Once the two closest reference images are stitched by the Mosaic Engine, the Survey Mapper locates the survey image in the mosaic image. Finally, the Change Detector uses a sequential combination of image differencing, binary image comparison and optical flow analysis to detect any changes occurring from one inspection to another. . . . . . . . . . . . . . . . . . .

4.19 Description of the sequential flowchart of the proposed SLAM module. Initial parameters assumption are introduced to the ray casting module and then the probabilistic approach is computed using the sensor model. After the robot's pose is estimated before closing the step for the next loop. . . . . . .

4.20 The occupancy grid map collected in real-time by the robot with the covered path. To notice the change of path when the environmental radioactivity was considered too high for the robot. . . . . . . . . . . . . . . . .

4.21 The SLAM architecture of the CERNTAURO framework. From left to right the flow goes in a first step from the hardware read-out and data fusion to kalman filter and direct sparse odometry as second step. In parallel the particle filter SLAM computes the localizaion and mapping. . . . . . . . . .

4.22 Schematic of the laser data acquisition. Laser points are gathered on the fixed x-z-plane and scanned almost $240^{\circ}$ with $0.36^{\circ}$ angular resolution, due to a blind spot in the rear containing the angle $\theta . . . . . . . . .$.

4.23 Point cloud cumulation over $\mathrm{m}$ keyframes (here $\mathrm{m}=3$ ). The previous three keyframes $(\mathrm{KFc} 3, \mathrm{KFc} 2, \mathrm{KFc} 1)$ generate a point cloud about the position of the current keyframe KFc. Each partial point cloud is characterized by the color of the respective keyframe. We assume that adding Pc would not meet the condition $|Q v| \leq \eta p . \ldots \ldots \ldots \ldots$

4.24 Vision-laser time synchronization. For the sake of simplicity in this figure, a keyframe is generated every four frames. In reality, this might heavily deviate, spanning from 5 to 20 frames per keyframe, depending on the marginalization rate (see Section 2) . . . . . . . . . . . . . . . 
4.25 Inter-frame transformations and planes. At each keyframe instant, we extract the $y_{c}$-direction from the current pose denoted by $T_{0, c}$ and subsequently span a plane with $y_{c}$ as the normal. . . . . . . . . . . . . . . .

4.26 Schematic block of the proposed system. The Fast Antenna Measurement (FAM) system is implemented on Linux low level APIs and allows acquiring the RSSI measurements for every single acquired packet by accessing to the 802.11 packets. The filtering module receives as input the four RSSI values of the received packet as well as the velocity of the relay node platform coming from the robot odometry, or the SLAM. The gradient computation module determines the future direction of the robot. The RSSI Kalman predictor is used to predict the RSSI in few meters, which allows to better determine the movement of the robot as well as to avoid black spots. The trajectory planning system, finds the best position of the robot and its navigation path, avoiding black spots and obstacles. . . . . . . . . . . . . . . . .

4.27 Filtered signal quality $(\%)$ and strength $(\mathrm{dBm})$ in $(\mathrm{A})$ Wi-Fi Relay 1 robot and (B) Target Relay2 robot. When the Relay 1 approximates to the Relay 2 the signal strength is increased and more stable when filtered, which grants a better performance for the different tasks the robots are assigned to. . . . .

4.28 Architectural overview of the relay robot use case. The relay nodes are used to extend the network coverage. . . . . . . . . . . . . . . .

4.29 A scenario of several robots working together for a given teleoperation task. The relay robots ensure a higher operational distance range even without line-of-sight. . . . . . . . . . . . . . . . . . .

4.30 YouBot (left) and CERNbot (right) integrating the Wi-Fi source and the SLAM sensors. . . . . . . . . . . . . . . . . . .

4.31 The cooperative mission plan scheme. The same operation can be performed and tested in simulation and then applied on the real robots seamlessly. . . .

5.1 The HRI automatically adapts to the robot configuration in order to control in the same modality all CERN's robots pool. . . . . . . . . . . . . . .

5.2 The control view of the HRI, reserved to the operator. Different views are available for cameras, 3D reconstruction, statistics etc. . . . . . . . . . .

5.3 A 3D reconstruction of a collimator with the radioactivity values collected in

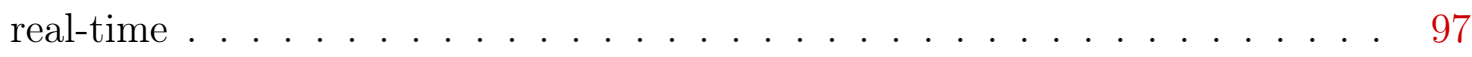

5.4 CERNTAURO Human Robot Interface Views . . . . . . . . . . . . . . . . . 98

5.5 The CERNTAURO scheme of the communication via web with the robots. . 99

5.6 CERNTAURO web pages to monitor the robots and their measurements. The mobile version is shown on the right. . . . . . . . . . . . . . 100

5.7 Example of geometrical and observational wrong prospective. . . . . . . . . 100

5.8 Overview of the proposed CERNTAURO system that is used to maintain the visualized reference frame of the camera jointed with the robot arm reference frame and compensate their misalignment . . . . . . . . . . . . 101 
5.9 Bilateral control system using the master-slave interaction to control a robotic arm. $F_{O}$ is the force applied to the master arm by the operator, $F_{e}$ is the environmental force applied to the slave arm, $x_{m}$ is the position of the master arm, $x_{s}$ is the position of the slave arm, $K_{f}$ is the gain factor for the environmental force feedback and $K_{s}$ is the gain factor for the position-error feedback. . . . . . . . . . . . . . . . . . . .

5.10 Plot of the time-delay passivation function using the parameters defined in Table 5.1. On the $\mathrm{x}$ axis, the control energy is represented in the range $[0 ; 1]$. The $\mathrm{y}$ axis shows the time delay in seconds in the range $[0 ; 500]$. Finally, the $\mathrm{z}$ axis represents the output energy in the range $[0 ; 1] \ldots \ldots$. . . . . . 108

5.11 The architecture of DnCNN network . . . . . . . . . . . . . . . 109

5.12 The compressed image with 50\% JPEG compression and the recovered image via $\mathrm{DnCNN}$. e numbers in parentheses represent the peak signal-to-noise ratio (PSNR) and the structural similarity (SSIM), respectively. . . . . . . . . 110

5.13 The architecture of ESPCN network. . . . . . . . . . . . . . . . 110

5.14 The $4 \mathrm{X}$ down-sampled image with 50\% JPEG compression and the recovered image via DnCNN and ESPCN. The numbers in parentheses represent the peak signal-to-noise ratio (PSNR) and the structural similarity (SSIM), respectively. . . . . . . . . . . . . . . . . . . . 111

5.15 Landscape field flow representation for non-linear trajectory . . . . . . . . 112

5.16 Dynamic change of canonical system's parameter $\alpha_{s} \ldots \ldots . . \ldots 114$

5.17 Learning workflow diagram . . . . . . . . . . . . . . . . . . . . 114

5.18 Robot's learned dynamics control scheme . . . . . . . . . . . . . . . . . 115

5.19 The joints and links comprising a robotic arm example (Telemax) . . . . . . 117

5.20 Joint-by-joint control scheme. . . . . . . . . . . . . . . . . . . . . . . . 118

5.21 Operational-space control scheme. . . . . . . . . . . . . . . . . . 118

5.22 Robotic operation simulation using visual feedback from the on-board cameras. . . . . . . . . . . . . . . . . . . . . . 120

5.23 The GUI used with the simulator. . . . . . . . . . . . . . . 120

5.24 Virtual reality system . . . . . . . . . . . . . . . . . . . . . . 122

5.25 Input and output devices . . . . . . . . . . . . . . . . . . . . 122

5.26 Type of input data of VERO . . . . . . . . . . . . . . . . . . . . 124

5.27 Flowchart interventions planning with VERO . . . . . . . . . . . . . 124

5.28 Four functions displayed in HTC VIVE controller. Teleport(Up), Map(Left), Navigation(Right) and laser(Down). . . . . . . . . . . . . . . . 125

5.29 Navigation map . . . . . . . . . . . . . . . . . . . . . . . 126

5.30 Example of laser in virtual environment . . . . . . . . . . . . . . . . . 127

5.31 Example of selecting a navigation point. . . . . . . . . . . . . . 127

5.32 Electrical driller (left) and electrical handsaw (right) models imported to Unity 128

5.33 Primitive collider. From left to right, box collider, sphere collider and capsule collider . . . . . . . . . . . . . . . . . . . . . . . . . . . 128

5.34 Comparison between box collider with mesh collider . . . . . . . . . . . 129 
5.35 Box collider added to the end of the driller and capsule collider added to the screw . . . . . . . . . . . . . . . . . . .

5.36 Operator tanning in simulated LHC tunnel; arm movement tracking incorporated and reproduced in the animated human body model (Left). Danger estimation system incorporated in TDE maintenance planning; radiation map and animated body are incorporated (Right). . . . . . . . . . . . . . 130

5.37 Module master-slave (left). Module robot controller (Right). . . . . . . . . . 130

5.38 Number of interventions performed from September 2014 until January 2019. The dashed line represents the period since when CERNTAURO solutions were applied. . . . . . . . . . . . . . . . . 131

5.39 Types of interventions performed from September 2014 until January 2019. . 132

5.40 Dose saved to personnel from September 2014 until January 2019. The dashed line represents the period since when CERNTAURO solutions were applied. . 133

5.41 Robots used for interventions from September 2014 until January 2019. . . . 133

5.42 A burndy connector used for the test . . . . . . . . . . . . . . . . . 134

5.43 Picture of the Lemo B-type standard self-latching multipole connectors, with alignment key, used for the test (left), the connection task being performed by CERNbot in single arm configuration (center) and an operator at the master station (right). . . . . . . . . . . . . . . . . . 136

5.44 Time performance comparison between non-experts and experts operators using the Telemax robot in doing the Lemo connection task. Each attempt shown is a time average taken from 5 different operators' attempts. The highlighted final parts in green contains the tentative done with the addition of an environmental camera that helped to improve the operational time performances stepping-out from the steady-state conditions reached beforehand.136

5.45 Time performance comparison between non-experts and experts operators using the CERNbot robot in doing the Lemo connection task. Each attempt shown is a time average taken from 5 different operators' attempts. The highlighted final parts in green contains the tentative done with the addition of an environmental camera that helped to improve the operational time performances stepping-out from the steady-state conditions reached beforehand.138

5.46 Time performance comparison between non-experts and experts operators using the Telemax and the CERNbot robot in doing the Lemo connection task. Each attempt shown is a time average taken from 5 different operators' attempts. The highlighted final parts in green contains the tentative done with the addition of an environmental camera that helped to improve the operational time performances stepping-out from the steady-state conditions reached. It is evident that the user-friendliness of the proposed novel master-thesis telemanipulation system allows the operators to operate and learn faster with respect to the Telemax industrial system. Non expert operators using the CERNTAURO system reach similar time performances in comparison with expert operators using Telemax robot, after only few trials 
5.47 Picture of the tasks being performed by CERNbot in dual arms configuration. Overview of the scene (top left) and the different views seen by the 3 main CERNbot cameras. In-hand left arm camera (bottom left), main base pantilt-zoom (PTZ) camera (top right), in-hand right arm camera (bottom right).140

6.1 CERNBot 1.0 while navigating autonomously in the environment . . . . . . 142

6.2 The occupancy grid map of the reconstructed environment . . . . . . . . . . 142

6.3 CERNBot 1.0 while approaching the radioactive object discovered in the area 143

6.4 Accuracy test. Blue and gray columns are the range of the real distance and orange one the estimation. Red circles show the two errors obtained over 1 cm. but always under $2 \mathrm{~cm}$. . . . . . . . . . . . . . . . . . . 143

6.5 Stability comparative between SURF and KCF based solutions proposed: X axis shows the distance to the target, and $\mathrm{Y}$ axis represents the percentage of frames that provide the correct measurement. . . . . . . . . . . . . . . . . 144

6.6 Relationship among translation and rotation to reach triangulation. . . . . . 145

6.7 A sample image from the Query dataset at time $t_{1} \ldots \ldots$. . . . . . . . 146

6.8 A sample image from the Survey dataset at time $t_{2} \ldots \ldots$. . . . . . . 147

6.9 This screenshot shows the change detection result from the sample set of two temporal images, query image in Figure 6.7 and survey image in Figure 6.8. The bounding boxes envelope the areas detected as change. . . . . . . . . . . 147

6.10 Manually marked TP showing a correctly identified change, FP marking an area incorrectly detected as a change and FN marking missed changes. . . . 148

6.11 Prototype system for test. Above installed X4M200 respiration sensor, in the middle Logitech C920 web-cam, below 12V light source. . . . . . . . . . . . . 150

6.12 System performance at $2 \mathrm{~m}$ distance. A,E,H,K,N represent five postures and bounding boxes around detected victims, generated by MobileNet. B, F, I, $\mathrm{L}, \mathrm{O}$ show acquired respiration data (subject was breathing normally at all times). C, G, J, M, P are pulse-doppler maps for each posture. . . . . . . . . 151

6.13 Multi-person view . . . . . . . . . . . . . . . . . . 152

6.14 Detection performance of MobileNet at various postures and corresponding distances . . . . . . . . . . . . . . . . . . . 153

6.15 Person on side turned away from camera at $4 \mathrm{~m}$. Green bounding box generated by MobileNet. . . . . . . . . . . . . . . . . . . . . . . . . 154

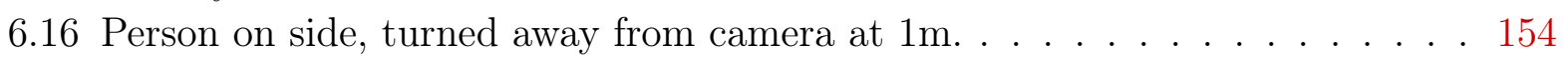

6.17 TCP's X position prediction . . . . . . . . . . . . . . 157

6.18 TCP's Alpha orientation prediction . . . . . . . . . . . . . 158

6.19 Kuka robot finishing Reach-Object-Vicinity Primitive . . . . . . . . . . . . 159

6.20 Full path of TCP performed by KUKA robot with 3 primitives for task com-

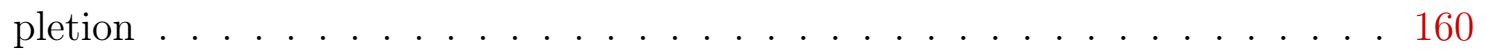

6.21 A Collimator in the LHC tunnel. . . . . . . . . . . . . . . . . . . 161

6.22 Reconstructions with different views of different point clouds of the separator (first and second row) and the collimator (third and forth row). . . . . . 163 
6.23 i-TIM website data display showing the corresponding LHC machine on the right side . . . . . . . . . . . . . . . . . . . 164

6.24 Data comparison between the different i-TIM radiation inspection missions in the Long Straight Section of Point 7 of the LHC, during 2018 . . . . . . . 164

6.25 CERNBot v1.0 and EXTRM in North Area beamline . . . . . . . . . . . . 165

6.26 Location of the water leak . . . . . . . . . . . . . . . . 166

6.27 Water leak area before (a) and after the intervention with the resin injected (e). Metal cover cut (b). Building the recipient to confine the leak area (c). CERNbot running CERNTAURO framework below the collimator. . . . . . 166

6.28 Filling of oil recipient done using industrial solution (left) and custom made solution using CERNTAURO framework (right). . . . . . . . . . . . 167

6.29 CERNbot in dual arm configuration (left pictures) and the iridium radioactive source handling (right pictures) . . . . . . . . . . . 167

A.1 The Intelligent Train Inspection Monorail (I-TIM) . . . . . . . . . . . . . . 173

A.2 The robotic arms integrated on the i-TIM robot. A one degree of freedom arm has been integrated for the first robot version (left). A six degrees of freedom arm is integrated in the current robot version (right). . . . . . . . 175

A.3 3D model of I-TIM mechanical configuration . . . . . . . . . . . . . . 175

A.4 I-TIM passing a sector door in the LHC and dimensions of one wagon . . . . 176

A.5 I-TIM control architecture in layers . . . . . . . . . . . . . . . 177

A.6 Possible operational scenario with a robotic arm deployed from I-TIM . . . . 178

A.7 Coverage provided by 8 sensors installed on a i-TIM wagon . . . . . . . . . 178

A.8 I-TIM Human Robot Interface . . . . . . . . . . . . . . . . . . . . . 179

A.9 Multi-functional reconfigurable CERNbot platform . . . . . . . . . . . . 180

A.10 CERNbot modular architecture . . . . . . . . . . . . . . . . 181

A.11 CERNbot in different configurations . . . . . . . . . . . . . . . . 182

A.12 The CERNBot1 with one robot arm. . . . . . . . . . . . . . 183

A.13 The CERNBot1 with cutter. . . . . . . . . . . . . . . . . . . 184

A.14 The CERNBot1 with the robotic arm in front position. . . . . . . . . . . . 184

A.15 The CERNBot1 with the robotic arm in one side. . . . . . . . . . . . 185

A.16 The CERNBot1 ready for inspection. . . . . . . . . . . . . . . . . 185

A.17 The CERNBot1 with helium injector system attached. . . . . . . . . . . 186

A.18 The CERNBot release for visual inspection. . . . . . . . . . . . . . . 187

A.19 The CERNBot2 with the stage up carrying dual robotic arms. . . . . . . . . 188

A.20 The CRANEBot carrying dual robotic arms. . . . . . . . . . . . . . 189

B.1 LHC tunnel mock-up with TIM on monorail and CERNbot. . . . . . . . . . 190

B.2 Two CERNbot units in LHC mock-up doing collaborative tasks. . . . . . . . 191

B.3 Robotic arms used and integrated by the CERTAURO framework. . . . . . . 192

C.1 Haptic friendly handler for master side arm. . . . . . . . . . . . . . . . . . 194 
C.2 Operator performing an intervention by the Master-Slave system with dual arms. . . . . . . . . . . . . . . . . . . . . . 195

C.3 Cat-design of the handler . . . . . . . . . . . . . . . . . . 195

C.4 Electric diagram of the first release . . . . . . . . . . . . . 196

C.5 Electric diagram of the current release . . . . . . . . . . . . . . . 197

C.6 Box-diagram of handler behaviour . . . . . . . . . . . . . . . . . 198

C.7 Final PCB layout . . . . . . . . . . . . . . . . . . . . . . . . . 199

C.8 Bottom polygon pour net GND . . . . . . . . . . . . . . . . 200

C.9 Top polygon pour net VCC ～. . . . . . . . . . . . . . . . 200

E.1 Main parts of the bolt. . . . . . . . . . . . . . . . . . . . 212

E.2 Socket-head (left), Hexagonal-head (right) _ . . . . . . . . . . . . . 213

E.3 Socket Head.Opposite flanks of hexagon must be parallel and internal corners must be sharp. . . . . . . . . . . . . . . . . . . . . . 213

E.4 Hex Head Modifications . . . . . . . . . . . . . . . . . . . . . . . 213

E.5 Bi-Hex Head . . . . . . . . . . . . . . . . . . . . . . . . . . . . . . 214

E.6 Example of point to avoid cross-threading. . . . . . . . . . . . . . . . . . . 214

E.7 Free captive bolts (left), close-up view of spring-loaded captive bolt (right). . 215

E.8 Guide Pin . . . . . . . . . . . . . . . . . . . . . . . . . . . . 217

E.9 Parallel Dowel Pin . . . . . . . . . . . . . . . . . . . . . . . . . . . . 218

E.10 Ball-End Dowel Arrangement . . . . . . . . . . . . . . . . . . . . . 219

E.11 Dowel Dimensioning and Tolerancing. This design incorporates a long ballended dowel that locates in a circular hole and a short parallel dowel that locates in a short slot. On top, it is shown the round shape design. In the middle an alternative design to help the insertion. On the bottom it is indicated the maximum approach angle. . . . . . . . . . . . . . . . . 220

E.12 Quick Disconnect System example from Technetics . . . . . . . . . . . . 224

E.13 Example of bolting flanges . . . . . . . . . . . . . . . . . . . . . . 225

E.14 Axial \& Azimuthal Flange Location . . . . . . . . . . . . . . . . . . . . . 226

E.15 Flange Spigots . . . . . . . . . . . . . . . . . . . . . . 226

E.16 RH Lemo Connector. . . . . . . . . . . . . . . . . . . . . . . 228

E.17 Kinematic Constraint Stages . . . . . . . . . . . . . . . . . . . . . 229

E.18 Example of CERNTAURO interventions at CERN where custom designed tools, described RH procedures, plug-and-play connectors (left) and quick disconnect systems have been used (right).

F.1 LHC tunnel's cross-section,, consisting of two parts, the one hosting the beamline and the other that is available for personnel and other machinery to be moved in the tunnel such as the TIM monorail robot on the ceiling. . . . . .

F.2 Light variation in the LHC tunnel, some areas are darker than the others because of no light tubes in the area or because of shadow cast from the existing equipment . . . . . . . . . . . . . . . . . . 232

F.3 TIM passing through sector doors passageways. . . . . . . . . . . . . 233 
F.4 TIM dimension constraints . . . . . . . . . . . . . . . . . . . . . . 233

F.5 Camera in the proposed orientation, such that it is facing the wall, capturing images containing a 'plain' background with wall cracks and other defects as foreground. . . . . . . . . . . . . . . . . . . . . 234

F.7 Image after enhancement using global histogram equalization . . . . . . . . 236

F.6 Original image before pre-processing . . . . . . . . . . . . . . 236

F.8 Image after enhancement using CLAHE . . . . . . . . . . . . . . . . . 237

F.9 Image after enhancement using the proposed shading correction . . . . . . 237

F.10 Position offset correction using mosaic . . . . . . . . . . . . . . . . 239

F.11 Pre-processed images used for mosaicing. Such images are closest in location in relation to the current survey image to be inspected. . . . . . . . . . . 240

F.12 The edge images used in template matching involved in the image registration step, (a) is used as the template and (b) is used as the source . . . . . . . 240

F.13 Mosaic image without blending. . . . . . . . . . . . . . . . . . . . 240

F.14 Survey image . . . . . . . . . . . . . . . . . . . . . . . . . . . . 242

F.15 Edge-based survey image mapping . . . . . . . . . . . . . . . . . 242

F.16 A sample image from the Query dataset at time $t_{1} \ldots \ldots$. . . . . . . 242

F.17 A sample image from the Survey dataset at time $t_{2} \ldots \ldots . . . . .243$

F.18 Simple Image Differencing when using different thresholds. When the threshold was set to a low value such as 10 and 30 , the difference image contains a lot of 'image noise'. Higher threshold values, suppress such noise as well as alignment errors better.

F.19 Neighbourhood Image Differencing when using different thresholds showing that for the same threshold values, neighbourhood image differencing suppresses alignment errors and image noise better than simple image differencing. 245

F.20 Neighbourhood image differencing with a low threshold value as used in the algorithm. . . . . . . . . . . . . . . . . . . . 246

F.21 Difference image after closing morphological operation, further suppressing irrelevant changes due to image noise . . . . . . . . . . . . . . 246

F.22 Connected components labelling whereby neighbouring pixels are grouped and labelled as separate objects. . . . . . . . . . . . . . . . . . 247

F.23 Connected components labelling, when applying morphological closing operation and not. . . . . . . . . . . . . . . . . . . . . 248

F.24 Merging components scenario . . . . . . . . . . . . . . . . . . 248

F.25 Bounding boxes of candidate changes before merging . . . . . . . . . 249

F.26 Bounding boxes of candidate changes after merging . . . . . . . . . . 249

F.27 Possible change components . . . . . . . . . . . . . . . . . 250

F.28 Binary comparison of sub-image with a registration misalignment (no change) 251

F.29 Binary comparisons of sub-images with a true change . . . . . . . . . . 251

F.30 Optical Flow between two images . . . . . . . . . . . . . . . . 252

F.31 Result after optical flow calculated from Figure F.16 and Figure F.17 . . . . 253

F.32 Filtered optical flow, showing only the significant vectors according to their magnitude . . . . . . . . . . . . . . . . . . 254 
F.33 Optical flow vector magnitude map using the colour scale on the right . . . . 256 F.34 Optical flow vector orientation map using the colour scale on the right . . . . 256

G.1 Check collision algorithm. . . . . . . . . . . . . . . . . . . . . 257

G.2 Tracking-Based Depth Estimation algorithm. . . . . . . . . . . . . . . 258

G.3 Scaling Process in L-DSO. V and L are the number of points in $Q_{v}^{*}$ and $Q_{l}^{*}$, respectively. $x_{i, v}^{(t *)}$ and $z_{i, v}^{(t *)}$ are coordinates of $Q_{v}^{*}$ at time $t^{*} . \tilde{x}_{j, l}^{*}$ and $\tilde{z}_{i, l}^{*}$ are coordinates of the transformed point cloud $\tilde{Q}_{l}^{*}$. $x_{K F}$ and $z_{K F}$ are the translational elements of the transformation from origin to current keyframe camera pose $T_{0, K F}$. w is the weight of the scale, the inverse distance of a point to the pose origin. $\mathrm{q}$ is the unweighted scale vector of accepted distance quotients. 


\section{List of Tables}

1.1 Robotic Revolution for Past and Future Years . . . . . . . . . . . . . . . 3

1.2 Semi-structured and Harsh Environments Challenges and the unique CERNTAURO control features to tackle them . . . . . . . . . . . . . . 6

3.1 SOME CERNTAURO ADDED VALUES WITH RESPECT TO ROS . . . . 37

3.2 A reduced example of a database fixed table of the CERNTAURO Robotic Pool . . . . . . . . . . . . . . . . . . . . . 37

3.3 A reduced example of a database table of the CERNTAURO Tooling Pool . 38

3.4 Available networks in the underground accelerator . . . . . . . . . . . . . 51

3.5 Network offset estimation using different network delays. It is evident how the proposed filtering using a moving average on the offset and compensating negative delays, gives better results than then standard NTP protocol. . . .

4.1 Technical specifications of X4M200 respiration sensor currently used by the CERNTAURO framework. . . . . . . . . . . . . . . . . . . 64

5.1 Network Latency Passivation Parameters . . . . . . . . . . . . . . . . . 107

5.2 Specifications of Oculus . . . . . . . . . . . . . . . . . . . . 123

5.3 Specifications of HTC Vive . . . . . . . . . . . . . . . . . . 123

5.4 Results obtained during the fulfilling of the first task . . . . . . . . . 135

5.5 Results obtained during the fulfilling of the second task . . . . . . . . . 135

5.6 Hearthbeat percentage increase during the fulfilling of the first task . . . . . 135

5.7 Hearthbeat percentage increase during the fulfilling of the first task . . . . . 135

6.1 Accuracy and stability of SURF and KCF based solutions . . . . . . . . . 144

6.2 Dataset information, including camera exposure settings: A-Aperture, SShutter speed and ISO and the type of the manually created change: P-Pencil, M-Marker, S-Scratches . . . . . . . . . . . . . . . . . . . . 146

6.3 Attributes of the detected 'change areas' including location and description information. The location of the center of the bounding box as well as its width and height are recorded. . . . . . . . . . . . . . . . . 148

6.4 Average TPR and PDR ratios for each of the datasets that were used for testing 148

6.5 Respiration detection rate. Male in t-shirt. . . . . . . . . . . . . . 155

6.6 Respiration detection rate. Male in thick clothing. . . . . . . . . . . . 155

6.7 RMSE of Dynamic Movement Primitive and Robot's end-effector real position 158

7.1 Main added values of the CERNTAURO framework with respect to other particle accelerators and power plants. . . . . . . . . . . . . 171 
D.1 Tooling pool database . . . . . . . . . . . . . . . . . . . . . . . . . 204

E.1 Staged Kinematic Constraint . . . . . . . . . . . . . . . . . . . . . . 229

F.1 Attributes of the detected 'change areas' . . . . . . . . . . . . . . . . 255

F.2 Dataset information, including camera exposure settings: A-Aperture, S-

Shutter speed and ISO and the type of change: P-Pencil, M-Marker, S-Scratches255 


\section{Bibliography}

[1] Klaus Schwab. The fourth industrial revolution. Crown Business, 2017.

[2] Adel H Alhamedi, Vaclav Snasel, Hamoud M Aldosari, and Ajith Abraham. Internet of things communication reference model. In Computational Aspects of Social Networks (CASoN), 2014 6th International Conference on, pages 61-66. IEEE, 2014.

[3] Zhijing Qin, Grit Denker, Carlo Giannelli, Paolo Bellavista, and Nalini Venkatasubramanian. A software defined networking architecture for the internet-of-things. In Network Operations and Management Symposium (NOMS), 2014 IEEE, pages 1-9. IEEE, 2014.

[4] Yongseong Cho, Jongsun Choi, Jaeyoung Choi, and Young-Jae Ryoo. Robot software platform for iot-based context-awareness. International Journal of Humanoid Robotics, 14(02):1750012, 2017.

[5] Tomoaki Yoshida, Keiji Nagatani, Satoshi Tadokoro, Takeshi Nishimura, and Eiji Koyanagi. Improvements to the rescue robot quince toward future indoor surveillance missions in the fukushima daiichi nuclear power plant. In Field and Service Robotics, pages 19-32. Springer, 2014.

[6] JP Friconneau, V Beaudoin, A Dammann, C Dremel, JP Martins, and CS Pitcher. Iter hot cell - remote handling system maintenance overview. Fusion Engineering and Design, 124:673-676, 2017.

[7] Chao Alexander Wu, Tigner Maury, and Zimmermann Frank. Handbook of accelerator physics and engineering. World Scientific, 2013.

[8] Christiane Lefevre. The cern accelerator complex. Technical report, 2008.

[9] R Brinkmann et al. The european xfel project. In FEL, volume 6, page 24, 2006.

[10] J Peters. The new desy rf-driven multicusp h- ion source. In AIP Conference Proceedings, volume 1097, pages 171-180. AIP, 2009.

[11] TE Mason, D Abernathy, I Anderson, J Ankner, T Egami, G Ehlers, A Ekkebus, G Granroth, M Hagen, K Herwig, et al. The spallation neutron source in oak ridge: A powerful tool for materials research. Physica B: Condensed Matter, 385:955-960, 2006.

[12] Lillian Hoddeson, Adrienne W Kolb, and Catherine Westfall. Fermilab: Physics, the frontier, and megascience. University of Chicago Press, 2009. 
[13] Mads Hvilshøj, Simon Bøgh, Oluf Skov Nielsen, and Ole Madsen. Autonomous industrial mobile manipulation (aimm): past, present and future. Industrial Robot: An International Journal, 39(2):120-135, 2012.

[14] Rob Buckingham and Antony Loving. Remote-handling challenges in fusion research and beyond. Nature Physics, 12(5):391, 2016.

[15] Alejandro Gutiérrez-Giles and Marco A Arteaga-Pérez. Transparent bilateral teleoperation interacting with unknown remote surfaces with a force/velocity observer design. International Journal of Control, pages 1-18, 2017.

[16] William J Manion and Thomas S LaGuardia. Decommissioning handbook. Technical report, Nuclear Energy Services, 1980.

[17] Mark E Rosheim. Robot evolution: the development of anthrobotics. John Wiley \& Sons, 1994.

[18] Luis Govinda García-Valdovinos, Tomás Salgado-Jiménez, Manuel Bandala-Sánchez, Luciano Nava-Balanzar, Rodrigo Hernández-Alvarado, and José Antonio CruzLedesma. Modelling, design and robust control of a remotely operated underwater vehicle. International Journal of Advanced Robotic Systems, 11(1):1, 2014.

[19] John J Leonard and Alexander Bahr. Autonomous underwater vehicle navigation. In Springer Handbook of Ocean Engineering, pages 341-358. Springer, 2016.

[20] Tomás Lozano-Perez. Autonomous robot vehicles. Springer Science \& Business Media, 2012.

[21] Justin E Manley. Unmanned surface vehicles, 15 years of development. In OCEANS 2008, pages 1-4. IEEE, 2008.

[22] G De Novi, Claudio Melchiorri, JC Garcia, PJ Sanz, Pere Ridao, and Gabriel Oliver. A new approach for a reconfigurable autonomous underwater vehicle for intervention. In Systems conference, 2009 3rd annual IEEE, pages 23-26. IEEE, 2009.

[23] Amit Shukla and Hamad Karki. Application of robotics in onshore oil and gas industry - a review part i. Robotics and Autonomous Systems, 75:490-507, 2016.

[24] Amit Shukla and Hamad Karki. Application of robotics in offshore oil and gas industry-a review part ii. Robotics and Autonomous Systems, 75:508-524, 2016.

[25] Maurizio Rossi, Davide Brunelli, Andrea Adami, Leandro Lorenzelli, Fabio Menna, and Fabio Remondino. Gas-drone: Portable gas sensing system on uavs for gas leakage localization. In SENSORS, 2014 IEEE, pages 1431-1434. IEEE, 2014.

[26] Eric Feron and Eric N Johnson. Aerial robotics. In Springer Handbook of Robotics, pages 1009-1029. Springer, 2008. 
[27] David W Matolak and Ruoyu Sun. Unmanned aircraft systems: Air-ground channel characterization for future applications. IEEE Vehicular Technology Magazine, 10(2):79, 2015.

[28] Javier Irizarry, Masoud Gheisari, and Bruce N Walker. Usability assessment of drone technology as safety inspection tools. Journal of Information Technology in Construction (ITcon), 17(12):194-212, 2012.

[29] Noel Sharkey. The automation and proliferation of military drones and the protection of civilians. Law, Innovation and Technology, 3(2):229-240, 2011.

[30] Peter M Asaro. The labor of surveillance and bureaucratized killing: new subjectivities of military drone operators. Social Semiotics, 23(2):196-224, 2013.

[31] Kimon P Valavanis and George J Vachtsevanos. Future of unmanned aviation. In Handbook of unmanned aerial vehicles, pages 2993-3009. Springer, 2015.

[32] Andrew Callam. Drone wars: Armed unmanned aerial vehicles. International Affairs Review, 18, 2015.

[33] Graham Wild, John Murray, and Glenn Baxter. Exploring civil drone accidents and incidents to help prevent potential air disasters. Aerospace, 3(3):22, 2016.

[34] Liam Pedersen, David Kortenkamp, David Wettergreen, I Nourbakhsh, and David Korsmeyer. A survey of space robotics. 2003.

[35] Mark Yim, Kimon Roufas, David Duff, Ying Zhang, Craig Eldershaw, and Sam Homans. Modular reconfigurable robots in space applications. Autonomous Robots, 14(2-3):225-237, 2003.

[36] Max Bajracharya, Mark W Maimone, and Daniel Helmick. Autonomy for mars rovers: Past, present, and future. Computer, 41(12), 2008.

[37] Kazuya Yoshida. Achievements in space robotics. IEEE Robotics $\mathscr{G}$ Automation Magazine, 16(4), 2009.

[38] Bret G Drake, Stephen J Hoffman, and David W Beaty. Human exploration of mars, design reference architecture 5.0. In Aerospace Conference, 2010 IEEE, pages 1-24. IEEE, 2010.

[39] David W Hainsworth. Teleoperation user interfaces for mining robotics. Autonomous Robots, 11(1):19-28, 2001.

[40] Peter Corke, Jonathan Roberts, Jock Cunningham, and David Hainsworth. Mining robotics. In Springer handbook of robotics, pages 1127-1150. Springer, 2008.

[41] Jessie YC Chen. Uav-guided navigation for ground robot tele-operation in a military reconnaissance environment. Ergonomics, 53(8):940-950, 2010. 
[42] PJ Springer. Military robots and drones: A reference handbook. abc. CLIO, 2013.

[43] Haris Balta, Holger Wolfmayr, Jürgen Braunstein, Yvan Baudoin, et al. Integrated mobile robot system for landmine detection. HUDEM2014, 2014.

[44] Geert De Cubber, Haris Balta, and Claude Lietart. Teodor: a semi-autonomous search and rescue and demining robot. In Applied Mechanics and Materials, volume 658, pages 599-605. Trans Tech Publ, 2014.

[45] Mario Di Castro, Luca Rosario Buonocore, Manuel Ferre, Simone Gilardoni, Roberto Losito, Giacomo Lunghi, and Alessandro Masi. A dual arms robotic platform control for navigation, inspection and telemanipulation. In 16th Int. Conf. on Accelerator and Large Experimental Control Systems (ICALEPCS'17), Barcelona, Spain, 8-13 October 2017, pages 709-713. JACOW, Geneva, Switzerland, 2018.

[46] Mario Di Castro, Maria Laura Baiguera Tambutti, Simone Gilardoni, Roberto Losito, Giacomo Lunghi, and Alessandro Masi. Lhc train control system for autonomous inspections and measurements. In 16th Int. Conf. on Accelerator and Large Experimental Control Systems (ICALEPCS'17), Barcelona, Spain, 8-13 October 201\%. JACOW, Geneva, Switzerland, 2018.

[47] JV Draper, WE Moore, JN Herndon, and BS Weil. Effects of force reflection on servomanipulator task performance. Technical report, Oak Ridge National Lab., 1986.

[48] Ray C Goertz. Manipulator systems developed at anl. In Proceedings, The 12th Conference on Remote Systems Technology, pages 117-136, 1964.

[49] Thomas B Sheridan. Telerobotics, automation, and human supervisory control. MIT press, 1992.

[50] Joachim Schuler. Integration von Förder-und Handhabungseinrichtungen, volume 104. Springer-Verlag, 2013.

[51] Evert Helms, Rolf Dieter Schraft, and M Hagele. rob@ work: Robot assistant in industrial environments. In Robot and Human Interactive Communication, 2002. Proceedings. 11th IEEE International Workshop on, pages 399-404. IEEE, 2002.

[52] Mads Hvilshøj, Simon Bøgh, Ole Madsen, and Morten Kristiansen. The mobile robot "little helper": concepts, ideas and working principles. In Emerging Technologies 83 Factory Automation, 2009. ETFA 2009. IEEE Conference on, pages 1-4. IEEE, 2009.

[53] Willow Garage. Willow garage. [Online]. Available: www.willowgarage.com/pages/pr2/overview, 2010.

[54] TUM-Rosie. Tum-rosie. [Online]. Available: http://ias.cs.tum.edu/robots/tumrosie/, 2011. 
[55] Fei Chenf, Boyang Gao, Mario Selvaggio, Zhijun Li, Darwin Caldwell, Keith Kershaw, Alessandro Masi, Mario Di Castro, and Roberto Losito. A framework of teleoperated and stereo vision guided mobile manipulation for industrial automation. In Mechatronics and Automation (ICMA), 2016 IEEE International Conference on, pages 16411648. IEEE, 2016.

[56] S Costo and R Molfino. A new robotic unit for onboard airplanes bomb disposal. In 35th International symposium on robotics ISR, volume 2004, pages 23-26. Citeseer, 2004.

[57] Bruno Siciliano and Oussama Khatib. Springer handbook of robotics. Springer, 2016.

[58] Jean-Arcady Meyer and David Filliat. Map-based navigation in mobile robots:: Ii. a review of map-learning and path-planning strategies. Cognitive Systems Research, 4(4):283-317, 2003.

[59] Manuel Ferre, Rafael Aracil, Carlos Balaguer, Martin Buss, and Claudio Melchiorri. Advances in telerobotics, volume 31. Springer, 2007.

[60] Peter F Hokayem and Mark W Spong. Bilateral teleoperation: An historical survey. Automatica, 42(12):2035-2057, 2006.

[61] First-MM. Flexible skill acquisition and intuitive robot tasking for mobile manipulation in the real world. [Online]. Available: http://www.first-mm.eu/, 2007-2013.

[62] TAPAS. Robotics-enabled logistics and assistive services for the transformable factory of the future. [Online]. Available: http://www.tapasproject.eu/, 2007-2013.

[63] TAPAS. Validation of advanced, collaborative robotics for industrial applications. [Online]. Available: http://www.valeri-project.eu, 2007-2013.

[64] RK Sinha and Anil Kakodkar. Design and development of the ahwr - the indian thorium fuelled innovative nuclear reactor. Nuclear Engineering and Design, 236(78):683-700, 2006.

[65] CMS Collaboration et al. The cms experiment at the cern lhc. Jinst, 3:S08004, 2008.

[66] Joost Broekens, Marcel Heerink, Henk Rosendal, et al. Assistive social robots in elderly care: a review. Gerontechnology, 8(2):94-103, 2009.

[67] Scott B Nokleby. Singularity analysis of the canadarm2. Mechanism and Machine Theory, 42(4):442-454, 2007.

[68] Jamshed Iqbal, Raza Ul Islam, Syed Zain Abbas, Abdul Attayyab Khan, and Syed Ali Ajwad. Automating industrial tasks through mechatronic systems-a review of robotics in industrial perspective. Tehnički vjesnik, 23(3):917-924, 2016. 
[69] Gary Anderson and Gary Anderson. The Economic Impact of Technology Infrastructure for Advanced Robotics. US Department of Commerce, National Institute of Standards and Technology, 2016.

[70] Iter. [Online] https://en.wikipedia.org/wiki/ITER.

[71] C Damiani, J Palmer, N Takeda, C Annino, S Balagué, P Bates, S Bernal, J Cornellá, G Dubus, $\mathrm{S}$ Esqué, et al. Overview of the iter remote maintenance design and of the development activities in europe. Fusion Engineering and Design, 2018.

[72] Y Noguchi, M Saito, T Maruyama, and N Takeda. Design progress of iter blanket remote handling system towards manufacturing. Fusion Engineering and Design, 2018.

[73] Salvador Esqué, Carine van Hille, Roberto Ranz, Carlo Damiani, Jim Palmer, and David Hamilton. Progress in the design, r\&d and procurement preparation of the iter divertor remote handling system. Fusion Engineering and Design, 89(9-10):2373-2377, 2014.

[74] Darren Locke, Carmen González Gutiérrez, Carlo Damiani, Jean-Pierre Friconneau, and Jean-Pierre Martins. Progress in the conceptual design of the iter cask and plug remote handling system. Fusion Engineering and Design, 89(9-10):2419-2424, 2014.

[75] Gregory Dubus, Adrian Puiu, Philip Bates, Carlo Damiani, Roger Reichle, and Jim Palmer. Progress in the design and $r \& d$ of the iter in-vessel viewing and metrology system (ivvs). Fusion Engineering and Design, 89(9-10):2398-2403, 2014.

[76] R Shuff, M Van Uffelen, C Damiani, A Tesini, C-H Choi, and R Meek. Progress in the design of the iter neutral beam cell remote handling system. Fusion Engineering and Design, 89(9-10):2378-2382, 2014.

[77] Makiko Saito, Katsunori Anzai, Takahito Maruyama, Yuto Noguchi, Kenichi Ueno, Nobukazu Takeda, and Satoshi Kakudate. Development of radiation hard components for iter blanket remote handling system. Fusion Engineering and Design, 109:15021506, 2016.

[78] M Keilhacker, A Gibson, C Gormezano, PJ Lomas, PR Thomas, ML Watkins, P Andrew, B Balet, D Borba, CD Challis, et al. High fusion performance from deuteriumtritium plasmas in jet. Nuclear Fusion, 39(2):209, 1999.

[79] Stephen Sanders. Remote operations for fusion using teleoperation. Industrial Robot: An International Journal, 33(3):174-177, 2006.

[80] AC Rolfe. Remote handling jet experience. 1999.

[81] Mark J Rennich and Thomas W Burgess. Remote handling in the spallation neutron source target facility. Nuclear News, 49(2):32-36, 2006. 
[82] Keiji Nagatani, Seiga Kiribayashi, Yoshito Okada, Kazuki Otake, Kazuya Yoshida, Satoshi Tadokoro, Takeshi Nishimura, Tomoaki Yoshida, Eiji Koyanagi, Mineo Fukushima, et al. Emergency response to the nuclear accident at the fukushima daiichi nuclear power plants using mobile rescue robots. Journal of Field Robotics, 30(1):44-63, 2013.

[83] Shinji Kawatsuma, Mineo Fukushima, and Takashi Okada. Emergency response by robots to fukushima-daiichi accident: summary and lessons learned. Industrial Robot: An International Journal, 39(5):428-435, 2012.

[84] David Sands. Cost effective robotics in the nuclear industry. Industrial Robot: An International Journal, 33(3):170-173, 2006.

[85] Bing Lam Luk, KP Liu, Arthur A Collie, David S Cooke, and S Chen. Tele-operated climbing and mobile service robots for remote inspection and maintenance in nuclear industry. Industrial Robot: An International Journal, 33(3):194-204, 2006.

[86] Anthony Andrews. Nuclear fuel reprocessing: Us policy development. LIBRARY OF CONGRESS WASHINGTON DC CONGRESSIONAL RESEARCH SERVICE, 2006.

[87] Philippe Desbats, Franck Geffard, Gérard Piolain, and Alain Coudray. Force-feedback teleoperation of an industrial robot in a nuclear spent fuel reprocessing plant. Industrial Robot: An International Journal, 33(3):178-186, 2006.

[88] Günter Niemeyer, Carsten Preusche, Stefano Stramigioli, and Dongjun Lee. Telerobotics. In Springer Handbook of Robotics, pages 1085-1108. Springer, 2016.

[89] Russell H Taylor, Arianna Menciassi, Gabor Fichtinger, Paolo Fiorini, and Paolo Dario. Medical robotics and computer-integrated surgery. In Springer handbook of robotics, pages 1657-1684. Springer, 2016.

[90] Sylvain Calinon. Learning from demonstration (programming by demonstration). Encyclopedia of Robotics, 2019.

[91] Ioannis Havoutis and Sylvain Calinon. Learning from demonstration for semiautonomous teleoperation. Autonomous Robots, pages 1-14, 2018.

[92] Ai (artificial intelligence). /Online] https://searchenterpriseai.techtarget.com/definition/AIArtificial-Intelligence.

[93] Yann LeCun, Yoshua Bengio, and Geoffrey Hinton. Deep learning. nature, 521(7553):436, 2015.

[94] Alex Krizhevsky, Ilya Sutskever, and Geoffrey E Hinton. Imagenet classification with deep convolutional neural networks. In Advances in neural information processing systems, pages 1097-1105, 2012. 
[95] K. Simonyan and A. Zisserman. Very Deep Convolutional Networks for Large-Scale Image Recognition. ArXiv e-prints, September 2014.

[96] C. Szegedy, W. Liu, Y. Jia, P. Sermanet, S. Reed, D. Anguelov, D. Erhan, V. Vanhoucke, and A. Rabinovich. Going Deeper with Convolutions. ArXiv e-prints, September 2014 .

[97] S. Ioffe and C. Szegedy. Batch Normalization: Accelerating Deep Network Training by Reducing Internal Covariate Shift. ArXiv e-prints, February 2015.

[98] K. He, X. Zhang, S. Ren, and J. Sun. Delving Deep into Rectifiers: Surpassing HumanLevel Performance on ImageNet Classification. ArXiv e-prints, February 2015.

[99] K. He, X. Zhang, S. Ren, and J. Sun. Deep Residual Learning for Image Recognition. ArXiv e-prints, December 2015.

[100] J. Huang, V. Rathod, C. Sun, M. Zhu, A. Korattikara, A. Fathi, I. Fischer, Z. Wojna, Y. Song, S. Guadarrama, and K. Murphy. Speed/accuracy trade-offs for modern convolutional object detectors. ArXiv e-prints, November 2016.

[101] W. Liu, D. Anguelov, D. Erhan, C. Szegedy, S. Reed, C.-Y. Fu, and A. C. Berg. SSD: Single Shot MultiBox Detector. ArXiv e-prints, December 2015.

[102] J. Dai, Y. Li, K. He, and J. Sun. R-FCN: Object Detection via Region-based Fully Convolutional Networks. ArXiv e-prints, May 2016.

[103] R. Gokberk Cinbis, J. Verbeek, and C. Schmid. Weakly Supervised Object Localization with Multi-fold Multiple Instance Learning. ArXiv e-prints, March 2015.

[104] S. Bell, C. L. Zitnick, K. Bala, and R. Girshick. Inside-Outside Net: Detecting Objects in Context with Skip Pooling and Recurrent Neural Networks. ArXiv e-prints, December 2015.

[105] J. Redmon, S. Divvala, R. Girshick, and A. Farhadi. You Only Look Once: Unified, Real-Time Object Detection. ArXiv e-prints, June 2015.

[106] R. Stewart and M. Andriluka. End-to-end people detection in crowded scenes. ArXiv e-prints, June 2015.

[107] K. Lenc and A. Vedaldi. R-CNN minus R. ArXiv e-prints, June 2015.

[108] Ross Girshick, Jeff Donahue, Trevor Darrell, and Jitendra Malik. Rich feature hierarchies for accurate object detection and semantic segmentation. In Proceedings of the IEEE conference on computer vision and pattern recognition, pages 580-587, 2014.

[109] R. Girshick. Fast R-CNN. ArXiv e-prints, April 2015. 
[110] Shaoqing Ren, Kaiming He, Ross Girshick, and Jian Sun. Faster r-cnn: Towards real-time object detection with region proposal networks. In Advances in neural information processing systems, pages 91-99, 2015.

[111] K. He, X. Zhang, S. Ren, and J. Sun. Spatial Pyramid Pooling in Deep Convolutional Networks for Visual Recognition. ArXiv e-prints, June 2014.

[112] N. Ballas, L. Yao, C. Pal, and A. Courville. Delving Deeper into Convolutional Networks for Learning Video Representations. ArXiv e-prints, November 2015.

[113] M. Mathieu, C. Couprie, and Y. LeCun. Deep multi-scale video prediction beyond mean square error. ArXiv e-prints, November 2015.

[114] Naiyan Wang and Dit-Yan Yeung. Learning a deep compact image representation for visual tracking. In Advances in neural information processing systems, pages 809-817, 2013.

[115] Chao Ma, Jia-Bin Huang, Xiaokang Yang, and Ming-Hsuan Yang. Hierarchical convolutional features for visual tracking. In Proceedings of the IEEE international conference on computer vision, pages 3074-3082, 2015.

[116] H. Nam and B. Han. Learning Multi-Domain Convolutional Neural Networks for Visual Tracking. ArXiv e-prints, October 2015.

[117] Xingyin Fu, Feng Zhu, Qingxiao Wu, Yunlei Sun, Rongrong Lu, and Ruigang Yang. Real-time large-scale dense mapping with surfels. Sensors (Basel, Switzerland), 18(5), 2018.

[118] Eduardo Moscoso Rubino, Diego Centelles, Jorge Sales Gil, Martí Avilés, José Vicente, Raúl Marín Prades, Pedro José Sanz Valero, and Alberto J Álvares. Progressive image compression and transmission with region of interest in underwater robotics. 2017.

[119] Christian Osendorfer, Hubert Soyer, and Patrick Van Der Smagt. Image superresolution with fast approximate convolutional sparse coding. In International Conference on Neural Information Processing, pages 250-257. Springer, 2014.

[120] C. Ledig, L. Theis, F. Huszar, J. Caballero, A. Cunningham, A. Acosta, A. Aitken, A. Tejani, J. Totz, Z. Wang, and W. Shi. Photo-Realistic Single Image SuperResolution Using a Generative Adversarial Network. ArXiv e-prints, September 2016.

[121] J. Johnson, A. Alahi, and L. Fei-Fei. Perceptual Losses for Real-Time Style Transfer and Super-Resolution. ArXiv e-prints, March 2016.

[122] Zhaowen Wang, Ding Liu, Jianchao Yang, Wei Han, and Thomas Huang. Deep networks for image super-resolution with sparse prior. In Proceedings of the IEEE International Conference on Computer Vision, pages 370-378, 2015. 
[123] J. Kim, J. K. Lee, and K. M. Lee. Deeply-Recursive Convolutional Network for Image Super-Resolution. ArXiv e-prints, November 2015.

[124] J. Kim, J. K. Lee, and K. M. Lee. Accurate Image Super-Resolution Using Very Deep Convolutional Networks. ArXiv e-prints, November 2015.

[125] C. Dong, C. Change Loy, K. He, and X. Tang. Image Super-Resolution Using Deep Convolutional Networks. ArXiv e-prints, December 2015.

[126] K. He, G. Gkioxari, P. Dollár, and R. Girshick. Mask R-CNN. ArXiv e-prints, March 2017.

[127] Alexander Kolesnikov and Christoph H Lampert. Seed, expand and constrain: Three principles for weakly-supervised image segmentation. In European Conference on Computer Vision, pages 695-711. Springer, 2016.

[128] Z. Liu, X. Li, P. Luo, C. Change Loy, and X. Tang. Semantic Image Segmentation via Deep Parsing Network. ArXiv e-prints, September 2015.

[129] J. Long, E. Shelhamer, and T. Darrell. Fully Convolutional Networks for Semantic Segmentation. ArXiv e-prints, November 2014.

[130] Abhishek Sharma, Oncel Tuzel, and David W Jacobs. Deep hierarchical parsing for semantic segmentation. In Proceedings of the IEEE Conference on Computer Vision and Pattern Recognition, pages 530-538, 2015.

[131] V. Badrinarayanan, A. Kendall, and R. Cipolla. SegNet: A Deep Convolutional Encoder-Decoder Architecture for Image Segmentation. ArXiv e-prints, November 2015 .

[132] Hamid Izadinia, Fereshteh Sadeghi, Santosh K Divvala, Hannaneh Hajishirzi, Yejin Choi, and Ali Farhadi. Segment-phrase table for semantic segmentation, visual entailment and paraphrasing. In Proceedings of the IEEE international conference on computer vision, pages 10-18, 2015.

[133] I. Kokkinos. Pushing the Boundaries of Boundary Detection using Deep Learning. ArXiv e-prints, November 2015.

[134] Richard S Sutton and Andrew G Barto. Reinforcement learning: An introduction. MIT press, 2018.

[135] Volodymyr Mnih, Koray Kavukcuoglu, David Silver, Alex Graves, Ioannis Antonoglou, Daan Wierstra, and Martin Riedmiller. Playing atari with deep reinforcement learning. arXiv preprint arXiv:1312.5602, 2013. 
[136] Volodymyr Mnih, Koray Kavukcuoglu, David Silver, Andrei A Rusu, Joel Veness, Marc G Bellemare, Alex Graves, Martin Riedmiller, Andreas K Fidjeland, Georg Ostrovski, et al. Human-level control through deep reinforcement learning. Nature, 518(7540):529, 2015.

[137] David Silver, Julian Schrittwieser, Karen Simonyan, Ioannis Antonoglou, Aja Huang, Arthur Guez, Thomas Hubert, Lucas Baker, Matthew Lai, Adrian Bolton, et al. Mastering the game of go without human knowledge. Nature, 550(7676):354, 2017.

[138] D. Silver, T. Hubert, J. Schrittwieser, I. Antonoglou, M. Lai, A. Guez, M. Lanctot, L. Sifre, D. Kumaran, T. Graepel, T. Lillicrap, K. Simonyan, and D. Hassabis. Mastering Chess and Shogi by Self-Play with a General Reinforcement Learning Algorithm. ArXiv e-prints, December 2017.

[139] Oriol Vinyals, Timo Ewalds, Sergey Bartunov, Petko Georgiev, Alexander Sasha Vezhnevets, Michelle Yeo, Alireza Makhzani, Heinrich Küttler, John Agapiou, Julian Schrittwieser, et al. Starcraft ii: A new challenge for reinforcement learning. arXiv preprint arXiv:1708.04782, 2017.

[140] OpenAI. Openai five. [Online] https://blog.openai.com/openai-five/, 2018.

[141] D. Quillen, E. Jang, O. Nachum, C. Finn, J. Ibarz, and S. Levine. Deep Reinforcement Learning for Vision-Based Robotic Grasping: A Simulated Comparative Evaluation of Off-Policy Methods. ArXiv e-prints, February 2018.

[142] S. Levine, P. Pastor, A. Krizhevsky, and D. Quillen. Learning Hand-Eye Coordination for Robotic Grasping with Deep Learning and Large-Scale Data Collection. ArXiv eprints, March 2016.

[143] A. Rajeswaran, V. Kumar, A. Gupta, G. Vezzani, J. Schulman, E. Todorov, and S. Levine. Learning Complex Dexterous Manipulation with Deep Reinforcement Learning and Demonstrations. ArXiv e-prints, September 2017.

[144] H. Zhu, A. Gupta, A. Rajeswaran, S. Levine, and V. Kumar. Dexterous Manipulation with Deep Reinforcement Learning: Efficient, General, and Low-Cost. ArXiv e-prints, October 2018.

[145] OpenAI, :, M. Andrychowicz, B. Baker, M. Chociej, R. Jozefowicz, B. McGrew, J. Pachocki, A. Petron, M. Plappert, G. Powell, A. Ray, J. Schneider, S. Sidor, J. Tobin, P. Welinder, L. Weng, and W. Zaremba. Learning Dexterous In-Hand Manipulation. ArXiv e-prints, August 2018.

[146] Ben Kehoe, Akihiro Matsukawa, Sal Candido, James Kuffner, and Ken Goldberg. Cloud-based robot grasping with the google object recognition engine. In IEEE Int'l Conf. on Robotics and Automation, page 8, 2013. 
[147] T. Zhang, Z. McCarthy, O. Jow, D. Lee, X. Chen, K. Goldberg, and P. Abbeel. Deep Imitation Learning for Complex Manipulation Tasks from Virtual Reality Teleoperation. ArXiv e-prints, October 2017.

[148] Yan Duan, Marcin Andrychowicz, Bradly Stadie, OpenAI Jonathan Ho, Jonas Schneider, Ilya Sutskever, Pieter Abbeel, and Wojciech Zaremba. One-shot imitation learning. In Advances in neural information processing systems, pages 1087-1098, 2017.

[149] DeepMind. Safety-first ai for autonomous data centre cooling and industrial control. [Online] https://deepmind.com/blog/safety-first-ai-autonomous-data-centrecooling-and-industrial-control/, 2018.

[150] Elvis Saravia and Soujanya Poria. Modern deep learning techniques applied to natural language processing. [Online] https://nlpoverview.com/.

[151] Oriol Vinyals, Łukasz Kaiser, Terry Koo, Slav Petrov, Ilya Sutskever, and Geoffrey Hinton. Grammar as a foreign language. In Advances in Neural Information Processing Systems, pages 2773-2781, 2015.

[152] Hao Zhou, Yue Zhang, Chuan Cheng, Shujian Huang, Xinyu Dai, and Jiajun Chen. A neural probabilistic structured-prediction method for transition-based natural language processing. Journal of Artificial Intelligence Research, 58:703-729, 2017.

[153] C. Dyer, M. Ballesteros, W. Ling, A. Matthews, and N. A. Smith. Transition-Based Dependency Parsing with Stack Long Short-Term Memory. ArXiv e-prints, May 2015.

[154] D. Weiss, C. Alberti, M. Collins, and S. Petrov. Structured Training for Neural Network Transition-Based Parsing. ArXiv e-prints, June 2015.

[155] Danqi Chen and Christopher Manning. A fast and accurate dependency parser using neural networks. In Proceedings of the 2014 conference on empirical methods in natural language processing (EMNLP), pages 740-750, 2014.

[156] D. Andor, C. Alberti, D. Weiss, A. Severyn, A. Presta, K. Ganchev, S. Petrov, and M. Collins. Globally Normalized Transition-Based Neural Networks. ArXiv e-prints, March 2016.

[157] A. Kumar, O. Irsoy, P. Ondruska, M. Iyyer, J. Bradbury, I. Gulrajani, V. Zhong, R. Paulus, and R. Socher. Ask Me Anything: Dynamic Memory Networks for Natural Language Processing. ArXiv e-prints, June 2015.

[158] Z. Huang, W. Xu, and K. Yu. Bidirectional LSTM-CRF Models for Sequence Tagging. ArXiv e-prints, August 2015.

[159] Cicero D Santos and Bianca Zadrozny. Learning character-level representations for part-of-speech tagging. In Proceedings of the 31st International Conference on Machine Learning (ICML-14), pages 1818-1826, 2014. 
[160] A. Vaswani, N. Shazeer, N. Parmar, J. Uszkoreit, L. Jones, A. N. Gomez, L. Kaiser, and I. Polosukhin. Attention Is All You Need. ArXiv e-prints, June 2017.

[161] J. Gehring, M. Auli, D. Grangier, D. Yarats, and Y. N. Dauphin. Convolutional Sequence to Sequence Learning. ArXiv e-prints, May 2017.

[162] Y. Wu, M. Schuster, Z. Chen, Q. V. Le, M. Norouzi, W. Macherey, M. Krikun, Y. Cao, Q. Gao, K. Macherey, J. Klingner, A. Shah, M. Johnson, X. Liu, Ł. Kaiser, S. Gouws, Y. Kato, T. Kudo, H. Kazawa, K. Stevens, G. Kurian, N. Patil, W. Wang, C. Young, J. Smith, J. Riesa, A. Rudnick, O. Vinyals, G. Corrado, M. Hughes, and J. Dean. Google's Neural Machine Translation System: Bridging the Gap between Human and Machine Translation. ArXiv e-prints, September 2016.

[163] Ilya Sutskever, Oriol Vinyals, and Quoc V Le. Sequence to sequence learning with neural networks. In Advances in neural information processing systems, pages 3104$3112,2014$.

[164] Xiangyang Zhou, Daxiang Dong, Hua Wu, Shiqi Zhao, Dianhai Yu, Hao Tian, Xuan Liu, and Rui Yan. Multi-view response selection for human-computer conversation. In Proceedings of the 2016 Conference on Empirical Methods in Natural Language Processing, pages 372-381, 2016.

[165] J. Dodge, A. Gane, X. Zhang, A. Bordes, S. Chopra, A. Miller, A. Szlam, and J. Weston. Evaluating Prerequisite Qualities for Learning End-to-End Dialog Systems. ArXiv e-prints, November 2015.

[166] R. Lowe, N. Pow, I. Serban, and J. Pineau. The Ubuntu Dialogue Corpus: A Large Dataset for Research in Unstructured Multi-Turn Dialogue Systems. ArXiv e-prints, June 2015.

[167] J. Li, M. Galley, C. Brockett, J. Gao, and B. Dolan. A Diversity-Promoting Objective Function for Neural Conversation Models. ArXiv e-prints, October 2015.

[168] Claudio Melchiorri. Robotic telemanipulation systems: An overview on control aspects. In Proceedings of the 7th IFAC Symposium on Robot Control, volume 1, pages 707-716, 2003.

[169] Suseong Kim, Seungwon Choi, and H Jin Kim. Aerial manipulation using a quadrotor with a two dof robotic arm. In Intelligent Robots and Systems (IROS), 2013 IEEE/RSJ International Conference on, pages 4990-4995. IEEE, 2013.

[170] Carmine Dario Bellicoso, Luca Rosario Buonocore, Vincenzo Lippiello, and Bruno Siciliano. Design, modeling and control of a 5-dof light-weight robot arm for aerial manipulation. In Control and Automation (MED), 2015 23th Mediterranean Conference on, pages 853-858. IEEE, 2015. 
[171] Nathan Michael, Jonathan Fink, and Vijay Kumar. Cooperative manipulation and transportation with aerial robots. Autonomous Robots, 30(1):73-86, 2011.

[172] Pedro J Sanz, Pere Ridao, Gabriel Oliver, Giuseppe Casalino, Carlos Insaurralde, Carlos Silvestre, Claudio Melchiorri, and Alessio Turetta. Trident: Recent improvements about autonomous underwater intervention missions. IFAC Proceedings Volumes, 45(5):355-360, 2012.

[173] Patryk Cieslak, Pere Ridao, and Mariusz Giergiel. Autonomous underwater panel operation by girona500 uvms: A practical approach to autonomous underwater manipulation. In Robotics and Automation (ICRA), 2015 IEEE International Conference on, pages 529-536. IEEE, 2015.

[174] Giacomo Marani, Song K Choi, and Junku Yuh. Underwater autonomous manipulation for intervention missions auvs. Ocean Engineering, 36(1):15-23, 2009.

[175] Enrico Simetti, Giuseppe Casalino, Sandro Torelli, Alessandro Sperinde, and Alessio Turetta. Floating underwater manipulation: Developed control methodology and experimental validation within the trident project. Journal of Field Robotics, 31(3):364$385,2014$.

[176] Oussama Khatib, Xiyang Yeh, Gerald Brantner, Brian Soe, Boyeon Kim, Shameek Ganguly, Hannah Stuart, Shiquan Wang, Mark Cutkosky, Aaron Edsinger, et al. Ocean one: A robotic avatar for oceanic discovery. IEEE Robotics $\mathcal{E}$ Automation Magazine, 23(4):20-29, 2016.

[177] Eduardo M Rubino, Alberto J Álvares, Raúl Marín, and Pedro J Sanz. Real-time rate distortion-optimized image compression with region of interest on the arm architecture for underwater robotics applications. Journal of Real-Time Image Processing, pages $1-33,2018$.

[178] Katherine J Kuchenbecker, Jamie Gewirtz, William McMahan, Dorsey Standish, Paul Martin, Jonathan Bohren, Pierre J Mendoza, and David I Lee. Verrotouch: Highfrequency acceleration feedback for telerobotic surgery. In International Conference on Human Haptic Sensing and Touch Enabled Computer Applications, pages 189-196. Springer, 2010.

[179] Alejandro Jarillo Silva, Omar A. Domínguez Ramirez, Vicente Parra Vega, and Jesus P. Ordaz Oliver. PHANToM OMNI Haptic Device: Kinematic and Manipulability. In 2009 Electronics, Robotics and Automotive Mechanics Conference (CERMA), pages 193-198. IEEE, 92009.

[180] T. Hulin. A practically linear relation between time delay and the optimal settling time of a haptic device. IEEE Robotics and Automation Letters, 2(3):1632-1639, July 2017. 
[181] Sandra Hirche, Andrea Bauer, and Martin Buss. Transparency of haptic telepresence systems with constant time delay. In Control Applications, 2005. CCA 2005. Proceedings of 2005 IEEE Conference on, pages 328-333. IEEE, 2005.

[182] Markus Rank, Zhuanghua Shi, Hermann J. Müller, and Sandra Hirche. Perception of delay in haptic telepresence systems. Presence: teleoperators and virtual environments, 19(5):389-399, 2010.

[183] Rajinder Sodhi, Ivan Poupyrev, Matthew Glisson, and Ali Israr. AIREAL. ACM Transactions on Graphics, 32(4):1, 72013.

[184] Tom Carter, Sue Ann Seah, Benjamin Long, Bruce Drinkwater, and Sriram Subramanian. UltraHaptics: Multi-Point Mid-Air Haptic Feedback for Touch Surfaces.

[185] M Di Castro, C Veiga Almagro, G Lunghi, R Marin, M Ferre, and A Masi. Trackingbased depth estimation of metallic pieces for robotic guidance. In 2018 IEEE/RSJ International Conference on Intelligent Robots and Systems (IROS), pages 5503-5508. IEEE, 2018.

[186] Giacomo Lunghi, Raul Marin Prades, and Mario Di Castro. An advanced, adaptive and multimodal graphical user interface for human-robot teleoperation in radioactive scenarios. In Proceedings of the 13th International Conference on Informatics in Control, Automation and Robotics, pages 224-231. SCITEPRESS-Science and Technology Publications, Lda, 2016.

[187] Jens Rasmussen. Human errors. A taxonomy for describing human malfunction in industrial installations. Journal of Occupational Accidents, 4(2-4):311-333, 91982.

[188] J. T. Reason. Human error. Cambridge University Press, 1990.

[189] Alan Baddeley. Working memory and conscious awareness. In Theories of Memory, pages 11-28. Lawrence Erlbaum Associates, 1993.

[190] P. Lin, K. Abney, and G. Bekey. Robot Ethics: The Ethical and Social Implications of Robotics. MIT press, 2012.

[191] M. K. Habib, Y. Baudoin, and F. Nagata. Robotics for rescue and risky intervention. In IECON 2011 - 37th Annual Conference of the IEEE Industrial Electronics Society, pages 3305-3310, Nov 2011.

[192] C. Wong, E. Yang, X. Yan, and D. Gu. An overview of robotics and autonomous systems for harsh environments. In 2017 23rd International Conference on Automation and Computing (ICAC), pages 1-6, Sept 2017.

[193] Puck Rombach, Mason A Porter, James H Fowler, and Peter J Mucha. Core-periphery structure in networks (revisited). SIAM Review, 59(3):619-646, 2017. 
[194] M Di Castro, A Masi, G Lunghi, and R Losito. An incremental slam algorithm for indoor autonomous navigation.

[195] Mario Di Castro, David Blanco Mulero, Manuel Ferre, and Alessandro Masi. A realtime reconfigurable collision avoidance system for robot manipulation. In Proceedings of the 3rd International Conference on Mechatronics and Robotics Engineering, pages 6-10. ACM, 2017.

[196] Mario Di Castro, Jorge Camarero Vera, Alessandro Masi, and Manuel Ferre. Novel pose estimation system for precise robotic manipulation in unstructured environment. In ICINCO (2), pages 50-55, 2017.

[197] Morgan Quigley, Ken Conley, Brian Gerkey, Josh Faust, Tully Foote, Jeremy Leibs, Rob Wheeler, and Andrew Y Ng. Ros: an open-source robot operating system. In ICRA workshop on open source software, volume 3, page 5. Kobe, Japan, 2009.

[198] Sebastian Thrun, Wolfram Burgard, and Dieter Fox. Probabilistic robotics. MIT press, 2005.

[199] MWM Gamini Dissanayake, Paul Newman, Steve Clark, Hugh F Durrant-Whyte, and Michael Csorba. A solution to the simultaneous localization and map building (slam) problem. IEEE Transactions on robotics and automation, 17(3):229-241, 2001.

[200] Y. Wu, B. Zhang, S. Yang, X. Yi, and X. Yang. Energy-efficient joint communicationmotion planning for relay-assisted wireless robot surveillance. In IEEE INFOCOM 2017 - IEEE Conference on Computer Communications, pages 1-9, May 2017.

[201] J. Jerald, P. Giokaris, D. Woodall, A. Hartbolt, A. Chandak, and S. Kuntz. Developing virtual reality applications with unity. In 2014 IEEE Virtual Reality (VR), pages 1-3, March 2014.

[202] Jeff W Murray. Building Virtual Reality with Unity and Steam VR. AK Peters/CRC Press, 2017.

[203] Lentin Joseph. Mastering ROS for robotics programming. Packt Publishing Ltd, 2015.

[204] Onur Özyeşil, Vladislav Voroninski, Ronen Basri, and Amit Singer. A survey of structure from motion*. Acta Numerica, 26:305-364, 2017.

[205] Michael L Dingus, Walter P Zoch, Thomas R Mayfield, Alan Bray, and Rock A Rushing. Methods and compositions for cleaning and decontamination, September 23 1997. US Patent 5,670,469.

[206] KF Langley and J Williams. Decontamination and waste minimisation techniques in nuclear decommissioning. Nuclear Energy, 40(03):189-195, 2001. 
[207] Andrew Hostler, Bridget Benson, and Joshua Warner. Budgetrov: An ultra low cost robotics platform for education and research. In OCEANS 2017-Aberdeen, pages 1-4. IEEE, 2017.

[208] GJ Butterworth. Low activation structural materials for fusion. Fusion Engineering and Design, 11(1-2):231-244, 1989.

[209] Cor Claeys and Eddy Simoen. Radiation effects in advanced semiconductor materials and devices, volume 57. Springer Science \& Business Media, 2013.

[210] Giorgio Apollinari, O Brüning, Tatsushi Nakamoto, and Lucio Rossi. High luminosity large hadron collider hl-lhc. arXiv preprint arXiv:1705.08830, 2017.

[211] Wen-Yeau Chang. The state of charge estimating methods for battery: A review. ISRN Applied Mathematics, 2013, 2013.

[212] Apostolos Kottas, Andrew Drenner, and Nikolaos Papanikolopoulos. Intelligent power management: Promoting power-consciousness in teams of mobile robots. In Robotics and Automation, 2009. ICRA'09. IEEE International Conference on, pages 11401145. IEEE, 2009.

[213] Bruno Belvedere, Michele Bianchi, Alberto Borghetti, Carlo Alberto Nucci, Mario Paolone, and Antonio Peretto. A microcontroller-based power management system for standalone microgrids with hybrid power supply. IEEE Transactions on Sustainable Energy, 3(3):422-431, 2012.

[214] Matthew G Earl and Raffaello D'andrea. Iterative milp methods for vehicle-control problems. IEEE Transactions on Robotics, 21(6):1158-1167, 2005.

[215] Yongguo Mei, Yung-Hsiang Lu, Y Charlie Hu, and CS George Lee. Deployment strategy for mobile robots with energy and timing constraints. In Robotics and Automation, 2005. ICRA 2005. Proceedings of the 2005 IEEE International Conference on, pages 2816-2821. IEEE, 2005.

[216] A Barili, M Ceresa, and C Parisi. Energy-saving motion control for an autonomous mobile robot. In Industrial Electronics, 1995. ISIE'95., Proceedings of the IEEE International Symposium on, volume 2, pages 674-676. IEEE, 1995.

[217] Hongjun Kim and Byung Kook Kim. Online minimum-energy trajectory planning and control on a straight-line path for three-wheeled omnidirectional mobile robots. IEEE Trans. Industrial Electronics, 61(9):4771-4779, 2014.

[218] Zhongbei Tian, Paul Weston, Stuart Hillmansen, Clive Roberts, and Ning Zhao. System energy optimisation of metro-transit system using monte carlo algorithm. In Intelligent Rail Transportation (ICIRT), 2016 IEEE International Conference on, pages 453-459. IEEE, 2016. 
[219] Oskar Von Stryk and Roland Bulirsch. Direct and indirect methods for trajectory optimization. Annals of operations research, 37(1):357-373, 1992.

[220] Misel Brezak and Ivan Petrovic. Time-optimal trajectory planning along predefined path for mobile robots with velocity and acceleration constraints. In Advanced Intelligent Mechatronics (AIM), 2011 IEEE/ASME International Conference on, pages 942-947. IEEE, 2011.

[221] Peter Iring and Peter Schreiber. Use of sigmoid functions in genetic algorithms for velocity profile optimization of mobile robot. In Intelligent Engineering Systems (INES), 2015 IEEE 19th International Conference on, pages 201-206. IEEE, 2015.

[222] Antti Lajunen. Energy-optimal velocity profiles for electric city buses. In Automation Science and Engineering (CASE), 2013 IEEE International Conference on, pages 886891. IEEE, 2013.

[223] Zhu Yu, Chen Han, and Mu Haihua. A novel approach of tuning trapezoidal velocity profile for energy saving in servomotor systems. In Control Conference (CCC), 2015 34th Chinese, pages 4412-4417. IEEE, 2015.

[224] Pratap Tokekar, Nikhil Karnad, and Volkan Isler. Energy-optimal trajectory planning for car-like robots. Autonomous Robots, 37(3):279-300, 2014.

[225] Yebin Wang, Yiming Zhao, Scott A Bortoff, and Koichiro Ueda. A real-time energyoptimal trajectory generation method for a servomotor system. IEEE Transactions on Industrial Electronics, 62(2):1175-1188, 2015.

[226] Michele Pirovano. Kinfu - an open source implementation of kinect fusion. 2012.

[227] Otmar Hilliges David Molyneaux Richard Newcombe Pushmeet Kohli Jamie Shotton Steve Hodges Dustin Freeman Andrew Davison Andrew Fitzgibbon Shahram Izadi, David Kim. Kinectfusion: Real-time 3d reconstruction and interaction using a moving depth camera. pages 559-568. ACM, October 2011.

[228] Thomas Whelan, Renato F Salas-Moreno, Ben Glocker, Andrew J Davison, and Stefan Leutenegger. Elasticfusion: Real-time dense slam and light source estimation. The International Journal of Robotics Research, 35(14):1697-1716, 2016.

[229] Stefan Hinterstoisser, Stefan Holzer, Cedric Cagniart, Slobodan Ilic, Kurt Konolige, Nassir Navab, and Vincent Lepetit. Multimodal templates for real-time detection of texture-less objects in heavily cluttered scenes. In Computer Vision (ICCV), 2011 IEEE International Conference on, pages 858-865. IEEE, 2011.

[230] Victor A Prisacariu and Ian D Reid. Pwp3d: Real-time segmentation and tracking of 3d objects. International journal of computer vision, 98(3):335-354, 2012. 
[231] Shuran Song and Jianxiong Xiao. Sliding shapes for 3d object detection in depth images. In European conference on computer vision, pages 634-651. Springer, 2014.

[232] Andy Zeng, Kuan-Ting Yu, Shuran Song, Daniel Suo, Ed Walker, Alberto Rodriguez, and Jianxiong Xiao. Multi-view self-supervised deep learning for $6 \mathrm{~d}$ pose estimation in the amazon picking challenge. In Robotics and Automation (ICRA), 2017 IEEE International Conference on, pages 1386-1383. IEEE, 2017.

[233] Shuran Song and Jianxiong Xiao. Deep sliding shapes for amodal 3d object detection in rgb-d images. In Proceedings of the IEEE Conference on Computer Vision and Pattern Recognition, pages 808-816, 2016.

[234] Mario Di Castro, Jorge Camarero Vera, Alessandro Masi, and Manuel Ferre. Object detection and $6 \mathrm{~d}$ pose estimation for precise robotic manipulation in unstructured environments. In Lecture Notes in Electrical Engineering, Springer, Volume 495, Chapter 20, 2018.

[235] Tsung-Yi Lin, Michael Maire, Serge Belongie, James Hays, Pietro Perona, Deva Ramanan, Piotr Dollár, and C Lawrence Zitnick. Microsoft coco: Common objects in context. In European conference on computer vision, pages 740-755. Springer, 2014.

[236] S. Tadokoro R. R. Murphy and A. Kleiner. Disaster Robotics. Springer, 2016.

[237] Mykhaylo Andriluka, Paul Schnitzspan, Johannes Meyer, Stefan Kohlbrecher, Karen Petersen, Oskar Von Stryk, Stefan Roth, and Bernt Schiele. Vision based victim detection from unmanned aerial vehicles. IEEE/RSJ 2010 International Conference on Intelligent Robots and Systems, IROS 2010 - Conference Proceedings, pages 1740$1747,2010$.

[238] N. Dalal and B. Triggs. Histograms of oriented gradients for human detection. In 2005 IEEE Computer Society Conference on Computer Vision and Pattern Recognition (CVPR'05), volume 1, pages 886-893 vol. 1, June 2005.

[239] Paul Viola and Michael Jones. Robust real-time face detection. 57:747, 012001.

[240] Alexander Meisel. Feature based Sensor Fusion for Victim Detection in the Rescue Robotics Domain. (May), 2014.

[241] R. P. Saputra and P. Kormushev. Casualty detection from 3d point cloud data for autonomous ground mobile rescue robots. In 2018 IEEE International Symposium on Safety, Security, and Rescue Robotics (SSRR), pages 1-7, Aug 2018.

[242] C. Castillo and C. Chang. A method to detect victims in search and rescue operations using template matching. In IEEE International Safety, Security and Rescue Rototics, Workshop, 2005., pages 201-206, June 2005. 
[243] Alex Krizhevsky, I Sutskever, and G E Hinton. ImageNet Classification with Deep Convolutional Neural Networks. In F Pereira, C J C Burges, L Bottou, and K Q Weinberger, editors, Advances in Neural Information Processing Systems (NIPS 2012), pages 1097-1105. Curran Associates, Inc., 2012.

[244] G. L. Oliveira, A. Valada, C. Bollen, W. Burgard, and T. Brox. Deep learning for human part discovery in images. In 2016 IEEE International Conference on Robotics and Automation (ICRA), pages 1634-1641, May 2016.

[245] C. H. Setjo, B. Achmad, and Faridah. Thermal image human detection using haarcascade classifier. In 2017 7th International Annual Engineering Seminar (InAES), pages 1-6, Aug 2017.

[246] Wei Li, Dequan Zheng, Tiejun Zhao, and Mengda Yang. An effective approach to pedestrian detection in thermal imagery. Proceedings - International Conference on Natural Computation, (Icnc):325-329, 2012.

[247] Vijay John, Seiichi Mita, Zheng Liu, and Bin Qi. Pedestrian detection in thermal images using adaptive fuzzy C-means clustering and convolutional neural networks. 2015 14th IAPR International Conference on Machine Vision Applications (MVA), pages 246-249, 2015.

[248] S. Kim, S. Jun, and J. Park. Thermal stereo system for visible range extension of disaster robot. In 2018 IEEE International Symposium on Safety, Security, and Rescue Robotics (SSRR), pages 1-2, Aug 2018.

[249] C Pereira, X Yu, T Goos, I Reiss, T Orlikowsky, K Heimann, B Venema, V Blazek, S Leonhardt, and D Teichmann. Noncontact Monitoring of Respiratory Rate in Newborn Infants Using Thermal Imaging. IEEE Trans Biomed Eng, PP(XX):1, 2018.

[250] A. Kleiner and R. Kummerle. Genetic mrf model optimization for real-time victim detection in search and rescue. In 2007 IEEE/RSJ International Conference on Intelligent Robots and Systems, pages 3025-3030, Oct 2007.

[251] Dušan Kocur, Peter Kažimír, Jana Fortes, Daniel Novák, Miloš Drutarovský, Pavol Galajda, and Rudolf Zetik. Short-range UWB radar: Surveillance robot equipment of the future. Conference Proceedings - IEEE International Conference on Systems, Man and Cybernetics, 2014-Janua(January):3767-3772, 2014.

[252] R. Salman and I. Willms. A mobile security robot equipped with uwb-radar for super-resolution indoor positioning and localisation applications. In 2012 International Conference on Indoor Positioning and Indoor Navigation (IPIN), pages 1-8, Nov 2012.

[253] Amer Nezirovic, Alexander G. Yarovoy, and Leo P. Ligthart. Experimental study on human being detection using UWB radar. 2006 International Radar Symposium, 2006 . 
[254] Changzhi Li, Yanming Xiao, and Jenshan Lin. Experiment and spectral analysis of a low-power $\mathrm{K}$ a-band heartbeat detector measuring from four sides of a human body. IEEE Transactions on Microwave Theory and Techniques, 54(12):4464-4471, 2006.

[255] Jeffrey A. Nanzer. A Review of Microwave Wireless Techniques for Human Presence Detection and Classification. IEEE Transactions on Microwave Theory and Techniques, 65(5):1780-1794, 2017.

[256] Kun Mu Chen, Devendra Misra, Huei Wang, Huey Ru Chuang, and Elliot Postow. An X-Band Microwave Life-Detection System. IEEE Transactions on Biomedical Engineering, BME-33(7):697-701, 1986.

[257] Victor C. Chen, Fayin Li, Shen Shyang Ho, and Harry Wechsler. Micro-doppler effect in radar: Phenomenon, model, and simulation study. IEEE Transactions on Aerospace and Electronic Systems, 42(1):2-21, 2006.

[258] Time-frequency Spectrogram Ground, Lan Du, Linsen Li, Baoshuai Wang, and Jinguo Xiao. Micro-Doppler Feature Extraction Based on Low-Resolution Radar. Ieee Sensors Journal, 16(10):3756-3763, 2016.

[259] Olee Hoi Ying Lam, Reinhard Kulke, Manfred Hagelen, and Gregor Mollenbeck. Classification of moving targets using mirco-Doppler radar. Proceedings International Radar Symposium, 2016-June:0-5, 2016.

[260] Youngwook Kim, Senior Member, and Taesup Moon. Human Detection and Activity Classification Based on Micro-Doppler Signatures Using Deep Convolutional Neural Networks. Ieee Geoscience and Remote Sensing Letters, 13(1):1-5, 2015.

[261] R. Pérez, F. Schubert, R. Rasshofer, and E. Biebl. Single-frame vulnerable road users classification with a $77 \mathrm{ghz}$ fmcw radar sensor and a convolutional neural network. In 2018 19th International Radar Symposium (IRS), pages 1-10, June 2018.

[262] C. Brüser, C. H. Antink, T. Wartzek, M. Walter, and S. Leonhardt. Ambient and unobtrusive cardiorespiratory monitoring techniques. IEEE Reviews in Biomedical Engineering, 8:30-43, 2015.

[263] X4m200 respiration sensor description.

[264] Andrew G. Howard, Menglong Zhu, Bo Chen, Dmitry Kalenichenko, Weijun Wang, Tobias Weyand, Marco Andreetto, and Hartwig Adam. MobileNets: Efficient Convolutional Neural Networks for Mobile Vision Applications. 2017.

[265] Mobilenet github repository.

[266] Imagenet large scale visual recognition challenge 2012. 
[267] Xinxin Du, Marcelo H Ang, and Daniela Rus. Car detection for autonomous vehicle: Lidar and vision fusion approach through deep learning framework. In Intelligent Robots and Systems (IROS), 2017 IEEE/RSJ International Conference on, pages 749754. IEEE, 2017.

[268] Constantine P Papageorgiou, Michael Oren, and Tomaso Poggio. A general framework for object detection. In Computer vision, 1998. sixth international conference on, pages 555-562. IEEE, 1998.

[269] Paul Viola and Michael Jones. Rapid object detection using a boosted cascade of simple features. In Computer Vision and Pattern Recognition, 2001. CVPR 2001. Proceedings of the 2001 IEEE Computer Society Conference on, volume 1, pages I-I. IEEE, 2001.

[270] Christopher H Messom and Andre L Barczak. Stream processing for fast and efficient rotated haar-like features using rotated integral images. International journal of intelligent systems technologies and applications, 7(1):40-57, 2009.

[271] William T Freeman and Michal Roth. Orientation histograms for hand gesture recognition. In International workshop on automatic face and gesture recognition, volume 12, pages 296-301, 1995.

[272] Navteen Dalal and Bill Triggs. Object detection using histograms of oriented gradients. In Pascal VOC Workshop, ECCV, 2006.

[273] João F Henriques, Rui Caseiro, Pedro Martins, and Jorge Batista. High-speed tracking with kernelized correlation filters. IEEE Transactions on Pattern Analysis and Machine Intelligence, 37(3):583-596, 2015.

[274] Tae-Kyun Kim, Thomas Woodley, Bjorn Stenger, Björn Stenger, and Roberto Cipolla. Online multiple classifier boosting for object tracking. In Computer Vision and Pattern Recognition Workshops (CVPRW), 2010 IEEE Computer Society Conference on, pages 1-6. IEEE, 2010.

[275] Thomas G Dietterich, Richard H Lathrop, and Tomás Lozano-Pérez. Solving the multiple instance problem with axis-parallel rectangles. Artificial intelligence, 89(12):31-71, 1997.

[276] Zdenek Kalal, Jiri Matas, and Krystian Mikolajczyk. Online learning of robust object detectors during unstable tracking. In Computer Vision Workshops (ICCV Workshops), 2009 IEEE 12th International Conference on, pages 1417-1424. IEEE, 2009.

[277] Per-Erik Danielsson. Euclidean distance mapping. Computer Graphics and image processing, 14(3):227-248, 1980.

[278] H Christopher Longuet-Higgins. A computer algorithm for reconstructing a scene from two projections. Nature, 293(5828):133, 1981. 
[279] Herbert Bay, Andreas Ess, Tinne Tuytelaars, and Luc Van Gool. Speeded-up robust features (surf). Computer vision and image understanding, 110(3):346-359, 2008.

[280] Shaoqing Ren, Kaiming He, Ross Girshick, and Jian Sun. Faster r-cnn: towards realtime object detection with region proposal networks. IEEE transactions on pattern analysis and machine intelligence, 39(6):1137-1149, 2017.

[281] Kaiming He, Xiangyu Zhang, Shaoqing Ren, and Jian Sun. Deep residual learning for image recognition. arXiv preprint arXiv:1512.03385, 2015.

[282] Christian Szegedy, Vincent Vanhoucke, Sergey Ioffe, Jonathon Shlens, and Zbigniew Wojna. Rethinking the Inception Architecture for Computer Vision. 2015.

[283] D. M. Ebert and D. D. Henrich. Safe human-robot-cooperation: image-based collision detection for industrial robots. In IEEE/RSJ International Conference on Intelligent Robots and Systems, volume 2, pages 1826-1831 vol.2, Sept 2002.

[284] D. Gandhi and E. Cervera. Sensor covering of a robot arm for collision avoidance. In SMC'03 Conference Proceedings. 2003 IEEE International Conference on Systems, Man and Cybernetics. Conference Theme - System Security and Assurance (Cat. No.03CH37483), volume 5, pages 4951-4955 vol.5, Oct 2003.

[285] A. D. Luca, A. Albu-Schaffer, S. Haddadin, and G. Hirzinger. Collision detection and safe reaction with the dlr-iii lightweight manipulator arm. In 2006 IEEE/RSJ International Conference on Intelligent Robots and Systems, pages 1623-1630, Oct 2006.

[286] Y. Chua, K. P. Tee, and R. Yan. Robust optimal inverse kinematics with self-collision avoidance for a humanoid robot. In 2013 IEEE RO-MAN, pages 496-502, Aug 2013.

[287] K. Steinbach, J. Kuffner, T. Asfour, and R. Dillmann. Efficient collision and selfcollision detection for humanoids based on sphere trees hierarchies. In 2006 6th IEEERAS International Conference on Humanoid Robots, pages 560-566, Dec 2006.

[288] F. Seto, K. Kosuge, and Y. Hirata. Self-collision avoidance motion control for human robot cooperation system using robe. In 2005 IEEE/RSJ International Conference on Intelligent Robots and Systems, pages 3143-3148, Aug 2005.

[289] J. Pan, S. Chitta, and D. Manocha. Fcl: A general purpose library for collision and proximity queries. In 2012 IEEE International Conference on Robotics and Automation, pages 3859-3866, May 2012.

[290] CERN. LHC Design Report Volume II Chapter 3, 2004. Online; accessed January 2018.

[291] CERN. Safety Code F, Protection against Ionizing Radiation, Radiation Safety Manual, 1996. Online; accessed January 2018. 
[292] Leanne Attard, Carl James Debono, Gianluca Valentino, and Mario Di Castro. Visionbased change detection for inspection of tunnel liners. Automation in Construction, 91:142-154, 2018.

[293] M. Ukai. Advanced inspection system of tunnel wall deformation using image processing. Quarterly report of railway technical research institute (rtri), 2007.

[294] S. Stent, C. Girerd, P. Long, and R. Cipolla. A low-cost robotic system for the efficient visual inspection of tunnels. In Proceedings of the 32nd International Symposium on Automation and Robotics in Construction 83 Mining (ISARC), Oulu, Finland, 2015.

[295] S. N. Yu, J. H. Jang, and C. S. Han. Auto inspection system using a mobile robot for detecting concrete cracks in a tunnel. Automation in Construction, 16:255-261, 2007.

[296] W. Zhang, Z. Zhang, D. Qi, and Y. Liu. Automatic crack detection and classification method for subway tunnel safety monitoring. Sensors, 2014.

[297] H. Huang, Y. Sun, Y. Xue, and F. Wang. Inspection equipment study for subway tunnel defects by grey-scale image processing. Advanced Engineering Informatics, 32:188-201, 2017.

[298] C. Balaguer, R. Montero, JG. Victores, S. Martínez, and A. Jardon. Towards fully automated tunnel inspection: A survey and future trends. In Proceedings of the 31st International Symposium on Automation and Robotics in Construction \& Mining (ISARC), Sydney, Australia, 2014.

[299] S. Y. Lee, S. H. Lee, D. I. Shin, Y. K. Son, and S. Han, C. Development of an inspection system for cracks in a concrete tunnel lining. Canadian Journal of Civil Engineering, 34:966-975, 2007.

[300] B. Ko, Y. K. Son, D. Shin, and C. S. Han. Development of an inspection system for cracks on the lining of concrete tunnels. In Proceedings of the 20th International Symposium on Automation and Robotics in Construction 83 Mining (ISARC), pages 457-463, Eindhoven, Holland, 2003.

[301] P. Hansen, H. Alismail, P. Rander, and B. Browning. Visual Mapping for Natural Gas Pipe Inspection. International Journal of Robotics Research, 34:532-558, 2015.

[302] C. H. Lee, Y. C. Chiu, T. T. Wang, and T. H. Huang. Application and validation of simple image-mosaic technology for interpreting cracks on tunnel lining. Tunnelling and Underground Space Technology, 34:61-72, 2013.

[303] S. Stent, R. Gherardi, B. Stenger, K. Soga, and R. Cipolla. Visual change detection on tunnel linings. Machine Vision and Applications, 27:319-330, 2016. 
[304] M. Gavilan, F. Sanchez, J. A. Ramos, and O. Marcos. Mobile inspection system for high resolution assessment of tunnels. In Proceedings of the 6th International Conference on Structural Health Monitoring of Intelligent Infrastructure, Hong Kong, China, 2013. Online; accessed January 2018.

[305] S. Pravenaa and R. Menaka. A methodical review on image stitching and video stitching techniques. International Journal of Applied Engineering Research, 11:34423448, 2016. Online; accessed January 2018.

[306] S. Arya. A review on image stitching and its different methods. International Journal of Advanced Research in Computer Science and Software Engineering, 5:299-303, 2015. Online; accessed January 2018.

[307] K. Shashank, N. S. Chatianya, G. Manikanta, C. Balaji, and V. Murthy. A survey and review over image alignment and stitching methods. International Journal of Electronics \& Communication Technology (IJECT), 5:50-52, 2014. Online; accessed January 2018.

[308] D. Ghosh and N. Kaabouch. A survey on image mosaicing techniques. Journal of Visual Communication and Image Representation, 34:1-11, 2016.

[309] D. Vaghela and K. Naina. A review of image mosaicing techniques. International Journal of Advanced Research in Computer and Management Studies, 2:431-437, 2014. Online; accessed January 2018.

[310] B. Zitova, J. Flusser, and F. Sroubek. Image registration: A survey and recent advances. In Proceedings of 12th IEEE International Conference on Image Processing, Genova, Italy, 2005. Online; accessed January 2018.

[311] L. G. Brown. A survey of image registration techniques. ACM Computing Surveys, 24:325-376, 1992.

[312] Z. Xiong and Y. Zhang. A critical review of image registration methods. International Journal of Image and Data Fusion, 1:137-158, 2010.

[313] R. Szeliski. Image alignment and stitching: A tutorial, 2004. Online; accessed January 2018.

[314] M. Brown and D. G. Lowe. Automatic panoramaic image stitching using invariant features. International Journal of Computer Vision, 74:59-73, 2007.

[315] V. Rankov, R. J. Locke, R. J. Edens, P. R. Barber, and B. Vojnovic. An algorithm for image stitching and blending. In Proceedings of Conference of SPIE - the international society for optics and photonics, volume 5701, 2005.

[316] P. Burt and E. Adelson. A multiresolution spline with application to image mosaics. ACM Transactions on Graphics, 2:217-236, 1983. 
[317] D. Lu, P. Mausel, E. Brondizio, and Moran.E. Change detection techniques. International Journal of Remote Sensing, 25:2365-2407, 2004.

[318] S. Minu and A. Sheetya. A comparative study of image change detection algorithms in matlab. In Proceedings of the International Conference on Water Resources, Coastal and Ocean Engineering (ICWRCOE), pages 1366-1373, Mangalore, India, 2015.

[319] Brian Paden, Michal Č́p, Sze Zheng Yong, Dmitry Yershov, and Emilio Frazzoli. A survey of motion planning and control techniques for self-driving urban vehicles. IEEE Transactions on intelligent vehicles, 1(1):33-55, 2016.

[320] Tim Bailey and Hugh Durrant-Whyte. Simultaneous localization and mapping (slam): Part ii. IEEE Robotics \&5 Automation Magazine, 13(3):108-117, 2006.

[321] Stefan Kohlbrecher, Oskar Von Stryk, Johannes Meyer, and Uwe Klingauf. A flexible and scalable slam system with full 3d motion estimation. In Safety, Security, and Rescue Robotics (SSRR), 2011 IEEE International Symposium on, pages 155-160. IEEE, 2011.

[322] Armin Hornung, Kai M Wurm, Maren Bennewitz, Cyrill Stachniss, and Wolfram Burgard. Octomap: An efficient probabilistic 3d mapping framework based on octrees. Autonomous Robots, 34(3):189-206, 2013.

[323] Steven M LaValle. Rapidly-exploring random trees: A new tool for path planning. 1998.

[324] Dimitri P Bertsekas. Nonlinear programming. Athena scientific Belmont, 1999.

[325] Jakob Engel, Vladlen Koltun, and Daniel Cremers. Direct sparse odometry. IEEE transactions on pattern analysis and machine intelligence, 40(3):611-625, 2018.

[326] Pratik Agarwal, Wolfram Burgard, and Luciano Spinello. Metric localization using google street view. In Intelligent Robots and Systems (IROS), 2015 IEEE/RSJ International Conference on, pages 3111-3118. IEEE, 2015.

[327] Ji Zhang and Sanjiv Singh. Loam: Lidar odometry and mapping in real-time. In Robotics: Science and Systems, volume 2, page 9, 2014.

[328] David Droeschel, Jörg Stückler, and Sven Behnke. Local multi-resolution surfel grids for mav motion estimation and 3d mapping. In Intelligent Autonomous Systems 13, pages 429-442. Springer, 2016.

[329] Chi Hay Tong, Sean Anderson, Hang Dong, and Timothy D. Barfoot. Pose interpolation for laser-based visual odometry. Journal of Field Robotics, 31(5):731-757, 2014. 
[330] Robert Zlot and Michael Bosse. Efficient large-scale three-dimensional mobile mapping for underground mines. Journal of Field Robotics, 31(5):758-779, 2014.

[331] Oleksandr Zadorozhnyi, Gunthard Benecke, Stephan Mandt, Tobias Scheffer, and Marius Kloft. Huber-norm regularization for linear prediction models. In Joint European Conference on Machine Learning and Knowledge Discovery in Databases, pages 714-730. Springer, 2016.

[332] K. Nagatani, S. Kiribayashi, Y. Okada, S. Tadokoro, T. Nishimura, T. Yoshida, E. Koyanagi, and Y. Hada. Redesign of rescue mobile robot quince. In 2011 IEEE International Symposium on Safety, Security, and Rescue Robotics, pages 13-18, Nov 2011.

[333] R. H. André, M. Martucci, C. G. V. Thomas, M. A. Silvio, and C. V. L. José. Wireless image transmission in electric power hostile environment. In IEEE Local Computer Network Conference, pages 760-763, Oct 2010.

[334] R. Recanatini, F. Moglie, and V. Mariani Primiani. Performance and immunity evaluation of complete wlan systems in a large reverberation chamber. IEEE Transactions on Electromagnetic Compatibility, 55(5):806-815, Oct 2013.

[335] A. Kadri. Performance of ieee 802.15.4-based wireless sensors in harsh environments. In 2012 8th International Wireless Communications and Mobile Computing Conference (IWCMC), pages 526-530, Aug 2012.

[336] N. Bezzo and R. Fierro. Tethering of mobile router networks. In Proceedings of the 2010 American Control Conference, pages 6828-6833, June 2010.

[337] S. Yarkan, S. Guzelgoz, H. Arslan, and R. R. Murphy. Underground mine communications: A survey. IEEE Communications Surveys Tutorials, 11(3):125-142, rd 2009.

[338] Y. Mostofi, A. Gonzalez-Ruiz, A. Gaffarkhah, and D. Li. Characterization and modeling of wireless channels for networked robotic and control systems - a comprehensive overview. In 2009 IEEE/RSJ International Conference on Intelligent Robots and Systems, pages 4849-4854, Oct 2009.

[339] G. Tuna, K. Gulez, T. V. Mumcu, and V. C. Gungor. Mobile robot aided self-deploying wireless sensor networks for radiation leak detection. In 2012 5th International Conference on New Technologies, Mobility and Security (NTMS), pages 1-5, May 2012.

[340] Stelios Timotheou and Georgios Loukas. Autonomous networked robots for the establishment of wireless communication in uncertain emergency response scenarios. In SAC, 2009.

[341] X. Long and B. Sikdar. A real-time algorithm for long range signal strength prediction in wireless networks. In 2008 IEEE Wireless Communications and Networking Conference, pages 1120-1125, March 2008. 
[342] Károly Farkas, Theus Hossmann, Franck Legendre, Bernhard Plattner, and Sajal K. Das. Link quality prediction in mesh networks. Computer Communications, 31(8):1497 - 1512, 2008. Special Issue: Modeling, Testbeds, and Applications in Wireless Mesh Networks.

[343] Avraham Leff and James T Rayfield. Web-application development using the model/view/controller design pattern. In Enterprise Distributed Object Computing Conference, 2001. EDOC'01. Proceedings. Fifth IEEE International, pages 118-127. IEEE, 2001.

[344] Günter Niemeyer and J-JE Slotine. Stable adaptive teleoperation. IEEE Journal of oceanic engineering, 16(1):152-162, 1991.

[345] Amir Haddadi, Kamran Razi, and Keyvan Hashtrudi-Zaad. Operator dynamics consideration for less conservative coupled stability condition in bilateral teleoperation. IEEE/ASME Transactions on Mechatronics, 20(5):2463-2475, 2015.

[346] Atsushi Suzuki and Kouhei Ohnishi. Frequency-domain damping design for timedelayed bilateral teleoperation system based on modal space analysis. IEEE Transactions on Industrial Electronics, 60(1):177-190, 2013.

[347] Lila San Roque, Kobin H Kendrick, Elisabeth Norcliffe, Penelope Brown, Rebecca Defina, Mark Dingemanse, Tyko Dirksmeyer, N J Enfield, Simeon Floyd, Jeremy Hammond, Giovanni Rossi, Sylvia Tufvesson, Saskia Van Putten, and Asifa Majid. Vision verbs dominate in conversation across cultures, but the ranking of non-visual verbs varies. Cognitive Linguistics, 26(1):31-60, 2015.

[348] Horace Barlow. Vision Science: Photons to Phenomenology by Stephen E. Palmer. Trends in Cognitive Sciences, 4(4):164-165, 42000.

[349] Susan J Lederman and Roberta L Klatzky. Hand movements: A window into haptic object recognition. Cognitive Psychology, 19(3):342-368, 71987.

[350] Rogelio Lozano, Nikhil Chopra, and Mark Spong. Passivation Of Force Reflecting Bilateral Teleoperators With Time Varying Delay. In IN PROCEEDINGS OF THE 8. MECHATRONICS FORUM, pages 24-26, 2002.

[351] N. Chopra, M.W. Spong, S. Hirche, and M. Buss. Bilateral teleoperation over the internet: the time varying delay problem. In Proceedings of the 2003 American Control Conference, 2003., volume 1, pages 155-160. IEEE, 2003.

[352] Katerina Mania, Bernard D. Adelstein, Stephen R. Ellis, and Michael I. Hill. Perceptual sensitivity to head tracking latency in virtual environments with varying degrees of scene complexity. In Proceedings of the 1st Symposium on Applied perception in graphics and visualization - APGV '04, page 39, New York, New York, USA, 2004. ACM Press. 
[353] J. Corde Lane, C.R. Carignan, B.R. Sullivan, D.L. Akin, T. Hunt, and R. Cohen. Effects of time delay on telerobotic control of neutral buoyancy vehicles. In Proceedings 2002 IEEE International Conference on Robotics and Automation (Cat. No.02CH37292), volume 3, pages 2874-2879. IEEE, 2002.

[354] Richard Held, Aglaia Efstathiou, and Martha Greene. Adaptation to displaced and delayed visual feedback from the hand. Journal of Experimental Psychology, 72(6):887891, 1966.

[355] I. Scott MacKenzie and Colin Ware. Lag as a determinant of human performance in interactive systems. In Proceedings of the SIGCHI conference on Human factors in computing systems - CHI '93, pages 488-493, New York, New York, USA, 1993. ACM Press.

[356] Chao Dong, Chen Change Loy, Kaiming He, and Xiaoou Tang. Image super-resolution using deep convolutional networks. IEEE transactions on pattern analysis and machine intelligence, 38(2):295-307, 2016.

[357] Thomas Huang and Jianchao Yang. Image super-resolution: Historical overview and future challenges. In Super-resolution imaging, pages 19-52. CRC Press, 2010.

[358] Chih-Yuan Yang, Chao Ma, and Ming-Hsuan Yang. Single-image super-resolution: A benchmark. In European Conference on Computer Vision, pages 372-386. Springer, 2014.

[359] Jiwon Kim, Jung Kwon Lee, and Kyoung Mu Lee. Deeply-recursive convolutional network for image super-resolution. In Proceedings of the IEEE conference on computer vision and pattern recognition, pages 1637-1645, 2016.

[360] Jiwon Kim, Jung Kwon Lee, and Kyoung Mu Lee. Accurate image super-resolution using very deep convolutional networks. In Proceedings of the IEEE conference on computer vision and pattern recognition, pages 1646-1654, 2016.

[361] Xiaojiao Mao, Chunhua Shen, and Yu-Bin Yang. Image restoration using very deep convolutional encoder-decoder networks with symmetric skip connections. In Advances in neural information processing systems, pages 2802-2810, 2016.

[362] Kai Zhang, Wangmeng Zuo, Yunjin Chen, Deyu Meng, and Lei Zhang. Beyond a gaussian denoiser: Residual learning of deep cnn for image denoising. IEEE Transactions on Image Processing, 26(7):3142-3155, 2017.

[363] Wenzhe Shi, Jose Caballero, Ferenc Huszár, Johannes Totz, Andrew P Aitken, Rob Bishop, Daniel Rueckert, and Zehan Wang. Real-time single image and video superresolution using an efficient sub-pixel convolutional neural network. In Proceedings of the IEEE Conference on Computer Vision and Pattern Recognition, pages 1874-1883, 2016. 
[364] Stefan Schaal. Dynamic Movement Primitives -A Framework for Motor Control in Humans and Humanoid Robotics, pages 261-280. Springer Tokyo, Tokyo, 2006.

[365] H. Hoffmann, P. Pastor, D. Park, and S. Schaal. Biologically-inspired dynamical systems for movement generation: Automatic real-time goal adaptation and obstacle avoidance. In 2009 IEEE International Conference on Robotics and Automation, pages 2587-2592, 2009.

[366] A. J. Ijspeert, J. Nakanishi, H. Hoffmann, P. Pastor, and S. Schaal. Dynamical movement primitives: Learning attractor models for motor behaviors. Neural Computation, 25(2):328-373, Feb 2013.

[367] C. Lauretti, F. Cordella, E. Guglielmelli, and L. Zollo. Learning by demonstration for planning activities of daily living in rehabilitation and assistive robotics. IEEE Robotics and Automation Letters, 2(3):1375-1382, July 2017.

[368] Lorenzo Sciavicco and Bruno Siciliano. Modelling and control of robot manipulators. Springer Science \& Business Media, 2012.

[369] Orocos. Orocos (2017a) kdl wiki. [Online]. Available: http://www.orocos.org/kdl, 2017.

[370] Orocos. Orocos (2017b) the orocos project. [Online]. Available: http://www.orocos.org, 2017.

[371] Patrick Beeson and Barrett Ames. Trac-ik: An open-source library for improved solving of generic inverse kinematics. In Humanoid Robots (Humanoids), 2015 IEEERAS 15th International Conference on, pages 928-935. IEEE, 2015.

[372] Pramit Dutta, Naveen Rastogi, and Krishan Kumar Gotewal. Virtual reality applications in remote handling development for tokamaks in india. Fusion Engineering and Design, 118:73-80, 2017.

[373] Stanislav Pidhorskyi, Michael Morehead, Quinn Jones, George Spirou, and Gianfranco Doretto. syglass: Interactive exploration of multidimensional images using virtual reality head-mounted displays. arXiv preprint arXiv:1804.08197, 2018.

[374] Tomas Blazauskas, Rytis Maskeliunas, Reda Bartkute, Vitalija Kersiene, Indre Jurkeviciute, and Mindaugas Dubosas. Virtual reality in education: New ways to learn. Communications in Computer and Information Science Information and Software Technologies, page 457-465, 2017.

[375] Joseph Psotka. Immersive training systems: Virtual reality and education and training. Instructional science, 23(5-6):405-431, 1995. 
[376] Zahira Merchant, Ernest T Goetz, Lauren Cifuentes, Wendy Keeney-Kennicutt, and Trina J Davis. Effectiveness of virtual reality-based instruction on students' learning outcomes in k-12 and higher education: A meta-analysis. Computers 83 Education, 70:29-40, 2014.

[377] Abdul-Hadi G Abulrub, Alex N Attridge, and Mark A Williams. Virtual reality in engineering education: The future of creative learning. In Global Engineering Education Conference (EDUCON), 2011 IEEE, pages 751-757. IEEE, 2011.

[378] Rory McCloy and Robert Stone. Science, medicine, and the future: Virtual reality in surgery. BMJ: British Medical Journal, 323(7318):912, 2001.

[379] Neal E Seymour, Anthony G Gallagher, Sanziana A Roman, Michael K O'brien, Vipin K Bansal, Dana K Andersen, and Richard M Satava. Virtual reality training improves operating room performance: results of a randomized, double-blinded study. Annals of surgery, 236(4):458, 2002.

[380] J. M. Rosen, H. Soltanian, R. J. Redett, and D. R. Laub. Evolution of virtual reality [medicine]. IEEE Engineering in Medicine and Biology Magazine, 15(2):16-22, March 1996.

[381] Cuan M Harrington, Dara O Kavanagh, John F Quinlan, Donncha Ryan, Patrick Dicker, Dara O'Keeffe, Oscar Traynor, and Sean Tierney. Development and evaluation of a trauma decision-making simulator in oculus virtual reality. The American Journal of Surgery, 215(1):42-47, 2018.

[382] Naveen Rastogi, Amit Kumar Srivastava, Pramit Dutta, and Krishan Kumar Gotewal. Virtual reality based monitoring and control system for articulated in-vessel inspection arm. 2017 IEEE International Conference on Power, Control, Signals and Instrumentation Engineering (ICPCSI), 2017.

[383] Pablo Martinez-Gonzalez, Sergiu Oprea, Alberto Garcia-Garcia, Alvaro Jover-Alvarez, Sergio Orts-Escolano, and Jose Garcia-Rodriguez. Unrealrox: An extremely photorealistic virtual reality environment for robotics simulations and synthetic data generation. arXiv preprint arXiv:1810.06936, 2018.

[384] Mohamed Elobaid, Yue Hu, Jan Babic, and Daniele Pucci. Telexistence and teleoperation for walking humanoid robots. arXiv preprint arXiv:1809.01578, 2018.

[385] Tobias Rodehutskors, Max Schwarz, and Sven Behnke. Intuitive bimanual telemanipulation under communication restrictions by immersive $3 \mathrm{~d}$ visualization and motion tracking. In Humanoid Robots (Humanoids), 2015 IEEE-RAS 15th International Conference on, pages 276-283. IEEE, 2015.

[386] Darpa robotics challenge (drc) (archived). 
[387] Balazs Vagvolgyi, Wenlong Niu, Zihan Chen, Paul Wilkening, and Peter Kazanzides. Augmented virtuality for model-based teleoperation. 2017 IEEE/RSJ International Conference on Intelligent Robots and Systems (IROS), 2017.

[388] Durand R Begault and Leonard J Trejo. 3-d sound for virtual reality and multimedia. 2000.

[389] William A McNeely. Robotic graphics: a new approach to force feedback for virtual reality. In Virtual Reality Annual International Symposium, 1993., 1993 IEEE, pages 336-341. IEEE, 1993.

[390] Hironao Yamada and Takayoshi Muto. Development of a hydraulic tele-operated construction robot using virtual reality: New master-slave control method and an evaluation of a visual feedback system. International Journal of Fluid Power, 4(2):3542, 2003.

[391] Facebook technologies. Oculus rift | oculus. [Online]. Available: https://www.oculus.com/rift/\#oui-csl-rift-games=robo-recall, 2018.

[392] HTC. Vice | discover virtual reality beyond imagination. [Online]. Available: https://www.vive.com/eu/, 2018.

[393] Unity Technologies. Unity - manual: Tags. [Online]. Available: https://docs.unity3d.com/Manual/Tags.html, 2018.

[394] Unity Technologies. Unity - manual: Colliders. [Online]. Available: https://docs.unity3d.com/Manual/CollidersOverview.html, 2018.

[395] Unity Technologies. Unity - manual: Rigidbody. [Online]. Available: https://docs.unity3d.com/Manual/class-Rigidbody.html, 2018.

[396] Z. Nick. Fluka | knowledge transfer. [Online]. Available: https://kt.cern/technologies/fluka, 2018.

[397] Bill Triggs, Philip F McLauchlan, Richard I Hartley, and Andrew W Fitzgibbon. Bundle adjustment - a modern synthesis. In International workshop on vision algorithms, pages 298-372. Springer, 1999.

[398] Monica Carfagni, Rocco Furferi, Lapo Governi, Michaela Servi, Francesca Uccheddu, and Yary Volpe. On the performance of the intel sr300 depth camera: metrological and critical characterization. IEEE Sensors Journal, 17(14):4508-4519, 2017.

[399] Leonid Keselman, John Iselin Woodfill, Anders Grunnet-Jepsen, and Achintya Bhowmik. Intel realsense stereoscopic depth cameras. arXiv preprint arXiv:1705.05548, 2017. 
[400] Andrzej Dworak, Felix Ehm, Pierre Charrue, and Wojciech Sliwinski. The new cern controls middleware. In Journal of Physics: Conference Series, volume 396, page 012017. IOP Publishing, 2012.

[401] Andrzej Dworak, M Sobczak, Felix Ehm, Wojciech Sliwinski, and P Charrue. Middleware trends and market leaders 2011. In Conf. Proc., volume 111010, page FRBHMULT05, 2011.

[402] RW Assmann, M Magistris, O Aberle, M Mayer, F Ruggiero, JM Jiménez, S Calatroni, A Ferrari, G Bellodi, I Kurochkin, et al. The final collimation system for the lhc. Technical report, 2006.

[403] Fabienne CERN Marcastel. Cern's accelerator complex. Technical report, 2013.

[404] L Ducimetière, U Jansson, GH Schroder, Eugène B Vossenberg, Michael J Barnes, and GD Wait. Design of the injection kicker magnet system for cern's 14 tev proton collider lhc. In Pulsed Power Conference, 1995. Digest of Technical Papers., Tenth IEEE International, volume 2, pages 1406-1411. IEEE, 1995.

[405] A Kellerbauer, M Amoretti, AS Belov, G Bonomi, I Boscolo, RS Brusa, Matthias Büchner, VM Byakov, L Cabaret, C Canali, et al. Proposed antimatter gravity measurement with an antihydrogen beam. Nuclear Instruments and Methods in Physics Research Section B: Beam Interactions with Materials and Atoms, 266(3):351-356, 2008.

[406] M Di Castro, ML Baiguera Tambutti, M Ferre, R Losito, G Lunghi, and A Masi. itim: A robotic system for safety, measurements, inspection and maintenance in harsh environments. In 2018 IEEE International Symposium on Safety, Security, and Rescue Robotics (SSRR), pages 1-6. IEEE, 2018.

[407] Mario Di Castro, Alessandro Masi, Simone Gilardoni, Roberto Losito, Maria Laura Baiguera Tambutti, and Giacomo Lunghi. Jacow: Lhe train control system for autonomous inspections and measurements. 2018.

[408] Leander Grech, Gianluca Valentino, Mario Di Castro, and Carlos Veiga Almagro. Collision avoidance system for the rp survey and visual inspection train in the cern large hadron collider. In 2018 IEEE 14th International Conference on Automation Science and Engineering (CASE), pages 817-822. IEEE, 2018.

[409] Mario Di Castro, Alessandro Masi, Luca Rosario Buonocore, Manuel Ferre, Roberto Losito, Simone Gilardoni, and Giacomo Lunghi. Jacow: A dual arms robotic platform control for navigation, inspection and telemanipulation. 2018.

[410] I. Aljarrah, A. Al-Amareeen, A. Idries, and O. Al-Khaleel. Image mosaicing using binary edge detection. In Proceedings of the International Conference on Computing Technology and Information Management, pages 186-190, Dubai, UAE, 2014. Online; accessed January 2018. 
[411] G. Farneback. Two-frame motion estimation based on polynomial expansion. In 13th Scandinavian Conference on Image Analysis, pages 363-370, Halmstad, Sweden, 2003. 